\title{
Laboratory Analysis of Fluid Flow and Solute Transport Through a Variably Saturated Fracture Embedded in Porous Tuff
}

Manuscript Completed: November 1989

Date Published: February 1990

Prepared by

Y. Chuang, W. R. Haldeman, T. C. Rasmussen, D. D. Evans

T. J. Nicholson, NRC Project Manager

Department of Hydrology and Water Resources

University of Arizona

Tucson, AZ 85721

Prepared for

Division of Engineering

Office of Nuclear Regulatory Research

U.S. Nuclear Regulatory Commission

Washington, DC 20555

NRC FIN D1662 


\section{DISCLAIMER}

This report was prepared as an account of work sponsored by an agency of the United States Government. Neither the United States Government nor any agency thereof, nor any of their employees, make any warranty, express or implied, or assumes any legal liability or responsibility for the accuracy, completeness, or usefulness of any information, apparatus, product, or process disclosed, or represents that its use would not infringe privately owned rights. Reference herein to any specific commercial product, process, or service by trade name, trademark, manufacturer, or otherwise does not necessarily constitute or imply its endorsement, recommendation, or favoring by the United States Government or any agency thereof. The views and opinions of authors expressed herein do not necessarily state or reflect those of the United States Government or any agency thereof. 


\section{DISCLAIMER}

Portions of this document may be illegible in electronic image products. Images are produced from the best available original document. 
Evans, D.D., 1983, Unsaturated Flow and Transport Through Fractured Rock - Related to High-Level Waste Repositories, NUREG/CR-3206, 231 pp.

Schrauf, T.W. and D.D. Evans, 1984, Relationship Between the Gas Conductivity and Geometry of a Natural Fracture, NUREG/CR-3680, 131 pp.

Huang, C. and D.D. Evans, 1985, A 3-Dimensional Computer Model to Simulate Fluid Flow and Contaminant Transport Through a Rock Fracture System, NUREG/CR-4042, 109 pp.

Green, R.T. and D.D. Evans, 1987, Radionuclide Transport as Vapor Through Unsaturated Fractured Rock, NUREG-CR-4654, 163 pp.

Rasmussen, T.C. and D.D. Evans, 1987, Unsaturated Flow and Transport Through Fractured Rock - Related to High-Level Waste Repositories, NUREG/CR-4655, 474 pp.

Yeh, T.C.J., T.C. Rasmussen and D.D. Evans, 1988, Simulation of Liquid and Vapor Movement in Unsaturated Fractured Rock at the Apache Leap Tuff Site: Models and Strategies, NUREG/CR-5097, 73 pp.

Weber, D.S. and D.D. Evans, 1988, Stable Isotopes of Authigenic Minerals in Variably-Saturated Fractured Tuff, NUREG/CR-5255, 70 pp.

Rasmussen, T.C. and D.D. Evans, 1989, Fluid Flow and Solute Transport Modeling Through Three-Dimensional Networks of Variably Saturated Discrete Fractures, NUREG/CR-5239, 193 pp. 


\begin{abstract}
Laboratory techniques are developed that allow concurrent measurement of unsaturated matrix hydraulic conductivity and fracture transmissivity of fractured rock blocks. Two blocks of Apache Leap tuff containing natural fractures were removed from a site near Superior, Arizona, shaped into rectangular prisms, and instrumented in the laboratory. Porous ceramic plates provided solution to the top of the test blocks at regulated pressures. Infiltration tests were performed on both test blocks. Steady-state flow testing of the saturated first block allowed the determination of matrix hydraulic conductivity and fracture transmissivity. Fifteen centimeters of suction were applied to the top of the second block throughout an imbibition test. Analysis of infiltration into that block indicates that fracture flow at the low compressive stress applied during the test was minimal and matrix hydraulic conductivity at fifteen centimeters of suction was an order of magnitude less than the saturated matrix hydraulic conductivity of the first block.

Coated-wire ion-selective electrodes were used to monitor the breakthrough of aqueous chloride concentrations in a tuff test block with a natural fracture. Minute samples of tracer solution were collected with filter paper. Preliminary results indicate the technique worked well for studying transport behavior in fractured rock at near-saturated flow conditions. It also appears to be a promising technique for unsaturated conditions. Breakthrough curves in the fracture and matrix, and a spatial concentration distribution map of chloride concentrations within the fracture, suggest the existence of preferential flow paths in the fracture segment, and substantial diffusion into the matrix. Estimates of average travel velocity, dispersion coefficient and longitudinal dispersivity in the fracture, are obtained using temporal moments analysis. Estimates of transport parameters in the matrix are hampered by insufficient data and the complex solute concentration history. A case study of the test block using a boundary integral method computer model aided in the interpretation of the data.
\end{abstract}




\section{ACKNOWLEDGMENTS}

Professor Dan D. Evans was responsible for project management and direction. The hydraulic testing experiments were conducted by William $R$. Haldeman while the solute transport experiments were conducted by Yueh Chuang. Todd Rasmussen provided the Boundary Integral model simulations and also aided in the preparation of this manuscript. Priscilla J. Sheets was responsible for providing the excellent illustrations. Funding for this report was provided by the U.S. Nuclear Regulatory Commission, Office of Nuclear Regulatory Research, Division of Engineering under contract NRC-04-86-114, FIN D1662. The NRC project technical monitor was Mr. Thomas J. Nicholson. 
OTHER REPORTS IN THIS SERIES ................. 2

ABSTRACT . . . . . . . . . . . . . . . . . 3

ACKNOWLEDGMENTS . . . . . . . . . . . . . . 4

TABLE OF CONTENTS .................... . 5

LIST OF ILLUSTRATIONS . . . . . . . . . . . . . . 8

LIST OF TABLES . . . . . . . . . . . . . . . . . . . 13

EXECUTIVE SUMMARY . . . . . . . . . . . . . 15

1. INTRODUCTION ....................... . . 18

1.1 Description of Problem . . . . . . . . . . . 18

1.2 Research Objectives... . . . . . . . . . . . 19

1.3 Experimental Approach . . . . . . . . . . 20

2. PROCUREMENT AND PREPARATION OF TEST BLOCKS . . . . . . 25

2.1 Description of the Apache Leap Tuff Site . . . . . 25

2.2 Test Block 1 Procurement . . . . . . . . . . 27

2.3 Test Block 2 Procurement . . . . . . . . . 29

3. FLUID FLOW . . . . . . . . . . . . . . . . 35

3.1 Saturated Flow Through Porous Media and Fracture . . . 35

3.2 Unsaturated Flow Through Porous Media and Fracture . . 38

3.3 Coupled Matrix/Fracture Flow -

Boundary Integral Method .............. . 43

3.4 Preparation of Rocks . . . . . . . . . . . . 43

3.5 Experiment Setup and Equipment Testing,
Calibration and Procedures . . . . . . . . 49

Calibration and Procedures . . . . . . . . . 49

3.5.1 Porous Ceramic Plates . . . . . . . . . 51

3.5.2 Head Contro1 . . . . . . . . . . . 53

3.5.3 Flow Rate Measurement . . . . . . . . . . 54

3.5.4 Water Potential Measurement

in the Fracture and the Matrix . . . . . . . . . 54

3.5.5 Displacement Transducers ... . . . . . 56

3.6 Test Block 1 Experiments . . . . . . . . . . . 56

3.6.1 Fracture Imbibition Tests . . . . . . . . . . . . 56

3.6.2 Three-Plate Imbibition Tests ... . . . . . . 58

3.6.3 Steady-State Flow Test . . . . . . . . 58

3.7 Test Block 2 Experiments . . . . . . . . . . . . . 59

3.8 Rock Characterization Tests .............. . 60

3.8.1 Matrix Saturated Hydraulic Conductivity . . . 60

3.8.2 Matrix Moisture Release Curves..... . . . . 60

3.8.3 Matrix Dry Bulk Density and Effective Porosity . . 61

3.8.4 Matrix Pore Size Distributions . . . . . . . . 61

3.9 Results and Discussion . . . . . . . . . 61

3.9.1 Equipment Calibration Results . . . . . . 62

3.9.2 Test Block 1 Experiments . . . . . . . 66

3.9.2.1 Fracture Imbibition Tests........ 66

3.9.2.2 Three-Plate Imbibition Tests . . . . . 67

3.9.2.3 Steady-State Flow Test . . . . . . 77 
TABLE OF CONTENTS (continued)

Page

3.9.3 Test Block 2 Experiments . . . . . . . . . . . . 84

3.9.4 Rock Characterization Tests . . . . . . . . . . . 88

3.9.4.1 Matrix Saturated Hydraulic

Conductivity . . . . . . . . . . . . . 88

3.9.4.2 Matrix Moisture Release Curves . . . . . 91

3.9.4.3 Matrix Dry Bulk Density

and Effective Porosity ........ . 91

3.9.4.4 Matrix Pore Size Distributions . . . . . 96

4. SOLUTE TRANSPORT . . . . . . . . . . . . . . . . . 97

4.1 Ion-Selective Electrodes . . . . . . . . . . . . 97

4.1.1 Background . . . . . . . . . . . . . . . . . . . . 98

4.1.2 Application to Filter Paper Environment . . . . 102

4.2 Transport Processes . . . . . . . . . . . . . 105

4.2.1 Major Mechanisms . . . . . . . . . . . . . 106

4.2.2 Solute-Medium Interaction . . . . . . . . . 109

4.2.3 Selection of Tracer . . . . . . . . . . . . . . 111

4.2.4 Effects on Breakthrough Curves . . . . . . . . . . 111

4.2.5 Estimation of Transport Parameters . . . . . . . . 112

4.2.6 Analytical Solution . . . . . . . . . . . . . 115

4.3 Coupled Matrix/Fracture Transport -

Boundary Integral Method . . . . . . . . . . . . 116

4.4 Water Penetration into Test Block Sampling Ports . . . . 117

4.5 Solute Transport Measurement . . . . . . . . . . . . . . 119

4.5.1 Preparation of Test Solutions, and Calibration of Electrodes . . . . . . . . . . 119

4.5.2 Sample Collection and Electrode Measurements . . 120

4.5.3 Chloride Breakthrough in the Porous Plates . . . . 125

4.6 Experimental and Computer Model Results . . . . . . . . . 125

4.6.1 Computer Model Case Study of Test Block 1 . . . . 127

4.6.2 Solute Transport in the Fracture . . . . . . . . . 136

4.6.3 Solute Transport in the Matrix . . . . . . . . . 152

5. CONCLUSIONS AND RECOMMENDATIONS FOR FUTURE STUDIES . . . . . 156

APPENDIX A: PREPARATION, CALIBRATION AND EXPERIMENTAL

PROCEDURES . . . . . . . . . . . . . . 165

Procedure 1: Preparation of Test Block for

Experimentation ........... . 166

Procedure 2: Preparation of Calcium Chloride Test

Solutions . . . . . . . . . . . . . . . 171

Procedure 3: Porous Plate Conductance Measurement . . . . 173

Procedure 4: Flow Measurement and Head Control . . . . . . 175

Procedure 5: Pressure Transducer Calibration . . . . . . . 177

Procedure 6: Microtensiometer Construction, Assembly

and Use . . . . . . . . . . . . . . . . . 179

Procedure 7: LVDT Calibration . . . . . . . . . . 183 


\section{TABLE OF CONTENTS (continued)}

$\underline{\text { Page }}$

Procedure 8: Calibration of Chloride Ion-Selective

Electrode . . . . . . . . . . . . 185

Procedure 9: Measurement of Potential Differences and

Sample Collection Using Filter Paper . . . . 190

Procedure 10: Determination of Effect of Suction on

Electrode Potential Measurements . . . . . . 192

Procedure 11: Determination of Chloride Breakthrough in

Porous Plates . . . . . . . . . . . . 194

APPENDIX B: FLUID FLOW CALIBRATION AND TEST RESULTS . . . . . 196

APPENDIX C: ANALYSIS OF WATER PENETRATION INTO CAVITIES . . . 258

APPENDIX D: CHLORIDE BREAKTHROUGH DATA OF POROUS PLATES . . . 261

APPENDIX E: SOLUTE TRANSPORT TEST DATA . . . . . . . . . 276

SELECTED REFERENCES . . . . . . . . . . . . . . . 325 


\section{LIST OF ILLUSTRATIONS}

Figure

1.1 Schematic diagram of the Apache Leap tuff site relative to the town of Superior, Arizona. Letters denote study locations. $A=$ Queen Creek road tunnel; $B=$ watershed study site; $\mathrm{C}=$ plateau site . . . . . . . . . . . 21

1.2 Test block number 1 experimental setup . . . . . . . . 22

1.3 Test block number 2 experimental setup. Frame $A$ is held by hooks from frame B................. 23

2.1 Physical characteristics of the Apache Leap tuff . . . . 24

2.2 Test block number 1 , prior to shaping, undergoing preliminary fracture conductivity test . . . . . . . . . 28

2.3 Test block number 1 , after being shaped, post single-plate infiltration test. . . . . . . . . . . . . . . . 30

2.4 Field exposure of partially welded tuff from which test block 2 was cut. Lens cap is $5.3 \mathrm{~cm}$ in diameter. The vertical trace to the left of the lens cap is the test fracture.................. . 31

2.5 Densely welded tuff block found along former route of U.S. 60. Test fracture runs diagonally from the upper right to the lower left of the block . . . . . . . . . . 32

3.1 Schematic of test block containing a single vertical

fracture. Coordinate axes used in text are shown at the rear of the block. Also shown is the aperture, e . 36

3.2 Water held in a film over particle surfaces and in capillary wedges between particles in unsaturated geologic media . . . . . . . . . . . . . . 40

3.3 Test block number 1 numbering system. Fracture intersects faces $1,2,3$, and 6 ................. . 45

3.4 Test block 1 sampling port locations . . . . . . . . . . . 46

3.5 Test block number 2 fracture sampling ports, face 4 . . . . 47

3.6 Test block number 2 matrix sampling ports, face $6 . . . . .48$

3.7 Test solution delivery system . . . . . . . . . . 50 
3.8 Porous ceramic plate. Length and thickness of all plates equals $20.2 \mathrm{~cm}$ and $0.7 \mathrm{~cm}$, respectively. Width of matrix plates equals $8.6 \mathrm{~cm}$, and width of plate covering fracture equals $3.0 \mathrm{~cm} \ldots \ldots . . . . . . .552$

3.9 Results of a conductance test performed on porous ceramic plate number 2. Head refers to total head drop across the plate......................... 63

3.10 Calibration results of LVDT number 2 . . . . . . 65

3.11 Monitoring of fracture aperture change in test block number 1 . . . . . . . . . . . . . . 69

3.12 Inflow to plate position $1-\mathrm{A}$, test block number $1 . . . .70$

3.13 Average pressure head beneath the plate located over position 1-A, test block number 1 . . . . . . . . 71

3.14 Inflow to plate position $1-B$, test block number 1 . . . 72

3.15 Average pressure head beneath the plate located over position 1-B, test block number $1 \ldots . . . . . .473$

3.16 Inflow to plate position $1-\mathrm{C}$, test block number $1 \ldots 74$

3.17 Average pressure head beneath the plate located over position 1-C, test block number 1......... . 75

3.18 For the boundary integral method computer model, the test block is divided into four domains: fracture, matrix, fracture plate and matrix plate. Except near the fracture opening, the streamlines are virtually vertical. The "zero-percent" or left most streamline should correspond to the left boundary of the matrix . . . . . . . . 81

3.19 Pertinent flow lines showing the fracture-matrix interaction near the top fracture opening. The streamlines are denoted as percentages of total flow $\left(Q_{t}\right)$ of the system. All the fracture plate flow enters the fracture within the top $3 \mathrm{~cm}$. However, nearly 19 percent of the matrix plate flow $\left(Q_{m p}\right)$ is also diverted to the fracture

3.20 Monitoring of fracture aperture change in test block number 2

3.21 Wetting front advancement during test block number 2 imbibition test. Numbers indicate days from beginning of test.......... 


\section{IIST OF ILLUSTRATIONS (continued)}

Figure

3.22 Test block number 2 imbibition test summary . . . . . . . . 87

3.23 Philip's infiltration analysis, test block number 2 . . . 89

3.24 Moisture release curves for cores obtained from rocks surrounding test block number 1. . . . . . . . . . 92

3.25 Moisture release curves for cores obtained from rocks surrounding test block number 1. . . . . . . . . . . . 93

3.26 Moisture release curves for cores obtained from rocks surrounding test block number 2... . . . . . . . . . 94

3.27 Moisture release curves for cores obtained from rocks surrounding test block number 2 . . . . . . . . . . . . 95

4.1 Minielectrodes used for experimentation: (A) chloride ion-selective electrode coated with silver chloride (AgC1), and (B) double-junction silver/silver chloride (Ag/AgCl) reference electrode . . . . . . . . . . .

4.2 Typical calibration curves for chloride electrode plotting potential difference (mV) versus $10 \mathrm{~g}$ chloride concentration (M) in an "aqueous" environment and a "filter paper" environment, with and without ionic strength buffer.................... . 101

4.3 Effects of different solute transport mechanisms on the shape of the breakthrough curve for a step input test plotting relative concentration, $C^{*}=\left(C-C_{1}\right) /\left(C_{0}-C_{1}\right)$, versus time elapsed $(\mathrm{hr}) . \mathrm{C}_{1}$ denotes the background solution concentration, and $\mathrm{C}_{0}$ the tracer solution concentration . . . . . . . . . . . . . . . . . . . . 113

4.4 Experimental apparatus used to assess the effects of suction on the reliability of the filter paper/chloride ion-selective electrode technique . . . . . . . . . . 122

4.5 Concentration $(\underline{M})$, as measured off filter paper, versus suction (cm of water) for a $1.40 \times 10^{-3} \mathrm{M}$ calcium chloride solution. The dashed lines indicate the full range of experimental error expected of \pm 20 percent . . . . . . . 123

4.6 Concentration $(\underline{M})$, as measured off filter paper, versus suction ( $\mathrm{cm}$ of water) for a $0.100 \mathrm{M}$ calcium chloride solution. The dashed lines indicate the full range of experimental error expected of \pm 20 percent . . . . . . . 124 


\section{LIST OF ILLUSTRATIONS (continued)}

Figure

4.7 Experimental setup used to measure chloride breakthrough in the porous plates. The plates are divided into four quadrants to determine possible spatial differences . . 126

4.8 Profile of fracture flow, $Q_{1}$ (fraction of total flow), as a function of longitudinal distance along fracture, $z$ (cm)

4.9 Profile of relative concentration, $\mathrm{C}^{*}$, as a function of longitudinal distance along the fracture, $z(\mathrm{~cm})$, with an instantaneous increase through the fracture plate. The time increments are $t_{1}=0.238 \mathrm{hrs}, t_{2}=1.302 \mathrm{hrs}$, $t_{3}=3.910 \mathrm{hrs}, t_{4}=12.70 \mathrm{hrs}$, and $t_{5} \geq 14.81 \mathrm{hrs} . .133$

4.10 Profile of relative concentration, $C^{*}$, as a function of longitudinal distance along the fracture, $z(\mathrm{~cm})$, taking into account the chloride breakthrough characteristic of the fracture plate. The time increments are $t_{1}=0.238 \mathrm{hrs}$, $t_{2}=1.302 \mathrm{hrs}, t_{3}=3.910 \mathrm{hrs}, t_{4}=12.70 \mathrm{hrs}$, and $t_{5} \geq 14.81$ hrs ................... . 134

4.11 Sampling ports, and sampling locations at the bottom fracture opening. The fracture extends from face 3 to face 6 . . . . . . . . . . . . . . . . 138

4.12 A complex solute concentration history in the test block is expected to result from introduction of calcium chloride solutions of different concentrations . . . . . . . . . 140

4.13 Breakthrough curves plotting the average relative concentration, $C^{*}$, versus time elapsed $(h r)$ for fracture transport test no. 1 at three distances along the fracture segment, $z=10 \mathrm{~cm}, z=35 \mathrm{~cm}$ and $z=50 \mathrm{~cm} . . . . .141$

4.14 Breakthrough curves plotting the average relative concentration, $C^{*}$, versus time elapsed (hr) for fracture transport test no. 2 at three distances along the fracture segment, $z=10 \mathrm{~cm}, z=35 \mathrm{~cm}$ and $z=50 \mathrm{~cm}$. . . . . 142

4.15 Breakthrough curves plotting the average relative concentration, $G^{*}$, versus time elapsed $(h r)$ for fracture transport test no. 3 at three distances along the fracture segment, $z=10 \mathrm{~cm}, z=35 \mathrm{~cm}$ and $z=50 \mathrm{~cm} . . . . . .143$

4.16 Breakthrough curves plotting the average relative concentration, $C^{*}$, versus time elapsed ( $h r$ ) for the first 24 hours of fracture transport test no. 2 at three distances along the fracture segment, $z=10 \mathrm{~cm}, z=35$ and $z=50 \mathrm{~cm}$. . . . . . . . . . . . . . . . . 144 
4.17 Breakthrough curves plotting the average relative concentration, $\mathrm{C}^{*}$, versus time elapsed $(\mathrm{hr})$ for fracture transport test no. 1 at $z=10 \mathrm{~cm}$ showing the full range of $C^{*}$ values for individual measurements . . . . . . . . 145

4.18 Spatial distribution map of relative concentrations of solution remaining on fracture surface immediately after fracture test no. 3. Two preferential flow paths are likely to exist with the dominant one closer to face 6 . The fracture sampling areas are denoted with circles . . 148

4.19 Breakthrough curves plotting the average relative concentration, $\mathrm{C}^{*}$, versus time elapsed (hr) for matrix transport test at two distances in the matrix, $z=5 \mathrm{~cm}$ and $z=30 \mathrm{~cm}$. . . . . . . . . . . . . . 153 
3.1 Plate Conductance Results ... . . . . . . ... 62

3.2 LVDT Calibration Summary . . . . . . . 66

3.3 Gamma Beam Attenuation Results, Post Fracture Test . . . 67

3.4 Gamma Beam Attenuation Results, Prior to Whole-Rock Test 68

3.5 Test Block Number 1 Water Potential Measurements . . . 78

3.6 Saturated Matrix Conductivity and Fracture Transmissivity, Test Block Number 1 . . . . . . 79

3.7 Mode1 Input Summary . . . . . . . . . . . . 80

3.8 Philip's Parameters, Test Block Number 2 . . . . . . . 88

3.9 Matrix Saturated Hydraulic Conductivity . . . . . . . . 90

3.10 Matrix Dry Bulk Density and Effective Porosity . . . . . 91

4.1 Results of sample critical seepage velocity calculations using hydraulic conductivity values of borehole tuff core samples found at Superior, Arizona . . . . . . . . . 118

4.2 Case study of idealized test block system: a comparison of computer model input parameters and experimental data . . 128

4.3 A comparison of flow and pressure head data obtained from using the computer model, and those measured or calculated from experimental data . . . . . . . . . . . . . . 129

4.4 Travel time calculations of selected streamlines contributing to fracture flow . . . . . . . . . . . 132

4.5 Relative concentrations in the fracture as functions of time and distance along the fracture . . . . . . . . 135

4.6 Pertinent information and test conditions of fracture transport tests... . . . . . . . . . . . 137

4.7 First and second temporal moments data using average relative concentration values for fracture transport test nos. 1 and 2 . . . . . . . . . . . . . . . . . . . . . . 146

4.8 First and second temporal moments data using average relative concentration values for fracture transport test no. 3 and the first twenty four hours of test no. 2 . . 147 
$\underline{\text { Table }}$

4.9 Estimates of average travel velocity, dispersion coefficient and longitudinal dispersivity using temporal moments analysis for fracture transport tests . . . . . . 149

4.10 Pertinent information and test conditions of matrix transport test . . . . . . . . . . . . . . 152

4.11 First and second temporal moments data using average relative concentration values for matrix transport test . 154

4.12 Estimates of average travel velocity using temporal moments analysis for matrix transport test . . . . . . 154

5.1 Summary of Test Blocks 1 and 2 . . . . . . . . . . . 157 


\section{EXECUTIVE SUMMARY}

\section{Introduction}

The hydraulic properties of partially saturated fractures are poorly understood even though the performance of a high level waste repository is critically dependent on whether water and radioactive solutes will flow through fractures under unsaturated conditions. Flow through fractures may result in accelerated velocities and reduced travel times from the repository to the accessible environment, thus breaching the geologic barrier which will be relied upon to isolate the nuclear waste. To understand and describe the processes that control flow and transport in unsaturated fractured rock, a series of experiments have been performed to quantify the relevant hydraulic and transport parameters. This document reports laboratory hydraulic and tracer tests conducted on natural fractures in rocks removed from a field site in unsaturated fractured tuff at the Apache Leap tuff site in central Arizona. The tests were conducted for the purpose of characterizing fluid flow and solute transport under conditions of negative matric potentials.

\section{Description of Fractured Rock Blocks}

Two blocks of Apache Leap tuff containing natural fractures were removed from the field site and shaped into regular solids measuring approximately $(0.2 \times 0.2 \times 0.5 \mathrm{~m})$ and $(0.2 \times 0.2 \times 0.7 \mathrm{~m})$. Rock bolts and exterior braces were used to stabilize the fracture in the field prior to shipment and shaping. The single fracture in each block traversed the solid along the longer dimension. The volumetric porosity of the rock blocks is approximately 17 percent and the bulk density is approximately $2100 \mathrm{~kg} / \mathrm{m}^{3}$. The fractured rock blocks were mounted in a support frame and enclosed within a chamber to prevent evaporation during the experiments. Ceramic porous plates were constructed and placed on the upper surface of the rock, with the center plate laying over the single vertical fracture.

\section{Hydraulic Tests}

The rate of fluid movement within a fractured rock parallel to the direction of the fracture depends upon the hydraulic conductivity of the rock matrix as well as on the transmissivity of the fracture. Experiments were performed to determine the matrix and fracture hydraulic properties by maintaining a positive fluid pressure in the porous plates above both the fracture and the matrix. The specific flux through the plate overlying the fracture was consistently larger than the flux through the plates overlying the matrix. Estimates of matrix and fracture hydraulic properties were obtained using a Boundary Integral numerical model which simulated flow through the plate-fracture-matrix flow domain. For the first rock block the hydraulic conductivity was estimated to be approximately $5.9 \times 10^{-8} \mathrm{~m} / \mathrm{s}$ and the fracture transmissivity was $7.2 \times 10^{-9} \mathrm{~m}^{2} / \mathrm{s}$.

A second series of experiments were performed using the second rock block by maintaining a fluid suction of $15 \mathrm{~cm}(1.5 \mathrm{kPa})$ on the upper surface of the ceramic plates. The hydraulic conductivity of the rock 
matrix was estimated to be $5.5 \times 10^{-9} \mathrm{~m} / \mathrm{s}$, which is approximately an order of magnitude lower than the first block. No apparent fracture flow was observed at the imposed suction of $15 \mathrm{~cm}$. This indicates that the fracture may not contribute to fluid flow at suctions greater than $15 \mathrm{~cm}$. Further tests over a wider range of fluid suctions are recommended.

\section{Tracer Tests}

Coated-wire ion-selective electrodes were used to monitor the breakthrough of chloride in the fracture and rock matrix. Measurements were made off filter paper used to collect small amounts of tracer solution prepared from calcium chloride. Independent tests using a porus cup in conjunction with a water column apparatus showed the filter paper/ion-selective electrode technique is capable of measuring concentration changes for unsaturated conditions up to a suction of $100 \mathrm{~cm}$ of water.

Ceramic porous plates were used to introduce test solutions to the upper surface. Solute transport parameters were estimated from results of three fracture transport tests and one matrix transport test using temporal moments analysis. Large variations in the results were observed for the same fracture segment among individual experiments. The average travel velocity in the fracture ranged from $2.12 \times 10^{-5} \mathrm{~m} / \mathrm{s}$ to $51.9 \times 10^{-5} \mathrm{~m} / \mathrm{s}$, the dispersion coefficient from $1.01 \times 10^{-6} \mathrm{~m}^{2} \mathrm{~m} / \mathrm{s}$ to $4.2 \times 10^{-3} \mathrm{~m}^{2} / \mathrm{s}$, and the longitudinal dispersivity from $2.07 \times 10^{-2} \mathrm{~m}$ to $8.01 \mathrm{~m}$. A spatial concentration distribution map of the fracture was also obtained at the end of the third fracture transport test. The map strongly suggests the existence of preferential flow paths under saturated conditions.

Parameter estimation in the matrix was hampered by insufficient data, and the complex two-dimensional solute concentration history in the test block. Monitoring of chloride breakthrough in the matrix sampling ports clearly indicated the influence of the fracture through matrix diffusion. The average travel velocity in the matrix ranged from $1.13 \mathrm{x}$ $10^{-7} \mathrm{~m} / \mathrm{s}$ to $2.86 \times 10^{-7} \mathrm{~m} / \mathrm{s}$. The dispersion coefficient and the longitudinal dispersivity in the matrix could not be calculated due to insufficient data.

\section{Computer Simulations}

Interpretation of flow and solute concentration data in the fracture was aided by the use of a Boundary Integral numerical model. The model showed fracture-matrix flow interaction, especially near the top fracture opening. Implications of the model results include:

- Approximately 20 percent of the flow through the matrix porous plates entered the fracture before reaching the bottom of the test block;

- A steady-state relative concentration of less than unity in the fracture is expected if the test solution is applied only to the plate directly over the fracture; and

- A constant solute concentration in the fracture for the bottom 30 to $40 \mathrm{~cm}$ of the fracture is expected if matrix diffusion is neglected. 


\section{Conclusions}

Procedures and instrumentation for estimating the hydraulic and solute transport properties of unsaturated fractured rock have been developed. Specific conclusions include:

- The filter paper/ion-selective electrode technique is a viable method for studying solute transport behavior under near-saturated conditions.

- Using the electrode technique, large variations in solute transport parameter estimates are observed for the same fracture segment. The variations probably result from fracture and matrix heterogeneities, differences in flow and pressure conditions, and solute concentration histories.

o Channeling of flow in the fracture is observed even for a relatively small fracture segment.

- Matrix diffusion effects are clearly observed for large concentration gradients during fracture transport tests. Matrix diffusion is expected to retard and to spread solute breakthrough curves.

- Fracture-matrix interactions are expected near the top fracture opening due to streamlines which originate in the rock matrix and transfer to the fracture.

- Water does not enter sampling ports evan at near-saturated flow conditions. The ports are expected to interfere with matrix flow, and to influence average matrix travel velocity calculations.

\section{$\underline{\text { Recommendations }}$}

To further extend existing procedures and instrumentation, the following recommendations are made:

- The acquisition of a superior $\mathrm{pH} / \mathrm{mV}$ meter with greater resolution and accuracy. With improved resolution will come more accurate chloride calibration curves for the ion-selective electrode.

- Experiments should be conducted to evaluate the accuracy of the filter paper/ion-selective electrode technique at suctions higher than $100 \mathrm{~cm}$.

- Additional fracture and matrix transport tests to monitor the solute concentration and suction in the rock matrix at various locations to determine the spatial variability of these variables. 


\section{CHAPTER ONE}

\section{INTRODUCTION}

The characterization of a proposed high-level radioactive waste repository site involves evaluating techniques to assess both the water flow and radionuclide transport properties of the surrounding geologic media. The U.S. Environmental Protection Agency has mandated that radionuclides must not exceed threshold concentration levels in the accessible environment within ten thousand years of disposal. The candidate repository horizon, which is located unsaturated fractured rock, should inhibit the outward movement of radionuclides in the event of leakage from the engineered barriers. In an unsaturated fractured rock setting, of particular interest are unsaturated fracture hydraulic transmissivity and permeability, and rock matrix unsaturated hydraulic conductivity and permeability. Also of interest are the transport properties of radionuclides in terms of time of travel, retardation and dispersion. Flow and transport as affected by the interaction between the fracture and adjacent rock matrix is an additional consideration. Because the repository may be located above the water table the hydraulic and transport properties, as well as the fracture-matrix interaction, require study as functions of water content, or negative water potential or suction.

\subsection{Description of Problem}

The hydraulic conductivity of a rock body is greatest when the body is saturated with water, that is, the rock pores are as full as is naturally possible with water. According to capillary theory, as a rock mass desaturates, the largest pores drain first. As more and more water is drained from the mass, smaller and smaller pores drain. The drainage of rock pores results, in theory and in practice, in a decreasing hydraulic conductivity. Depending on the pore size distribution, the drop off in hydraulic conductivity with decreasing water content can be several orders of magnitude.

Fractures in a rock body behave in a similar manner. Saturated fractures have a higher hydraulic conductivity than do unsaturated fractures. However, as the water potential is lessened, fractures drain according to the distribution of their aperture widths, and the hydraulic conductivity decreases accordingly. Under saturated conditions, fractures often act as flow conduits since their apertures are often considerably larger than the surrounding matrix pores. A fracture or fracture system that acts as a conduit under saturated conditions, however, may act as a barrier when the fractured rock mass is subjected to negative water potentials, or suction. The degree to which unsaturated fractures in a rock mass affect water flow through the media depends upon how the hydraulic conductivity of the fracture varies with suction or water content. Since groundwater movement is a possible mode of contaminant transport, a decreased hydraulic conductivity of fractured rock results in decreased flow and thus decreased contaminant transport. 
However, it is not enough to know in general terms that a given repository location will be subject to reduced groundwater flow and radionuclide transport rates due to its location in the vadose zone. One must characterize flow and transport around the repository for a variety of possible conditions, including those present at the site prior to waste emplacement. This assessment is currently feasible for saturated fractured geologic media. Three possible approaches may be used to analyze such media. The first is the deterministic continuum approach where the fractured rock media is viewed as a continuous porous media with properties that represent averages obtained in a representative elementary volume (de Marsily, 1986). The second possible approach is the deterministic non-continuum method. This approach involves individual analysis of each fracture in the region of interest. A stochastic representation of the fractured media is the third approach and involves obtaining statistical fracture parameters from a limited number of samples. These parameters are then used to simulate various scenarios.

Unfractured rock matrix may be studied using a stochastic approach by obtaining numerous field samples from the proposed waste disposal site. Angled boreholes and oriented rock cores can provide samples for laboratory analysis (Rasmussen and Evans, 1987) from which three-dimensional parameter distributions may be developed. Results from such a procedure may be put into a flow and transport model, yielding field scale and regional scale predictions for various conditions.

The addition of fractures to unsaturated rock mass complicates analysis, and currently no methods are available for water flow or transport characterization. If one could obtain intact samples with undisturbed rock fractures and develop techniques to measure unsaturated fracture parameters, a stochastic analysis could also be performed with the sample fractures. The fracture parameters thus obtained could then be linked to the matrix parameters through a computer model. Important fracture parameters and relationships that need to be developed include the variation of hydraulic conductivity with changing suction and the relationship between suction and water content. Solute transport properties such as time of travel, retardation and dispersion are also required.

Another possible fractured rock characterization method would involve the development of a field test similar to aquifer testing methods that are used to investigate saturated fractured rock. Such a continuum approach might involve the use of a membrane impeding layer placed inside of a borehole and held in place by a frame, allowing the imposition of a negative water potential on the rock matrix/fracture system surrounding the borehole.

\subsection{Research Objectives}

The purpose of this research is to develop techniques and procedures to study flow and transport behaviors in natural fractures at variably saturated conditions. This study adopts the stochastic approach to characterizing rock fractures. The objectives of the fluid flow part of the investigation are: (1) to develop procedures to determine the fracture transmissivity and the adjacent matrix hydraulic conductivity of blocks of rock in the laboratory, (2) to perform infiltration and percolation tests on each block being studied, and (3) to characterize, in 
the laboratory, rock cores obtained from rock surrounding the test blocks. The objectives of the transport part of the investigation are: (1) to test filter paper as a possible means of sample collection, (2) to assess the capability of coated-wire ion-selective electrodes by monitoring breakthrough of chloride tracer solutions collected using filter paper at near-saturated conditions, (3) to test the reliability of the filter paper/ion-selective electrode technique at higher suctions using a separate apparatus.

\subsection{Experimental Approach}

This research adopts the approach of studying blocks of rock brought to the laboratory. Previous experience in the laboratory and field indicates the need to perform experiments under controlled conditions. Fluid flow appears to be based on past wetting and drying history. The flow path within the fracture is expected to be tortuous. The nature of solute transport is understandably expected to be complicated as well. Because of the complexity of fracture flow and transport, a better understanding of the hydraulic and transport properties, and experimental techniques, can be achieved in the laboratory before pursuing in situ assessments.

Generally, the approach included location, removal, and shaping of two blocks of partially welded tuff, each containing one test fracture, from the plateau site at the Apache Leap tuff site near Superior, Arizona (see Figure 1.1 for location). The first of the two blocks used for this study was chosen both for its convenient size and its ease of removal. The test block has a dominant fracture and several minor fractures. The main fracture is not considered ideal because of its highly variable aperture. It also curves at approximately 30 degrees from verical four-fifths of the way along the fracture. The first test block was used primarily to develop testing methods while subsequent test blocks were being located. Transport experiments were performed for the first test block only. At a later date, the second test block was chosen and cut, along with two other blocks, from a larger sample.

For fluid flow experiments, the intent has been to develop procedures that would be applicable over a range of suctions and would allow concurrent measurement of fracture transmissivity and matrix hydraulic conductivity. However, steady-state flow tests were performed at nearly saturated conditions to allow relatively rapid development and testing of procedures and equipment. Infiltration and percolation tests were conducted by applying water to the top face of each block through porous ceramic plates. Monitoring of the water intake rates and wetting front advancement was performed throughout the imbibition tests. Various plate configurations, and thus water source locations, were used during these tests. Characterization of rock cores adjacent to the test blocks allows for comparison of the test block matrix with other rock cores recovered from the Apache Leap tuff site. The saturated hydraulic conductivity, dry bulk density, effective porosity, and pore size distribution were determined for each unfractured core. Additionally, matrix moisture release curves were prepared. 


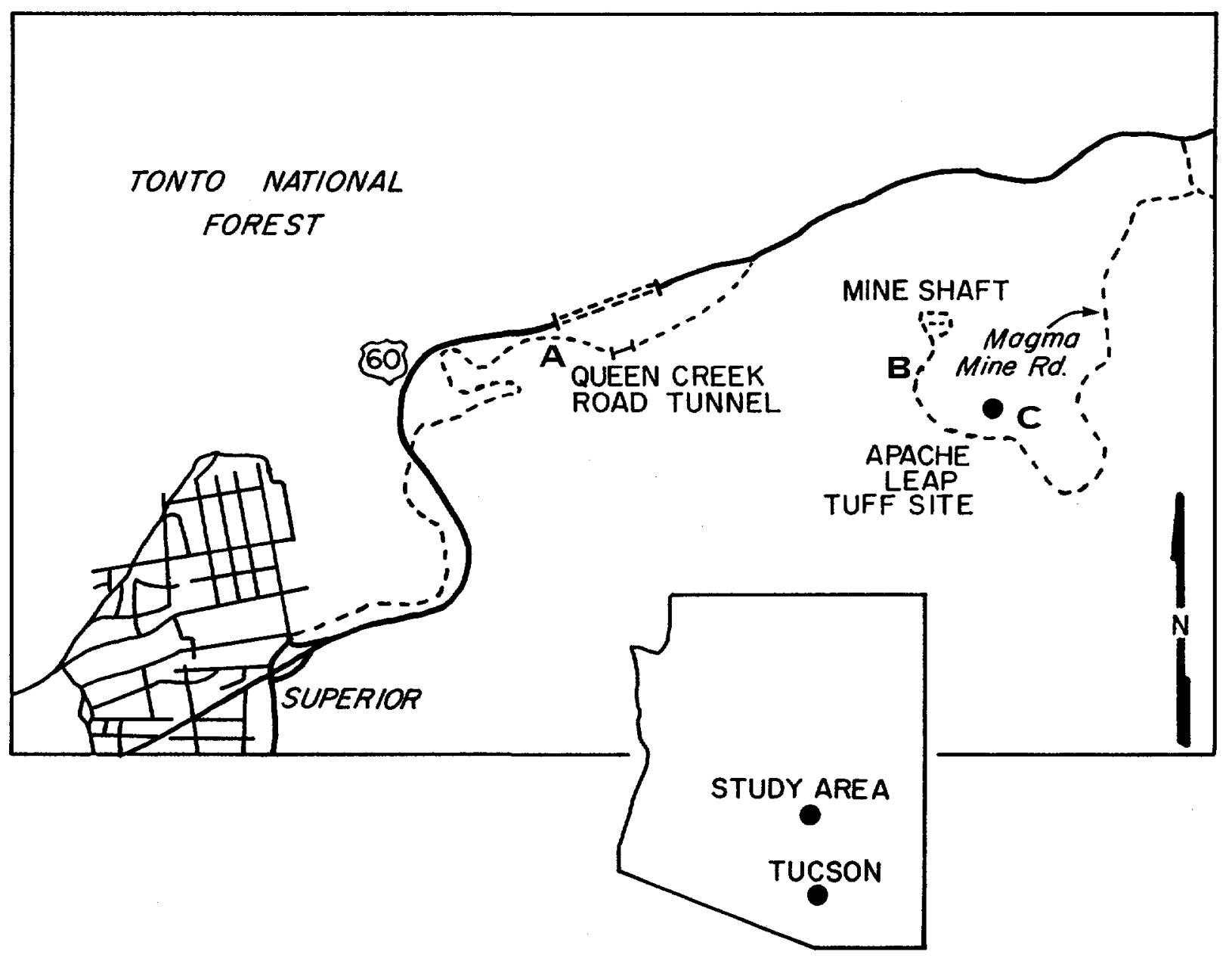

Figure 1.1 Schematic diagram of the Apache Leap tuff site relative to the town of Superior, Arizona. Letters denote study locations. $A=$ Queen Creek road tunnel; $B=$ watershed study site; $C=$ plateau site. 
The two rectangular test blocks (see Figures 1.2 and 1.3 for experimental setups), less than $0.1 \mathrm{~m}^{3}$ in size, were set up in the laboratory such that the test fracture lay in the vertical plane and were instrumented with custom-made porous ceramic plates, linear variable displacement transformers (displacement transducers or LVDTs), and a microtensiometer. Experiments were conducted by suspending the test blocks from metal frames. Evaporation was minimized with a plastic canopy. To measure water potential and solute transport in the fractures and rock matrix, sampling ports were installed. Sampling ports extending to the fracture plane, as well as ones which end in the rock matrix, were drilled for both test blocks.

The ceramic porous plates, with air entry values of $200 \mathrm{kPa}$, were used to introduce background and tracer solutions. Only the top rock surface was covered with plates for this phase of the research. A narrow plate covered the fracture and adjacent rock matrix. Two wider plates covered the rock matrix on either side of the fracture, and were hydraulically separated from the center fracture plate. They were also used to control pressure head at the upper boundary with constant-head Mariotte reservoirs. The arrangement provided for a rapid changeover of tracer solutions from the background solution of $10^{-3}$ Molar ( $\underline{M}$ ) calcium chloride $\left(\mathrm{CaCl}_{2}\right)$ to either $10^{-2} \underline{\mathrm{M}}$, or $10^{-1} \underline{\mathrm{M}} \mathrm{CaCl}_{2}$. The behavior of the chloride tracer in the porous plates was also assessed in order to distinguish between apparatus and rock matrix transport.

Whatman filter paper number 42 with a retention rating of $2.5 \mu \mathrm{m}$ (equivalent to an air entry value of $116 \mathrm{kPa}$ at $20^{\circ} \mathrm{C}$ using the capillary rise equation) was used to collect samples in the fracture and matrix.

Because of small sample sizes, ion-selective electrodes capable of measuring changes of potential differences by surface contact were used to monitor chloride tracer breakthrough. The chloride electrode is of the coated-wire type, and the reference electrode is a double-junction electrode. Although the transport tests were conducted at nearsaturated conditions, independent tests showed the filter paper/ionselective electrode method to be promising for studying transport behavior at suctions up to nearly $10 \mathrm{kPa}$ (i.e., $100 \mathrm{~cm}$ of water).

The discussion that follows is organized into four chapters and five appendices. Chapter two presents a review of the process used to recover the fractured rock blocks for analysis. Discussion of fluid flow and solute transport is separated into two chapters. Chapter three provides the theoretical background for study of unsaturated fracture flow. It also includes a description of the experimental setup and procedures, and the methodologies of the computer model used to help interpret the flow processes. Finally, the major results and recommendations for future work in the fluid flow part of the study are discussed and summarized in the chapter. Chapter four discusses the theoretical considerations of ion-selective electrodes and transport processes. Considerations of the computer model as it affects the transport processes, and the analysis of water penetration into the sampling ports are also discussed. The experimental setup for transport studies and the procedures developed are described. A continuation of the case study of an idealized test block using the computer model is presented in the chapter along with future studies and recommendations. The appendices contain more detailed discussions of the procedures and data. 


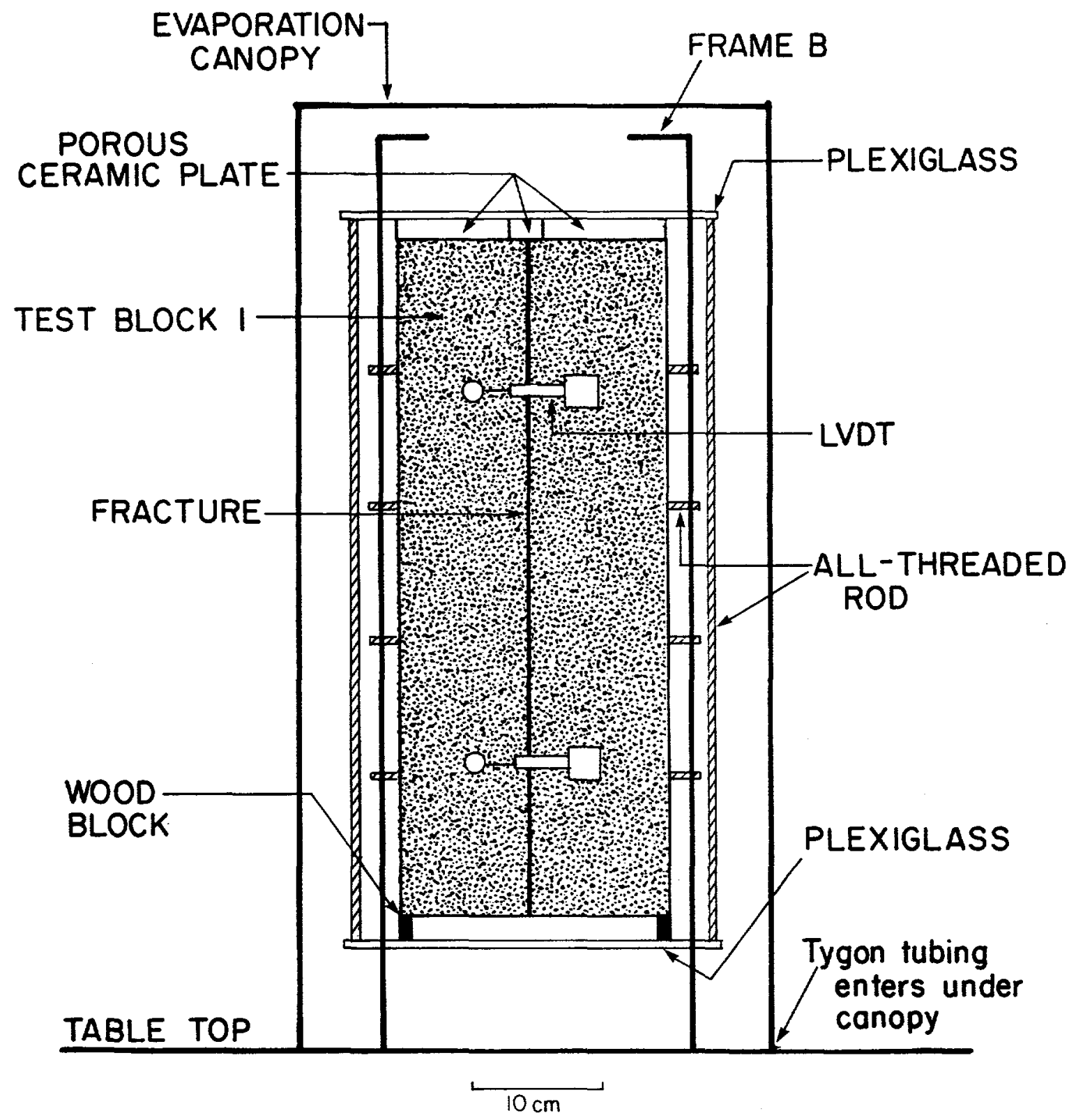

Figure 1.2 Test block number 1 experimental setup. 


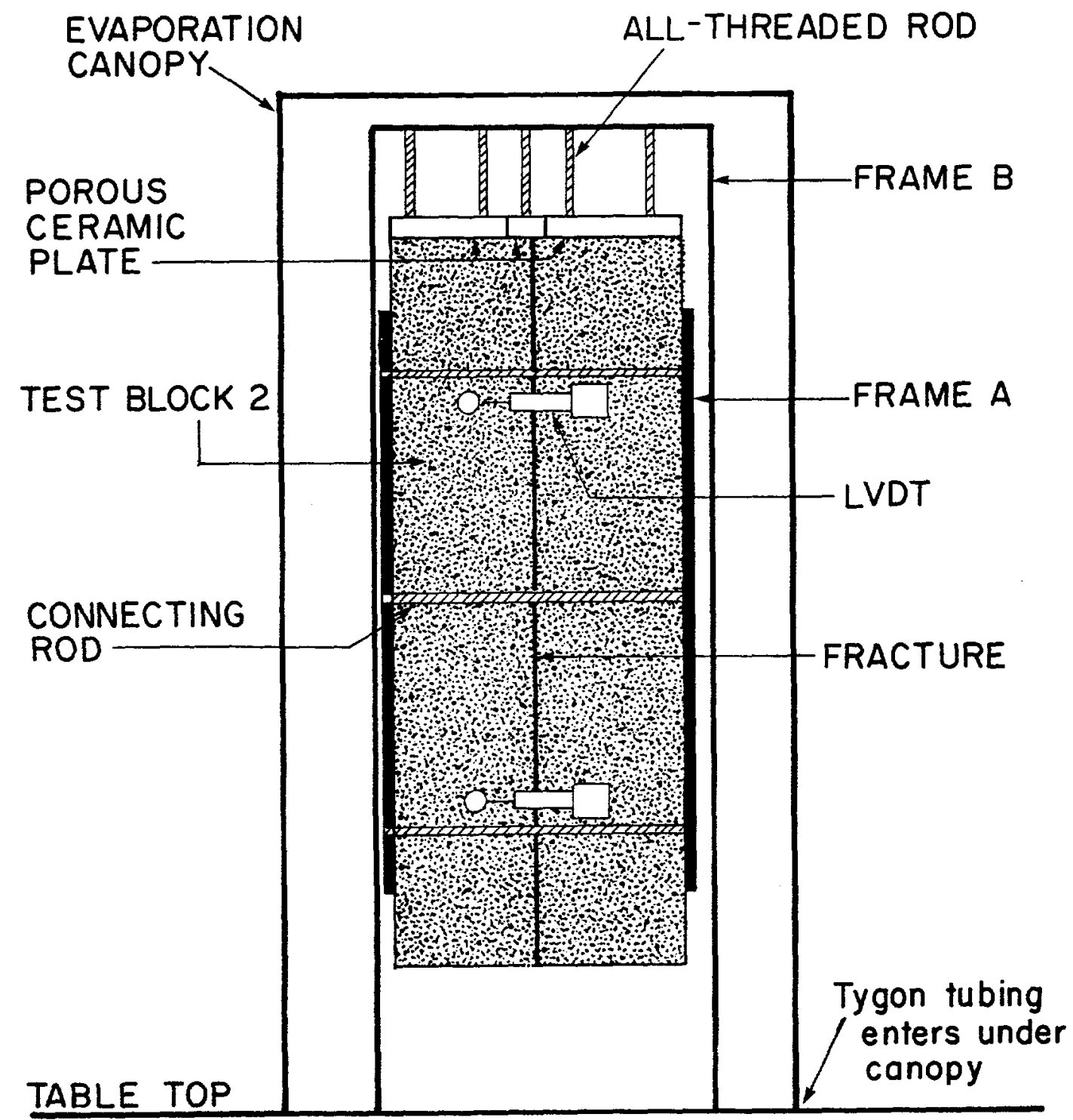

Figure 1.3 Test block number 2 experimental setup. Frame $A$ is held by hooks from frame B. 
CHAPTER TWO

PROCUREMENT AND PREPARATION OF TEST BLOCKS

Laboratory test blocks of partially welded and densely welded tuff were obtained from the Apache Leap test site, near Superior, Arizona. The Department of Hydrology and Water Resources at the University of Arizona, in conjunction with the Nuclear Regulatory Commission, operates the Apache Leap test site for the purpose of conducting hydrologic testing of partially welded tuff that is similar to that located at the Yucca Mountain site in Nevada. Following is a brief summary of the field site, the Apache Leap tuff, and the methods used to procure laboratory test blocks from the site. A brief discussion of selection and removal of a block of densely welded tuff is included. Preparation of the test blocks and a general description of the experiment setups are presented in this section. Detailed discussion of the procedures used for testing equipment, preparing calibration curves, and running experiments on the test blocks are included in Chapter Three.

\subsection{Description of the Apache Leap Tuff Site}

The Apache Leap tuff site is located at the western edge of the Pinal mountains of south-central Arizona, a few kilometers northeast of the town of Superior (see Figure 1.1). Steep mountains and deeply incised canyons characterize the local terrain. Three separate study locations compose the test site. The first, at the former Queen Creek road tunnel in Queen Creek Canyon on U.S. Route 60, lies at an elevation of about 1036 meters ( 3400 feet). It consists of both a weather station and a series of boreholes drilled in the abandoned tunnel. The second and third study locations are found along the Magma Mine Road overlooking Dak Flat, roughly at an elevation of 1262 meters (4140 feet). Two small watersheds compose the second study location; the third consists of an array of angled boreholes drilled on a small plateau.

Rock at the Apache Leap tuff site consists of ash-flow tuff. A pyroclastic deposit, ash-flow tuff results from the deposition, compaction, and consolidation of a mobile, high-density suspension of hot glass shards, pumice, rock fragments, and crystals. These airborne suspensions can travel more than $100 \mathrm{~km} / \mathrm{hr}$ and can be deposited on land or water. Once deposited, an ash-flow tuff begins to compact. Flattening of pumice fragments, a decrease in porosity, and deformation and welding of glass shards result from compaction of the hot mass. The degree of welding depends upon the ash-flow temperature at deposition and the rate of heat loss to the surrounding environment. Numerous ash-flows may be deposited on top of one another. If such deposition is closely spaced in time, the compaction and cooling of one unit may affect the compaction and cooling of units above and below it. Peterson (1961, 1968) studied the ash-flow tuff deposited east of Superior, Arizona. He found a sequence of welded tuffs that apparently cooled simultaneously. Welding of the tuff varies from nonwelded to densely welded (Figure 2.1), and maximum thickness of the sequence is 600 meters. Fracturing of the ash-flow tuff is roughly orthogonal; one set lies subhorizontally, and the other two lie at angles between 60 degrees and 90 degrees in the 


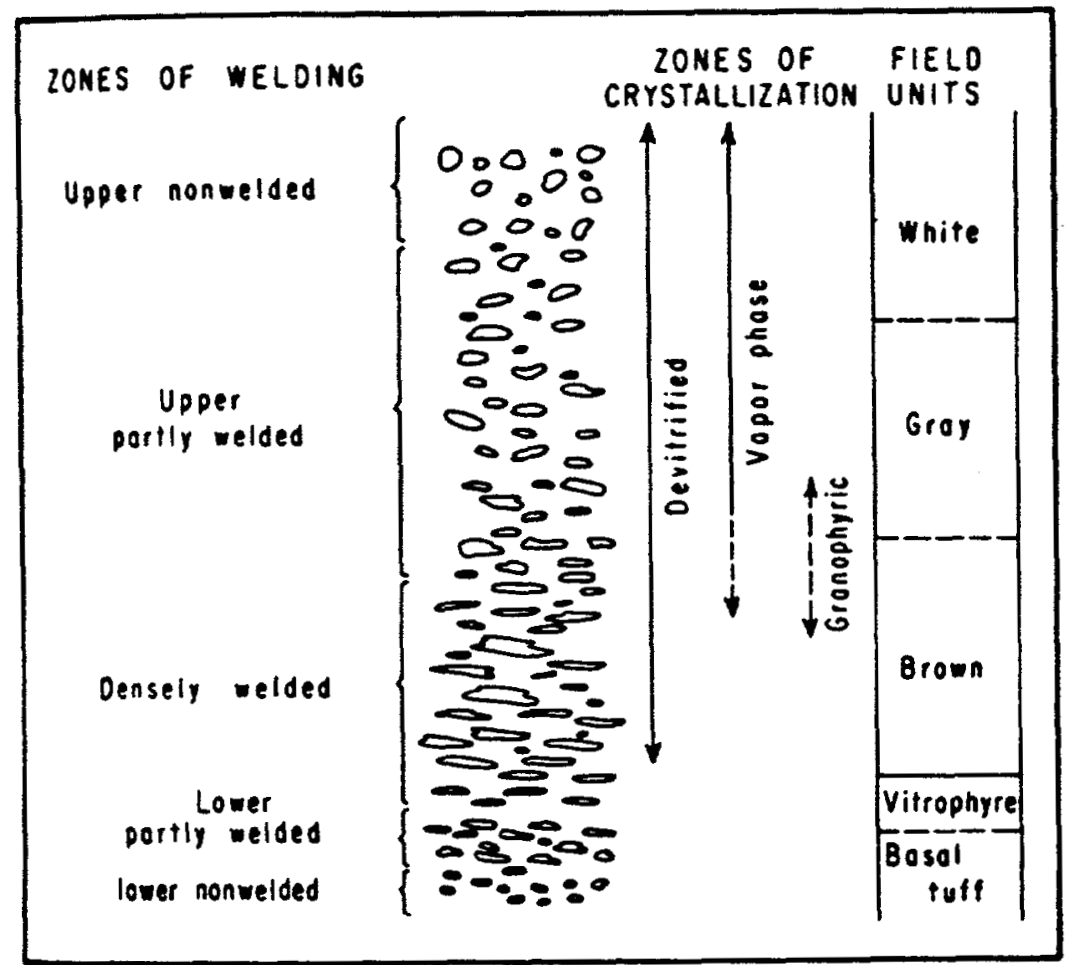

Figure 2.1 Physical characteristics of the Apache Leap tuff. 
vertical plane. Based on chemical composition, the tuff is classified as a porphyritic, quartz latite of middle Miocene age (about 20 million years old). From laboratory studies (Peterson, 1961; Rasmussen and Evans, 1987), the matrix porosity of the partially welded rock is between 17 percent and 20 percent. The matrix porosity of the densely welded tuff is estimated to be 9 percent. The Apache Leap test site lies in various parts of the sequence. The Queen Creek road tunnel study location lies in densely welded tuff. The watershed study location and the plateau site lie near the top of the sequence in a partially welded to nonwelded zone.

Weber (1986) performed a reconnaissance study of the hydrology of the region surrounding the Apache Leap tuff site. In comparing rainfall records of the town of Superior (elevation 910 meters) with those obtained at the Magma Copper Company shaft number 9 (elevation 1270 meters), he found discernible orographic effects over a ten year period of comparison. The average annual precipitation in the town of Superior was $538.8 \mathrm{~mm}$ (21.2 inches), and the average annual precipitation at the mine shaft was $639.6 \mathrm{~mm}$ (25.2 inches). Orographic effects were most significant during the winter and summer months, when rainfall is the highest in the area.

The region surrounding the study site is drained by ephemeral streams, the most prominent of which is Queen Creek. Small stock ponds along the Magma Mine road collect runoff and remain full into the summer months. Although the town of Superior obtains its water from wells near Florence Junction to the west, a significant amount of groundwater was pumped from shafts at the Magma Mine prior to its closure in 1986. Pumping from the mine shafts, the deepest of which was located at an elevation of -183 meters ( -600 feet) relative to Mean Sea Level, averaged 450 gallons per minute. Despite the fact that Weber (1986) had a difficult time obtaining useful groundwater quality data, the data which he obtained indicated that the groundwater in the area is highest in calcium, bicarbonate, and sulfate ions.

\subsection{Test Block 1 Procurement}

Test block 1 was found along the access road to the plateau at the Apache Leap tuff site. Presumably, the block had been loosened during construction of the road to the site. It appeared to be an appropriate size for initial experimentation; a relatively planar fracture bisected the sample, and the fracture appeared both conductive to water and wellcemented enough to withstand transportation to the laboratory. Using a backhoe, the rock was hoisted into the back of a pickup truck and transported back to the laboratory.

Very roughly a rectangular prism, the first rock's maximum field dimensions were $79 \mathrm{~cm}$ by $79 \mathrm{~cm}$ by $36 \mathrm{~cm}$ (Figure 2.2). The main fracture lay in the largest plane and measured about $70 \mathrm{~cm}$ by $58 \mathrm{~cm}$. However, due to the irregular and sloping nature of the rock near the fracture edges, the longest usable portion of the fracture, allowing the block to be shaped into a rectangular prism, was $50 \mathrm{~cm}$. After preliminary flow and transport tests, an outline of the optimal, finished block was drawn on the surface of the rock. Four $9.53-\mathrm{mm}$ (3/8-inch) diameter rock bolts were installed using a hand-held rotary hammer drill. 


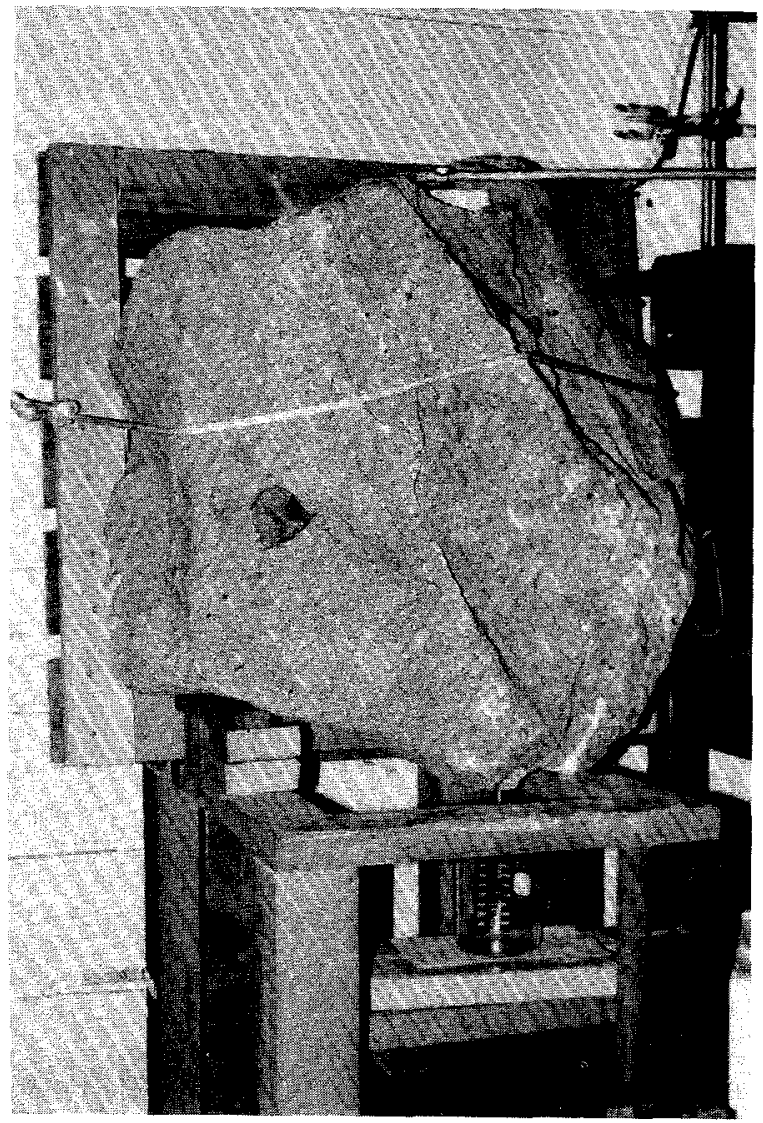

Figure 2.2 Test block number 1, prior to shaping, undergoing preliminary fracture conductivity test. 
A local concrete coring company shaped the block to dimensions of 20.3 $\mathrm{cm}$ by $20.3 \mathrm{~cm}$ by $49.4 \mathrm{~cm}$ (Figure 2.3), after which the porous plates were ordered. After shaping, the fracture lay roughly parallel to the block sides and measured about $50 \mathrm{~cm}$ by $21 \mathrm{~cm}$. It was noted that, for rock fragments cut off of the test block during the shaping process, about 5 percent of the fracture surface was cemented.

\subsection{Test Block 2 Procurement}

After location, removal, transportation, and shaping of the first test block, further criteria were developed for selection of additional test blocks. It was determined that two additional test blocks were desired, a partially welded test block that would be about $1 \mathrm{~m}^{3}$ in size and a densely welded block. Rocks in the desired size range were examined for quality of fracturing, ease of excavation, and simplicity of removal from the site. Desirable fractures were straight, continuous throughout the sample, and relatively free of clay, organic matter, and extensive cementation. It was also desired that the sample arrive in the laboratory as undisturbed as possible. Therefore, blocks were located in the field such that a minimum of work was needed to excavate and remove the sample.

Two other observations were made before selecting the final rock samples. First, the fracture along the center of the sample ideally was to be the only fracture in the rock. Practically, this was not possible. It was desirable, however, that the amount of subsidiary fracturing in the matrix of the sample be minimal. Second, the fracture being investigated needed to be conductive to water introduced at low positive pressure heads. Therefore, prior to removal, the field sample was investigated in a simple manner to determine if water moved through the fracture.

Two blocks were found that fit the above criteria. The first was a 1.2 $\mathrm{m}$ high by $1.0 \mathrm{~m}$ wide by $1.1 \mathrm{~m}$ deep partially welded tuff block located along the Magma Mine road, about 100 meters from the plateau site (Figure 2.4). The lower and right sides of this block were bounded by fractures, and the left, front, and top faces were open. The second block was an irregularly shaped, densely welded tuff $0.9 \mathrm{~m}$ ( 3 feet) high and $1.8 \mathrm{~m}$ ( 6 feet) long. It was found along the old U.S. 60 route, about 75 meters west of the old tunnel (Figure 2.5). From inspection of the rock wall on the north side of the road, it appeared that this block was dislodged from a location about 3.7 meters (12 feet) above the road.

Once these samples had been selected in the field, field flow tests were performed using a dilute calcium chloride solution. These tests indicated a conductive main fracture in each block. It appeared during the flow test on the partially welded block that a series of subsidiary fractures in the block would not allow the entire block to be used for testing. The decision was made to cut three smaller blocks from the rock during the shaping process. After the field flow tests were performed, $1.27-\mathrm{cm}$ ( $1 / 2-$ inch) diameter holes were drilled normal to the fracture plane to allow the installation of bolts. The holes were drilled with a rotary hammer drill and cleaned with air. The first $5 \mathrm{~cm}$ length of the holes were drilled with a $1.91-\mathrm{cm}(3 / 4-i n c h)$ diameter bit 


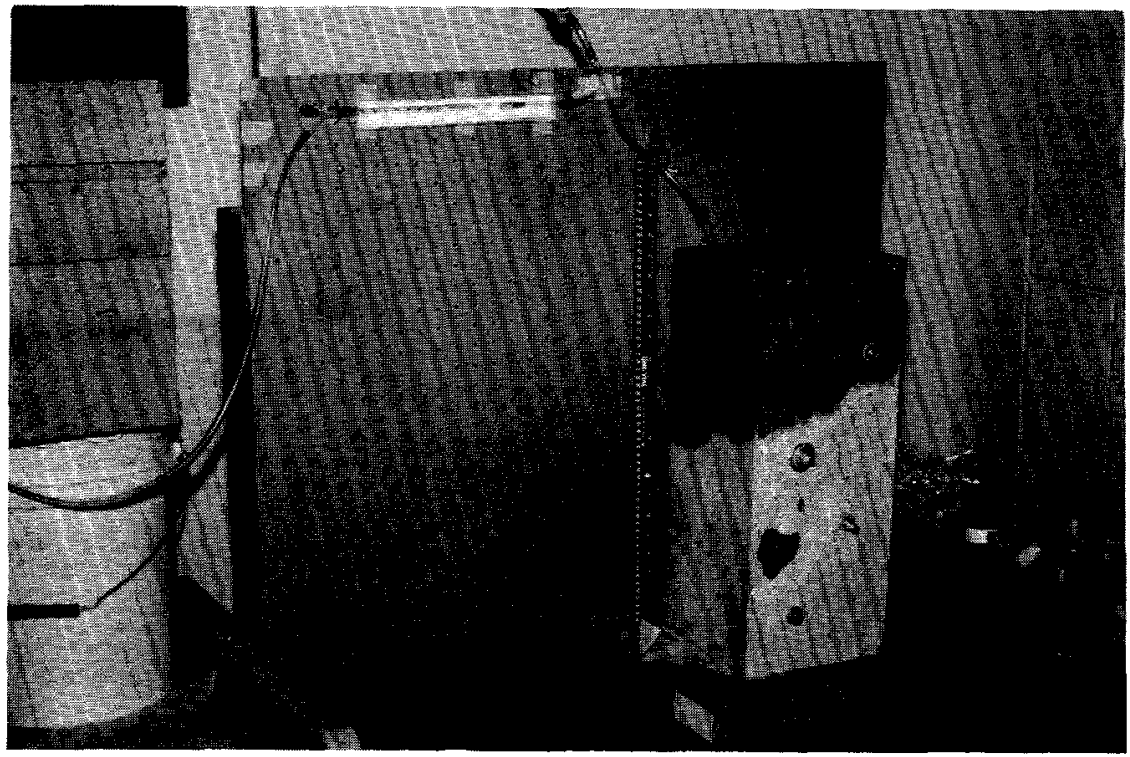

Figure 2.3 Test block number 1, after being shaped, post single-plate infiltration test. 


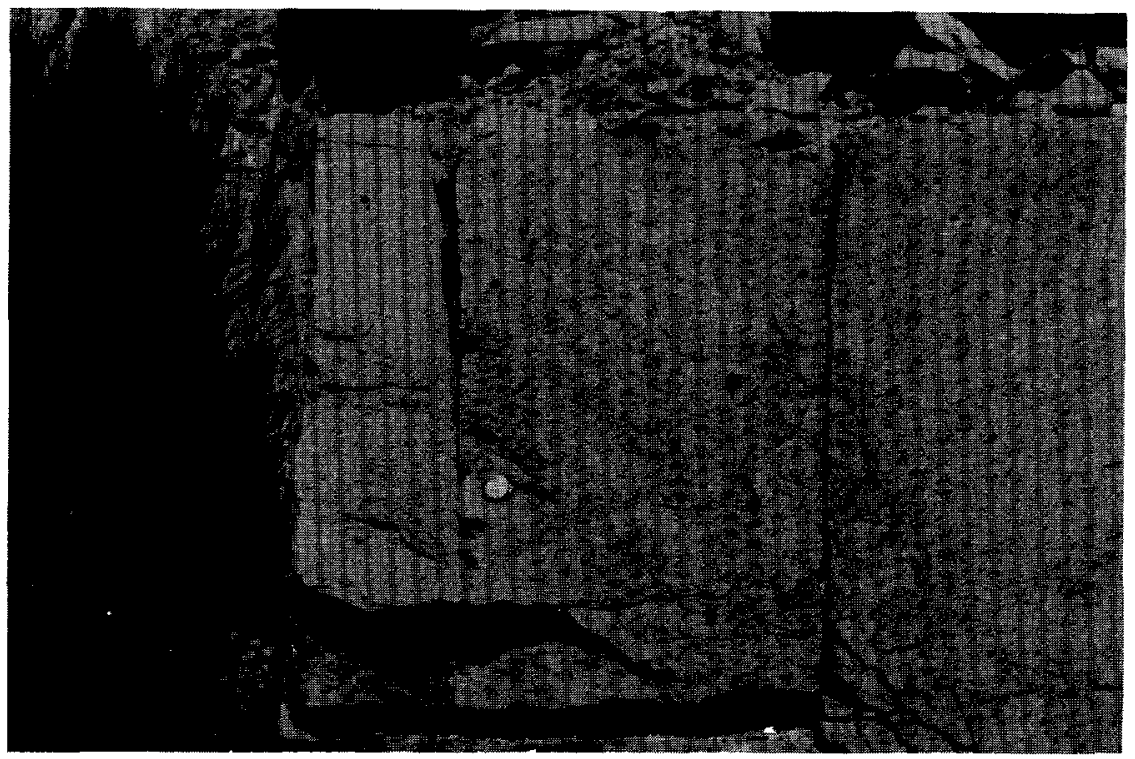

Figure 2.4 Field exposure of partially welded tuff from which test block 2 was cut. Lens cap is $5.3 \mathrm{~cm}$ in diameter. The vertical trace to the left of the lens cap is the test fracture. 


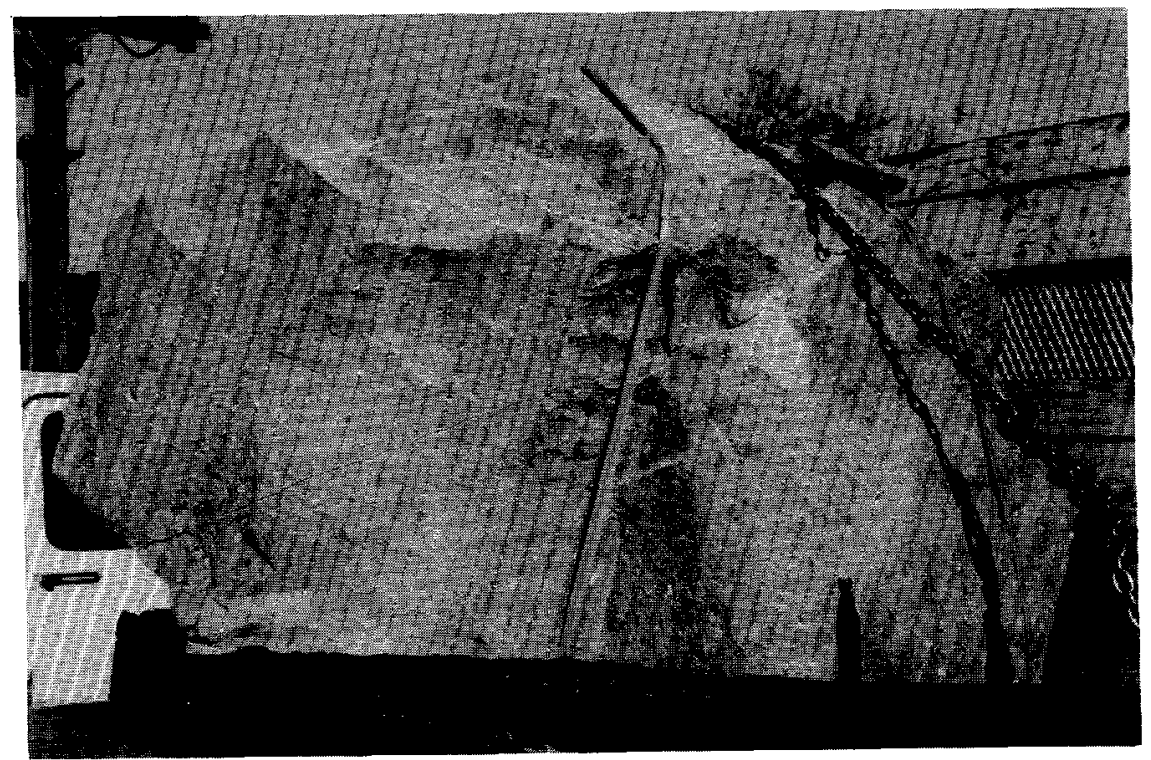

Figure 2.5 Densely welded tuff block found along former route of U.S. 60. Test fracture runs diagonally from the upper right to the lower left of the block. 
to allow recessing of the bolts in the rock during the shaping process. Consisting of $1.27-\mathrm{cm}(1 / 2$-inch) diameter all-threaded rod, the bolts were installed to help stabilize the fracture during removal and shaping. An adhesive was used to secure the rock bolts in the holes. It was used because, if later desired, the glue would break down upon heating and allow removal of the bolts. The glue was mixed such that it was of low enough viscosity to allow sufficient sealing of the annulus around the rock bolt and yet viscous enough not to invade the fracture of interest.

The densely welded tuff required no further preparation for removal. However, a fracture needed to be created along the back face of the partially welded block prior to removal. To accomplish this, sixteen $5.08-\mathrm{cm}$ (2-inch) diameter boreholes were drilled along the back face and parallel to the top surface of the rock. A local construction company was hired to drill these holes with a compressed air driven, rotary hammer drill. The boreholes were cleaned with compressed air, and the pneumatic packers that were to be inserted in the holes were tested for fit. Two pneumatic packers were then placed in boreholes, leaving one empty borehole between them, and inflated until a crack was induced. The pneumatic packers each consisted of a reinforced, rubber gland, or bladder, which expands radially when inflated by pneumatic pressure. The specific packers used in this method were chosen such that the maximum amount of pressure exerted by the inflated packer against the side of the borehole exceeded the estimated tensile strength of the rock. Compressed nitrogen was used to inflate the packers and was delivered to the packers through 4.8-mm (3/16-inch) outside diameter stainless steel tubing. It was expected that the induced crack would be short, and the procedure would need to be repeated a few times. However, the first time the procedure was attempted, at 950 psi inflation pressure, a crack was induced along the entire set of boreholes. When the crack appeared along the boreholes, the freed block of rock settled onto timbers that had been placed beneath it before inflating the packers. The settling of the block onto the timbers appeared to open the fracture slightly.

After both rock blocks were prepared for removal, a truck towing company was hired to lift each rock onto the back of a stake-bed truck. To accomplish this, woven straps were placed under the rocks, and the rocks were cradled from the tow truck boom. Timbers were placed below each rock on the truck to provide cushioning, and chains and binders were used to secure the load. During the lifting process of the partially welded block, a corner of the block hit another boulder, causing the corner to break off. This shortened the potential length of one of the test blocks.

The two blocks of tuff were then transported to Belen, New Mexico to be shaped. New Mexico Travertine, whose cutting and shaping plant is located in Belen, shaped the test blocks in a two-stage process that required four days to complete. The first stage consisted of separating excess rock by making saw cuts parallel to the fracture. Two parallel saw cuts, approximately $21 \mathrm{~cm}$ ( 8.25 inches) apart, were made using a $9.5-\mathrm{mm}$ diameter cable saw. The cable saw consisted of a continuous loop of cable which held diamond-impregnated steel cylinders. Although the cable saw was able to cut blocks up to 2.7 meters ( 9 feet) across, the 
accuracy of the cable sawing procedure was less than that using the blade saw that was available.

After a slab of rock had been cut with the test fracture running down the middle of the slab, the block was transferred to a computer-driven laser-guided blade saw for the second stage of cutting. Each test block was separated from the slab and trimmed to the final dimensions. One test block was obtained from the densely welded tuff block, and three test blocks were cut from the partially welded tuff block. Rock number 2 was one of the three partially welded test blocks. Final dimensions after shaping were $66.0 \mathrm{~cm}$ high by $20.9 \mathrm{~cm}$ wide by $20.2 \mathrm{~cm}$ deep. After shaping, the test blocks were strapped to pallets, the excess rock pieces were loaded onto the stake-bed truck, the pallets were chained to the truck, and the blocks and excess rock were transported back to the University of Arizona. 
CHAPTER 3

FLUID FLOW

Flow through saturated porous media has been extensively studied, and much has been written about the flow equations which describe flow processes. A brief review of saturated flow in porous media and fracture, and unsaturated flow in porous media, is provided here as a basis for discussion of unsaturated fracture flow. A description of a modeled case study using the boundary integral method is included. The experimental setups and procedures are discussed. Finally, major results and recommendations are presented. Appendix A details the procedures used, and Appendix B tabulates the test data.

\subsection{Saturated Flow Through Porous Media and Fracture}

Darcy's law provides the fundamental basis for analyzing steady groundwater flow through saturated porous media. It can be written in the form:

$$
\mathrm{q}=\mathrm{Q} / \mathrm{A}=-\mathrm{K} \operatorname{grad}(\phi)
$$

where

$$
\begin{aligned}
\mathrm{q} & \text { flux or specific discharge of water, } \mathrm{m} / \mathrm{s} ; \\
\mathrm{Q} & \text { volumetric flow, } \mathrm{m}^{3} / \mathrm{s} ; \\
\mathrm{A} & \text { cross-sectional area normal to direction of flow, } \mathrm{m}^{2} ; \\
\mathrm{K} & \text { hydraulic conductivity, } \mathrm{m} / \mathrm{s} ; \\
\text { grad } & \text { differential operator, } 1 / \mathrm{m} ; \\
\phi & \text { total hydraulic head, } \mathrm{m} .
\end{aligned}
$$

Hydraulic conductivity is a function of both the media through which flow takes place and the fluid which flows. It can be stated as:

$$
\mathrm{K}=\mathbf{k} \boldsymbol{\gamma} / \mu
$$

where

$\mathrm{k} \quad$ intrinsic permeability, $\mathrm{m}^{2}$;

$\gamma \quad$ specific weight of the reference manometer fluid, $\mathrm{Pa} / \mathrm{m}$;

$\mu$ dynamic viscosity of the test fluid, $\mathrm{Pa} s$.

Intrinsic permeability depends only on the properties of the media. Darcy's law will be applied in a number of instances in this study. It will be used to calculate the average pressure head at the bottom, or rock-facing, side of each porous plate. It will also be used to calculate the hydraulic conductivity of the matrix and the transmissivity of the fracture during saturated flow tests.

The simplest method for viewing laminar water flow through a fracture is to assume that the fracture walls are planar and a constant distance, e, apart (Figure 3.1). From the Navier-Stokes equations, one can derive what is known as the cubic law, here shown for one-dimensional, vertical fracture flow: 


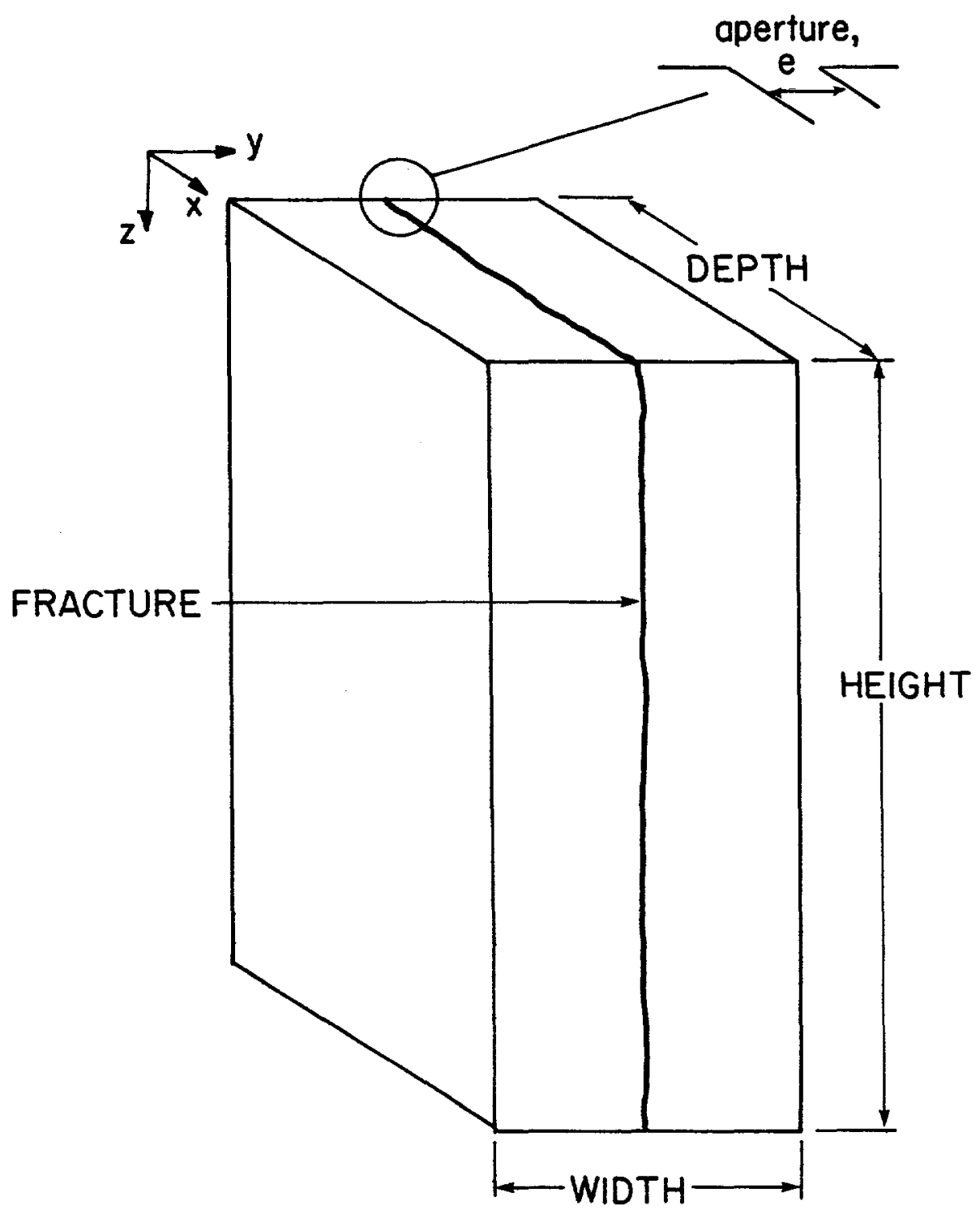

Figure 3.1 Schematic of test block containing a single vertical

fracture. Coordinate axes used in text are shown at the rear of the block. Also shown is the aperture, e. 
where

$Q_{f} \quad$ volumetric flow through the fracture, $\mathrm{m}^{3} / \mathrm{s}$;

$d_{f} \quad$ length along the $x$-axis of the fracture, $m$;

$e$ aperture of the fracture along the $y$-axis, $m$;

$z$ vertical direction, positive downward.

The cubic law is only valid for a homogeneous, incompressible fluid under isothermal conditions. Combining equations 3.1 and 3.3 yields:

(3.4) $\quad Q_{f}=A_{f} K_{f} d \phi / d z$

where

$A_{f} \quad$ fracture area, $m^{2}$, which equals $d_{f} e$;

$\mathrm{K}_{\mathrm{f}}$ fracture hydraulic conductivity, $\mathrm{m} / \mathrm{s}$, which equals $\gamma \mathrm{e}^{2} / 12 \mu$.

Note that fracture intrinsic permeability, $k_{f}\left(m^{2}\right)$ equals:

$$
k_{f}=e^{2} / 12 \text {. }
$$

If one knows the fracture aperture and imposed pressure gradients, equations 3.3 and 3.4 allow the prediction of expected flow through a given fracture. Similarly, measuring fracture flow under known gradients, one can calculate the fracture aperture.

As straightforward as the cubic law is for estimating aperture or flow rate from known parameters, natural rock fractures rarely have smooth, planar surfaces. More often, natural fractures have rough, wavey surfaces and appear curved or irregular in the field. Additionally, most fractures occur buried beneath sediments and rock and are thus subject to compressive stresses. According to Gale, et al. (1985), a number of researchers have proceeded to test the validity of the cubic law under a variety of conditions, from simulated to natural fractures. Lomize (1951) and later Louis (1969) used parallel glass plates to validate the cubic law for open smooth fractures. They also simulated rough parallel fractures and developed an empirical flow equation including a roughness coefficient. Other studies performed on natural or induced fractures (Sharp, 1970; Iwai, 1976) also indicate the applicability of the cubic law for open fractures.

Laboratory investigations of gas and water flow through rock fractures subjected to compressive stress that are normal to the fracture indicate that application of the cubic law may be limited. Engelder and Scholtz (1981) and Gale (1982) found that for compressive stresses above $10 \mathrm{MPa}$, the flow results differed from those predicted by the cubic law. Studies performed on a natural granodiorite fracture (Schrauf, 1984; Schrauf and Evans, 1986) also found significant deviations from the cubic law. They suggest that shear movement may significantly alter flow paths through a fracture as compressive stress increases. To fit the experimental data, they propose a pipe model of flow.

In summary, it appears that for essentially open fractures at low compressive stresses, the cubic law holds. At higher compressive stresses 
and higher resulting contact area, tortuosity increases (Tsang, 1984), and the cubic law is no longer valid.

Such results lead Tsang and Tsang (1987) to view fractures as tortuous channels rather than planes. They characterize the channel aperture density distribution, the effective channel length and width, and the aperture spatial correlation. They then statistically generate aperture systems through which flow and transport may be studied.

Another way to approach fracture flow study is to incorporate effective aperture into the term representing fracture hydraulic conductivity. First, fracture flow is expressed as flow per unit length along the $x$ axis (depth), $q_{f}\left(\mathrm{~m}^{2} / \mathrm{s}\right)$. Using equation 3.4 :

$$
q_{t}=Q_{f} / d_{f}=e K_{f} d \phi / d z
$$

Then, since the aperture of a given test fracture is not often known and may vary considerably throughout a fracture, transmissivity, $T_{f}\left(\mathrm{~m}^{2} / \mathrm{s}\right)$, rather than fracture hydraulic conductivity is discussed:

$$
\text { (3.7) } \quad T_{t}=e K_{f}
$$

and

$$
\text { (3.8) } \quad q_{f}=T_{f} d \phi / d z \text {. }
$$

Equation 3.8 allows the characterization of a fracture by transmissivity obtained from the volumetric flow rate and the imposed total head gradient. This dispenses with the need to characterize a fracture by the effective aperture, which is often not known and which may not equal the aperture determined by tracer tests or volume balance calculations (Smith, et al., 1987; Schrauf and Evans, 1986). If desired, an intrinsic transmissivity term could also be determined.

\subsection{Unsaturated Flow Through Porous Media and Fracture}

The nature of flow through unsaturated porous media depends to a great degree on the water content of the media through which flow takes place. Water content in turn depends upon the water potential of the porous media. The media water potential contains gravitational, pressure (suction), osmotic, and temperature components. Since the experiments performed in this study are all carried out in nearly isothermal conditions, the temperature component of total moisture potential will be ignored. Likewise, osmotic potential will not be considered due to the absence of a membrane or diffusion barrier in the experiments conducted during this study.

As in saturated flow, gravitational and suction potentials may be expressed as energy per unit weight, or head, in meters. The gravitationa1 potential at any given point in a saturated porous medium, when measured in energy per unit weight, equals the elevation of the point relative to an arbitrary reference height.

Suction, or matric, potential results from both capillary and adsorptive forces in the media matrix. Capillary forces represent the dominant 
component of matric potential in relatively wet environments and can be expressed by:

$$
\psi=2 \tau \cos \alpha / \mathrm{r}
$$

where

$$
\begin{aligned}
& \psi \text { suction, } \mathrm{kgm} / \mathrm{s}^{2} \text { or } \mathrm{Pa} ; \\
& \tau \quad \text { water-air surface tension, } \mathrm{kg} / \mathrm{s}^{2} ; \\
& \alpha \quad \text { liquid-matrix contact angle, usually taken as zero for water } \\
& \text { and soil or rock; } \\
& \mathbf{r} \text { radius of capillary tube, } \mathrm{m} .
\end{aligned}
$$

Expressing pressure in meters of water, $h_{c}$, yields:

$$
\mathrm{h}_{\mathrm{c}}=2 \mathrm{~T} \cos \alpha / \mathrm{\gamma} \mathrm{r} \text {. }
$$

The term $h_{c}$ represents the height water will rise in a capillary tube or cylindrical pore of radius, $r$. The term $P$ indicates the pressure at which a given pore size will drain. As equations 3.9 and 3.10 indicate, larger pores drain at smaller suctions. For example, at $20^{\circ} \mathrm{C}, \tau=$ $0.0727 \mathrm{~kg} / \mathrm{s}^{2}$ and $\gamma=9790 \mathrm{~Pa} \mathrm{~s}$. Assuming that $\alpha=0$, a pore of $100 \mu \mathrm{m}$ radius would drain at $0.15 \mathrm{~m}$ of suction, and a pore of $10 \mu \mathrm{m}$ radius would drain at a suction of $1.5 \mathrm{~m}$.

Geologic media also exhibit adsorptive forces which form hydration envelopes, or a film of water, over the particles in the media (Figure $3.2)$. The volume of water in a porous medium held to particle surfaces by adsorptive forces tends to be rather small compared to the volume of water held by capillary forces, especially at low suction. However, at higher suction, when many pores have drained, film flow of water may be quite substantial relative to flow through water-filled pores.

The prediction of pore size drainage using the capillary equation is complicated by irregularly shaped pores and branching pore networks. Any neck or branch that leads to a smaller pore size will increase the drainage suction for a given pore or system of pores. Therefore, it is necessary to know the effective pore size distribution of a porous medium, that is, the distribution of pores that yield water under a range of applied suctions. Two methods are available for producing such a distribution. The first consists of a moisture release curve plotting suction versus water content. Experimentally, water content is measured after equilibrium is achieved at various suctions. Ideally, one should prepare two curves for each medium, one desorption curve and one sorption curve. Typically, the two curves will not be the same; this phenomenon is called hysteresis. It is thought that hysteresis may be due to factors such as the "ink-bottle" effect (necking of the pores), entrapped air, the wetting contact angle differing from the drying contact angle, and swelling or shrinking of the medium. The second method consists of mercury intrusion porosimetry, which produces a pore size distribution by forcing mercury into the rock pores under pressure. A nonwetting fluid, such as mercury, under pressure simulates a wetting fluid, such as water, under suction. By noting that the hydraulic conductivity is a function of suction, $\mathrm{K}(\psi)(\mathrm{m} / \mathrm{s})$, Darcy's law may be applied to unsaturated porous media: 


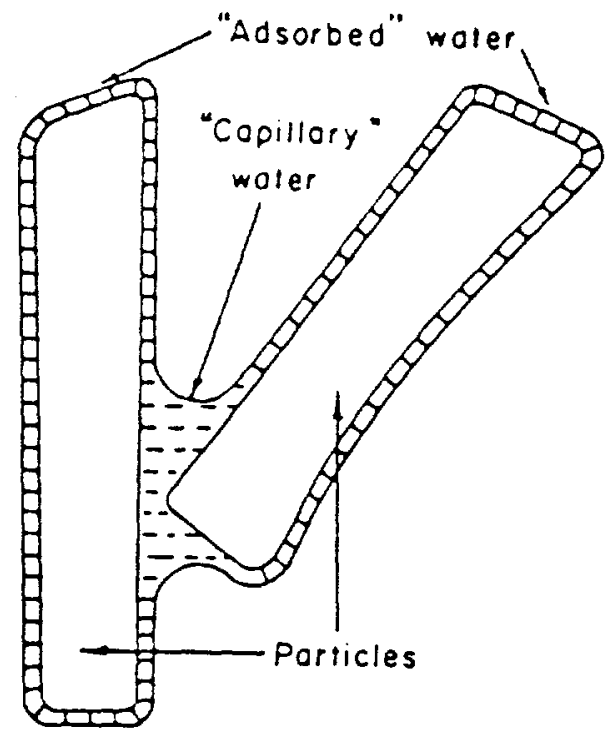

Figure 3.2 Water held in a film over particle, surfaces and in capillary wedges between particles in unsaturated geologic media. 
As moisture characteristic curves are developed to characterize the effective pore size distribution, unsaturated hydraulic conductivity curves may also be developed, plotting $K(\psi)$ versus either suction or water content. Since $K(\psi)$ may be hysteretic, typically the relationship is used for either continuously decreasing or continuously increas ing suction. Hydraulic conductivity decreases with increasing suction, and depending on the effective pore size distribution, may decrease rapidly with only slight increases in suction.

Downward infiltration and percolation into an initially unsaturated porous medium has received much study in the field of soil science. In general, both the suction gradient and the gravity gradient affect the rate at which water is imbibed into the medium. The suction gradient influences infiltration rate early in the infiltration process. As the water content of the medium increases, the average suction gradient decreases, and the gravity gradient plays a more important role. Philip (1969) presents a solution to Richards' equation for vertical infiltration into a porous medium, which is presented here in an approximate form:

$$
I(t)=s t^{1 / 2}+A t
$$

where

$$
\begin{array}{cl}
I(t) & \text { cumulative infiltration, } m ; \\
t & \text { time, } s ; \\
s & \text { sorptivity, } m /\left(\mathrm{s}^{1 / 2}\right) ; \\
\text { A } & \text { infiltration rate at large time, } \mathrm{m} / \mathrm{s} .
\end{array}
$$

Equation 3.12 can also be written in terms of infiltration rate, $i(t)$ $(\mathrm{m} / \mathrm{s})$ :

$$
i(t)=1 / 2\left(s t^{-1 / 2}\right)+A \text {. }
$$

From equation 3.13 , it can be seen that at small times, infiltration rate varies according to $t^{-1 / 2}$. At large times, infiltration rate approaches A. Since the gravity gradient drives infiltration and percolation at large times, the parameter $A$ approximates the hydraulic conductivity of the medium corresponding to the applied suction head. If $I / t$ is plotted against $t^{-1 / 2}$, one can obtain an estimate of hydraulic conductivity from the intercept of the straight portion of the curve.

Flow through unsaturated rock fractures is subject to the water content, and thus water potential, of the system in a manner similar to the matrix surrounding it. Both capillary and adsorptive forces in a rock fracture work to hold water in a fracture as suction is applied to the system. Equation 3.10 is modified for drainage from smooth planar fractures such that:

$$
h_{c}=2 \tau \cos \alpha / \gamma e
$$


with all variables previously defined. Equation 3.14 shows that as suction is increased, the largest diameter fractures in a rock body will drain before smaller fractures drain. However, natural fractures are rarely smooth and planar. More likely, natural fractures consist of variably rough fractures that are locally cemented and only approximately planar on a laboratory scale. As with unfractured geologic media, the development of the moisture characteristic curve is necessary to adequately predict moisture content of an unsaturated fracture.

Assuming that the flow is laminar, Darcy's law may also be applied to unsaturated rock fractures. Modifying equations 3.7 and 3.8 for unsaturated flow yields:

$$
T_{f}(\psi)=\text { e } K_{f}(\psi)
$$

and

$$
q_{f}=T_{f}(\psi) d \phi / d z
$$

where

$\mathrm{K}_{\mathrm{f}}(\psi) \quad$ unsaturated fracture hydraulic conductivity, $\mathrm{m} / \mathrm{s}$;

$\mathrm{T}_{\mathrm{f}}(\psi)$ unsaturated fracture transmissivity, $\mathrm{m}^{2} / \mathrm{s}$.

Because both $K_{f}(\psi)$ and $T_{f}(\psi)$ depend upon the suction present in the system, one can see the necessity of developing $K_{f}$ or $T_{f}$ versus $\psi$ relationships for individual fractures or fracture systems of interest.

For a partially saturated fracture, transmissivity can also be expressed as a function of water content. If $\theta_{1}$, the water content of the fracture, is defined as:

$$
\text { (3.17) } \quad \theta_{\mathrm{t}}=2 \mathrm{~b}_{\mathrm{a}} / \mathrm{e}
$$

where $b_{a}(m)$ is the average thickness of a water film, then the transmissivity for a partially saturated fracture, $T_{f}(\psi)$, can be written as:

$$
T_{f}(\psi)=\text { e } K_{f}(\psi) .
$$

Without such relationships as $K_{f}$ or $T_{f}$ versus $\psi$ and the moisture characteristic curve, prediction of fracture flow under given imposed suctions would be difficult.

Because of the difficulty in measuring fracture transmissivity versus suction relationships, little experimental work has been done with unsaturated fractures. The closest related work that has been accomplished has been that of soil physicists working with soil macropores. Wang and Narasimhan (1985) in their modeling study of fluid flow through partially saturated, fractured porous media summarize the current thinking on the subject, much of it based upon soil physics work. Saturated flow through a low-conductivity porous medium that is highly fractured will most likely take place predominantly through the fractures. As suction in the medium increases, only those locations within the fractures where the aperture is smaller than the drainage aperture for the suction present will remain saturated. As the fracture dries out, the 
transmissivity of the fracture decreases, leading to decreased flow in the fracture. Depending upon the effective aperture of the fracture and the change in suction in the medium, this decrease can be abrupt.

often, the effective pore size distribution of the matrix is considerably smaller than the effective aperture of fractures in the system. Over much of the suction range that a fractured rock body may experience, the hydraulic conductivity of the matrix may be higher than the effective hydraulic conductivity of the fracture. As Wang and Narasimhan (1985) point out, desaturation of the fracture reduces the area of the fracture across which flow may take place to points of contact or necks. Thus, at higher suctions, the tortuosity of flow through an unsaturated fractured rock body is increased.

\subsection{Coupled Matrix/Fracture Flow - Boundary Integral Method}

To provide a means of understanding the nature of flow through fractured rock, the test setup used to analyze block number 1 was modeled using a two-dimensional boundary integral model. A numerical model of the first test block aids the study of flow through a coupled matrix/fracture system in at least three ways. First, it allows the estimation of pressure head gradients, inflow rates, and streamline locations for the applied pressure heads at the top of the block. Second, parameters such as the matrix hydraulic conductivity and the fracture transmissivity can be varied so that different scenarios can be evaluated. Third, it

improves the interpretation of laboratory data. This steady-state model was developed by Rasmussen (1988) and assumes that the hydraulic conductivity within a flow domain is constant in space and time. The model

functions by discretizing the boundary surrounding the area of interest. Laplace's equation is solved along the boundary using a weighted residual function. Since the focus of this study is on the development of laboratory procedures, the reader is referred to Rasmussen and Evans (1988) for a detailed discussion of the model and its theoretical background. Further discussion from a solute transport stand point is deferred till Chapter 4.

\subsection{Preparation of Rocks}

Each test block needed to be prepared for experimentation prior to the start of the first test. Preparation involved installing the test block in a frame to hold the fracture together, tightening the fracture aperture as desired for testing, drilling the matrix and fracture sampling ports, drilling the holes that held the displacement transducer (LVDT) posts, cleaning both the boreholes and the rock surface, and assembling the test block in its testing location. A detailed procedure outlining this preparation is provided in Appendix A, Procedure 1 . This section will provide a brief summary of that procedure.

Because rock number 1 contained four galvanized all-threaded rods, a steel inner frame was not necessary. One at a time, each rod was taken out of the test block and replaced with a pre-weighed rod, allowing later weighing of the test block. The bolts were then tightened to a torque of 5 foot pounds using a torque wrench.

Rock number 2 was first prepared for experimentation by attaching a painted steel inner frame to the rock, denoted frame $A$. The purpose of 
this frame was to hold the test block together across the test fracture. The frame was held together by all-threaded rods and nuts that were threaded through holes drilled in the overhanging frame. To ensure that the frame load was evenly distributed across the rock face, brass shim was placed where needed between the frame and the rock surface. Using a torque wrench, the bolts were tightened to a torque of 30 foot pounds.

Each face of the test block was labeled. On both blocks, face 1 and face 2 were the top and bottom of the block, respectively. Figure 3.3 diagrams test block 1 ; test block 2 face numbers are shown later in Figure 3.21. Matrix and fracture sampling ports and displacement transducer (LVDT) post holes were drilled in each test block (Figures 3.4 through 3.6). The purpose of the sampling ports was to allow the measurement of in-situ water potentials and the sampling of test solution for tracer movement. The sampling ports and LVDT post holes were drilled using a diamond-studded coring bit. Tap water was used to cool the drill bit during this process. The rock was leveled so that the port was perpendicular to the face parallel with the fracture plane. The matrix ports and LVDT post holes extended approximately $4 \mathrm{~cm}$ into the rock matrix.

The fracture sampling ports were paired such that variations in flow and transport could be studied in the direction lateral to the general direction of flow. Care was exhibited during the drilling of the fracture sampling ports not to drill past the fracture surface. When the fracture was neared, drilling proceeded slowly. The sampling port was frequently inspected, both visually and with a wire, and drilling was stopped when the water in the vertically oriented sampling port drained out the bottom of the hole, which was assumed to be the fracture surface. Additionally, the cores taken from the sampling ports were inspected, and often the intersection of a weathered fracture surface could be seen on the end of the cores. In rock number 1, three ports extended from a face parallel to the fracture to the fracture face. The fourth port, 5F-LC, intersected a subsidiary fracture and was not drilled to the main fracture. When the ports were not being used to obtain samples, rubber stoppers sealed off the port entrances.

After drilling was completed, the rock was moved to a table and cleaned. The cleaning procedure consisted of flushing the sampling ports and post holes repeatedly with a dilute calcium chloride solution from a squirt bottle and removing any cuttings with a test tube brush. A chisel was used to chip out any rock pieces still attached to the end of the port after the drilling process. The test block surface was then cleaned using a dilute calcium chloride solution and a soft bristle brush, removing any silt or clay accumulated on the rock surface in the shaping and port drilling. Throughout the preparation of the block for testing, touching of the end faces (faces 1 and 2) was minimized, to avoid adding oil to the surface pores.

Installation of the LVDT posts followed. The LVDT posts consisted of solid cylindrical aluminum. One of the two posts making up a set contained a tapped hole which accepted the threaded LVDT core (see section 3.5.5). The other post contained a square head in two parts which could be tightened over the LVDT coil with four screws. The posts were glued into the rock using Depend Adhesive which, upon heating above about 


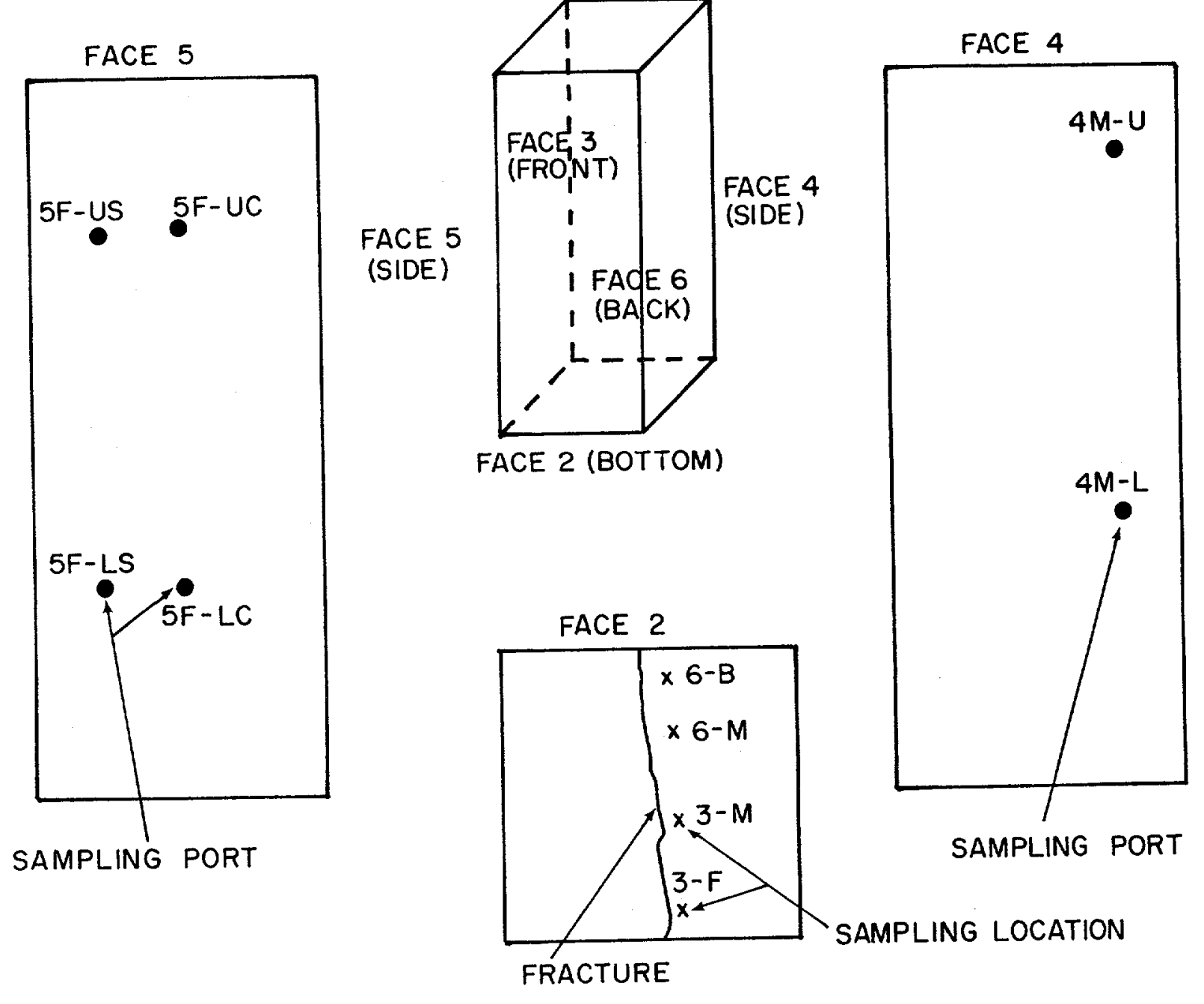

Figure 3.3 Test block number 1 numbering system. Fracture intersects faces $1,2,3$, and 6 . 
MATRIX PORTS

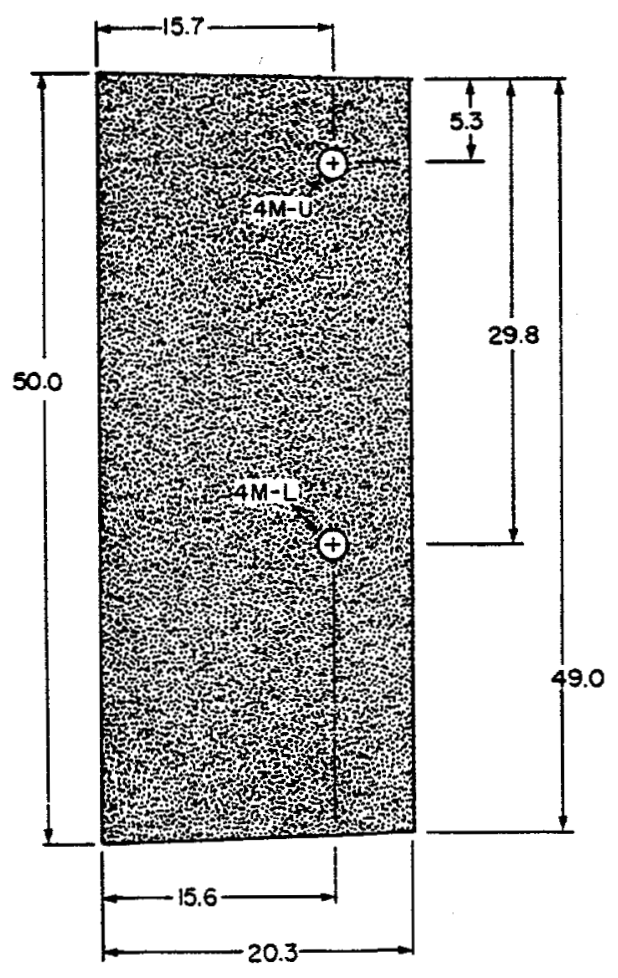

FACE 4
FRACTURE PORTS

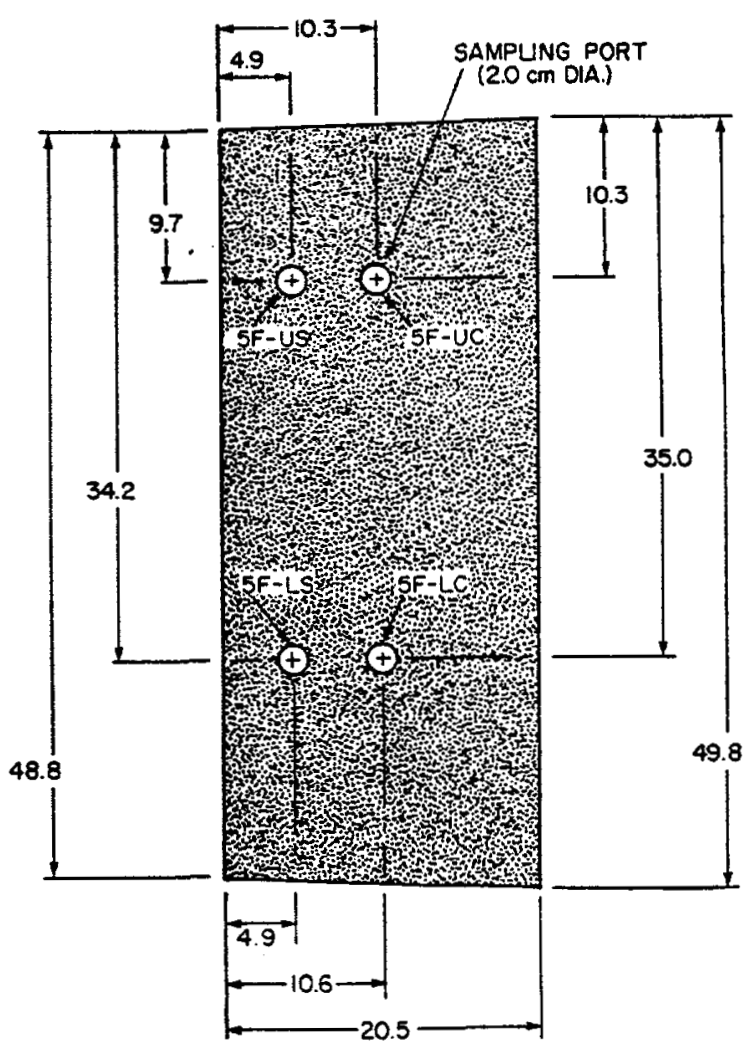

FACE 5

Figure 3.4 Test block 1 sampling port locations. 


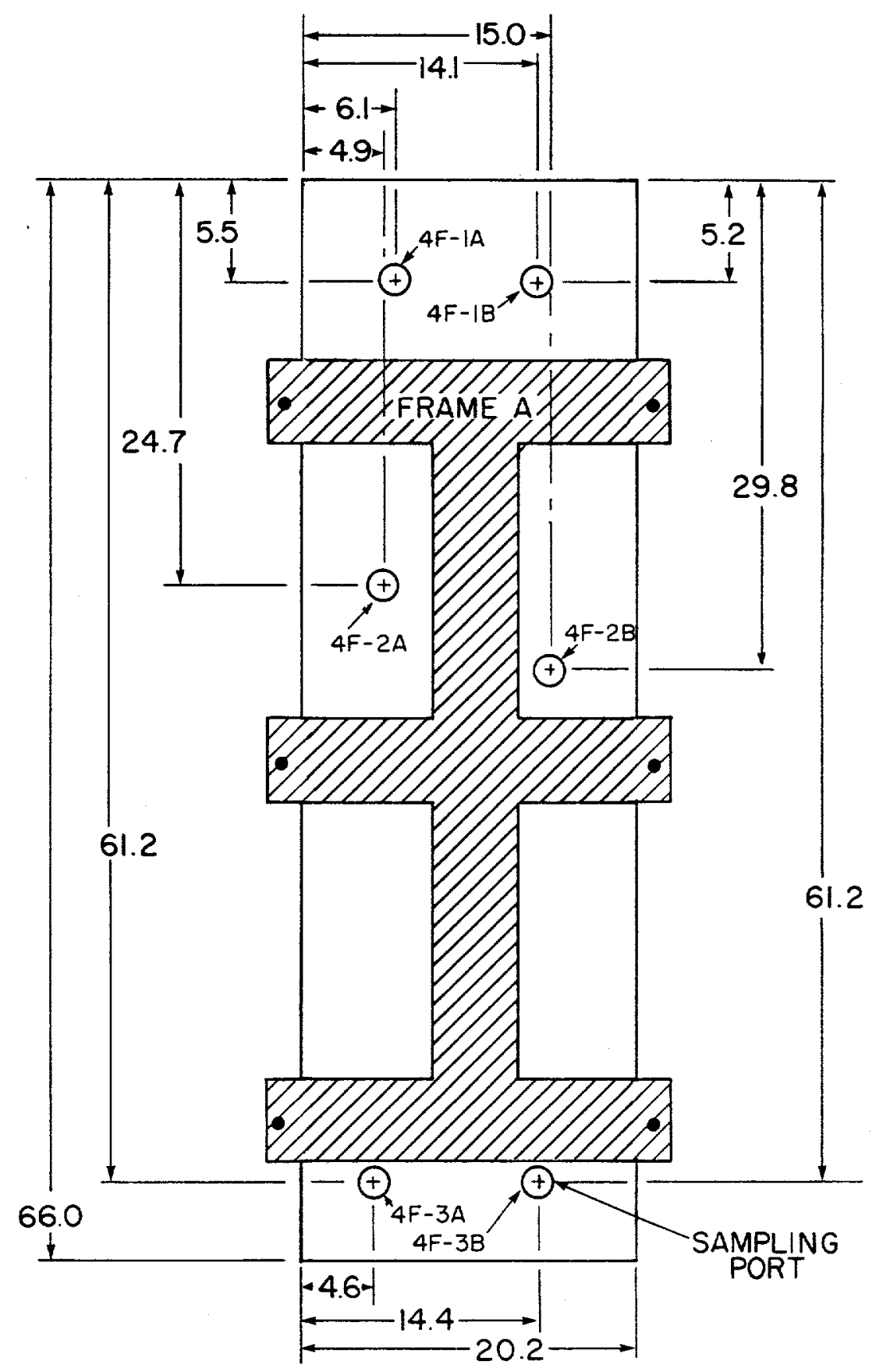

Figure 3.5 Test block number 2 fracture sampling ports, face 4 . 


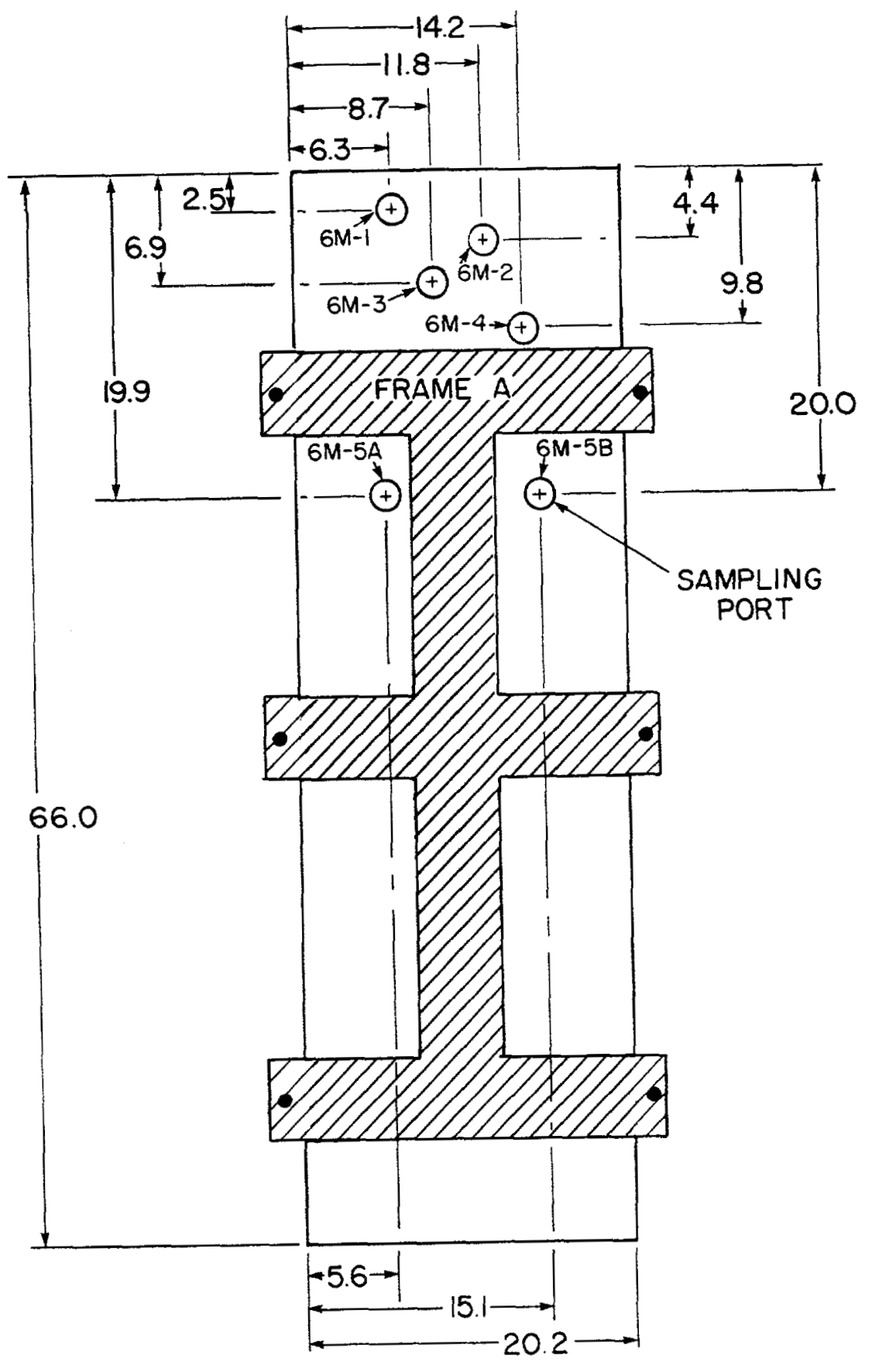

Figure 3.6 Test block number 2 matrix sampling ports, face 6 . 
$120^{\circ} \mathrm{C}$, broke down and allowed removal of the aluminum posts when the experiment was concluded.

The test blocks were mounted in a frame which stood on a laboratory table top. For sake of discussion, this second frame is called frame B. The frame material was composed of thick galvanized angle iron, with pre-drilled holes; the frame design consisted of four independently standing posts, with footings welded on, and separate cross strips that were bolted to the corner posts. By standing the test block in its testing orientation and elevation on top of blocks of wood, the cross members of frame $B$ were attached to the protruding all-threaded rod, in the case of rock 1 , and frame $A$, in the case of rock 2. The wood blocks were then removed and the frame and rock assembly positioned in its testing location.

To minimize evaporation, it was necessary to enclose the test block assembly in a plastic canopy. This was accomplished by welding $6.4-\mathrm{mm}$ (1/4-inch) diameter steel rod together to form a canopy frame. The frame was built big enough to fit over the entire test assembly. Clear polyethylene covered the canopy frame that was placed over rock 1 . Duct tape secured the polyethylene to the frame and the laboratory table top. Access patches were cut in the canopy, allowing entry to the sampling ports and faces 1 and 2. Duct tape closed the access patches when the ports were not being sampled and was used to tape on the polyethylene top.

Using polyethylene as a canopy material had two disadvantages: it could not be glued using any available adhesives, and it was hard to see through. Therefore, clear vinyl was used to enclose rock 2. Vinyl adhesive sealed the seams, and as before, duct tape sealed the bottom edges to the table top. Rubber bands were used to secure the polyethylene top that covered the top of the frame. The access patches in rock number 1 also proved to be inadequate. Clear PVC tubing was used to access the sampling ports in rock 2 . The tubing was caulked to the evaporation canopy using lightweight washers to give added support. Epoxy glued to the lead end of the tubing provided a tight fit in the ends of the sampling ports. Less than $1 \mathrm{~cm}$ of the tubing was allowed to rest in a sampling port. Rubber stoppers sealed the ends of the access tubes when they were not in use.

\subsection{Experiment Setup and Equipment Testing. Calibration and Procedures}

Figures 1.2 and 1.3 show the basic setup for experiments performed using test blocks 1 and 2. Both setups involved the assemblies described above and a test solution delivery system which is detailed in Figure 3.7. The calibration and use of each of the components in the test block experimentation is described below. In general, the upper surface of each test block was fit with specially designed rectangular porous ceramic plates to provide a water source under a controlled pressure head. Each plate was hydraulically separated from adjacent plates. Narrow plates were placed along the fracture/surface intersections to more precisely measure fracture inflow. Test solution inflow for each plate was measured. Additional test data acquisition included water potential measurements at the sampling port locations at the fracture surface and in the matrix, and fracture displacement monitoring. 


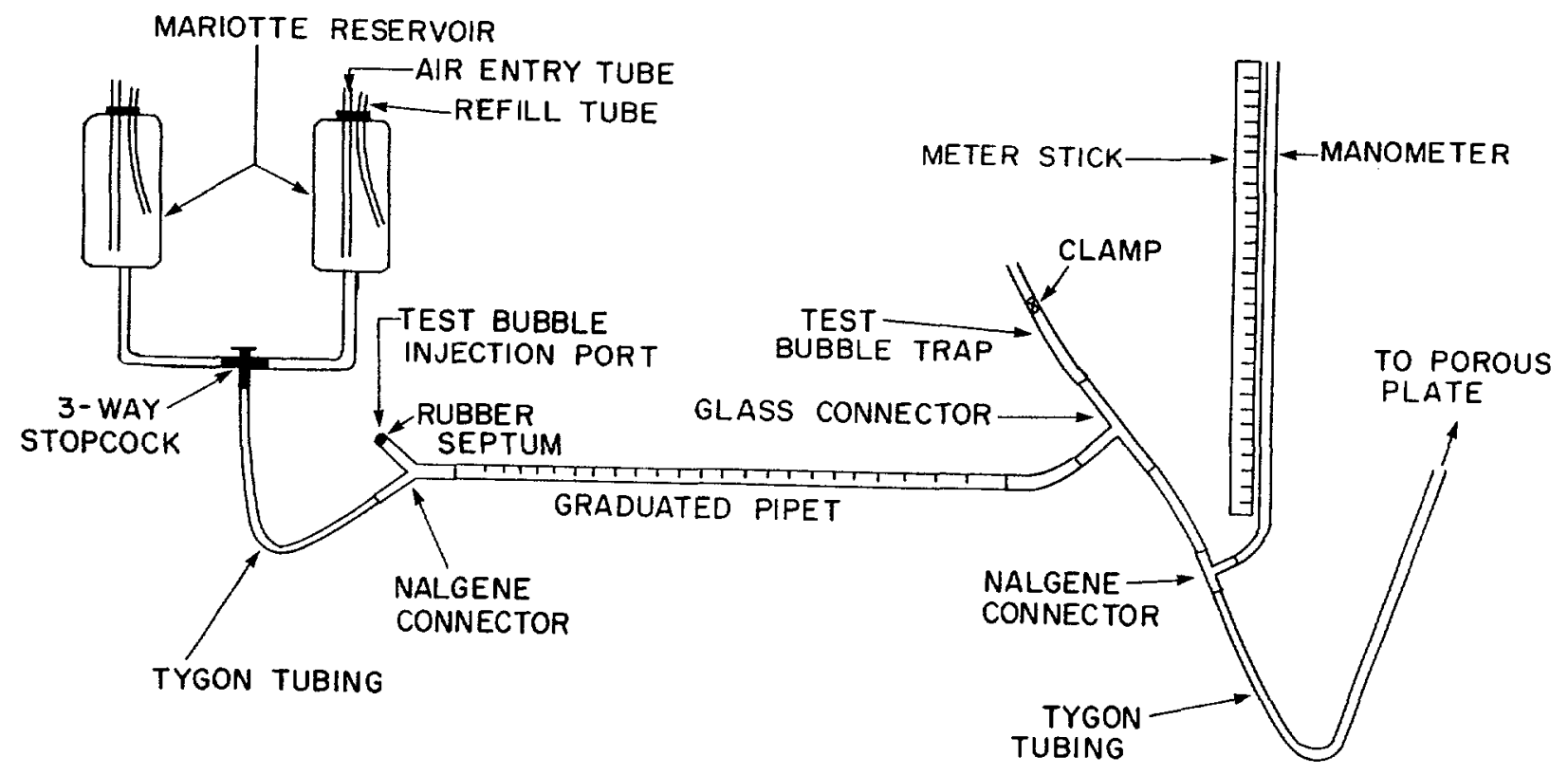

Figure 3.7 Test solution delivery system. 
The test solution used during the experiments performed on the test blocks consisted of deaerated $0.001 \mathrm{M} \mathrm{CaCl}_{2}$, with 0.1 gram of thymol added per liter of solution. Procedure 2 of Appendix A outlines the preparation of the solution. This particular test solution was chosen because calcium stabilizes the diffuse double layer surrounding any clay present in the test fracture or present in any heavily weathered portions of the block. Chloride was chosen as the tracer to be used in the transport portion of the study. A concentration of $0.001 \underline{M}$ was selected based on the standard test solution concentrations used in soil science studies (Klute and Dirksen, 1986). Thymol acted as a bacteriological inhibitor (Klute and Dirksen, 1986). The test solution was deaerated by boiling to minimize air bubble formation in the porous plate matrix, backing, and associated tubing.

The principal equipment used in the test block experiments included the porous plates, the constant-head reservoirs, the pipet flow tubes, the microtensiometer, and the displacement transducers. The following sections describe each device.

\subsubsection{Porous Ceramic Plates}

Soilmoisture Equipment Corp. of Goleta, California manufactured the porous ceramic plates. Each plate consisted of a $0.7-\mathrm{cm}$ thick rectangle of baked ceramic attached to a $1.2 \mathrm{~cm}$ thick, clear polyvinyl chloride (PVC) base, or backing (Figure 3.8). Standard plate length was $20.2 \mathrm{~cm}$ ( 7.950 inches). The porous plate was held to the PVC by epoxy around the four, notched edges. The pore sizes of the ceramic plate were small enough that the plate provided at least $200 \mathrm{kPa}$ (2 bars) of suction, that is, the air entry value was at least $200 \mathrm{kPa}$ of suction. A continuous groove cut the plate side of the PVC backing and connected two brass nipples. This construction allowed both the supply of water to the ceramic plate and, since the groove was continuous, the instantaneous flushing out of the water delivery system.

One narrow ceramic plate $(3.0 \mathrm{~cm}$, or 1.190 inches wide) was used to provide solution to the top of the fracture. Two ceramic plates ( 8.6 $\mathrm{cm}$, or 3.380 inches wide) were required to deliver solution to the top of the rock matrix. For the porous plates to be effective, good contact was required between the plates and the rock matrix surface or fracture. Whatman filter paper number 42 , with a $2.5 \mu \mathrm{m}$ retention rating was cut to fit the ceramic side of each plate and was placed between the plate and the matrix surface. Additionally, filter paper pulp, derived from Whatman filter paper number 42, was placed in the top of the fracture to aid contact between the porous plate and the fracture. The porous ceramic plates were held to the rock face by one of two methods. For rock number $1,1.27-\mathrm{cm}$ (1/2-inch) thick plexiglas was placed on top of the plates above the rock. The plexiglas was held down by all-threaded rods connecting to a second piece of plexiglas underneath the test block. Small blocks of wood were used to distance the second piece of plexiglas from the bottom of the rock (see Figure 1.3). For rock number 2 , 6.4-mm (1/4-inch) o.d. all-threaded rod cut into appropriate lengths was used to hold the plates down onto the top of the rock. Threaded couplings were used to tighten the rod against galvanized steel strips that were bolted to rock frame $B$. 


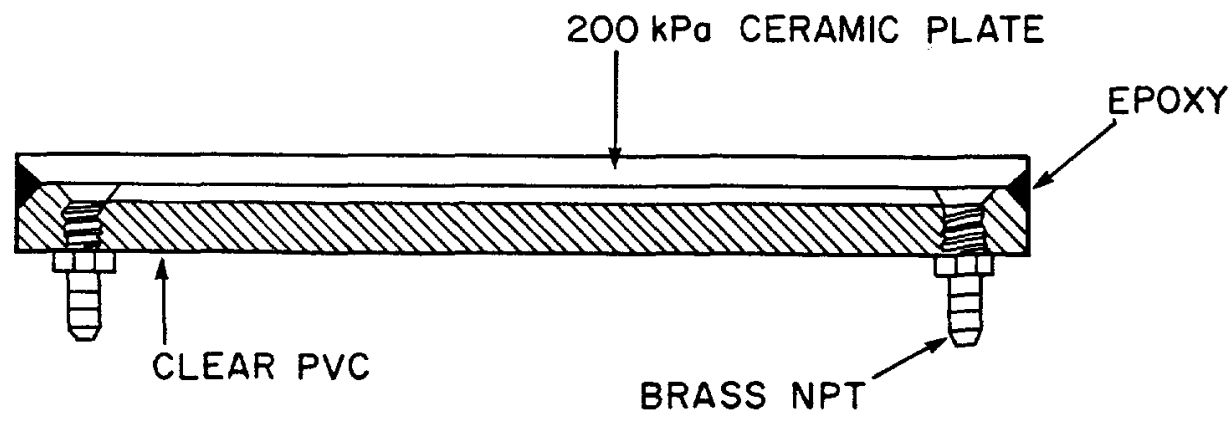

SIDE VIEW

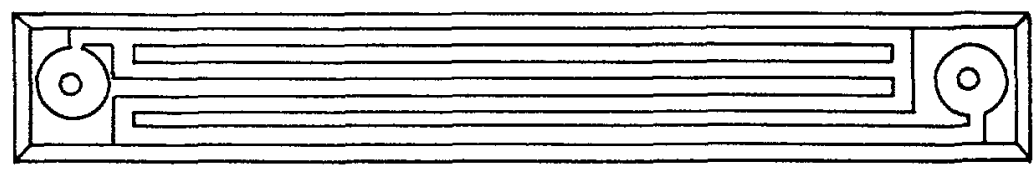

PLAN VIEW SHOWING GROOVES IN PVC

Figure 3.8 Porous ceramic plate. Length and thickness of all plates equals $20.2 \mathrm{~cm}$ and $0.7 \mathrm{~cm}$, respectively. Width of matrix plates equals $8.6 \mathrm{~cm}$, and width of plate covering fracture equals $3.0 \mathrm{~cm}$. 
Prior to use, each porous ceramic plate was tested for plate conductance (Appendix A, Procedure 3). Each porous plate was set up with tubing, a pipet flow tube, a manometer, and a Mariotte reservoir as described in the procedure for setting up the rock and supporting equipment. Instead of placing the porous plate on a rock block, it was placed horizontally in a plastic tub, ceramic side down. Enough solution was added to the tub to cover the plate. A second manometer measured the solution level in the tub. The elevation of the Mariotte reservoir was varied, and the flow rate through the porous plate was measured at least twice at each reservoir elevation. The conductance, which includes the plate hydraulic conductivity, area, and ceramic thickness, was then determined by plotting flow rate versus the total head drop across the plate.

\subsubsection{Head Control}

The constant-head reservoirs supplying test solution to the porous ceramic plates consisted of Mariotte reservoirs (Figure 3.3). Each reservoir was composed of a sealed one-liter nalgene bottle with a screw top. A solution tube exited the bottom of the bottle, and two tubes entered the top of the reservoir. One of the tubes remained clamped off when the bottle was in use and served as a solution refill tube. The other tube was open to the atmosphere and allowed air to bubble into the bottom of the reservoir. Prior to use, each reservoir was marked along the outside in $100 \mathrm{~mL}$ increments and was pressure tested up to $13.8 \mathrm{kPa}$ (2 psi).

Mariotte reservoirs maintain a nearly constant pressure head by balancing out the decreasing positive solution pressure at the bottom of the air entry tube with an increasing negative gas pressure in the air space above the solution. When the bottle is first filled, the solution pressure at the bottom of the air entry tube is at its highest, and the air pressure is at its most negative. As a small amount of solution drains from the reservoir, the solution pressure decreases slightly, an air bubble is induced to enter the reservoir through the open air entry tube, and the air pressure increases, maintaining a nearly constant pressure head over time. The pressure drop required to cause a bubble to enter the reservoir depends upon the size of the bubble or number of bubbles that enter the reservoir at a time, which in turn depends upon the type of air entry tube used.

Since the size or number of bubbles entering the Mariotte reservoir at a given time controls the pressure variation around the constant pressure that one is trying to maintain, it is best to have an air entry tube that produces a steady bubbling rate and a small bubble size. Various materials were tested as air entry tubes, and two were eventually used in the experiments. The first was used primarily with test block 1 and consisted simply of a $3.2-\mathrm{mm}(1 / 8$-inch) o.d. stiff plastic tube. The second type of air entry tube, used mainly with rock 2, contained a set of needles assembled in series. Stiff plastic tubing, as described previously, was glued to the bottle top, and two needles were joined in series from the tubing with appropriately sized tygon tubing.

During the experiments performed with the test blocks, each porous plate was supplied solution from a separate Mariotte bottle. This was required since each porous plate had a unique conductance. To vary the total 
head on the top of a porous plate, the Mariotte bottles were simply raised or lowered accordingly. A manometer was used to measure the pressure head, or total head if the manometer elevation is measured relative to the bottom of the plate, at the top of the plate. Once a flow rate through the plate was obtained (see below), the average pressure head at the bottom of the plate was calculated using Darcy's Law applied across the plate:

$$
\mathrm{h}_{\mathrm{p}}=\phi-\mathrm{Q} / \mathrm{C}
$$

where

$h_{p}$ average head at the bottom of the plate, $\mathrm{cm}$;

$\phi$ average total head at the top of the plate, $\mathrm{cm}$, if the manometer elevation is measured from the bottom of the plate;

Q flow rate, $\mathrm{cm}^{3} / \mathrm{min}$;

C plate conductance, $\mathrm{cm}^{2} / \mathrm{min}$.

\subsubsection{Flow Rate Measurement}

Two methods were used to measure flow into the test blocks. The first consisted of an air-bubble flow meter, or pipet flow tube (Appendix A, Procedure 4). This device was constructed using a 1.0-mL graduated pipet with a bubble entrance port and a bubble exit port attached on opposite ends of the pipet. The ports were constructed from nalgene or glass elbows, flexible tubing, and rubber septa. Flow rate was measured by injecting an air bubble through the entry port and monitoring its movement along the pipet.

In the course of testing the pipet flow tube, it was found that injection of an air bubble into the system temporarily raised the pressure head at the top of the plate. To lower the pressure back to the controlled pressure range, solution was extracted out of the bubble inlet tube once the bubble moved into the pipet. Enough solution was extracted to force an air bubble into the system through the air inlet tube of the Mariotte bottle, ensuring that the pressure in the system was in the range that the Mariotte reservoir would hold it.

On the average, a pressure drop of less than $5 \mathrm{~mm}$ across the test bubble was measured during flow measurement. To ensure that no solution was flowing around the test bubble in the pipet, producing faulty results, two tests were performed. In the first, dye was injected in front of the bubble and a flow test performed. Dye was injected behind the test bubble in the second test. In both cases, the dye did not move around the test bubble. These tests demonstrated that the test bubble moved at the same rate as the solution around it in the pipet and was thus an adequate method of measuring flow.

The second method of inflow measurement involved measuring the change in volume in the Mariotte reservoir. When flow measurements were made using the air bubble flow meter, the volume of solution in the reservoir was also noted. Since times and dates were also recorded, the average flow rate since the last measurement could also be calculated. 


\subsubsection{Water Potential Measurement in the Fracture and the Matrix}

Water potential measurement in the test blocks was made possible using a microtensiometer. This device was constructed of a porous ceramic cup ( $100 \mathrm{kPa}$ air entry value) which was epoxied to a short aluminum rod through which a hole was drilled. A stainless steel tube was slipped through the hole in the rod and epoxied in place. A thimble, of the type used in Soxhlet extractions, was epoxied along its base onto the end of the porous cup. The thimble was obtained from Whatman, Inc. of Clifton, New Jersey, and consisted of a 1-mm layer of cotton cellulose. The stainless steel tubing was slipped through a rubber stopper which contained a hole drilled through the center lengthwise. After the inside of the assembly was filled under vacuum with deaerated distilled water, it was joined to a pressure transducer (MICRO SWITCH 142PC15D) with a vacuum tubing attachment. A syringe was used to fill the pressure transducer port with deaerated distilled water prior to assembly of the tensiometer.

Prior to their use, both the pressure transducer and the microtensiometer required calibration. The pressure transducer was first calibrated by applying a partial vacuum to the low pressure port (Appendix A, Procedure 5). The vacuum was varied, and the transducer output was measured. A calibration curve was then prepared that minimized the error in the low pressure differential range. This was accomplished by determining an average zero differential output, subtracting this value from all of the readings, and then determining an average pressurecorrected output ratio.

The microtensiometer was calibrated by two methods. The first involved putting the cup of the instrument in test solution in a sealed chamber, decreasing the air pressure over the test solution, and measuring the transducer output. The second method involved placing the cup of the microtensiometer against a vertical porous plate, varying the suction head applied to the plate, and measuring the transducer output. To minimize evaporation, the porous plate was placed in partially sealed box. Sampling ports were installed in the box, allowing the tensiometer to rest solidly against the ceramic surface of the porous plate.

Calibrating and using the microtensiometer involved the use of a regulated power supply to provide a direct current to the pressure transducer, a precision voltage regulator, and a Hewlett Packard data acquisition unit to measure the voltage output from the pressure transducer. Additionally, since the pressure transducer used for this study required that the low pressure port remain dry, a hand-operated vacuum pump and associated tubing was necessary to impose a partial vacuum on the transducer. A detailed description of the type of equipment used is provided in Appendix A, Procedure 6.

Use of the microtensiometer in test fracture water potential measurement involved placing the instrument in a sampling port with the tensiometer tip against the fracture surface. The stopper was slipped into the sampling port until it held the tensiometer tip against the fracture. The pressure transducer output was monitored until a stable reading, or range of readings, was obtained. An average value was recorded. The tensiometer was then moved to another sampling port. Obtaining a matrix 
water potential from either the matrix sampling ports or the rock surface was more difficult and less precise. The matrix sampling ports were too short to allow the stopper to hold the tensiometer in place. Therefore, the tensiometer had to be held by hand which put abnormal pressure on the vacuum tubing connection between the pressure transducer and the stainless steel tubing. The readings obtained by this method varied more about the average value recorded at a given time than did those obtained when the tensiometer was held by the stopper.

\subsubsection{Displacement Transducers}

Linear variable differential transformers (LVDTs) were used to measure displacement perpendicular to the fracture plane. The electronics of the LVDT were contained in a stainless steel housing and were referred to as a coil, or coil assembly. A stainless steel core fit into a hole that ran lengthwise through the coil. The unit required a $D C$ input, and a DC output was yielded. The core, when displaced axially within the coil assembly, produced a voltage change in the output directly proportional to the core displacement from the electrical center of the coil. The polarity of the output voltage was a function of the direction of the core displacement with respect to the electrical center. The maximum working range of coil displacement from the electrical center was plus or minus $6.4 \mathrm{~mm}$ ( $1 / 4$ inch).

Prior to use, the displacement transducers required calibration. A detailed account of the calibration procedure is provided in Appendix A, Procedure 7. It involved installing two square-head aluminum posts into a scrap block of partially welded tuff, cut from test block 1 during the shaping process. The coil assembly of the LVDT to be calibrated was then secured in one post, and a micrometer that held the core was installed in the other post. Output voltage readings were then recorded for various core positions inside of the coil. The micrometer was used to measure the amount of movement of the core within the coil assembly. A calibration curve was then plotted, and the data were fit using the least squares method, yielding a displacement to voltage ratio for the tested coil assembly.

Two displacement transducers were used to monitor fracture movement in test block 1 . One was placed on each side of the block, $34.5 \mathrm{~cm}$ down from the top on face 3 and $12 \mathrm{~cm}$ down from the top on face 6 . Three LVDTs were installed in test block 2. Two were placed on face $3,11 \mathrm{~cm}$ and $52 \mathrm{~cm}$ down from the top. One LVDT was also installed $31 \mathrm{~cm}$ down from face 1 on face 5 . Frequent LVDT readings were taken throughout the experiments performed on each block.

\subsection{Test Block 1 Experiments}

Three types of tests were performed on the first test block. The first involved flowing test solution down the fracture only. The second test type consisted of an imbibition test using three porous plates. A steady state flow test was the third type of analysis performed on the first test block. A brief description of each test follows. 


\subsubsection{Fracture Imbibition Tests}

Two fracture imbibition tests were performed on the first test block. The first consisted of a simple fracture conductivity test. Prior to shaping the first block, a test was performed to determine if the proposed test fracture was conductive to solution movement. The rock was strapped to a pallet and positioned on a frame such that the fracture lay in a vertical plane. Moldable, caulking compound formed a surface reservoir, and a Mariotte bottle supplied tap water to the rock through tygon tubing. The water was dripped on the exposed fracture on the upper rock surface.

The porous plates for test block number 1 were not designed and ordered until after the block was shaped. After the porous plates arrived, the second fracture imbibition test was performed on the rock sample. The purpose of the second test was to determine whether a test solution would travel vertically downward through the test fracture when the solution was applied to the surface or whether horizontal solution movement and leakage out the side of the fracture would be a problem. To test this, the block was oriented such that the test fracture lay in the vertical plane, and a narrow porous plate was placed over the upper fracture surface. The plate had been previously saturated with deaerated test solution. Whatman filter paper number 42 was used to provide good contact between the porous plate and the rock surface. Deaerated test solution was supplied to the rock through tygon tubing from a 1 liter beaker; an air-bubble flow meter was used to measure the flow rate. Inflow rate and wetting front position were monitored through time. Initially, the solution reservoir surface was held at the same elevation as the top of the rock. The solution reservoir elevation was raised throughout the experiment.

Solution was applied and the wetting front monitored for 149 hours, at which time the block was subjected to analysis with the gamma attenuation apparatus. Rasmussen and Evans (1987) describe the gamma attenuation equipment in detail. Such analysis involves passing a monoenergetic gamma radiation beam through the test block. The reduction in intensity of the gamma beam can be related to either the water content or the bulk density of the sample. The effective diameter of rock that affects the gamma beam is about $1 \mathrm{~cm}$. To solve for water content:

$$
\theta=-\left[\ln \left(I / I_{0}\right)-\mathrm{x} \mu_{\mathrm{r}} \rho_{\mathrm{r}}\right] / \mathrm{x} \mu_{\mathrm{w}} \rho_{\mathrm{w}}
$$

where

$\theta \quad$ volumetric water content of the rock;

I measured beam intensity after attenuation by the test block;

Io source intensity;

$x$ thickness of the test block in the path of the gamma beam;

$\mu_{r}$ gamma absorption coefficient for the matrix of the test block;

$\rho_{r}$ dry bulk density of the test block;

$\mu_{w}$ gamma absorption coefficient for water;

$P_{w}$ density of the pore water. 
A 110 millicurie Cs -137 source was used in this analysis. It was enclosed in a $5-\mathrm{cm}$ thick lead shield. A sodium-iodide crystal gamma detector, and electronics to process and record the detector signal were also used. The test block was oriented such that the gamma beam was perpendicular to the fracture, and readings were taken at seven locations in the block.

\subsubsection{Three-plate Imbibition Test}

Test block number 1 was air dried for 102 days between the fracture imbibition tests and the whole-rock imbibition test. During this time, equipment was calibrated, further test blocks were obtained from the field, the laboratory was set up for testing on multiple blocks, and the sampling ports and LVDT post holes were drilled in rock number 1 . Ten days prior to beginning the three-plate imbibition test, a gamma beam attenuation analysis of the block was performed. The test block was positioned so that the gamma beam was parallel to the fracture; readings were taken at ten locations in the block, five on each side of the fracture.

The purpose of the three-plate, or whole-rock, imbibition test was to investigate the infiltration and percolation characteristics of the test fracture and surrounding matrix when test solution was applied to the test block at atmospheric pressure. In conjunction with this purpose, test instruments and procedures to perform such an experiment were also developed. The experiment involved standing the test block such that the test fracture lay in the vertical plane. Face 1 was the top face, and face 2 was the bottom face. A narrow plate was placed over the test fracture, and two wide plates were placed over the matrix on either side of the test fracture. A $500-\mathrm{mL}$ beaker of tap water was placed on the table top inside of the evaporation canopy to provide a source for maintaining saturated airspace in the canopy. Throughout the test, the Mariotte reservoirs were adjusted to maintain the pressure head at the bottom of each plate as close to atmospheric pressure as possible. Inflow rate, visual observations of the wetting front position, room temperature, and fracture displacement were monitored through time. Barometric pressure was not monitored in any of the tests.

Improvements were made in experimental procedures throughout the test. Manometers, which were not used at the beginning of the experiment, were added to the inflow tubing to monitor total head at the top of the plate. Improvements to the evaporation barrier were made, and an upgraded evaporation canopy frame was built. Minor adjustments in the flow rate procedure were also made.

\subsubsection{Steady-State Flow Test}

Once the matrix of test block number 1 was completely wet and solution was dripping from the face 2 , the steady state flow test was begun. The purpose of this experiment was to determine the saturated, or nearsaturated fracture transmissivity and matrix hydraulic conductivity of the test block. As in the previous experiment, test instruments and techniques were developed to perform the test. The flow test involved continued application of test solution through the configuration of plates used in the imbibition test. The constant-head reservoirs were 
adjusted to maintain the pressure head at face 1 near atmospheric pressure. Inflow rate was measured using both the pipet flow meters and the volume change in the Mariotte reservoirs. Outflow rate was monitored by collecting in beakers the solution that dripped from face 2 . As in the imbibition test, a beaker of water was placed inside of the evaporation canopy to saturate the airspace, minimizing evaporation from the test block. Room temperature and fracture displacement were monitored until the data acquisition unit failed to operate properly.

Improvements and additions were made in experimental procedures throughout the test, the most significant of which involved the construction and use of the microtensiometer. Two-thirds of the way through the steady-state flow test, the microtensiometer was built, tested, and calibrated. Frequent readings were made throughout the remainder of the test. The fracture sampling ports were the focus of the monitoring since the tensiometer could not be used in the short matrix ports.

At the end of the steady-state flow test, the evaporation canopy and frame B were disassembled. The test block was separated along the test fracture, and a transport analysis was performed along the fracture surface (see Section 4.6.2). The microtensiometer would have been used to obtain water potential measurements at various points along the fracture surface, but the pressure transducer malfunctioned as the final stage of the test was begun. Another student is currently studying the surface roughness characteristics of the separated fracture of test block 1 .

\subsection{Test Block 2 Experiments}

One experiment was performed on the second test block. It consisted of a three-plate imbibition test similar to the rock number 1 test. Due to time limitations, the wetting front was less than half way down the test block at the time this study ended. Other students are continuing the study and will present the remaining imbibition results in a future publication.

The three-plate imbibition test investigated the infiltration and percolation characteristics of the test fracture and surrounding matrix when test solution was applied to the rock at subatmospheric pressure. With rock number 2 , an added purpose in the experiment was to see at what applied suction head the fracture specific discharge decreased below the matrix specific discharge.

After the preliminary setup of the test block 2, the porous plates were attached to the top of the rock (face 1), and the imbibition test was initiated. One narrow plate was used to introduce test solution to the test fracture, and two wide porous plates provided solution to the matrix. A nearly constant negative pressure head, or suction head, of $15 \mathrm{~cm}$ was maintained along face 1 by adjusting the elevations of the Mariotte reservoirs. A beaker filled with water provided a saturated airspace inside of the evaporation barrier. Inflow rate, wetting front advance, fracture displacement, and room temperature were monitored throughout the experiment. Water potential in the fracture and matrix would have been monitored behind the wetting front, but the pressure 
transducer needed to quantify the amount of suction in the microtensiometer remained on backorder through this phase of the imbibition test.

Two methods were used to derive the relationship of fracture flow rate to matrix flow rate. The first involved visually comparing the influence of the fracture on the wetting front shape. It was assumed that if the wetting front did not protrude downward at the fracture relative to the matrix wetting front that the fracture specific discharge was less than or equal to the matrix specific discharge. The second method involved comparing the inflow rates to the fracture and matrix porous plates. If the average specific discharge of the fracture plate, defined as the volumetric flow rate, $Q$, divided by the cross-sectional area of the plate, did not exceed the average specific discharge of the matrix plate, it was assumed that the fracture specific discharge was less than or equal to that of the matrix.

\subsection{Rock Characterization Tests}

Rock samples cut from test blocks 1 and 2 during the shaping process were analyzed for pore size distribution, dry bulk density, effective porosity, and saturated hydraulic conductivity. Additionally, moisture release curves were prepared. A diamond-studded coring bit was used to obtain $5.65-\mathrm{cm}$ diameter cores for testing. Cores $1.2 \mathrm{~cm}$ in diameter were used in the mercury porosimeter. Rasmussen and Evans (1987) provide detailed procedures for all of the characterization tests but the mercury intrusion test. Vogt (1988) presents the mercury intrusion procedure used in this study. Brief summaries of the methods follow.

\subsubsection{Matrix Saturated Hydraulic Conductivity}

The saturated hydraulic conductivity was determined for six samples each from test blocks 1 and 2 using a modified Tempe pressure cell. The saturated sample was confined in an inflatable bladder, placed in the cell, and confined between 0 -rings on the top and the bottom. The bladder was inflated to $689 \mathrm{kPa}$ (100 psi). A precisely-regulated pressurizing system supplied nitrogen pressure above a test solution supply tank. Flow rate through the sample was monitored using a pipet flow meter installed at the Tempe cell exit port. The matrix saturated hydraulic conductivity, $K_{s}(m / s)$, was calculated from:

$$
\text { (3.21) } \quad \mathrm{K}_{\mathrm{s}}=\mathrm{QL} / \mathrm{A} \phi
$$

where

$$
\begin{aligned}
& \text { Q flow rate, } \mathrm{m}^{3} / \mathrm{s} ; \\
& \mathrm{L} \text { sample length, } \mathrm{m} ; \\
& \text { A sample area, } \mathrm{m}^{2} ; \\
& \phi \quad \text { total head imposed on the upper surface of the sample, } \mathrm{m}, \\
& \text { relative to total head imposed on lower surface. }
\end{aligned}
$$

The intrinsic permeability of the sample was determined from:

$$
\mathrm{k}_{\mathrm{w}}=\mathrm{K}_{\mathrm{s}} \mu / \gamma
$$

where $k_{w}$ is the intrinsic permeability, $\mathrm{m}^{2}$. 


\subsubsection{Matrix Moisture Release Curves}

Moisture release curves were prepared for six core samples retrieved from rock surrounding block number 1 and five samples from rock surrounding test block 2. Compressed nitrogen, supplied at a regulated pressure, was used to force solution out of sample cores that were placed on top of a porous ceramic plate inside of a pressure extractor. To start the test, saturated cores were weighed and placed in the pressure extractor. Nitrogen gas pressurized the container to $10 \mathrm{kPa}$. When equilibrium was reached, the samples were removed, weighed, and returned to the pressure extractor. The process was then repeated at pressures of $25 \mathrm{kPa}, 50 \mathrm{kPa}$, and $100 \mathrm{kPa}$.

\subsubsection{Matrix Dry Bulk Density and Effective Porosity}

The matrix effective porosity was determined by first oven drying the sample for at least 48 hours. The dry mass and volume were then measured, using a balance and calipers, respectively. After saturating the sample, the wet mass was measured. Effective porosity equals:

$$
\mathbf{n}_{e}=\left(\mathrm{m}_{\text {sat }}-\mathrm{m}_{\mathrm{dry}}\right) / \rho_{\mathrm{w}} \mathrm{V}
$$

where

$\mathrm{n}_{\mathrm{e}} \quad$ effective porosity, dimensionless;

$\mathrm{m}_{\text {sat }} \quad$ saturated sample mass, $\mathrm{kg}$;

$\mathrm{m}_{\mathrm{dry}}$ oven dry sample mass, $\mathrm{kg}$;

$\rho_{w} \quad$ density of water, $\mathrm{kg} / \mathrm{m}^{3}$;

$\mathrm{V}$ sample volume, $\mathrm{m}^{3}$.

Dry bulk density, $\rho_{\mathrm{b}}\left(\mathrm{kg} / \mathrm{m}^{3}\right)$, equals:

(3.24) $\quad \rho_{\mathrm{b}}=\mathrm{m}_{\mathrm{dry}} / \mathrm{V}$.

Six samples taken from rock number 1 were analyzed for dry bulk density and effective porosity. Ten samples from block 2 were tested.

\subsubsection{Matrix Pore Size Distributions}

Mercury porosimetry is based on the capillary equation discussed in section 3.1 above. A positive pressure which intrudes the non-wetting fluid, mercury, into rock pores represents a negative pressure which forces the wetting fluid, water, from rock pores. In general, an ovendried pre-weighed sample core was evacuated in a Micromeritics Pore Sizer, model 9310, and the sample chamber gravity-filled with mercury. In two stages, the sample was then intruded with mercury. This was accomplished by reducing the vacuum in the first stage and by applying hydraulic pressure to the sample chamber stem in the second stage. These steps forced mercury from the stem into the pores of the rock sample. At each measurement step during the intruding process, the capacitance of the sample chamber stem was recorded. The capacitance thus recorded was later converted to a volume of mercury that had left the sample chamber stem and entered the sample pores for a given applied 
pressure. Pore size distributions were determined for 5 core samples from test block 1 and 6 core samples from block 2 .

\subsection{Results and Discussion}

This section discusses the results of the imbibition and steady-state flow tests using test blocks 1 and 2. Also included are discussions of the equipment calibrations and the rock characterization analyses. All tables referred to as "B." in this chapter appear in Appendix B.

\subsubsection{Equipment Calibration Results}

Table B.1 presents the results of the porous ceramic plate conductance tests. Table 3.1 summarizes those results. Volumetric flow rate was plotted versus the total head drop across the plate, and the least squares method was used to fit the data with a straight line. The slope of the best-fit line was taken as the plate conductance. Findings from a typical conductance test are provided in Figure 3.9. As seen in the case of porous plate number 2, a straight line fits the data we11. The sample coefficients of determination $\left(r^{2}\right)$ show a good straight-line relationship and range from 0.966 to 0.995 for the six plates tested. Factoring out the thickness and area of the plate yields the hydraulic conductivity of the porous ceramic used in the plates. For the six plates used in the study, the hydraulic conductivity varied from $1.8 \mathrm{x}$ $10^{-9} \mathrm{~m} / \mathrm{s}$ to $6.6 \times 10^{-9} \mathrm{~m} / \mathrm{s}$. The mean value was $4.0 \times 10^{-9} \mathrm{~m} / \mathrm{s}$.

Table 3.1: Plate Conductance Results.

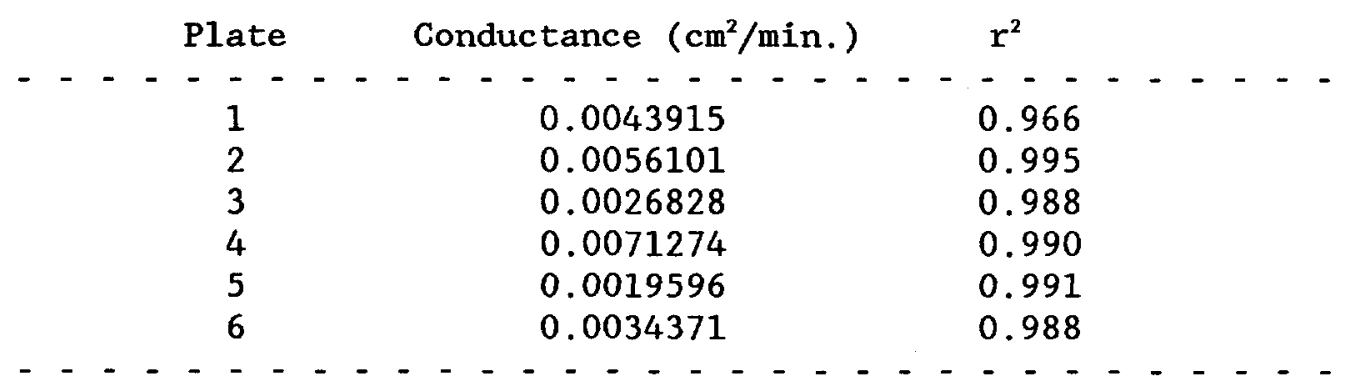

The method used to obtain the plate conductance data contains a number of sources of variability. First, is the measurement of pressure head. In this method, pressure head is measured using a manometer and a meter stick, graduated in millimeters. Potential errors include a variable meniscus due to dirty manometer tubing and incorrect sighting of the meniscus location with a hand level. In addition, the pressure head on top of the porous plate fluctuates with time due to pressure variation in the Mariotte reservoir. All effects combined, the probable variation in the pressure head reading is plus or minus $0.5 \mathrm{~mm}$. A second source of variation lies in the flow rate reading itself. Starting and stopping the stopwatch as the test bubble crosses the pipet graduations leads to variability in the time recorded. Variations from this source are difficult to quantify, but a rough estimate might be plus or minus 0.5 percent of the flow rate measured. From equation 3.19 , one can see that the pressure head calculated at the bottom of the porous plate is 


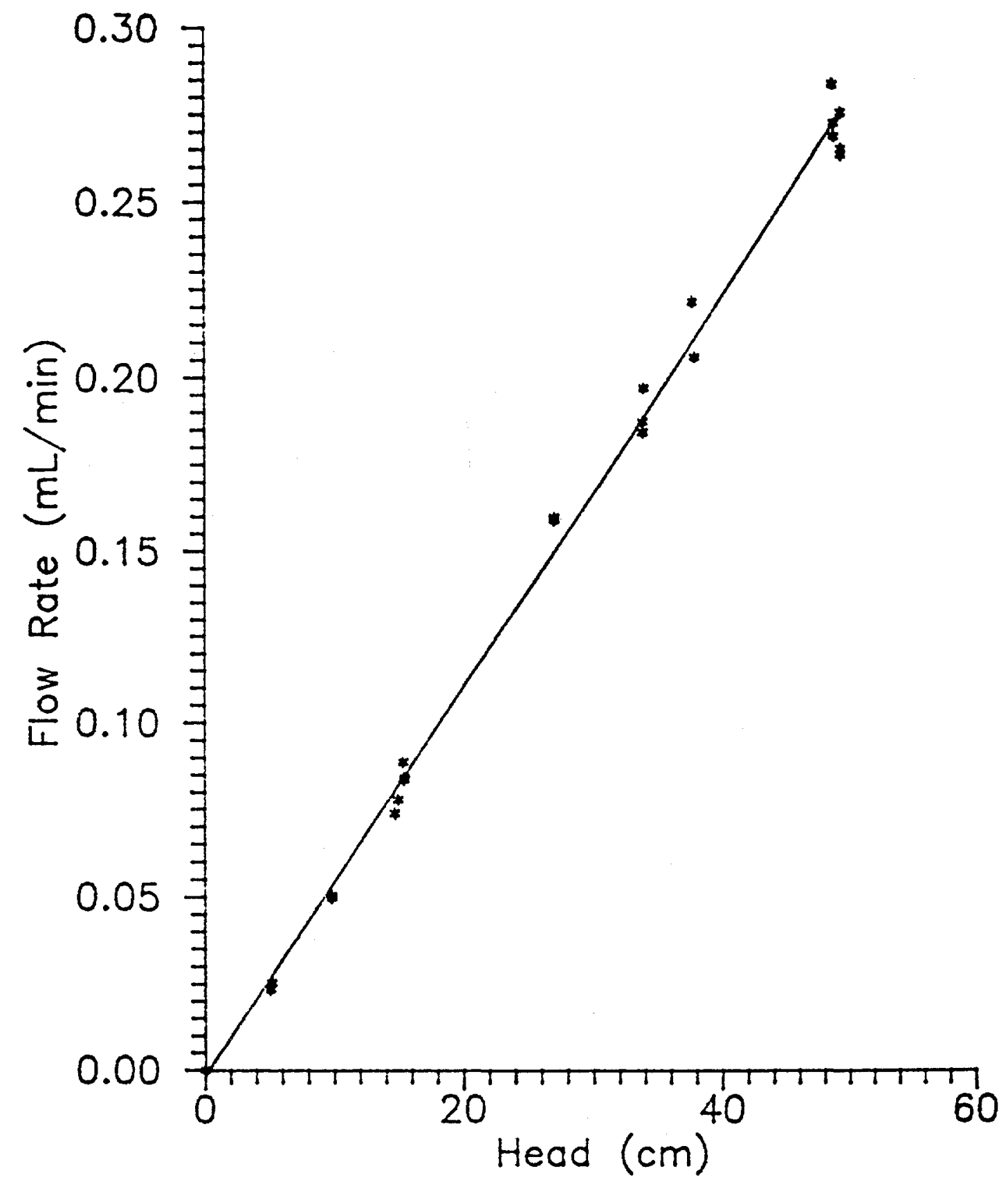

Figure 3.9 Results of a conductance test performed on porous ceramic plate number 2. Head refers to total head drop across the plate. 
highly sensitive to the value of the plate conductance used in the equation. Although it is essential to obtain accurate plate conductances, the variability in the slope of the plate conductance curve due to the aforementioned sources is difficult to quantify. As these errors are not systematic, the effects of variability in flow and pressure measurements of one point may cancel such variability of another point.

After the first set of plates was removed from test block number 1 , they were cleaned with an ultrasonic bath. Plate conductances determined for one of the plates before and after cleaning were the same, and plate clogging was not considered throughout the rest of the study. However, in work performed after the completion of this study, it was found by another student that reduction in plate conductance of up to 10 percent may take place after a plate has been idle for a few months in a solution bath.

Tables B. 2 through B. 7 provide the pressure transducer and microtensiometer calibration results. Two methods were used to analyze the pressure transducer output. The first involved plotting the imposed pressure differential versus output voltage and, using the least squares method, fitting a straight line to the data. The second, as described in section 3.5.4, involved minimizing the error in the low pressure differential range, which was the principal range in which the transducer was used. The second method, for sake of discussion, is referred to as the J.B. method. The results in Tables B. 2 and B. 3 show that the J.B. method of analysis provides a lower mean squared percentage error, due to the smaller percentage error for low pressure differential measurements.

Of the two methods used to calibrate the microtensiometer, the method employing the porous ceramic plate provides more usable results. Tables B. 4 and B.5 show that, after considerable start-up difficulties, the microtensiometer readings obtained in the chamber method of calibration were within about $0.5 \mathrm{~cm}$ of suction head of the suctions applied to the chamber. However, Tables B. 6 and B.7 indicate that a correction factor of $2.88 \mathrm{~cm}$ of suction is required to compare tensiometer readings with suction heads present in the porous plate against which the tensiometer was placed. The correction factor was used to obtain fracture or matrix suctions during analysis of test block 1 . In summary, the results of the microtensiometer calibration indicate that 90 percent of the microtensiometer readings, after application of the correction factor, should be within about $1 \mathrm{~cm}$ of the actual water potential. This neglects any variations which are possible in measuring the suction imposed on pressure transducer port P1. Another, perhaps easier, method of calibrating the microtensiometer would be to eliminate the pressure transducer calibration and employ the porous plate method, subsequently plotting applied pressure versus output voltage. The slope of a straight line fit to the data would then be used to translate voltage output from the tensiometer to water potential.

Calibration of the LVDT units was simple and straightforward. Table B.8 provides the raw data and the least squares regression results. Figure 3.10 shows a typical plot of micrometer readings versus LVDT output. Table 3.2 summarizes the calibration results. The smallest amount of movement that could be detected by the LVDTs was not determined. Dis- 


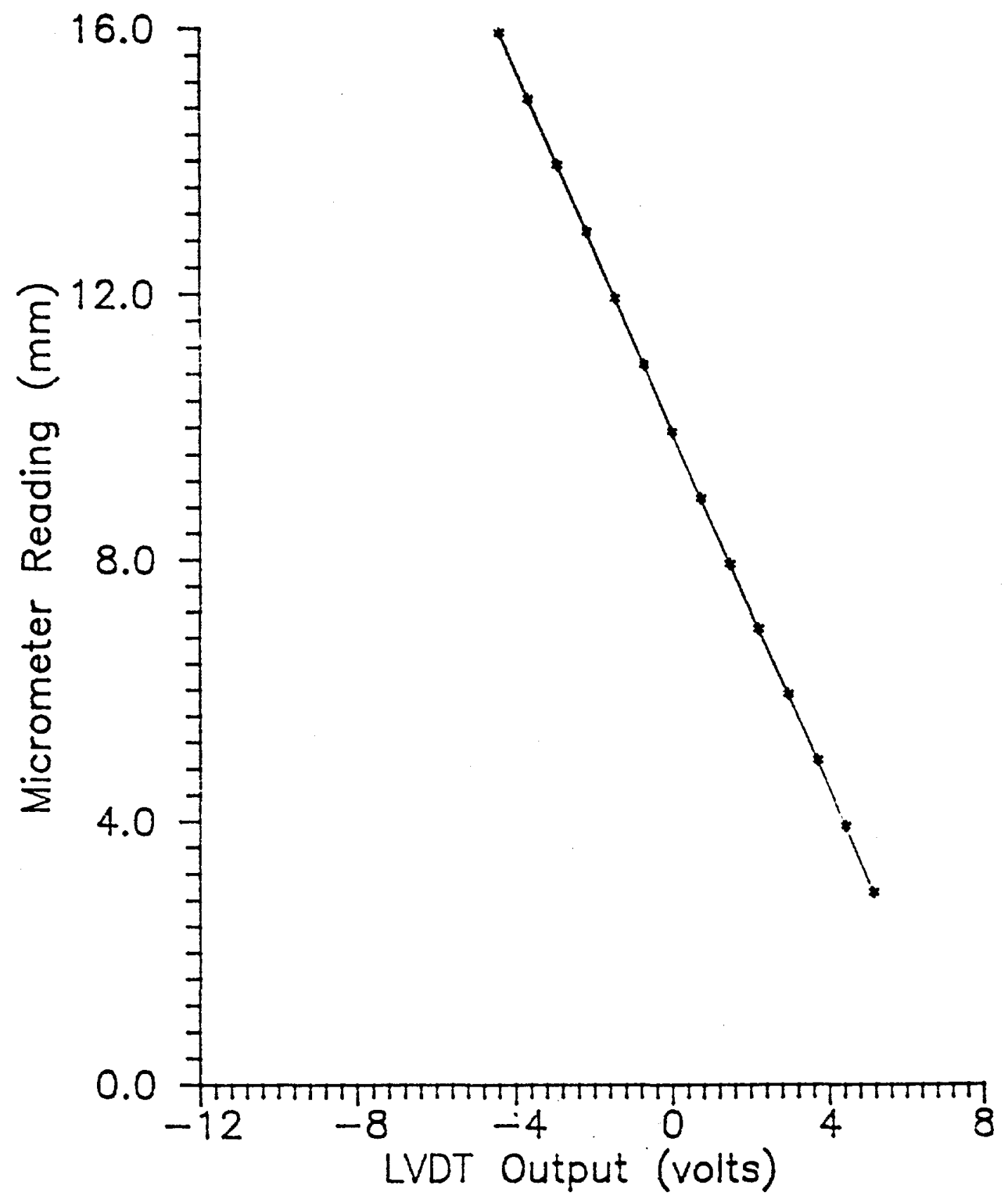

Figure 3.10 Calibration results of LVDT number 2 . 
placement transducer 6 was tested twice on different days, and virtually the same results were obtained. Voltage output from the LVDTs increases as the core is pushed into the coil assembly. Therefore, an increase in LVDT output indicates fracture closing, and a decrease in LVDT output shows fracture opening.

Table 3.2: LVDT Calibration Summary.

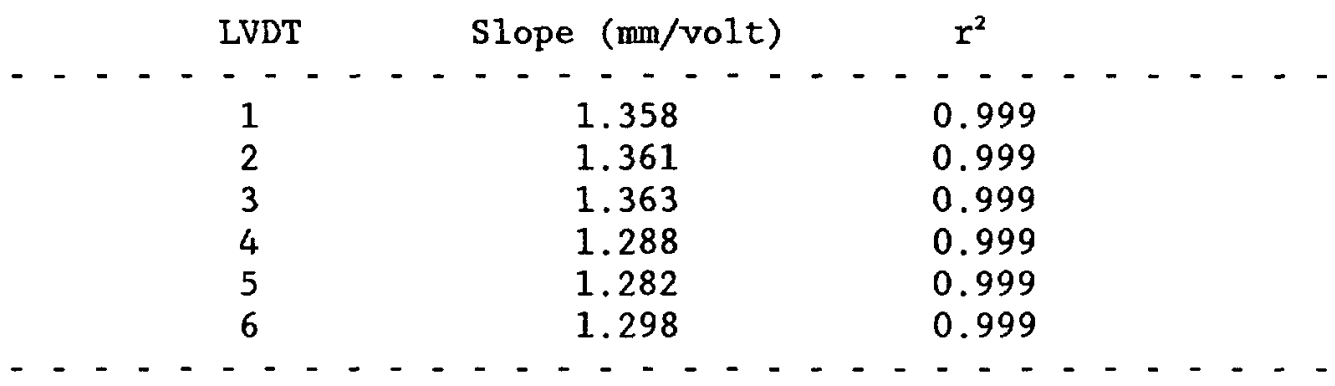

\subsubsection{Test Block 1 Experiments}

The results of experiments conducted using test block 1 are discussed below, including the fracture imbibition tests, the three-plate imbibition tests and the steady-state flow test. Modeled results are included in section 3.9 .2 .3 .

\subsubsection{Fracture Imbibition Tests}

In the first test fracture imbibition test, tap water was dripped on the exposed fracture trace on top of the rough test block. When dripped in such a manner, water immediately flowed into the fracture. The drip rate was held at about $3 \mathrm{ml} / \mathrm{minute}$ for 100 minutes at which time the rate was increased tenfold. Water began flowing from the bottom of the fracture shortly after the drip rate increased. This test indicated that the fracture was conductive to water and that the rock was suitable for experimentation. After this initial test, the test block was shaped to its test dimensions.

The second fracture imbibition test consisted of applying test solution to the top of the fracture trace through a narrow porous plate.

Initially, the surface of the reservoir supplying solution to the plate was held at the same elevation as the top of the rock. Fracture flow was monitored by visually inspecting the wetting front. If the wetting front contained a significant lobe protruding downward along the fracture trace, it was assumed that solution was flowing through the fracture at a greater rate than through the matrix. After 21 hours of applying solution, no fracture flow was observed, and the reservoir surface was raised to an elevation $10 \mathrm{~cm}$ above the top of the rock. Four hours later, the reservoir was raised to an elevation $20 \mathrm{~cm}$ above the upper rock surface. It was maintained at this elevation for 21 hours, and since no fracture flow was observed, the system was disconnected. 
It was suspected that the conductance of the porous plate was high enough that the pressure head at the top of the rock was negative. The plate conductance, with the filter paper in place, was roughly measured, and the system was reconnected. At that point, the reservoir surface elevation was placed at $26 \mathrm{~cm}$ above the upper rock surface. At $t=73$ hours the average pressure head calculated at the bottom of the porous plate was still less than atmospheric. Based on this, the solution supply surface was raised to $42 \mathrm{~cm}$ above the top of the rock. Five hours later, after similar calculations, the reservoir was raised to an elevation of $51 \mathrm{~cm}$. Solution movement had been noted in the fracture by the movement of the wetting front an hour earlier. This movement continued on one side of the block. No fracture flow was noted on the other side of the rock. Solution was applied and the wetting front monitored for three more days, bringing the total experiment time to 149 hours, at which time the block was subjected to analysis with the gamma ray apparatus. Table 3.3 presents the gamma ray results, with the distance, $z$, measured from the top of the block. At that time, the wetting front had advanced between $11 \mathrm{~cm}$ and $28 \mathrm{~cm}$ down the sample block, averaging about $20 \mathrm{~cm}$. Average pressure head at the bottom of the plate increased from $-37 \mathrm{~cm}$ at the beginning of the test to $8 \mathrm{~cm}$ at the end of the test. Since only one porous ceramic plate was used to supply test solution to the sample block, test block flow parameters could not be determined.

Table 3.3: Gamma Beam Attenuation Results, Post Fracture Test.

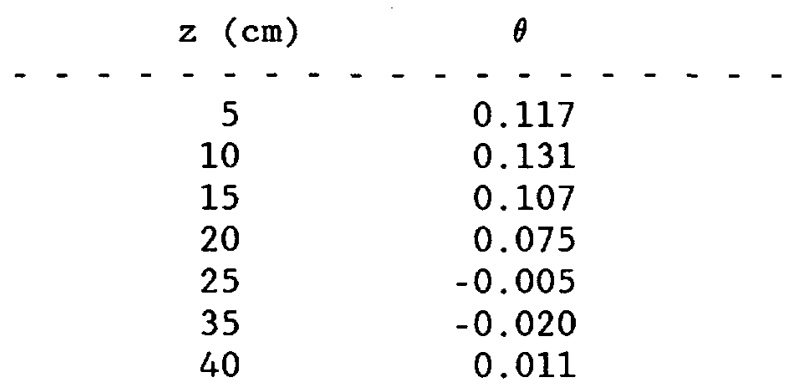

From the initial tests, it was determined that leakage from the side of the fracture did not present difficulties during analyses of flow through the fracture and matrix of the sample. Additionally, it was found that fracture flow did not occur until the suction at the porous plate-fracture interface was less than that required to allow filling of the fracture. Some sand-sized rock fragments had been plucked from the top of the fracture during the shaping process, and it was suspected that hydraulic contact between the porous plate and the fracture was not adequate. During the subsequent tests, filter paper pulp was placed in the top of the fracture to aid contact between the porous plate and the fracture. Based on the preliminary analyses, it was decided that a three-plate imbibition test would be performed on a partially dry test block. 


\subsubsection{Three-plate Imbibition Test}

Ten days prior to beginning the whole-rock imbibition test, the test block was subjected to gamma attenuation analysis. Table 3.4 presents a summary of the water content values determined at various points throughout the block. Despite the preparations performed on the test block, nearly all of the water in the test block had evaporated prior to the start of the imbibition experiment. Negative values in the table result from probable local variations in dry bulk density and reflect the minimum amount of error to be expected in the results.

Table 3.4: Gamma Beam Attenuation Results, Prior to Whole-rock Test.

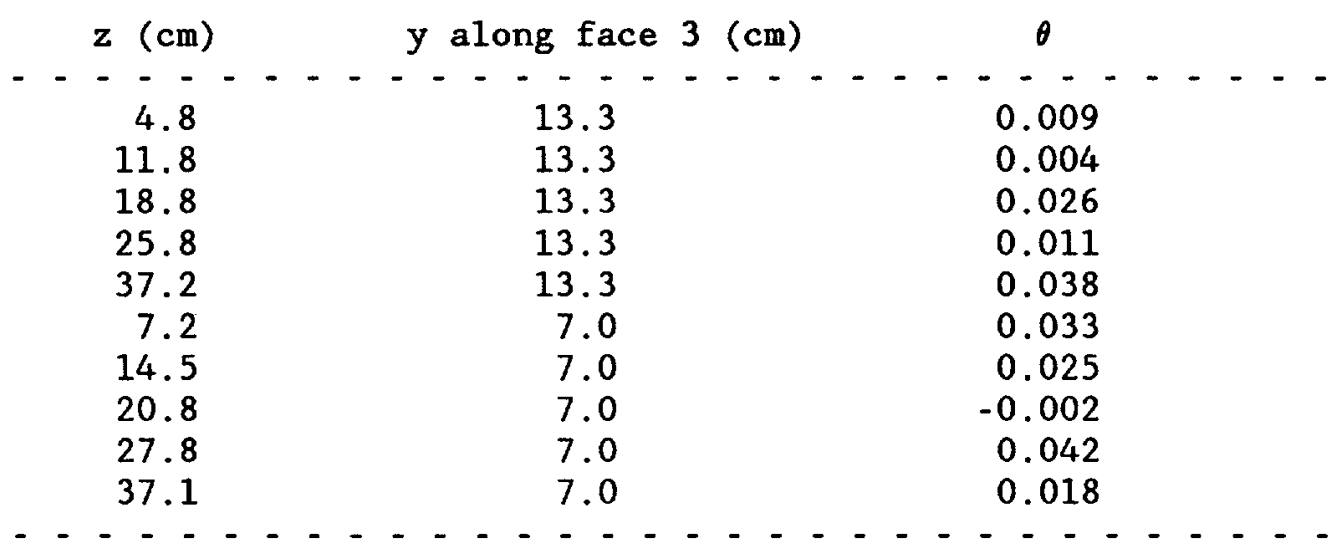

Seven days prior to the start of the test, monitoring of the displacement transducers was begun. Table B.9 provides the LVDT data collected during the imbibition and steady state flow tests. Figure 3.11 presents a plot of the LVDT output versus time. The large break in the data through the middle of the imbibition test occurred because the electronics ceased to function correctly and required repairing. As soon as the repairs were made, the system was reconnected, and final readings were taken. From Figure 3.11, it can be seen that the displacement transducer output varied throughout the test. Around the beginning of the imbibition test, $179 \mu \mathrm{m}$ of fracture closure were indicated in a short period of time by LVDT 3 . This suggests that during the placement and securing of the porous plates movement in the fracture took place or that LVDT 3 was bumped at the time. By about 20 days into the imbibition test, the LVDT readings had stabilized. Overall, output from LVDT 2 dropped about 0.163 volts, indicating an opening of about $222 \mu \mathrm{m}$ in the fracture aperture by the end of the test. Output from LVDT 3 increased 0.1 volts during the imbibition test, representing a fracture closure of $136 \mu \mathrm{m}$. Given that only 5 foot pounds of torque were applied to the bolts holding the test fracture together, it is likely that the fracture movement indicated by the displacement transducers occurred due to lack of sufficient confining pressure normal to the fracture. 
+++++ LVDT $3(1.363 \mathrm{~mm} /$ volt $)$

$\Delta \Delta \Delta \Delta \Delta$ LVDT 2 (1.361 mm/volt)

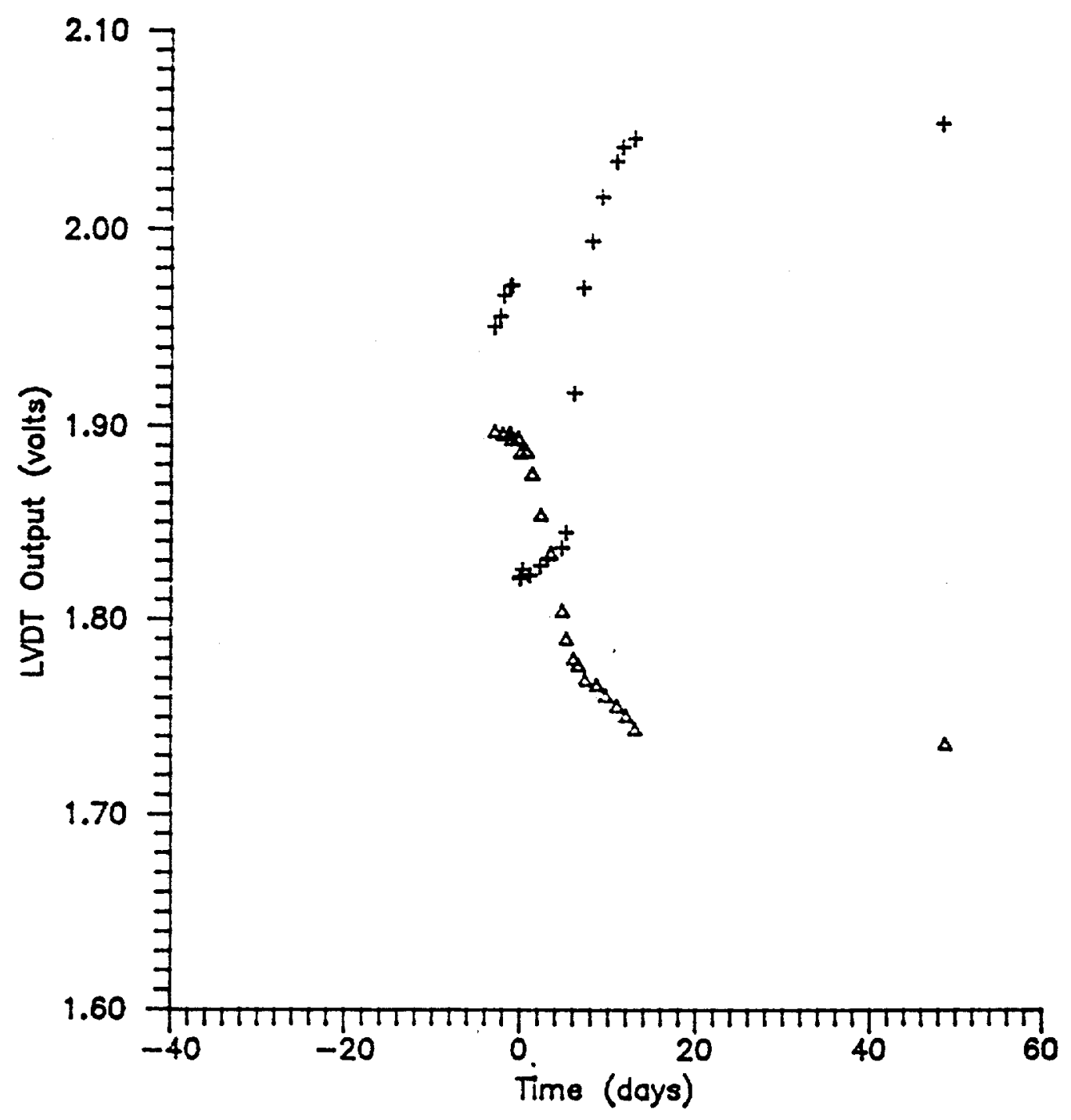

Figure 3.11 Monitoring of fracture aperture change in test block 1. 
The flow rates obtained using the pipet flow tube taken through the imbibition test are provided in Table B.10. Reliable mariotte reservoir volume measurements were not made during the imbibition test. A graphical presentation of the flow through each plate and the pressure head maintained at the bottom of the plates appears in Figures 3.12 to 3.17 .

Significant fracture flow was noted throughout the early portion of the imbibition test. Up through three days after the beginning of the test, the wetting front extended up to $20 \mathrm{~cm}$ farther down the fracture trace than down the matrix near the edges of the block. The wetting front lobe along the fracture trace was more pronounced on face 6 than face 3 . Although the all-threaded rods extending through the rock were tightened to the same torque, the fracture aperture may have been greater near one face than the other. Sand grains, other debris in the fracture, or loosened rock fragments may have prevented even tightening of the fracture over its entire area. Small wetting front lobes were observed along fracture splays on face 3 . One such fracture, which terminated in the matrix, acted as a solution conduit to the matrix along its length.

After two days of solution flow into the test block, test solution was observed along the upper portions of the fracture traces on faces 3 and 6. After three days of flow, test solution was seen flowing from one of the plates covering the rock matrix down the outside of the rock along face 6 . The effects of this external flow were noted in subsequent days as the wetting front wrapped around the bottom of the test block (face 2 ) and up face 3. Possible reasons for the external flow include poor contact between the plate in position 1-C, a rough fracture surface that channeled flow to the edge of the test block, and a sloping top surface of the test block. Most likely, a combination of these three mechanisms acted to channel test solution over the edge of the test block rather than allowing the solution to infiltrate. In an attempt to improve the plate-rock surface contact, all three plates were replaced 33 days into the test. After seven days of flow, solution began to drip from the fracture along face 2. It was collected in beakers placed beneath the test block. Interpretation of the solution volumes that were collected was hampered by the intermittent leakage of solution both from beneath one of the matrix plates and out of the fracture on face 6 . The volume of solution collected beneath the test block was always less than the inflow volume, however. Nine days into the test, the surface of the test block was completely moist.

As seen in the tables and figures, flow rates decreased with time during the earliest portion of the imbibition test. This was most noticeable for plates covering rock positions $1-A$ and $1-C$. The narrow fracture plate showed a general decrease in flow rate with time during the imbibition test; however, the flow rates through this plate varied somewhat from that trend over short time intervals. Figures $3.12,3.14$, and 3.16 show that about 35 days after the infiltration test was begun, steady state flow was achieved. The measured inflow rates and thus the average pressure head calculated at the bottom of the plate varied considerably in the test. It was expected that after a fairly steady imbibition rate was achieved both the inflow rate and the pressure head would stabilize throughout the remainder of the imbibition test. From Figures 3.12 through 3.17 , it can be seen that although these measurements fluctuated 


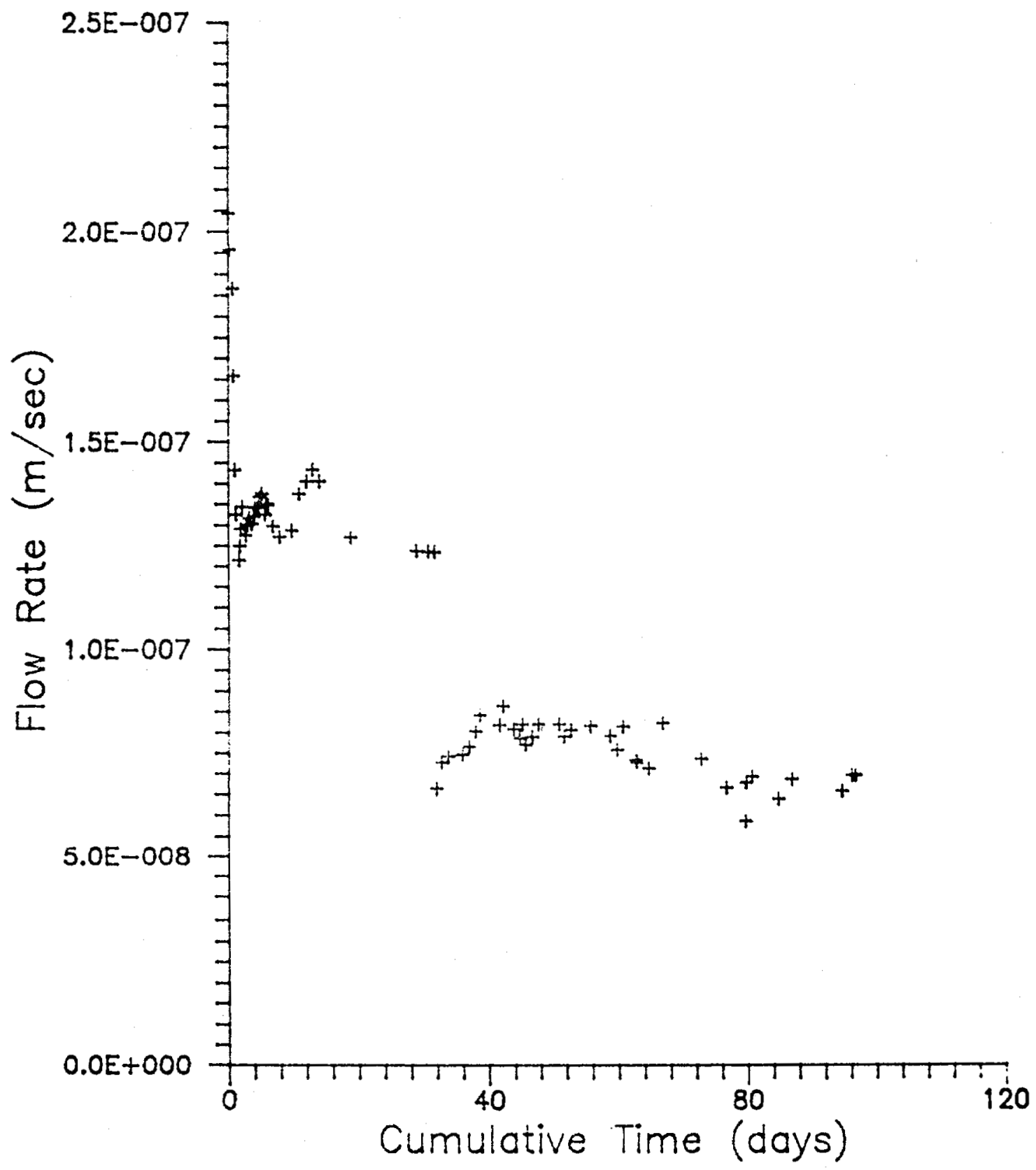

Figure 3.12 Inflow to plate position 1 -A, test block number 1 . 


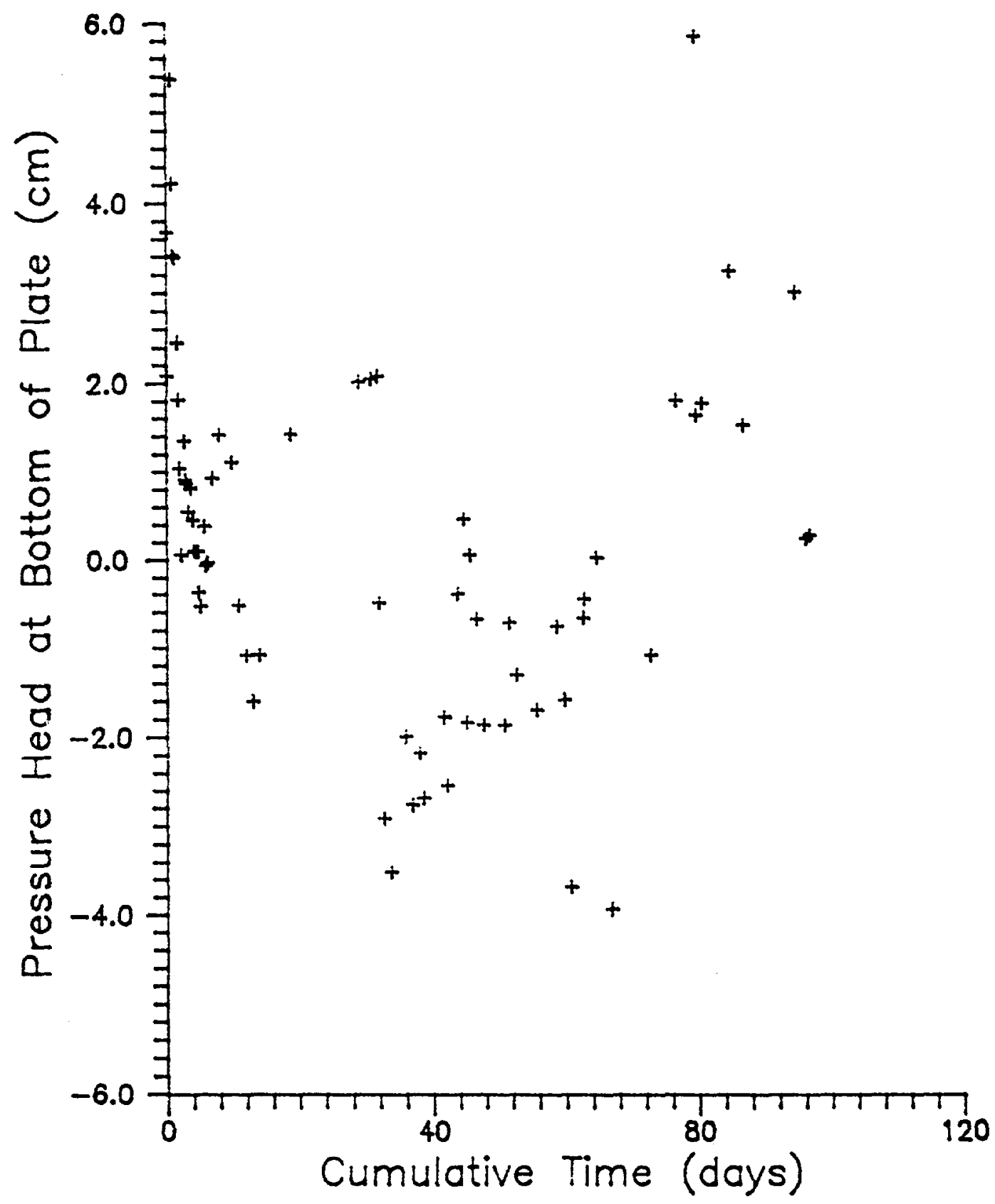

Figure 3.13 Average pressure head beneath the plate located over position 1-A, test block number 1 . 


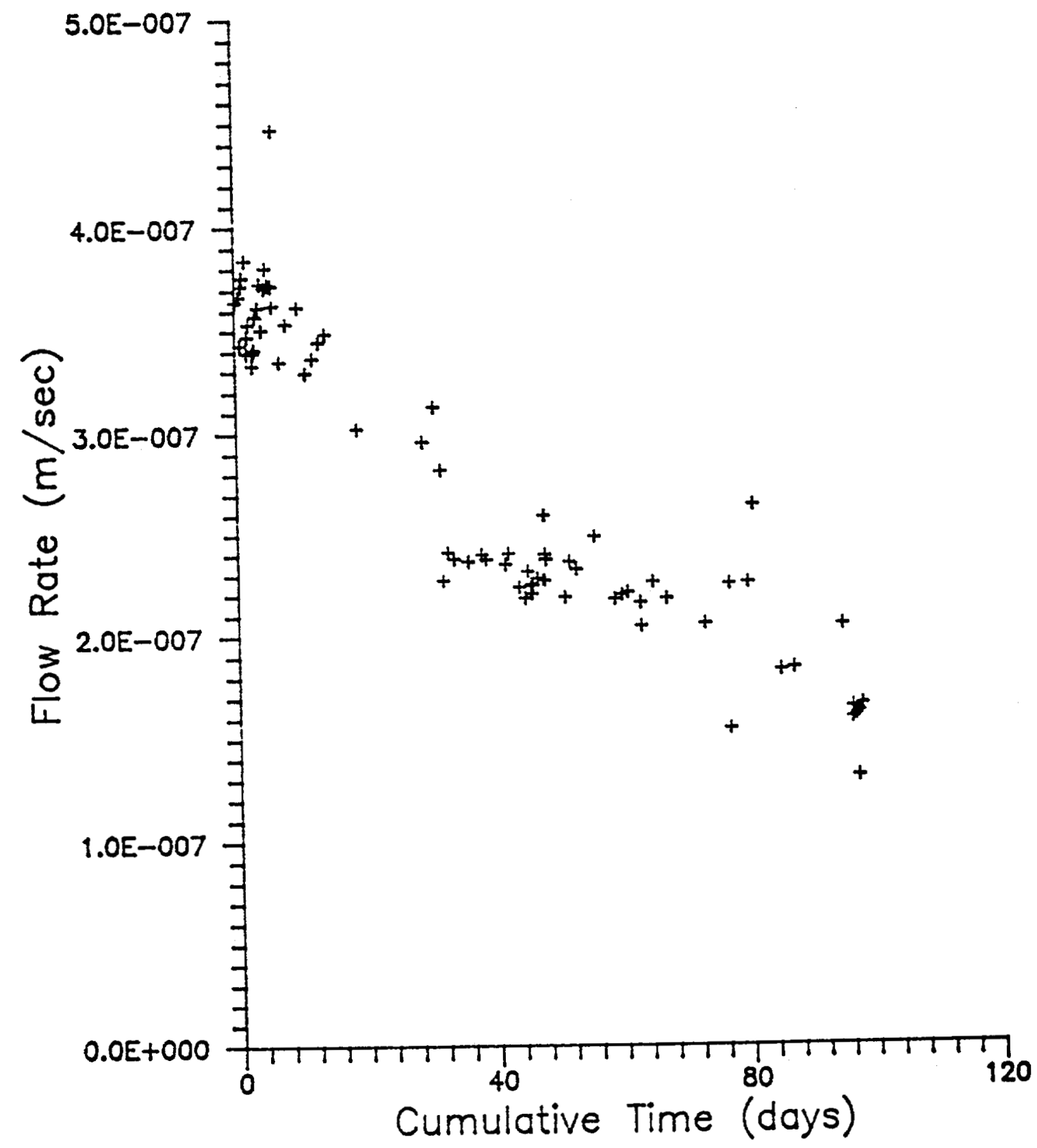

Figure 3.14 Inflow to plate position 1-B, test block number 1 . 


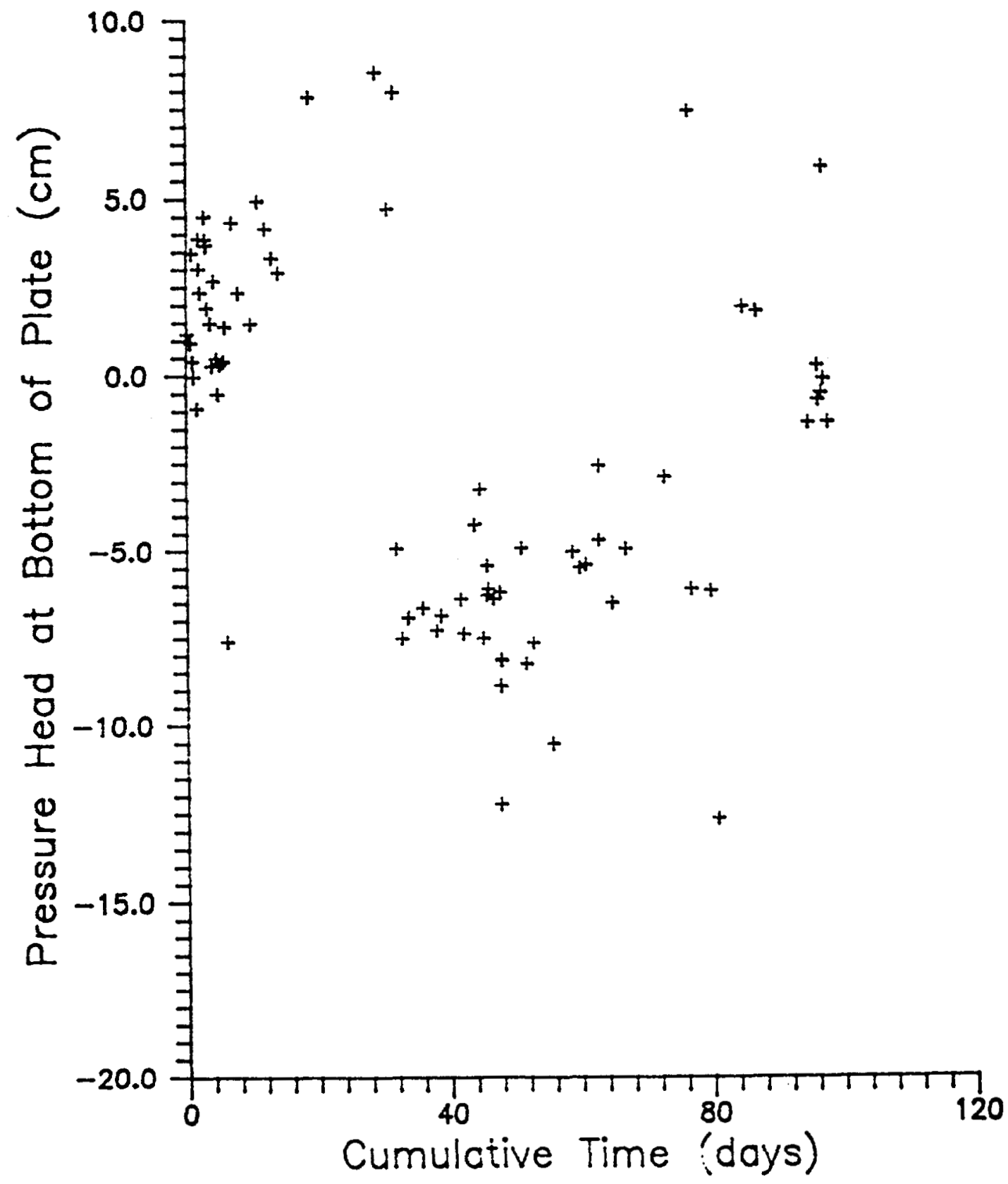

Figure 3.15 Average pressure head beneath the plate located over position 1-B, test block number 1 . 


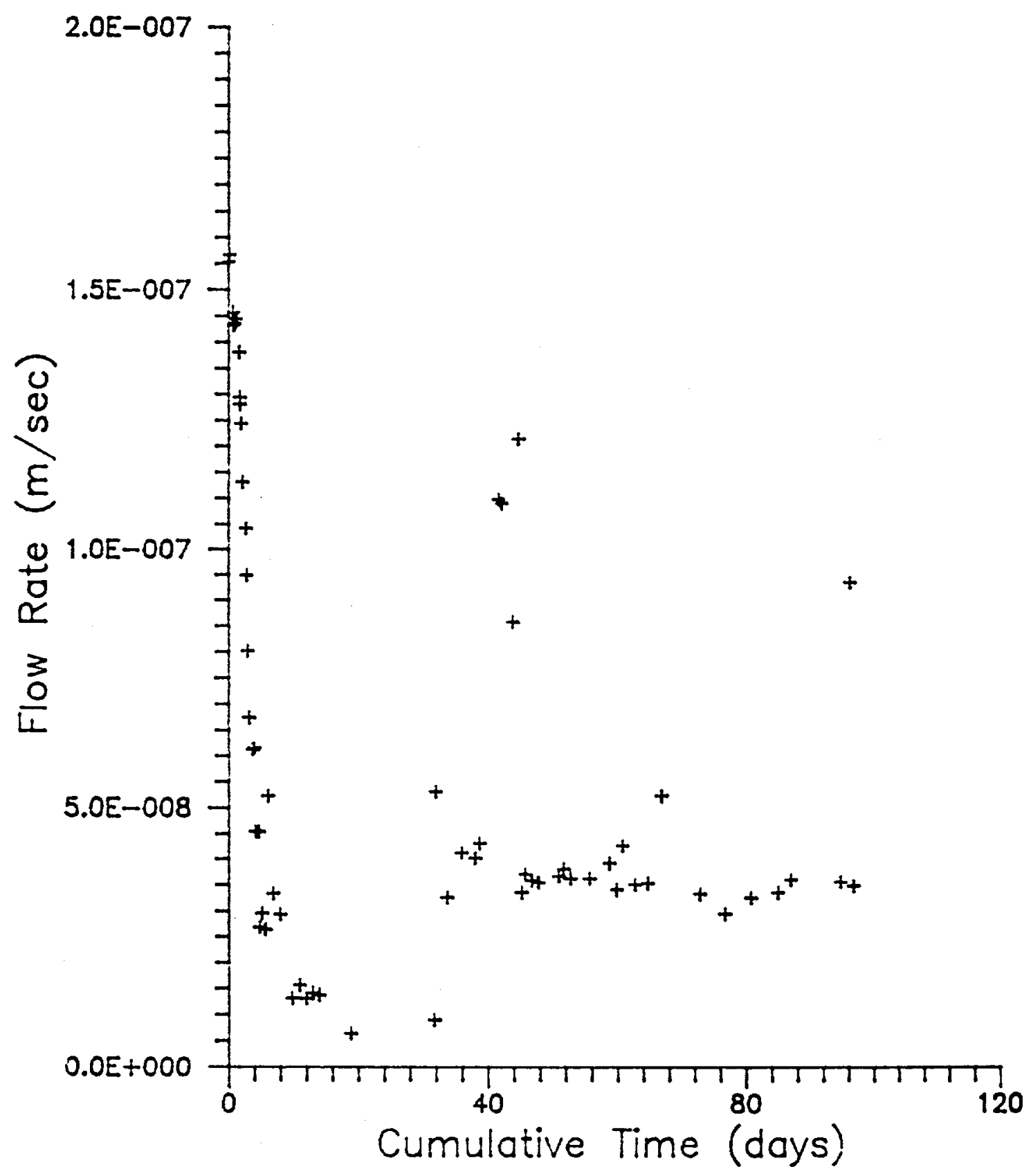

Figure 3.16 Inflow to plate position $1-C$, test block number 1 . 


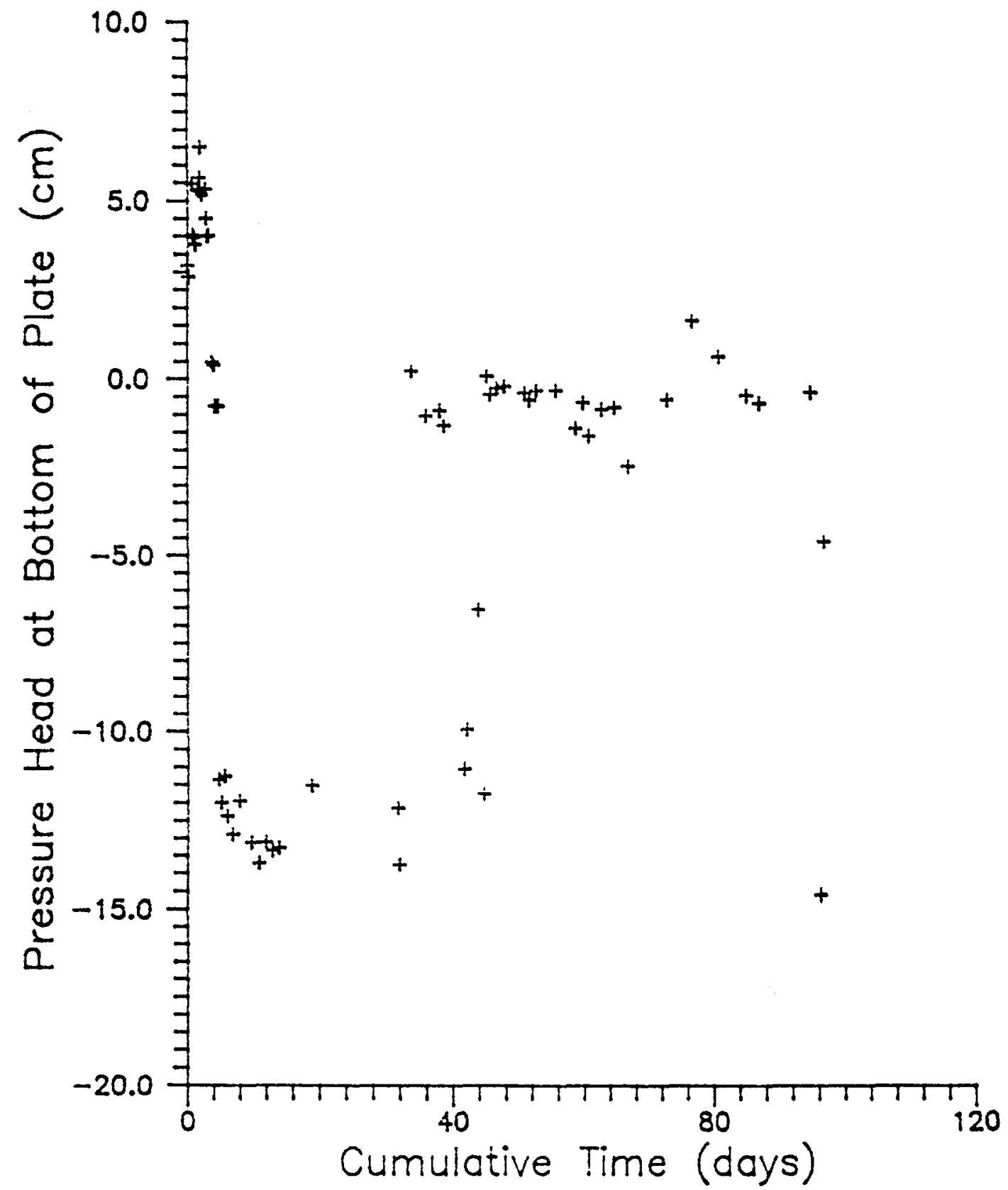

Figure 3.17 Average pressure head beneath the plate located over position 1-C, test block number 1 . 
throughout the test, only the data obtained from the plate positioned over the fracture trended significantly after the early days of the test.

Equation 3.19 shows the relationship between $\phi$, pressure head, and flow rate. As flow rate decreases with time during the early stages of infiltration, the calculated $h_{p}$ increases. The Mariotte reservoir is lowered, and both $\phi$ and $Q$ in equation 3.19 change. How these variables change depends on how the matrix and fracture imbibe solution and interact with each other. Based on calculations using equation 3.19 , the Mariotte reservoirs were raised or lowered, with the intent of maintaining a constant average $h_{p}$ across the top of the test block. Much of the variation in both flow rate and $h_{p}$ can be explained as a result of attempts to maintain a constant pressure head along the top of the test block. External leakage of solution also contributed to variation in measured flow rates and calculated pressure heads.

\subsubsection{Steady-State Flow Test}

As implied above, the three-plate imbibition test and the steady-state flow test ran sequentially with no break in between tests. Results of the steady-state flow test are presented in Table B.10 and Figures 3.12 through 3.17. LVDT output for the steady state test is presented above in Table 3.3; the output remained steady through the latter stages of the infiltration test and throughout the steady state flow test. Flow through plate positions $1-\mathrm{A}$ and $1-C$ was the most stable; the narrow plate in position 1-B decreased in flow rate slightly over the steadystate portion of the test. Possibly, the fracture drained slightly over the course of the test or less leakage from the side of the fracture occurred during later stages of the steady-state test. The sudden increases in flow rate for plate position 1-C occurred when solution leaked over the edge of the rock and ran down face 6 .

Outflow solution from the bottom of the test block was collected throughout the steady-state flow test. A mass balance was routinely performed. It indicated that, in general, the outflow was about 95 percent of the inflow. Since direct solution volume extractions for transport analysis or microtensiometer measurement were insignificantly small, the solution loss was probably due to evaporation. Despite attempts through the test to improve the evaporation canopy surrounding the test block, sampling and water potential measurement required opening of the canopy, increasing the airflow around the test block.

The microtensiometer was first employed on the seventy-fifth day after the three-plate imbibition test was begun, and it was used until the steady-state test was completed. Output from the pressure transducer was allowed to equilibrate before a reading was recorded. Equilibration time varied from 15 minutes to 90 minutes. To calculate the water suction potential, the pressure transducer output was multiplied by the pressure-voltage ratio developed in the J.B. calibration method. The correction factor obtained in calibrating the microtensiometer with a porous plate was then applied to the initial water potential estimate, yielding the corrected suction. Tables B.11 and B.12 present the microtensiometer data, and Table 3.5 summarizes the results in each of the sampling locations. 
Table 3.5: Test Block Number 1 Water Potential Measurements.

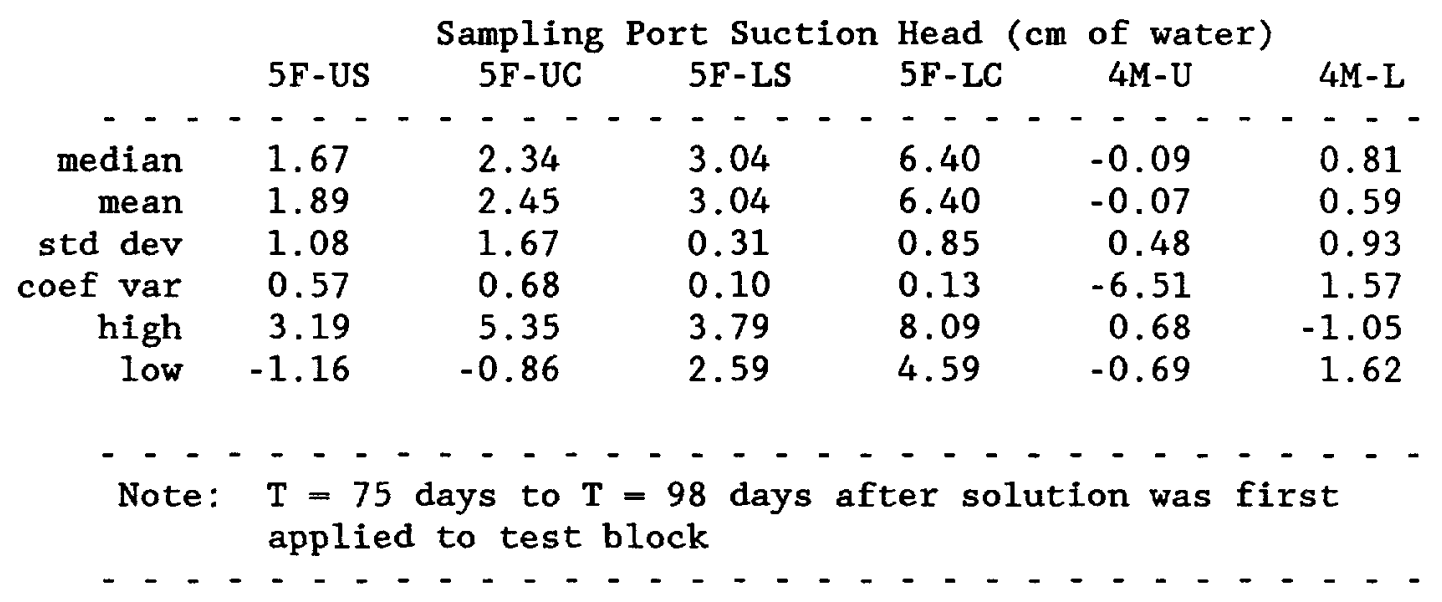

As seen in Table 3.5, suction in the fracture varied from $-1.2 \mathrm{~cm}$ (positive pressure) to $8.1 \mathrm{~cm}$. The upper two fracture sampling ports yielded lower suctions and more variable results than did the lower two ports. The least variable results were obtained from the two matrix ports, whose standard deviations were the lowest. Considering that the tensiometer was held in the matrix sampling ports with a wrench, these results are quite good. Sampling port 5F-LC, which intercepted a fracture splay rather than the main test fracture, yielded the highest mean suction, indicating that less flow may have occurred through the subsidiary fracture. Data from only one sampling port, 5FUS, showed a trend with time. Suction increased with time in this port. During transport sampling and other periods when the evaporation canopy was open, the short-term suctions obtained from a given port increased, possibly due to increased air flow around the edges of the fracture.

When test block number 1 was separated after the flow test, it was observed that fine sand- and silt-sized particles had accumulated, or been left unentrained, in tortuous paths down the fracture face. One such path lay near sampling port 5F-UC. These debris paths may represent the locations of preferential solution flow. Such an interpretation is supported by transport data taken immediately after the test block was broken apart.

Matrix hydraulic conductivity and fracture transmissivity were calculated for the steady state portion of the test. Data used in the calculations were obtained after the forty-fifth day since the start of the imbibition test. Two methods were used to estimate the fracture trans missivity. In the first, it was assumed that all of the solution which flowed into the test block through the narrow center plate entered the fracture at the top surface. No other solution was assumed to flow into the fracture. Outflow from the fracture was assumed to be at the bottom of the rock only. Darcy's law was applied from the top to the bottom of the fracture. The pressure head at the top of the fracture was assumed to be the average pressure head calculated at the bottom of the plate, and the pressure head at the bottom of the fracture was assumed to be 
atmospheric. Transmissivities calculated by this method are designated $\mathrm{T}(1)$ in Table B.13.

The second method of determining fracture transmissivity assumed that vertical flow lines occurred throughout the test block. An average specific discharge was calculated from data taken from the matrix plates. This value was multiplied by the fracture plate area and subtracted from the volumetric flow rate through the fracture plate:

$$
Q_{f}=Q_{f p}-q_{m p} A_{f p}
$$

where

$Q_{1} \quad$ inflow to the top of the fracture, $\mathrm{m}^{3} / \mathrm{s}$;

$Q_{i p} \quad$ inflow through the fracture $\mathrm{plate}, \mathrm{m}^{3} / \mathrm{s}$;

$\mathrm{q}_{\mathrm{mp}}$ average specific discharge through the matrix plates, $\mathrm{m} / \mathrm{s}$;

$A_{f p} \quad$ surface area of the fracture plate contacting the test block, $\mathrm{m}^{2}$.

The volumetric flow rate calculated by this method was then inserted into Darcy's law as in the first method. Table B.13 designates transmissivities determined in this manner $T(2)$. Table 3.6 summarizes the results of the above calculations.

Table 3.6. Saturated Matrix Conductivity and Fracture Transmissivity, Test Block Number 1.

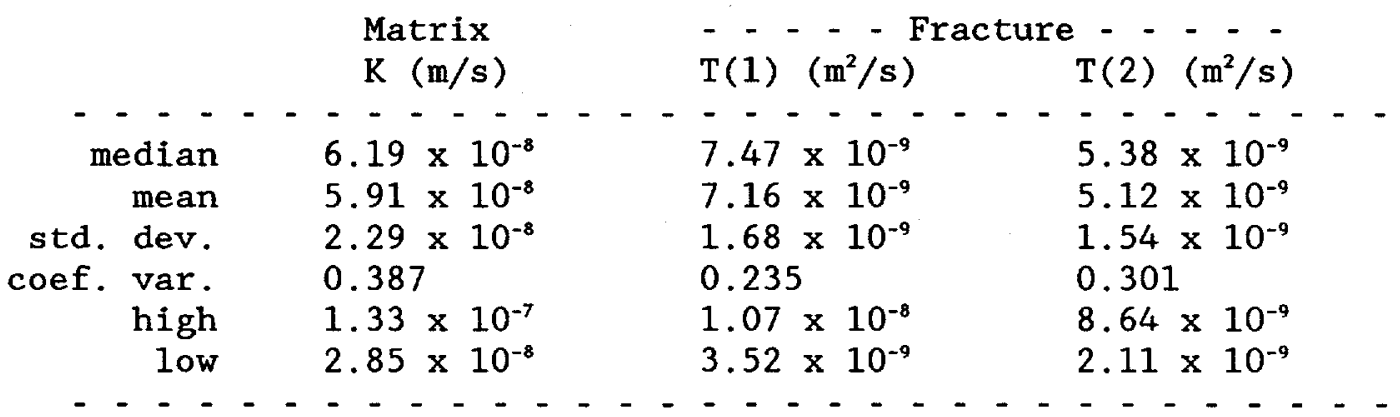

Table 3.6 indicates that both methods of calculating the fracture transmissivity yield similar results. As expected, the first method gives a higher mean transmissivity than does the second. The standard deviations and ranges of $\mathrm{T}(1)$ and $\mathrm{T}(2)$ are also reasonable. The matrix hydraulic conductivities were calculated using data collected from both matrix plates and varied somewhat more than did the fracture transmissivities. The higher range and coefficient of variation of the matrix conductivities reflect this. Probably, the increased variation in the matrix hydraulic conductivities was due to the intermittent solution leakage from the top of the test block.

Rather than calculate either matrix hydraulic conductivity or fracture transmissivity, one can determine a bulk test block hydraulic conductivity which includes both fracture and matrix. Bulk hydraulic conductivities were determined in instances where flow rates were available for each plate on the same day; due to time limitations and equipment malfunctions, flow rates for all three inflow plates were not taken on the 
same day. Twenty such bulk hydraulic conductivity values were calculated. The mean bulk hydraulic conductivity for twenty test block 1 data points was $3.39 \times 10^{-7} \mathrm{~m} / \mathrm{s}$, and the standard deviation was $3.74 \times 10^{-8}$ $\mathrm{m} / \mathrm{s}$. Tidwell (1988) performed such analyses in angled boreholes drilled at the Apache Leap tuff site. By two analytical methods, he calculated bulk hydraulic conductivities for borehole segments. The mean conductivity of all of the data collected using the first method was $2.75 \mathrm{x}$ $10^{-7} \mathrm{~m} / \mathrm{s}$ and using the second method was $5.61 \times 10^{-7} \mathrm{~m} / \mathrm{s}$. Variation on the order of 5 decimal places was found, reflecting fractured and nonfractured zones in the boreholes. Since no compressive stress was applied to the block to simulate field conditions, the mean bulk hydraulic conductivity calculated for test block 1 is not really comparable to those calculated by Tidwell. However, the bulk conductivity of test block 1 lies well within the range of values he calculated.

Figures 3.18 and 3.19 present the flow and sampling port pressure head data generated from the boundary integral model. Because the test block was roughly symmetrical about the test fracture, half of the block was modeled. Four domains were used to characterize the block. Looking at a vertical face containing the fracture trace, the first domain contained the rock matrix to the left of the test fracture, and the second contained the left half of the test fracture. During laboratory testing of the block, three porous ceramic plates were used to supply prepared test solution to the matrix and fracture. Two plates covered the matrix, and one covered the fracture. Therefore, two domains were modeled to represent the porous plates; the third domain included the porous plate covering the matrix, and the fourth contained left half of the plate covering the test fracture. Domains 1,3 , and 4 were two dimensional; domain 2 was effectively modeled in one dimension, since properties were considered constant across the fracture aperture. All boundaries were linear, and contact between the porous ceramic plates and the matrix or fracture domains was considered perfect.

Table 3.7 summarizes the relevant domain dimensions and parameters. The hydraulic conductivities of the matrix and the porous plates were obtained from laboratory test data. The fracture transmissivity to be used in the final model was determined iteratively by running the model and matching the output (inflow rate and pressure head) with data obtained in testing block number 1 . Although the final fracture transmissivity used in the model generated output that matched the laboratory data the best, the inflow rates and pressure heads created by the model varied somewhat from those measured in the laboratory.

Table 3.7: Model Input Summary.

$\begin{array}{rrrr}\text { Domain } & \mathrm{y}(\mathrm{cm}) & \mathrm{z}(\mathrm{cm}) & \text { Hydraulic Conductivity }(\mathrm{m} / \mathrm{s}) \\ -- & - & - & - \\ 1 & 10.20 & 50.0 & 5 \times 10^{-8} \\ 2 & - & 50.0 & \mathrm{~T}=5 \times 10^{-9} \mathrm{~m}^{2} / \mathrm{s} \\ 3 & 8.60 & 0.7 & 2 \times 10^{-9} \\ 4 & 1.51 & 0.7 & 5 \times 10^{-9} \\ - & - & -\ldots\end{array}$




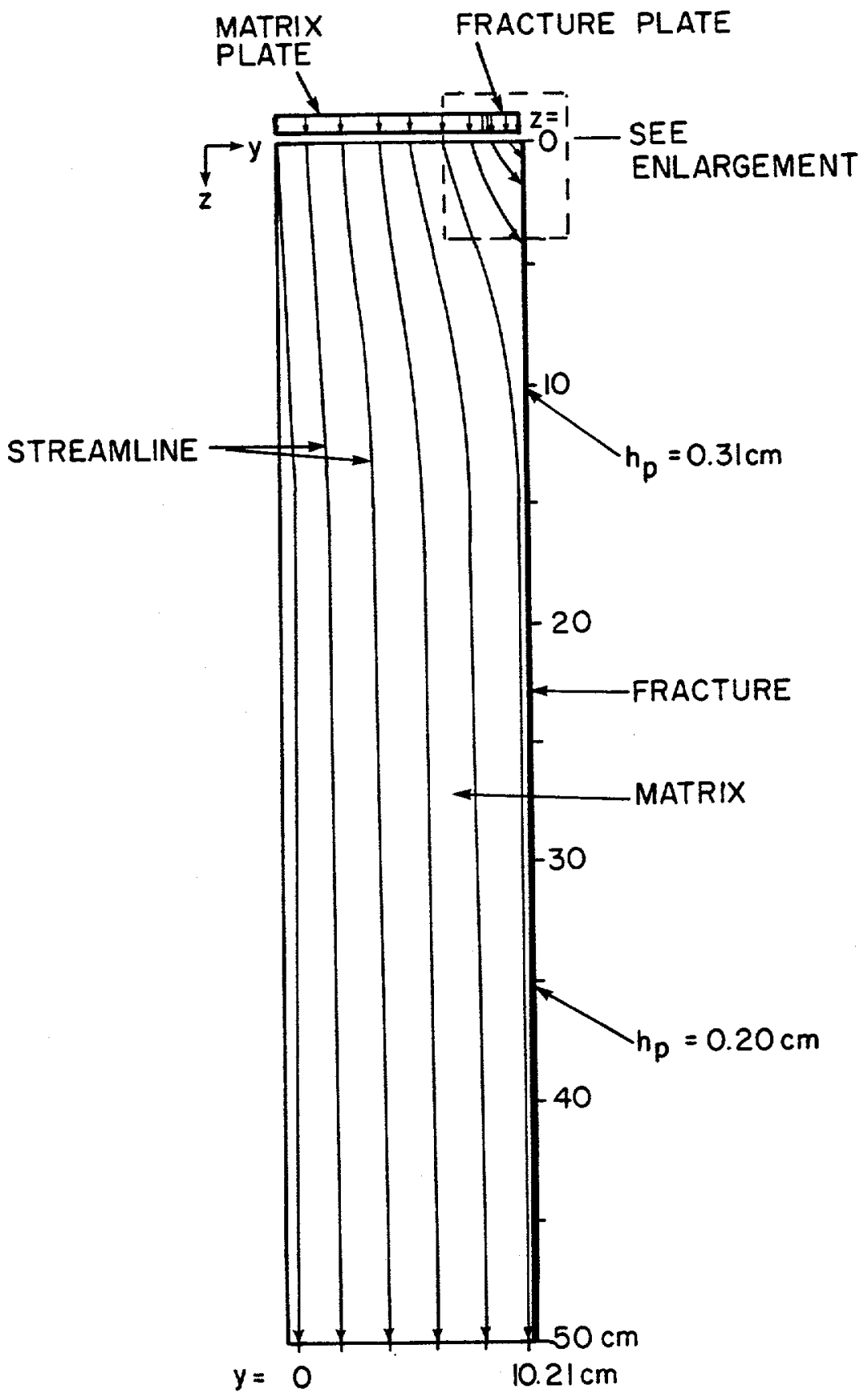

Figure 3.18 For the boundary integral method computer model, the test block is divided into four domains: fracture, matrix, fracture plate and matrix plate. Except near the fracture opening, the streamlines are virtually vertical. The "zero-percent" or left most streamline should correspond to the left boundary of the matrix. 


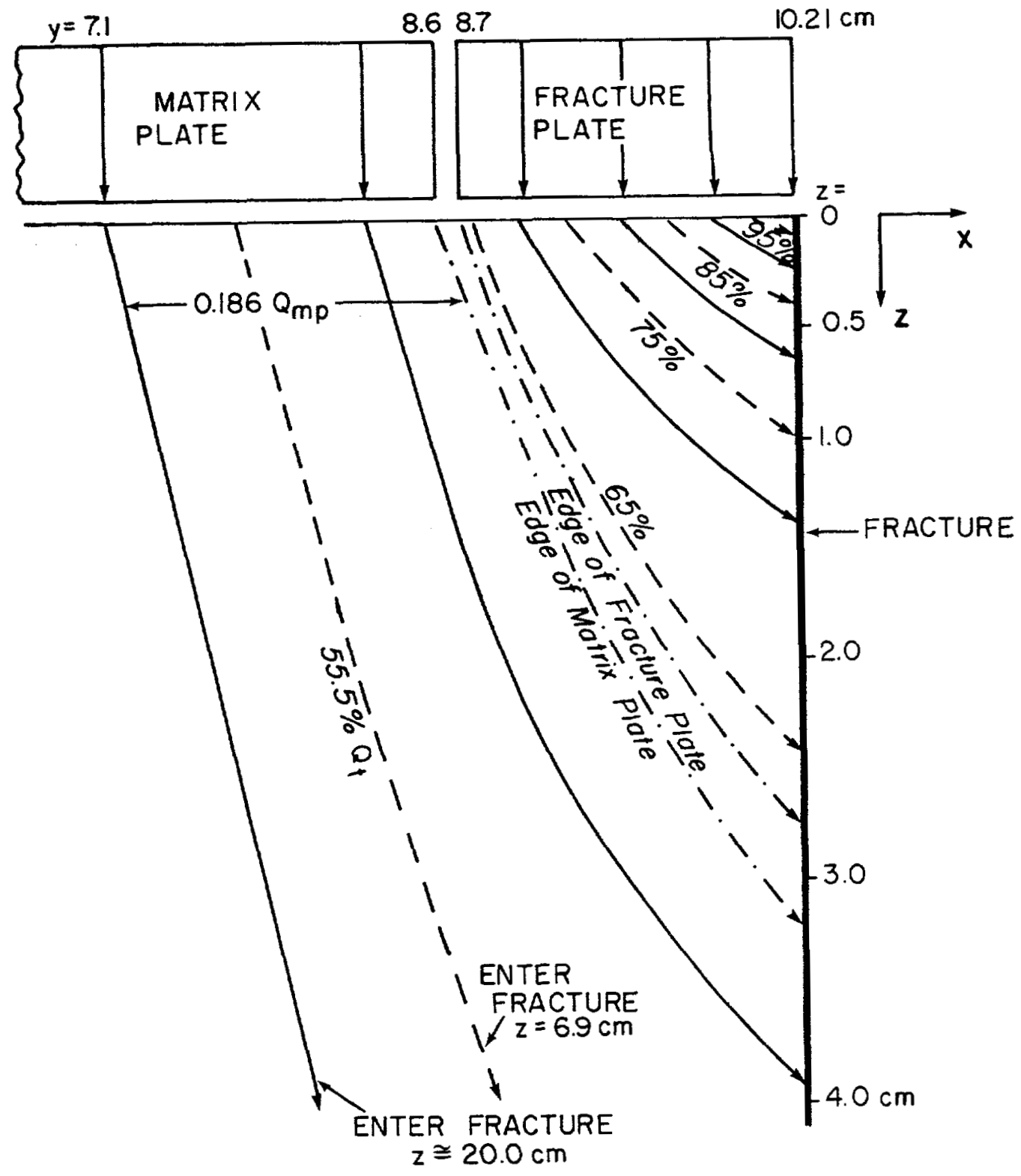

Figure 3.19 Pertinent flow lines showing the fracture-matrix interaction near the top fracture opening. The streamlines are denoted as percentages of total flow $\left(Q_{t}\right)$ of the system. All the fracture plate flow enters the fracture within the top $3 \mathrm{~cm}$. However, nearly 19 percent of the matrix plate flow $\left(Q_{m p}\right)$ is also diverted to the fracture. 
Ten streamtubes encompass the matrix. Although the leftmost streamline should be at the edge of the block, it is located in the rock matrix due to model numerical oscillations and errors. In the model, the streamlines are roughly vertical towards the outside edge of the block. Towards the top of the block, the streamlines bend towards the fracture. Figure 3.19 shows the upper right-hand corner of the block and the strong influence that the fracture has on fluid flow through the matrix. Under the conditions imposed in the model, all of the solution flowing into the block through the center plate enters the fracture within 3.5 $\mathrm{cm}$ of the top. Additionally, about 19 percent of the solution flowing through the outer plate enters the fracture by the bottom of the test block. Streamlines in the porous plates were essentially vertical, with those nearest the fracture in the center plate bending slightly towards the fracture.

Based upon these results, it would seem that the two methods used to estimate fracture transmissivity are inadequate. Neither accounts for fluid entering the main test fracture from subsidiary fractures or the matrix plates, and neither considers the effect of solution entering the fracture but at the top. To accurately calculate fracture transmissivity, one should determine the amount of solution entering different fracture segments, calculate the transmissivity of each segment, and average the transmissivities thus determined. In practice, it is not possible to measure the flow rate entering the fracture through the walls, and one of the estimates used above needs to be employed.

It was assumed in the model that no filter paper aided plate-rock contact. In fact, filter paper was used in the laboratory experiment. Although the hydraulic conductivity of the filter paper was not measured, based upon its retention rating, it was more conductive than the test block or the porous plates. As such, it would provide a preferential flow conduit for solution to move from the center plate to the fracture. Without the filter paper, it would be expected that results similar to the modeled case would exist. However, with the filter paper present, most of the fluid exiting the center porous plate was most likely shunted directly to the top of the fracture. This suggests that the first method of calculating fracture transmissivity is the most accurate. The influence of using filter paper as a contact material has not been quantitatively studied, nor its impact upon the streamlines beneath the matrix plates considered.

The modeled case produced pressure heads along the fracture very near zero. At $z=10 \mathrm{~cm}, h_{p}$ was $0.31 \mathrm{~cm}$, and at $z=35 \mathrm{~cm}, h_{p}$ was $0.2 \mathrm{~cm}$. Given the numerical accuracy of the mode1, these two values are equivalent. In the laboratory study, the mean suction heads at the fracture sampling ports varied from $1.9 \mathrm{~cm}$ to $3.0 \mathrm{~cm}$, suggesting that the suction head at the top of the fracture was greater than zero. A pressure head drop of about $5 \mathrm{~cm}$ occurred along the base of the center porous plate in the modeled case, leaving a suction head of $1.7 \mathrm{~cm}$ at the top of the fracture. The suction at the fracture was about $4 \mathrm{~cm}$ greater than the areal average for the modeled plate. This also indicates that the suction at the top of the test fracture was greater than the average suction beneath the plate and probably greater than zero. If the fracture transmissivities are recalculated, using a suction head at the top of the fracture $4 \mathrm{~cm}$ greater than the fracture plate average, the mean 
values of transmissivity using both calculation methods increase. $T(1)$ increases to $7.69 \times 10^{-9} \mathrm{~m}^{2} / \mathrm{s}$, and $\mathrm{T}(2)$ increases to $5.20 \times 10^{-9} \mathrm{~m}^{2} / \mathrm{s}$.

\subsubsection{Test Block 2 Experiments}

Nine days before the initial application of test solution to rock number 2 , the LVDTs were connected. The three displacement transducers were monitored from that point throughout the test. Table B.14 provides complete LVDT data, which is summarized in Figure 3.20. LVDT 1 was the most stable, varying only 3.7 millivolts throughout the test. This corresponds to a fracture closure of 5 microns at that location. LVDT 2 indicated a fracture closure of 44 microns. The third displacement transducer was the most variable and, not counting accidental bumping, showed 148 microns of closure. Since LVDT 3 output continued to increase through the entire test, it is not known if it was working properly. In general, less fracture aperture change occurred during testing of block number 2 compared to block number 1, most likely because the frame holding the test fracture together in block 2 was tightened to 30 foot-pounds of torque. Apparently, 5 foot-pounds, and even 30 foot-pounds, of torque is not enough to maintain the test fracture at a nearly constant aperture.

Figure 3.21 presents a composite diagram of the wetting front advancement with time. Significant fluctuation of the wetting front occurred during the first five days of the test. Contributing factors to this included poor plate-rock contact along the upper edge of face 4 during the first few days of the test and adjustments made to flow rate to obtain the desired suction below the porous plates. By ten days into the test, the wetting front had smoothed out considerably. Subsequent$1 y$, it proceeded rather evenly down the rock. The wetting front along face 4 lagged behind the front along face 6 due to the poor plate contact early in the test at the top of face 4 .

Flow data obtained during the imbibition test are presented in Tables B.15 and B.16. Flow rate was monitored throughout the test both by using the pipet flow meters and by recording volume changes in the Mariotte reservoirs. Despite the similarity in flow rates obtained by the two methods, the pipet flow tubes were difficult to use at the low flow rates encountered in this test, and at lower flow rates may cease to function entirely. A graphical summary of the cumulative volume of inflow versus time is presented in Figure 3.22. After one day of flow, the flow rate was nearly constant, and by four days, the flow rate roughly stabilized, yielding a straight-line plot. This indicates that, after four days of flow, the suction gradient in the zone of transmission was negligible. By the end of the imbibition test, the specific discharge of the porous plate covering the test fracture was less than the specific discharge of either of the two matrix plates. This suggested that the specific discharge of the fracture was less than or equal to that of the matrix. This was visually confirmed by the lack of a wetting front lobe throughout the test.

An infiltration and percolation analysis was performed on three sets of data: wetting front, pipet flow tube inflow, and Mariotte reservoir inflow. The data used are tabulated in Tables B.17, B.18, and B.19. To use the wetting front data, it was assumed that the test block was 


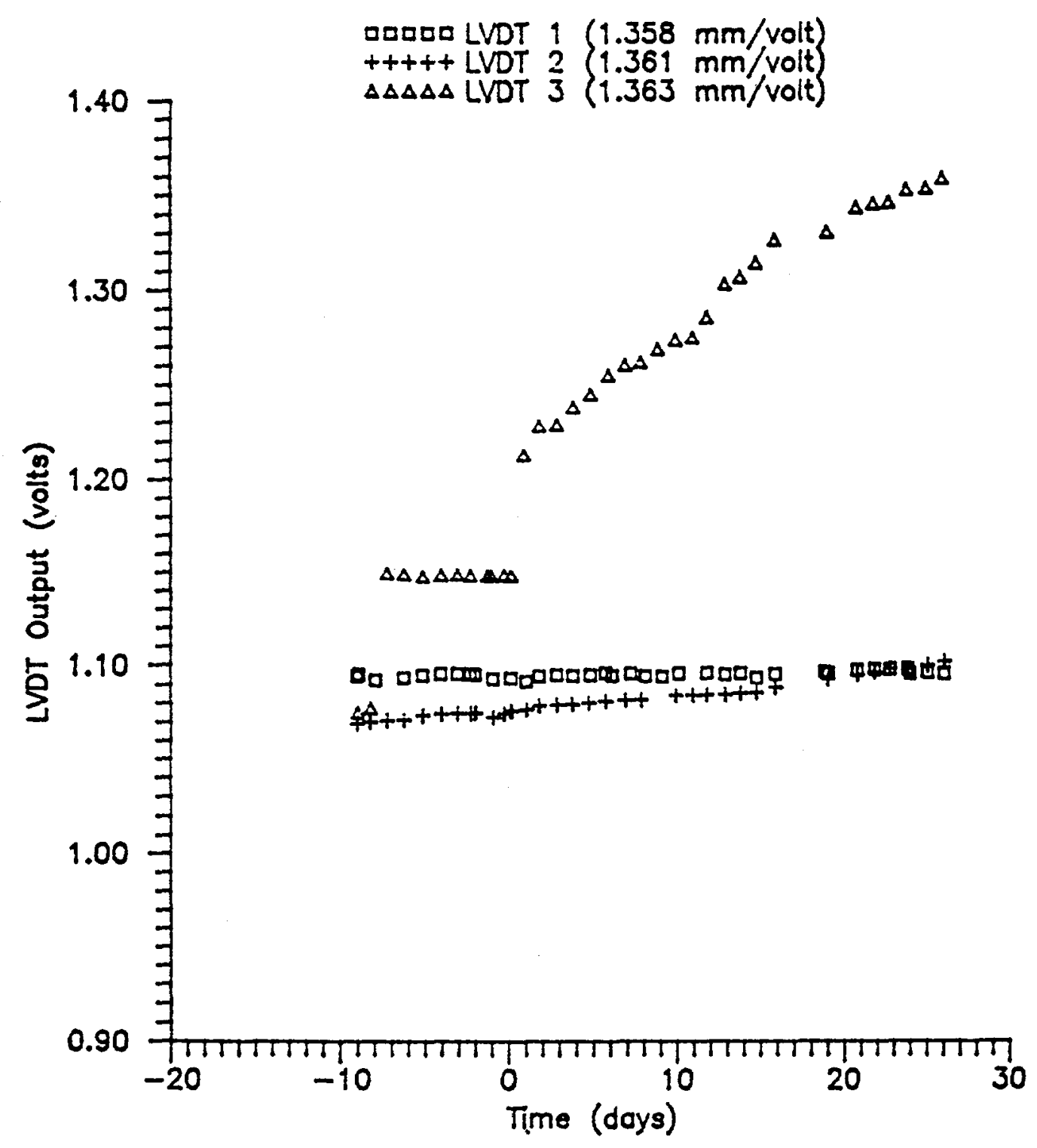

Figure 3.20 Monitoring of fracture aperture change in test block 2 . 


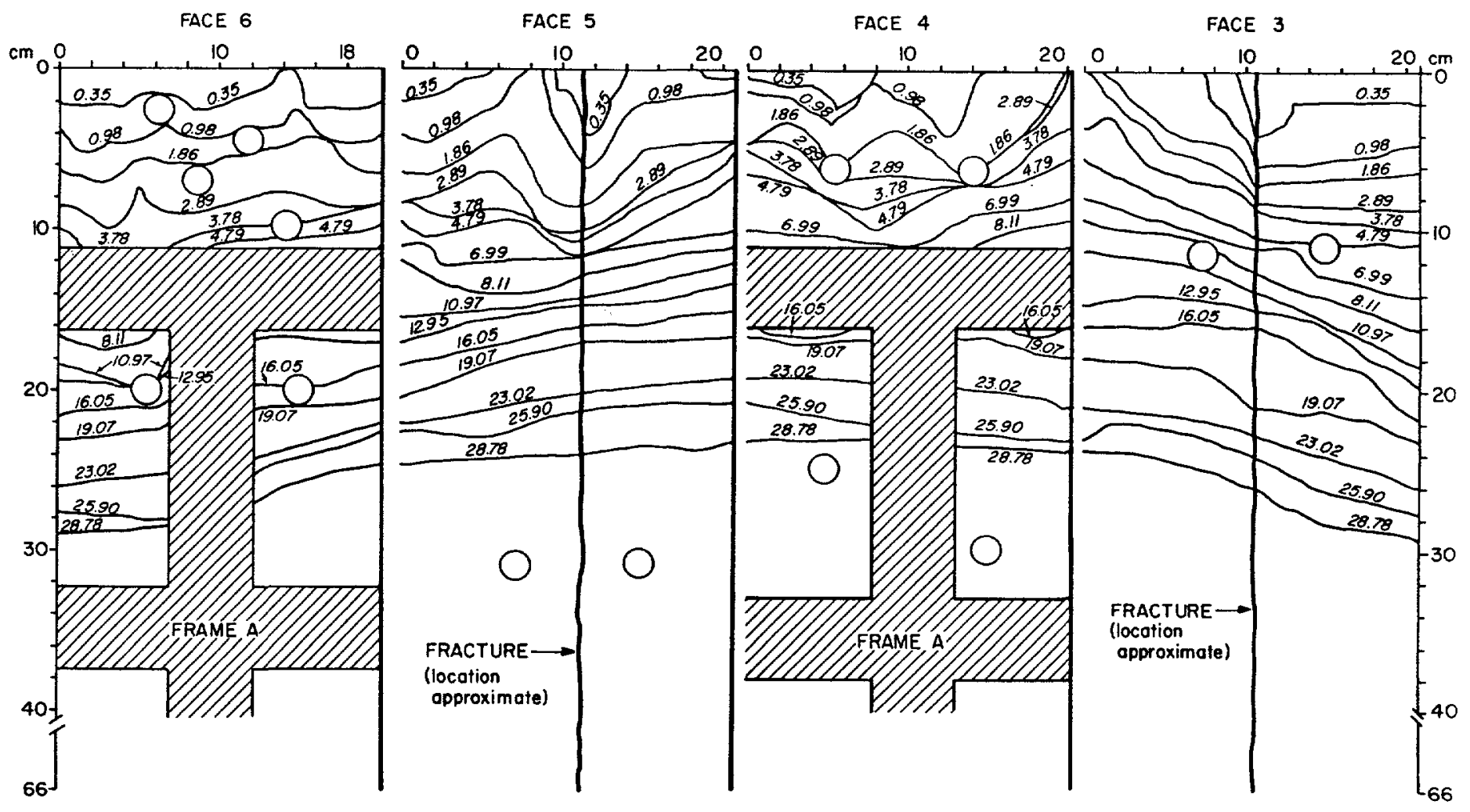

Figure 3.21 Wetting front advancement during test block number 2 imbibition test. Numbers indicate days from beginning of test. 


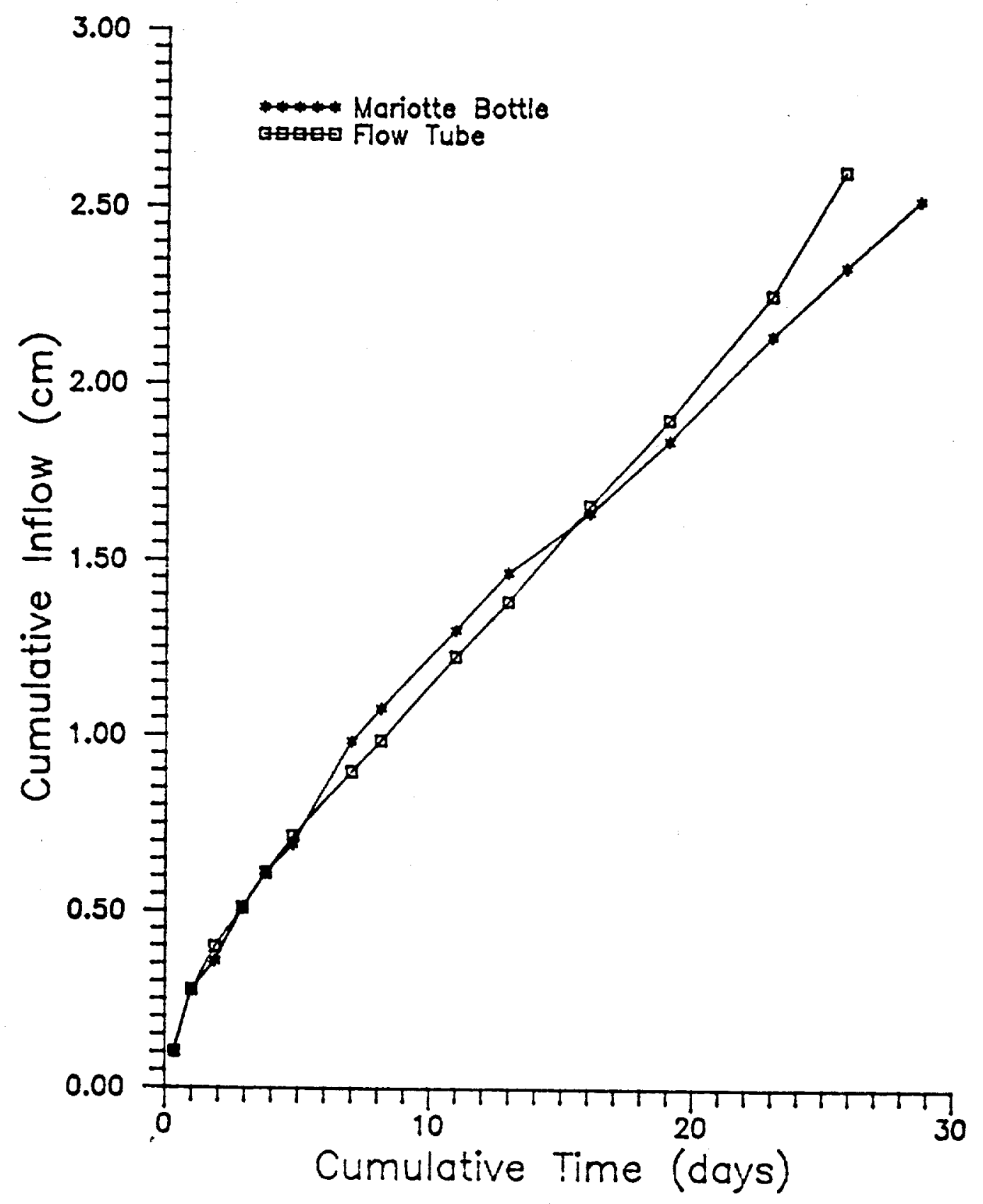

Figure 3.22 Test block number 2 imbibition test summary 
saturated behind the wetting front. Cumulative inflow and inflow rate were then calculated. Based on the inflow data, the two unknown parameters of the Philip's infiltration equation were determined (Philip, 1969). Figure 3.23 provides a plot of $I(t) / t$ versus $t^{-1 / 2}$ for pipet flow tube and constant-head reservoir data. The wetting front data were used to prepare a similar plot but it is not shown because the assumption that the matrix was saturated behind the wetting front proved unsatisfactory; a saturated average wetting front was determined from the Mariotte reservoir data and consistently was less than the actual mean wetting front. The slope of the linear part of the Philip's curve equals the sorptivity, $s$. The $y$-intercept equals the parameter $A$, or since the data along the linear part of the curve represents relatively late-time data, the hydraulic conductivity at about $15 \mathrm{~cm}$ of applied suction. Little fracture flow occurred during the infiltration test. Thus, the hydraulic conductivity determined by this method may be equivalent to or less than the matrix hydraulic conductivity at $15 \mathrm{~cm}$ of suction, depending on how the fracture influenced the adjacent matrix flow. Table 3.8 summarizes the calculations, which were based on a least squares fit of data taken after $t=2.9$ days. The hydraulic conductivities calculated for the case of $15 \mathrm{~cm}$ of applied suction are an order of magnitude less than the matrix conductivities determined for test block 1 at roughly $0 \mathrm{~cm}$ of applied suction.

Table 3.8: Philip's Parameters.

Flow Tube Data

Mariotte Reservoir Data

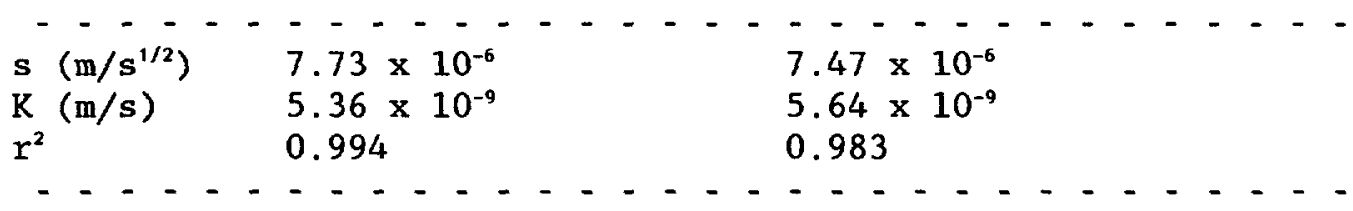

\subsubsection{Rock Characterization Tests}

This section presents the results of rock characterization tests using core samples adjacent to and directly from the test blocks. Characteristics evaluated include the matrix saturated hydraulic conductivity, the matrix moisture release curves, the matrix dry bulk density and effective porosity, and the matrix pore size distributions.

\subsubsection{Matrix Saturated Hydraulic Conductivity}

The complete results of the matrix saturated conductivity tests described in section 3.8.1 are presented in Tables B.20 and B.21. Initially, numerous flow tests were performed for each rock core, with the intent that sample statistics could be developed. However, in the course of the tests, it was observed that the flow rate through a given core decreased with time, despite the fact that none of the test conditions were changed. The flow rates did not stabilize within two days of beginning the test. It was postulated that the air pressure exerted on the sleeve used to prevent flow down the side of the core reduced the pore volume over time. Given enough time, the flow rates should have stabilized and would have represented the hydraulic conductivity at 689 


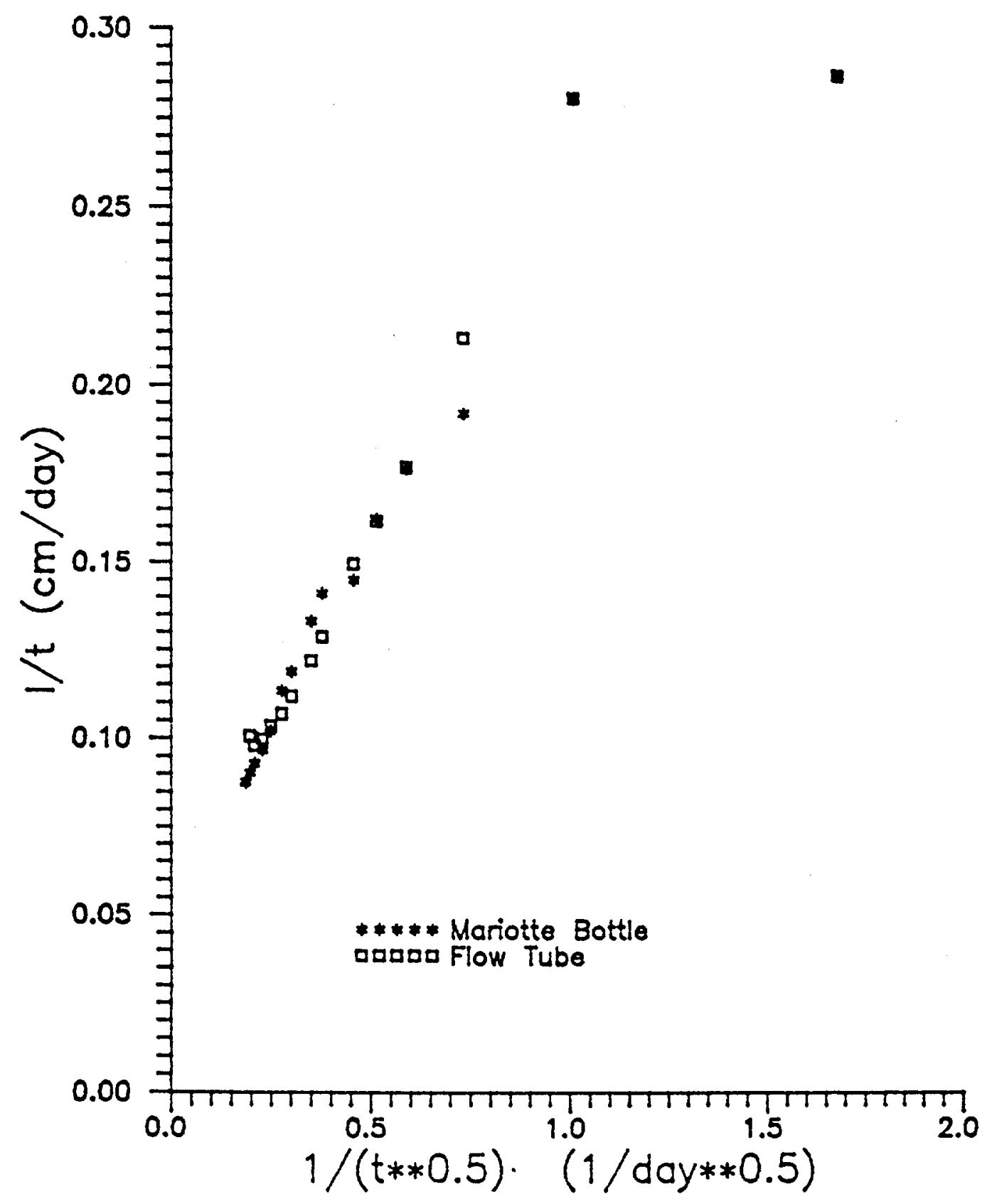

Figure 3.23 Philip's infiltration analysis, test block number 2 
$\mathrm{kPa}$ (100 psi) applied stress. Since little stress was applied to either test block, it was decided to use the first measurement taken as a rough estimate of saturated matrix hydraulic conductivity taken under no applied stress. Gores analyzed later in the program were therefore only tested once. Table 3.9 summarizes those results for cores obtained near test blocks 1 and 2 .

Table 3.9: Matrix Saturated Hydraulic Conductivity.

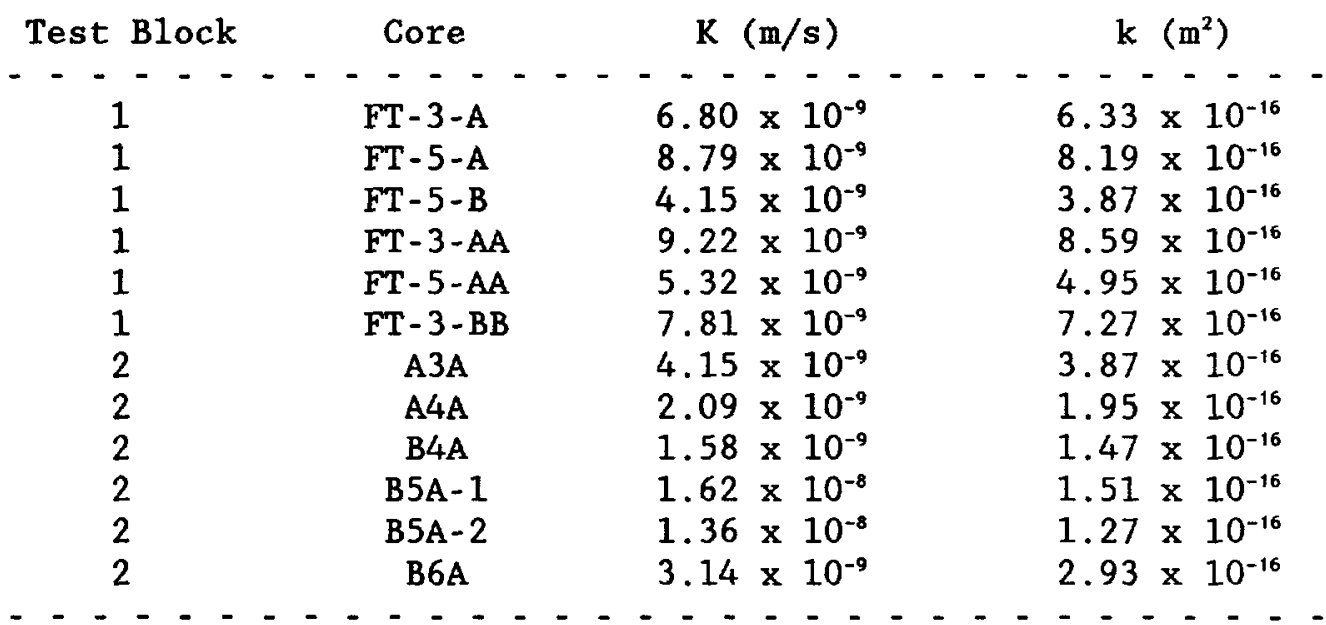

The mean saturated matrix hydraulic conductivities, determined using the core samples from near test blocks number 1 and 2 were $7.02 \times 10^{-9} \mathrm{~m} / \mathrm{s}$ and $6.79 \times 10^{-9} \mathrm{~m} / \mathrm{s}$, respectively; the standard deviations were $1.99 \mathrm{x}$ $10^{-9} \mathrm{~m} / \mathrm{s}$ and $6.39 \times 10^{-9} \mathrm{~m} / \mathrm{s}$, respectively; the median values were $7.30 \mathrm{x}$ $10^{-9} \mathrm{~m} / \mathrm{s}$ and $3.65 \times 10^{-9} \mathrm{~m} / \mathrm{s}$, respectively. Compared to the near-

saturated matrix hydraulic conductivity determined from the analysis of test block 1 , the conductivities determined from the rock cores are low. The most 1ikely explanation for the unexpectedly low conductivities determined using the modified Tempe cell lies in the method itself. Apparently, clogging of the cores from microbiological sources almost immediately reduces the pore space available to flow and greatly reduces the resulting hydraulic conductivity. Thus, not even readings taken immediately after beginning the tests are comparable to matrix hydraulic conductivities determined in flow tests on the fractured blocks.

Cores recovered from the angled boreholes drilled on the plateau at the Apache Leap tuff site have been analyzed for saturated hydraulic conductivity (Evans, 1988) using the same technique that was used in this study. Only $550 \mathrm{kPa}$ ( $80 \mathrm{psi}$ ) were applied to the bladder surrounding the core. The mean conductivity was $1.69 \times 10^{-8} \mathrm{~m} / \mathrm{s}$, with a coefficient of variation of 2.89. However, a range of over two orders of magnitude was found, and the median hydraulic conductivity was $4.20 \times 10^{-9} \mathrm{~m} / \mathrm{s}$. Since a wide range of conductivities were determined in the study of Evans, it is not surprising that one order of magnitude variation was found in samples taken near test block 2 or that the test block mean matrix hydraulic conductivities determined using the Tempe cell in this 
study are lower than those calculated by Evans (1988). The median hydraulic conductivity values compare well.

\subsubsection{Matrix Moisture Release Curves}

Table B. 22 provides the data used to construct the matrix moisture release curves. Figures 3.24 through 3.27 show the curves, plotted as suction versus relative saturation, for test blocks 1 and 2. Table B.22 includes the water content at each pressure step. Little moisture was lost from the samples at low applied chamber pressures. Due to measurement error, two samples gained mass after the first pressure step. The pressure step from $50 \mathrm{kPa}$ to $100 \mathrm{kPa}$ induced the most drainage from the samples, but noticeable drops in water content also occurred in the pressure step from $25 \mathrm{kPa}$ to $50 \mathrm{kPa}$. Since data were not generated which would allow construction of curves showing hydraulic conductivity versus suction or relative saturation, it is not known how the slight drop in relative saturation at the first pressure step would affect the water transmitting properties of the matrix during unsaturated tests performed on the test blocks. Additionally, the first pressure step was significantly greater than the entire suction range that has been, and likely will be, analyzed with the test blocks.

\subsubsection{Matrix Dry Bulk Density and Effective Porosity}

The results of the matrix dry bulk density and effective porosity characterizations are presented in Table B.23. A summary of the results is provided in Table 3.10. Similar analyses were performed on other core samples retrieved from the plateau boreholes at the Apache Leap tuff site (Evans, 1988). They found a mean dry bulk density of $2.12 \mathrm{~g} / \mathrm{cm}^{3}$ and a mean effective porosity of 0.161 . Their median values were close to the mean, but their range of results was slightly larger than in this study. Given the few samples used in this study and the variable nature of ash flow tuffs, the results obtained in this study appear to compare favorably with those found in other cores obtained from the field site.

Table 3.10: Matrix Dry Bulk Density and Effective Porosity.

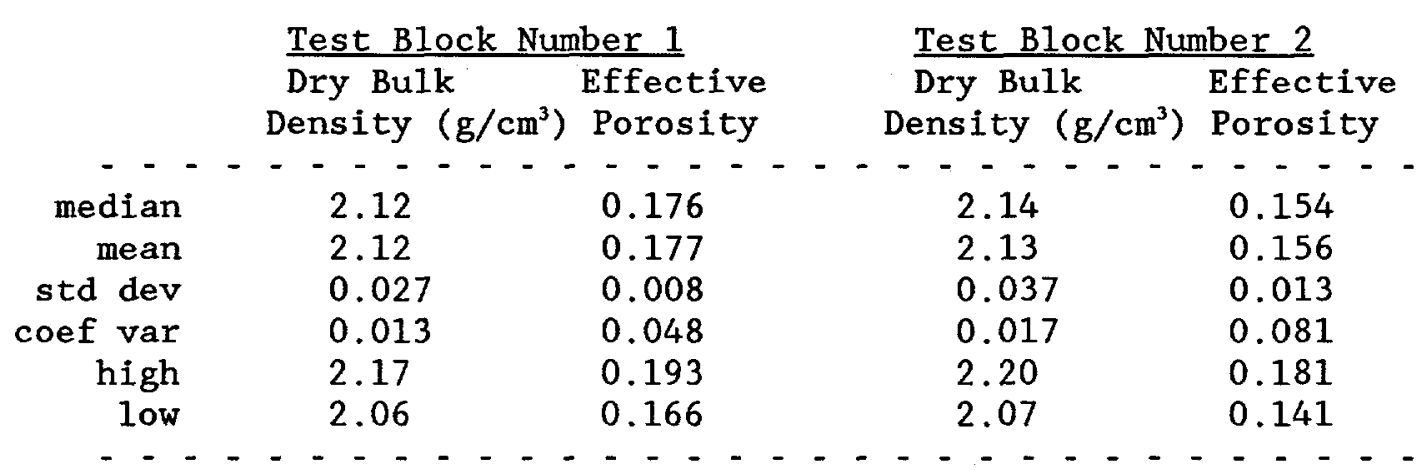




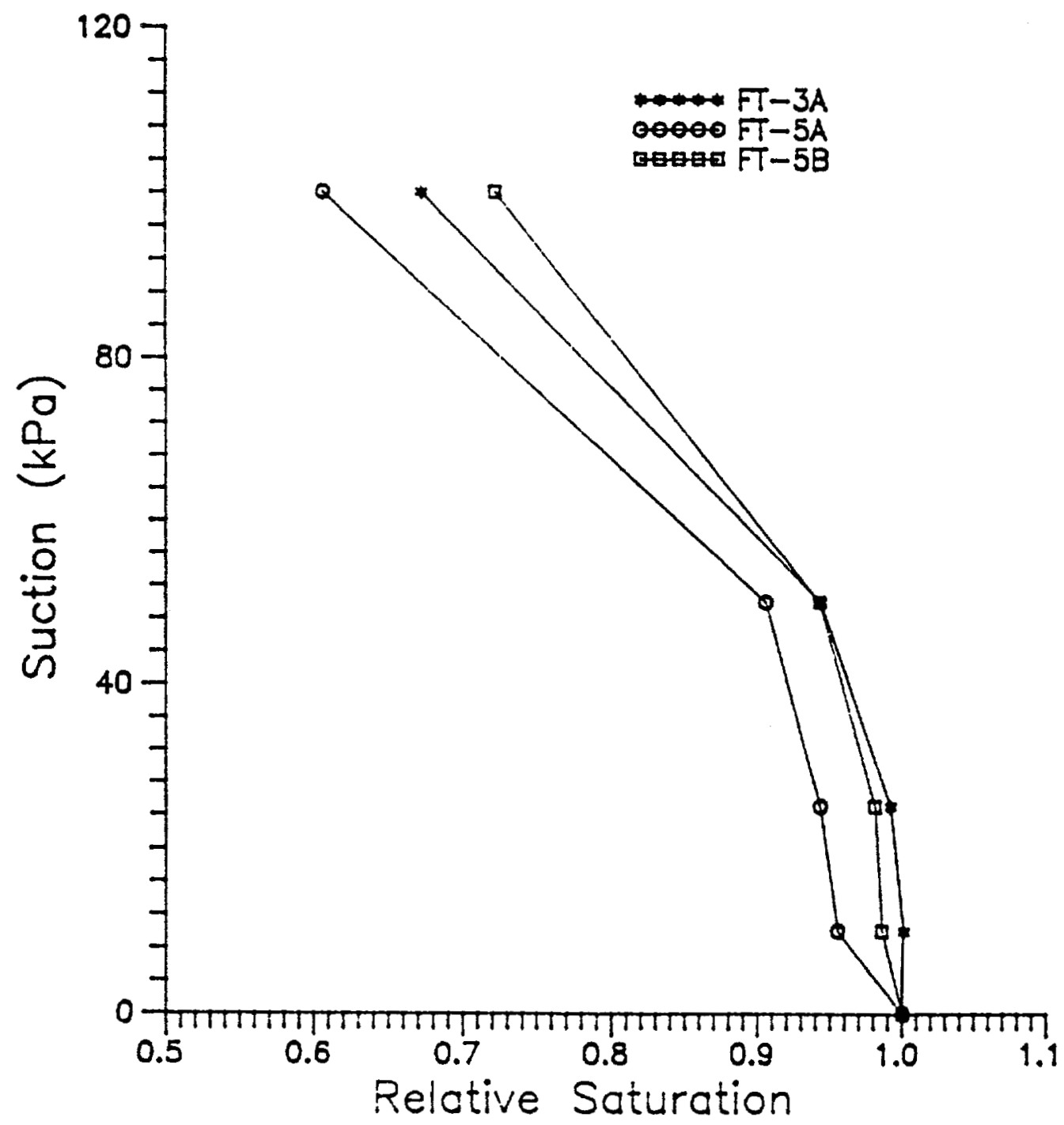

Figure 3.24 Moisture release curves for cores obtained from rocks surrounding test block number 1 . 


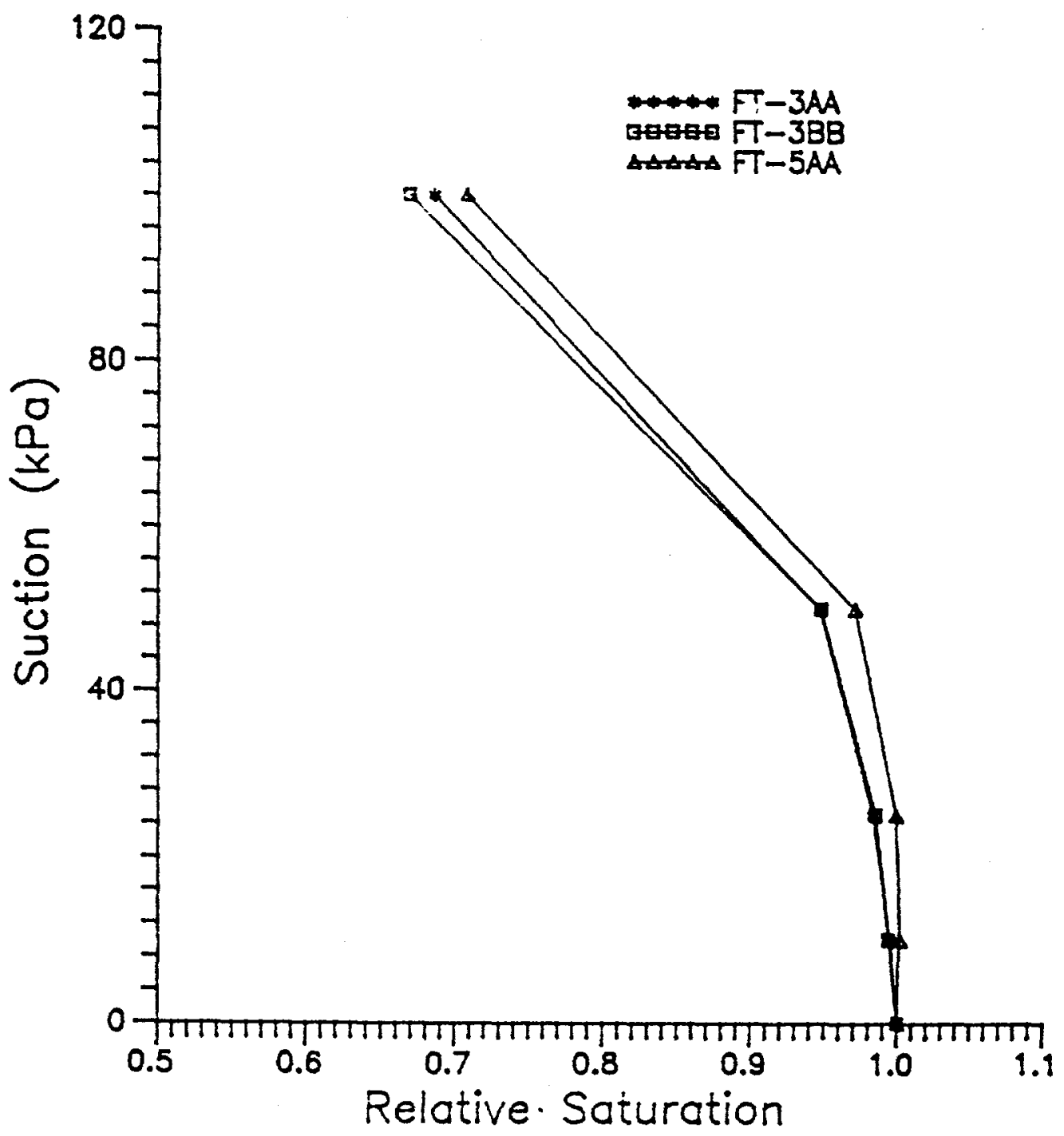

Figure 3.25 Moisture release curves for cores obtained from rocks surrounding test block number 1 . 


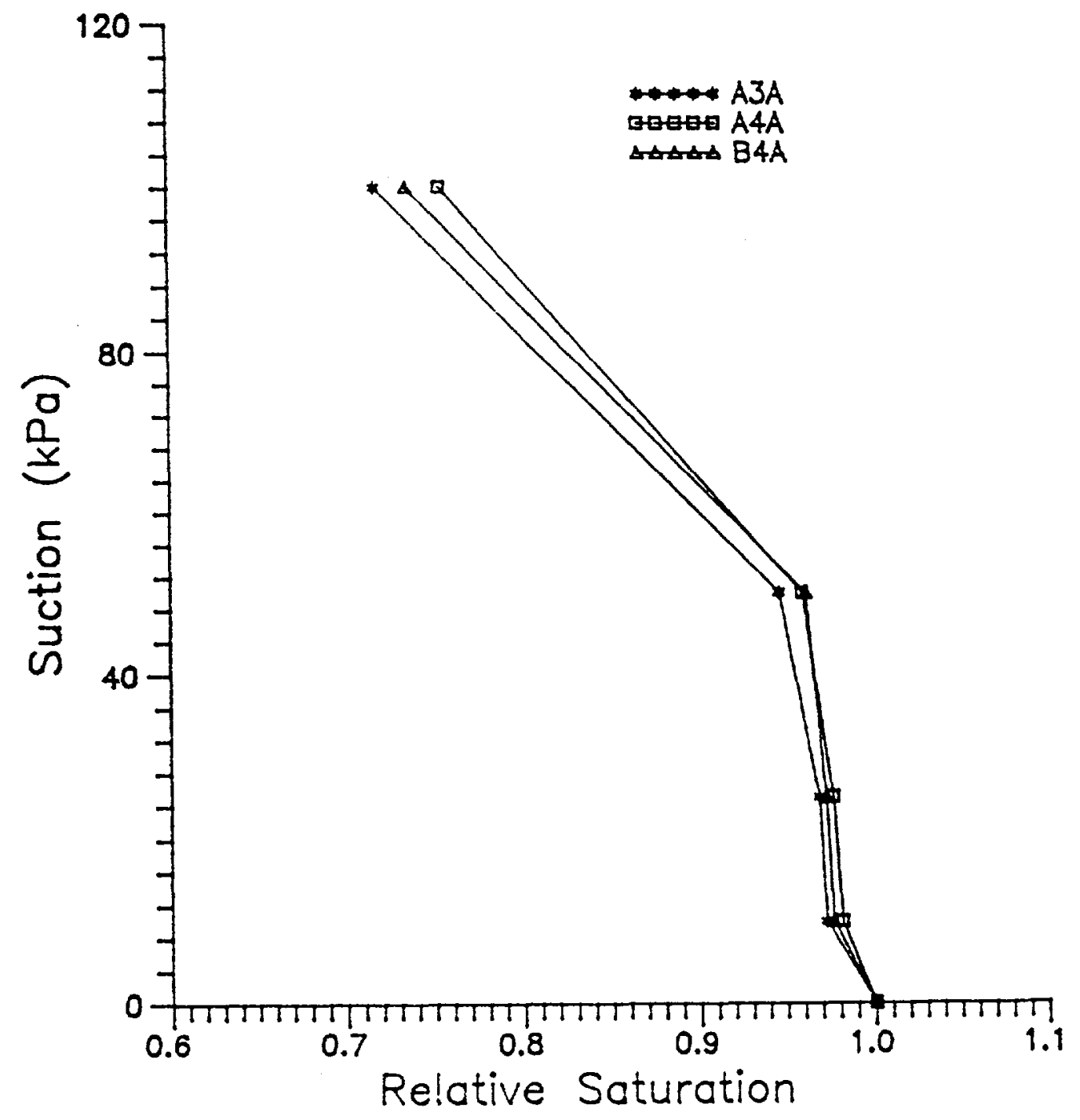

Figure 3.26 Moisture release curves for cores obtained from rocks surrounding test block number 2 . 


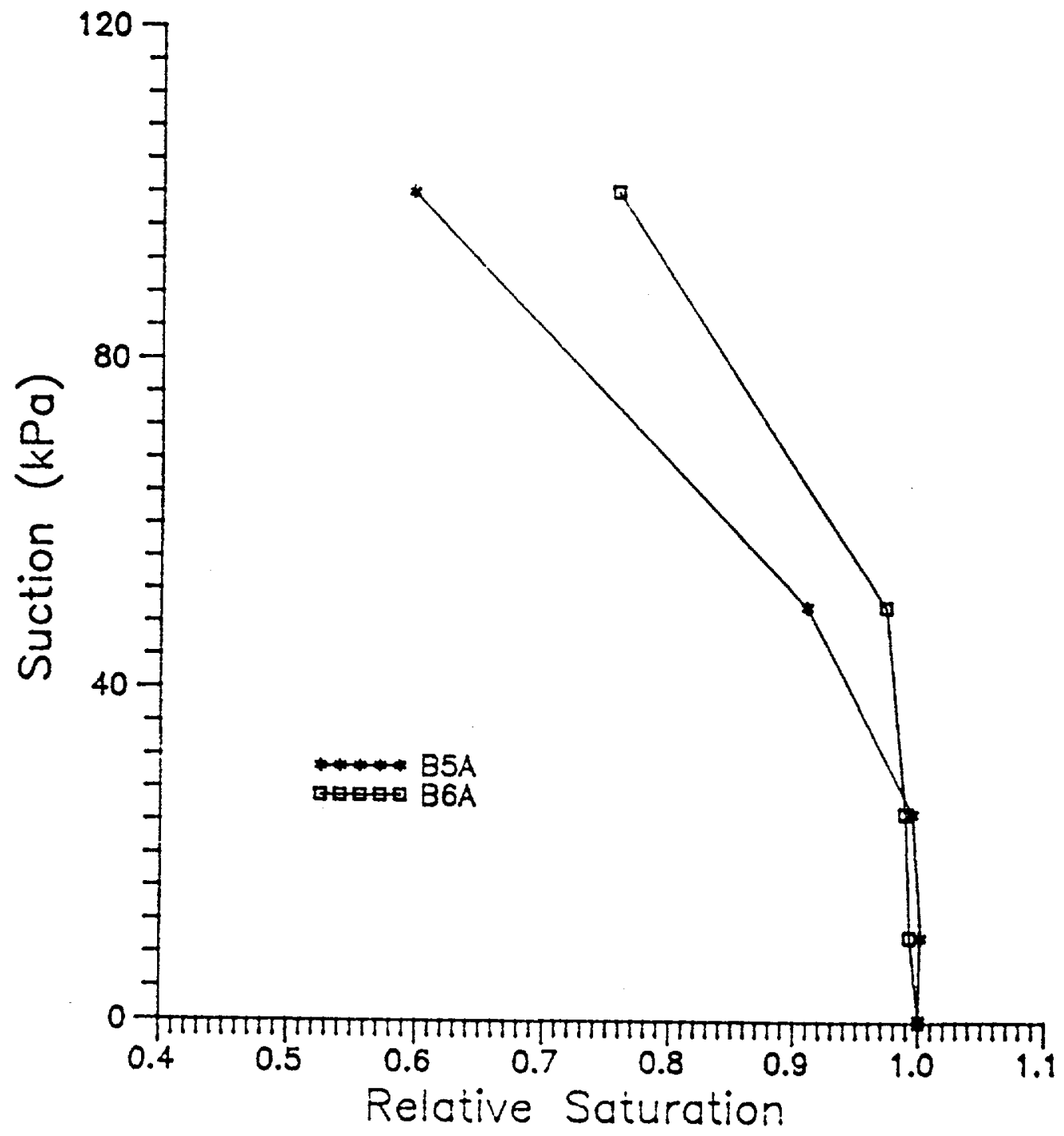

Figure 3.27 Moisture release curves for cores obtained from rocks surrounding test block number 2 . 


\subsubsection{Matrix Pore Size Distributions}

Output from the porosimeter consisted of a table of raw data, a set of curves showing pore volume and pore surface area plotted against pore diameter, and a summary data table. Since the present and future laboratory work does not employ suction heads exceeding $200 \mathrm{kPa}$, the porosimeter was not used up to the $207 \mathrm{MPa}$ (30,000 psi) 1imit. Therefore, the output obtained from this study is only visually comparable to other porosimeter work performed on core samples obtained from the Apache Leap test site.

Vogt (1988) found a bimodal pore size distribution. The mean large pore size class mode was $2.91 \mu \mathrm{m}$, and the mean small pore size class mode was $0.07 \mu \mathrm{m}$. All of the pore size distribution curves plotted from this study showed a bimodal distribution through the range tested. One pore size mode corresponded to the large pore size class noted by Vogt. For test blocks 1 and 2 , this peak averaged $2.97 \mu \mathrm{m}$ and $3.00 \mu \mathrm{m}$, respective$1 y$, which compares well with the peak noted by Vogt. Another larger mode in the pore size distribution was also noted in all but one of the ten samples. It was located at $68.6 \mu \mathrm{m}$ for all samples in which it appeared. Unlike the study of Vogt, this study performed a low pressure test which identified the low pressure pore size distribution. The two pore size peaks in this study, $2.98 \mu \mathrm{m}$ and $68.6 \mu \mathrm{m}$, correspond to suction heads of about $5.0 \mathrm{~m}$ and $20 \mathrm{~cm}$, respectively. Although drainage of the smaller peak requires a suction head beyond that used in this phase of this project, the larger pore size peak represents the upper end of suctions applied to test block number 2. According to the data, a considerable number of pores exist that are larger than $68.6 \mu \mathrm{m}$, and it is conceivable that some pore drainage of the matrix could occur at low suctions, reducing the hydraulic conductivity. 


\section{CHAPTER 4}

\section{SOLUTE TRANSPORT}

The study of solute transport in fractured rock systems under unsaturated conditions requires special considerations, especially with regard to sampling of water under conditions of negative pressures. Of primary interest to this study is the feasibility of coated-wire ion-selective electrodes (ISE's) to make reliable potential measurements of tracer solutions under unsaturated conditions. While ISE's have been used in diverse applications for determining solute concentrations, this investigation explores the ability of filter papers to absorb samples of pore fluids which are then used to measure solute concentrations. As part of the experimental procedure, three porous plates placed on the upper surface of the fractured rock were used to impose the upper boundary condition. Ports were drilled through the rock matrix to access the fracture for sampling. Ports were also drilled to sample matrix waters. While the ports are expected to affect flow and transport due to their presence, sampling at different points along the fracture and matrix would be impossible without them. Filter papers were then used to extract pore fluids from the ports for ISE analysis.

Once primary data sets related to the movement of solutes in unsaturated fractured rock have been obtained, theories developed to describe transport in porous media can be examined for their relevancy to fractured media. While transport in unsaturated environments are more complex than in saturated conditions, transport in unsaturated fractures present even greater complexity.

The following sections review the current understanding of ion-selective electrodes, and transport in porous media. These topics are then addressed as they apply to unsaturated flow and transport in fractured rock systems, and in particular, to this research. The influence of the porous plates, and fracture-matrix flow interaction, on solute data interpretation is also discussed. An analysis of rock water penetration into cavities is used to assess the effects of the sampling ports on flow and transport.

\subsection{Ion-Selective Electrodes}

Chloride ion-selective electrodes (ISEs) are being examined to study the transport behavior of solutes in unsaturated fractured rock. The measurement technique requires sensitivity and selectivity. Ionselective electrode potentiometry has been used successfully in many applications ranging from copper activity measurements in soil solutions, to stack gas measurements for nitrogen species, to intracellular measurements of major cation species (Minnich and McBride, 1987;

Freiser, 1978; Covington, 1979; Ammann, 1986). There is strong indication that ISEs of the coated-wire type can be adapted for solute transport studies in unsaturated fractured rock. These ISEs, which are also minielectrodes, require minute sample sizes for measurements, with surface contact often being sufficient. Sample sizes of $0.01 \mathrm{~mL}$, and depths of immersion of $0.1 \mathrm{~mm}$, are typically measured by these elec- 
trodes. Even with the small amounts of rock water available for sample collection, they enable measurements to be made of tracer solution collected by filter paper.

\subsubsection{Background}

Ion-selective electrodes refer to a variety of membrane electrodes which respond preferentially to certain ion(s) in the presence of other ions in solution. There are numerous solid state and liquid membranes currently available. The electrodes are classified and organized into several types depending on membrane types, functions and number of interfaces (Freiser, 1978; Covington, 1979). The ion-selective electrodes (see Figure 4.1) being evaluated are electrodes of the "second type" in which metal wires, such as copper or platinum, are coated with low solubility salts of the appropriate ion species (Cattrall and Hamilton, 1984; Cattral1 and Freiser, 1971; James et a1., 1972). Construction of the electrodes involves first preparing the wire surfaces and then dipping them into polymer solutions of the appropriate salts, and often with several coatings of different solutions. These coated-wire electrodes are operationally similar to conventional "liquid membrane" electrodes but are novel arrangements from a thermodynamic viewpoint.

Operationally, an electrical circuit is established when a reference electrode and ISE come into contact with the sample solution. The double-junction reference electrode used is constructed of two compartments. The upper compartment consists of a silver wire coated with silver chloride ( $\mathrm{AgCl}$ ), and immersed in a saturated solution of $3 \underline{\mathrm{M}}$ potassium chloride (KCl) (see Figure 4.1). The KCl filling solution provides a fixed activity of chloride, and therefore, constant potential. The lower compartment consists of a potassium nitrate $\left(\mathrm{KNO}_{3}\right)$ bridge solution which separates the filling solution from the sample solution. This is important especially when contamination of the sample by the filling solution is undesirable.

The electrodes form part of an electrochemical cell and can be analyzed as two half-cells. The conventional cell arrangement for potentiometric measurements is:

\begin{tabular}{|c|c|c|c|c|}
\hline $\begin{array}{l}\text { inter } \\
\text { refer } \\
\text { elen }\end{array}$ & $\begin{array}{c}\text { internal } \\
\text { aqueous reference } \\
\text { solution }\end{array}$ & $\begin{array}{l}\text { ion- } \\
\text { selective } \\
\text { membrane }\end{array}$ & $\begin{array}{l}\text { sample } \\
\text { tracer } \\
\text { solution }\end{array}$ & $\begin{array}{l}\text { al } \\
\text { nce } \\
\text { ode }\end{array}$ \\
\hline
\end{tabular}

The two reference elements provide constant potentials. The potential difference across the ion-selective membrane is a function of the boundary potentials at the membrane/sample solution interfaces, and the diffusion potential resulting from the differences in chloride ion concentrations (Cattrall and Hamilton, 1984).

For coated-wire electrodes, the cell arrangement is:

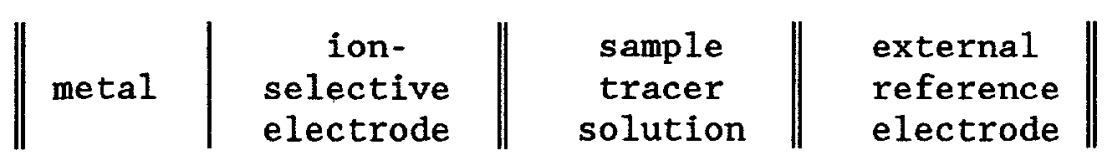



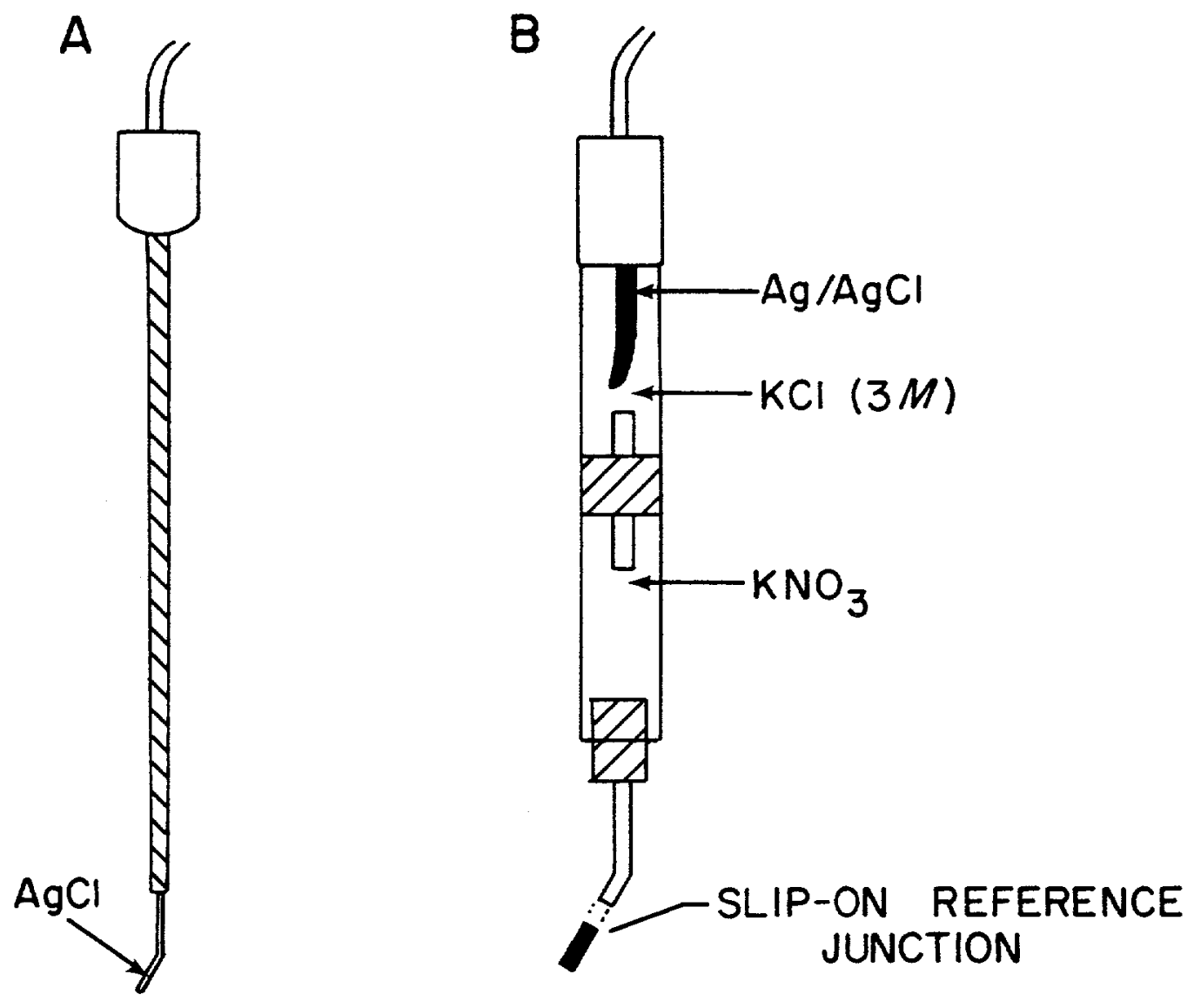

Figure 4.1 Minielectrodes used for experimentation: (A) chloride ionselective electrode coated with silver chloride (AgCl), and (B) double-junction silver/silver chloride (Ag/AgCl) reference electrode. 
There is no internal reference solution, yet the metal/membrane junction does maintain a constant potential. Current theories to explain this anomaly focuses on the role of oxygen in determining the potential difference measured at the membrane. The asymmetry of the coated-wire electrode results in deviations from the behavior expected of conventional ISEs. However, the consensus is the coated-wire electrode performs superior to more traditional electrodes in terms of selectivity towards the ion of interest versus interfering ion(s), but are inferior with respect to drift in potential measurements and reproducibility of results (Cattrall and Hamilton, 1984; Cattrall and Freiser, 1971; James et al., 1972).

The electronic transfer that occurs can be described as a reductionoxidation (redox) reaction. For the single electrode, the redox potential is related to the activity of the ion species according to the Peters-Nernst equation (Stumm and Morgan, 1981):

$$
\text { (4.1) } \quad E=E^{0}+R T / n F \ln \left(\Pi a_{o x}{ }^{n 1} / \Pi a_{r e d}{ }^{n j}\right)
$$

where

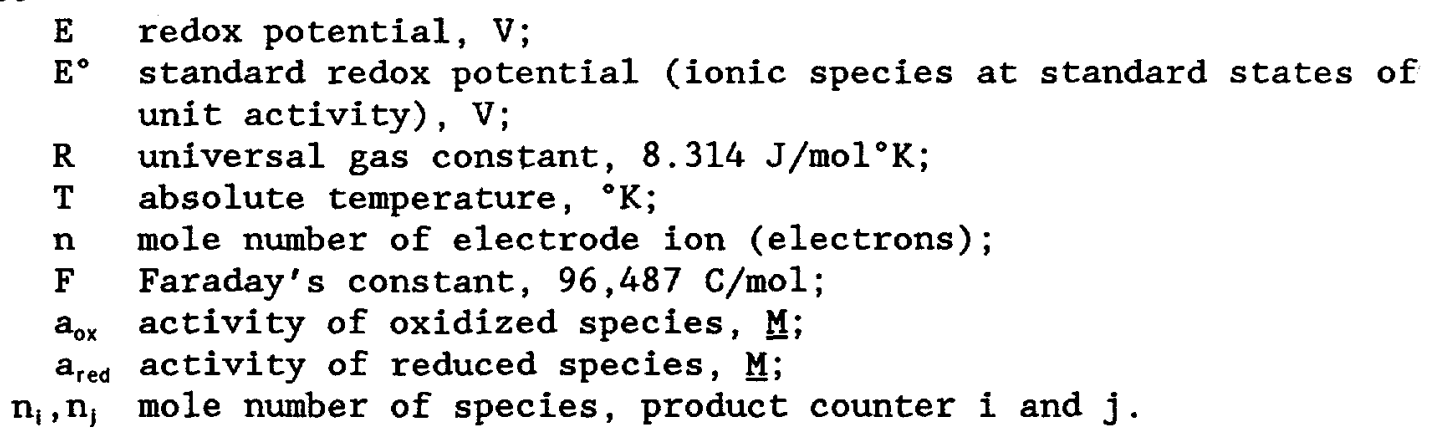

Changes in tracer solutions are measured using calibration curves plotting potential difference against solution concentration (see Figure 4.2). The electrodes actually measure changes in activity and not concentration. Although the two terms are often used interchangeably, as adopted here, they are only equivalent at very dilute concentrations. The differences are explained below in Section 4.1.2.

Interferences by other ions are measured using the Nicolsky-Eisenman equation to calculate the selectivity coefficients (Covington, 1979):

$$
E=R T / n F \ln \left(1+K_{1} a_{1}^{n / z l} / a\right)
$$

where

$$
\begin{aligned}
& K_{i} \text { selectivity coefficient, dimensionless; } \\
& a_{i} \text { activity of interfering ion, } \underline{M} ; \\
& a \text { activity of electrode ion, } \underline{M} ; \\
& z_{1} \text { mole number of the interfering ion. }
\end{aligned}
$$

The coated-wire electrodes generally exhibit favorable selectivity towards the ions of interest as compared to conventional electrodes. Major interferents of chloride electrodes are bromide, nitrate and sulfate. 


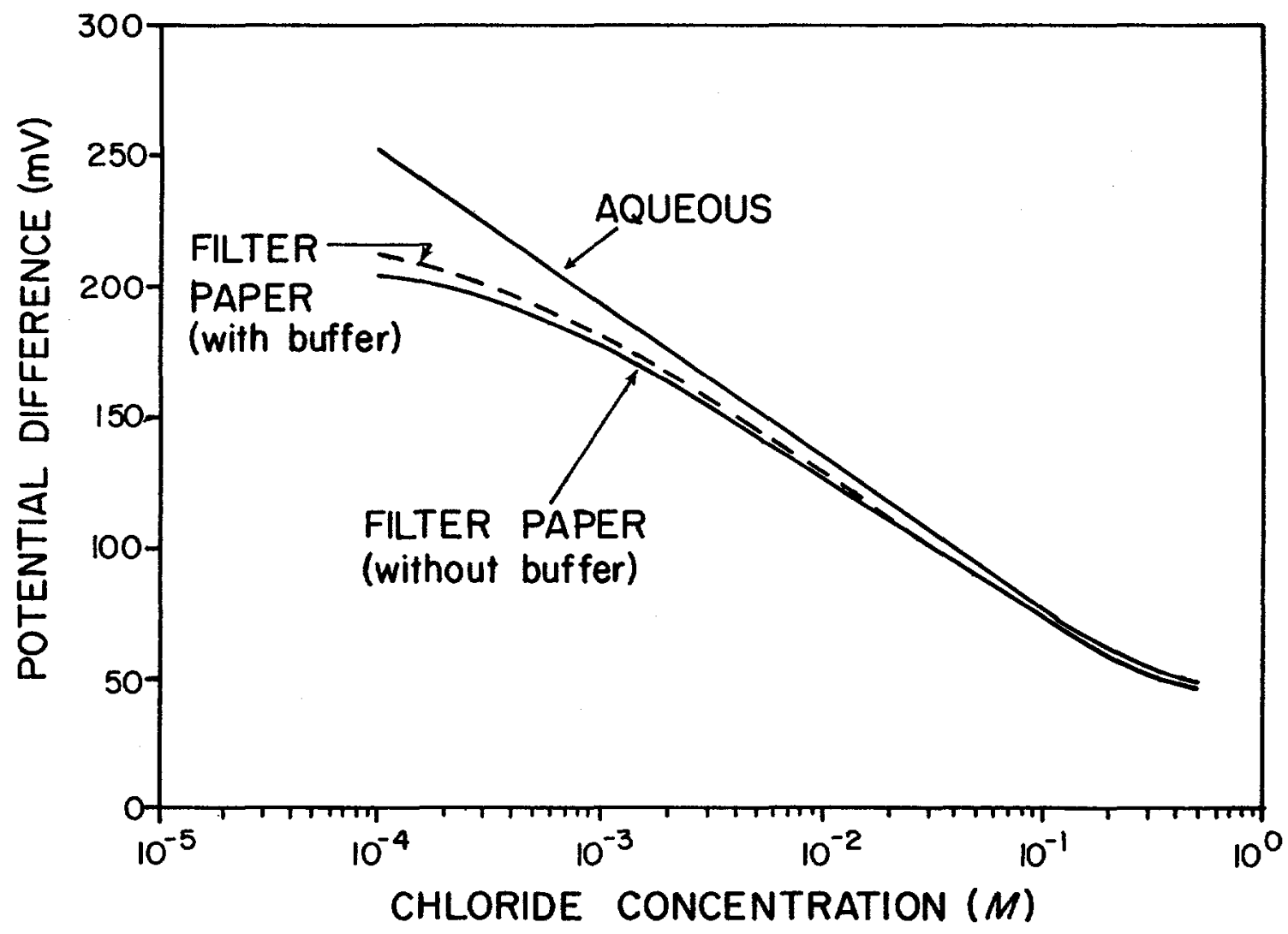

Figure 4.2 Typical calibration curves for chloride electrode plotting potential difference (mV) versus $\log$ chloride concentration ( $\underline{M}$ ) in an "aqueous" environment and a "filter paper" environment, with and without ionic strength buffer. 


\subsubsection{Application to Filter Paper Environment}

Successful application of coated-wire ion-selective electrode technology to transport studies in unsaturated fractured rock depends on several considerations. Although normal use of the electrodes require only small amounts of sample, no known applications have been extended to a filter paper environment. An understanding of the operation of ISEs in more conventional aqueous measurement environments is necessary to gage the performance of the electrodes in a filter paper environment. The term "aqueous" is used here to differentiate from measurements made off filter paper.

Main advantages of ion-selective electrodes lie in their short response times and sensitivity (Freiser, 1978). Depending on the type of electrode and concentration range, the response times vary from seconds to minutes. Many electrode sensors are useful over a wide dynamic operating range, in which linear response occurs over much of the operating range. Their sensitivity to ionic activity requires the use of "titrations" or "ionic strength buffering" to obtain useful concentration data from potential difference measurements.

The sensitivity of the ISEs may be different in the two measurement environments. Figure 4.2 shows typical calibration curves for chloride in both environments. A $\mathrm{CaCl}_{2}$ solution of $10^{-4} \mathrm{M}$ appears to be the lower limit of sensitivity for the chloride ISE in a filter paper environment as compared to $10^{-5} \underline{M}$, or less, in an aqueous environment. The range of linearity also decreases. A straight-line relationship holds between certain ranges of concentration, usually between $10^{-4} \underline{\mathrm{M}}$ and $10^{-1} \underline{\mathrm{M}}$ for chloride in an aqueous environment, but decreases to approximately $10^{-3}$ $\underline{M}$ and $10^{-1} \underline{M}$ in a filter paper environment. Theoretically, at $25^{\circ} \mathrm{C}$ the slope of the line in equation 4.1 is equal to $59 \mathrm{mV}$ per $\log$ activity for ionic species of valence -1 , such as chloride. These values are usually less for practical applications, and certainly in a filter paper environment.

Another consideration is the possible effects of different ionic strengths of the background and tracer solutions in the two environments. In very dilute solutions, the potential difference can be plotted against either the -log of the concentration or activity of a specific ion without loss in accuracy. However, an increase in concentration, and therefore ionic strength, of the tracer solutions results in the departure of the thermodynamic activities of ionic species from their concentrations. The activity $\left(a_{1}\right)$ of ionic species $i$ is its effective concentration in solution and defined as:

$$
a_{1}=\alpha_{i} c_{1}
$$

where

$\alpha_{1} \quad$ activity coefficient of species $i$, dimensionless;

$c_{i}$ concentration of species $i$, $\underline{M}$.

The ionic strength (I) of a solution with $N$ ionic specie is: 
(4.4) $\quad I=0.5 \sum_{i=1}^{N} c_{i} n_{1}{ }^{2}$

where $c_{1}$ and $n_{i}$ are terms defined above for species 1 . For example, a $10^{-3} \mathrm{M} \mathrm{CaCl}_{2}$ solution, with no other significant ionic specie, has an $\mathrm{I}=$ 0.0025 , and $\alpha_{c 1}=0.945$, using the Debye-Huckel equation at $25^{\circ} \mathrm{C}$ (Freeze and Cherry, 1979). A $10^{-1} M$ solution has an $I=0.25$, and $\alpha_{c 1}$ decreases to 0.860 , using the Davis equation (Stumm and Morgan, 1981). The increase in ionic strength leads to greater differences between the activity and concentration of an ionic species.

Normally, ion concentration is measured when an appropriate ionic buffer solution is added both to the sample and standard solutions. This ensures that the ionic strength, and therefore activity coefficient, is constant for all ionic species in the solution. However, because filter paper is used to collect samples of the tracer solution, the amount of sample volume, and therefore the proper volume of buffer solution, cannot be determined easily. If the ionic strengths of the tracer solutions, as compared to the standard solutions, do not change over the course of an experiment, the differences in solution ionic strengths are reflected in the nonbuffered calibration curves. This may not be the situation in the fractured rock if ion exchange, and to a lesser extent, chemical reactions are significant. Calibration of standard solutions (except the $0.5 \mathrm{M}$ standard) adjusted to an $\mathrm{I}=0.25 \mathrm{M}$ indicates the curve deviates from that of nonbuffered standards in the filter paper environment (see Figure 4.2). The adjusted ionic strength corresponds to $10^{-1} \underline{\mathrm{M}} \mathrm{CaCl}_{2}$ solution. A decrease in time to equilibration in the buffered solutions for the more dilute standards is also observed. If a sample is not buffered during potential measurement, its ionic strength is not known. From the calibration curves, the errors associated with measurements at the more dilute concentrations are expected to be larger if the ionic strengths change.

Although ion-selective electrodes have distinct advantages, direct potentiometry using the electrodes are not considered to be a highly precise analytical technique (Freiser, 1978; Cattrall and Hamilton, 1984). According to Freiser (1978), the analytical precision is seldom better than one percent. Aside from the obvious instrumental problems, the major sources of error include electrode interferences, reference electrode instability, electrode drift, and poor calibration of measurement system.

With the availability of solid-state $\mathrm{pH} / \mathrm{mV}$ meters which are equipped with extremely high input impedance, the errors associated with voltage drops and drift caused by current flow through the ce11, are virtually eliminated. However, the resolution of the $\mathrm{pH} / \mathrm{mV}$ meter used for the study is relatively poor at $\pm 2 \mathrm{mV}$, or approximately \pm 88 in concentration. Because of the instrument restrictions, the precision of the calibration curves is expected to be poor, and experimental errors are expected to be large. The error associated with individual potential readings can vary. From the calibration data, it is not unusual to experience as much as $\pm 3 \mathrm{mV}$, even in the aqueous environment (refer to Section 4.2 for further detail). The associated concentration range is \pm 10 to 15 percent. The error is expected to be greater for measure - 
ments off filter paper, often depending on how wet the sample is. A $\mathrm{pH} / \mathrm{mV}$ meter with greater resolution can easily rectify this problem.

The most common errors associated with the ion-selective electrode are heterogeneous distributions of ionic species in the sample solution, presence of interferents in the sample solution, sensor poisoning, and existence of "bound" water. Collection of samples using filter paper can result in spatial variations in the measured concentrations due to flow patterns in the rock at the sampling ports, and the wetting process of the filter paper. A range of potential readings is expected unless the filter paper collects a completely homogeneous sample.

With a double-junction reference electrode employing a $\mathrm{KNO}_{3}$ bridge solution, diffusion of the bridge solution into the sample will interfere with measurements. Because nitrate $\left(\mathrm{NO}_{3}^{-1}\right.$ is a major interferent of chloride, potential drift occurs with time in a confined sample volume. The decrease in potential has been observed to be as much as $10 \mathrm{mV}$ in a 6 -hour period for $30 \mathrm{~mL}$ of distilled water $\left(\leq 10^{-5} \underline{\mathrm{M}}\right.$ chloride). The corresponding apparent increase in chloride concentration is one and a half times.

It is also not uncommon for the electrode to experience fouling and poisoning of the membrane leading to a general loss of membrane integrity. Fouling and poisoning are generally due to water penetration or irreversible reactions of solution ions with the membrane. A drastic shift in potential is a good indication of the end of the electrode's useful life.

Whatman filter paper number 42 is made of cellulosic material from cotton linter. As shown in Figure 4.2, "bound" water may be responsible for the apparent increase of ionic activity or concentration as indicated by the "filter paper" calibration curves. The filter paper may have caused some water to be no longer "free" to act as a solvent.

The operation of the reference electrode can also be a factor. A variety of problems, usually between the liquid junction of the reference half-cell and the sample solution, can occur. These errors are poorly understood. Use of a salt-bridge which does not contain interfering ions should minimize their effects. The nitrate bridge solution of the current reference electrode prevents contamination of the $\mathrm{KCl}$ filling solution, but is also an interferent of chloride.

Potential drift, the shift in potential difference values over time (shift in calibration curve), and the associated problem of time to equilibration, are also difficulties encountered. Under the best of controlled laboratory conditions, potential drift occurs over time. The reasons are numerous and varied, with the major cause the change in temperature (Freiser, 1978). Time to equilibration presents the problem of when to take a reading. Most researchers recommend a set time period, or set minimum error such as $\pm 0.2 \mathrm{mV}$ or even greater errors for ion-selective electrodes (Freiser, 1978; Covington, 1979). The drift over prolonged periods are usually much worse, possibly in the range of several millivolts. Depending on the type and severity of the drift, correction may be difficult to impossible. 
With the current reference electrode, especially in a filter paper environment, nitrate interference with timely and accurate potential measurements poses a substantial problem. For sampling reasons, a small piece of filter paper is used. The problem of outward diffusion of $\mathrm{KNO}_{3}$ is exacerbated by the proximity of the electrode junctions. Furthermore, depending on the moisture content of the filter paper sample, the $\mathrm{KNO}_{3}$ may be drawn out by the filter paper. Potential measurements are restricted within a short time period, and the recommended placement of the electrodes is for as far apart as possible. The use of a reference electrode with a bridge solution other than $\mathrm{KNO}_{3}$ will negate this problem.

The above discussion touches upon major considerations in ion-selective electrochemistry as applied to this research. There are other factors to be considered, many of which are discussed in Freiser (1978). In order that the electrodes are used properly, the magnitude of potential errors can be minimized with a superior $\mathrm{pH} / \mathrm{mV}$ meter, controlled experimental environment, regular calibration of electrodes, and confirmation of standards. The judicious interpretation of data should be accompanied with observations of the operational integrity of the electrode, and the moisture content of the filter paper sample.

\subsection{Transport Processes}

Much of past and current research has concentrated on obtaining relevant hydraulic and solute transport parameters of fluid flow through saturated and unsaturated porous media. Estimates of transport parameters have been obtained from large-scale field tests of fractured rock systems (de Marsily, 1986) by visualizing the existence of equivalent porous media. Research on solute transport through single fracture segments has been confined to single phase flow (Neretnieks et al., 1982; Moreno et al., 1985). Although conceptual models, such as Wang and Narasimhan's (1985), explored the hydraulic behavior of partially saturated fractures, no measurements of flow parameters, and for that matter, transport parameters are available. To describe the phenomenon where dissolved substances are carried along by fluid displacement, the laws governing transport of miscible fluids must be coupled with that of fluid movement.

The transport of solutes through partially saturated rock fractures and adjacent rock matrices involves several processes which are governed by laws of transport, and laws of interaction between the transported substances and the medium. The mechanisms of transport for nonreacting species include advection, molecular diffusion, and mechanical dispersion. Sorption due to ion exchange, and chemical and redox reactions are also mechanisms to be considered for reacting species, as is radioactive decay for radionuclides.

Solute transport characterization in a fractured rock system is complicated by the fracture-rock matrix interaction. The effects of solute diffusion in the matrix can be considered in a manner conceptually similar to 'double-porosity' or 'Intra-aggregate' transport models (Grisak and Pickens, 1980). Solute transport is a multi-dimensional process because of solute concentration gradients from the difference in rate of transport in the fracture compared to the surrounding rock matrix. For 
partially saturated flow, the moisture content or suction is also a factor.

\subsubsection{Major Mechanisms}

The advection-dispersion transport equation for reactive constituents in both the fracture and porous media can be written as (Bear, 1979):

$$
\partial(n C) / \partial t=-\operatorname{div}(q C)+\operatorname{div}[n D \operatorname{grad}(C)]+S^{*} n
$$

where

$$
\begin{array}{cl}
\text { n effective porosity or water content, dimensionless; } \\
\text { C volumetric concentration, } \underline{M} ; \\
\text { q Darcian velocity, m/s; } \\
\text { div differential operator, } 1 / \mathrm{m} ; \\
\text { D combined diffusion-dispersion coefficient, } \mathrm{m}^{2} / \mathrm{s} ; \\
\mathrm{s}^{*} \text { composite source-sink rate, } \underline{\mathrm{M}} / \mathrm{s} .
\end{array}
$$

For saturated flow, $\mathrm{n}$ is the effective porosity, and for partially saturated flow the water content. The composite source-sink rate term is a catchall to account for solute-medium interactions, and is discussed in the subsequent section. The advective flux of solutes is simply the product of $\mathrm{q}$ and $\mathrm{C}$, in which $\mathrm{q}$ is a gross average of actual velocities. The Darcian velocity is usually expressed as:

$$
\text { (4.6) } \quad q=u_{s} n
$$

where $u_{s}$ is the linear velocity of water. In partially saturated fractures and rock matrices, $q$ is strongly influenced by water content, and therefore, suction.

Solute movement is not only influenced by advection but by molecular diffusion and mechanical dispersion. Molecular diffusion is described by Brownian motion theory, and mechanical dispersion occurs as a result of nonuniformity in the flow velocity. Diffusion is caused by molecular agitation, dispersion by differences in aperture and travel distance from one pore to another, or one section of fracture to another. The two processes cause spreading in all directions. Diffusion and dispersion can be viewed as scale-dependent, but similar to processes described by Fick's first law in which diffusion describes small-scale effects and dispersion large-scale effects. The twin processes are difficult to separate for large-scale problems, and a composite diffusion-dispersion coefficient:

$$
\text { (4.7) } \quad D(\psi)=D_{d}\left(\psi, u_{s}\right)+D_{m}(\psi)
$$

where

$D_{d}$ mechanical dispersion coefficient, $\mathrm{m}^{2} / \mathrm{s}$;

$D_{m}$ effective diffusion coefficient, $\mathrm{m}^{2} / \mathrm{s}$;

describes the phenomenon of hydrodynamic dispersion. Both processes are functions of water content. The effective diffusion coefficient is related to the diffusion coefficient in free water, $d_{0}$, which is (de Marsily, 1986): 
where

$$
\begin{aligned}
& \mathrm{N} \text { Avogadro's number, } 6.023 \times 10^{23} \text {; } \\
& \mathrm{r} \text { mean radius of ion or diffusing molecular aggregate, m } \\
& \text { (infinite solution only). }
\end{aligned}
$$

The effective diffusion coefficient is usually less than $d_{o}$ due to tortuosity. The mechanical dispersion coefficient is often represented by:

$$
D_{d}=\alpha(\psi) u_{s}
$$

where the dynamic dispersivity $\alpha$ (m) is a characteristic property of the rock medium and also a function of water content. For multi-dimensional flow, longitudinal $\left(\alpha_{1}\right)$ and transverse $\left(\alpha_{\mathrm{T}}\right)$ dispersivities are described as :

$$
D_{L}=\alpha_{L}(\psi) u_{s}
$$

and

$$
D_{\mathrm{T}}=\alpha_{\mathrm{T}}(\psi) \mathrm{u}_{\mathrm{s}} .
$$

Walter (1985) showed $D_{m}$ to be a function of pore-size distribution of the rock matrix. With increasing tortuosity and decreasing water fluxes, hydrodynamic dispersion is expected to increase for both fracture and matrix flows. The magnitude of $D_{d}$ is expected to be larger than $D_{m}$, but with decreasing water content (increasing suction) the relative importance of diffusion may increase with respect to mixing. Nielsen and Biggar (1961, 1962), and Biggar and Nielsen (1962) showed that diffusion becomes more important in soils with decreasing moisture content.

Statistical theories describe molecular diffusion and mechanical dispersion as random or stochastic processes (Fischer et al., 1979; de Marsily, 1986). For diffusion, when a slug of tracer is introduced, Fick's first law describes the velocity or displacement of a solute particle as a function of time. The function may only be specified as a probability distribution. If the probability distribution is not a function of time, the process is known as a stationary process which is described by statistical mean, variance, and an autocorrelation function of the process. The rate of spreading is a constant after an initial development period, and can be related to the diffusion coefficient:

$$
\text { (4.10) } \quad \partial \operatorname{Var}(\mathrm{x}) / \partial \mathrm{t}=2 \mathrm{D}_{\mathrm{m}}
$$

and

$$
\operatorname{Var}(x)=\int_{0}^{\infty} \int_{0}^{\infty} \int_{0}^{\infty} x^{2} c(x, y, z, t) d x d y d z
$$


where $\operatorname{Var}(x)$ is the spatial variance of solute particles having undergone a random walk process. The probability function is described by the concentration distribution.

Mechanical dispersion is conceptually Fickian if the deviation of velocity from the mean is treated as a diffusive process. G.I. Taylor (Fischer et al., 1979) proposed that the velocity and concentration profiles which developed over the cross section of a pipe can be described as such a process. This means equation 4.10 is valid for mechanical dispersion as well. Therefore, hydrodynamic dispersion is referred to as Fickian dispersion. Equation 4.10 can be written by substituting $D_{m}$ with $D$ :

$$
\partial \operatorname{Var}(\mathrm{x}) / \partial \mathrm{t}=2 \mathrm{D}
$$

However, Taylor's analysis is valid only if the solute is displaced after a long enough period.

Subsequent extensions of the statistical approach to describe dispersion (Mercado, 1967; Gelhar et al., 1979) indicate that the process is a function of time. Taylor's mechanism is said to occur only after a time corresponding to a travel distance much larger than the lateral correlation scale. The statistical theories maintain the classical advectiondispersion equation is not valid even when Taylor's mechanism takes place. A dispersion coefficient fitting data obtained at one observation point is invalid for data at another observation point further away from the input source (de Marsily, 1986). This suggests scale- dependency.

The concept of macrodispersion uses "mean" and "perturbation" advectiondispersion equations to describe the spreading phenomenon. But different parameters are of importance. Important parameters include the mean $(K)$ and variance $(\operatorname{Var}(K))$ of hydraulic conductivity, integral scale $(\epsilon)$, and transverse dispersivity $\left(a_{T}\right)$ estimated from the mean equation. The longitudinal macrodispersion coefficient is then broken down into two regimes (Mercado, 1967; Gelhar et a1., 1979):

$$
\text { (4.13) } \quad D_{L}=\left[\operatorname{Var}(K) / K^{2}\right] x^{*} u^{*}, \quad x^{*} \ll \epsilon
$$

and

$$
D_{L}=1 / 3\left[\operatorname{Var}(K) / K^{2}\right]\left(\epsilon^{2} / \alpha_{T}\right) u^{*}, x^{*} \gg \epsilon
$$

where $\mathrm{x}^{*}$ and $\mathrm{u}^{*}$ are the mean displacement and velocity specified by the mean equation formulations.

Mixing in the fracture is not only influenced by variations in fluid velocity, but also velocity variations between channels in the fissure (Neretnieks et al., 1982; Moreno et al., 1985). Tsang and Witherspoon (1985) suggested a rough fracture can be envisioned as a collection of voids defined in terms of an aperture density distribution. Channeling dispersion assumes the existence of different channel pathways with different aperture openings, $e_{f}(m)$, and fluid velocities differ in each channel. However, each fracture channel is still assumed to be of a 
parallel plate configuration. If the temporal concentration distribution, or breakthrough curve, for each channel is given as $C_{f}\left(e_{f}, t\right)$, then the concentration of the mixed effluent from all the channels is (Neretnieks et al., 1982):

$$
\frac{C(t)}{C_{0}}=\frac{\int_{0}^{\infty} f\left(e_{f}\right) Q\left(e_{f}\right) C_{f}\left(e_{f}, t\right) d e_{f}}{\int_{0}^{\infty} f\left(e_{f}\right) Q\left(e_{f}\right) d e_{f}}
$$

where $f\left(e_{f}\right)$ is the fissure frequency or density function. For nonsorbing tracers, Moreno et al. (1985) found the results to be similar for both hydrodynamic dispersion and channeling dispersion. However, for longer distances, the channeling dispersion model showed greater dispersion and earlier arrival of the solute front than the hydrodynamic dispersion model.

Diffusion into and out of the rock matrix contributes to the retardation of solute transport within the fracture. Grisak et al. (1980) indicated that diffusion into the low permeability pore spaces of fractured clayey glacial till contributes significantly to the retardation of solute movement in the fracture. The matrix hydraulic conductivity of the till was estimated as $6 \times 10^{-11} \mathrm{~m} / \mathrm{s}$ compared to $6 \times 10^{-8} \mathrm{~m} / \mathrm{s}$ of the tuff used in this study. A theoretical diffusion coefficient, $D_{m}$, for chloride of $5 \times 10^{-11} \mathrm{~m}^{2} / \mathrm{s}$ was estimated from model results. The net result of the continued strong flux of solute from the fracture into the matrix, caused by removal of ions from solution, is to reduce the mean solute velocity in the fracture.

Neretnieks et al. (1982) also found matrix diffusion in granite cores to be a significant process for solute transport in the fracture. Expanding the work done by Neretnieks et al., Moreno et al. (1985) compared their fracture tracer test data against two dispersion models, one using hydrodynamic dispersion, the other channeling dispersion; the experimental data for both models compared well only when diffusion into the matrix and sorption were considered. The effects of sorption is discussed in further detail below.

\subsubsection{Solute-Medium Interactions}

The third term on the right-hand side of equation 4.5 contains a composite source-sink rate term, $S *$, which accounts for interactions between the solute and medium. Sorption, and chemical and redox reactions, and radioactive decay for radionuclides can retard transport and attenuate solute concentration. Neretnieks et al. (1982) and Moreno et al. (1985) found sorption onto the surface of the fracture, as well as the intercrystalline microfissures of the matrix, of granite cores to be significant. Sorption is especially important for transport of cationic species, and probably radionuclides.

Sorption is caused by ion exchange. Ion exchange is expected to occur between water flowing through the rock and the mineral surfaces. Attraction between ions with opposite charge results in an electrical 
(diffuse) double layer (Kemper, 1960). The mechanisms of ion exchange usually fall into one of two categories (de Marsily, 1986): (1) imperfections or ion substitutions in the crystal lattice of the mineral causes electrical imbalance, and the creation of two electrical layers, one stable, the other mobile; and (2) the specific adsorption of ions by a mineral initially uncharged creates a stable electrical layer to which other ions become attached. In general, divalent cations have stronger affinities than monovalent ions, therefore greater selectivity in preferential ion exchange.

The effects of sorption in the rock matrix, in which the matrix is in local equilibrium with the fluid, can be described by (Freeze and Cherry, 1979):

$$
R_{v}=u_{s} / u_{t}=1+\left(\rho_{b} / n\right) K_{d}
$$

where

$R_{v}$ retardation factor due to volume sorption;

$u_{t}$ mean velocity of the solute front. $\mathrm{m} / \mathrm{s}$;

$\rho_{\mathrm{b}}$ rock matrix bulk density, $\mathrm{kg} / \mathrm{m}^{3}$;

$\mathrm{K}_{\mathrm{d}}$ distribution coefficient, $\mathrm{mL} / \mathrm{g}$.

The distribution coefficient quantifies the mass of solute sorbed on the solid phase in relation to that in the liquid phase. For fracture flow, a more appropriate expression is based on a per-unit-surface-area basis. Retardation is then (Neretnieks et al., 1982):

$$
\text { (4.17) } \quad \mathrm{R}_{\mathrm{a}}=1+\mathrm{a}(\psi) \mathrm{K}_{\mathrm{a}}
$$

where

$$
\begin{aligned}
& R_{a} \text { retardation factor due to surface sorption; } \\
& a \quad \text { specific surface (fracture surface per unit volume of rock), } \\
& \mathrm{m}^{2} / \mathrm{m}^{3} ; \\
& \mathrm{K}_{\mathrm{a}} \text { surface equilibrium constant, m. }
\end{aligned}
$$

Retardation in both the matrix and fracture is a function of water content.

Compositionally, the rock matrix and fracture of the test block are dominated by calcium; cationic exchange sites are also expected to be calcium-dominated. The introduction of a dilute calcium chloride solution into the rock matrix and fracture serves to saturate the exchange sites over time as calcium ions preferentially replace other common cations that may be present, such as magnesium (Mg) and sodium ( $\mathrm{Na}$ ). The exchange of calcium from subsequent tracer solutions can occur with no significant net change in its base concentration. If the temperature, $\mathrm{pH}$ and redox conditions in the rock matrix and fracture remain fairly stable, the ionic strength which affects electrode measurements also remains fairly constant. Ion exchange then becomes less of a variable in solute transport.

Other geochemical and radiological mechanisms can attenuate solute concentrations during transport. The extent of chemical reactions such as precipitation and dissolution depends on factors such as the minerals 
present in the fracture, and the kinetics of such reactions. Weber (1986) found that fracture-filling minerals in tuff include not only quartz and opalline silica but calcite and clay minerals as well. Acidbase and redox reactions depend on the $\mathrm{pH}$ and redox potential, respectively. Radioactive decay of radionuclides can result in the disappearance of the original species and/or formation of daughter products. The significance of radiological mechanisms is determined by the parent material and decay rates of all chain members (Wilson and Dudley, 1987). Such reactions are generally described by:

$$
S^{*}=\partial C / \partial t=-k_{s} C
$$

if the process is linearly proportional to the concentration of the solute species, and $\mathrm{k}_{\mathrm{s}}(1 / \mathrm{s})$ is the decay constant. The equation is applicable for both chemical loss and radioactive decay. Characterizing only the transport behavior of the original solute species may be inadequate as the transport properties of the new species are potentially different from the original ones.

\subsubsection{Selection of Tracer}

The proper choice of tracers is dictated by the objective of the experiment. Experimental methods to characterize transport behavior of solutes in the unsaturated fractured rock environment is hampered by the small quantities of water for sampling. Chloride is chosen initially because it is relatively conservative, and it can be detected easily by coated-wire electrode potentiometry. The development of techniques to study conservative tracers provides insight into the more complicated nature of radionuclide transport behavior in unsaturated fractured rock.

Chloride is considered conservative. However, chloride ions may travel faster than the average water velocity, particularly if significant amounts of negatively-charged exchange sites, such as commonly found on clay, exist. Biggar and Nielsen (1962) found that chloride breakthrough occurred ahead of the average water velocity even in a glass bead medium without attempting to explain the phenomenon. James and Rubin (1972) claimed the shift in breakthrough to be a result of apparatus-induced dispersion. With these qualifications, chloride is considered wel1suited for initial solute transport assessments in fractured rock systems.

Other suitable tracers available as coated-wire ion-selective electrodes include bromide, sulfate, nitrate, calcium and magnesium. The anions, with the possible exception of sulfate, can serve as conservative tracers in tuff. Of course, when nitrate is the tracer ion, the current double-junction reference electrode cannot be used. An electrode with a different bridge solution, or a single-junction electrode will have to be used. The use of cations, under the proper conditions, can provide valuable information. They are most appropriately used to study ion exchange and sorption to rock surfaces. A better understanding of the solute transport processes can be achieved with different tracers. 


\subsubsection{Effects on Breakthrough Curves}

Solute transport is the displacement of miscible fluids in time and space. Breakthrough curves plot the change in concentration with displacement. Estimates of transport parameters such as solute travel time, dispersion coefficient and dispersivity can be obtained from these curves. A tracer solution usually introduced as a slug or step input, replaces the background solution flowing through the fracture and porous matrix. The change in relative concentration, $C^{*}$ or $\left(C-C_{1}\right) /\left(C_{0}-C_{1}\right)$, is measured over time or space, where $C_{1}$ is the concentration of the background solution, and $\mathrm{C}_{0}$ is the concentration of the tracer solution. If the background solution has a $C_{1}=0$, the relative concentration collapses to the commonly used ratio of $\mathrm{C} / \mathrm{C}_{0}$. Both analytical solutions and numerical schemes have been developed for various boundary conditions to estimate solute transport parameters by finding the best fits to the observed breakthrough curves.

The effect of different transport processes on the shape of the breakthrough curve for both fracture and porous matrix transport is illustrated in Figure 4.3. For simplicity, breakthrough plotting $\mathrm{C}^{*}$ versus time for a step input function is discussed. If neither diffusion nor dispersion occurs, the displacement of two liquids results in the classical case of piston flow. However, all fluids are miscible and mixing occurs to some extent at the front between the two solutions. The spread about the mean position of the front gives rise to the $S$ shape (sigmoid) of the breakthrough curve. Attenuation of the tracer ions is manifested in the end-tail reaching a plateau at $\mathrm{C}^{*}<1.0$. Retardation due to sorption on rock surfaces results in a delay of breakthrough, or a shift of the S-shaped curve to the right. Ideally, the breakthrough curve is symmetrical about the advancing front with the deflection at the mean relative concentration, or $C^{*}=0.5$. The spread of the two tails are expected to increase dramatically with decreasing water content (or increasing suction) due to the increase in tortuosity of the flow path and the decrease of the rate of water percolation.

For fracture flow in which a concentration gradient exists between the fracture and rock matrix, multi-dimensional transport occurs due to slow matrix diffusion. This generally results in the flattening of the endtail of the S-shaped curve at less than $C^{*}=1.0$. Matrix diffusion also retards the breakthrough of the tracer front. Additional insights are provided by Grisak and Pickens (1980), who examined the effects of several different variables on fracture transport including fracture aperture size, fracture water velocity, dispersivity, and porosity and distribution coefficient of the rock matrix.

The effects of the different solute transport processes on breakthrough curves of slug or impulse inputs, in which a finite mass of solute is introduced at a given time, are not as immediately evident at a glance. In general, the solutions to the classical advection-dispersion equation result in symmetrical bell-shaped concentration profiles for spatial distributions, and skewed profiles for temporal distributions. 


\subsubsection{Estimation of Transport Parameters}

Solute transport through fractured rock, especially under unsaturated conditions, offer new challenges to interpretation. Estimates of solute transport parameters are generally obtained by fitting analytical or numerical solutions of the classic advection-dispersion model to experimental data. Because of the expected spatial variability of point

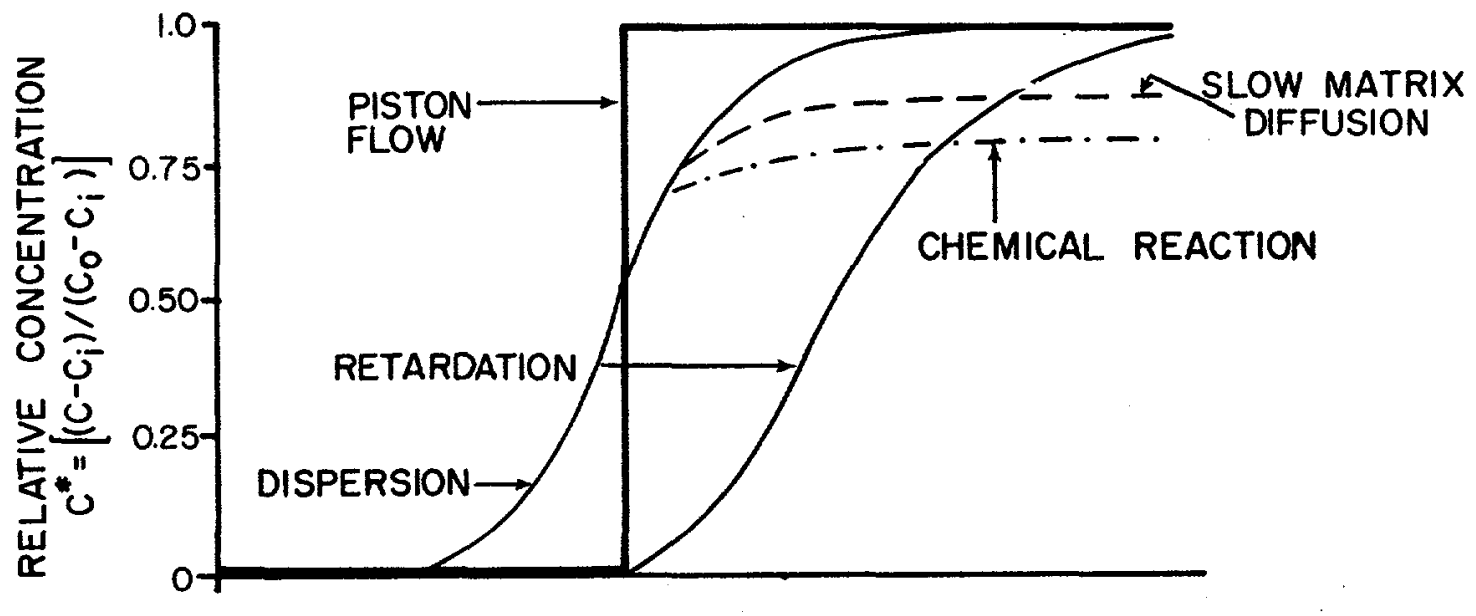

TIME

Figure 4.3 Effects of different solute transport mechanisms on the shape of the breakthrough curve for a step input test plotting relative concentration, $C^{*}=\left(C-C_{1}\right) /\left(C_{0}-C_{1}\right)$, versus time elapsed (hr). $C_{1}$ denotes the background solution concentration, and $C_{0}$ the tracer solution concentration. 
sampling due to heterogeneities, the physically-based classical approach with constant coefficients, and assumptions of steady, homogeneous pore water velocity, appears inadequate. To demonstrate the validity of the advection-dispersion model using such parameters is difficult as they are dependent on the model assumptions in the first place.

An alternative is to use stochastic models such as Gelhar and Axness (1983), and Dagan (1984) for the interpretation of experimental data. Stochastic models describe the experimental observations as realizations, and estimates of transport parameters are expectations over the ensemble of possible realizations. These models may be better predictors of transport parameters, but inherent weighing of individual observations still occurs with the model assumptions.

The use of porous plates in this research, especially a fracture plate which covers the fracture and adjacent matrix, may result in varying concentration boundary conditions at the top of the fracture segment. A method of data analysis which is independent of the physical or chemical behavior of transport, and does not require special knowledge of the input function is required. That method may be found in spatial or temporal moments analysis.

Freyberg (1986) proposed the use of lower-order spatial moments of the concentration distribution to characterize a solute plume and to estimate transport parameters. The moments are defined independently of the physical or chemical behavior of transport. The zeroth-, first-, and second-order spatial moments of the concentration distributions are calculated from point observations requiring few model assumptions.

The center of mass and the spread of a plume about its center of mass can be estimated with first- and second-order spatial moments. The two moments correspond to the mean and variance of the spatial concentration distribution. Temporal moments are then directly used to estimate the travel time and dispersion coefficient for temporal concentration distributions. For one-dimensional vertical flow, the mean and variance for the continuous case are:

$$
E(t)=\int_{-\infty}^{\infty} t C(x, t) d t / \int_{-\infty}^{\infty} C(x, t) d t
$$

and

$$
\operatorname{Var}(t)=\int_{-\infty}^{\infty}(t-E(t))^{2} C(x, t) d t / \int_{-\infty}^{\infty} C(x, t) d t
$$

For the discrete case, the mean and variance are:

$$
t_{m}=\sum_{i=1}^{N} t_{i} C_{i} / \sum_{i=1}^{N} C_{1}
$$

and

$$
s_{t}^{2}=\sum_{i=1}^{N}\left(t_{i}-t_{m}\right)^{2} C_{i} / \sum_{i=1}^{N} C_{i} \text {. }
$$


The travel time is simply the first moment of the temporal concentration distribution. By assuming Fickian dispersion theory is valid, the dispersion coefficient for the discrete case is described as:

$$
d s_{x}^{2} / d t=2 D
$$

Knowing the relationship between the second moments for the spatial and temporal concentration distributions:

$$
d s_{x}^{2}=u_{t}^{2} d s_{t}^{2}
$$

the dispersion coefficient is estimated by:

$$
D=\left(u_{t}{ }^{2} / 2\right)\left[\left(s_{t, 2}{ }^{2}-s_{t, 1}{ }^{2}\right) /\left(t_{m, 2}-t_{m, 1}\right)\right]
$$

where $t_{m, 1}$ and $t_{m, 2}$ are the means, and $s_{t, 1}{ }^{2}$ and $s_{t, 2}{ }^{2}$ are the variances calculated at two different observation points.

Estimates of fracture transport parameters are calculated in Section 4.6.2, and a possible analytical solution is presented below in section 4.2.6 which assumes all the flow from the fracture plate enters the fracture near the fracture opening with minimal rock matrix interaction. The moments calculated can be interpreted using either the classic or stochastic models, and the predictive ability of these models validated. While no attempt is made to fit the parameters to any of these models for this research effort, it is recommended that this be done.

\subsubsection{Analytical Solution}

With the porous plate controlling the input of tracer solution, neither analytical solutions to a slug nor a step input are appropriate. Input to the fracture and rock matrix is a function of chloride breakthrough in the plate, which in turn is a function of time. If one assumes the fracture and rock matrix to be homogeneous, then the input function, for any point, can be written as:

$$
\text { Mass input }=\begin{array}{cc}
0 & t<0 \\
f(t) & t \geq 0
\end{array}
$$

where $f(t)$ is expressed in units of mass/time. At any time $t_{1}$, if the slug of mass $\left(M_{i}\right)$ introduced is:

$$
M_{i}=f\left(t_{i}\right) d t
$$

then the breakthrough of the plate can be treated as a series of slug inputs, and the contribution for each time increment $d t_{i}$ is summed up. Assuming the superposition principle holds, then for a one-dimensional discrete case, the concentration at time $t$ and position $x, C^{\prime}(x, t)$, is (Fischer et al., 1979):

$$
C^{\prime}(x, t)=\sum_{i=1}^{N} \frac{f\left(t_{1}\right) d t_{1} \exp \left[-\left(x-u_{s}\left(t-t_{1}\right)\right)^{2} / B\right]}{\operatorname{rA~}(\pi B)^{0.5}}
$$


where $B=4 D\left(t-t_{i}\right)$. If $f(t)$ is expressed as $\mathrm{mg} / \mathrm{s}$, then $C^{\prime}(x, t)$ has units of $\mathrm{mg}$ of solute $/ \mathrm{Kg}$ of water, or ppm (parts-per-miliion). The results can be converted to concentration units of knowing the molecular weight of chloride. For more in depth discussions on possible solutions, the reader is referred to texts such as Fischer et al. (1979), Bear (1979), and de Marsily (1986).

\subsection{Coupled Matrix/Fracture Transport - Boundary Integral Method}

There are several advantages to applying the experimental results to a computer model. From a solute transport stand point, the interpretation of the chloride breakthrough data is affected by fracture-matrix flow interaction. Estimates of transport parameters are also affected by the contribution of fracture plate flow entering the fracture through the matrix. Strictly speaking, equation 4.28 is valid if flow from the fracture plate enters the fracture at the opening. However, the use of temporal moments analysis preempts the need to know the exact input function, as long as the fracture segment of interest is below the region where fracture-matrix flow interaction occurs. A model can determine how far along the fracture the interaction essentially ceases.

Calculation of the suctions imposed at the top of the test block assumes a constant average value under the porous plates. However, the variation in suction over the top boundary of the rock may be large enough, especially under the fracture plate, such that the exact suction over the fracture opening is different from that at the edges of the plate. A model can calculate the pressure heads under the plates by neglecting the presence of any material used as contact. of course, with filter paper between the plates and rock surface, the suction distribution may be smaller, and more flow may be diverted to the fracture near the opening.

Estimates of the fracture transmissivity assumes one of two scenarios: all the flow from the fracture plate, or only the flow proportional to the aperture opening, enters the fracture. The second scenario subtracts the flow attributed to the matrix by using an average matrix conductivity. Whether either value is reasonable can be determined by varying the transmissivity, and fixing all input parameters already determined experimentally by other means.

A11 the above reasons, and possibly more, argue for the use of a computer model such as the one employing the boundary integral method (BIM) developed by Rasmussen (1988). By dividing the test block and plates into appropriate domains, experimentally determined data on flow rates and imposed head gradients can be used in a case study. The model can provide information not only on streamlines and travel times of solute, but also the head distribution at the boundaries and within the test block. The methodologies of BIM are described briefly below again, and a continuation of the case study (see Section 3.9.2.3) for an idealized test block 1 system is presented in Section 4.6.1 with the experimental results.

As a reminder, methodologies developed employ the boundary integral method to calculate water flow and solute transport properties of unsaturated fractured rock. The method discretizes the boundaries of two- 
dimensional flow domains and solves the relationship between fluid flow and head within them. Solutions are obtained by solving Laplace's equation using Gauss's formula, and the weighted residual statement which minimizes error between true and estimated head and flux over a domain. The method is capable of accounting for flow between the rock matrix and fracture. Solute travel times and breakthrough curves are determined by integrating the inverse velocity along constant streamlines. The stream function is equated with cumulative discharge. The boundary integral method, unlike finite element and finite difference methods, provides estimates of travel time by defining smooth functions of velocity and streamline at points internal to the discretized flow domain. The reader is encouraged to refer to Rasmussen (1988) for greater detail of the methodologies developed.

\subsection{Water Penetration into Test Block Sampling Ports}

Six circular cylindrical sampling ports were drilled in the test block. The ports are expected to affect the flow and the study of solute transport in the test block. However, they serve as obstacles to flow, and the water pressure is expected to be greater over parts of the cavity surface. Water entry into, or exclusion from, the ports from steady downward unsaturated seepage was explored by Philip (1988). For a given shape and size of the cavity, knowing the hydraulic conductivity or seepage velocity, characteristic cavity length and two hydrologic parameters of the porous medium, one can establish whether seepage water enters the cavity. The analysis also offered maps of dimensionless potential and stream functions, and velocity fields to aid in understanding the flow into and around cavities such as the ports.

Philip (1988) solved for $\nu_{\max }$, a maximum dimensionless Kirchhoff potential using a quasilinear form of the basic flow equation in unsaturated porous medium. Knowing $\nu_{\max }$, which is a function of the dimensionless characteristic cavity length $s$, one can determine whether water enters the cavity for a given combination of unsaturated hydraulic conductivity or seepage velocity, $k_{0}$, characteristic cavity length, $1_{c}$, saturated conductivity, $\mathrm{K}_{\mathrm{m}}$, and sorptive number, $\alpha_{\mathrm{s}}$. The seepage velocity occurs at $\psi_{0}$, a water potential less than atmospheric pressure $\left(\psi_{0}<0\right)$. The $1_{c}$ for a circular-cylindrical cavity is its radius. The critical seepage velocity $k_{0}$. is calculated as:

$$
\mathrm{K}_{0} \cdot=\mathrm{K}_{\mathrm{m}}\left[\nu_{\max }(\mathrm{s})\right]^{-1} \text {, }
$$

with

$$
s=0.5 \alpha_{s} 1_{c}
$$

and $\alpha_{s}$ entering the exponential representation of $K(\psi)$,

$$
K(\psi)=K_{0} \exp \left[\alpha_{s}\left(\psi-\psi_{0}\right)\right] \text {, }
$$$$
0 \geq \psi \geq-\infty \text {. }
$$

Water is defined to have entered the cavity for $k_{0}>k_{0}$.

The applicability of this method is summarized in Table 4.1 which presents the results using hydraulic conductivity values of several tuff 
cores obtained from boreholes at the study site. The hydraulic conductivities correspond to the saturated case and at $\psi=10 \mathrm{kPa}(1.0197 \mathrm{~m})$. For a $l_{c}=1.0 \mathrm{~cm}$, which is the radius of the sampling ports, the tuff cores have $\nu_{\max }$ values very close to 1.0 indicating $\mathrm{K}_{0}$. is very nearly the saturated conductivity with correspondingly low suction or moisture potential values. Water is essentially excluded from the sampling ports except at near-saturated flow conditions in the rock matrix.

Table 4.1: Results of sample critical seepage velocity calculations using hydraulic conductivity values of borehole tuff core samples found at Superior, Arizona.

Borehole I.D.

Core Sample I.D.
$\mathrm{X} 1$

$A A$

143.1
X3

EP

50.5

5.27

2.22

0.0111

0.0073
1.45 $\left(\mathrm{cm}^{-1}\right)$
32.62

$\mathrm{K} \times 10^{10}(\mathrm{~m} / \mathrm{s})$

$10^{2}$

Dimensionless Characteristic

$$
\text { Cavity Length, } \mathbf{s}^{(2)}
$$

Maximum Dimensionless

$$
\text { Potential, } \nu_{\max }{ }^{(3)}
$$

Critical Seepage Velocity, $\mathrm{K}_{0} \times 10^{10}(\mathrm{~m} / \mathrm{s})$

49.4

141.1

$\left(0.986 \mathrm{~K}_{\mathrm{m}}\right)$

$\mathrm{X} 2$

$\mathrm{CH}$

375.9

10.02

Critical moisture potential ${ }^{(4)}$, $\psi_{0} \times 10^{2}(\mathrm{kPa}) \quad 9.5$

9.7

3.55

0.0178

1) Conductivity data from NRC project file.

(2) The value of $s$ is calculated using $1_{c}=0.01 \mathrm{~m}$.

(3) At small $s, \nu_{\max }$ approaches $1+2 s$.

(4) Critical moisture potential, $\psi_{0^{*}}=\left[\ln \left(\mathrm{K}_{\mathrm{m}} / \mathrm{K}_{0^{*}}\right) / \alpha_{\mathrm{s}}\right](0.0981 \mathrm{kPa} / \mathrm{cm})$.

There are several implications for studying transport behavior with the sampling ports. From Philip's analysis, the greatest water potential is at the apex of the circular cylindrical port where water entry occurs under the appropriate conditions. A "dry" zone develops under the port as flow lines are affected by the presence of the port. The placement of ports should take this into consideration. The downward flow velocity is smallest in the two regions, but greatest to the sides of the 
port. As water entry occurs at the apex, a slower solute travel time is expected. However, for estimates of the average travel velocity in the matrix, the ports are not significantly different from rock inclusions, and other structures that can obstruct flow.

Depending on the size and location of the port, water may not enter at prevailing flow conditions. As for test block 1, water entry into the port occurs at nearly saturated conditions for a $1_{c}=1.0 \mathrm{~cm}$. Sample collection at higher suctions in the rock matrix may entail placing the filter paper against the walls of the ports. An alternative scheme may be the enlargement of the sampling port because a larger $1_{c}$ corresponds to a larger $s$, and therefore smaller $k_{0}$. An increase in sampling time is necessary unless a larger port is installed. However, at higher suctions, the possibility of contamination by matrix waters is less of a problem when collecting samples in the fracture ports.

\subsection{Solute Transport Measurement}

A $10^{-3} \underline{\mathrm{M}} \mathrm{CaCl}_{2}$ solution was used as the background solution for experimentation. Tracer solutions were either $10^{-1} \underline{\mathrm{M}} \mathrm{CaCl}_{2}$ for the fracture transport tests, or $10^{-2} \mathrm{MaCl}_{2}$ for the matrix transport test. A three-way $\mathrm{T}$ shape glass stopcock was used to switch between $\mathrm{CaCl}_{2}$ solutions. Changeover from one concentration to another was ensured by completely flushing the test solution delivery system and grooves above the porous plates. The following section describes the preparation of test solutions used, the calibration of the electrodes, the methods used to collect samples and to make electrode measurements, and the procedures used to measure chloride breakthrough of the plates.

\subsubsection{Preparation of Test Solutions and Calibration of Electrodes}

Test solutions were prepared with calcium chloride $\left(\mathrm{CaCl}_{2} \cdot \mathrm{H}_{2} \mathrm{O}, \mathrm{FW}=\right.$ 147.02 , assay $=74.68$ ) and distilled water. Thymol was added to the solutions ( $0.1 \mathrm{~g}$ per $1000 \mathrm{~mL}$ solution) to prevent biological growth, and deaerated by boiling to minimize air bubble formation in the flow system (Klute and Dirksen, 1986). Test solutions of $10^{-2} \underline{M}$ and $10^{-3} \underline{M}$ were prepared from $10^{-1} \mathrm{M}$ stock solution using serial dilution to minimize error. Evaporation of water during boiling was accounted for by adjusting the stock solution concentration. No adjustment was made when $0.1 \mathrm{M}$ solution was used directly as the tracer solution.

The electrodes were calibrated in an "aqueous" and a "filter paper" environment. Eight standard solutions of half-decade concentration from $10^{-4} \mathrm{M}$ to $0.5 \mathrm{M} \mathrm{CaCl}_{2}$ were prepared for calibration. Because of poor $\mathrm{pH} / \mathrm{mV}$ meter resolution, the calibration errors are relatively large. Without the use of more sophisticated analytical methods, the concentration of the standard solutions was verified independently with Hach kits. Titrations were performed for both calcium and chloride concentrations.

The performance of the electrodes were also tested in both environments with sodium nitrite $\left(\mathrm{NaNO}_{2}, \mathrm{FW}=69.00\right.$, assay $\left.=97.78\right)$ as an ionic buffer. A second set of standard solutions was buffered to a common ionic strength of $0.25 \underline{\mathrm{M}}$ (corresponds to ionic strength of $10^{-1} \mathrm{M} \mathrm{CaCl}_{2}$ solution). The ionic strength of $0.5 \underline{\mathrm{M}} \mathrm{CaCl}_{2}$ ( $I=1.25 \underline{\mathrm{M}}$ ) was consider- 
ed too high and the standard was not buffered further in both sets of calibrations. The results are presented graphically in Figure 4.2 plotting potential difference (mV) against chloride concentration ( $\underline{M})$. It shows only the best curves drawn for average potential difference values of each standard solution.

The change in potential difference per decade concentration in the aqueous environment is linear from approximately $1.00 \times 10^{-4} \underline{M}$ to $1.00 \times$ $10^{-1} \mathrm{M}$ at $58 \mathrm{mV} /$ decade. In the filter paper environment, the calibration curve is nonlinear except in the range between $1.00 \times 10^{-3} \mathrm{M}$ and $1.00 \times 10^{-1} \mathrm{M}$. In general, the change in potential difference per decade in the aqueous environment is greater than the change in the filter paper environment. This phenomenon may be due to "bound" water resulting in an apparent increase in ionic activity or concentration. The calibration curves are exactly the same in the aqueous environment for both buffered and nonbuffered standards. However, the curve is steeper for the buffered standards in the filter paper environment. The buffer solution may have minimized the "bound" water effect. One noteworthy difference between the nonbuffered and buffered standards is the shorter time to equilibration for the latter set.

\subsubsection{Sample Collection and Electrode Measurements}

Sample collection in the fracture and matrix was made possible with Whatman filter paper number 42 (retention rating $=2.5 \mu \mathrm{m}$ ). According to the capillary rise equation (Hillel, 1980), the air entry value for a $2.5 \mu \mathrm{m}$ pore is $116 \mathrm{kPa}$ at $20^{\circ} \mathrm{C}$. This suggests that the filter paper can be a good absorber with the greatest efficiency in collecting samples when dry. Samples were collected by inserting pieces of filter paper with forceps into the sampling ports, and pressed against the exposed fracture surface, or the end of the ports in the matrix. The optimum filter paper size was determined to be $1.0 \mathrm{~cm}$ by $0.5 \mathrm{~cm}$, both for sampling in the $2.0-\mathrm{cm}$ ports, as well as electrode measurement purposes.

Potential differences were measured using one of three chloride ionselective electrodes (LAZAR ISM-146CL), and a double-junction Ag/AgC1 reference electrode (LAZAR DJM-146). A Corning $610 \mathrm{~A} \mathrm{pH} / \mathrm{mV}$ meter, with expanded scale for $\mathrm{mV}$ measurement, was used. For better resolution, measurements were made with a HP-41CV calculator which was connected to the HP data acquisition unit in turn connected to the Corning meter. Calibration of the HP-41CV indicates the actual potential difference is 70 times the value of the calculator. Individual potential readings are usually rounded off to the nearest $\mathrm{mV}$ because of the combined resolution of the $\mathrm{pH} / \mathrm{mV}$ meter and calculator. The nonbuffered calibration curve was used to convert potential readings to concentrations. With calibration errors of \pm 10 to 15 percent under controlled conditions, the experimental error in concentration is estimated to be \pm 20 percent. Aside from instrument problems, the single most important means of minimizing experimental error is to ensure long enough sampling time so that the filter paper is sufficiently wet.

The optimum sampling period depends on the availability of water for sampling. Under the near-saturated test conditions, most fracture samples required no more than two minutes, and matrix samples almost instantaneous because of water infiltration into the sampling ports. 
The reliability of electrode measurements is expected to be affected strongly by the moisture content of the filter paper.

Once the sample was collected, excess test solution was dabbed off with Kimwipe, if necessary, to ensure the potential readings were for a filter paper and not an aqueous environment. The electrodes were then touched against the filter paper for approximately 5 seconds before a reading was made. The chloride electrode was then moved to a different spot on the filter paper, and the measurement repeated. The potential reading, or range of readings, were recorded. The time to make potential readings was limited as the spread of the $\mathrm{KNO}_{3}$ bridge solution due to outward diffusion from the reference electrode would cause erroneous ly low potential readings (high concentration) when the chloride electrode came into contact with it. The electrodes were placed as far apart as possible.

The transport tests were conducted under near-saturated conditions. Ample sample volumes were collected often within seconds to minutes of sampling. However, the reliability of the electrode measurements at higher suctions remains untried under test conditions. The performance of the filter paper was assessed at greater suctions to confirm its reliability under drier conditions using a separate apparatus.

Figure 4.4 shows the setup used to test the filter paper up to a suction of $100 \mathrm{~cm}$ of water. A Buchner funnel equipped with a ceramic porous plate (50-kPa air entry value) was connected to a buret with Tygon tubing, setting up a water column filled with $\mathrm{CaCl}_{2}$ solution. An ovendried slice of tuff core was placed on the porous plate with filter paper acting as contact between the plate and rock. A cover made of plastic and duct tape with two acrylic access tubes for the electrodes was fitted over the mouth of the funnel. Two small air holes in the cover were provided. The access tubes were stoppered to minimize evaporation whenever they were not in use.

The water column was used to completely saturate the rock core, and to apply the suctions. After equilibration periods commensurate to the suction applied, measurements were made off filter paper pieces still contacting the rock core, as well as ones removed to the external environment. Two $\mathrm{CaCl}_{2}$ solutions, $1.40 \times 10^{-3} \underline{\mathrm{M}}$ and $1.00 \times 10^{-1} \underline{\mathrm{M}}$, at four suctions, $10 \mathrm{~cm}, 20 \mathrm{~cm}, 50 \mathrm{~cm}$, and $100 \mathrm{~cm}\left(90 \mathrm{~cm}\right.$ for $1.00 \times 10^{-1}$ M), were examined. Separate rock cores were used for the two solutions. The sampling period was 10 minutes for suctions up to and including $50 \mathrm{~cm}$, and 20 minutes for the highest suction. Figures 4.5 and 4.6 show the results by plotting suction (cm of water) against chloride concentration (M) . The dotted lines show the range of possible concentrations accounting for measurement errors.

In view of factors such as variability in rock properties and evaporation of the $\mathrm{CaCl}_{2}$ solutions at the surface, the results indicate a certain degree of reliability up to $100 \mathrm{~cm}$ of suction. The scatter in data is most likely due to samples not sufficiently wet despite their apparent appearances. In general, greater deviation from the expected range of concentrations is seen for the more dilute solution. Coupled 


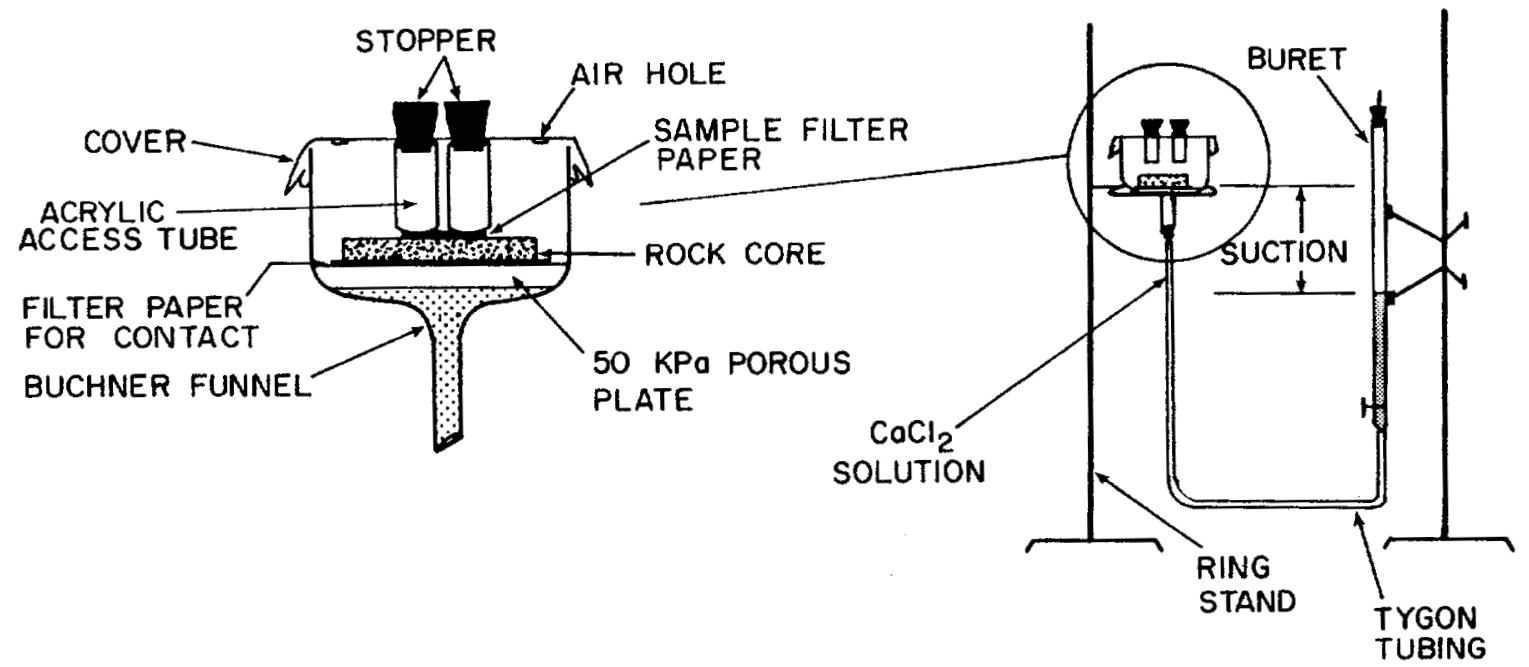

Figure 4.4 Experimental apparatus used to assess the effects of suction on the reliability of the filter paper/chloride ion-selective electrode technique. 

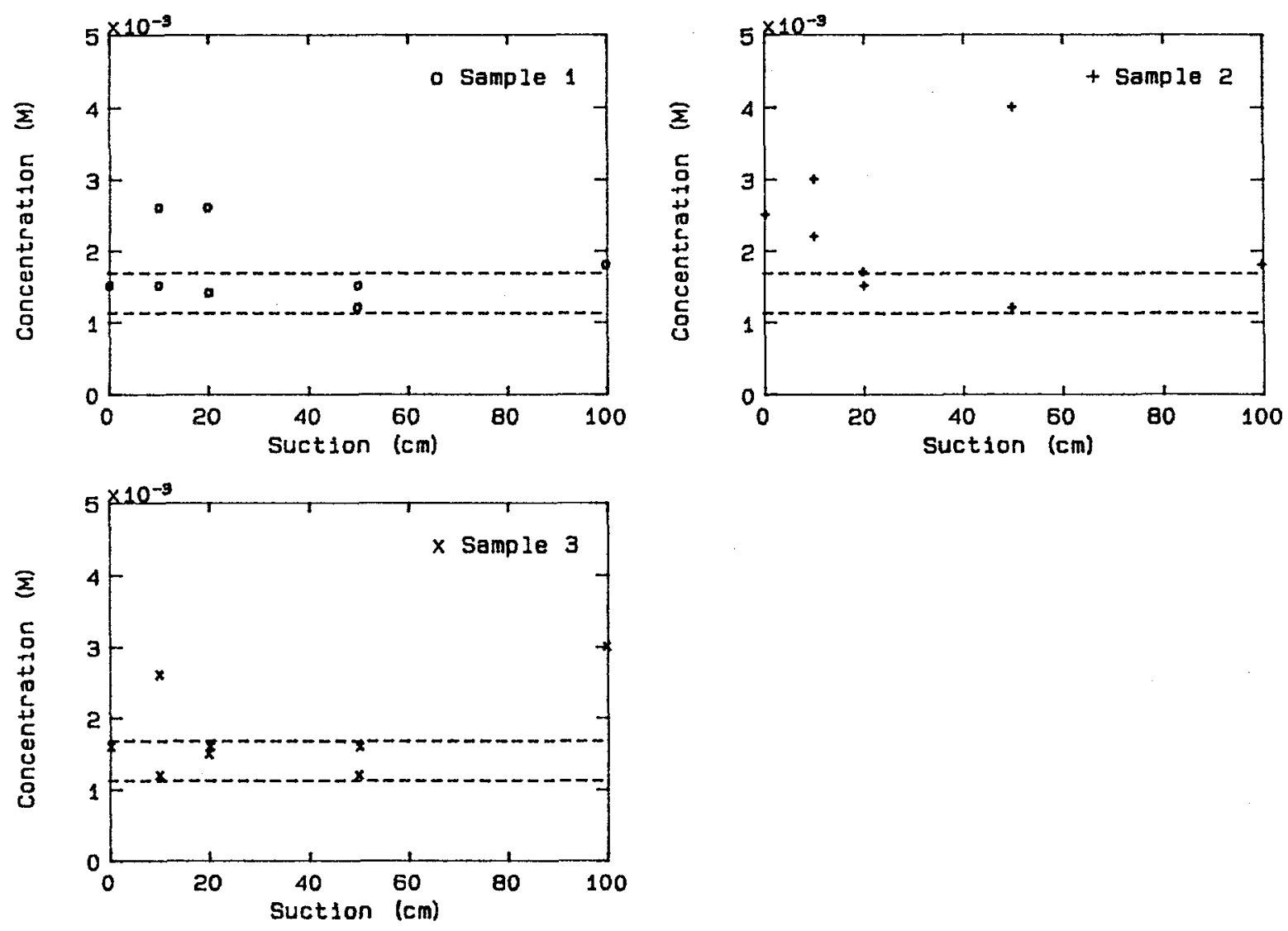

Figure 4.5 Concentration $(\underline{M})$, as measured off filter paper, versus suction (cm of water) for a $1.40 \times 10^{-3} \mathrm{M}$ calcium chloride solution. The dashed lines indicate the full range of experimental error expected of \pm 20 percent. 

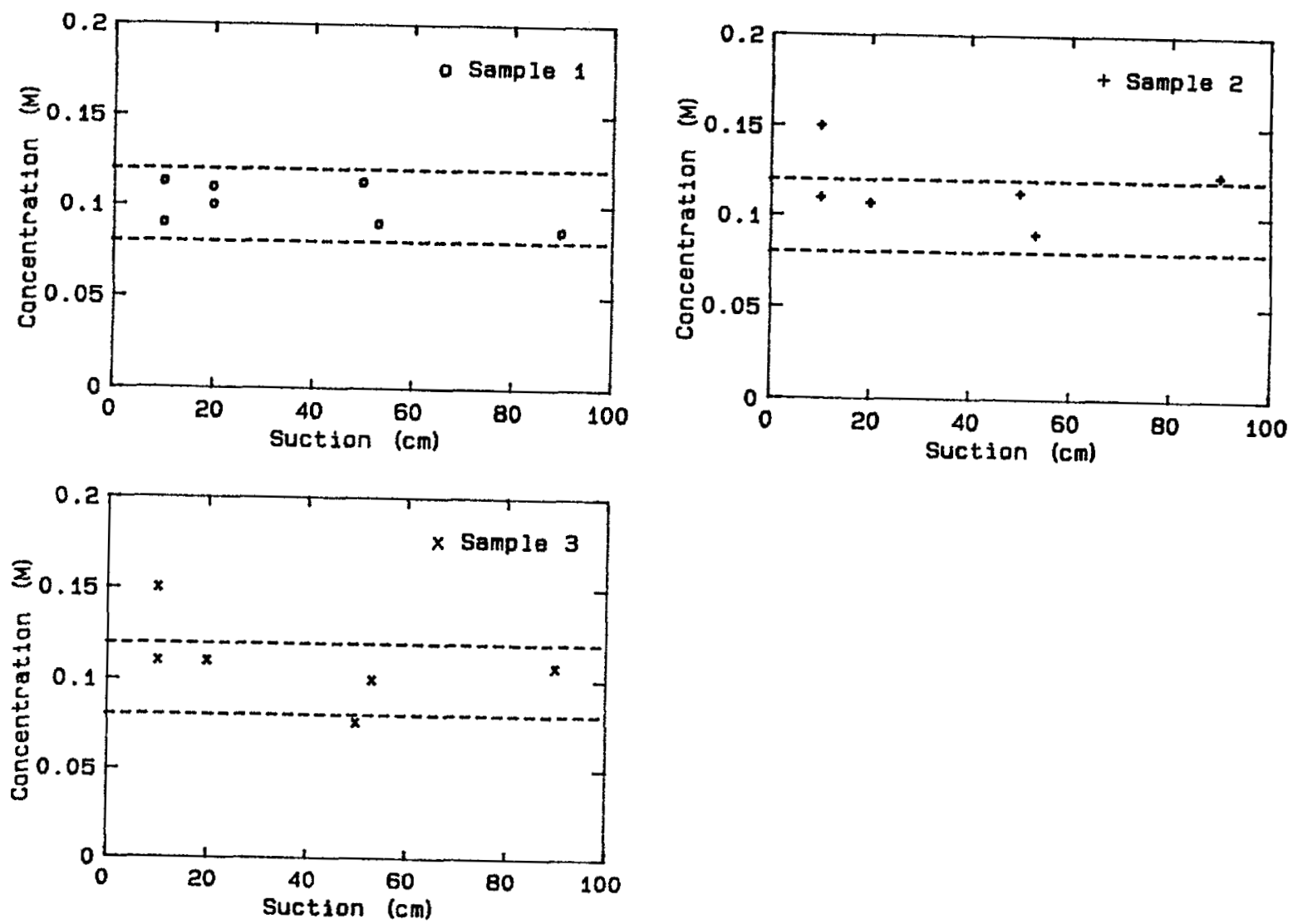

Figure 4.6 Concentration $(\underline{M})$, as measured off filter paper, versus suction ( $\mathrm{cm}$ of water) for a $0.100 \mathrm{M}$ calcium chloride solution. The dashed lines indicate the full range of experimental error expected of \pm 20 percent. 
with the result of Philip's analysis indicating no water entry into the sampling port at these higher suctions, a longer sampling period to collect matrix samples will be necessary during experimentation. of course, at suctions greater than the critical suction, fracture flow is negligible and collection of fracture samples is a moot point. However, at suctions close to, but less than, the critical suction, when some fracture flow still exists, sampling for a "pure" fracture sample may be difficult. The collection of a fracture sample may in reality be one of the matrix adjacent to the fracture surface. The limits of the technique may depend on the flow condition in the fracture.

\subsubsection{Chloride Breakthrough in the Porous Plates}

The porous plates were used to impose suctions at the top of the rock, and therefore, to control flow. However, the upper boundary condition, in terms of concentration, depends on the transport properties of the plates. The input of chloride is a function of its breakthrough in the plates. The breakthrough of each plate was measured experimentally using the setup shown in Figure 4.7. The setup basically consisted of the experimental flow system, without the plate in contact with rock. The plate was suspended with clamps attached to ring stands. Changeover from one test solution to another was accomplished with the three-way stopcock, and flushing of the solution delivery system and plate grooves. A plastic cover equipped with two windows for electrode measurements helped to minimize evaporation during testing. The plate was divided into four quadrants to account for any spatial variation, and potential readings were reported as a range of values. Potential measurements were made by touching the electrodes directly against the ceramic surface. Because it is important to protect the sensor tip of the chloride electrode, measurements made off filter paper placed on the plate is recommended for future testing. Tests were conducted at different head gradients, and for certain plates, different tracer and background solutions.

The breakthrough curves of the plates were obtained with constant average head gradients imposed. The measured breakthrough curves are intended to be reference curves for interpolation or extrapolation to different breakthrough curves associated with different imposed head gradients. The effective porosity $\left(n_{e}\right)$ of each plate is also determined from:

$$
n_{e}=Q t^{*} / L_{b} A_{p}=q / v^{*}=K_{p} t^{*} d H / L_{b}^{2}
$$

where $v^{*}(\mathrm{~m} / \mathrm{s})$ is the average travel, velocity of the solute, and equal to $L_{p} / t^{*}$. The travel time, $t^{*}$, is the time to $C^{*}=0.5$. The results of plate no. 6 indicate differences among tests, and the need to obtain an average $n_{e}$ from several tests. Because of scheduling and availability of the plates, single tests (including step-up and step-down portions) were conducted for plate nos. 1 to 3 , and two tests were conducted for plate nos. 4 and 5. Further testing is recommended to characterize the breakthrough of chloride in the porous plates. 


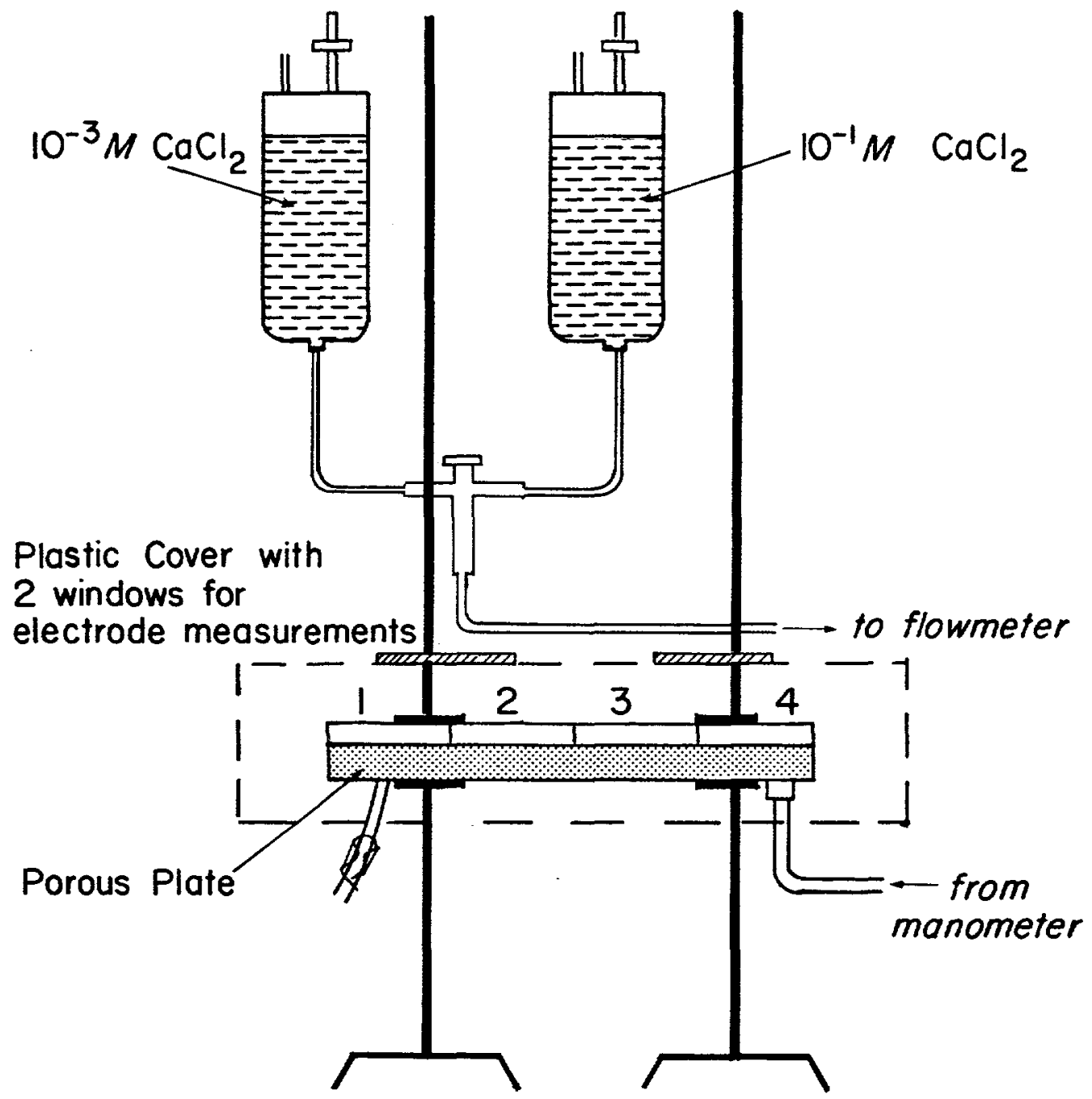

Figure 4.7 Experimental setup used to measure chloride breakthrough in the porous plates. The plates are divided into four quadrants to determine possible spatial differences. 


\subsection{Experimental and Computer Model Results}

The experimental data and computer model case study are discussed in this section. For an idealized test block system, flow and transport results from application of the boundary integral method computer model are also discussed. The model provides insights to the possible effect of fracture-matrix interaction on transport data interpretation.

Results of three fracture transport tests, and one rock matrix transport test conducted using only the top porous plates are also presented. A spatial relative concentration distribution of the fracture obtained immediately after the third fracture transport test contributes to an understanding of fracture flow.

\subsubsection{Computer Model Case Study of Test Block}

Valuable insights to fractured rock flow and transport processes are provided by an application of the boundary integral method computer model to the experimental test block. Of special interest is the interaction of the rock matrix and the fracture. The test block is conceptualized as being bisected by the fracture, the plane of symmetry. It also possesses homogeneous properties in both the matrix and fracture. The system is then divided into four domains: fracture, matrix, fracture plate and matrix plate, as shown in Figure 3.18. The test block matrix and plates are treated as two-dimensional, and the fracture onedimensional, by assuming constant properties over their respective depths, and lengths (x-direction). Steady-state flow is also assumed. By assigning independently determined plate conductivities and matrix conductivity, and actual total heads applied at the top of the plates, fracture transmissivity can be varied to obtain total and pressure heads at any point in the four domains. Ten stream tubes (eleven streamlines) divide the total flux equally. The model results reflect the attempted matching of the actual flows observed, and the pressure heads measured in the fracture with the microtensiometer, as well as average calculated pressure heads at the top of the test block. However, the matching of the model and experimental results is considered less important than the information on the effects of the fracture-matrix interaction on flow and transport, and pressure head distribution at the top boundary and in the fracture.

Table 4.2 summarizes the input parameters for the case study, and compares them to experimentally determined values. Figures 3.18 and 3.19 show the streamlines obtained. Note the conductivity used in this analysis is the larger value. The transmissivity corresponds to the smaller of the two values determined. The left-most, or "zero-percent," streamline should correspond to the left boundary of the matrix $(y=0$ $\mathrm{cm})$. The deviation is due to the errors involved with the numerical scheme. Table 4.3 presents the flows of each domain and pertinent pressure heads calculated, and compares them to experimental data whenever available. 
Table 4.2: Case study of idealized test block system:

A comparison of computer model input parameters and experimental data.

Input Parameters

Average Matrix Hydraulic

Conductivity,

$\mathrm{K}_{\mathrm{m}} \times 10^{9}(\mathrm{~m} / \mathrm{s})$
Mode1

Experimental

\section{Average Fracture \\ Transmissivity, \\ $\mathrm{T}_{\mathrm{f}} \times 10^{9}\left(\mathrm{~m}^{2} / \mathrm{s}\right)$}

Fracture Half-Aperture, $e_{b}$ (um)

Average Matrix Plate Conductivity, $\mathrm{K}_{\mathrm{mp}} \times 10^{9}(\mathrm{~m} / \mathrm{s})$

Applied Head above Matrix Plate, $\mathrm{H}_{\mathrm{mp}}(\mathrm{cm})$

\section{Average Fracture Plate} Conductivity, $\mathrm{K}_{\mathrm{pp}} \times 10^{9}(\mathrm{~m} / \mathrm{s})$

Applied Head above Fracture Plate, $\mathrm{H}_{\mathrm{fp}}(\mathrm{cm})$
59.1

(1) Transmissivity is calculated assuming all the flow from fracture plate and no flow from the matrix plate enter the fracture; mean = $7.16 \times 10^{-9} \mathrm{~m}^{2} / \mathrm{s}$ and Std $=1.68 \times 10^{-9} \mathrm{~m}^{2} / \mathrm{s}$.

(2) Transmissivity is calculated assuming flow from fracture plate is split between fracture and rock matrix; mean $=5.12 \times 10^{-9} \mathrm{~m}^{2} / \mathrm{s}$ and $\mathrm{std}=1.54 \times 10^{-9} \mathrm{~m}^{2} / \mathrm{s}$. Proportion of flow in matrix is calculated using an average $K_{m}$, and subtracted from the total flow to determine $T_{f}$.

(3) NA - Not applicable.

(4) Period monitored is between 6-13-88 and 8-3-88. 
Table 4.3: A comparison of flow and pressure head data obtained from using the computer model, and those measured or calculated from experimental data.

Model

Experimental

Matrix Plate Flow,

$Q_{\text {mp }} \times 10^{9}\left(\mathrm{~m}^{2} / \mathrm{s}\right)$

6.33

6.65

Fracture Plate Flow,

$Q_{f p} \times 10^{9}\left(\mathrm{~m}^{2} / \mathrm{s}\right)$

3.77

$3.15^{(1)}$

Flow Exiting Fracture,

$Q_{f} \times 10^{9}\left(\mathrm{~m}^{2} / \mathrm{s}\right)$

5.06

$\mathrm{NDM}^{(2)}$

Flow Exiting Matrix,
Q $\times 10^{9}\left(\mathrm{~m}^{2} / \mathrm{s}\right)$
5.15
NDM

Pressure Head under

Matrix Plate,

$\mathrm{h}_{\mathrm{mp}}(\mathrm{cm})$

$$
+2.6 \text { to }+3.7
$$

$($ mean $=+3.2)$

-3.9 to $+5.9^{(3)}$

Pressure Head under

Fracture Plate,

$h_{\mathrm{fp}}(\mathrm{cm})$

-1.7 to $+3.2^{(4)}$

$($ mean $=+0.1)$

-12.6 to $+7.4^{(3)}$

Pressure Head in

Fracture $(\mathrm{cm})$

at $z=10 \mathrm{~cm}$

at $z=35 \mathrm{~cm}$

$+0.3$

-1.8 to -5.6

$+0.2$

-3.1 to -8.4

Pressure Head in

Matrix (cm)

at $\mathrm{x}=4 \mathrm{~cm}$

and $z=5 \mathrm{~cm}$

at $\mathrm{x}=4 \mathrm{~cm}$

and $z=30 \mathrm{~cm}+0.3 \quad+0.6$ to +1.9

(1) Fracture plate flow is half the actual observed because the model divides the test block into two identical halves with the fracture as the plane of symmetry.

(2) NDM - not directly measured.

(3) Pressure head calculated using equation 3.19.

(4) The pressure head directly over the fracture opening is $-1.7 \mathrm{~cm}$ (suction of $1.7 \mathrm{~cm}$ ). 
It is readily apparent from the streamlines that flow from the fracture plate is diverted to the fracture through the matrix within the first 3 cm. However, at a transmissivity of $5 \times 10^{-9} \mathrm{~m}^{2} / \mathrm{s}$, part of the matrix plate flow $\left(Q_{m p}\right)$ is also diverted to the fracture. Streamlines in the matrix are affected most dramatically near the fracture opening. Approximately 19 percent of $Q_{m p}$, or 12 percent of the total flow $\left(Q_{t}\right)$ reach the fracture within the top $20 \mathrm{~cm}$, and exit through the fracture. Insignificant exchange of fracture and matrix flows occur between $z=20$ $\mathrm{cm}$, and the fracture opening at $z=50 \mathrm{~cm}$. A plot of the fracture flow (calculated as fraction of $Q_{t}$ ) as a function of distance is shown in Figure 4.8. The model $Q_{m p}$ is slightly less, and fracture plate flow $\left(Q_{i p}\right)$ slightly greater, than those measured experimentally. The difference between the sums of $Q_{m p}$ and $Q_{i p}$, and actual matrix $\left(Q_{m}\right)$ and fracture flows $\left(Q_{1}\right)$, is due to mass balance errors.

The simulated pressure heads under the porous plates match those calculated, as a whole, but the pressure heads in the fracture are greater than those measured with the tensiometer. The pressure heads in the matrix compare well to those measured. Observations during testing seemed to indicate the actual pressure heads under the plates were less than atmospheric as seepage did not occur, with few exceptions. The discrepancies between model and experimental pressure heads should be viewed in light of the expected experimental variabilities. The flow discrepancies are probably due in part to the differences in pressure heads, as well as to heterogeneities of the test block. An examination of the test block clearly showed the existence of inclusions and microfractures. Another explanation may be the presence of filter paper between the plates and rock surface which is not taken into account by the model. The possible effects of filter paper is explored in more detail below.

Due to the fracture-matrix interaction near the fracture opening, the concentration profile in the fracture is of interest as functions of both time and longitudinal distance (in the $z$ direction). At nodal points close to the triple point (point at the upper right shared by the fracture plate, matrix and fracture domains), the simulated travel times of the streamlines become increasingly unstable. This is due to the nature of numerical schemes such as ones used in the BIM. A simple averaging is applied to calculate travel times of the streamlines.

Table 4.4 presents the travel times of selected streamlines entering the fracture as calculated by assuming straight streamlines, and using the average gradient between the end nodal points. Piston pump displacement is also assumed for transport in the matrix by neglecting the effects of diffusion into the matrix, and hydrodynamic dispersion. Matrix diffusion will retard the breakthrough, and dispersion will spread out the front. With 49 percent of $Q_{t}$ entering the fracture, the travel times of five stream tubes, each representing ten percent of the flux, except for stream tube no. 5, are calculated. The calculations are performed for an "average" streamline representative of each stream tube. They are arbitrarily chosen as the five-percent streamlines of each stream tube (except 4.5-percent for stream tube no. 5), e.g., the 95 percent streamline is chosen to represent the properties of stream tube no. 1 . The time increments are chosen as the travel times of the average streamlines for simplicity. 


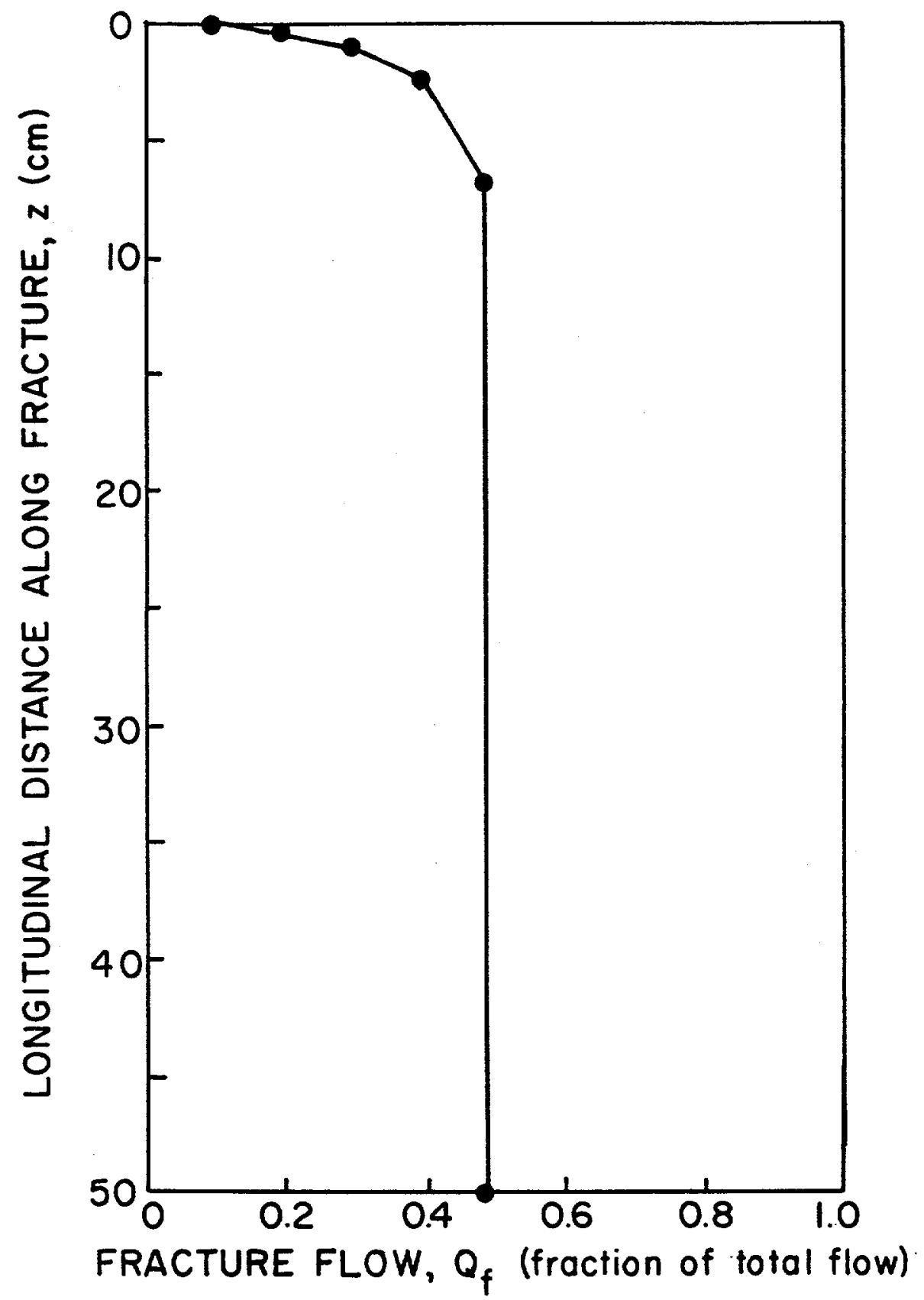

Figure 4.8 Profile of fracture flow, $Q_{f}$ (fraction of total flow), as a function of longitudinal distance along fracture, $z(\mathrm{~cm})$. 
Table 4.4: Travel time calculations of selected streamlines contributing to fracture flow.

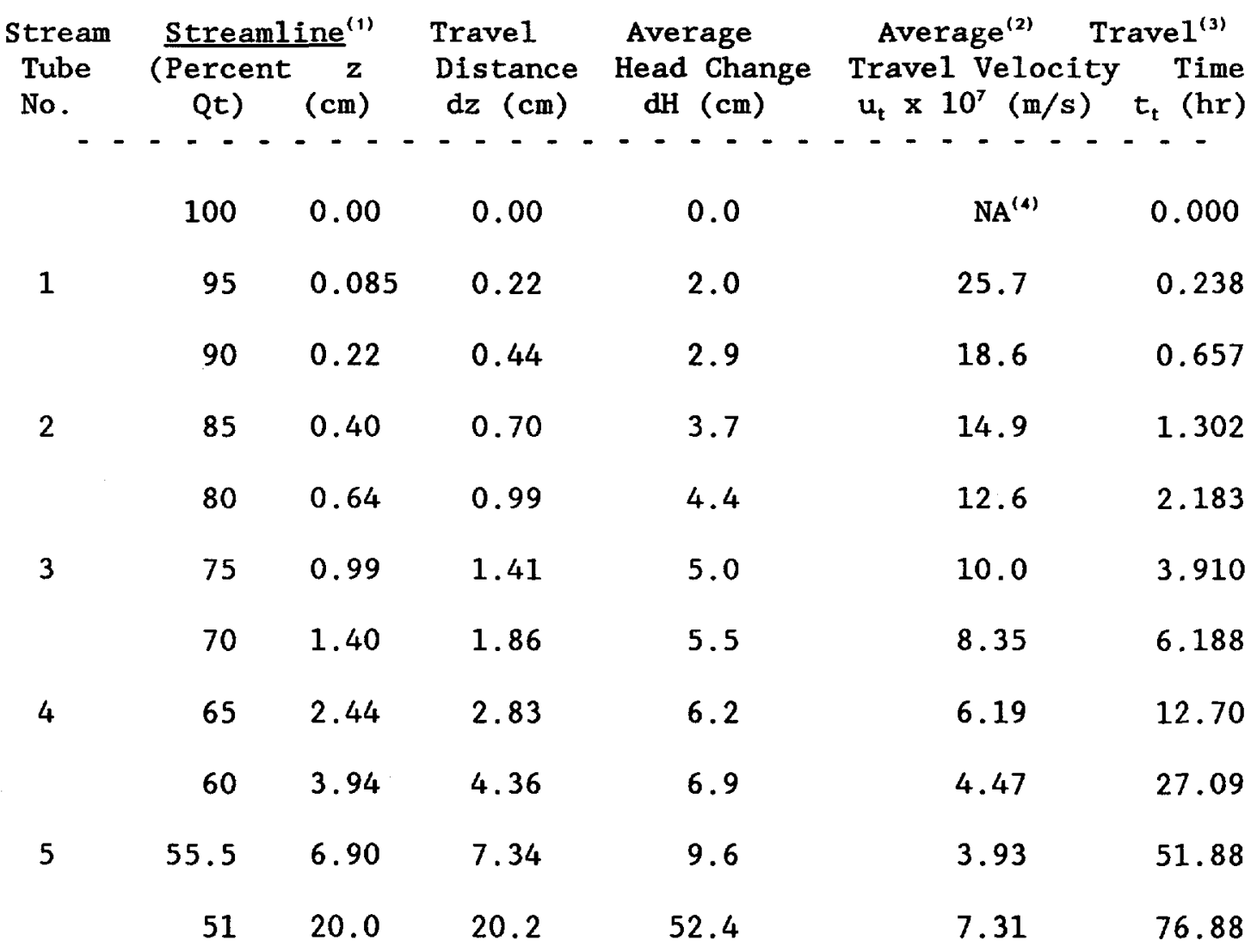

(1) The average streamline of each stream tube is chosen to represent the properties of the stream tube, e.g., travel time and concentration of 95 -percent streamline represents those of stream tube no. 1 bounded by 90 - and 100-percent streamlines.

(2) Average travel velocity, $u_{t}=\left(K_{w} / n_{e}\right)(d H / d z)$.

(3) Travel time, $t_{t}=d z / u_{t}$.

(4) NA - Not applicable.

The relative concentration profiles in the fracture over time, for a step increase in concentration from $C_{1}\left(=0.01 C_{0}\right)$ to $C_{o}$ in the fracture plate only, are plotted in Figures 4.9 and 4.10. The concentration in the matrix plate remains at $C_{i}$. A summary of the concentration and $C^{*}$ calculations are included in Table 4.5. The first plot shows the profiles for an instantaneous increase of concentration through the plate, and the second takes into account the solute breakthrough characteristic of the plate. For plotting purposes, the midpoints of the stream tubes (along the $z$-direction) are connected. The chloride breakthrough of plate no. 5 is used for the second set of profiles, after adjusting for the proper flow rate. The difference in the two cases is confined to the first two time increments after which the background solution is 


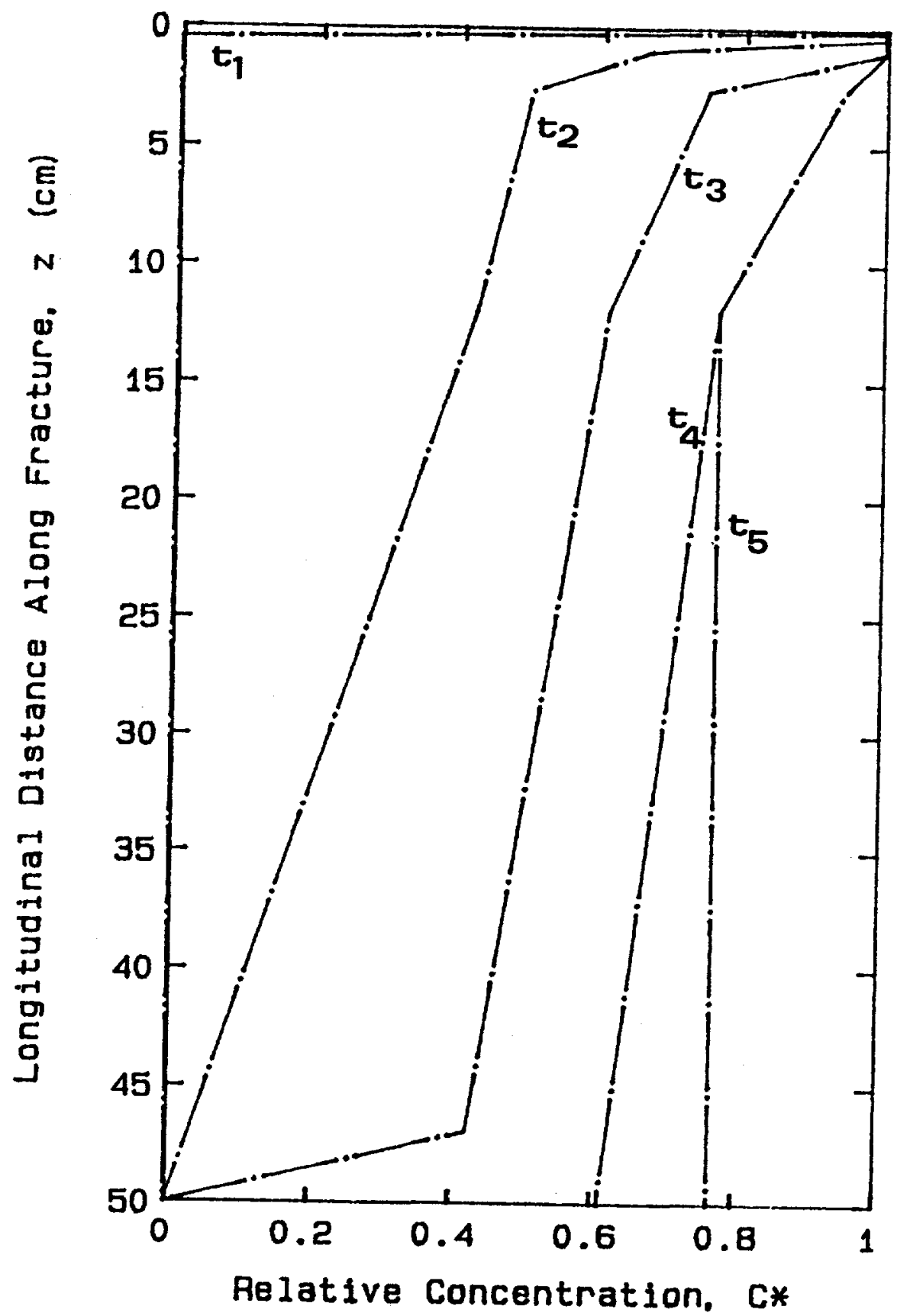

Figure 4.9 Profile of relative concentration, $\mathrm{C}^{*}$, as a function of longitudinal distance along the fracture, $z(\mathrm{~cm})$, with an instantaneous increase through the fracture plate. The time increments are $t_{1}=0.238 \mathrm{hrs}, t_{2}=1.302 \mathrm{hrs}, t_{3}=3.910 \mathrm{hrs}, t_{4}=12.70 \mathrm{hrs}$, and $t_{5} \geq 14.81 \mathrm{hrs}$. 


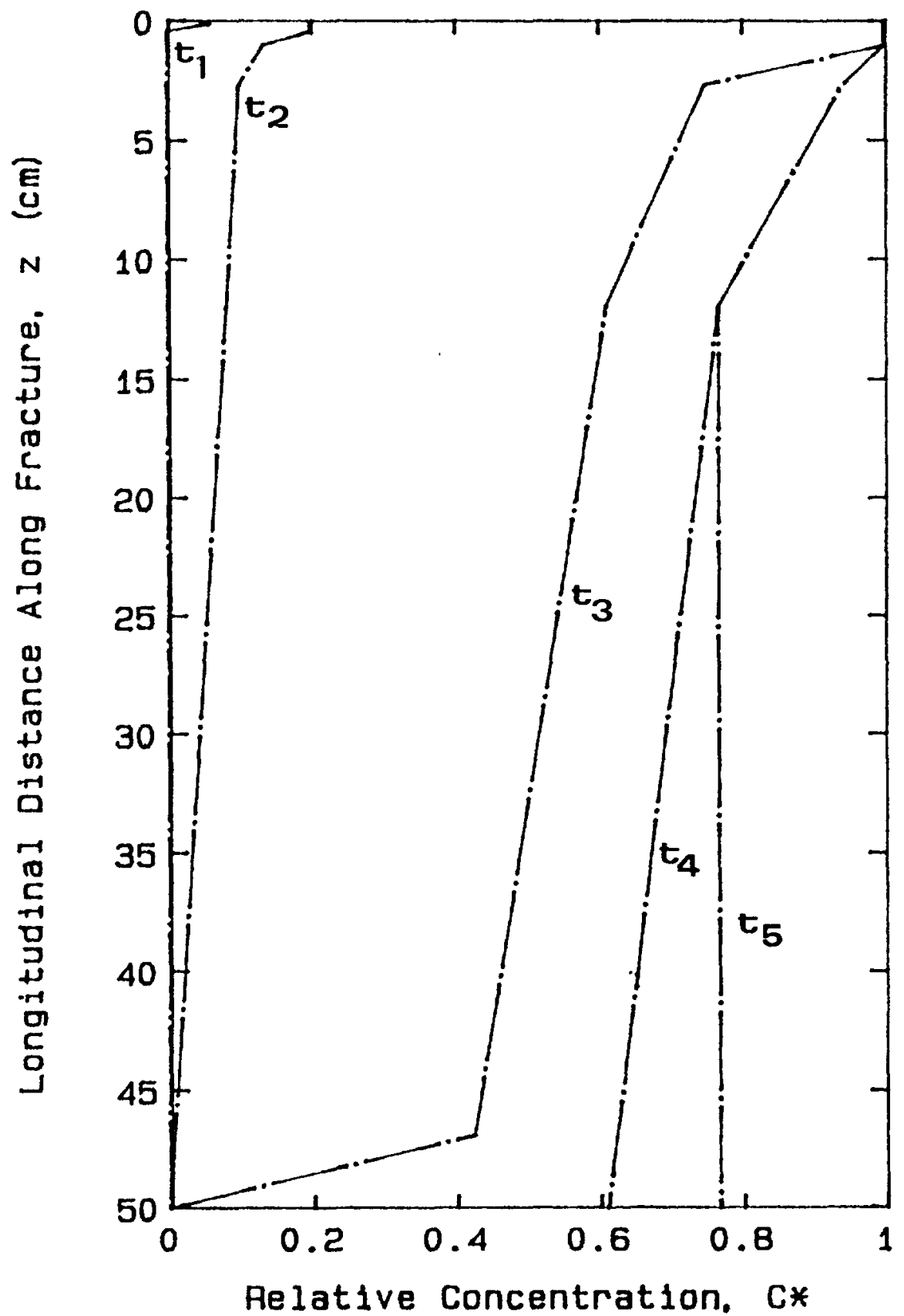

Figure 4.10 Profile of relative concentration, $\mathrm{C}^{*}$, as a function of longitudinal distance along the fracture, $z(\mathrm{~cm})$, taking into account the chloride breakthrough characteristic of the fracture plate. The time increments are $t_{1}=0.238 \mathrm{hrs}, t_{2}=1.302 \mathrm{hrs}, t_{3}=$ $3.910 \mathrm{hrs}, t_{4}=12.70 \mathrm{hrs}$, and $t_{5} \geq 14.81 \mathrm{hrs}$. 
Table 4.5: Relative concentrations in the fracture as functions of time and distance along the fracture.

\begin{tabular}{|c|c|c|c|c|c|c|}
\hline \multirow{4}{*}{$\begin{array}{l}\text { Elapsed } \\
\text { Time, } t_{\mathrm{F}} \\
(\mathrm{hr})\end{array}$} & \multicolumn{2}{|c|}{$\underline{\text { Stream }}^{(1)}$} & Concentration & $\mathrm{C} \times 1 / \mathrm{C}_{0}{ }^{(2)}$ & \multicolumn{2}{|c|}{ Relative Conc., $\mathrm{C}^{\prime}$} \\
\hline & \multicolumn{2}{|c|}{ Tube } & instant. & plate & instant. & plate \\
\hline & No. & $z(\mathrm{~cm})$ & increase & effect & increase & effect \\
\hline & - & --- & ------ & ---- & ---- & - - \\
\hline 0.238 & 1 & 0.11 & 1.000 & 0.069 & 1.000 & 0.060 \\
\hline & 2 & 0.43 & 0.010 & 0.010 & 0.000 & 0.000 \\
\hline & 3 & 1.02 & 0.010 & 0.010 & 0.000 & 0.000 \\
\hline & 4 & 2.67 & 0.010 & 0.010 & 0.000 & 0.000 \\
\hline & 5 & 11.97 & 0.010 & 0.010 & 0.000 & 0.000 \\
\hline & - & 50.00 & 0.010 & 0.010 & 0.000 & 0.000 \\
\hline 1.302 & 1 & 0.11 & 1.000 & 0.208 & 1.000 & 0.200 \\
\hline & 2 & 0.43 & 1.000 & 0.208 & 1.000 & 0.200 \\
\hline & 3 & 1.02 & 0.670 & 0.142 & 0.667 & 0.133 \\
\hline & 4 & 2.67 & 0.505 & 0.109 & 0.500 & 0.100 \\
\hline & 5 & 11.97 & 0.431 & 0.091 & 0.425 & 0.082 \\
\hline & - & 50.00 & 0.010 & 0.010 & 0.000 & 0.000 \\
\hline 3.910 & 1 & 0.11 & 1.000 & 1.000 & 1.000 & 1.000 \\
\hline & 2 & 0.43 & 1.000 & 1.000 & 1.000 & 1.000 \\
\hline & 3 & 1.02 & 1.000 & 1.000 & 1.000 & 1.000 \\
\hline & 4 & 2.67 & 0.753 & 0.753 & 0.750 & 0.750 \\
\hline & 5 & 11.97 & 0.616 & 0.616 & 0.612 & 0.612 \\
\hline & - & 46.94 & 0.431 & 0.431 & 0.425 & 0.425 \\
\hline & - & 50.00 & 0.010 & 0.010 & 0.000 & 0.000 \\
\hline 12.70 & 1 & 0.11 & 1.000 & 1.000 & 1.000 & 1.000 \\
\hline & 2 & 0.43 & 1.000 & 1.000 & 1.000 & 1.000 \\
\hline & 3 & 1.02 & 1.000 & 1.000 & 1.000 & 1.000 \\
\hline & 4 & 2.67 & 0.943 & 0.943 & 0.942 & 0.942 \\
\hline & 5 & 11.97 & 0.771 & 0.771 & 0.769 & 0.769 \\
\hline & - & 50.00 & 0.616 & 0.616 & 0.612 & 0.612 \\
\hline$\geq 14.81$ & 1 & 0.11 & 1.000 & 1.000 & 1.000 & 1.000 \\
\hline & 2 & 0.43 & 1.000 & 1.000 & 1.000 & 1.000 \\
\hline & 3 & 1.02 & 1.000 & 1.000 & 1.000 & 1.000 \\
\hline & 4 & 2.67 & 0.943 & 0.943 & 0.942 & 0.942 \\
\hline & 5 & 11.97 & 0.771 & 0.771 & 0.769 & 0.769 \\
\hline & - & 50.00 & 0.771 & 0.771 & 0.769 & 0.769 \\
\hline
\end{tabular}

(1) The midpoint of each stream tube along the $z$-direction is chosen for plotting, which is different from where the average streamline enters the fracture.

(2) Concentrations are measured as fractions of the tracer solution concentration, $\mathrm{C}_{\mathrm{o}}$. 
displaced by the tracer solution. At $t_{3}=3.910 \mathrm{hrs}$, note the tracer front is only at $z=47 \mathrm{~cm}$ and has not reached the bottom of the fracture. The breakthrough curve at any distance along the fracture can be constructed by simply connecting the $C^{*}$ values for each time increment for that distance.

There are several observations of importance to understanding the results of the fracture transport tests discussed in the next section. As shown in Figures 4.9 and 4.10 , the concentration in the fracture is attenuated initially because part of the fracture plate flow traversed through the matrix. Furthermore, the steady-state $C^{*}$ value is not expected to reach 1.0 as the matrix plate contributes flow at the lower concentration of $C_{1}$. Both fracture flow and concentration are a function of distance along the fracture. Data interpretation is necessarily confined to the bottom 30 to $40 \mathrm{~cm}$, where $Q_{f}$ remains constant, and $C^{*}$ reaches a steady-state value over time, in this case after approximately 15 hours.

An omission in the case study is the use of filter paper as contact between the porous plates and the rock. Perfect contact between the plates and rock surface is assumed in the analysis. Results may be different because if the permeability of the filter paper is greater than those of the plates and rock matrix, flow from the fracture plate may converge towards the fracture opening via the filter paper. For this case, less flow will pass through the matrix resulting in a decrease in travel time for a greater proportion of flux at the higher concentration. The presence of the filter paper may act similarly to the case of a plate which just covers the fracture. The $C^{*}$ profile will approach steady state much faster, and analysis of data is then applicable for a greater segment of fracture. However, the permeability of the filter paper will depend on the compressive force applied against it. Its permeability is expected to be smaller with greater applied force. Additionally, the suction which develops under the plates is also expected to affect filter paper permeability. The porous plates and filter paper are just two factors affecting the transport of solute in the fracture. An understanding of their effects in an ideal test block provides background to interpreting experimental results.

\subsubsection{Solute Transport in the Fracture}

Three fracture transport tests were performed, two slug input tests of varying duration (nos. 1 and 2), and one step input test (no. 3). A "slug" test is defined here as one in which the tracer solution is introduced at the top of the plate over a period of time, e.g., 12 hours, followed by the background solution. A "step" test is one in which only the introduction of tracer solution occurs without subsequent flushing with the background solution. Therefore, a "step" test is simply the first part of a "slug" test. Information on the three tests including test conditions are summarized in Table 4.6. Sampling locations are shown in Figure 4.11. 
Table 4.6: Pertinent information and test conditions of fracture transport tests.

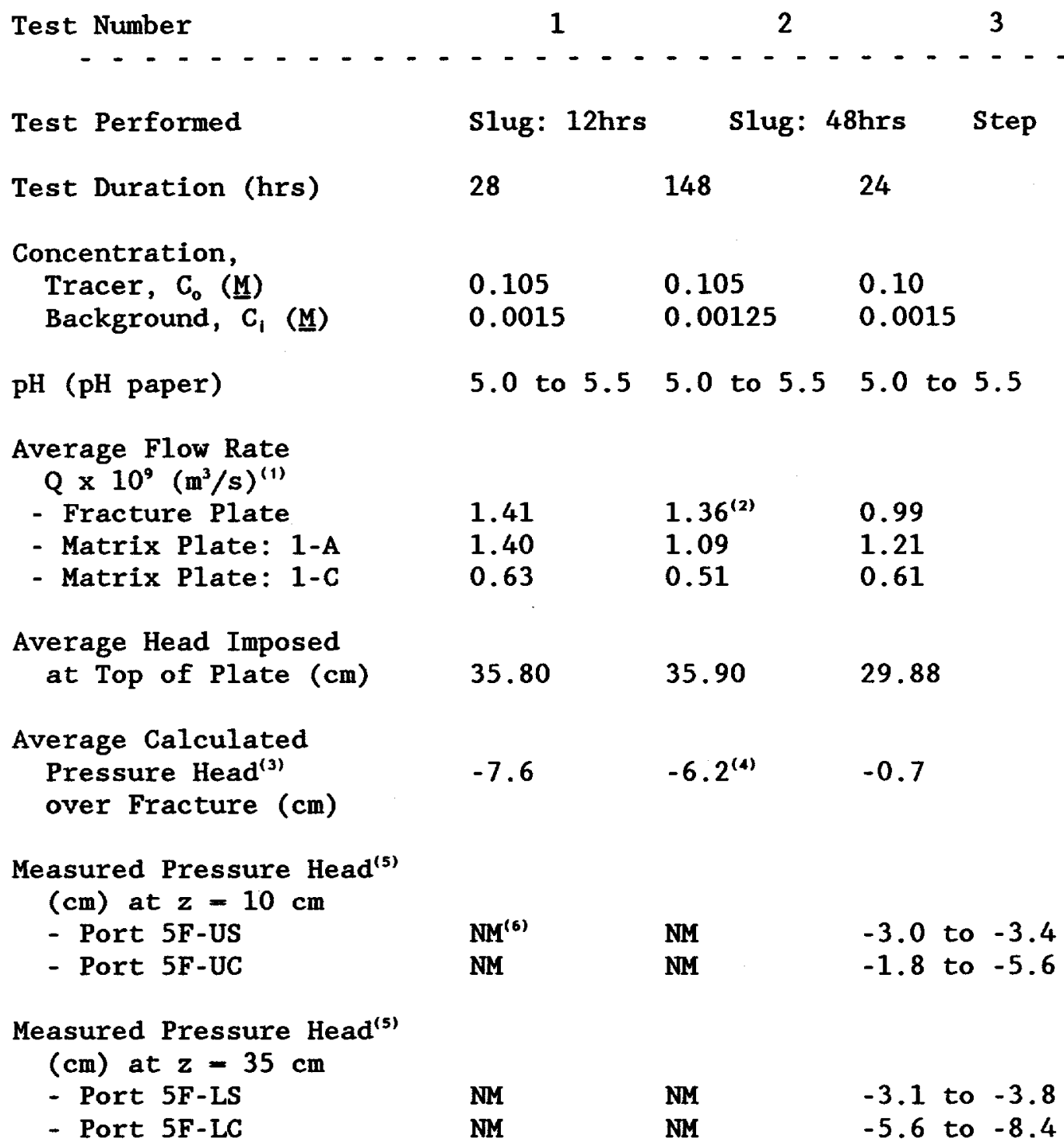

(1) Flow rate variation during tests was $\pm 3.0 \times 10^{-11} \mathrm{~m}^{3} / \mathrm{s}$.

(2) Actual flow rate was probably less due to seepage observed from the porous plate down test block face 6 during test.

(3) Values are estimated from flow rates and heads applied at the top of the porous plate.

(4) Actual pressure head was probably greater due to seepage observed from the porous plate down test block face 6 during test.

(5) Pressure heads were monitored during the following periods: 5F-US (8-3-88), 5F-UC (8-1-88 to 8-3-88), 5F-LS (7-26-88 to 7-28-88 and 8-1-88), and 5F-LC (7-27-88 to $8-1-88)$.

(6) NM - not measured. 


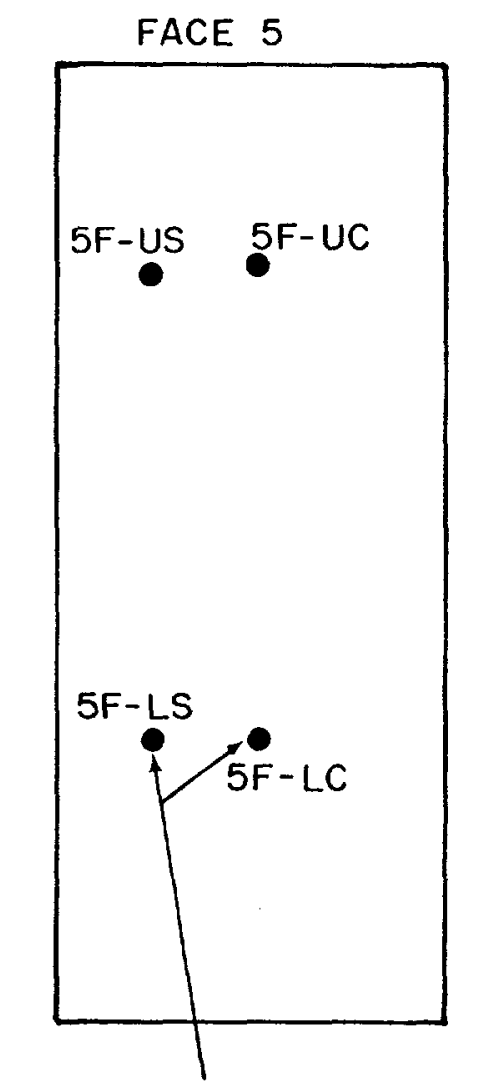

SAMPLING PORT

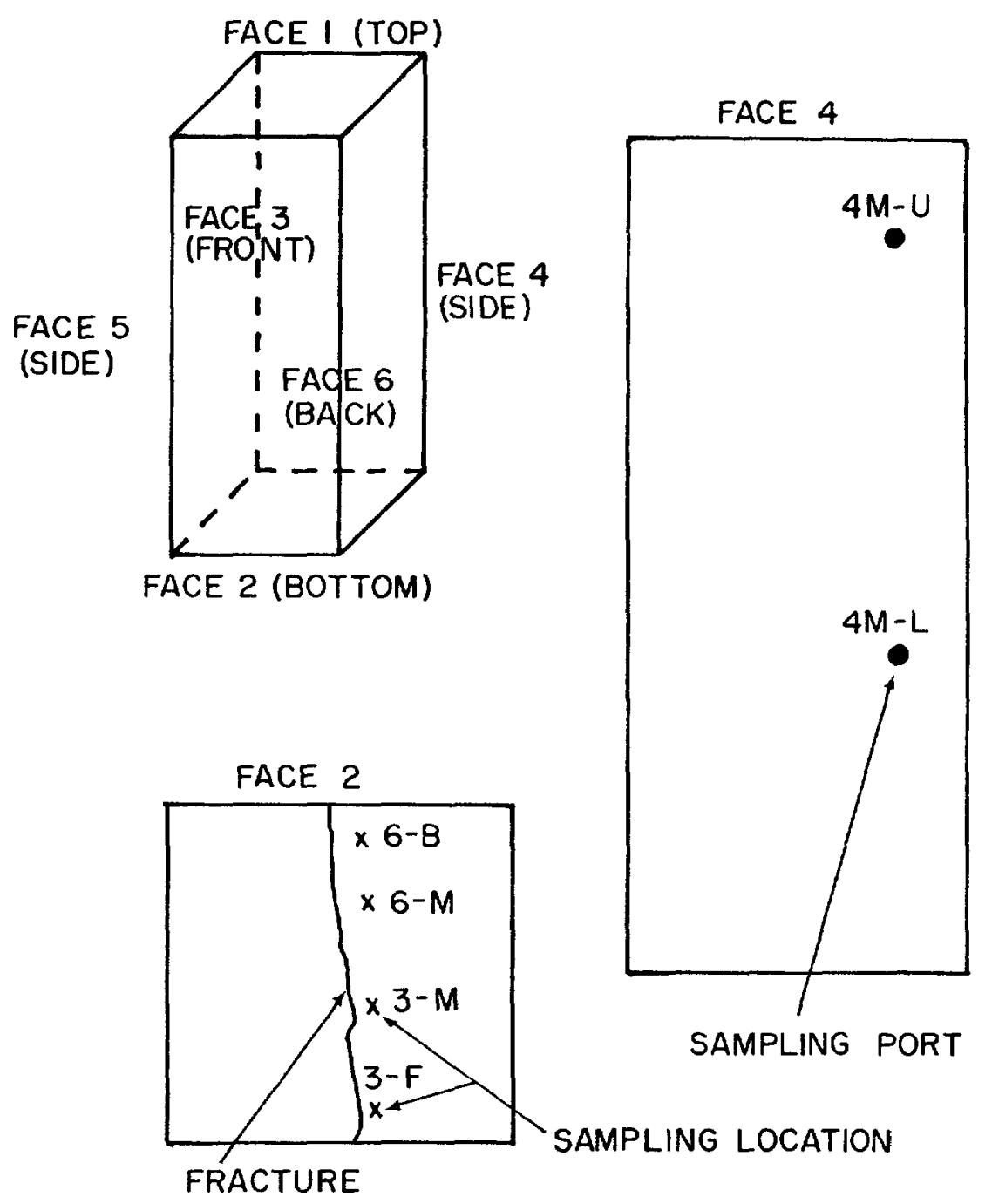

Figure 4.11 Sampling ports, and sampling locations at the bottom fracture opening. The fracture extends from face 3 to face 6 . 
During the fracture transport tests, a step input test in matrix side 1 $C$ (see Figure 4.12) was also conducted using a $1.25( \pm 0.10) \times 10^{-2} \mathrm{M}$ $\mathrm{CaCl}_{2}$ solution, while matrix side $1-\mathrm{A}$ continued to receive the background solution of $1.30( \pm 0.20) \times 10^{-3} \mathrm{M} \mathrm{CaCl} l_{2}$. The flowing of two $\mathrm{CaCl}_{2}$ solutions, both more dilute than what the fracture receives, through the matrix is expected to result in interesting two-dimensional transport scenarios. The concentration gradient is away from the fracture during the step-up portion of tests. However, on the step-down portion, the gradient is towards both the fracture and the side boundaries of the matrix. The tracer front moving down matrix side 1-C complicates the scenario. For discussion of the matrix transport test results, refer to Section 4.6 .3 below. Results of the transport tests are expected to be influenced by the concentration history in the fracture and matrix.

Breakthrough curves plotting the temporal concentration distributions for the three tests are presented in Figures 4.13 to 4.15 . Figure 4.16 plots the first 24 hours of test no. 2 (referred to as no. 2 - 24 hrs) for comparison against test no. 3, which was a 24-hour step input test. For clarity, the graphs only plot the average $C^{*}$ values. Figure 4.17 is a detailed graph of breakthrough at $z=10 \mathrm{~cm}$ for test no. 1 showing the typical range of $C^{*}$ values encountered. The first and second temporal moments of the average $C^{*}$ values are summarized in Tables 4.7 and 4.8. The breakthrough curves and temporal moments analyses show channeling flow in the fracture segment probably occurred with one or more preferential flow path(s). Not only flow conditions and local heterogeneities, but previous solute concentration history, are likely to affect solute transport. Results of all three tests indicate earlier arrival at sampling port $5 F-U C(x=10 \mathrm{~cm})$ than $5 F-U S(x=5 \mathrm{~cm})$ even though the ports are at the same distance along the fracture $(z=10 \mathrm{~cm})$. The first two tests alsu show earlier arrival at the bottom of the fracture segment $(z=50 \mathrm{~cm})$ than at sampling ports $5 F-L S$ and $5 F-L C$, both at a distance of $\mathrm{z}=35 \mathrm{~cm}$. This seems to indicate the tracer front bypassed the sampling ports at $\mathrm{z}=35 \mathrm{~cm}$ probably due to channeling. This discussion includes sampling port $5 F-L C$, even though it apparently intercepted a secondary fracture, because data collected at the port are not significantly different from those of port 5F-LS. However, the third test shows the earlier arrival of the center of mass at $z=35 \mathrm{~cm}$ than at $z=10 \mathrm{~cm}$. The breakthrough curves at $5 \mathrm{~F}-\mathrm{UC}$ all share a similar, but distinctive, decrease in $C^{*}$ after an initial steady increase in $C^{*}$ to approximately 0.6 . This phenomenon may have resulted from the intrusion of a secondary front moving through. The first moment data of test nos. 1 and 2 suggest a preferential flow path connecting $5 F-U C$ and $6-M$, whereas first moment data of test no. 3 indicate an alternative flow path connecting 5F-UC and 3-F possibly. The similarity of breakthrough curves of all four sampling locations at $z=50 \mathrm{~cm}$ is likely due to a "smearing effect" as the tracer solution front mixed at atmospheric pressure at the exit boundary. 
$\underline{1-A} \quad \underline{1-B} \quad 1-C$

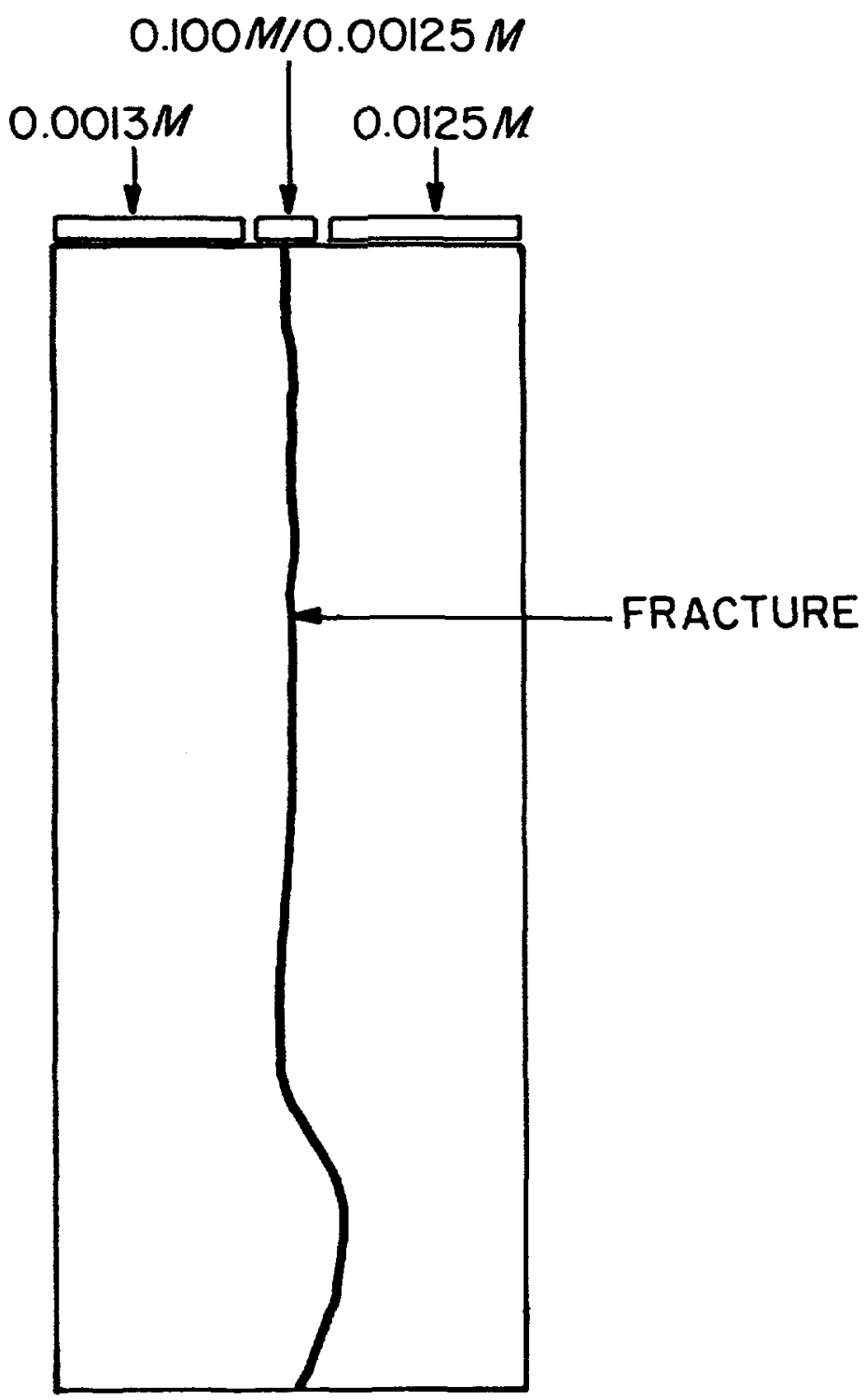

Figure 4.12 A complex solute concentration history in the test block is expected to result from introduction of calcium chloride solutions of different concentrations. 

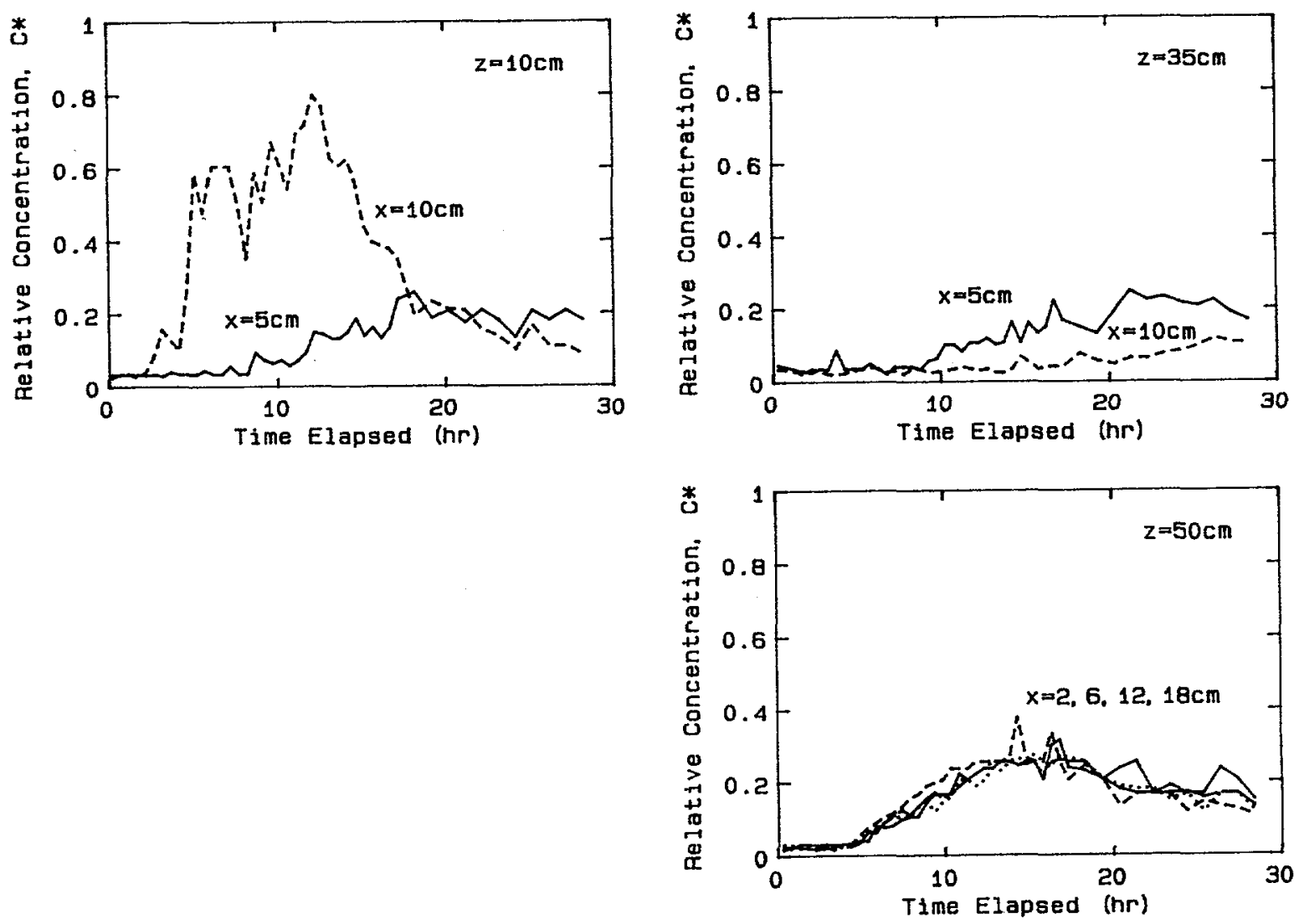

Figure 4.13 Breakthrough curves plotting the average relative concentration, $C^{*}$, versus time elapsed $(h r)$ for fracture transport test no. 1 at three distances along the fracture segment, $z=10 \mathrm{~cm}, z=$ $35 \mathrm{~cm}$ and $z=50 \mathrm{~cm}$. 

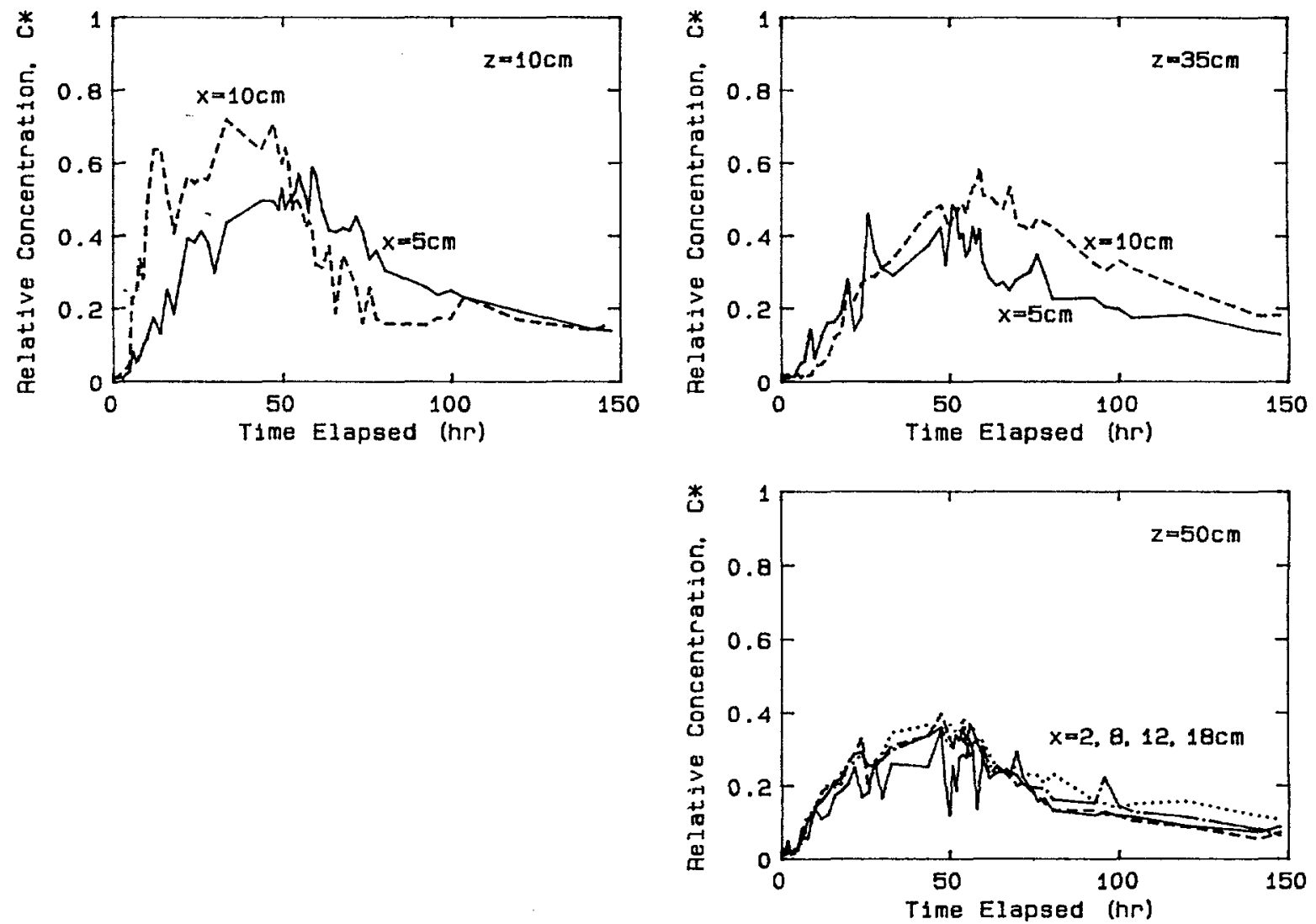

Figure 4.14 Breakthrough curves plotting the average relative concentration, $C^{*}$, versus time elapsed (hr) for fracture transport test no. 2 at three distances along the fracture segment, $z=10 \mathrm{~cm}, z=$ $35 \mathrm{~cm}$ and $\mathrm{z}=50 \mathrm{~cm}$. 

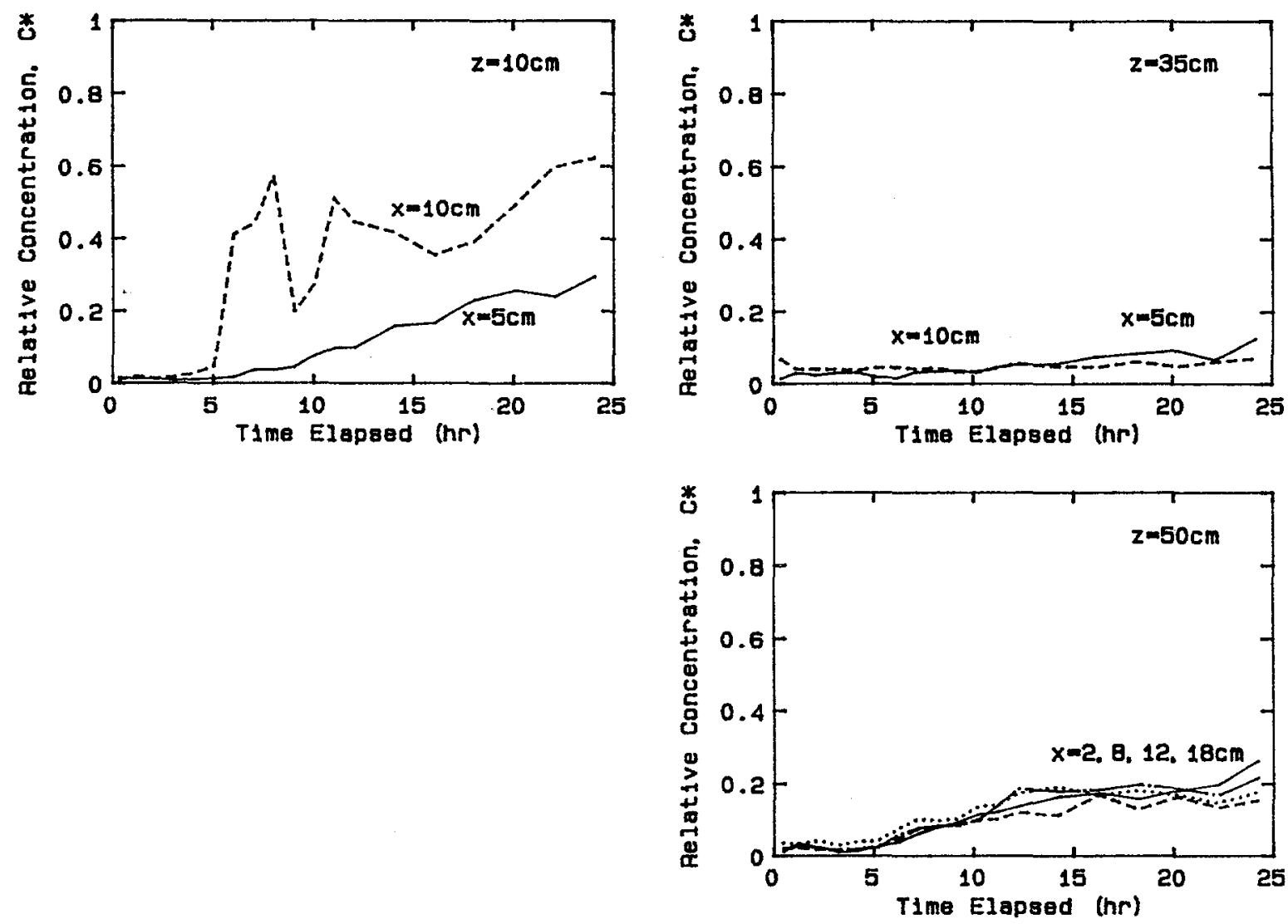

Figure 4.15 Breakthrough curves plotting the average relative concentration, $\mathrm{C}^{*}$, versus time elapsed (hr) for fracture transport test no. 3 at three distances along the fracture segment, $z=10 \mathrm{~cm}, z=$ $35 \mathrm{~cm}$ and $z=50 \mathrm{~cm}$. 

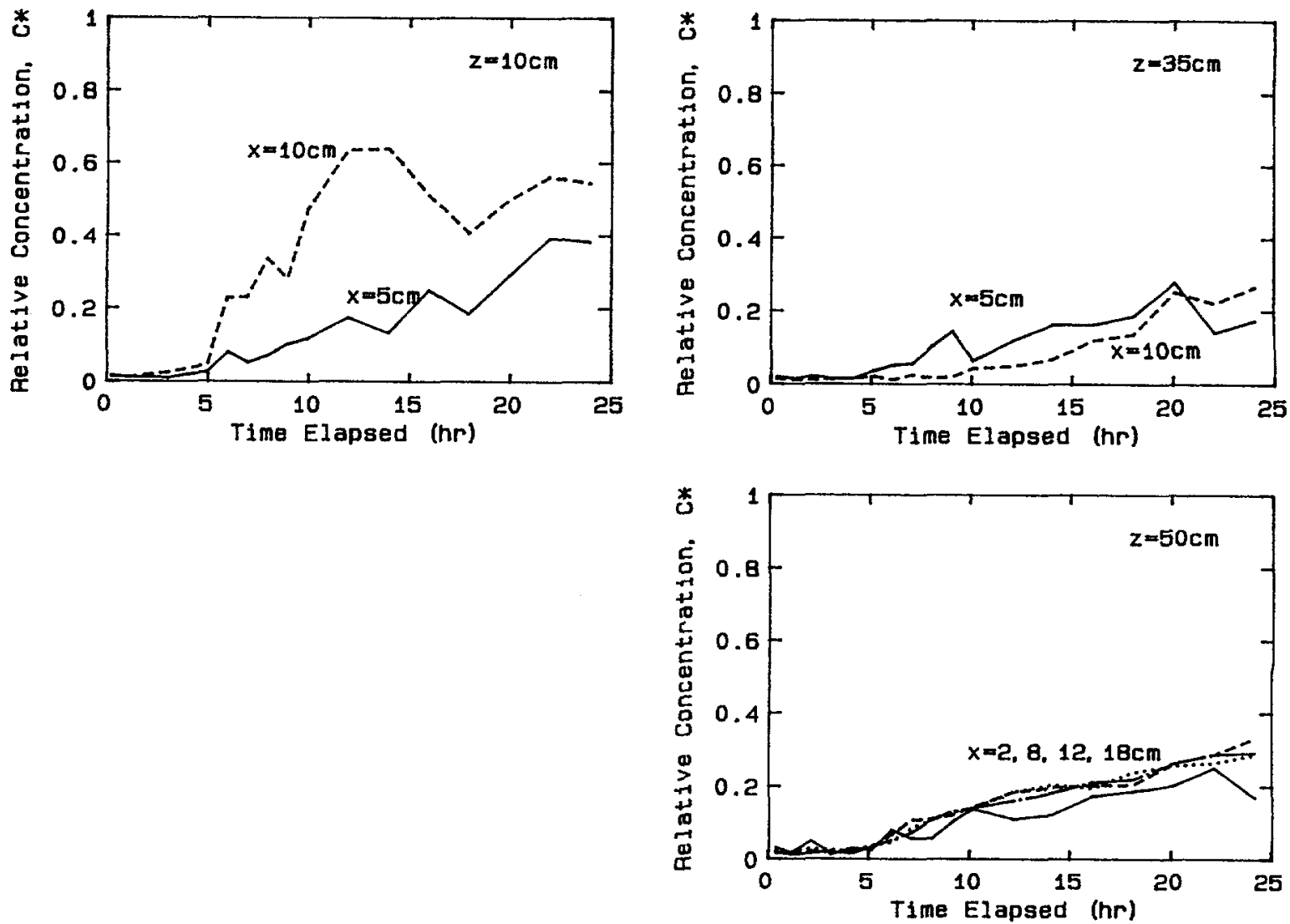

Figure 4.16 Breakthrough curves plotting the average relative concentration, $C^{*}$, versus time elapsed (hr) for the first 24 hours of fracture transport test no, 2 at three distances along the fracture segment, $z=10 \mathrm{~cm}, z=35$ and $z=50 \mathrm{~cm}$. 


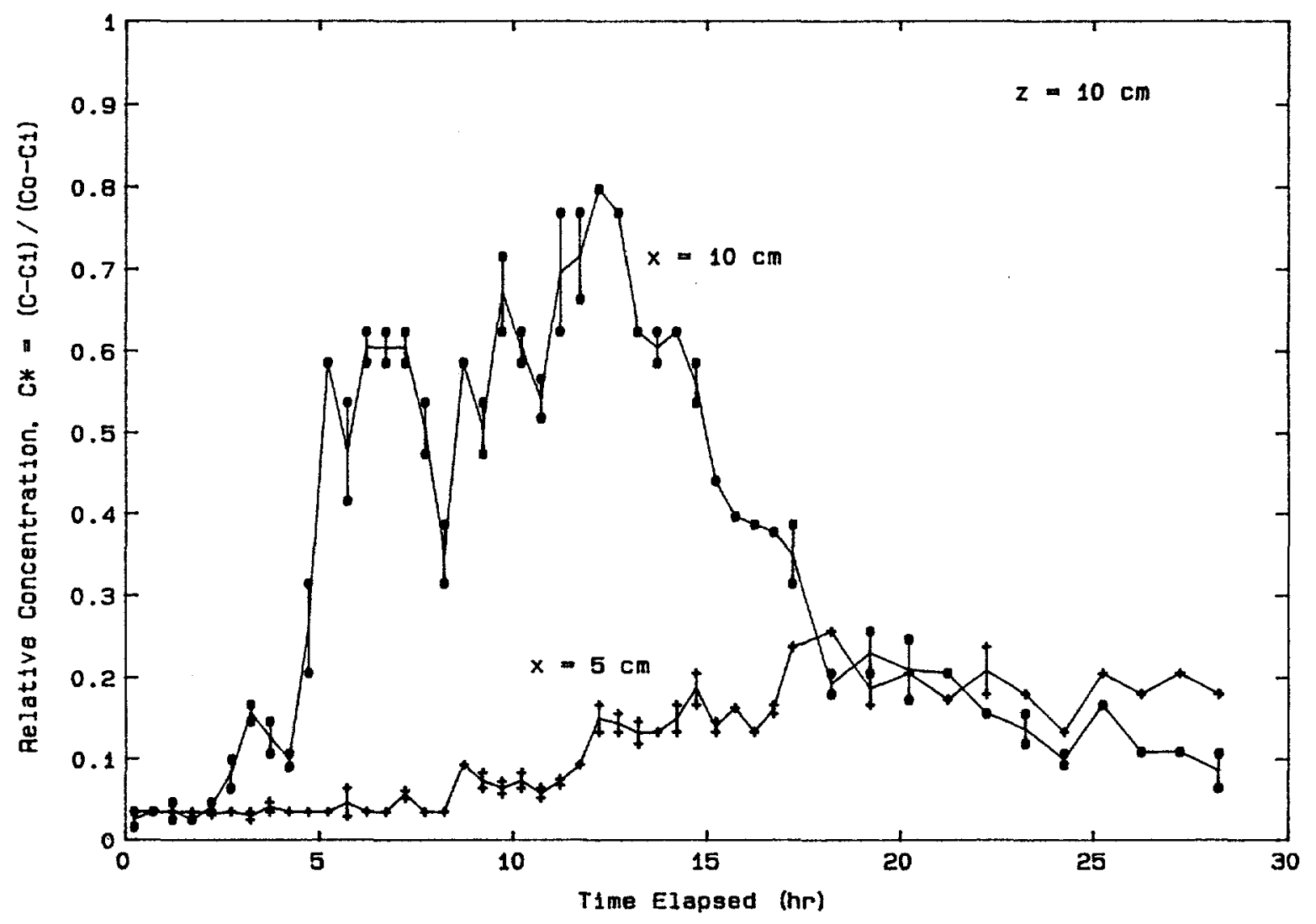

Figure 4.17 Breakthrough curves plotting the average relative concentration, $\mathrm{C}^{*}$, versus time elapsed (hr) for fracture transport test no. 1 at $z=10 \mathrm{~cm}$ showing the full range of $\mathrm{C}^{*}$ values for individual measurements. 
Table 4.7: First and second temporal moments data using average relative concentration values for fracture transport test nos. 1 and 2 .

Sampling Port/Location

$$
t_{\mathrm{m}} \frac{\text { Test No. } 1}{(h r) \quad s_{t}{ }^{2}\left(h r^{2}\right)}
$$$$
t_{\mathrm{m}}(\mathrm{hr}) \mathrm{st}_{\mathrm{t}}{ }^{2}\left(\mathrm{hr}^{2}\right)
$$

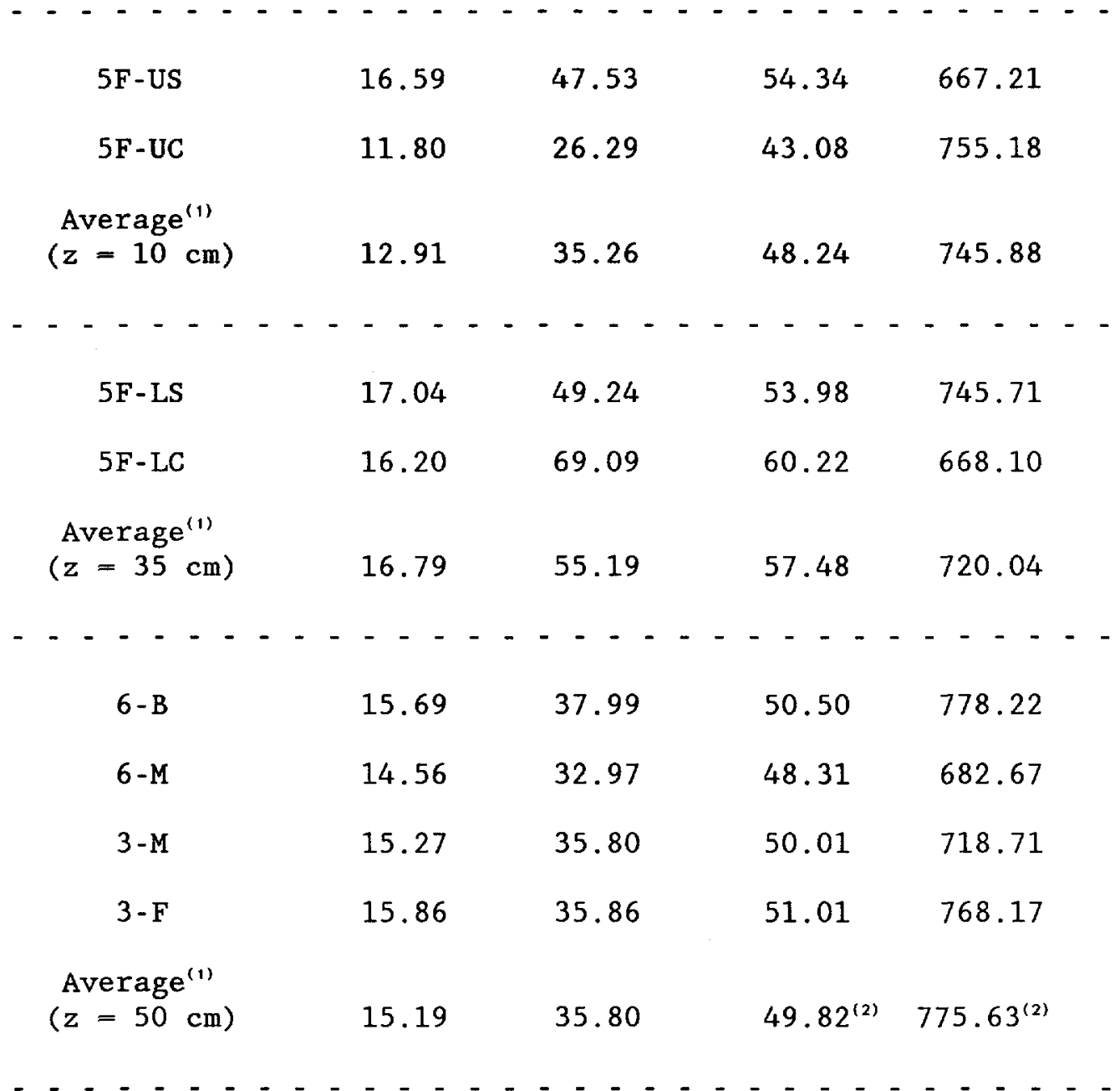

(1) First and second temporal moments are average values for each longitudinal distance, $z$.

(2) Average is calculated for sampling locations 6-M, 3-M and 3-F only. 
Table 4.8: First and second temporal moments data using average relative concentration values for fracture transport test no. 3 and the first twenty four hours of test no. 2 .

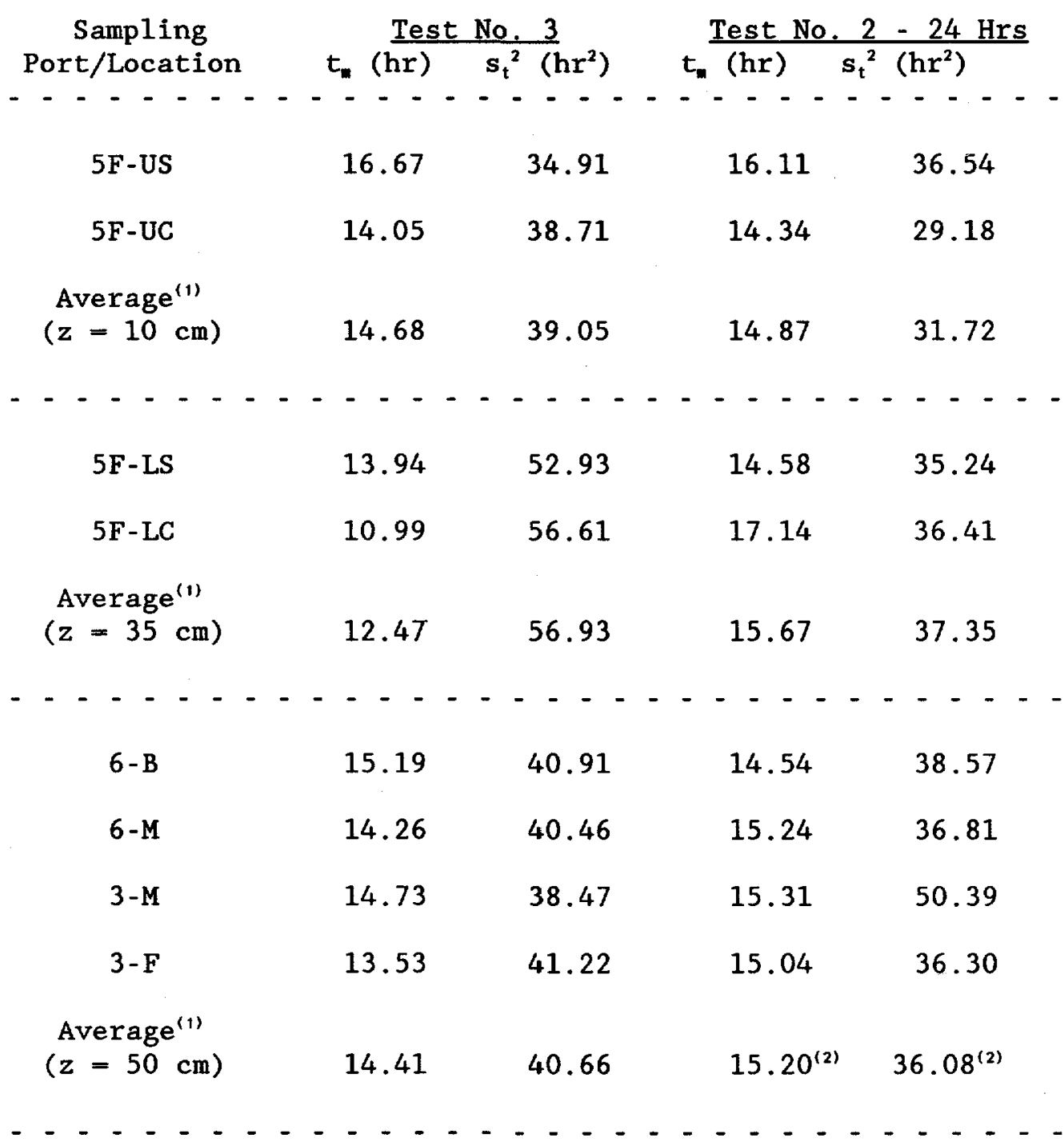

(1) First and second temporal moments are average values for each longitudinal distance, $z$.

(2) Average is calculated for sampling locations 6-M, 3-M and $3-F$ only. 
The first two tests were slug input tests with apparently similar flow conditions and average pressure heads over the top fracture opening (see Table 4.6). A 12-hour slug of tracer solution was introduced for the first test with a monitoring period of 28 hours, and 48-hour slug for the second with a monitoring period of 148 hours. Major differences resulting from the longer slug input is apparent in the breakthrough curves at 5F-US, 5F-LS and 5F-LC (see Figure 4.14) for test no. 2. The breakthrough curve at $5 F-U S$ is similar to that of $5 F-U C$ but with a time delay. Both breakthrough curves at $z=35 \mathrm{~cm}$ indicate a dramatic increase in relative concentration occurred at 5F-LS (maximum $C^{*}=$ 0.45 ), and at 5F-LC (maximum $C^{*}-0.60$ ). The shorter slug of tracer solution in test no. 1 may be the cause for the correspondingly flatter breakthrough curves at the three sampling ports. This bolsters the conjecture of an uneven tracer front due to channeling flow.

Results of the second test indicate a more slowly moving tracer front. During the test, tracer solution was observed to be seepage down face 6 . The earlier increase in $\mathrm{C}^{*}$ at sampling location $6-\mathrm{B}$ supports this contention. The test was not interrupted as the exact time the seepage started to occur was not known. The actual flow rate in the fracture and pressure head over the fracture are expected to be different from the apparent values presented in Table 4.6.

Although the exact flow conditions of the second test are not known, the results of the first 24 hours of the test can be compared with the third (see Table 4.8; Figures 4.15 and 4.16). The breakthrough curves, with few exceptions, are remarkably similar. The first and second moments at $z=10 \mathrm{~cm}$ and $\mathrm{z}=50 \mathrm{~cm}$ compare well, but not at $\mathrm{z}=35 \mathrm{~cm}$. Results of the third test at $z=35 \mathrm{~cm}$ show a center of mass which has a slower average velocity, but much less spread, than the first 24 hours of the second test. The discrepancy at $z=35 \mathrm{~cm}$ may be due to differences in flow conditions between the tests not manifested at $z=10 \mathrm{~cm}$ or $z=50$ $\mathrm{cm}$.

It is interesting to note that at 48 hours into the second test, the maximum $C^{*}$ is only 0.4 at the bottom fracture opening, which is slightly greater than half of the value calculated in the case study. This suggests several possibilities. A first guess is the effects of dispersion and matrix diffusion, which were not included in the model, cause the lower $\mathrm{C}^{*}$ measured. The contribution of matrix flow may be greater than anticipated. However, port $5 \mathrm{~F}-\mathrm{UC}$ at $\mathrm{z}=10 \mathrm{~cm}$ shows maximum values similar to the calculated $\mathrm{C}^{*}$ of 0.769 . Another is a slower than anticipated travel velocity due to the tortuosity of fracture flow. Estimates of travel velocity from the fracture tests, as discussed below, show this not to be the case. Precipitation of calcium chloride is ruled out from preliminary X-ray diffraction test results (Vickers, 1988).* Without tests in which the step-up portion is maintained for a longer period, the exact reason(s) may not be known.

Immediately at the end of the third test, the experimental setup was disassembled, and the test block broken apart at the fracture. A spatial concentration distribution of the fracture was measured of the solution remaining on the fracture surface. A discussion of the concentration distribution map of the fracture surface (Figure 4.18), in addition to the breakthrough curves and temporal moments data, is helpful in

*Vickers, B. C., 1988, "Rock Fracture Surface Roughness and Water Retention Properties", Unpublished Data. 
understanding the fracture solute transport parameter estimates obtained in Table 4.9. There appears to be two preferential flow paths, with the primary one closer to face 6 . Coupled with the concentration measurements, darker coloration of the fracture surface, and remnants of materials washed loose, seem to confirm this observation. The ridge, due to a curving of the main fracture at approximately four-fifths of the distance, probably controls the flow path direction to a certain extent. The liberties taken on the exact flow paths beyond the ridge are based partially on the first temporal moments calculated for the three tests (see sampling locations 6-B to 3-F, Tables 4.7 and 4.8).

Table 4.9: Estimates of average travel velocity, dispersion coefficient and longitudinal dispersivity using temporal moments analysis for fracture transport tests.

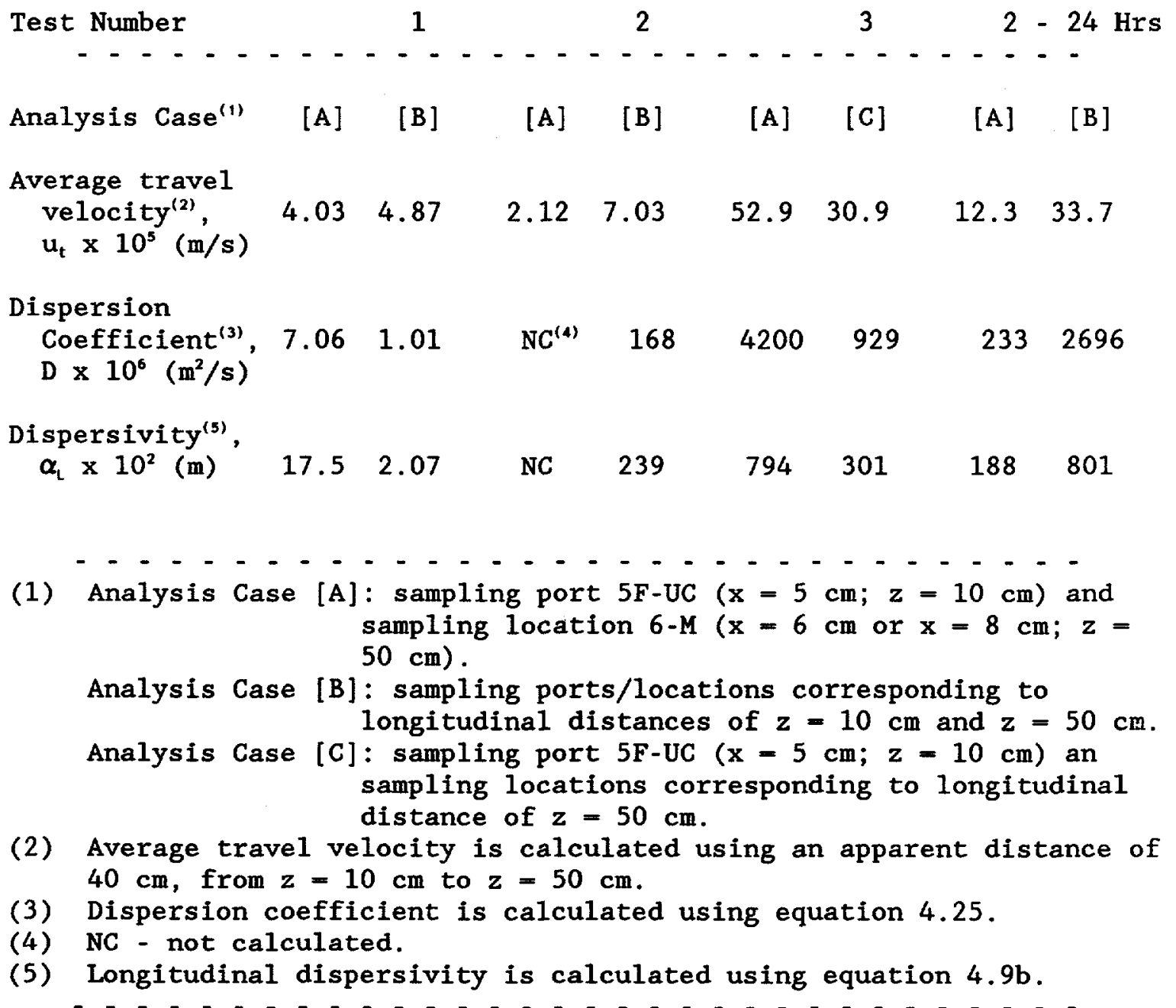




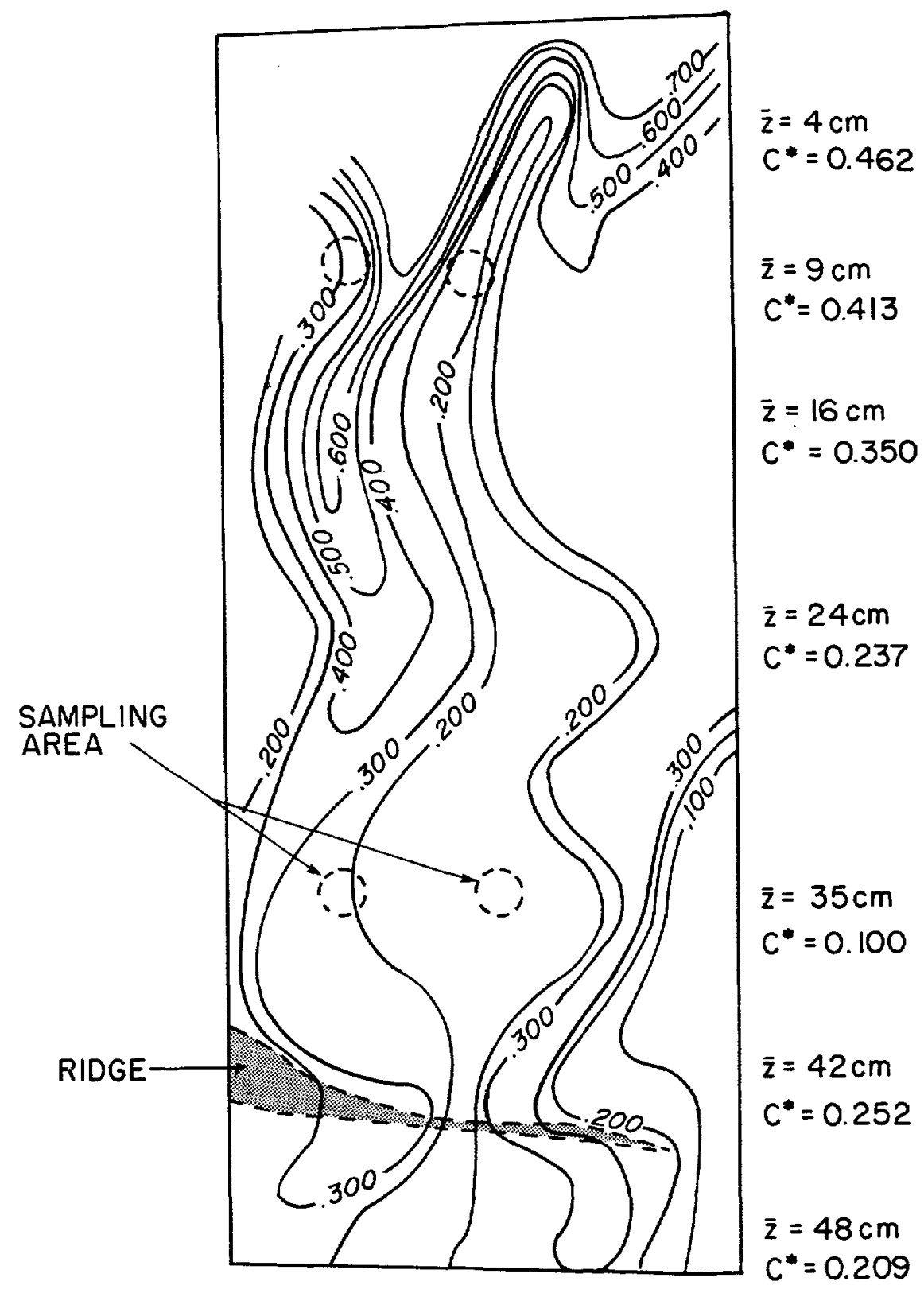

Figure 4.18 Spatial distribution map of relative concentrations of solution remaining on fracture surface immediately after fracture test no. 3. Two preferential flow paths are likely to exist with the dominant one closer to face 6 . The fracture sampling areas are denoted with circles. 
Estimates of the average travel velocity $\left(u_{t}\right)$ are determined using an apparent travel distance of $40 \mathrm{~cm}$, from the $5 \mathrm{~F}$-series ports at $z=10 \mathrm{~cm}$ to the bottom of the fracture at $z=50 \mathrm{~cm}$. From the computer model results, the analysis is seen as best confined to approximately this distance. The dispersion coefficient (D) and dispersivity $\left(\alpha_{L}\right)$ are determined using equations 4.25 and $4.9 \mathrm{~b}$. Based on the breakthrough curves, temporal moments data and spatial concentration distribution map, three cases are analyzed for solute transport in the fracture. Analysis case 'A' assumes a major flow path connecting sampling port $5 \mathrm{~F}$ $\mathrm{UC}$ and sampling location 6-M. Analysis case ' $B$ ' takes a laterallyaveraged approach (average for particular distance, $z$ ) and examines the temporal moments at $z=10 \mathrm{~cm}$ and $z=50 \mathrm{~cm}$. Analysis case ' $C$ ', which examines the temporal moments between sampling port 5F-UC and the laterally-averaged values at $z=50 \mathrm{~cm}$, is adopted as the only possible means of interpreting experimental data of the third test.

The average travel velocity ranges from $2.12 \times 10^{-5} \mathrm{~m} / \mathrm{s}(7.6 \mathrm{~cm} / \mathrm{hr})$ to $52.9 \times 10^{-5} \mathrm{~m} / \mathrm{s}(190 \mathrm{~cm} / \mathrm{hr})$, a one-order magnitude difference. The dispersion coefficient and longitudinal dispersivity vary from $1.01 \mathrm{x}$ $10^{-6} \mathrm{~m}^{2} / \mathrm{s}$ to $4.2 \times 10^{-3} \mathrm{~m}^{2} / \mathrm{s}$, and $2.07 \times 10^{-2} \mathrm{~m}$ to $8.01 \mathrm{~m}$, respectively. These values correspond to three-order and two-order magnitude differences. The travel velocity of the third test, which is a step input test, seems to have increased from the first two tests, which are slug tests, with a corresponding increase in dispersion as well. However, by isolating the first 24 hours of the second test, and comparing the results to the third test, the travel velocities and dispersion coefficients are much more similar. According to the temporal moments analysis, parameter estimation is not dependent on the type of test conducted. But without further testing, it appears the results may be a function of the type of test conducted.

In the computer model case study of the previous section, for a $T_{f}=5 \mathrm{x}$ $10^{-9} \mathrm{~m}^{2} / \mathrm{s}$, and a fracture half-aperture of $10^{-4} \mathrm{~m}(100 \mu \mathrm{m})$, the corresponding $K_{f}$ is $5 \times 10^{-5} \mathrm{~m} / \mathrm{s}(18 \mathrm{~cm} / \mathrm{hr})$. If the fracture is assumed to be fully saturated, then the travel velocity equals $k_{f}$, which matches we11 with results of test nos. 1 and 2. Longitudinal dispersivity is expected to be of a scale close to the length of the fracture segment, $0.5 \mathrm{~m}$ in this case. If these parameters have any validity to them, the $D$ is then $2.5 \times 10^{-5} \mathrm{~m}^{2} / \mathrm{s}$, which is within the range of values estimated. These values are the best estimates for the fracture segment.

The results suggest large variations for the same fracture segment among individual experiments, possibly due to fracture heterogeneities, magnified even at apparently similar flow conditions, and previous solute concentration histories. The results also suggest the importance of channeling flow. Moreno et al. (1985) found their parameter estimates to be similar assuming either hydrodynamic dispersion or channeling dispersion. However, they also concluded that for longer fracture segments the channeling dispersion model would show greater dispersion and earlier arrival of the solute front than the hydrodynamic dispersion model. If channel flow dominates, estimates of dispersion are then a function of the length of fracture segment. 


\subsubsection{Solute Transport in the Matrix}

While the fracture transport tests were conducted, a step input test was also started in matrix side 1-C. A tracer solution of $1.20( \pm 0.10) \mathrm{x}$ $10^{-2} \mathrm{M} \mathrm{CaCl} l_{2}$ was introduced beginning approximately one week prior to fracture test no. 1. Monitoring continued for nearly 51 days. However, insufficient data were collected at sampling port $4 \mathrm{M}-\mathrm{L}$ up until fracture test no. 2. Data collected after fracture test no. 2 should be viewed in light of prolonged periods of handling of the canopy flaps and placement of the tensiometer in the sampling ports for pressure readings.

Pertinent information including test conditions are presented in Table 4.10. Once again, the breakthrough curves are plotted using average $C^{\prime}$ values (see Figure 4.19). The curves for the two sampling ports, 4M-U and $4 \mathrm{M}-\mathrm{L}$, are expected to reflect conditions in the fracture. The periods corresponding to the three fracture transport tests are also indicated in the figure.

Table 4.10: Pertinent information and test conditions of matrix transport test.

Test Performed

Period Monitored (hrs)

Concentration, Tracer, $\mathrm{C}_{\mathrm{o}}(\underline{\mathrm{M}})$ Background, $C_{1}(\underline{M})$ $\mathrm{pH}(\mathrm{pH}$ paper)
Step

1222.25

$0.0125( \pm 0.0010)$

$0.0013( \pm 0.0002)$

5.0 to 5.5

1.79 to 3.18

4.30 to 5.95

Head Imposed at Top of Plate (cm)

Calculated Pressure Head ${ }^{(2)}$ at

Top of Matrix $(\mathrm{cm})$

$$
-2.45 \text { to }+1.64
$$

Measured Pressure Head ${ }^{(3)}(\mathrm{cm})$ at

- Port $4 \mathrm{M}-\mathrm{U}(z=5 \mathrm{~cm}) \quad-0.4$ to +1.0

- Port $4 \mathrm{M}-\mathrm{L}(\mathrm{z}=30 \mathrm{~cm})+0.6$ to +1.9

(1) Flow rate variation during tests was $\pm 5.0 \times 10^{-12} \mathrm{~m}^{3} / \mathrm{s}$.

(2) Values are estimated from flow rates and heads applied at the top of the porous plate.

(3) Pressure heads were monitored on 8-4-88. 


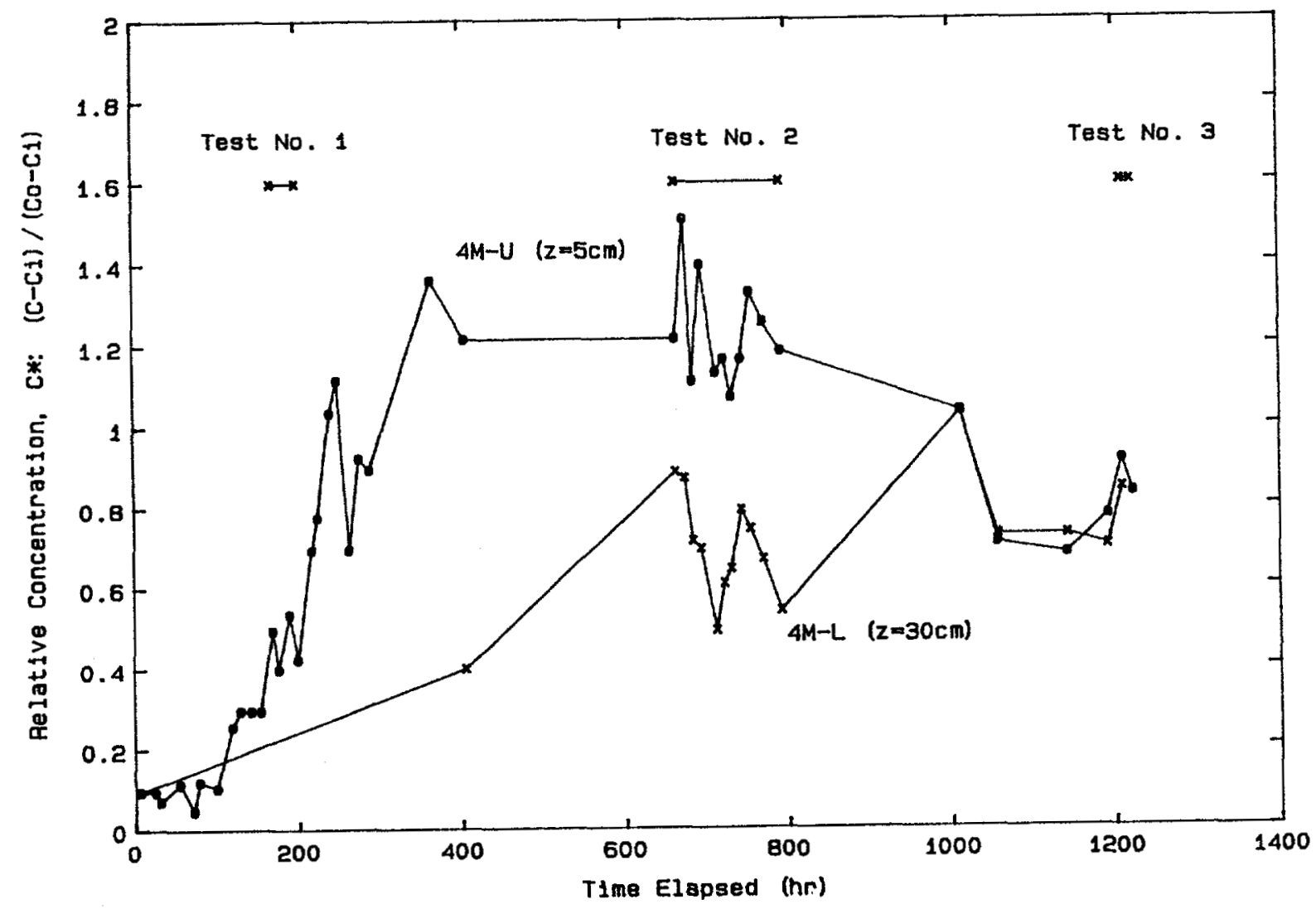

Figure 4.19 Breakthrough curves plotting the average relative concentration, $C^{*}$, versus time elapsed $(\mathrm{hr})$ for matrix transport test at two distances in the matrix, $z=5 \mathrm{~cm}$ and $z=30 \mathrm{~cm}$. 
From temporal moments analysis (see Tables 4.11 and 4.12 ), the average travel velocity from $z=0 \mathrm{~cm}$ to $z=5 \mathrm{~cm}$ is $1.13 \times 10^{-7} \mathrm{~m} / \mathrm{s}(0.97$ $\mathrm{cm} /$ day), and from $z=5 \mathrm{~cm}$ to $z=30 \mathrm{~cm}$ is $2.86 \times 10^{-7} \mathrm{~m} / \mathrm{s}(2.47$ $\mathrm{cm} /$ day). The first calculation assumes, at $z=0 \mathrm{~cm}$, an input from plate no. 4 as measured experimentally. The travel time appears to have decreased from the first $5 \mathrm{~cm}$ to the next $25 \mathrm{~cm}$. These values compare with a travel velocity of $3.34 \times 10^{-7} \mathrm{~m} / \mathrm{s}(2.88 \mathrm{~cm} /$ day) assuming an average $\mathrm{K}_{\mathrm{m}}=5.91 \times 10^{-8} \mathrm{~m} / \mathrm{s}, \mathrm{n}_{\mathrm{e}}=0.177$, and unit hydraulic gradient. The difference between the travel velocities calculated using the solute versus flow data may be attributed to the effect of the sampling ports. However, the differences are small and the effects, if any, of the ports should be assessed separately.

Table 4.11: First and second temporal moments data using average relative concentration values for matrix transport test.

$$
\text { Sampling Port } t_{m}(h r) \quad s_{t}{ }^{2}\left(h r^{2}\right)
$$

$\begin{array}{rrrr}\text { Plate No. } 4 & \text { Input }^{(1)} & 475.54 & 139237.8 \\ 4 \mathrm{M}-\mathrm{U} & 598.83 & 107109.3 \\ & 4 \mathrm{M}-\mathrm{L} & 841.40 & 44012.2\end{array}$

(1) Temporal moments analysis is performed using the experimentally determined breakthrough curve of plate no. 4 as the input function at $z=0 \mathrm{~cm}$.

Table 4.12: Estimates of average travel velocity using temporal moments analysis for matrix transport test.

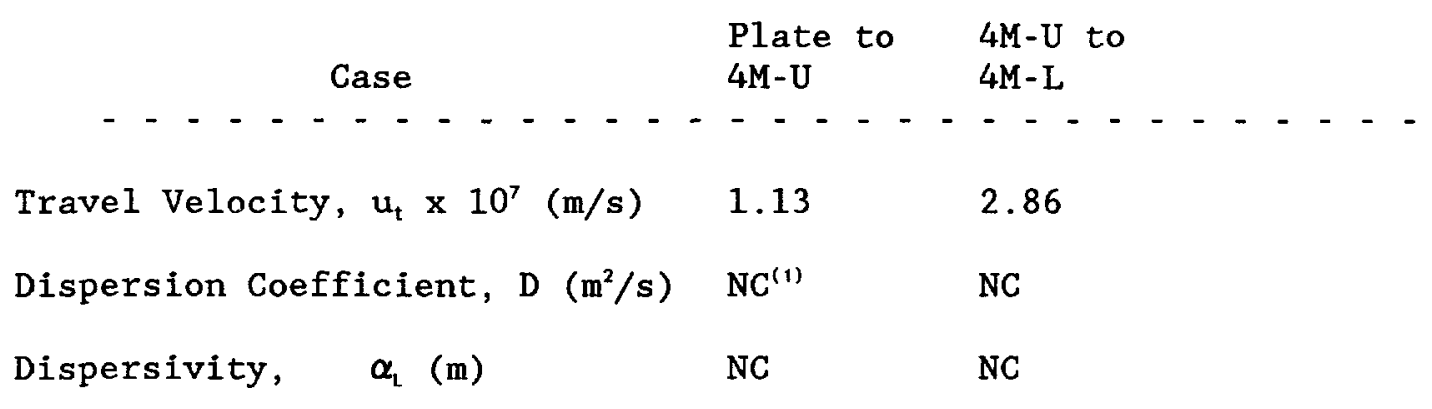

(1) NC - not calculated because of insufficient data and other data problems. 
The travel velocity calculations neglect the effects of matrix diffusion. During fracture transport tests, the concentration gradients are expected to result in lateral movement of chloride possibly affecting the concentration observed at the matrix ports. Such multi-dimensional transport due to fracture-matrix interactions, other than matrix heterogeneities, may explain the higher concentrations observed at the ports compared to a simple step input test. Figure 4.19 indicates the periods associated with the first two fracture tests, especially immediately after the step-up portions, show anomalous concentration increases. Additionally, the similarity in behavior at both ports during fracture test no. 2 argues for lateral as opposed to longitudinal influence of chloride movement. The period just prior to the last fracture test also show remarkably similar behavior at the two ports. These trends are believed to be more than experimental variabilities. However, the average travel velocity estimates are believed appropriate. The lateral movement of chloride should affect results at both ports, with a delay of several hours between the two ports.

A dispersion coefficient, and therefore, longitudinal dispersivity is not calculated because of the variance, or second moment, data. Because of the lack of data points at port $4 \mathrm{M}-\mathrm{L}$, and possibly the assumed input at $z=0 \mathrm{~cm}$, the smallest variance is seen at $z=30 \mathrm{~cm}$, making it impossible to apply equations 4.25 and $4.9 \mathrm{~b}$. The dispersion coefficient in the matrix is expected to be considerably greater than the effective diffusion coefficient of $5 \times 10^{-11} \mathrm{~m}^{2} / \mathrm{s}$ reported by Grisak et al. (1980), or $2 \times 10^{-11}$ to $17 \times 10^{-11} \mathrm{~m}^{2} / \mathrm{s}$ (for sodium halide salts) reported by Walter (1985). It is probably of the same magnitude as the fracture dispersion coefficient, if not greater, because of the tortuosity of flow paths. The quantification of the diffusion effect using the current experimental setup is complicated by the different fracture transport tests performed, each having different durations of tracer and background test solutions flowing through the fracture. Estimates of D and $\alpha_{l}$ in the matrix, and $D_{m}$ can be made more appropriately without fracture test interactions. 


\section{CHAPTER 5}

\section{CONCLUSIONS AND RECOMMENDATIONS FOR FUTURE STUDIES}

For the fluid flow component of this study, the principal objective was to develop procedures to determine both the unsaturated transmissivity of a fracture under controlled laboratory conditions and the unsaturated hydraulic conductivity of the adjacent matrix. Although the unsaturated transmissivity of the test fractures in either of the two blocks was not determined over a range of applied suctions, procedures were developed allowing such experimentation in future studies. Additional purposes of this study included the investigation of the infiltration and percolation characteristics of a fractured laboratory test block and the analysis of the physical properties of the tested blocks. This section will summarize how this study accomplished the above objectives and will suggest changes that might improve the ongoing investigation.

During this investigation, instruments and equipment were developed to facilitate the objectives of the study. Porous ceramic plates, Mariotte reservoirs, pipet flow meters, an effective evaporation canopy, and a microtensiometer were among the more important of the instruments used in the study.

This study found that accurate measurement of the porous ceramic plate conductances was necessary to minimize the error in calculation of pressure head at the bottom of the porous plate. This was particularly true when the applied suction of the test block was near atmospheric pressure. Since the hydraulic conductivity of the plates was only an order of magnitude less than rock matrix at saturation, the importance of the plate as an impeding layer should diminish at the lower matrix hydraulic conductivities expected under unsaturated conditions. Given that the study of fracture flow takes place at relatively wet conditions, porous plates of lower air entry values than those used in this study could be employed. After the first set of plates was removed from test block number 1 , they were cleaned with an ultrasonic bath. Plate conductances determined for one of the plates before and after cleaning were the same, and plate clogging was not considered throughout the rest of the study. In work progressing after the completion of this study, it was found by another student that reduction in plate conductance of up to 10 percent may take place after a plate has been idle for a few months in a solution bath. It is not known whether biological activity or precipitation of solutes contributed to the clogging that has recently been observed. Future work will have to consider the change in plate conductance with time for plates that are idle and plates that are being used in flow tests.

Once the Mariotte reservoirs were calibrated, it was found that the flow measurements obtained using the reservoir volume measurements agreed well with those determined from the pipet flow tubes. Since measuring the reservoir volume is significantly less time consuming than taking a pipet flow reading and more accurate at low flow rates, it is recommended that the Mariotte reservoirs be used to monitor inflow. Installing pre-calibrated burets sized according to the expected flow rate would 
significantly improve accuracy. A small yet non-restrictive air entry tube in the Mariotte burets will minimize the head variation on top of the porous plate also.

About five percent evaporative loss occurred during steady state-flow tests performed with test block number 1 . Laboratory temperature remained relatively constant throughout the study, varying from about $18^{\circ} \mathrm{C}$ to $22^{\circ} \mathrm{C}$. The access to the sampling ports was redesigned for test block 2, and significantly less evaporation should occur. If the improved vinyl evaporation canopy and access tubes do not lessen evaporation in the future, it is recommended that a humidifying system be designed and implemented to maintain the test environment at a constant humidity.

Although reasonable microtensiometer results were obtained during steady-state flow tests on block number 1, improvements in design could be made. Given that the pressure transducer failed during use and has been difficult to replace, it is recommended that a different type of transducer be employed. Instead of a differential transducer, a vacuum transducer should be obtained. Additionally, a stiffer connection between the tensiometer stem and the transducer could be designed to reduce outside pressure variations on the tensiometer assembly. If available, porous ceramic cups of lower air entry suction than the 100 $\mathrm{kPa}$ cup used in this study would also speed equilibration between measurements. Equilibration time for the tensiometer used on test block 1 varied from 15 minutes to 90 minutes.

Table 5.1 summarizes the test results for blocks 1 and 2 . Mean values are reported unless otherwise stated. A number of imbibition test results are worth noting. First, infiltration and percolation tests performed on both trst blocks indicate that the fracture influenced the shape of the wetting front curve only during early portions of the test. Later on, the fracture contributed solution to the matrix, slowing the advance of the wetting front near the fracture, relative to the matrix. Second, when the suction gradient was strongest in the early stages of imbibition, the shape of the wetting front was influenced by less

Table 5.1: Summary of Test Blocks 1 and 2.

\begin{tabular}{|c|c|c|}
\hline Parameter & Test Block 1 & Test Block \\
\hline$--\overline{-}--\overline{-}-\overline{-}$ & $-\overline{12}$ & $-\overline{2}-$ \\
\hline $\begin{array}{l}\text { Dry Bulk Density }\left(\mathrm{g} / \mathrm{cm}^{3}\right) \\
\text { Effective Porosity }\end{array}$ & $\begin{array}{l}2.12 \\
0.177\end{array}$ & $\begin{array}{l}2.13 \\
0.156\end{array}$ \\
\hline Saturated Matrix $\mathrm{K}(\mathrm{m} / \mathrm{s})$ & $5.91 \times 10^{-8}$ & - \\
\hline Saturated Matrix $\mathrm{k}\left(\mathrm{m}^{2}\right)$ & $5.51 \times 10^{-15}$ & - \\
\hline Matrix K, Applied $\psi=15 \mathrm{~cm}(\mathrm{~m} / \mathrm{s})$ & - & $5.50 \times 10^{-9}$ \\
\hline ceady State Matrix Suction (cm) & 0.2 & - \\
\hline aturated $T_{f}\left(m^{2} / \mathrm{s}\right)$ & $7.16 \times 10^{-9}$ & - \\
\hline dy State Fracture & 1.9 to 3.0 & - \\
\hline
\end{tabular}


permeable inclusions in the tuff and had a jagged appearance. Third, both the gamma beam attenuation tests performed on test block number 1 and wetting front analysis of test block 2 suggest that the test blocks did not have a uniform water content behind the wetting front.

Fourth, Philip's equation fit the infiltration data from test block 2 we11. The strong linear trend of the late-time data suggests that flow through the transmission zone was driven by the gravity gradient only and was primarily through the matrix, with little fracture flow occurring. Dominant matrix flow was also seen in both the shape of the test block 2 wetting front and the comparison of specific discharges from the plates covering the fracture and the matrix. The Philip's infiltration analysis of the total inflow yielded a matrix hydraulic conductivity of $5.50 \times 10^{-9} \mathrm{~m} / \mathrm{s}$, an order of magnitude less than in the saturated flow tests performed on test block 1 . If the saturated hydraulic conductivity of the matrix of block 2 equals that of block 1 , a significant decrease in the matrix conductivity occurs with relatively little applied suction.

From the plots of inflow rate versus time, it was determined that steady-state flow in test block number 1 was achieved about 35 days after solution was first introduced to the test block. Despite steadystate conditions, inflow rates continued to vary around a mean value due to Mariotte reservoir adjustments and solution leakage from beneath one of the matrix plates and from the side of the fracture. The inflow rate of the fracture plate decreased slightly with time, suggesting that either the fracture may have drained slightly over the course of the test or less leakage from the side of the fracture occurred during later stages of the steady-state test. Table 5.1 shows the test block parameters determined during the steady-state portion of testing on block 1 . The fracture transmissivity shown was calculated by assuming that all of the solution flowing into the test block through the fracture plate went into the top of the fracture. Although modeling of steady-state flow through test block number 1 found about 20 percent of the flow through the matrix plates entered the fracture before reaching the bottom of the test block, it was assumed in the model that no filter paper aided plate-rock contact. In fact, filter paper was used in the laboratory experiment. Filter paper improved contact between the porous plates and the matrix or fracture, and most of the fluid exiting the center porous plate was likely shunted directly to the top of the fracture. Although the hydraulic conductivity of the filter paper was not measured, based upon its retention rating, it was more conductive than the test block or the porous plates. As such, it would provide a preferential flow conduit for solution to move from the center plate to the fracture. This suggests that the assumption used to calculate fracture transmissivity shown in Table 5.1 is a good one. Future work on this project will have to consider further how using filter paper as a contact material influences flow into the test fracture.

Results of the block 1 tests indicate a number of limitations to this method of study. First, due to leakage from beneath both the porous plates and out of the fracture, the method does not lend itself to saturated fracture study. If the fracture traces along the sides of the block could be sealed, and if a gasket could be placed around the edges 
of the porous plate, saturated flow could more accurately be investigated. Second, monitoring of the displacement transducers suggests that over 30 foot-pounds of torque need to be applied to the fracture to minimize aperture change during the course of flow testing. It is not known what maximum torque can be applied to the current innermost frame. Third, if comparisons of the matrix hydraulic conductivity or bulk hydraulic conductivity of each test block with the hydraulic conductivity of Apache Leap tuff determined elsewhere are to be made, a new method of applying compressive stress to the entire rock matrix needs to be developed. The saturated matrix hydraulic conductivity calculated from the block analysis was an order of magnitude greater than those determined on cores. Apparently, the compressive stress applied during testing of the cores reduced the pore space available to flow and reduced the resulting hydraulic conductivity. This suggests that a compressive stress will need to be applied to the test blocks that is equivalent to that present in the medium to which the results will be compared. However, more can be said about the comparability of results once more data has been gathered over a range of applied suction heads using the current setup.

Results of the rock characterization tests indicate that the partially welded test blocks used in this study are of similar porosity, dry bulk density, and pore size distribution to the cores obtained from the plateau location at the Apache Leap tuff site. However, additional moisture release curves will be needed to precisely characterize the rock matrix in the range of suctions to be used during flow tests. Although significant variability occurs throughout the entire tuff sequence, the partially welded test blocks were removed from locations near to the borehole locations, and thus have similar physical properties. It is expected that the densely welded test block will differ considerably in physical properties from the partially welded tuff.

In conclusion, by using porous ceramic plates to apply a relatively constant suction along the upper surface of a fractured block of tuff, it is possible to determine important unsaturated flow properties. By varying the applied suction along the top and bottom of the test block and the applied stress perpendicular to the test fracture, it should be possible to analyze flow through a combined matrix-fracture system over a variety of conditions.

A number of other studies have been completed or are in progress which investigate the nature of flow and transport through unsaturated fractured tuff. The transport of chloride ion in test block number 1 using ion-selective electrodes is explored in Chapter 4. By using filter paper to obtain solution samples from both the matrix and fracture sampling ports, the movement of pulse inputs of chloride transport through the block is monitored.

Since the end of this study, other students have continued the work with test block number 2 . Once the wetting front has reached the bottom of the test block, porous plates will be placed against the bottom face of the block, allowing removal of solution from the block. A controlled pressure head will be maintained along the bottom of the test block in a manner similar to that used at the top face of the block. Attempts will be made to maintain the same pressure head at the top and bottom of the 
block. After steady-state flow has been reached, fracture transmissivity and matrix hydraulic conductivity will be determined. Additionally, the transport of chloride or other tracer will be investigated. The controlled pressure head at the top and bottom of the test block will then be changed. When steady-state flow has again been achieved, the test block parameters will again be determined. This process will be repeated throughout a controlled suction range less than the critical suction where fracture flow is less than matrix flow. Once test block parameters have been defined for one applied stress level, the compressive stress across the fracture will be increased and the analysis repeated.

Similar studies will also be performed by graduate students on three other test blocks retrieved from the Apache Leap tuff site. One is a densely welded tuff block, and two are partially welded tuff blocks with different vertical lengths than the test blocks used in this study. Various plate configurations may be used during the imbibition tests performed on these blocks, and the test fracture may be oriented differently than it was during this study. Porous plates will only be placed over the test fracture of the densely welded block, since preliminary laboratory tests indicate that the matrix of the densely welded tuff is one to two orders of magnitude less conductive than the matrix of the partially welded tuff.

Two other related studies are investigating fractured tuff. The first consists of a fracture profile study on the test fracture from block number 1. A computer-controlled profiling device is being used to characterize the roughness and tortuosity of the fracture. The second is an extension of this study. It involves the use of a cellulose membrane that will serve as an impeding layer in a field flow test. Development of a membrane and frame to hold it in the borehole will allow a positive pressure head inside the angled boreholes at the Apache Leap tuff site to result in a negative pressure head around the boreholes. This will allow the analysis of unsaturated flow characteristics in situ.

Transport studies through single fracture segments have been confined to single phase flow. Techniques are being developed to obtain hydraulic and solute transport parameters in natural fractures at variably saturated conditions. This portion of the research focused on developing techniques to study transport behavior, testing them under nearsaturated conditions, and also evaluating them for further studies at unsaturated conditions.

Preliminary transport tests were conducted on a partially welded tuff test block with a natural fracture at near-saturated conditions. Four additional test blocks with more representative fractures are being prepared and instrumented for further studies. The first test block was selected for convenience to test the techniques and procedures developed, but its fracture may be more variable than the other test blocks.

Coated-wire ion-selective electrodes were used to monitor the breakthrough of chloride in the fracture and rock matrix. Measurements were made on filter paper used to collect small amounts of tracer solution prepared from calcium chloride. Special considerations were necessary for the proper use of the electrodes in an unconventional sampling 
environment such as filter paper. Calibration curves were prepared comparing potential difference measurements in a more conventional "aqueous" environment versus a "filter paper" environment. Independent tests using a porous cup/water column apparatus showed the filter paper/ion-selective electrode technique is capable of measuring concentration changes under unsaturated conditions, i.e., up to a suction of $100 \mathrm{~cm}$ of water.

Sample collection at various points along the fracture and matrix was made possible with six sampling ports. Four of the sampling ports extend to the fracture plane, and two end in the matrix. The ports were necessary to obtain data, but are expected to affect flow and transport.

Ceramic porous plates were used to control the upper boundary pressure heads, and to introduce test solutions. The hydraulic and transport properties of the plates were determined experimentally. Plate conductance, a direct measurement of the plate's ability to conduct water, and Mariotte reservoirs were used to control suction at the top of the rock. Chloride breakthrough in the plate provided information on the input function of chloride at the top of the rock.

Solute transport parameters were estimated from results of three fracture transport tests and one matrix transport test using temporal moments analysis. Large variations in the results were observed for the same fracture segment among individual experiments. The average travel velocity in the fracture ranges from $2.12 \times 10^{-5} \mathrm{~m} / \mathrm{s}(7.6 \mathrm{~cm} / \mathrm{hr})$ to 52.9 $\times 10^{-5} \mathrm{~m} / \mathrm{s}(190 \mathrm{~cm} / \mathrm{hr})$, the dispersion coefficient from $1.01 \times 10^{-6} \mathrm{~m}^{2} / \mathrm{s}$ to $4.2 \times 10^{-3} \mathrm{~m}^{2} / \mathrm{s}$, and longitudinal dispersivity from $2.07 \times 10^{-2} \mathrm{~m}$ to $8.01 \mathrm{~m}$. Interpretation of the data in the fracture was aided with a computer model case study of the test block. The model showed fracturematrix flow interaction occurs near the top fracture opening. The implications of the model results include a steady-state relative concentration of less than 1.0 in the fracture, and a constant concentration for the bottom 30 to $40 \mathrm{~cm}$ of fracture if matrix diffusion is neglected. A spatial concentration distribution map of the fracture was also obtained at the end of the third fracture transport test strongly suggesting the existence of preferential flow paths.

Parameter estimation in the matrix was hampered by insufficient data, and the complex two-dimensional solute concentration history in the test block. Monitoring of chloride breakthrough in the matrix sampling ports clearly indicated the influence of the fracture through matrix diffusion. The average travel velocity in the matrix ranges from $1.13 \times 10^{-7}$ $\mathrm{m} / \mathrm{s}(0.97 \mathrm{~cm} /$ day $)$ to $2.86 \times 10^{-7} \mathrm{~m} / \mathrm{s}(2.47 \mathrm{~cm} /$ day $)$. The dispersion coefficient and longitudinal dispersivity in the matrix were not calculated.

Based upon the evaluation of the filter paper/ion-selective electrode technique, and the transport tests, the major conclusions of this study are:

- The filter paper/ion-selective electrode technique is a viable method to study solute transport behavior under near-saturated conditions in fractured rock systems. Although the combined experimental error for the study can be as much as \pm 20 percent, use of a 
superior $\mathrm{pH} / \mathrm{mV}$ meter with greater resolution and accuracy can reduce the error to acceptable values.

o The technique appears to be promising for studying unsaturated fractured rock systems. Independent tests show acceptable experimental errors up to suctions of $100 \mathrm{~cm}$ of water, a level probably greater than the critical suction when flow in fractures of interest will have drained.

- Large variations in solute transport parameter estimates are observed for the same fracture segment. The variations probably result from fracture and matrix heterogeneities, differences in flow and pressure conditions, and solute concentration history. Use of temporal moments analysis to estimate the parameters appears appropriate, but may require further confirmation.

- Channeling flow in the fracture is observed even for the relatively small fracture segment. The implications for a longer fracture segment may be greater dispersion and earlier arrival of the solute front.

- Under large concentration gradients, the effects of matrix diffusion are clearly seen through monitoring of matrix sampling ports during fracture transport tests. Matrix diffusion is expected to retard breakthrough, and also to spread out the breakthrough curve. The experimental data in the fracture appears to support this contention.

o With the experimental arrangement employing only the top porous plates, fracture-matrix flow interaction occurs. However, it is confined to the region near the top fracture opening. Use of bottom plates are expected to minimize the interaction.

o Water does enter the sampling ports at near-saturated flow conditions. However, the ports are expected to interfere with flow, and to influence the average travel velocity calculations. For $2.0-\mathrm{cm}$ diameter ports, water is expected to be excluded even at slightly unsaturated flow conditions, requiring longer sampling periods than at saturated flow conditions during sample collection in the matrix. The problem of mixing with matrix waters during collection of fracture samples is reduced under the same conditions.

Four additional test blocks, three of which are slightly welded tuff and one densely welded tuff, are being prepared for further testing. Imbibition tests and preliminary monitoring of chloride concentration are currently under way on a second slightly welded tuff test block. Based upon experiences with the filter paper/ion-selective electrode technique and ceramic porous plates, and transport test results, the following recommendations are made:

- With the acquisition of a superior $\mathrm{pH} / \mathrm{mV}$ meter with greater resolution and accuracy, a top priority is the quantitative assessment of individual sources of error of the $\mathrm{pH} / \mathrm{mV}$ meter, ion-selective electrode, reference electrode, and electrode drift. New and more accurate calibration curves can also be constructed. 
o If experimentation is to continue with chloride tracers, a doublejunction reference electrode with a different bridge solution, i.e., other than $\mathrm{KNO}_{3}$, will be a necessary addition. A bridge solution that is not a major interferent of chloride will improve the reliability of the technique, and allows for a longer period to measure potential differences. Otherwise, a single-junction (no bridge solution) reference electrode is recommended.

- The reliability of the filter paper/ion-selective electrode technique requires confirmation at higher suctions. It tested well with a porous plate/water column apparatus, but needs to be evaluated in actual transport tests.

- Chloride breakthrough in the porous plates requires further testing at different flow conditions, and background and tracer concentrations. More tests are necessary to obtain representative breakthrough behavior of the plates. Use of filter paper placed on the plate during measurements is highly recommended to prevent electrode wear.

- To ensure the porous plates are hydraulically separated, an impermeable barrier to be placed between the plates, or a depression to be cut into the rock along the entire length of the plates to physically separate them, is recommended. This will allow for equalization of pressure head under each plate.

- The study of transport behavior in the fracture and the matrix is best accomplished with separate test blocks, or possibly different tracers with the same test block. Several options are available. For different test blocks:

- Conduct fracture tests, and monitor effects in the matrix at different locations to account for spatial variability.

- Conduct tests in the matrix on one side of the fracture, and monitor the effects in the fracture, as well as the other side of the matrix.

- Conduct fracture tests for a densely welded tuff block with negligible matrix permeability to isolate fracture transport behavior.

For the same test block:

- Conduct fracture and matrix tests simultaneously with different tracer solutions, or by monitoring different tracer ions. Careful selection of tracers is needed to take into account compatibility of the different ionic specie, and also compatibility of the tracers with the host rock environment.

- In addition to slug input tests, step input fracture transport tests of longer duration are of interest to assess the long-term concentrations in the fracture for a finite sized test block.

- Evaluation of the temporal moments analysis method is recommended by fitting the parameters to either the classical advection- dispersion model, or statistical models, if enough data is available. Also compare results of slug versus step input tests. The variation in 
parameter estimates is believed to be independent of the tests conducted, but confirmation is still needed.

- To account for spatial variability in the fracture and rock matrix, drill sampling ports extending to the fracture and ending in the matrix from both sides of the fracture plane. Additional sampling ports should be drilled to obtain more data points for any transect at a given longitudinal distance.

- For studying matrix transport properties, place the sampling ports in a staggered fashion to minimize the "dry shadow" effect directly below the ports. This is especially important under more unsaturated conditions. 
APPENDIX A

PREPARATION, CALIBRATION AND EXPERIMENTAL PROCEDURES 
PROCEDURE 1

\section{PREPARATION OF TEST BLOCK FOR EXPERIMENTATION}

\section{Equipment}

1. Drill press.

2. Rotating drill assembly, with hose connection to water source.

3. Longyear diamond-edged coring bit, $1.91 \mathrm{~cm}(3 / 4-i n$.) outside diameter (o.d.).

4. Leve1(s).

5. Wood blocks, planks, shims or similar support and wedging imple ments.

6. Large clamps, $30 \mathrm{~cm}$.

7. Flashlight.

8. Tygon tubing, $3.2-\mathrm{mm}(1 / 8-$ inch) inside diameter (i.d.), 6.4-mm (1/4-inch) o.d.

9. Suction pump, hand-held.

10. Syringe.

11. Metal wire.

12. Squirt bottle.

13. Chise1.

14. Hammer.

15. Test tube brush.

16. Rock Frame A (frame lying against rock surface), made of $1.59-\mathrm{cm}$ (5/8-inch) thick steel, with vertical rib.

17. Rock Frame B (frame holding rock above table), made of $3.2-\mathrm{mm}$ (1/8-inch) thick angle iron containing pre-drilled holes or equivalent, with footing welded on each post.

18. Aluminum U-tubing, 3.2-mm (1/8-inch) thick, appropriately sized to fit around rock frame $A$ and instrumentation.

19. Galvanized steel, 2.54-cm (1-inch) wide, long enough to connect rock frame $B$ corner posts, with bolts to connect on aluminum Utubing.

20. Evaporation canopy frame, $6.4-\mathrm{mm}$ (1/4-inch) diameter galvanized steel, canopy dimensions large enough to contain test block and instrumentation. Clear vinyl $0.36 \mathrm{~mm}$ (0.014-inches) thick to cover frame.

21. Clear PVC tubing, $1.59-\mathrm{cm}(5 / 8-$ inch) i.d., $1.91-\mathrm{cm}(3 / 4-$ inch) o.d., enough to reach each sampling port from the evaporation canopy frame. Stoppers to fit tubing, caulking, light-weight washers to fit over PVC tubing.

22. Approximately 2.75 meters ( 9 feet) of $6.4-\mathrm{mm}$ (1/4-inch) i.d. tygon tubing, series $R-3603$ per porous plate.

23. One $6.4-\mathrm{mm}(1 / 4-i n c h)$ o.d. glass " $T$ " per porous plate.

24. One 6.4-mm (1/4-inch) o.d. Nalgene " $\mathrm{T}$ " per porous plate.

25. One $6.4-\mathrm{mm}(1 / 4-\mathrm{inch})$ o.d. Nalgene "Y" per porous plate.

26. Two 6.4-mm (1/4-inch) o.d. Nalgene quick connects per porous plate.

27. One 1-1iter Nalgene, wide-mouth bottle with screw lid or buret with stopper and needle entry per porous plate.

28. One 3.2-mm (1/8-inch) o.d. hard plastic tube per porous plate.

29. One 1-m1 graduated pipet (100 graduations) per porous plate.

30. One ringstand per porous plate. 
31. One ring and one ringstand clamp per ringstand.

32. One meter stick with $\mathrm{mm}$ graduations per two porous plates.

33. Three to four plastic hose clamps per porous plate.

34. One rubber $9 \mathrm{~mm}$ septum.

35. Epoxy.

36. Whatman 42 filter paper, $2.5 \mu \mathrm{m}$ retention rating.

37. Custom-built porous ceramic plates, appropriate size and number to perform the desired experiment.

38. Thin all-threaded rod and couplings for holding porous plate to test block.

\section{Solutions}

1. $\mathrm{CaCl}_{2} 0.001 \mathrm{M}$, deaerated, with $0.1 \mathrm{~g} / \mathrm{L}$ thymol.

\section{$\underline{\text { Procedure }}$}

1. Attach rock frame A to the rock using the all-threaded rods and nuts. The preferred method is to attach the frame while the rock is lying with the fracture parallel to the table. To ensure that the frame load is evenly distributed across the rock face, use brass shim or other non-corroding material to build up low spots on the rock surface. This procedure may require standing the rock up.

2. Using a torque wrench, tighten bolts to a torque not exceeding the initial testing torque. Be sure that the torque is enough to prevent the rock from separating or moving along the fracture.

3. Drill the ports which extend to the fracture surface:

a. Attach the coring bit to the rotating drill assembly and then attach both to the drill press. Connect the hose to the water source.

b. Position the rock so the coring bit is directly over the intended port. Level the rock such that the port is perpendicular to the face parallel with the fracture plane. (Note: This is important because the port should not be sloping. Such sloping may result in preferential flow to one end of the circular cylindrical port should the test solution flow into the cavity during flow and transport tests.) Use wood blocks, planks, shims or other similar implements to accomplish this.

c. Measure the exposed fracture plane to approximate the required depth of drilling. Plan to drill to a "safe" depth, up to 1.0 $\mathrm{cm}$ to $1.5 \mathrm{~cm}$ less than the required depth at faster speeds, e.g., 5 to 7 on the speed dial. Mark the length corresponding to the "safe" depth and required depth on the coring bit with a waterproof marker.

d. Clamp the rock down to avoid movement during drilling.

e. Drill, with the water on, to the "safe" depth while constantly watching for any unusual change in the amount of water flushing cuttings out of the port. A sudden decrease of the water flow usually means the fracture plane has been reached and drilling should cease immediately. Raise the coring bit and watch for any drop in water level in the port for several minutes, e.g., 5 minutes.

f. Beyond the "safe" depth, drill slowly and at no more than twotenths of a centimeter at a time at first, and then one-tenth 
of a centimeter as the required depth is approached. Raise the coring bit and repeat the water-level check in the port each time.

g. Check that the required depth is reached by draining the port of the drilling water which may be laden with cuttings with the tygon tubing and the syringe. Fill the port back up with water and watch for changes in the water level. Also check the port visually with a flashlight, as well as by feel, with a metal wire down the walls of the port for the fracture plane. Examine the rock core drilled for evidence that the fracture plane is reached.

h. After drilling, move the rock to a well-lit area to clean the port. Use a chisel to chip out any rock pieces still attached to the end of the port which may obstruct flow and impede sampling. Flush the port repeatedly with the test solution in a squirt bottle and bail with the test tube brush cleaner to remove cuttings. Turn the rock as necessary to ensure the rock bits and cuttings are flushed out completely. Watch for wetting of the fracture trace. If several ports are drilled, observe the influence of the ports on each other by filling the ports in appropriate patterns.

4. Drill the ports which end in the rock matrix:

a. Repeat Section 3, Steps a and $b$ above. Mark the length corresponding to the required depth of the port on the coring bit with a waterproof marker.

b. Repeat Section 3, Step d above and drill at faster speeds, slowing down when the required depth is approached.

c. Move the rock to a well-lit area to clean the port by chise1ing and flushing with the test solution.

5. Drill holes in which the LVDT posts will be glued as in section 4 .

6. Clean test block using test solution and a soft bristle brush, removing any silt or clay accumulated in the shaping and port drilling.

7. Install the LVDT posts:

a. Use Depend Adhesive only, allowing the posts to be removed at a later date.

b. Apply the activator to the aluminum post set to be placed in the rock. One male and one female post constitute a set. Squeeze in enough adhesive to fill the volume of hole not to be occupied by the post.

c. Quickly position both posts in the holes, and place both the LVDT core and coil into their respective posts, checking the fit of the entire setup.

d. Allow to dry at least 48 hours. The curing time of the glue varies with how much is used and how the posts are installed. Be sure the glue is dry before obtaining an initial LVDT reading.

8. Install the test block and frame $A$ in frame $B$. This is best accomplished by standing the block in its testing position on top of blocks of wood. Stand it such that it is at its testing elevation. Stand up the corner posts of frame B. Cut the aluminum U-tube into short lengths (about $5 \mathrm{~cm}$ long), and drill holes in them to accept the bolts. Assemble the galvanized cross pieces and $U$-tube pieces, and slide them under the bolts holding together frame A. Bolt the crossmembers onto the cornerposts. Ensure that 
there is no slack beneath the frame A bolts. The wood blocks may then be pulled out from underneath the rock.

9. Position the entire setup in its testing location.

10. Attach the vinyl to the evaporation canopy frame. Use Weld On 1909 vinyl adhesive to seal the seams. Leave off the top until the plates have been put on and are operating properly.

11. Cut the sampling port PVC tubing to fit each port, and epoxy a rim on the front end of the tube to hold against the port. Position the PVC tubing in the sampling port and through the canopy. Caulk the tubing-canopy interface, using the washers to provide permanent support. Always keep a stopper in the end of the sampling port access tube.

12. Connect the LVDTs, and tape the bottom of the vinyl canopy to the table, sealing off the airspace inside of the evaporation canopy.

13. Start taking LVDT readings.

14. Make sufficient test solution to start experiment.

15. Set up the Mariotte (constant head) reservoirs:

a. Drill holes on the bottom and top of the reservoir if a nalgene bottle is used and epoxy quick connect fittings, nipple side out. Mark graduations on side of reservoir.

b. Drill hole for air entry tube, insert tube through hole, and epoxy, if necessary, in place.

c. Position Mariotte reservoir on ringstand or on pegboard.

d. Attach 6.4-mm (1/4-inch) i.d. tygon tubing, at least one meter in length, from the bottom nipple of the reservoir, fill reservoir, and clamp off.

16. Set up the flow tube:

a. Break off small end of pipet using a file.

b. Trim one arm and one leg of the nalgene "Y" so that tygon tubing will just fit over it. Connect leg of "Y" to the uncut end of the pipet. Attach a nipple over the cut arm of the "Y", using a small piece of tygon tubing if necessary. Be sure that a syringe with a bent needle can be inserted through the needle into the pipet. Attach the free end of tygon from the reservoir to the free arm of the "Y".

c. Attach a short piece of tygon tubing from the free end of the pipet to the leg of the " $\mathrm{T}$ ". Attach a $20 \mathrm{-cm}$ length of tygon tubing to the upper arm of the "T", clamping off the free end, and connect a long (at least one meter) piece of $6.4-\mathrm{mm}(1 /$ inch) i.d. tygon tubing to the remaining arm.

d. Wire the flow tube assembly to a white backing on the pegboard, with both the nipple and the bubble trap facing up.

17. Set up the porous plate:

a. Cut a piece of Whatman 42 filter paper to fit the ceramic side of the plate.

b. Soak the filter paper in test solution, and then position the filter paper on the bottom of the plate.

c. Place the plate on rock in desired location.

d. Elevating reservoir, fill tubing with test solution. Clamp off tubing, and then connect the free end up to the plate. Use a short piece of tubing and a clamp to clamp off the other end of the plate.

e. Take off clamps blocking flow through tubing and allow test solution to displace the air in the plate backing. Carefully observe the base of each nipple for air bubbles that might be 
caught. A flashlight is helpful for this. This procedure could also be carried out before the plate is positioned on the test block.

f. Once all of the air is out of the plate backing, reclamp the exit tube from the plate.

g. Using thin all-threaded rod and corresponding threaded couplings, tighten the plate to the rock. Use galvanized steel bolted to the top of frame $B$ as a reaction for the tightening rod.

h. Temporarily tape vinyl over the top of the evaporation canopy. Leave no air passages around the edges.

i. Make sure there is good contact between the porous plate (including the filter paper) and the top of the rock. Extra strips of filter paper should be used to fill in low spots on the rock surface.

j. Keep track of all volumes of test solution flowing through the plate. This is especially difficult at the beginning of an experiment due to adjustments that need to be made to flush out bubbles.

k. Begin experiment measurements. 
PROCEDURE 2

\section{PREPARATION OF CALCIUM CHLORIDE TEST SOLUTION}

\section{Equipment}

1. Weighing scale, with accuracy to milligrams (minimum 0.1 gram).

2. Weighing paper.

3. Spatula.

4. Volumetric flask, $2000 \mathrm{~mL}$.

5. Beaker, $2000 \mathrm{~mL}$.

6. Graduated cylinder, $100 \mathrm{~mL}$.

7. Watch glass, 7 in.

8. Stirring plate.

9. Stir bar.

10. Hot plate.

11. Plastic wrap.

12. Rubber bands.

\section{Chemicals}

1. Calcium chloride, $\mathrm{CaCl}_{2} 2 \mathrm{H}_{2} \mathrm{O}$.

2. Thymol chips.

\section{Procedure}

1. If the $0.1 \mathrm{M}$ calcium chloride $\left(\mathrm{CaCl}_{2} \cdot 2 \mathrm{H}_{2} \mathrm{O}, \mathrm{FW}=147.02\right)$ solution is used as stock solution, make up $2000 \mathrm{~mL}$ by weighing out $29.404 \mathrm{~g}$ of the chemical and transfer to a $2000-\mathrm{mL}$ volumetric flask. Fill the flask up to the mark with distilled water. Stir to dissolve crystals completely. Transfer and store in an appropriately labeled container. Proceed to Step 5 below.

2. If the $0.1 \mathrm{M}$ calcium chloride solution is used as test solution, make up $2000 \mathrm{~mL}$ by weighing out $29.110 \mathrm{~g}$ of the chemical and transfer to a $2000-\mathrm{mL}$ volumetric flask. Fill the flask up to the mark with distilled water. Stir to dissolve crystals completely.

3. Transfer the solution to a $2000-\mathrm{mL}$ beaker and add $0.2 \mathrm{~g}$ of thymol to the solution. Cover the beaker with a watch glass and heat the solution to a boil. Continue to boil the solution for an additional two minutes to allow for sufficient deaeration. Watch that the solution does not boil over by lifting the watch glass occasionally. (Note: During boiling, approximately two percent of the water will be lost due to evaporation.)

4. Remove the beaker from the hot plate and replace the watch glass with plastic wrap. Secure the plastic wrap cover with a rubber band. Allow the solution to cool sufficiently, e.g. overnight, before transferring to an appropriately labeled container.

Note: $0.01 \mathrm{M}$ and $0.001 \mathrm{M}$ calcium chloride test solutions are made up using the "serial dilution" method to minimize error.

5. Make up $2000 \mathrm{~mL}$ of $0.01 \mathrm{M}$ calcium chloride solution by measuring out $198.0 \mathrm{~mL}$ of $0.1 \mathrm{M}$ solution and transfer to a $2000-\mathrm{mL}$ volumetric flask. Fill with distilled water and transfer the solution to 
a $2000-\mathrm{mL}$ beaker. Add $0.2 \mathrm{~g}$ of thymol to the solution and follow the procedures detailed in Steps 3 and 4 above.

6. Make up $2000 \mathrm{~mL}$ of $0.001 \mathrm{M}$ calcium chloride solution by measuring out $198.0 \mathrm{~mL}$ of $0.01 \underline{\mathrm{M}}$ solution and transfer to a $2000-\mathrm{mL}$ volumetric flask. Fill with distilled water and transfer the solution to a $2000-\mathrm{mL}$ beaker. Add $0.2 \mathrm{~g}$ of thymol to the solution and follow the procedures detailed in Steps 3 and 4 above. 
PROCEDURE 3

\section{POROUS PLATE CONDUCTANCE MEASUREMENT}

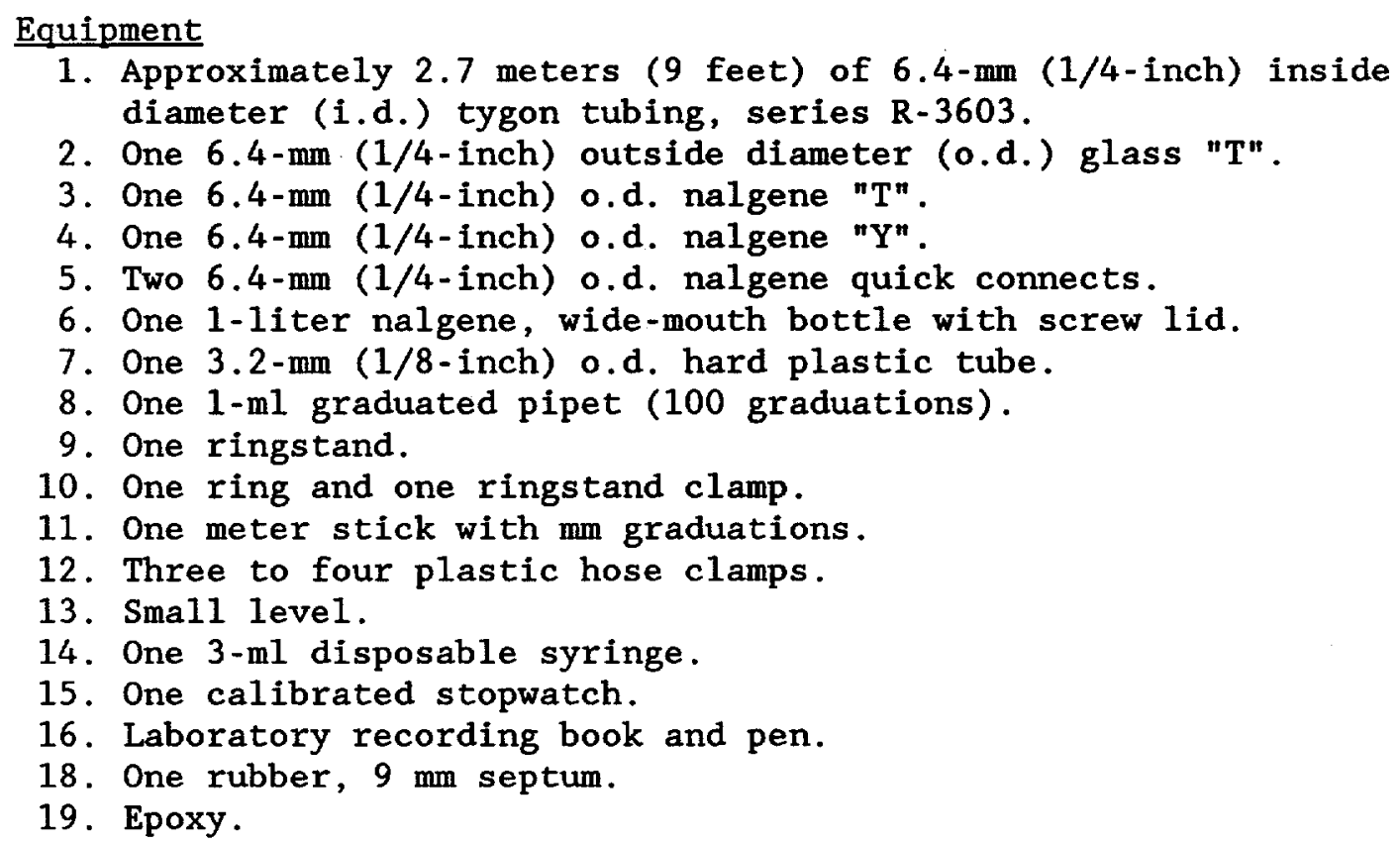

\section{Solution}

1. $\mathrm{CaCl}_{2}, 0.001 \mathrm{M}$, with $0.1 \mathrm{~g} / \mathrm{L}$ thymol.

\section{Procedure}

1. Set up the porous plate, tubing, pipet flow tube, manometer, and Mariotte bottle as described in the procedure for setting up the rock and supporting equipment. Instead of placing the porous plate on a rock block, place it in the plastic tub, ceramic side down. Support the plate from the bottom of the tub with washers or stoppers, and use rock cores to hold the plate down. Make sure that the plate is level. Install a second manometer to the tub, allowing measurement of the pressure head on the bottom of the plate. Add enough solution to the tub to cover the plate.

2. Fill the system with solution, and work out any air bubbles. A flashlight may be helpful in determining if any air is caught in the plate nipples.

3. Set the Mariotte bottle such that the manometer recording the pressure head on the top of the plate is $5 \mathrm{~cm}$ above the manometer recording the pressure head on the bottom of the plate, that is, $\mathrm{dh}_{\mathrm{p}}$ is $5 \mathrm{~cm}$. Allow the flow system to equilibrate. This may be hastened by using the syringe to extract solution through the septum until a bubble is forced from the air entry tube in the Mariotte bottle.

4. Set up an appropriate recording table in the lab book.

5. Record the manometer level(s) prior to injection of the test bubble. 
6. Inject a bubble through the septum into the nalgene "Y". Inject enough air to create a bubble about 1 to $2 \mathrm{ml}$ in the pipet.

7. When the test bubble has passed beyond the injection arm of the nalgene "Y", extract enough air and solution to force an air bubble through the air inlet tube of the Mariotte bottle. This ensures that the pressure in the system is not overly elevated due to the injection of the test bubble. Be sure the test bubble has not been sucked into the injection arm of the nalgene "Y".

8. Start the stopwatch when either the front or the back of the test bubble has crossed the first graduation. Make sure that the entire test bubble is in the pipet when a measurement is being made.

9. Record the times at which the test bubble crosses the $0.2,0.4$, $0.6,0.8$, and $1.0 \mathrm{ml}$ graduations and the manometer heads at these times. This allows analysis of the bubble movement if desired and the ability to calculate a time-weighted average $\mathrm{dh}_{\mathrm{p}}$.

10. Repeat the above procedure at least once at the same $d h_{p}$ after the test bubble has passed into the trap. Variation will occur from reading to reading.

11. After two to three runs have been performed at the lowest $\mathrm{dh}_{\mathrm{p}}$, raise the Mariotte bottle about $5 \mathrm{~cm}$ to $10 \mathrm{~cm}$ and repeat the test. This process should be repeated through $a \mathrm{dh}_{\mathrm{p}}$ of about $50 \mathrm{~cm}$.

\section{Calculations}

1. Average flow rate over $1 \mathrm{~mL}=1.0 \mathrm{~mL}$ divided by the $1.0 \mathrm{~mL}$ time in minutes. Flow rate is then in $\mathrm{cm}^{3} / \mathrm{min}$.

2. Average head at the bottom of the plate is calculated by:

where

$$
\mathrm{H}_{\mathrm{bP}}=\mathrm{dH}-(\mathrm{Q} / \mathrm{C}) \text {, }
$$

$\mathrm{H}_{\mathrm{bp}}=$ average head at the bottom of the plate in $\mathrm{cm}$,

$\mathrm{dH}=$ total head drop across the plate in $\mathrm{cm}$, which can also be expressed as $\mathrm{dh}_{\mathrm{p}}+0.7 \mathrm{~cm}$, also in $\mathrm{cm}$,

$\mathrm{Q}=$ flow rate in $\mathrm{cm}^{3} / \mathrm{min}$, and

$\mathrm{C}=$ plate conductance in $\mathrm{cm}^{2} / \mathrm{min}$. 
PROCEDURE 4

FLOW MEASUREMENT AND HEAD CONTROL

\section{Equipment}

1. Small level.

2. One 3-ml disposable syringe.

3. Calibrated stopwatch.

4. Laboratory recording book and pen.

\section{Solution}

1. $\mathrm{CaCl}_{2}$, either $0.001 \underline{\mathrm{M}}$ or $0.1 \underline{\mathrm{M}}$, saturated with thymol.

\section{Procedure}

Note: See Procedure 1 for setup of test block and instrumentation.

1. Flow measurement using a pipet flow tube:

a. Set up an appropriate recording table in the lab book.

b. Record the manometer level(s) prior to injection of the test bubble. Measure the manometer from the bottom of the plate; it will then read total head at the top of the plate.

c. Inject a bubble through the septum into the nalgene "Y". Inject enough air to create a bubble about 1 to $2 \mathrm{ml}$ in the pipet.

d. When the test bubble has passed beyond the injection arm of the nalgene "Y", extract enough air and solution to force an air bubble through the air inlet tube of the Mariotte bottle. This ensures that the pressure in the system is not overly elevated due to the injection of the test bubble. Be sure the test bubble has not been sucked into the injection arm of the nalgene "Y".

e. Start the stopwatch when either the front or the back of the test bubble has crossed the first graduation. Choose the front or the back of the test bubble to measure such that the test bubble will always be in the pipet when a measurement is being made. Record the time and date when the test was started, that is when the bubble passes the first graduation.

f. Record the times at which the test bubble crosses the 0.2 , $0.4,0.6,0.8$, and $1.0 \mathrm{ml}$ graduations. This allows analysis of the bubble movement if desired.

g. Also record the variations in manometer levels during the test and a rough time-weighted average level.

$h$. If one desires to obtain another flow reading, repeat the above procedure once the test bubble has passed into the trap. Variation will occur from reading to reading.

2. Flow measurement using Mariotte reservoir:

a. Set up an appropriate recording table in the lab book.

b. Record the manometer level(s).

c. Measure the test solution level in the Mariotte reservoir.

d. Record time and date when the above measurements were taken. 
3. Head control:

a. Adjust the Mariotte reservoir up or down according to the desired head to be maintained at the top of the test block.

b. When additional test solution is added to the reservoir, first record the test solution level, clamp off the exit tube from the reservoir, fill the bottle or buret, unclamp the exit tube, and reestablish equilibrium by extracting solution through the septum until an air bubble enters the reservoir through the air entry tube. Make sure to record the amount of solution extracted and take it into account when using the second method of flow rate measurement.

\section{Calculations}

1. Flow measurement when pipet flow tube is used:

a. Average flow rate over $1 \mathrm{~mL}=1.0 \mathrm{~mL}$ divided by the $1.0 \mathrm{~mL}$ time in minutes. Flow rate is then in $\mathrm{cm}^{3} / \mathrm{min}$.

$b$. Average pressure head at the bottom of the plate is calculated by:

where

$$
h_{p}=\phi-(Q / C) \text {, }
$$

$h_{p}=$ average head at the bottom of the plate in $\mathrm{cm}$;

$\phi=$ average total head at the top of the plate in $\mathrm{cm}$, if measured from the bottom of the plate;

$\mathrm{Q}=$ flow rate in $\mathrm{cm}^{3} / \mathrm{min}$;

$\mathrm{C}=$ plate conductance in $\mathrm{cm}^{2} / \mathrm{min}$.

2. Flow measurement when Mariotte reservoir is used:

a. Average flow rate in the time period since the previous Mariotte reservoir level was taken is just the drop in reservoir level in $\mathrm{cm}^{3}$ divided by the time between readings in minutes.

b. Calculate the average head at the bottom of the plate as in section $1 b$ above.

c. Use an average of the $\phi^{\prime}$ 's measured at the two measuring times used in the calculation. 
PROCEDURE 5

\section{PRESSURE TRANSDUGER CALIBRATION}

\section{Equipment}

1. MICRO SWITCH 140PC series or 160PC series pressure transducer.

2. Water manometer and mercury manometer.

3. Vacuum pump.

4. About 2.7 meters ( 9 feet) of $6.4-\mathrm{mm}(1 / 4-\mathrm{inch}$ ) inside diameter (i.d.) tygon, or similar, tubing.

5. About 1.22 meters (4 feet) of $4.8-\mathrm{mm}(3 / 16-\mathrm{inch})$ i.d. tygon, or similar tubing.

6. One 6.4-mm (1/4-inch) outside diameter (o.d.) nalgene " $T^{n}$.

7. Three $6.4-\mathrm{mm}(1 / 4-\mathrm{inch})$ i.d. hose clamps.

8. One $6.4-\mathrm{mm}$ (1/4-inch) i.d. quick-connect.

9. One 8-volt regulated power supply.

10. One voltmeter.

11. One ribbed tygon tubing connector (the type used to connect tygon tubing to swagelock fittings).

\section{Procedure}

1. Divide the $6.4-\mathrm{mm}(1 / 4-\mathrm{inch})$ i.d. tubing into two pieces, and connect each piece onto an arm of the nalgene " $T$ " with a hose clamp. Using the quick-connect, attach the remaining end of one of the pieces of tubing to the vacuum pump. Fit the free end of the second section of tubing onto the water manometer, preferably with a water trap in the line.

2. Connect the $4.8-\mathrm{mm}(3 / 16-\mathrm{inch})$ i.d. tubing onto the remaining arm of the nalgene " $\mathrm{T}$ " with a hose clamp. Fit the other end of the $4.8-\mathrm{mm}(3 / 16-i n c h)$ i.d. tubing into the swagelock connector and then over one of the two pressure ports. Attach the tubing to the port designated to be the low pressure side of the chip. See the instruction sheet enclosed with the transducer or the MICRO SWITCH catalog \#15, issue 2 .

3. Hook up the regulated power supply and the voltmeter to the pressure transducer in the configuration specified in the instruction sheet. Note: If correct input and output connections are not made, the unit may be damaged. It is recommended that any connecting wires not be soldered directly to the leads protruding from the transducer, but that they be soldered to a removable multi-prong plate that can be held onto the transducer with a rubber band.

4. Prior to applying a partial vacuum to the transducer, turn on the power supply and voltmeter. Verify that the input to the transducer is 8 volts dc. Measure the voltage output with no pressure differential across the chip, that is, between the two pressure ports. This reading should be stable to at least two or three decimal points. Since this reading is very important, take it a number of times during the calibration.

5. Using the vacuum pump, carefully apply a small suction to the transducer, and measure the output when stable. Increase the suction slightly, and measure the output again. Continue 
this process until the limit of either the manometer or the transducer has been reached. The upper limit of the linear output of the 162PC01D transducer is 27.68 inches of water pressure across the chip. Under no circumstances should greater than 5 psi differential pressure be applied across the chip. Therefore, only the water manometer should be used with this unit. The upper limit of the linear output of the 142PC15D unit is 15 psi differential pressure. Twenty psi differential pressure should never be exceeded using this transducer. Once the limit of the water manometer has been reached with the 142PC15D transducer, the mercury manometer should be used to apply differential pressures up to $15 \mathrm{psi}$.

6. Repeat step 5 at least once.

\section{Calculations}

1. Determine a mean zero-pressure voltage (zpv) for readings taken when no partial vacuum was applied across the chip, i.e., $P_{1}-P_{2}=$ 0 .

2. Determine a "corrected" output voltage by subtracting this value from each of the output voltages obtained when suctions were applied to the transducer.

3. Calculate a mean pressure/corrected output voltage ratio ( $p / v)$.

4. To determine the pressure represented by a given output voltage: where$$
\text { Pressure }\left(\mathrm{cm} \mathrm{H} \mathrm{H}_{2} \mathrm{O}\right)=(\mathrm{ov}-\mathrm{zpv})(\mathrm{p} / \mathrm{v}) \text {, }
$$

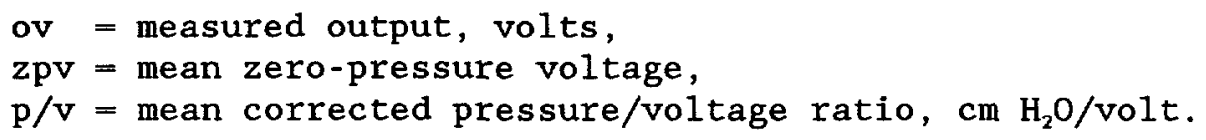




\title{
PROCEDURE 6
}

\author{
MICROTENSIOMETER CONSTRUCTION, ASSEMBLY, AND USE
}

\section{Equipment}

1. One Soilmoisture Equipment 1-bar porous ceramic cup, $10.2 \mathrm{~cm}$ (4 inches) in length, 11.1-mm (7/16-inch) outside diameter (o.d.), and $7.1-\mathrm{mm}(9 / 32-i n c h)$ inside diameter (i.d.).

2. One Whatman pure cotton cellulose extraction thimble, $10 \mathrm{~mm}$ i.d., either single-wall or double-wall thickness.

3. One two foot length of $3.2-\mathrm{mm}(1 / 8-\mathrm{inch}) 0 . \mathrm{d}$. stainless steel tubing.

4. One $6.4-\mathrm{mm}$ (1/4-inch) length piece of $12.7-\mathrm{mm}(1 / 2-$ inch) diameter solid aluminum rod.

5. One \#1 solid rubber stopper with a 3.2 -mm (1/8-inch) diameter hole drilled through the center of the stopper, lengthwise $(1 / 8$-inch diameter drill bit used).

6. Short length of $\# 22$ copper wire.

7. Epoxy.

8. One connection assembly, consisting of a $2.9-\mathrm{cm}(1-1 / 8-$ inch $)$ length of $6.4-\mathrm{mm}$ (1/4-inch) i.d. vacuum hose, two small hose clamps, one $7.9-\mathrm{mm}$ ( $5 / 16$-inch) i.d. ribbed, swagelock tubing coupling, and various short lengths of $2.4-\mathrm{mm}(3 / 32-\mathrm{inch})$ i.d. and $3.2-\mathrm{mm}(1 / 8-$ inch) i.d. tygon tubing.

9. One calibrated MICRO SWITCH 140PC series or 160PC series pressure transducer.

10. One saturation assembly, consisting of a saturation chamber, pressure gauge, vacuum pump and delivery hoses.

11. One disposable $3 \mathrm{~mm}$ syringe.

12. 8-volt regulated power supply with attached, precise voltage regulator.

13. Hewlett Packard (HP) 41CV calculator, with ROMPAC, HPIL, and time modules or sensitive voltmeter.

14. HP 3421 Data Acquisition unit or sensitive voltmeter.

15. Appropriate lengths of shielded \#18-\#22 wire.

16. One constant head reservoir/flow tube setup (see Procedure 1).

17. One plywood evaporation control box, large enough to contain one $20.2 \mathrm{~cm}$ by $8.6 \mathrm{~cm}$ porous ceramic plate. Two access tubes placed $10 \mathrm{~cm}$ apart.

18. One porous ceramic plate, $8.6 \mathrm{~cm}$ by $20.2 \mathrm{~cm}$, saturated with test solution.

If the pressure transducer requires that the high side of the chip be the wet side, the following will also be needed:

19. Water manometer and mercury manometer.

20. Hand-operated vacuum pump.

21. About 2.7 meters ( 9 feet) of $6.4-\mathrm{mm}(1 / 4-\mathrm{inch}$ ) inside diameter (i.d.) tygon, or similar, tubing.

22. About 1.22 meters (4 feet) of $4.8-\mathrm{mm}$ (3/16-inch) i.d. tygon, or similar tubing.

23. One 6.4-mm (1/4-inch) outside diameter (o.d.) nalgene " $T$ ".

24. Three 6.4- mm (1/4-inch) i.d. hose clamps. 
25. One 6.4-mm (1/4-inch) i.d. quick-connect.

26. One ribbed tygon tubing connector (the type used to connect tygon tubing to swagelock fittings).

\section{Reagents}

1. Sufficient amount of deaerated, distilled water to cover the porous cup in the saturation chamber.

2. Test solution: $10^{-3} \underline{\mathrm{M}} \mathrm{CaCl}_{2}$, with $0.1 \mathrm{~g} / \mathrm{L}$ thymol.

\section{Procedure}

1. Microtensiometer construction and assembly:

a. Cut enough of the stainless steel tubing to allow the tensiometer to reach the fracture from the outside of the evaporation canopy.

b. Drill a 3.2-mm (1/8-inch) diameter hole through the center of the flat edge of the aluminum rod.

c. Epoxy the stainless steel tube through the hole in the aluminum rod with one end of the steel tube flush with edge of the aluminum rod.

d. Cut the rounded, 6.4-mm (1/4-inch) end of the porous ceramic cup off with a hacksaw and epoxy the remaining cup onto the flush edge of the aluminum rod.

e. Fit the \#1 stopper over the open end of the stainless steel tubing.

$f$. Cut the cotton cellulose extraction thimble to fit snugly over the porous cup, and tie the thimble onto the cup with a small piece of \#22 copper wire.

g. Assemble the vacuum tubing connector that will join the stainless steel tube to the pressure transducer by inserting the ribbed, swagelock tubing connector into the vacuum tubing and sliding this end of the connector over the pressure transducer. Insert hose clamps over the free end of the vacuum tubing.

h. Place the tensiometer into the saturation chamber, and evacuate the chamber for at least 24 hours.

$i$. Turn off the vacuum pump, and introduce the deaerated, distilled water into the chamber. Cover at least the entire cup and aluminum rod of the tensiometer. Let the tensiometer fill with the distilled water for at least 8 hours.

j. If the tensiometer was not completely covered by distilled water in the saturation chamber, fill the remainder of the tensiometer stem by applying a suction with a hand vacuum pump to the open end of the stainless steel tubing.

1. Using a syringe, fill the pressure transducer port and vacuum tubing connector with deaerated, distilled water.

m. Gently join the vacuum tubing connector and the open end of the stainless steel tubing. Tighten the hose clamps on both ends of the vacuum tubing connector.

$\mathrm{n}$. Store the assembled tensiometer under deaerated, distilled water or in the rock, against the fracture.

2. Microtensiometer calibration: 
a. Assemble the porous ceramic plate in the evaporation control box with the ceramic side of the plate facing the access tubes.

b. Fill the Mariotte reservoir and tubing, connecting up the plate to the tubing. Bleed all air from the system as described in Procedure 1.

c. Use the microtensiometer in the access ports as described in sections 3 and 4 below. Take readings from both the upper and lower ports at various applied heads.

d. Prepare a calibration curve or develop a correction factor to allow use of the microtensiometer in the test blocks.

3. To use the microtensiometer if the low pressure side of the transducer chip is the wet side:

a. Hook up the regulated power supply and the voltmeter to the pressure transducer in the configuration specified in the instruction sheet.

Note: If correct input and output connections are not made, the unit may be damaged. If the HP system is used, be sure to turn off the calculator when the data acquisition unit is being hooked up. The system is rather delicate.

It is recommended that any connecting wires not be soldered directly to the leads protruding from the transducer, but that they be soldered to a removable multi-prong plate that can be held onto the transducer with a rubber band.

b. Place the tensiometer assembly in the access tube leading to the sampling port in which a reading is desired. Adjust the stopper such that the tip of the tensiometer lies against the back end of the sampling port.

c. Monitor the pressure transducer output until a stable reading is obtained. The microtensiometer may take a while to equilibrate, especially if much water is moving in or out through the porous cup. Apply the correction factor or calibration curve obtained in section 2 to obtain the suction in the sampling port.

d. Repeat steps a through d for additional sampling ports.

4. To use the microtensiometer if the low pressure side of the transducer chip is the dry side:

a. Divide the 6.4-mm (1/4-inch) i.d. tubing into two pieces, and connect each piece onto an arm of the nalgene " $T$ " with a hose clamp. Using the quick-connect, attach the remaining end of one of the pieces of tubing to the vacuum pump. Fit the free end of the second section of tubing onto the water manometer, preferably with a water trap in the line.

b. Connect the $4.8-\mathrm{mm}(3 / 16-$ inch) i.d. tubing onto the remaining arm of the nalgene " $\mathrm{T}$ " with a hose clamp. Fit the other end of the $4.8-\mathrm{mm}(3 / 16-\mathrm{inch})$ i.d. tubing into the swagelock connector and then over one of the two pressure ports. Attach the tubing to the port designated to be the low pressure side of the chip. See the instruction sheet enclosed with the transducer or the MICRO SWITCH catalog number 15, issue 2 .

c. Using the vacuum pump apply a partial vacuum to the transducer, and proceed as described in section $3 a$ through $3 e$. Remember, the upper limit of the linear output of the 162PC01D 
transducer is 27.68 inches of water pressure across the chip. Under no circumstances should greater than 5 psi differential pressure be applied across the chip. Therefore, only the water manometer should be used with this unit. The upper limit of the linear output of the 142PC15D unit is 15 psi differential pressure. Twenty psi differential pressure should never be exceeded using this transducer. Once the limit of the water manometer has been reached with the 142PC15D transducer, the mercury manometer should be used to apply differential pressures up to $15 \mathrm{psi}$. 
PROCEDURE 7

LVDT CALIBRATION

Equipment

1. TRANS*TEK 0242-0000 linear variable differential transformer (LVDT).

2. 15-volt regulated power supply with attached, precise voltage regulator.

3. Hewlett Packard (HP) $41 \mathrm{CV}$ calculator, with ROMPAC, HPIL, and time modules or sensitive voltmeter.

4. HP 3421 Data Acquisition unit or sensitive voltmeter.

5. Appropriate lengths of shielded \#18-\#22 wire.

6. Partially welded or welded tuff sample with two $1.91 \mathrm{~cm}(3 / 4-$ inch) holes drilled $7.62 \mathrm{~cm}$ ( 3 inches) apart and about $5.08 \mathrm{~cm}$ deep.

7. Mitutoyo $0-25 \mathrm{~mm}$ micrometer with hole tapped in end to receive the threaded end of the LVDT core.

8. Two aluminum LVDT holders with female heads.

9. One $1.91 \mathrm{~cm}(3 / 4$-inch) outside diameter (o.d.) aluminum ring. Inside diameter (i.d.) should be $1.20 \mathrm{~cm}$ ( 0.473 inches) to fit over the front end of the micrometer.

10. Loctite brand Depend Adhesive.

11. Blowtorch if aluminum LVDT holders are to be removed from rock.

\section{Procedure}

1. At least two days prior to calibration, glue the LVDT holders into the rock. Ensure that the LVDT coil will line up in the holders.

2. Slip the aluminum ring over the front end of the micrometer, and screw the LVDT core into the micrometer.

3. Slip the ring and micrometer into one of the LVDT holders, and tighten the screws to secure the assembly. Advance the micrometer to about half of its length.

4. Place the LVDT coil into the other LVDT holder, making sure that the core slides freely inside of the coil. Do not yet tighten the screws on the coil holder.

5. Connect the electronics according the TRANS*TEK instruction sheet and the instruction sheets to the HP system or the voltmeter. If the HP system is used to measure voltage, be sure that the calculator is off prior to hooking up the calculator to the data acquisition unit. The system is rather delicate.

6. Connect the power supply according to the TRANS*TEK instructions.

7. Find the zero point (the point at which the core is evenly spaced between the two output coils of the coil assembly, giving a zero output) by gently sliding the coil towards or away from the micrometer. Once this point has been found, tighten the screws holding the coil.

8. Record the exact voltage reading at the zero point.

9. Since the TRANS*TEK $0242-0000$ has a working range of $0.635 \mathrm{~cm}$ ( 0.25 inches) on either side of the zero point, take 6 readings on each side of the zero point, each reading $1 \mathrm{~mm}$ farther out from the last. Advance the micrometer, take a reading, and record the exact values of both the voltage and the micrometer distance. 
Occasionally, check the input voltage to ensure stability of the input.

\section{Calculations}

1. Prepare a graph of the results by plotting the micrometer readings $(y$-axis) versus the output voltage ( $x$-axis). The output should fall along a straight line if the LVDT is working correctly. Using the least squares method, determine the slope of the line.

2 . Use the slope, in $\mathrm{mm} / \mathrm{volt}$ or micrometers/mvolt, to interpret the relative movement of the LVDT during actual use. 


\section{PROCEDURE 8}

\section{CALIBRATION OF CHLORIDE ION-SELECTIVE ELECTRODE}

\section{Equipment/Material}

1. Weighing Scale, with accuracy to $0.001 \mathrm{~g}$ (minimum $0.01 \mathrm{~g}$ )

2. Weighing paper

3. Spatula

4. Volumetric flask, $250 \mathrm{~mL}$

5. Volumetric flask, $50 \mathrm{~mL}$

6. Graduated cylinder, $25 \mathrm{~mL}$ or $50 \mathrm{~mL}$

7. Erlenmeyer flasks (2), $125 \mathrm{~mL}$ and $250 \mathrm{~mL}$

8. Measuring pipet, $5 \times 1 / 10 \mathrm{~mL}$

9. Beakers, $50 \mathrm{~mL}(8)$ and $100 \mathrm{~mL}(6)$

10. Glass bottles, $250 \mathrm{~mL}$ (8) and $30 \mathrm{~mL}(2)$

11. Stirring plate

12. Stir bar

13. Hach Kit titrator

14. HP-41CV calculator

15. $\mathrm{pH} /$ Nolt meter, with expanded scale for $\mathrm{mV}$ measurement

16. Ion-selective electrode, chloride

17. Reference electrode, $\mathrm{Ag} / \mathrm{AgCl}$, double-junction

18. Forcep, 8 in.

19. Parafilm, flexible thermoplastic material, 4"

20. Kimwipe tissue

21. Whatman filter paper, no. 42

22. Semi-log paper, 4 cycles $\times 10$ to the inch

23. French Curve

\section{Reagents/Chemicals}

1. Calcium chloride, $\mathrm{CaCl}_{2} \cdot 2 \mathrm{H}_{2} \mathrm{O}$, reagent grade

2. Sodium nitrite, $\mathrm{NaNO}_{2}$, reagent grade

3. Hach Kit titrants, $0.0800 \mathrm{M}$ or $0.800 \mathrm{M}$ EDTA for total hardness (or calcium), and $0.2256 \underline{\mathrm{N}}$ or $2.256 \underline{\mathrm{N} \mathrm{Hg}}\left(\mathrm{NO}_{3}\right)_{2}$ for chloride

4. Hardness indicator, $10 \mathrm{~g} / \mathrm{L} \mathrm{CaCO}_{3}$

5. Potassium hydroxide, $\mathrm{KOH}$, standard solution

6. Calver 2 calcium-indicator powder pillow

7. Diphenylcarbazone reagent powder pillow

\section{Procedure}

\section{A. Preparation of Standard Solutions}

1. Prepare $250 \mathrm{~mL}$ of $0.5 \mathrm{M}$ calcium chloride $\left(\mathrm{CaCl}_{2} \cdot 2 \mathrm{H}_{2} \mathrm{O}, \mathrm{FW}=147.02\right.$, assay $=74.68$ ) solution by weighing out $18.5975 \mathrm{~g}$ of the chemical, and transfer to a $250-\mathrm{mL}$ volumetric flask. Fill the flask up to the mark with previously deaerated distilled water saturated with thymol. Stir until the crystals are completely dissolved. (Note: potential difference measurements are not affected by degree of aeration of distilled water.) 
2. Dilute the $0.5 \underline{M}$ solution to $0.0001 \underline{M}$, in serial fashion, to obtain eight standard solutions of half-decade concentration difference, i.e., $0.5 \underline{M}, 0.1 \underline{M}, 0.05 \underline{M}, 0.01 \underline{M}, 0.005 \underline{M}, 0.001 \underline{M}$, $0.0005 \mathrm{M}$, and $0.0001 \mathrm{M}$. (Note: the dilution factor is $4: 1$ from $0.5 \underline{M}$ to $0.1 \underline{M}$, and $1: 1$ from $0.1 \underline{M}$ to $0.05 \underline{M}$, and so on to 0.0001 $\underline{M})$. The dilution can also be done by first making $0.1 \underline{M}$ solution from the $0.5 \underline{M}$ solution, then dilute to obtain the whole-versus half-decade standard solutions separately. (Note: the dilution factor is $9: 1$ from $0.5 \mathrm{M}$ to $0.05 \mathrm{M}$, as is $0.1 \mathrm{M}$ to $0.01 \mathrm{M}$.) Store the standard solutions in separately labeled glass 250 -mL bottles.

3 . Check independently the concentration of the standard solutions by titrating with the appropriate Hach Kit titrants, $0.0800 \mathrm{M}$ or $0.800 \mathrm{M}$ EDTA for total hardness (or calcium), and $0.2256 \underline{\mathrm{N}}$ or $0.256 \mathrm{~N} \mathrm{Hg}\left(\mathrm{NO}_{3}\right)_{2}$ for chloride. Confirm the concentration of the standard solutions with more sophisticated analytical techniques if possible.

\section{B. Preparation of Ionic Strength Buffer Solution}

1. Prepare $50 \mathrm{~mL}$ of $5 \underline{\mathrm{M}}$ sodium nitrite $\left(\mathrm{NaNO}_{2}, \mathrm{FW}=69.00\right.$, assay $=$ 97.78 ) by weighing out $17.656 \mathrm{~g}$ of the chemical, and transfer to a 50-mL volumetric flask. Fill the flask to the mark with distilled water. Stir until the crystals dissolve completely.

2. Transfer the ionic strength buffer solution to two $30-\mathrm{mL}$ glass bottles.

\section{Calibration Without Ionic Strength Buffer Solution}

1. Pour out $30 \mathrm{~mL}$ to $35 \mathrm{~mL}$ of each of the eight standard solutions into the $50-\mathrm{mL}$ beakers, and cover with Parafilm to minimize evaporation.

2. First calibrate the electrodes in an "aqueous" environment by immersing them in the standard solutions always moving from the least to the most concentrated, i.e., $10^{-4} \underline{M}$ to $0.5 \mathrm{M}$. Agitate the beaker containing standard solution slightly, and wait for the reading to stabilize before recording the potential value (in $\mathrm{mV}$ ), or range of values. Record readings from both the $\mathrm{pH} / \mathrm{volt}$ meter on expanded scale, and the HP-41CV. Keep the electrodes approximately one centimeter apart. (Note: the potential values read off of the HP is 1/70-th that of the actual values. The more dilute the standard solution, the longer the time to stabilization: up to 30 seconds and more for $10^{-4} \mathrm{M}$, yet almost instantaneously for 0.1 $\underline{M}$ and $0.5 \underline{M}$.$) Rinse off both electrodes with distilled water, and$ wipe dry with a Kimwipe before proceeding to the next standard solution.

3. Soak the reference and chloride electrodes in distilled water after the $0.5 \underline{M}$ measurement for a minimum of 15 to 30 minutes or until the potential reading in distilled water stabilizes at approximately $315 \mathrm{mV}$ to $325 \mathrm{mV}$.

4. Then calibrate the electrodes in a "filter paper" environment by wetting a precut piece of whatman no. 42 filter paper (approximately $1.0 \mathrm{~cm} \times 0.5 \mathrm{~cm}$ ) by dipping it in the standard solution with a forcep. Partially dry the filter paper with a paper towel until no solution can stream down either face. Touch the electrodes to the filter paper, and record the potential reading after 
approximately 5 to 10 seconds. Move the chloride electrode to a different spot on the filter paper, and repeat the measurement. Record the range of potential readings observed.

Note: the spread of the potassium nitrate bridge solution due to outward diffusion from the reference electrode will cause erroneously low potential, i.e., high concentration, readings when the chloride electrode comes into contact with the potassium nitrate. Therefore, place the electrodes as far apart as possible over the filter paper as the time to make potential readings is limited.

5. Plot the potential difference against the $10 \mathrm{~g}$ of concentration. (Note: The change in potential difference per decade concentration in the "aqueous" environment is linear from approximately $1.00 \mathrm{x}$ $10^{-4} \underline{\mathrm{M}}$ to $1.00 \times 10^{-1} \mathrm{M}$ at $58 \mathrm{mV} /$ decade. The calibration curve exhibits nonlinearity at concentrations greater than approximately $1.00 \times 10^{-1} \mathrm{M}$. In the "filter paper" environment, the calibration curve is nonlinear except in the range between $1.00 \times 10^{-2} \underline{M}$ to $1.00 \times 10^{-1} \underline{\mathrm{M}}$. In general, the change in potential difference in the aqueous environment is greater than the change in the filter paper environment. In other words the calibration curve is flatter for filter paper.)

\section{Calibration With Ionic Strength Buffer Solution}

1. Measure out $50.0 \mathrm{~mL}$ of each of the six most dilute standard solutions, i.e., all except the $0.1 \underline{M}$ and $0.5 \underline{M}$ solutions, and pour into the $100-\mathrm{mL}$ beakers. Cover the beakers with Parafilm to minimize evaporation.

2. Measure out the specified volumes of buffer solution as detailed below, and add to the six standard solutions:

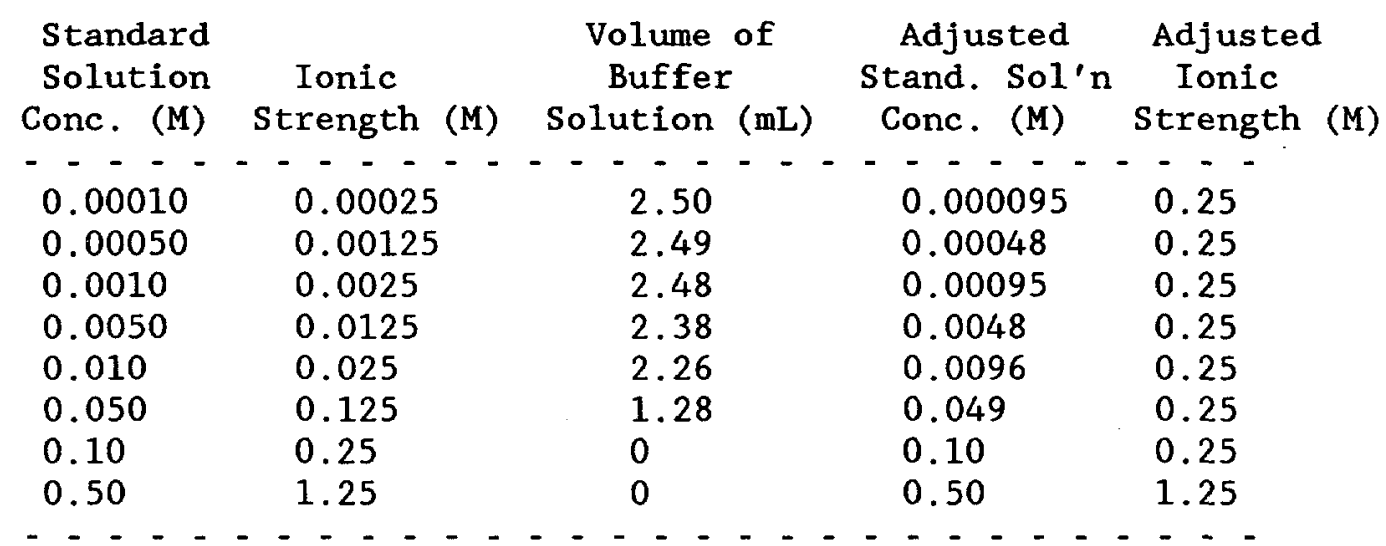

Ionic strength, I, for $\mathrm{N}$ specie is calculated as follows:

$$
I=0.5 \sum_{i=1}^{N} z_{1}{ }^{2} c_{i}
$$

where $z_{1}$ is charge of species $i$, dimensionless, and $c_{i}$ is concentration of species $i$, $\underline{M}$. Because the test solution concentrations 
are $0.001 \underline{\mathrm{M}}$ and $0.1 \underline{\mathrm{M}}$, the standard solutions are adjusted to the ionic strength of the more concentrated solution of the two.

3. Calibrate both in the "aqueous," and "filter paper" environments as specified above in Section $C$, steps 2,3 and 4 . (Note: With buffer solution added, the time to achieve a stable potential reading decreases for the more dilute standard solutions.)

4. Plot the potential difference against the concentration as in Section C, Step 5 .

Note: The calibration curve is exactly the same in the "aqueous" environment. However, the potential readings in the "filter paper" environment increases at more dilute concentrations, i.e., from approximately $0.05 \underline{M}$ to $0.0001 \underline{M}$, with the remainder of the curve the same.

The calibration data are presented in the table below. 
Calibration data for chloride ion-selective

electrodes measuring potential difference (mV) versus chloride concentration $(\underline{M})$.

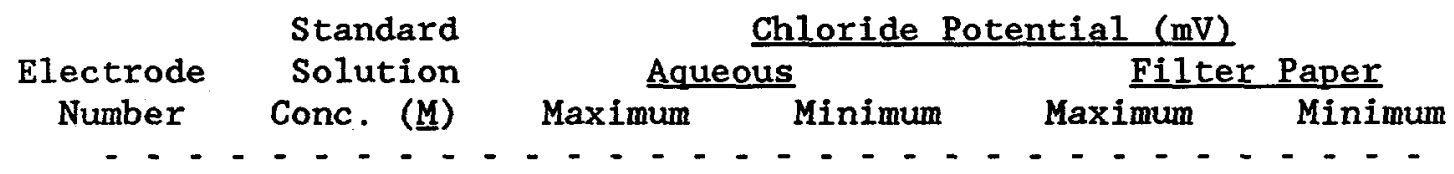

Without Ionic Strength Buffer Solution

$\begin{array}{clrrrr}\text { CL-1 } & 0.000095 & 255 & 247 & 203 & 200 \\ & 0.00050 & 213 & 207 & 180 & 180 \\ & & & & & \\ & 0.00095 & 205 & 196 & 175 & 168 \\ & 0.0050 & 153 & 147 & 148 & 145 \\ & 0.0095 & 138 & 135 & 138 & 138 \\ & 0.050 & 95 & 95 & 100 & 100 \\ & 0.095 & 83 & 79 & 83 & 83 \\ & 0.50 & 53 & 51 & 48 & 48 \\ \text { CL-2 } & 0.000095 & 255 & 245 & 198 & 200 \\ & 0.00050 & 213 & 210 & 185 & 183 \\ & 0.00095 & 200 & 198 & 178 & 178 \\ & 0.0050 & 153 & 150 & 143 & 143 \\ & 0.0095 & 140 & 136 & 135 & 133 \\ & 0.050 & 97 & 95 & 95 & 95 \\ & 0.095 & 82 & 77 & 78 & 78 \\ & 0.50 & 52 & 51 & 53 & 53 \\ & & & & & \\ \text { CL-3 } & 0.000095 & 258 & 247 & 209 & 202 \\ & 0.00050 & 207 & 202 & 190 & 182 \\ & 0.00095 & 198 & 189 & 182 & 175 \\ & 0.0050 & 149 & 144 & 145 & 134 \\ & 0.0095 & 139 & 133 & 136 & 123 \\ & 0.050 & 96 & 91 & 95 & 86 \\ & 0.095 & 80 & 77 & 80 & 71 \\ & 0.50 & 53 & 47 & 50 & 43\end{array}$

With Ionic Strength Buffer Solution (1)

$\begin{array}{rlrrrr}\text { CL-3 } & 0.000095 & 247 & 244 & 215 & 207 \\ & 0.00050 & 207 & 203 & 196 & 195 \\ 0.00095 & 196 & 193 & 186 & 185 \\ 0.0050 & 147 & 146 & 144 & 141 \\ 0.0095 & 137 & 136 & 133 & 132 \\ 0.050 & 92 & 89 & 87 & 86 \\ 0.095 & 80 & 77 & 80 & 71 \\ 0.50 & 53 & 47 & 50 & 43\end{array}$

(1) Electrodes CL-1 and CL-2 were not calibrated with ionic strength buffer solution because they have exceeded their useful life. 


\section{PROCEDURE 9}

\section{MEASUREMENT OF POTENTIAL DIFFERENCES AND SAMPLE COLLECTION}

\section{Equipment/Material}

1. Ion-selective electrode, chloride

2. Reference electrode, $\mathrm{Ag} / \mathrm{AgCl}$, double-junction

3. Bridge solution syringe dispenser, potassium nitrate

4. Reference junction, slip-on

5. $\mathrm{pH} /$ Volt meter, with expanded scale for $\mathrm{mV}$ measurement

6. HP-41CV calculator

7. Beaker, $30 \mathrm{~mL}$

8. Forceps, 8 in.

9. Parafilm, flexible thermoplastic material, 4"

10. Kimwipe tissue

11. Whatman filter paper, no. 42

\section{Procedure}

\section{A. Operating the Reference and Ion-Selective Electrodes}

NOTE: Ensure the microelectrodes are operating normally before making potential difference measurements. Refer to the operations manual.

1. Prior to testing, soak both the reference and chloride electrodes in distilled water for 15 minutes. Soak the reference electrode such that the whole blue "slip-on" reference junction is completely immersed. Check that no air bubbles are trapped in the narrow teflon tubing. If air bubbles are present, tap the tubing to purge the bubbles into the bottom compartment containing potassium nitrate $\left(\mathrm{KNO}_{3}\right)$ bridge solution. Keep the bottom compartment filled with bridge solution. If the reference junction appears fouled, replace it with a new junction. Soak the junction in distilled water for 15 minutes before use.

2. During testing, always soak both the reference and chloride electrodes in distilled water when measurements are not being made, even between measurements. Change the beaker of distilled water periodically as the potential reading decreases, i.e., indicating an apparent increase in chloride concentration. This occurs due to outward diffusion of the bridge solution into the beaker of distilled water. (Note: This drifting is natural but check, as necessary, the operation of the electrodes by calibrating against standard solutions and comparing the potential readings with the calibration curves.)

3. After each potential measurement, rinse off both electrode tips with distilled water and wipe dry with a Kimwipe before attempting the next measurement.

4. After testing, soak the reference electrode in distilled water. Leave the chloride electrode to air-dry. Store the electrodes in their shipping boxes if testing is not done on a regular basis. 


\section{B. Sampling with and Measuring Potential Differences off Filter Paper}

1. Cut the filter paper, Whatman no. 42, into pieces approximately $1.0 \mathrm{~cm} \times 0.5 \mathrm{~cm}$.

Note: Wear gloves if handling is not hampered. Otherwise, ensure hands are washed before handling and minimize the handling time.

2. Crimp the filter paper into halves, and then quarters. Affix to the end of the forceps, and clamp down on the filter paper with the aid of a wire wrapped around the perimeter of the forcep.

3. Insert the forcep into the sampling port, and press against the exposed fracture face, or the rock matrix. Sample until the filter paper is sufficiently wet for a reliable potential difference measurement.

Note: The length of sampling time varies depending on the moisture content of the sampling surface. At near-saturated conditions in the fracture, the optimum sampling time is approximately two minutes. Sampling time in the rock matrix ports varies from two minutes to instantaneous.

4. Remove the filter paper, and place it on a piece of Parafilm. Dab off any excess test solution from the filter paper if necessary.

Note: This ensures that the potential readings are for a "filter paper" environment, and not an "aqueous" environment.

5. Touch the electrodes to the filter paper, and note the potential reading(s) after approximately 5 to 10 seconds. Move the chloride electrode to a different spot on the filter paper, and repeat the measurement. Record the range of potential readings observed.

Note: the spread of the potassium nitrate bridge solution due to outward diffusion from the reference electrode will cause erroneously low potential, i.e., high concentration, readings when the chloride electrode comes into contact with the potassium nitrate. Therefore, place the electrodes as far apart as possible. The time to make potential readings is limited. 
PROGEDURE 10

\section{DETERMINATION OF EFFECT OF SUCTION ON POTENTIAL DIFFERENCE MEASUREMENTS}

\section{Equipment}

1. Buchner funnel with $50 \mathrm{kPa}$ ceramic porous plate, $150 \mathrm{~mL}$

2. Buret, straight teflon stopcock, $25 \mathrm{~mL}$

3. Ringstands (2)

4. Iron ring, 3-in. with support clamp

5. Buret clamp holder

6. Leve1

7. Flat top bolts (2)

8. Tygon tubing, $1 / 8-i n$. ID $\times 1 / 4-i n$. OD

9. Acrylic tubing, 5/8-in. ID $\times 3 / 4-i n .08$

10. Stoppers, no. 00 (1), no. 2 (2)

11. Duct tape

12. Whatman filter paper, no. 42

13. $\mathrm{pH} /$ Volt meter, with expanded scale for $\mathrm{mV}$

14. HP-41CV calculator

15. Ion-selective electrode, chloride

16. Reference electrode, $\mathrm{Ag} / \mathrm{AgC1}$, double-junction

17. Plastic beaker, $250 \mathrm{~mL}$

18. Kimwipe tissue

19. Forceps, 8 in.

\section{Reagent}

1. $\mathrm{CaCl}_{2}$ solutions, $0.001 \mathrm{M}$ and $0.1 \mathrm{M}$, deaerated, saturated with thymol (see Procedure 7 on preparation of test solution).

2. Distilled water

\section{Procedure}

1. Set up the apparatus by first filling the Tygon tubing and buret with $0.001 \mathrm{M} \mathrm{CaCl}_{2}$ solution. Then fill the funnel portion below the porous plate before connecting the Tygon tubing to the funnel. Ensure no air bubbles are present in the entire column of solution.

2. Measure and record the potential of the $\mathrm{CaCl}_{2}$ solution in the column.

3. Place the oven-dried rock core. (preferably as thin a slice of core as possible, e.g., $5 \mathrm{~mm}$ thick) against the plate with previously saturated filter paper as contact.

4. Attach the access tubes, cut to approximately $3.8 \mathrm{~cm}$ ( $1.5 \mathrm{in}$.) in length, to the duct tape cover, and poke two air holes in the cover.

5. Place the cover over the mouth of the funnel, secure the cover with rubber bands, and stopper the access tubes to minimize evaporation.

6. Saturate the rock core by raising the water column step-wise over several hours. Leave the rock immersed in the solution for 12 hours. 
7. Desaturate to the first suction value, e.g., $10 \mathrm{~cm}$, and let equilibrate for a minimum of 2 hours. Record the initial and final suction values at each step.

8. Place two filter paper pieces down the access ports and sample for 10 minutes with the bolts pressing on the filter paper to provide contact. Remove the samples one at a time and measure the potential difference.

9. Sample with another piece of filter paper for 10 minutes. Take a potential reading by inserting the electrodes down the access ports. Take another measurement with the same sample outside the funnel if a reliable reading is possible.

10. Rotate the whole cover so the access ports are over different parts of the rock core. Stopper the ports to minimize evaporation when not testing.

11. Repeat for a different suction level starting at step 7 again. Increase the sampling period to 15 or 20 minutes if necessary.

Note: at a suction of $100 \mathrm{~cm}$ and above, the minimum sampling period should be 20 minutes or more.

12. Repeat by resaturating the rock core to the different suctions starting at step 7 so the wetting, as well as the drying, effects can be studied.

13. Measure the potential difference of the test solution in the funne1, and the filter paper used as contact at the end of the test as checks on the concentrations.

14. Use a different rock core when testing with the $0.1 \underline{\mathrm{M}} \mathrm{CaCl}_{2}$ solution. 
PROCEDURE 11

\section{DETERMINATION OF SOLUTE BREAKTHROUGH IN POROUS PLATES}

\section{Equipment}

1. Test solution delivery system

2. Ringstands (2)

3. Utility clamps, 3-prong large (2), 3-prong medium (2)

4. Clamp holder (4), V-jaw large

5. Iron rings (2), 3 -in. with support clamps

6. Porous plate

7. Plastic cover with access flaps

8. Level(s), small single-bubble and larger

9. Duct Tape

10. $\mathrm{pH} /$ Volt meter, with expanded scale for $\mathrm{mV}$

11. $\mathrm{HP}-41 \mathrm{CV}$

12. Ion-selective electrode, chloride

13. Reference electrode, $\mathrm{Ag} / \mathrm{AgCl}$, double-junction

14. Plastic beaker, $250 \mathrm{~mL}$

15. Kimwipe tissue

16. Squirt bottle

17. Calibration curves

\section{$\underline{\text { Reagent }}$}

1. $\mathrm{CaCl}_{2}$ solutions, $0.001 \mathrm{M}, 0.01 \mathrm{M}$ and $0.1 \mathrm{M}$, deaerated, saturated with thymol (see Procedure 7 on Preparation of Test Solution).

2. Distilled water

\section{Procedure}

1. Vacuum saturate the porous plate is saturated with deaerated $\mathrm{CaCl}_{2}$ solution. The determination of solute breakthrough involves displacing the $\mathrm{CaCl}_{2}$ used to saturate the plate with a solution of different concentration, i.e., saturate the plate with $0.001 \underline{M}$ solution, displace with $0.1 \mathrm{M}$ solution.

2. Fill the flow system with the test solution by first filling the Mariotte reservoir (refer to Procedure 3 on flow measurement and head control for a better understanding of the operation of the system). Connect the plate to the flow system, then purge the system completely of air bubbles. Inspect the plate flow channels for entrapped air carefully before finally clamping onto the ringstand setup.

3. Clamp the plate with the ceramic side up using the medium utility clamps. Position the plastic cover over the plate, and tape down the wire support to the bottom side of the plate. Tape the rest of the plastic cover around the plate, and utility clamps to provide a tight seal. Level the four sides of the plate so that the test solution does not flow towards any one side during the test. At no time should the plastic or tape touch the ceramic plate. (Note: During setup, allow some test solution to flow to ensure that the plate does not dry out. Record the total time of flow during this initial period.) 
4. Set the desired head difference by adjusting the height of the Mariotte reservoir. The head difference should be measured from the water manometer to the plate surface.

5. Before starting the test, make sure the electrodes are functioning properly. Measure the potential difference of the test solutions by taking samples from the Mariotte bottle, and from the plate channels. If the plate was soaked in test solution in a vesse1, also measure the potential difference of that solution.

6. Divide up the plate into four quadrants, i.e., a one-by-four matrix. A range of values can be obtained for each plate. Measure with the reference and chloride electrodes, and record the potential values (in $\mathrm{mV}$ ) from both the $\mathrm{pH} /$ volt meter, and the HP41CV. As a check of the two potential measurements, the $\mathrm{pH} / \mathrm{vo} 1 \mathrm{t}$ meter values are 70 times that of the HP values.

7. Touch the reference and chloride electrodes to the plate surface by finding the wettest spot(s) of each quadrant, making sure the reference electrode tip is flush against the surface of the plate. Keep the distance separating the two electrodes to no more than a centimeter, preferably within one-half of a centimeter. The two sampling windows should be open only as long as necessary for the measurements. After each set of measurements, wipe off the plate initially with a paper towel, and dab the excess solution with a Kimwipe.

8. Measure the potential difference every one-half hour to one hour depending on the flow rate, and the amount of solution displaced. The measurements are most meaningful when a minimum of $1 \mathrm{~mm}$ of solution covers the plate. Continue to measure the potential difference until the relative concentration, $C^{*}=\left(C-C_{1}\right) /\left(C_{0}-C_{1}\right)$ $=1.0$ on a step-up test, and $C^{\circ}=0.0$ on a step-down test, or until the $C^{*}$ levels off. The background concentration is $C_{i}$, and tracer concentration is $C_{0}$.

9. During the test, measure the flow rate of the system periodically to ensure steady flow. Compare the flow rates to previously determined ones as a check against possible leaks in the Mariotte reservoir, or other flow system components.

10. Plot $C^{*}$ versus time elapsed to obtain the breakthrough curve. The effective porosity $\left(n_{e}\right)$ of the plate can be determined from the curve as shown below.

\section{Calculations}

The effective porosity $\left(n_{e}\right)$ of each plate is calculated as:

$$
n_{e}=q t_{0.5} / L
$$

where

$$
q=Q / A
$$

Q flow rate, $\mathrm{m}^{3} / \mathrm{s}$;

A cross-sectional area of plate, $\mathrm{m}^{2}$;

$t_{0.5}$ travel time, or time when $C^{*}=\left(C-C_{1}\right) /\left(C_{0}-C_{i}\right)=0.5, s$; and

$\mathrm{L}$ thickness of plate, $m$. 
APPENDIX B

FLUID FLOW CALCULATION AND TEST RESULTS 
TABLE B.1

PLATE CONDUCTANCE TESTS

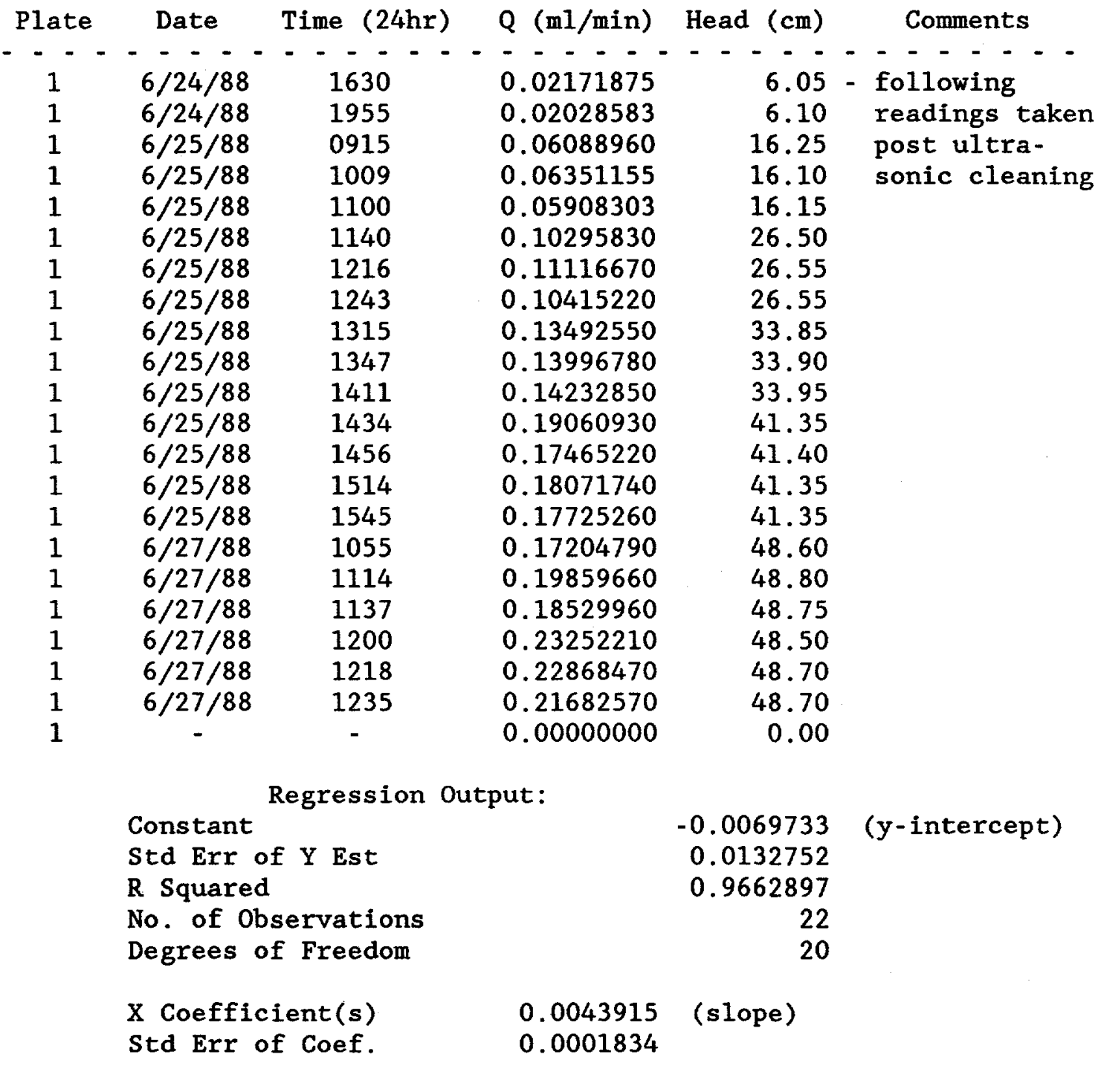


TABLE B.1 (continued)

\begin{tabular}{|c|c|c|c|c|c|}
\hline Plate & Date & Time (24hr) & $\mathrm{Q}(\mathrm{m} 1 / \mathrm{min})$ & Head (cm) & Comments \\
\hline$-\cdot-$ & $-\overline{-}-\overline{-}$ & $-\overline{-}-\overline{-}$ & $-\overline{0}-\overline{-}-\overline{1}$ & $--{ }^{-}-\overline{0}$ & $-\overline{-}-\overline{-}--\cdot$ \\
\hline 2 & $6 / 15 / 88$ & 1133 & 0.04981320 & $\begin{array}{l}9.80 \\
9.78\end{array}$ & - following \\
\hline 2 & $\begin{array}{l}6 / 15 / 88 \\
6 / 15 / 88\end{array}$ & $\begin{array}{l}1237 \\
1350\end{array}$ & $\begin{array}{l}0.05044009 \\
0.02545987\end{array}$ & $\begin{array}{l}9.78 \\
5.18\end{array}$ & readings taken \\
\hline $\begin{array}{l}2 \\
2\end{array}$ & $\begin{array}{l}6 / 15 / 88 \\
6 / 15 / 88\end{array}$ & $\begin{array}{l}1350 \\
1551\end{array}$ & $\begin{array}{l}0.02545987 \\
0.02344638\end{array}$ & $\begin{array}{l}5.18 \\
5.05\end{array}$ & prior to \\
\hline $\begin{array}{l}2 \\
2\end{array}$ & $6 / 16 / 88$ & $\begin{array}{l}1551 \\
1250\end{array}$ & 0.08370069 & $\begin{array}{r}5.05 \\
15.40\end{array}$ & ultra-sonic \\
\hline $\begin{array}{l}2 \\
2\end{array}$ & $6 / 16 / 88$ & $\begin{array}{l}1250 \\
1326\end{array}$ & 0.08890206 & $\begin{array}{l}15.40 \\
15.30\end{array}$ & \\
\hline 2 & & 1326 & & & \\
\hline 2 & $6 / 16 / 88$ & 1423 & 0.08418808 & 15.40 & \\
\hline 2 & $6 / 16 / 88$ & 1454 & 0.18744730 & 33.85 & \\
\hline 2 & $6 / 16 / 88$ & 1513 & 0.19712200 & 33.95 & \\
\hline 2 & $6 / 16 / 88$ & 1539 & 0.18449620 & 33.85 & \\
\hline 2 & $6 / 16 / 88$ & 1556 & 0.28401020 & 48.85 & \\
\hline 2 & $6 / 16 / 88$ & 1612 & 0.26890150 & 48.95 & \\
\hline 2 & $6 / 16 / 88$ & 1624 & 0.27275210 & 48.95 & \\
\hline 2 & $6 / 19 / 88$ & 1534 & 0.26532240 & 49.53 & following \\
\hline 2 & $6 / 19 / 88$ & 1548 & 0.27577330 & 49.48 & readings taken \\
\hline 2 & $6 / 19 / 88$ & 1604 & 0.26371310 & 49.50 & post ultra- \\
\hline 2 & $6 / 19 / 88$ & 1625 & 0.20594490 & 37.90 & sonic cleaning \\
\hline 2 & $6 / 19 / 88$ & 1640 & 0.22152480 & 37.75 & \\
\hline 2 & $6 / 19 / 88$ & 1705 & 0.16000430 & 27.00 & \\
\hline 2 & $6 / 19 / 88$ & 1725 & 0.15913010 & 27.00 & \\
\hline 2 & $6 / 20 / 88$ & 1215 & 0.07792208 & 14.90 & \\
\hline 2 & $6 / 20 / 88$ & 1300 & 0.07391894 & 14.65 & \\
\hline 2 & - & - & 0.00000000 & 0.00 & \\
\hline \multicolumn{6}{|c|}{ Regression Output: } \\
\hline \multicolumn{4}{|c|}{ Constant } & \multicolumn{2}{|l|}{-0.0018161} \\
\hline & \multicolumn{3}{|c|}{ Std Err of Y Est } & \multirow{2}{*}{\multicolumn{2}{|c|}{0.0068014}} \\
\hline & \multicolumn{3}{|l|}{ R Squared } & & 0.9951367 \\
\hline & \multicolumn{3}{|c|}{ No. of Observations } & 23 & \\
\hline & \multicolumn{3}{|c|}{ Degrees of Freedom } & 21 & \\
\hline & X Coeffici & ient (s) & 0.0056101 & & \\
\hline
\end{tabular}


TABLE B.1 (continued)

\begin{tabular}{|c|c|c|c|c|c|}
\hline Plate & Date & Time (24hr) & $\mathrm{Q}(\mathrm{ml} / \mathrm{min})$ & Head $(\mathrm{cm})$ & Comments \\
\hline$-{ }^{-}-$ & $-\overline{-}-\overline{-}$ & $-\frac{-}{1012}--$ & 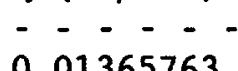 & $---\overline{6}$ & ----- \\
\hline $\begin{array}{l}3 \\
3\end{array}$ & $\begin{array}{l}8 / 15 / 88 \\
8 / 15 / 88\end{array}$ & $\begin{array}{l}1012 \\
1340\end{array}$ & $\begin{array}{l}0.01365763 \\
0.01219535\end{array}$ & $\begin{array}{l}6.07 \\
5.83\end{array}$ & \\
\hline 3 & $8 / 15 / 88$ & 1528 & 0.03259208 & 13.45 & \\
\hline 3 & $8 / 16 / 88$ & 1001 & 0.03091287 & 19.88 & \\
\hline 3 & $8 / 16 / 88$ & 1151 & 0.03280757 & 13.59 & \\
\hline 3 & $8 / 16 / 88$ & 1450 & 0.03104626 & 13.48 & \\
\hline 3 & $8 / 16 / 88$ & 1620 & 0.05508580 & 21.77 & \\
\hline 3 & $8 / 17 / 88$ & 1015 & 0.05334282 & 21.70 & \\
\hline 3 & $8 / 17 / 88$ & 1100 & 0.05138228 & 21.90 & \\
\hline 3 & $8 / 17 / 88$ & - & 0.07423629 & 30.93 & \\
\hline 3 & $8 / 17 / 88$ & - & 0.07588693 & 30.85 & \\
\hline 3 & $8 / 18 / 88$ & 1002 & 0.08088979 & 30.92 & \\
\hline 3 & $8 / 18 / 88$ & 1032 & 0.07239295 & 30.89 & \\
\hline 3 & $8 / 18 / 88$ & 1120 & 0.07662052 & 30.90 & \\
\hline 3 & $8 / 18 / 88$ & 1400 & 0.10797390 & 40.52 & \\
\hline 3 & $8 / 18 / 88$ & 1420 & 0.10675970 & 40.38 & \\
\hline 3 & $8 / 19 / 88$ & 1130 & 0.10450050 & 40.40 & \\
\hline 3 & $8 / 19 / 88$ & 1159 & 0.10406550 & 40.40 & \\
\hline 3 & $8 / 22 / 88$ & 1540 & 0.13724570 & 51.57 & \\
\hline 3 & $8 / 22 / 88$ & 1555 & 0.12954210 & 51.45 & \\
\hline 3 & $8 / 22 / 88$ & 1605 & 0.13407820 & 51.55 & \\
\hline 3 & $8 / 22 / 88$ & 1615 & 0.13317940 & 51.52 & \\
\hline 3 & - & - & 0.00000000 & 0.00 & \\
\hline
\end{tabular}

Constant

Regression Output:

Std Err of $Y$ Est

$-0.0052230$

0.0047078

$R$ Squared

0.9882314

No. of Observations

23

Degrees of Freedom

21

$\begin{array}{ll}\text { X Coefficient(s) } & 0.0026828 \\ \text { Std Err of Coef. } & 0.0000639\end{array}$ 
TABLE B.1 (continued)

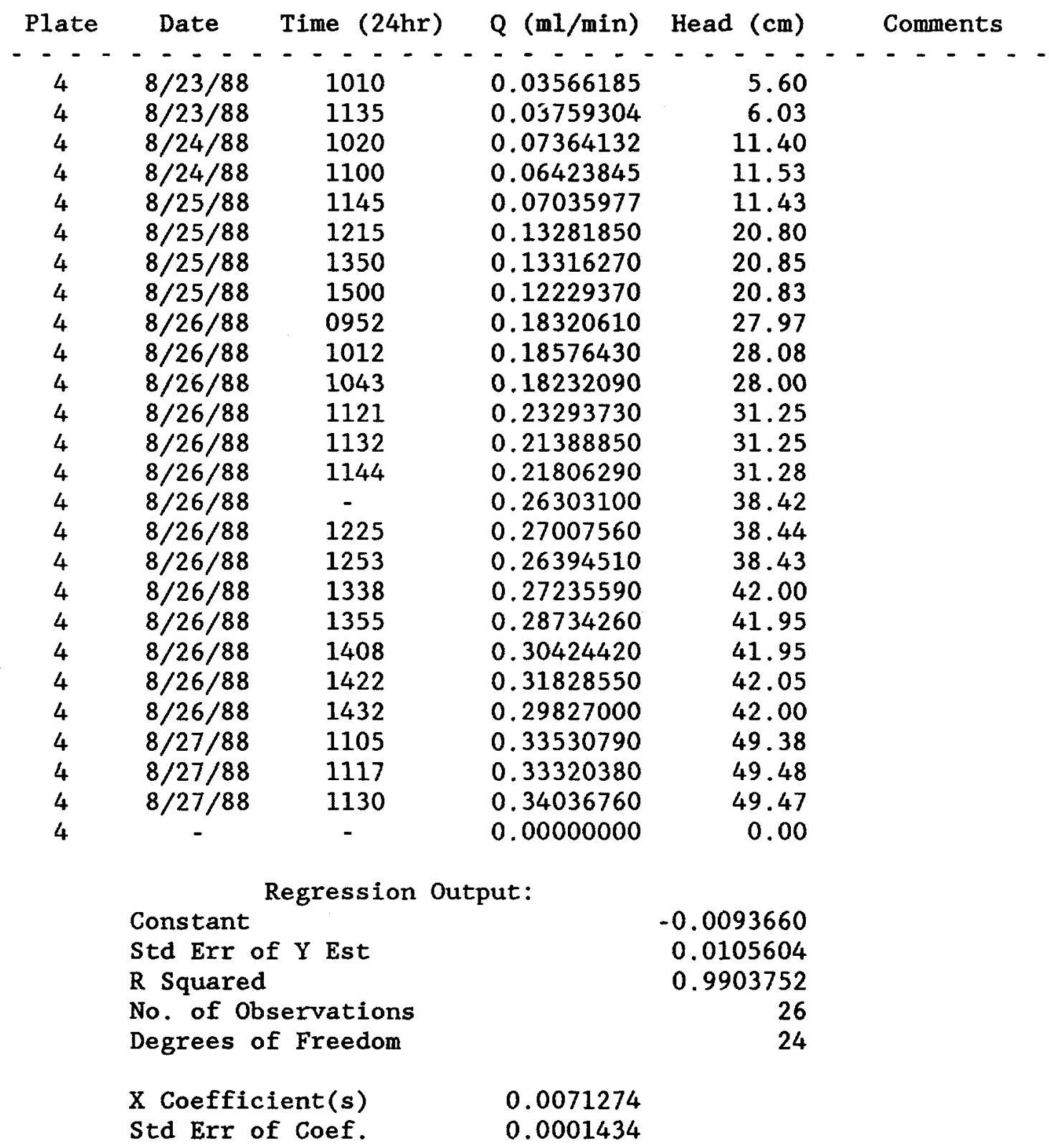


TABLE B.1 (continued)

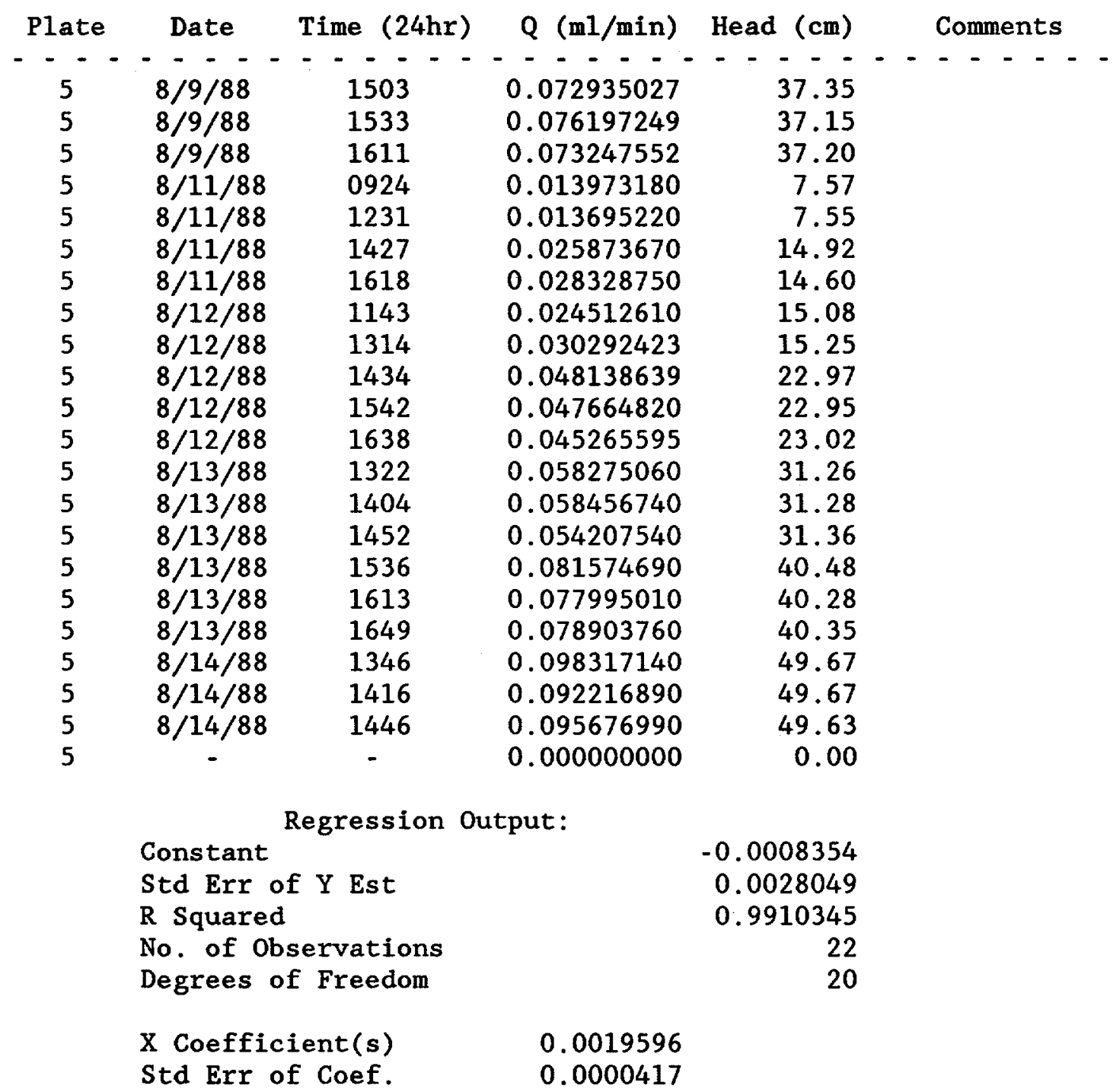


TABLE B.1 (continued)

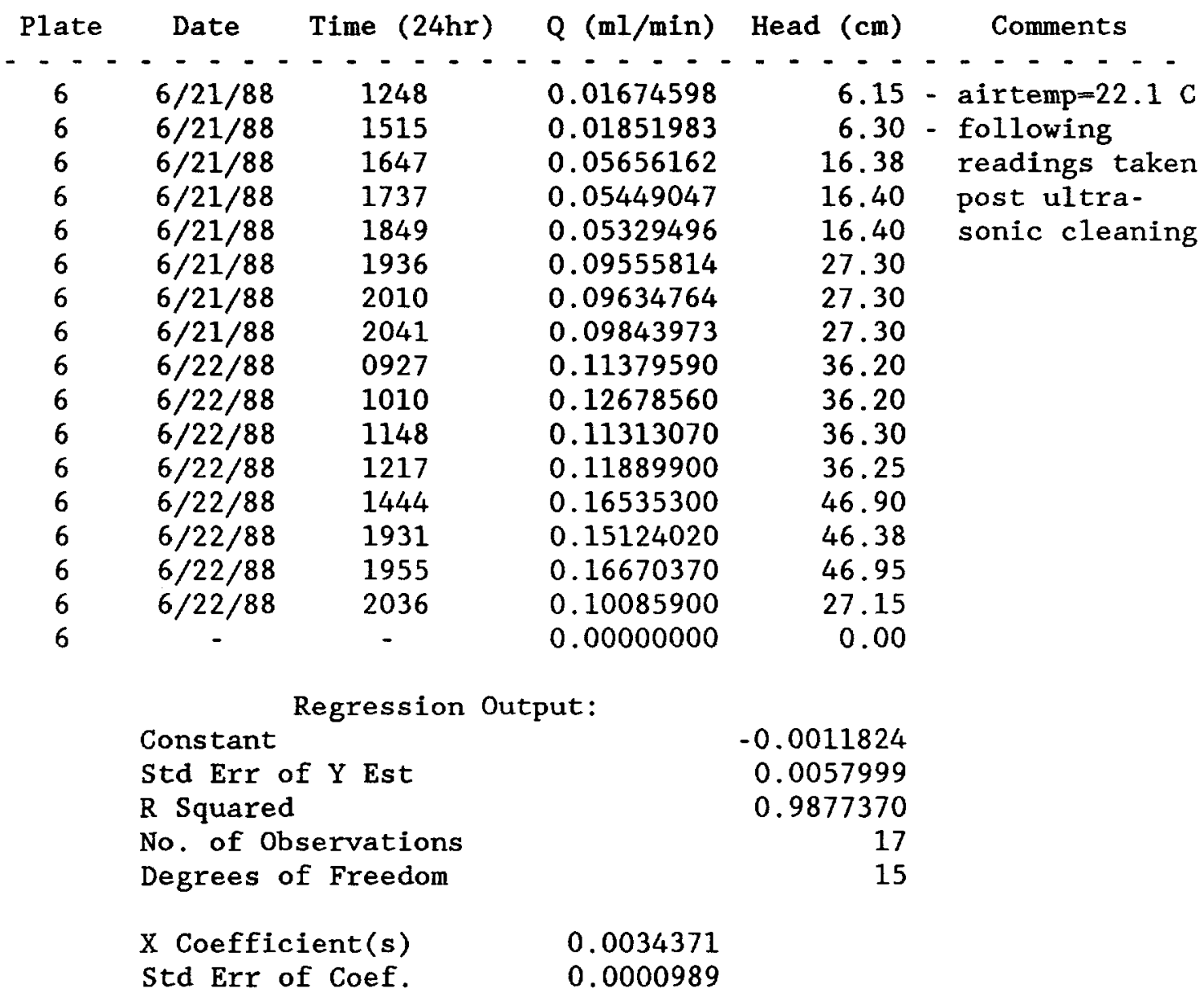


TABLE B. 2

PRESSURE TRANSDUCER CALIBRATION DATA

TRANSDUCER 5290035 - JULY 11, 1988

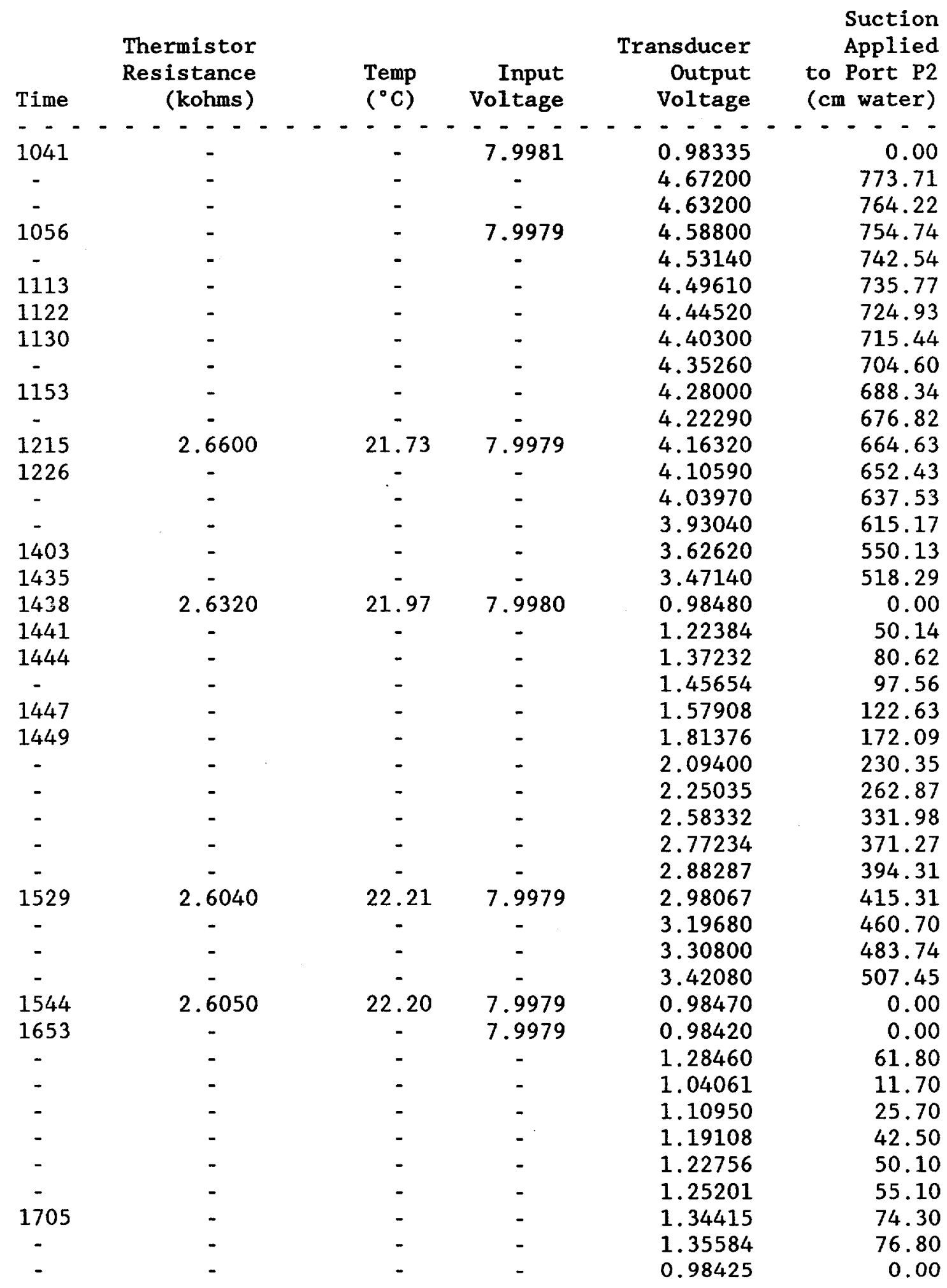


TABLE B.2 (continued)

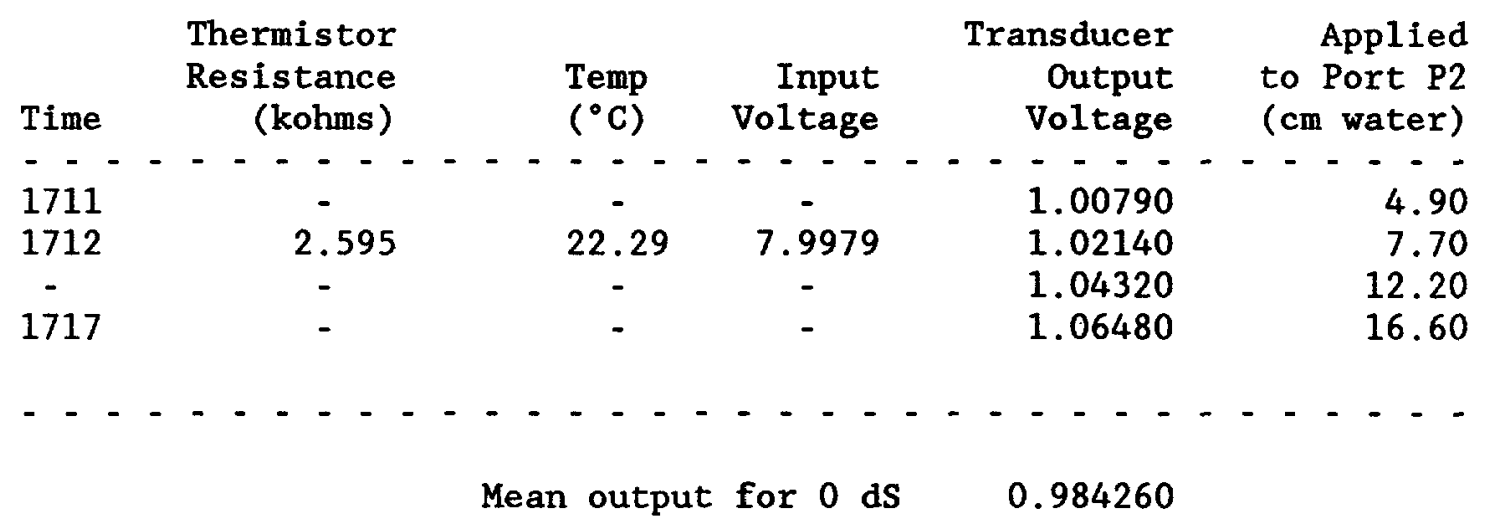


TABLE B. 3

PRESSURE TRANSDUCER CALIBRATION SUMMARY

TRANSDUCER 5290035 - JULY 11, 1988

J.B. Method

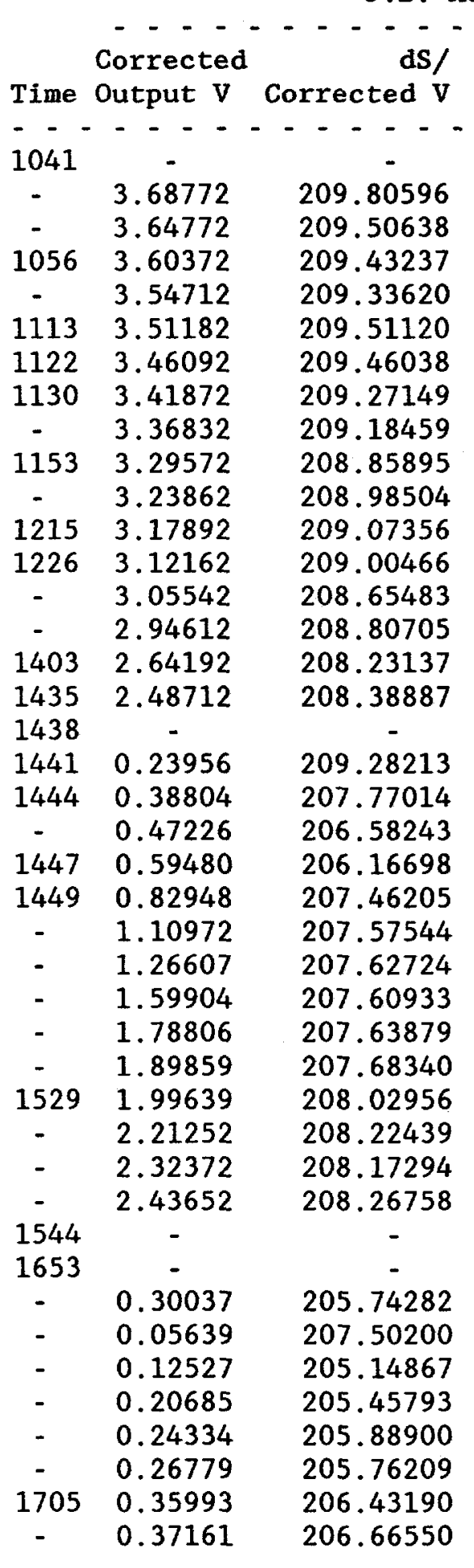

Least Squares Method

Predicted

dS

Error
dS

Error
766.44716

758.13365

748.98878

737.22516

729.88849

719.30954

710.53878

700.06375

684.97473

673.10719

660.69927

648.79016

635.03129

612.31462

549.09034

516.91704

49.78901

80.64877

98.15288

123.62133

172.39671

230.64119

263.13664

332.34041

371.62592

394.59824

414.92478

459.84477

482.95634

506.40045

62.42929

11.71894

26.03689

42.99230

50.57422

55.65586

74.80604

77.23566
$-0.00938$

$-0.00796$

$-0.00761$

$-0.00716$

$-0.00799$

$-0.00775$

$-0.00685$

$-0.00644$

$-0.00489$

$-0.00549$

$-0.00591$

$-0.00558$

$-0.00392$

$-0.00464$

$-0.00189$

$-0.00264$

$-0.00690$

0.00033

0.00608

0.00810

0.00181

0.00126

0.00101

0.00110

0.00096

0.00074

$-0.00092$

$-0.00186$

$-0.00161$

$-0.00206$

0.01018

0.00162

0.01311

0.01158

0.00947

0.01009

0.00681

0.00567
770.87126

762.50012

753.29187

741.44671

734.05919

723.40692

714.57537

704.02774

688.83412

676.88433

664.39041

652.39876

638.54453

615.67040

552.00791

519.61161

\section{24585}

80.31951

97.94493

123.58991

172.70336

231.35154

264.07222

333.75565

373.31345

396.44499

416.91242

462.14376

485.41552

509.02212

61.96161

10.89977

25.31696

42. 38989

50.02437

55.14122

74.42413

76.87060
$-0.00366$

$-0.00225$

$-0.00191$

$-0.00147$

$-0.00232$

$-0.00209$

$-0.00121$

$-0.00081$

0.00072

0.00009

$-0.00036$

$-0.00005$

0.00160

0.00081

0.00341

0.00255

$-0.01774$

$-0.00376$

0.00395

0.00785

0.00359

0.00435

0.00457

0.00536

0.00550

0.00543

0.00386

0.00313

0.00347

0.00310

0.00261

$-0.06840$

$-0.01490$

$-0.00259$

$-0.00151$

0.00075

0.00167

0.00092 
TABLE B.3 (continued)

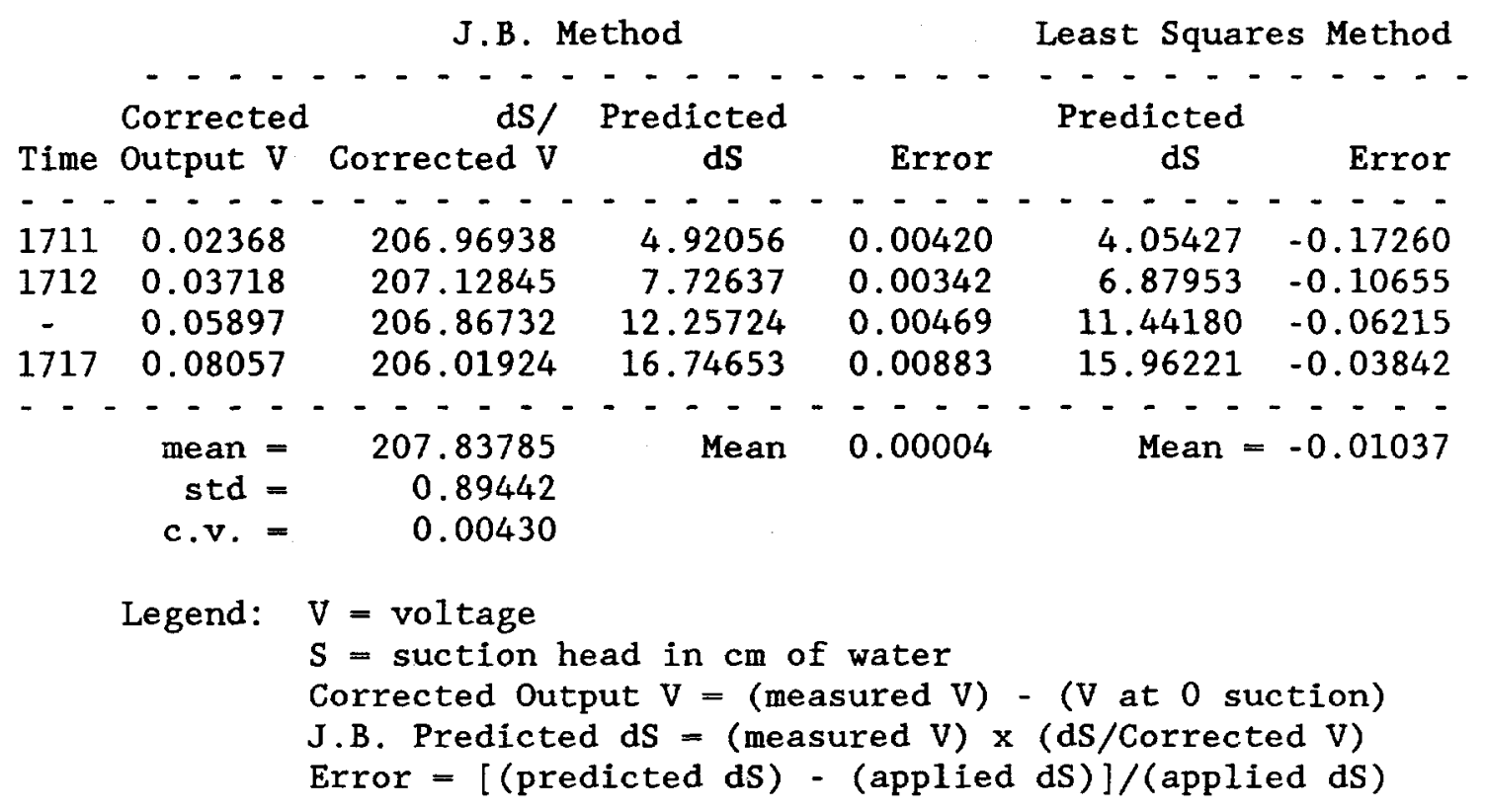


TABLE B. 4

TENSIOMETER CHAMBER DATA - TRANSDUCER 5290035

\begin{tabular}{|c|c|c|c|c|c|c|c|c|}
\hline \multirow{12}{*}{$\begin{array}{c}\text { Date } \\
-\overline{-}- \\
7 / 12 / 88\end{array}$} & \multirow{2}{*}{ Time } & $\begin{array}{l}\text { Therm. } \\
\text { Resist. } \\
\text { (kohms) }\end{array}$ & $\begin{array}{l}\text { Air } \\
\text { Temp } \\
\left({ }^{\circ} \mathrm{C}\right)\end{array}$ & $\begin{array}{l}\text { Input } \\
\text { voltage }\end{array}$ & $\begin{array}{l}\text { Tensio. } \\
\text { Output } \\
\text { Voltage }\end{array}$ & \multicolumn{2}{|r|}{$\begin{array}{l}\text { Suction } \\
\text { Applied } \\
\text { o Chamber }\end{array}$} & $\begin{array}{r}\text { Suction } \\
\text { Applied } \\
\text { to P1 }\end{array}$ \\
\hline & & $\therefore--$ & -- & $-\cdots$ & $-\cdots$ & - & 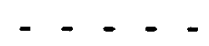 &.$--\cdot$ \\
\hline & - & - & - & - & 4.01790 & & 57.50 & 796.74 \\
\hline & - & - & - & - & 4.01000 & & 0.00 & 795.39 \\
\hline & - & - & - & - & 4.61500 & & 22.40 & 792.68 \\
\hline & 1635 & - & - & 7.9978 & 4.60920 & & 22.40 & 789.97 \\
\hline & 1644 & - & - & - & 4.60230 & & 22.60 & 788.61 \\
\hline & - & - & - & - & 1.37759 & & 0.00 & 89.91 \\
\hline & 1700 & - & - & 7.9978 & 385 & & 0.00 & 45.70 \\
\hline & 1720 & - & - & - & 160 & & 7.10 & 85.90 \\
\hline & 1727 & - & - & - & 300 & & 7.30 & .00 \\
\hline & 1736 & - & - & - & 1.32400 & & 7.50 & 86.00 \\
\hline above & data & questionab & le due & to start & up proble & $\mathrm{ms}+$ & + vertical & chamber \\
\hline $7 / 13 / 88$ & 1016 & - & - & 7.9981 & 1. 24155 & & 26.80 & 89.60 \\
\hline & 1033 & - & - & - & 1.24250 & & 26.80 & 89.80 \\
\hline & 1043 & - & - & 7.9981 & 1.24200 & & 26.80 & 89.80 \\
\hline & 1102 & 2.814 & 20.50 & - & 1.24170 & & 26.80 & 89.80 \\
\hline & 1106 & - & - & - & 1.19055 & & 37.25 & 89.80 \\
\hline & 1112 & - & - & - & 1.19055 & & 37.25 & 89.80 \\
\hline & 1116 & - & - & - & 1.11225 & & 53.30 & 89.40 \\
\hline & 1123 & - & - & - & 1.11245 & & 10 & 89.40 \\
\hline - & above & data ques & tionabl & e due to & vertical & test & t chamber- & $-\cdot$ \\
\hline & 1132 & - & - & - & 1.16860 & & & 89.00 \\
\hline & 1140 & - & - & - & 1.41635 & & 0.00 & 89.00 \\
\hline & 1144 & - & - & - & 60 & & 0.00 & 89.00 \\
\hline & 1147 & - & - & - & 1.3 & & 8.80 & 89.00 \\
\hline & & - & - & - & 1.3 & & 8.80 & 89.00 \\
\hline & & - & - & - & 1.3 & & 13.95 & 89.00 \\
\hline & & - & - & - & 00 & & & 89.00 \\
\hline & & - & - & 7.9980 & 90 & & & 89.60 \\
\hline & & 2.881 & 20.00 & - & 1.2 & & 10 & 89.40 \\
\hline & & - & - & - & 1.2 & & 41.20 & 89.20 \\
\hline & & - & - & - & 100 & & 46.80 & 89.20 \\
\hline & & - & - & 7.9980 & 20 & & 0.00 & 55 \\
\hline & & 2.855 & 20.19 & - & 51 & & 59.60 & 30 \\
\hline & & - & - & - & 20 & & 76.50 & 20 \\
\hline & & . & - & - & 092 & & 70.60 & 00 \\
\hline & & 2.821 & 20.45 & - & 550 & & 61.25 & .00 \\
\hline & & 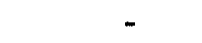 & - & - & 70 & & .80 & .75 \\
\hline & & 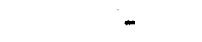 & - & 7.9980 & 1.2 & & 39.70 & .85 \\
\hline & & - & - & - & 1.2 & & 35.10 & 87.70 \\
\hline & & - & - & - & 1.2 & & 29.80 & 87.70 \\
\hline & & - & - & - & 1.29620 & & 23.60 & 87.50 \\
\hline & 1549 & - & - & - & 1.32330 & & 17.60 & 87.50 \\
\hline
\end{tabular}


TABLE B.4 (continued)

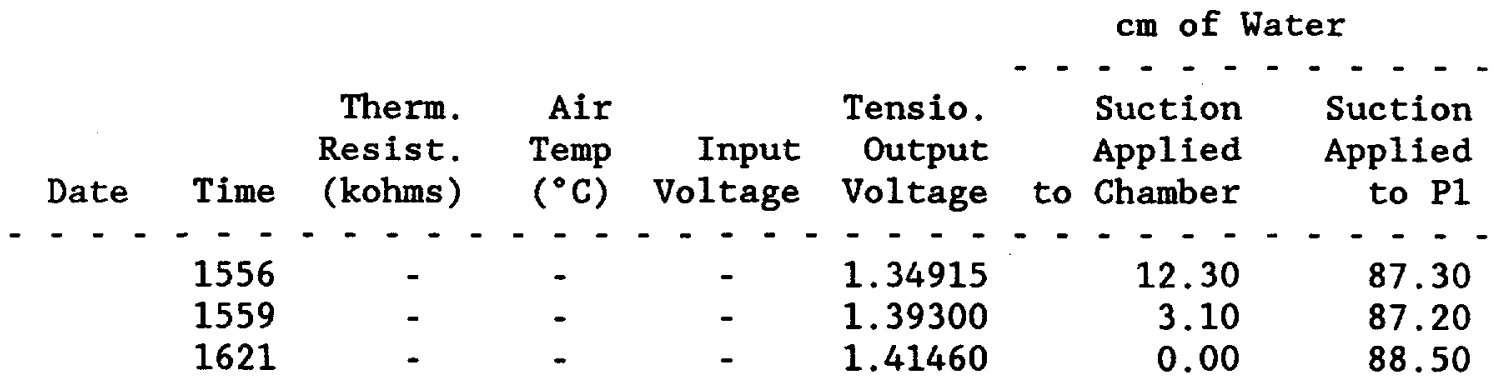


TABLE B.5

TENSIOMETER CHAMBER CALIBRATION SUMMARY TRANSDUCER 5290035

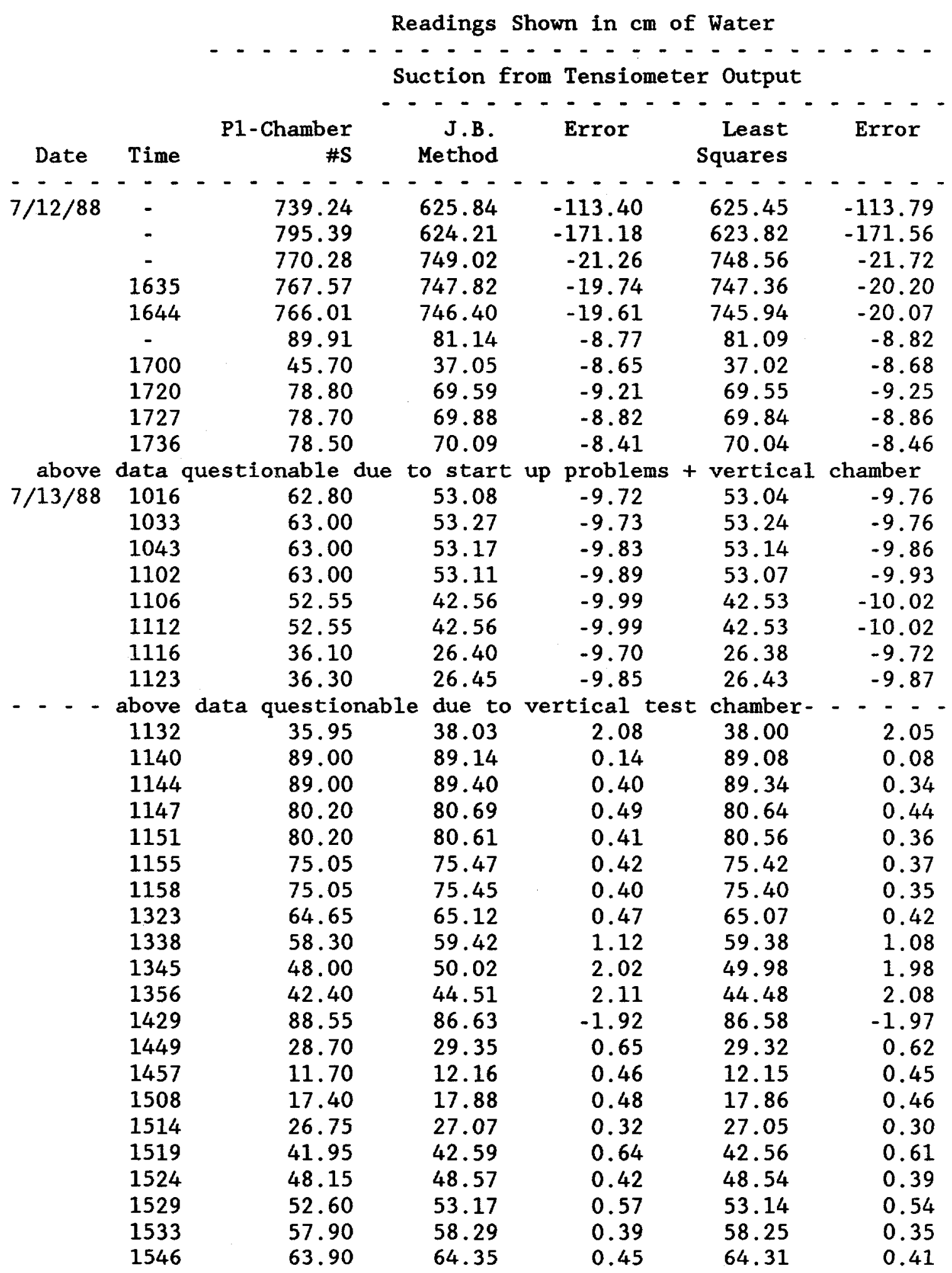


TABLE B.5 (continued)

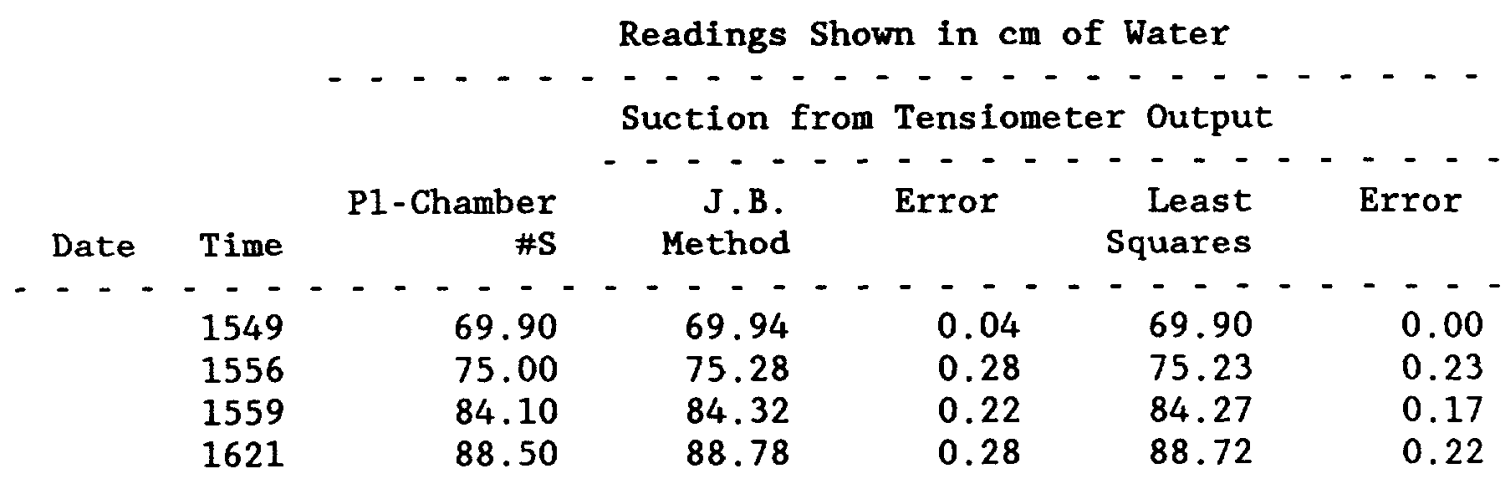


TABLE B. 6

TENSTOAETER GALIBRATION DATA GALIBRATED WITH POROUS PIATE - TRANSDUCER 5290035

\begin{tabular}{|c|c|c|c|c|c|c|}
\hline Date & Time & $\begin{array}{c}\text { Port } \\
\text { Location }\end{array}$ & $\begin{array}{c}\text { Thermistor } \\
\text { Resistance } \\
\text { (kohms) }\end{array}$ & $\begin{array}{l}\text { Air } \\
\text { Temp } \\
\left({ }^{\circ} \mathrm{C}\right)\end{array}$ & $\begin{array}{l}\text { Input } \\
\text { Voltage }\end{array}$ & $\begin{array}{l}\text { Tensio. } \\
\text { Output } \\
\text { (volts) }\end{array}$ \\
\hline & -- & $-\cdots$ & $---\cdot-$ & -- & $-\cdot-\cdot$ & $\therefore--$ \\
\hline \multirow[t]{2}{*}{$7 / 18 / 88$} & 2055 & upper & - & - & 8.0001 & 1.29600 \\
\hline & 2139 & upper & - & - & - & 1.29300 \\
\hline \multirow[t]{13}{*}{$7 / 19 / 88$} & 1010 & upper & 2.862 & & 7.9990 & 1.27840 \\
\hline & 1017 & upper & - & - & - & 1.30400 \\
\hline & 1119 & upper & 2.825 & & 7.9988 & 1.27930 \\
\hline & 1203 & upper & 2.810 & & 7.9986 & 1.23015 \\
\hline & 1322 & upper & - & - & - & 1.18050 \\
\hline & 1358 & upper & 2.801 & & 7.9986 & 1.19858 \\
\hline & 1421 & upper & - & - & - & 1.23975 \\
\hline & 1438 & upper & 2.740 & & 7.9989 & 1.24150 \\
\hline & 1520 & upper & 2.751 & & 7.9989 & 1.28660 \\
\hline & 1600 & upper & - & - & 7.9988 & 1.30665 \\
\hline & 1616 & upper & 2.717 & & - & 1.30800 \\
\hline & 1659 & upper & 2.716 & & 7.9989 & 1.33266 \\
\hline & 1724 & upper & 2.746 & & 7.9990 & 1.33650 \\
\hline \multirow[t]{9}{*}{$7 / 20 / 88$} & 0850 & upper & 2.845 & & 7.9987 & 1.34765 \\
\hline & 0938 & upper & 2.839 & & 7.9988 & 1.32320 \\
\hline & 1007 & upper & - & - & - & 1.32300 \\
\hline & 1114 & upper & 2.850 & & 7.9989 & 1.20150 \\
\hline & 1231 & upper & - & - & - & 1.19870 \\
\hline & 1401 & upper & 2.855 & & 7.9988 & 1.25120 \\
\hline & 1604 & upper & - & - & - & 1.33350 \\
\hline & 1656 & upper & 2.812 & & 7.9990 & 1.33380 \\
\hline & 2030 & lower & - & - & - & 1.36100 \\
\hline \multirow[t]{6}{*}{$7 / 21 / 88$} & 0825 & lower & 3.077 & & 7.9990 & 1.36330 \\
\hline & 1016 & lower & 2.841 & & 7.9984 & 1.35200 \\
\hline & 1148 & lower & - & - & - & 1.32650 \\
\hline & 1335 & lower & - & - & - & 1.32560 \\
\hline & 1451 & upper & 2.780 & & 7.9988 & 1.26600 \\
\hline & 1659 & upper & 2.790 & & 7.9988 & 1.21950 \\
\hline \multirow[t]{4}{*}{$7 / 22 / 88$} & 1049 & lower & 3.043 & & 7.9993 & 1.27175 \\
\hline & 1244 & lower & - & - & - & 1.22875 \\
\hline & 1501 & upper & 3.090 & & 7.9992 & 1.17340 \\
\hline & 1528 & upper & - & - & - & 1.16520 \\
\hline
\end{tabular}

Note: Upper and lower sampling ports located $10 \mathrm{~cm}$ apart 
TABLE B.7

\section{TENSIOMETER CALIBRATION SUMMARY CALIBRATED WITH POROUS PLATE - TRANSDUCER 5290035}

\begin{tabular}{|c|c|c|c|c|c|c|c|}
\hline \multirow{3}{*}{$\begin{array}{c}\text { Date } \\
-\overline{-}-\overline{8} \\
7 / 18 / 88\end{array}$} & \multirow{2}{*}{$\begin{array}{r}\text { Time } \\
-\end{array}$} & $\begin{array}{c}\text { Port } \\
\text { Location }\end{array}$ & $\begin{array}{l}\operatorname{Re} \\
\text { Suction } \\
\text { Applied } \\
\text { to Plate }\end{array}$ & $\begin{array}{c}\text { dings Sho } \\
----- \\
\text { Suction } \\
\text { Applied } \\
\text { to P1 }\end{array}$ & $\begin{array}{l}\mathrm{P} 1-\mathrm{P} 2 \\
\mathrm{dP}\end{array}$ & $\begin{array}{c}\text { of Water } \\
\text { Calc. } \\
\text { Plate } \\
\text { Suction }\end{array}$ & $\begin{array}{r}-- \\
\text { Diff. } \\
\text { from } \\
\text { Applied }\end{array}$ \\
\hline & & -- & --- & -- & -- & -- & --- \\
\hline & 2055 & upper & 19.60 & 86.30 & 64.31 & 21.99 & 2.39 \\
\hline & 2139 & upper & 19.60 & 86.00 & 63.69 & 22.31 & 2.71 \\
\hline$/ 19 / 88$ & 1010 & upper & 20.50 & 84.20 & 60.68 & 23.52 & 3.02 \\
\hline & 1017 & upper & 19.70 & 83.50 & 65.96 & 17.54 & -2.16 \\
\hline & 1119 & upper & 19.70 & 83.20 & 60.87 & 22.33 & 2.63 \\
\hline & 1203 & upper & 29.50 & 82.60 & 50.73 & 31.87 & 2.37 \\
\hline & 1322 & upper & 39.50 & 82.50 & 40.48 & 42.02 & 2.52 \\
\hline & 1358 & upper & 34.40 & 81.40 & 44.21 & 37.19 & 2.79 \\
\hline & 1421 & upper & 24.50 & 81.20 & 52.71 & 28.49 & 3.99 \\
\hline & 1438 & upper & 24.50 & 80.90 & 53.07 & 27.83 & 3.33 \\
\hline & 1520 & upper & 14.50 & 80.40 & 62.37 & 18.03 & 3.53 \\
\hline & 1600 & upper & 9.50 & 79.90 & 66.51 & 13.39 & 3.89 \\
\hline & 1 & upper & 9.50 & 80.00 & 66.79 & 13.21 & 3.71 \\
\hline & 1 & upper & 4.40 & 80.40 & 71.87 & 8.53 & 4.13 \\
\hline & 1724 & upper & 4.30 & 80.40 & 72.67 & 7.73 & 3.43 \\
\hline$/ 20 / 88$ & 0850 & upper & 4.40 & 82.50 & 74.97 & 7.53 & 3.13 \\
\hline & 09 & upper & 9.80 & 82.40 & 69.92 & 12.48 & 2.68 \\
\hline & 10 & pper & 9.80 & 82.40 & 69.88 & 12.52 & 2.72 \\
\hline & 11 & upper & 34.60 & 81.90 & 44.82 & 37.08 & 2.48 \\
\hline & 12 & upper & 34.60 & 81.70 & 44.24 & 37.46 & 2.86 \\
\hline & 14 & upper & 22.70 & 80.80 & 55.07 & 25.73 & 3.03 \\
\hline & 1 & upper & 4.70 & 79.80 & 72.05 & 7.7 & 3.05 \\
\hline & & upper & 4.70 & 79.70 & 72.11 & 7. & 2.89 \\
\hline & & lon & -5.25 & 80.90 & 77.72 & 3.18 & 8.43 \\
\hline$/ 21 / 88$ & 0825 & low & -0.70 & 81.30 & 78.20 & 3.10 & 3.80 \\
\hline & 10 & lon & 0.00 & 79.00 & 75.86 & 3.1 & 3.14 \\
\hline & & lon & 4.80 & 77.90 & 70.60 & 7.3 & 2.50 \\
\hline & 1335 & $10 \mathrm{r}$ & 4.80 & 77.80 & 70.42 & 7.38 & 2.58 \\
\hline & & & 14.80 & 75.55 & 58.12 & 17.43 & 2.63 \\
\hline & & upp & 23.20 & 74.50 & 48.53 & 25.97 & 2.77 \\
\hline 22100 & 1049 & lower & 13.20 & 75.30 & 59.31 & 15.99 & 2.79 \\
\hline & 12 & 10r & 20.85 & 74.10 & 50.44 & 23.66 & 2.81 \\
\hline & & up & 30.85 & 72.60 & 39.02 & 33.58 & 2.73 \\
\hline & 1 & upper & 30.85 & 71.00 & 37.33 & 33.67 & 2.82 \\
\hline
\end{tabular}


TABLE B. 8

\section{LVDT CALIBRATION}

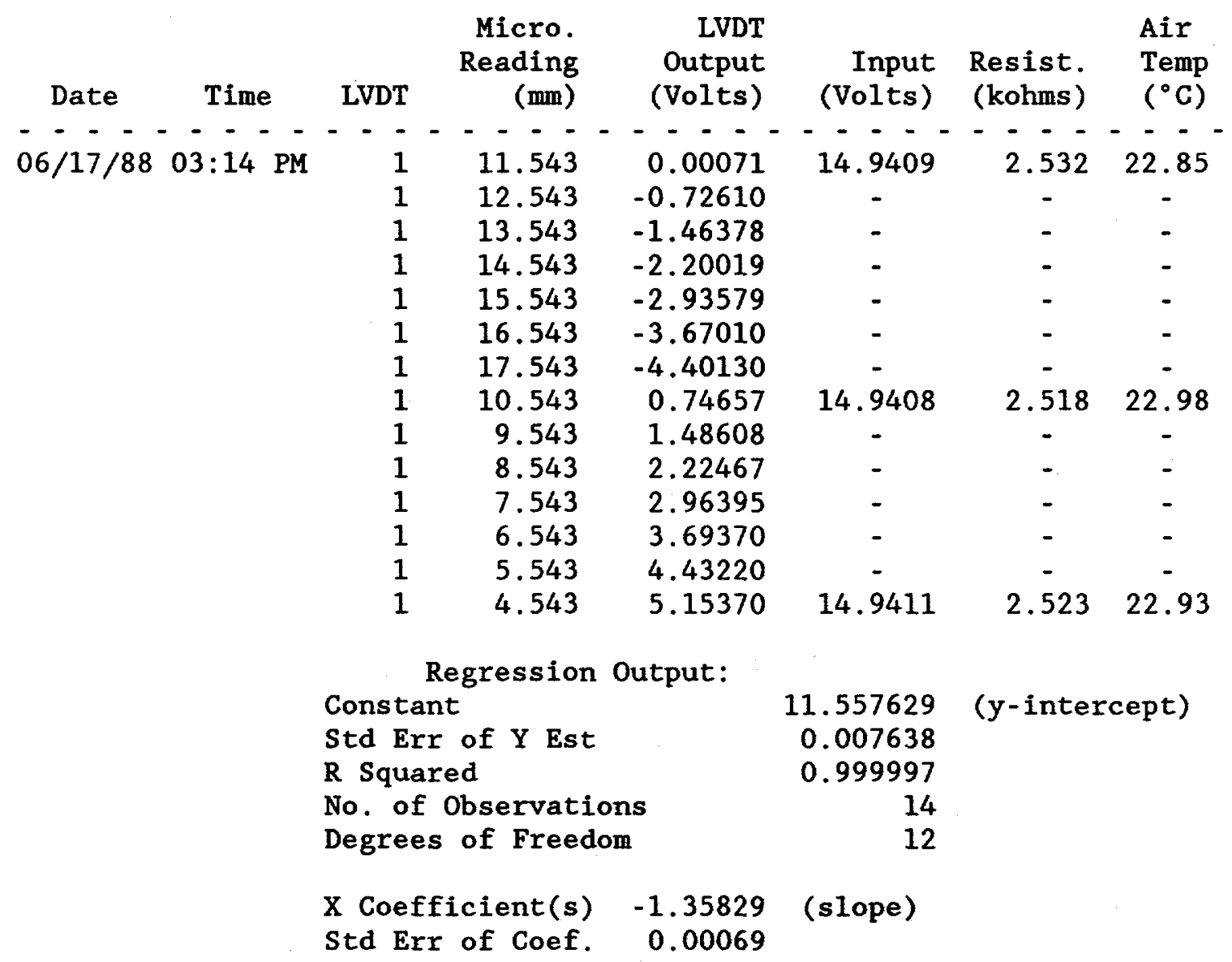




\section{TABLE B.8 (continued)}

\begin{tabular}{|c|c|c|c|c|c|c|c|c|}
\hline Date & Time & & LVDT & $\begin{array}{l}\text { Micro. } \\
\text { Reading } \\
(\mathrm{mm})\end{array}$ & $\begin{array}{r}\text { LVDT } \\
\text { Output } \\
\text { (Volts) }\end{array}$ & $\begin{array}{r}\text { Input } \\
\text { (Volts) }\end{array}$ & $\begin{array}{l}\text { Resist. } \\
\text { (kohms) }\end{array}$ & $\begin{array}{l}\text { Air } \\
\text { Temp } \\
\left({ }^{\circ} \mathrm{C}\right)\end{array}$ \\
\hline$--\cdot-$ & $--\cdot-$ & $\cdot-$ & $-\cdot-$ & $-\cdot-$ & $-\cdot--$ & $--\cdot-$ & --- & --- \\
\hline $06 / 17 / 88$ & $02: 17$ & PM & 2 & 9.936 & -0.00016 & 14.9409 & 2.558 & 22.61 \\
\hline & & & 2 & 10.936 & -0.72720 & - & - & - \\
\hline & & & 2 & 11.936 & -1.46370 & - & - & - \\
\hline & & & 2 & 12.936 & -2.19747 & - & - & - \\
\hline & & & 2 & 13.936 & -2.92885 & - & - & - \\
\hline & & & 2 & 14.936 & -3.65710 & - & - & - \\
\hline & & & 2 & 15.936 & -4.38560 & - & - & - \\
\hline & & & 2 & 8.936 & 0.73817 & 14.9410 & 2.548 & 22.70 \\
\hline & & & 2 & 7.936 & 1.47826 & - & - & - \\
\hline & $02: 48$ & PM & 2 & 6.936 & 2.21454 & - & - & - \\
\hline & & & 2 & 5.936 & 2.95200 & - & - & - \\
\hline & & & 2 & 4.936 & 3.68670 & - & - & - \\
\hline & & & 2 & 3.936 & 4.42260 & - & - & - \\
\hline & & & 2 & 2.936 & 5.14940 & 14.9410 & 2.542 & 22.76 \\
\hline \multicolumn{9}{|c|}{ Regression Output: } \\
\hline & & & \multicolumn{3}{|c|}{ Constant } & \\
\hline & & & \multirow{2}{*}{\multicolumn{3}{|c|}{$\begin{array}{l}\text { Std Err of Y Est } \\
\text { R Squared }\end{array}$}} & 0.008036 & & \\
\hline & & & & & & & & \\
\hline & & & \multicolumn{3}{|c|}{ No. of Observations } & 14 & & \\
\hline & & & \multicolumn{3}{|c|}{ Degrees of Freedom } & 12 & & \\
\hline & & & $\begin{array}{l}X \text { Coef } \\
\text { Std Er }\end{array}$ & $\begin{array}{c}\text { icient(s) } \\
\text { of Coef. }\end{array}$ & $\begin{array}{r}-1.36160 \\
0.00073\end{array}$ & & & \\
\hline
\end{tabular}




\section{TABLE B.8 (continued)}

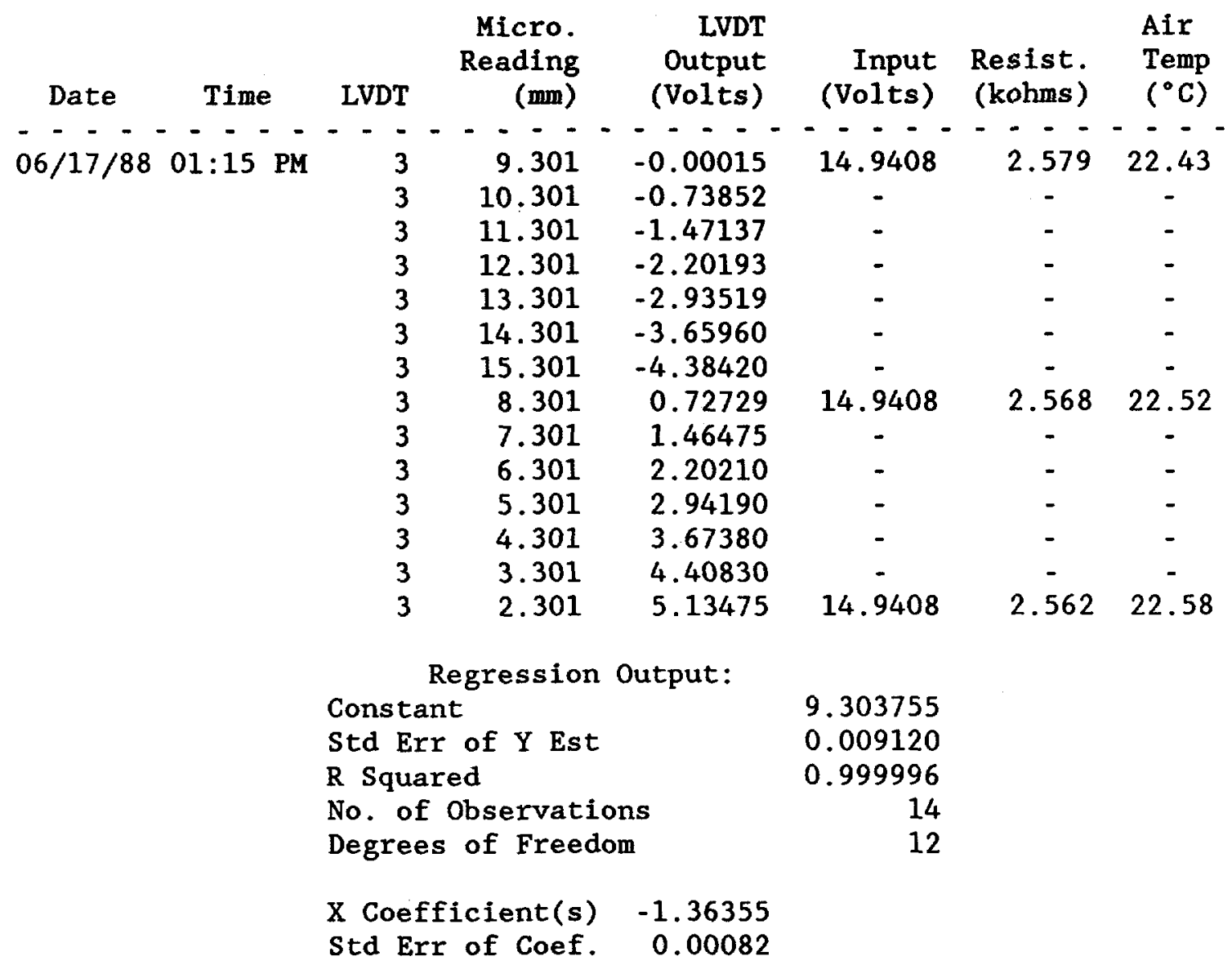


TABLE B.8 (continued)

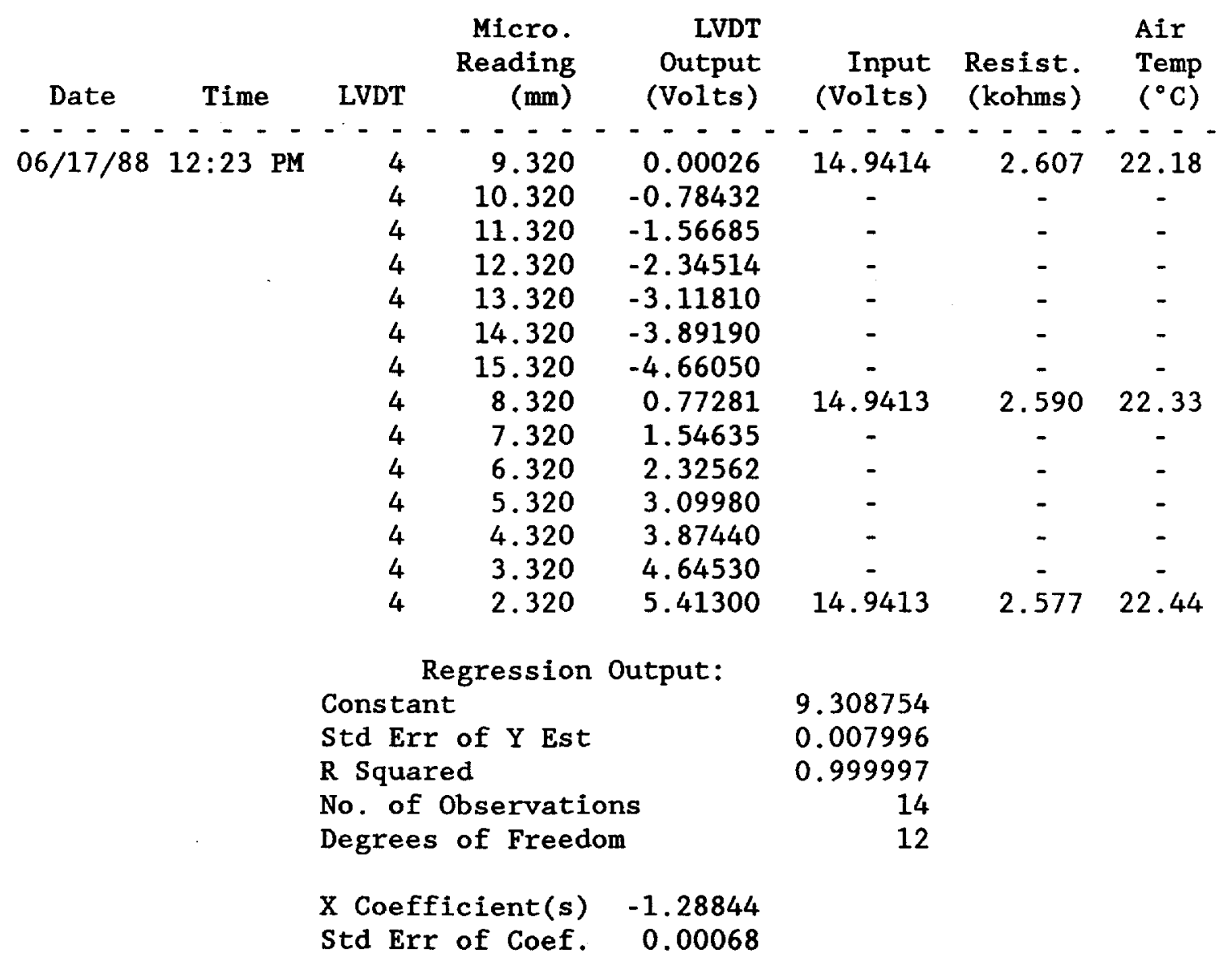




\section{TABLE B.8 (continued)}

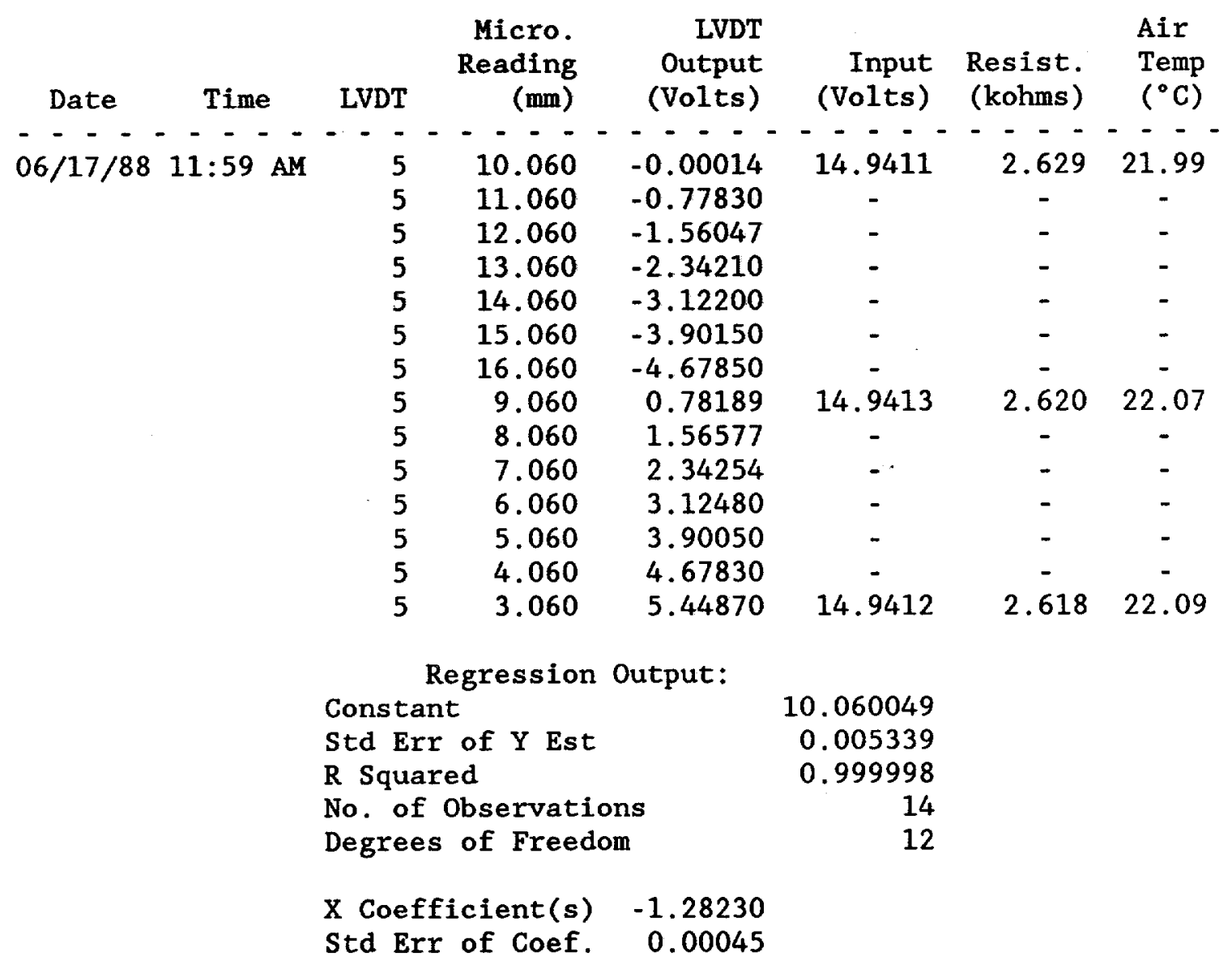


TABLE B.8 (continued)

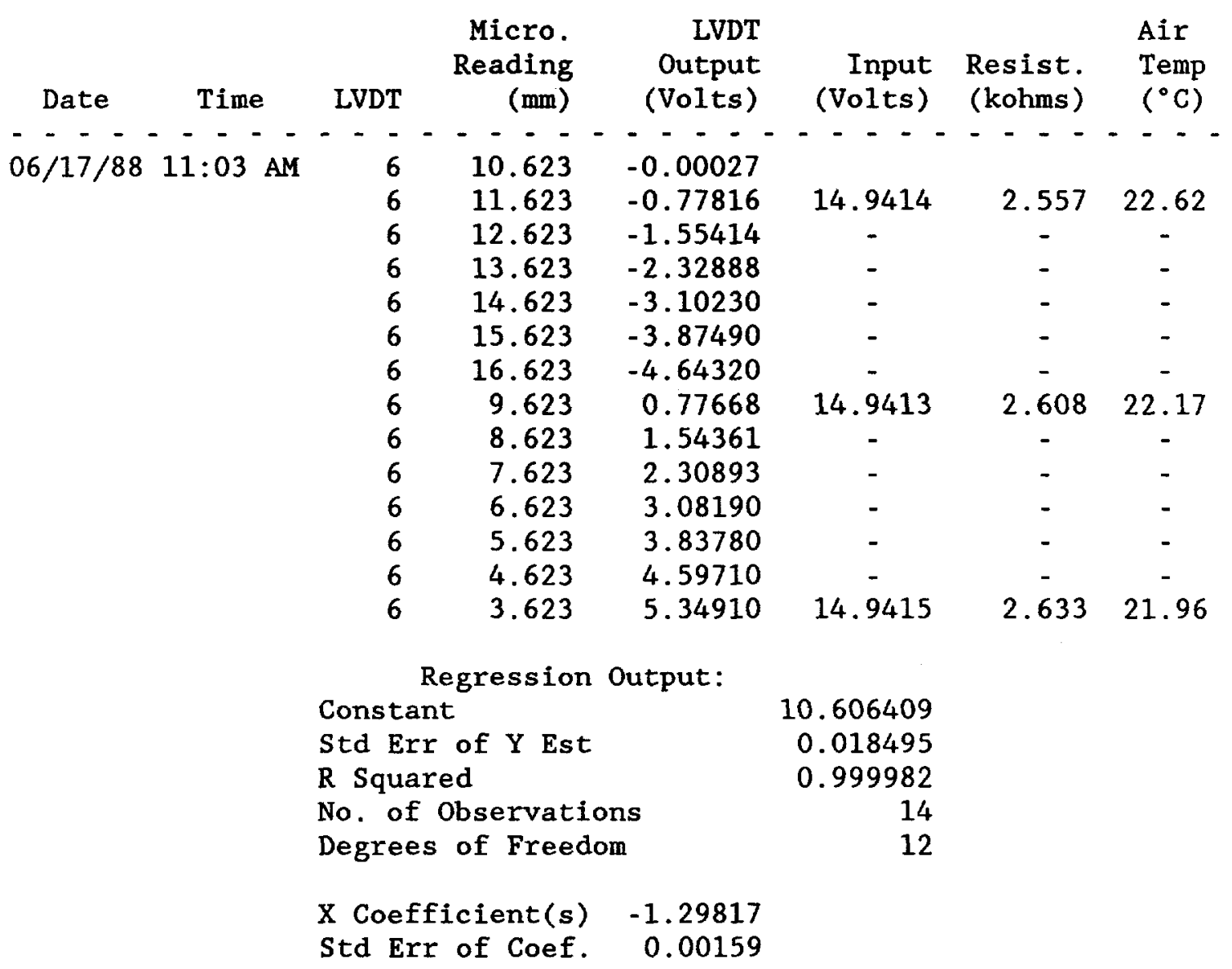


TABLE B.8 (continued)

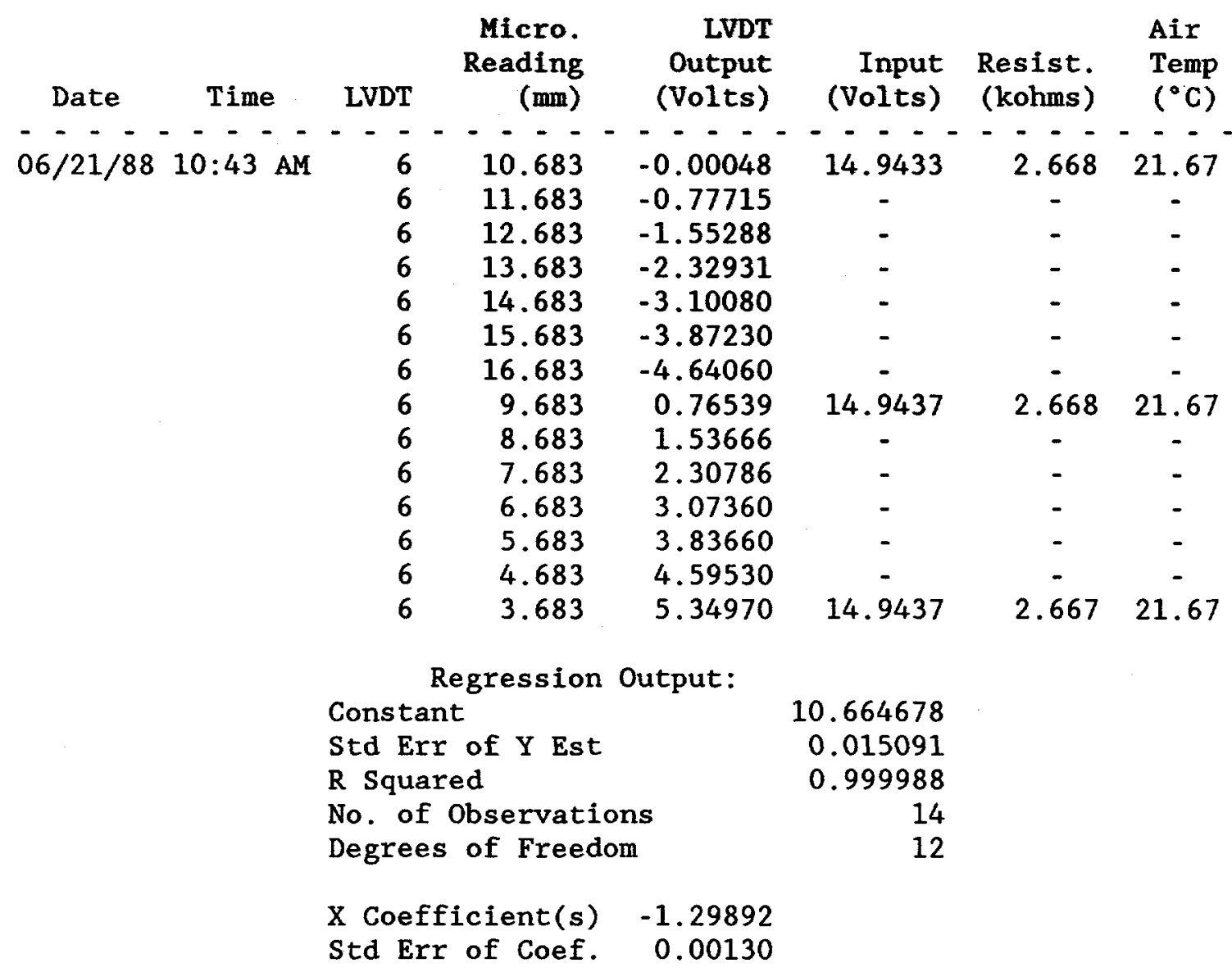


TABLE B. 9

ELECTRONICS DATA SUMMARY - TEST BLOCK 1

\begin{tabular}{|c|c|c|c|c|c|c|}
\hline Date & Time & $\begin{array}{l}\text { Therm. } \\
\text { Resist. } \\
\text { (kohms) }\end{array}$ & $\begin{array}{l}\text { Air } \\
\text { Temp } \\
\left({ }^{\circ} \mathrm{C}\right)\end{array}$ & $\begin{array}{c}\text { LVDT } \\
\text { Input } \\
\text { (volts) }\end{array}$ & $\begin{array}{c}\text { LVDT Output } \\
----\cdot-\end{array}$ & $\begin{array}{c}(\operatorname{volts}) \\
--- \\
3\end{array}$ \\
\hline---- & $-\quad-\quad-$ & --- & -- & --- & ----- & $-\quad-$ \\
\hline $04 / 22 / 88$ & $16: 11$ & - & - & 14.9396 & - & - \\
\hline $04 / 22 / 88$ & $18: 11$ & - & - & 14.9389 & - & - \\
\hline $04 / 22 / 88$ & $18: 41$ & - & - & 14.9388 & - & - \\
\hline $04 / 22 / 88$ & $19: 11$ & - & - & 14.9387 & - & - \\
\hline $04 / 22 / 88$ & $19: 41$ & - & - & 14.9386 & - & - \\
\hline $04 / 22 / 88$ & $20: 11$ & - & - & 14.9386 & - & - \\
\hline $04 / 22 / 88$ & $20: 41$ & - & - & 14.9387 & - & - \\
\hline $04 / 22 / 88$ & $21: 11$ & - & - & 14.9387 & - & - \\
\hline $04 / 22 / 88$ & $21: 41$ & - & - & 14.9388 & - & - \\
\hline $04 / 22 / 88$ & $22: 11$ & - & - & 14.9388 & - & - \\
\hline $04 / 22 / 88$ & $22: 41$ & - & - & 14.9387 & - & - \\
\hline $04 / 23 / 88$ & $14: 46$ & - & - & - & 5.89760 & - \\
\hline $04 / 23 / 88$ & $15: 16$ & - & - & - & 5.89430 & - \\
\hline $04 / 23 / 88$ & $15: 46$ & - & - & - & 5.89400 & - \\
\hline $04 / 23 / 88$ & $16: 46$ & - & - & - & 5.89390 & - \\
\hline $04 / 23 / 88$ & $17: 16$ & - & - & - & 5.89390 & - \\
\hline $04 / 23 / 88$ & $17: 46$ & - & - & - & 5.89410 & - \\
\hline $04 / 23 / 88$ & $18: 16$ & - & - & - & 5.89420 & - \\
\hline $04 / 23 / 88$ & $20: 16$ & - & - & - & 5.89560 & - \\
\hline $04 / 23 / 88$ & $20: 46$ & - & - & - & 5.89550 & - \\
\hline $04 / 23 / 88$ & $21: 16$ & - & - & - & 5.89500 & - \\
\hline $04 / 23 / 88$ & $21: 46$ & - & - & - & 5.89470 & - \\
\hline $04 / 23 / 88$ & $22: 16$ & - & - & - & 5.89450 & - \\
\hline $04 / 23 / 88$ & $22: 46$ & - & - & - & 5.89450 & - \\
\hline $04 / 23 / 88$ & $23: 16$ & - & - & - & 5.89450 & - \\
\hline $04 / 23 / 88$ & $23: 46$ & - & - & - & 5.89450 & - \\
\hline $04 / 24 / 88$ & $00: 16$ & - & - & - & 5.89470 & - \\
\hline $04 / 24 / 88$ & $00: 46$ & - & - & - & 5.89480 & - \\
\hline $04 / 24 / 88$ & $02: 16$ & - & - & - & 5.89540 & - \\
\hline $04 / 24 / 88$ & $02: 46$ & - & - & - & 5.89550 & - \\
\hline $04 / 24 / 88$ & $03: 16$ & - & - & - & 5.89560 & - \\
\hline $04 / 24 / 88$ & $03: 46$ & - & - & - & 5.89570 & - \\
\hline $04 / 24 / 88$ & $04: 16$ & - & - & - & 5.89590 & - \\
\hline $04 / 24 / 88$ & $04: 46$ & - & - & - & 5.89600 & - \\
\hline $04 / 24 / 88$ & $05: 16$ & - & - & - & 5.89610 & - \\
\hline $04 / 24 / 88$ & $06: 16$ & - & - & - & 5.89640 & - \\
\hline $04 / 24 / 88$ & $06: 46$ & - & - & - & 5.89650 & - \\
\hline $04 / 24 / 88$ & $08: 46$ & - & - & - & 5.89650 & - \\
\hline $04 / 24 / 88$ & $09: 46$ & - & - & - & 5.89630 & - \\
\hline $04 / 24 / 88$ & $10: 16$ & - & - & - & 5.89620 & - \\
\hline $04 / 24 / 88$ & $10: 46$ & - & - & - & 5.89620 & - \\
\hline $04 / 24 / 88$ & $11: 16$ & - & - & - & 5.89600 & - \\
\hline $04 / 24 / 88$ & $11: 46$ & - & - & - & 5.89600 & - \\
\hline $04 / 24 / 88$ & $12: 16$ & - & - & - & 5.89580 & - \\
\hline $04 / 24 / 88$ & $12: 46$ & - & - & - & 5.89620 & 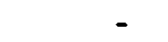 \\
\hline
\end{tabular}


TABLE B.9 (continued)

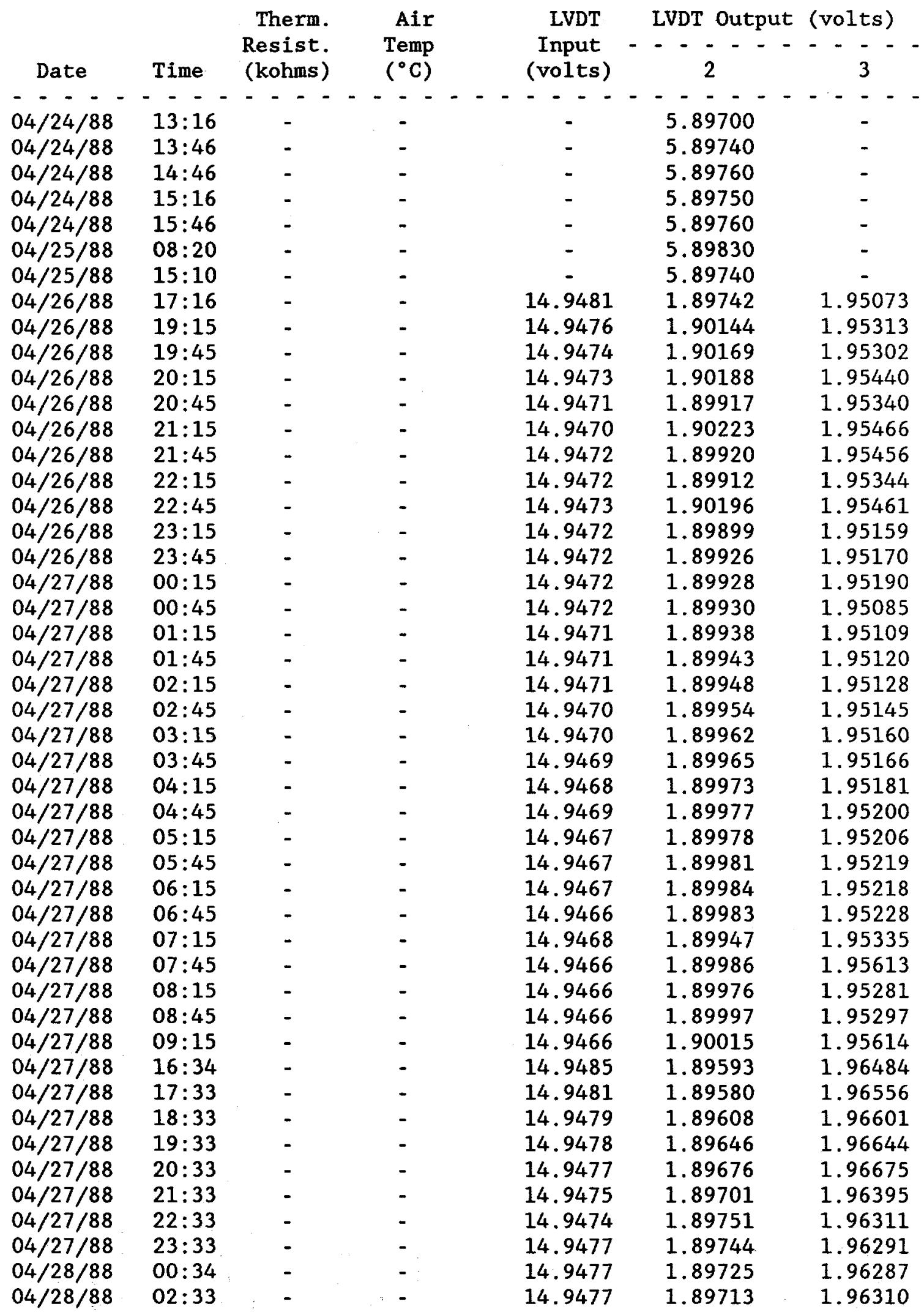


TABLE B.9 (continued)

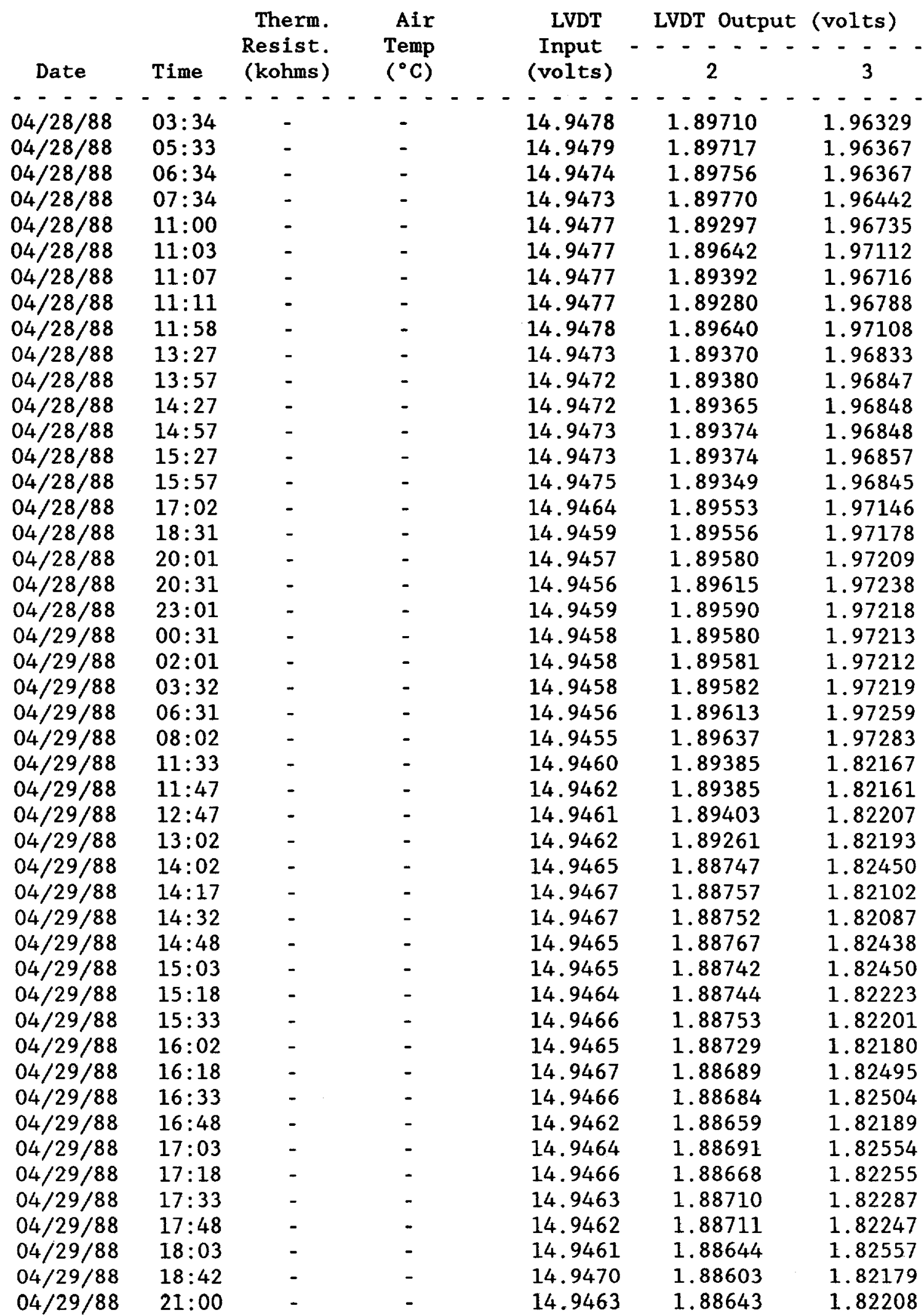


TABLE B.9 (continued)

\begin{tabular}{|c|c|c|c|c|c|c|}
\hline Date & Time & $\begin{array}{l}\text { Therm. } \\
\text { Resist. } \\
\text { (kohms) }\end{array}$ & $\begin{array}{l}\text { Air } \\
\text { Temp } \\
\left({ }^{\circ} \mathrm{C}\right)\end{array}$ & $\begin{array}{c}\text { LVDT } \\
\text { Input } \\
\text { (volts) }\end{array}$ & $\begin{array}{l}\text { LVDT Output } \\
----\cdot\end{array}$ & $\begin{array}{c}\text { (volts) } \\
---- \\
3\end{array}$ \\
\hline$-\quad-$ & --- & --- & - & --- & ----- & --- \\
\hline $04 / 29 / 88$ & $22: 30$ & - & - & 14.9460 & 1.88718 & 1.82555 \\
\hline $04 / 30 / 88$ & $00: 00$ & - & - & 14.9461 & 1.88707 & 1.82248 \\
\hline $04 / 30 / 88$ & $01: 30$ & - & - & 14.9459 & 1.88758 & 1.82672 \\
\hline $04 / 30 / 88$ & $10: 10$ & - & - & 14.9460 & 1.88700 & 1.82649 \\
\hline $04 / 30 / 88$ & $11: 41$ & - & - & 14.9462 & 1.88573 & 1.82267 \\
\hline $04 / 30 / 88$ & $13: 41$ & - & - & 14.9461 & 1.88508 & 1.82248 \\
\hline $04 / 30 / 88$ & $17: 28$ & - & - & 14.9462 & 1.87991 & 1.82251 \\
\hline $04 / 30 / 88$ & $19: 28$ & - & - & 14.9458 & 1.87989 & 1.82335 \\
\hline $04 / 30 / 88$ & $21: 28$ & - & - & 14.9461 & 1.87681 & 1.82332 \\
\hline $04 / 30 / 88$ & $23: 28$ & - & - & 14.9460 & 1.87542 & 1.82381 \\
\hline $05 / 01 / 88$ & $01: 28$ & - & - & 14.9459 & 1.87498 & 1.82405 \\
\hline $05 / 01 / 88$ & $03: 28$ & - & - & 14.9457 & 1.87475 & 1.82432 \\
\hline $05 / 01 / 88$ & $05: 28$ & - & - & 14.9455 & 1.87291 & 1.82456 \\
\hline $05 / 01 / 88$ & $16: 24$ & 2.5950 & 22.2856 & 14.9453 & 1.85731 & 1.82613 \\
\hline $05 / 01 / 88$ & $20: 24$ & 2.8812 & 19.9980 & 14.9448 & 1.85648 & 1.82717 \\
\hline $05 / 01 / 88$ & $22: 24$ & 2.9278 & 19.6517 & 14.9448 & 1.85424 & 1.82763 \\
\hline $05 / 02 / 88$ & $00: 24$ & 2.9691 & 19.3483 & 14.9447 & 1.85406 & 1.82817 \\
\hline $05 / 02 / 88$ & $02: 24$ & 3.0024 & 19.1049 & 14.9446 & 1.85369 & 1.82870 \\
\hline $05 / 02 / 88$ & $04: 24$ & 3.0360 & .18 .8601 & 14.9444 & 1.85337 & 1.82927 \\
\hline $05 / 02 / 88$ & $06: 24$ & 3.0597 & 18.6878 & 14.9443 & 1.85209 & 1.82920 \\
\hline $05 / 02 / 88$ & $08: 24$ & 3.0400 & 18.8310 & 14.9442 & 1.85059 & 1.83025 \\
\hline $05 / 02 / 88$ & $10: 24$ & 2.9395 & 19.5654 & 14.9446 & 1.84703 & 1.82996 \\
\hline $05 / 02 / 88$ & $12: 28$ & 2.9532 & 19.4646 & 14.9444 & 1.84068 & 1.82999 \\
\hline $05 / 02 / 88$ & $14: 28$ & 3.0301 & 18.9031 & 14.9443 & 1.84073 & 1.83101 \\
\hline $05 / 02 / 88$ & $16: 28$ & 2.9902 & 19.1935 & 14.9444 & 1.83936 & 1.83106 \\
\hline $05 / 02 / 88$ & $18: 28$ & 3.0310 & 18.8965 & 14.9443 & 1.83460 & 1.83126 \\
\hline $05 / 02 / 88$ & $20: 28$ & 3.0198 & 18.9780 & 14.9441 & 1.83516 & 1.83339 \\
\hline $05 / 02 / 88$ & $22: 28$ & 2.9513 & 19.4789 & 14.9443 & 1.83416 & 1.83433 \\
\hline $05 / 03 / 88$ & $00: 28$ & 2.9927 & 19.1756 & 14.9442 & 1.83384 & 1.83476 \\
\hline $05 / 03 / 88$ & $02: 28$ & 3.0490 & 18.7656 & 14.9440 & 1.83376 & 1.83507 \\
\hline $05 / 03 / 88$ & $04: 28$ & 3.0809 & 18.5338 & 14.9439 & 1.83374 & 1.83539 \\
\hline $05 / 03 / 88$ & $08: 28$ & 2.9855 & 19.2282 & 14.9440 & 1.83057 & 1.83523 \\
\hline $05 / 03 / 88$ & $13: 27$ & 2.9536 & 19.4615 & 14.9444 & 1.82658 & 1.83710 \\
\hline $05 / 03 / 88$ & $15: 33$ & 2.9002 & 19.8563 & 14.9443 & 1.82158 & 1.83810 \\
\hline $05 / 03 / 88$ & $16: 52$ & 2.8393 & 20.3131 & - & - & - \\
\hline $05 / 03 / 88$ & $17: 22$ & 2.8628 & 20.1355 & - & - & - \\
\hline $05 / 03 / 88$ & $17: 52$ & 2.8534 & 20.2065 & - & - & - \\
\hline $05 / 03 / 88$ & $18: 22$ & 2.8856 & 19.9651 & - & - & - \\
\hline $05 / 03 / 88$ & $18: 52$ & 2.9134 & 19.7583 & - & - & - \\
\hline $05 / 03 / 88$ & $19: 22$ & 2.9468 & 19.5119 & - & - & - \\
\hline $05 / 03 / 88$ & $19: 52$ & 2.9579 & 19.4300 & - & - & - \\
\hline $05 / 03 / 88$ & $20: 22$ & 2.9500 & 19.4883 & - & - & - \\
\hline $05 / 03 / 88$ & $20: 52$ & 2.9329 & 19.6142 & - & - & - \\
\hline $05 / 03 / 88$ & $20: 22$ & 2.9461 & 19.5169 & - & - & - \\
\hline $05 / 03 / 88$ & $21: 39$ & 2.8594 & 20.1610 & - & - & - \\
\hline $05 / 03 / 88$ & $22: 05$ & 2.8544 & 20.1989 & - & - & - \\
\hline $5 / 03 / 88$ & $23: 05$ & 2.8945 & 19.8985 & - & - & - \\
\hline
\end{tabular}


TABLE B.9 (continued)

\begin{tabular}{|c|c|c|c|c|c|c|}
\hline Date & Time & $\begin{array}{l}\text { Therm. } \\
\text { Resist. } \\
\text { (kohms) }\end{array}$ & $\begin{array}{l}\text { Air } \\
\text { Temp } \\
\left({ }^{\circ} \mathrm{C}\right)\end{array}$ & $\begin{array}{c}\text { LVDT } \\
\text { Input } \\
\text { (volts) }\end{array}$ & $\begin{array}{l}\text { LVDT Output } \\
---\overline{2}\end{array}$ & $\begin{array}{c}\text { (volts) } \\
---- \\
3\end{array}$ \\
\hline-- & -- & -- & --- & $--\cdot-\cdot$ & $--\cdot-$ & --- \\
\hline $05 / 03 / 88$ & $23: 35$ & 2.9002 & 19.8563 & - & - & - \\
\hline $05 / 04 / 88$ & $00: 05$ & 2.9060 & 19.8131 & - & - & - \\
\hline $05 / 04 / 88$ & $00: 35$ & 2.9182 & 19.7223 & - & - & - \\
\hline $05 / 04 / 88$ & $01: 35$ & 2.9403 & 19.5593 & - & - & - \\
\hline $05 / 04 / 88$ & 02 & 2.9 & 19. & - & - & - \\
\hline $05 / 04 / 88$ & 03 & 2.9719 & 19.3 & - & - & - \\
\hline $05 / 04 / 88$ & 5 & 8 & 19. & - & - & - \\
\hline $05 / 04 / 88$ & 5 & 6 & 1 & - & - & - \\
\hline $05 / 04 / 88$ & 5 & 2. & 303 & - & - & - \\
\hline $05 / 04 / 88$ & 5 & $3 .($ & 18.9736 & - & - & - \\
\hline $05 /$ & 5 & 3 & 18.9525 & - & - & - \\
\hline $05 /$ & 15 & 3 & 18.9598 & - & - & - \\
\hline $05 /$ & 5 & 16 & 1 & - & - & - \\
\hline 05 & 5 & 0 & 1 & - & - & - \\
\hline$/ 88$ & $07: 35$ & 2.9668 & 19.3652 & - & - & - \\
\hline $05 / 04 / 88$ & כ & 2.9363 & 19.5891 & - & - & - \\
\hline $05 / 04 / 88$ & 08: & 2.9410 & 19.5543 & - & - & - \\
\hline $05 / 04 / 88$ & $09: 05$ & 2.9411 & 19.5534 & - & - & - \\
\hline $05 / 04 / 88$ & $09: 53$ & 2.9122 & 19.7674 & 14.9443 & 1.80448 & 1.83666 \\
\hline $05 / 04 / 88$ & $09: 54$ & 2.9090 & 19.7908 & - & - & - \\
\hline $05 / 04 / 88$ & $10: 54$ & 2.9315 & 19.6246 & - & - & - \\
\hline $05 / 04 / 88$ & $11: 24$ & 2.9079 & .7989 & - & - & - \\
\hline $05 / 04 / 88$ & $11: 54$ & 2.8597 & .1586 & - & - & - \\
\hline $05 / 04 / 88$ & $12: 24$ & 2.8124 & 20.5175 & - & - & - \\
\hline $05 / 04 / 88$ & $13: 24$ & 2.6936 & 21.4552 & - & - & - \\
\hline $05 / 04 / 88$ & $20: 25$ & 2.8391 & 20.3146 & 14.9444 & 1.79057 & 1.84470 \\
\hline $05 / 04 / 88$ & $21: 10$ & 2.8622 & 20.1397 & 14.9447 & 1.79032 & 1.84551 \\
\hline $05 / 05 / 88$ & $00: 10$ & 2.8361 & 20.3369 & 14.9445 & 1.79038 & 1.85142 \\
\hline $05 / 05 / 88$ & $03: 10$ & 2.8489 & 20.2400 & 14.9444 & 1.79014 & 1.85883 \\
\hline $05 / 05 / 88$ & $06: 10$ & 2.9506 & 19.4841 & 14.9442 & 1.79006 & 1.86820 \\
\hline $05 / 05 / 88$ & $09: 10$ & 2.9346 & 19.6019 & 14.9438 & 1.78904 & 1.87761 \\
\hline $05 / c$ & $11: 26$ & 2.8787 & 20.0167 & 14.9442 & 1.78771 & 1.88564 \\
\hline $05 / c$ & $13: 05$ & 2.7777 & 20.7854 & 14.9446 & 1.78491 & 1.89406 \\
\hline $05 / c$ & $16: 45$ & 2.6747 & 21.6097 & 14.9448 & 1.78024 & 1.90406 \\
\hline $05 /$ & $19: 45$ & 2.826 & 20.4129 & 14.9445 & 1.77669 & 1.91699 \\
\hline$/ 88$ & $22: 45$ & 2.8625 & 20.1379 & 14.9442 & 1.77704 & 1.92810 \\
\hline $05 / 06 / 88$ & $01: 45$ & 2.8470 & 20.2548 & 14.9442 & 1.77685 & 1.93797 \\
\hline $05 / 06 / 88$ & $04: 45$ & 2.8470 & 20.2548 & 14.9440 & 1.77699 & 1.94447 \\
\hline $05 / c$ & $07: 45$ & 2.94 & 19.5468 & 14.9438 & 1.77713 & 1.94925 \\
\hline /88 & 1. & 2.92 & 19.6 & 14.9441 & 1.77347 & 1.96094 \\
\hline$/ 88$ & $15: 13$ & 2.90 & 19.8013 & - & - & - \\
\hline $05 / 06 / 88$ & $15: 58$ & 2.8606 & 20.1519 & 14.9442 & 1.77019 & 1.97064 \\
\hline $05 / 06 / 88$ & $19: 58$ & 2.9666 & 19.3668 & 14.9435 & 1.77036 & 1.97018 \\
\hline $05 / 06 / 88$ & $23: 58$ & 2.95 & 19.4751 & 14.9436 & 1.76943 & 1.97434 \\
\hline $05 / 07 / 88$ & $03: 58$ & 3.0327 & 18.8841 & 14.9434 & 1.76951 & 1.97869 \\
\hline $05 / 07 / 88$ & $07: 58$ & 3.05 & 18.7394 & 14.9432 & 1.76959 & 1.98268 \\
\hline 188 & $11: 58$ & .0300 & 18.9038 & 14.9434 & 1.76899 & 98599 \\
\hline
\end{tabular}


TABLE B.9 (continued)

\begin{tabular}{|c|c|c|c|c|c|c|}
\hline Date & Time & $\begin{array}{l}\text { Therm. } \\
\text { Resist. } \\
\text { (kohms) }\end{array}$ & $\begin{array}{l}\text { Air } \\
\text { Temp } \\
\left({ }^{\circ} \mathrm{C}\right)\end{array}$ & $\begin{array}{c}\text { LVDT } \\
\text { Input } \\
\text { (vo1ts) }\end{array}$ & $\begin{array}{l}\text { LVDT Output } \\
---\cdot-\end{array}$ & $\begin{array}{c}\text { (vo1ts) } \\
--- \\
3\end{array}$ \\
\hline$-\overline{7}-$ & $--\overline{15 \cdot 22}$ & -- & $\overline{19}-\overline{0}-\overline{-}$ & $-\overline{-}-\overline{-}$ & $--\overline{-}-\overline{-}$ & $-\overline{-}-\overline{-}$ \\
\hline $05 / 07 / 88$ & $19: 22$ & - & 18.8826 & 14.9433 & 1.76732 & 1.99396 \\
\hline $5 / 08 / 88$ & $03: 22$ & - & 18.8471 & 14.9432 & 1.76663 & 2.00134 \\
\hline $5 / 08 / 88$ & $07: 22$ & - & 18.6774 & 14.9431 & 1.76679 & 2.00443 \\
\hline $05 / 08 / 88$ & $23: 22$ & - & 20.1374 & 14.9439 & 1.76060 & 2.01640 \\
\hline $55 / 09 / 88$ & $03: 22$ & - & 19.5101 & 14.9435 & 1.76084 & 2.01938 \\
\hline $55 / 09 / 88$ & $07: 22$ & - & 19.2993 & 14.9434 & 1.76115 & 2.02160 \\
\hline $05 / 09 / 88$ & $11: 22$ & - & 19.4449 & 14.9437 & 1.76009 & 2.02294 \\
\hline $05 / 09 / 88$ & $15: 22$ & - & 20.1736 & 14.9439 & 1.75986 & 2.02523 \\
\hline $05 / 09 / 88$ & $19: 22$ & - & 19.7654 & 14.9437 & 1.75906 & 2.02738 \\
\hline $05 / 10 / 88$ & $14: 18$ & - & 20.7533 & 14.9442 & 1.75604 & 2.03399 \\
\hline $05 / 11 / 88$ & 08:01 & - & 19.8032 & 14.9437 & 1.75484 & 2.04123 \\
\hline $05 / 11 / 88$ & $15: 05$ & - & 22.5386 & 14.9451 & 1.75100 & 2.04128 \\
\hline $05 / 11 / 88$ & $16: 58$ & - & 22.3789 & 14.9456 & 1.74923 & 2.04128 \\
\hline $05 / 12 / 88$ & $13: 25$ & - & 23.0353 & 14.9458 & 1.74566 & 2.04595 \\
\hline $05 / 12 / 88$ & $14: 25$ & - & 23.1615 & 14.9458 & 1.74498 & 2.04585 \\
\hline $05 / 12 / 88$ & $15: 53$ & - & 23.4842 & 14.9460 & 1.74402 & 2.04555 \\
\hline $06 / 17 / 88$ & $09: 24$ & 2.7529 & 20.9799 & 14.9436 & 1.73693 & 2.05351 \\
\hline $06 / 17 / 88$ & $09: 28$ & . & . & 14.9437 & 1.73660 & 2.05320 \\
\hline
\end{tabular}


FLOW TUBE MEASUREMENTS - TEST BLOCK 1

Legend: $\mathrm{Ht}=$ total head on top of the plate $\mathrm{q}=$ specific discharge $=\mathrm{Q} / \mathrm{A}, \mathrm{A}=$ area of plate $\mathrm{hb}=$ pressure head on the bottom of the plate

\begin{tabular}{|c|c|c|c|c|c|c|}
\hline Date & Time & $\begin{array}{r}\text { Pla } \\
\text { Position }\end{array}$ & Number & $\begin{array}{l}\mathrm{Ht} \\
(\mathrm{cm})\end{array}$ & $\begin{array}{c}q \\
(m / \sec )\end{array}$ & $\begin{array}{l}\mathrm{hb} \\
(\mathrm{cm})\end{array}$ \\
\hline 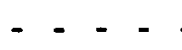 &.- & $-\ldots$ & 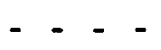 & 8 & $-\ldots-\ldots$ & -- \\
\hline $01 / 12 / 88$ & $11: 55$ & $1-B$ & 6 & -10.00 & $2.537713 \mathrm{E}-07$ & -37.02 \\
\hline $01 / 12 / 88$ & $12: 10$ & $1-B$ & 6 & -10.00 & $2.360958 \mathrm{E}-07$ & -35.14 \\
\hline $01 / 12 / 88$ & $12: 45$ & $1-B$ & 6 & -10.00 & $2.142758 \mathrm{E}-07$ & -32.82 \\
\hline $01 / 12 / 88$ & $13: 16$ & $1-B$ & 6 & -10.00 & $2.065457 \mathrm{E}-07$ & -32.00 \\
\hline $01 / 12 / 88$ & $13: 48$ & $1-B$ & 6 & -10.00 & $2.006088 \mathrm{E}-07$ & -31.36 \\
\hline $01 / 12 / 88$ & $14: 18$ & $1-B$ & 6 & -10.00 & $1.980502 \mathrm{E}-07$ & -31.09 \\
\hline $01 / 12 / 88$ & $14: 48$ & $1-B$ & 6 & -10.00 & $1.940341 \mathrm{E}-07$ & -30.66 \\
\hline $01 / 12 / 88$ & $15: 15$ & $1-B$ & 6 & -10.00 & $1.930217 \mathrm{E}-07$ & -30.56 \\
\hline $01 / 12 / 88$ & $15: 42$ & $1-B$ & 6 & -10.00 & $1.947473 E-07$ & -30.74 \\
\hline $01 / 12 / 88$ & $16: 10$ & 1-B & 6 & -10.00 & $1.907513 \mathrm{E}-07$ & -30.31 \\
\hline $01 / 12 / 88$ & $19: 47$ & $1-B$ & 6 & -10.00 & $1.821539 \mathrm{E}-07$ & -29.40 \\
\hline $01 / 13 / 88$ & $08: 18$ & $1-B$ & 6 & -10.00 & $1.734917 \mathrm{E}-07$ & -28.48 \\
\hline $01 / 13 / 88$ & $09: 07$ & $1-B$ & 6 & -10.00 & $1.939739 \mathrm{E}-07$ & -30.66 \\
\hline $01 / 13 / 88$ & $11: 43$ & $1-B$ & 6 & -10.00 & $1.893277 \mathrm{E}-07$ & -30.16 \\
\hline $01 / 13 / 88$ & $13: 31$ & $1-B$ & 6 & 20.00 & $2.292415 E-07$ & -4.41 \\
\hline $01 / 13 / 88$ & $15: 43$ & $1-B$ & 6 & 20.00 & $2.329189 \mathrm{E}-07$ & -4.80 \\
\hline $01 / 13 / 88$ & $21: 33$ & $1-B$ & 6 & 20.00 & $2.344480 E-07$ & -4.97 \\
\hline $01 / 14 / 88$ & $09: 15$ & $1-B$ & 6 & 20.00 & $2.290877 E-07$ & -4.40 \\
\hline $01 / 14 / 88$ & $11: 56$ & $1-B$ & 6 & 26.20 & $2.757743 \mathrm{E}-07$ & -3.17 \\
\hline $01 / 14 / 88$ & $13: 34$ & $1-B$ & 6 & 26.20 & $2.680595 E-07$ & -2.35 \\
\hline $01 / 14 / 88$ & $15: 42$ & $1-B$ & 6 & 26.20 & $2.778506 \mathrm{E}-07$ & -3.39 \\
\hline $01 / 14 / 88$ & $20: 56$ & $1-B$ & 6 & 25.60 & $2.850906 \mathrm{E}-07$ & -4.76 \\
\hline $01 / 15 / 88$ & $08: 14$ & $1-B$ & 6 & 25.20 & $2.760748 \mathrm{E}-07$ & -4.20 \\
\hline $01 / 15 / 88$ & $08: 40$ & $1-B$ & 6 & 27.40 & $2.906913 E-07$ & -3.56 \\
\hline $01 / 15 / 88$ & $11: 20$ & $1-B$ & 6 & 27.40 & $2.815662 E-07$ & -2.58 \\
\hline $01 / 15 / 88$ & $12: 40$ & 1-B & 6 & 27.40 & $2.827410 \mathrm{E}-07$ & -2.71 \\
\hline $01 / 15 / 88$ & $15: 41$ & 1-B & 6 & 42.30 & $4.089513 E-07$ & -1.25 \\
\hline $01 / 15 / 88$ & $16: 33$ & 1-B & 6 & 50.00 & $4.676852 E-07$ & 0.20 \\
\hline $01 / 15 / 88$ & $17: 37$ & 1-B & 6 & 50.00 & $4.683136 \mathrm{E}-07$ & 0.13 \\
\hline $01 / 15 / 88$ & $22: 11$ & 1-B & 6 & 51.50 & $4.740017 \mathrm{E}-07$ & 1.02 \\
\hline $01 / 15 / 88$ & $22: 28$ & 1-B & 6 & 51.50 & $4.813072 E-07$ & 0.24 \\
\hline $01 / 16 / 88$ & $10: 02$ & 1-B & 6 & 50.20 & $4.417470 \mathrm{E}-07$ & 3.16 \\
\hline $01 / 16 / 88$ & $14: 40$ & $1-B$ & 6 & 49.80 & $4.371052 \mathrm{E}-07$ & 3.25 \\
\hline $01 / 16 / 88$ & $14: 55$ & $1-B$ & 6 & 52.00 & $4.542462 \mathrm{E}-07$ & 3.63 \\
\hline $01 / 16 / 88$ & $21: 52$ & $1-B$ & 6 & 51.20 & $4.422606 \mathrm{E}-07$ & 4.10 \\
\hline $01 / 17 / 88$ & $11: 01$ & $1-B$ & 6 & 49.40 & $4.108364 \mathrm{E}-07$ & 5.65 \\
\hline $01 / 17 / 88$ & $16: 05$ & $1-B$ & 6 & 52.00 & $4.298407 E-07$ & 6.23 \\
\hline $01 / 17 / 88$ & $21: 00$ & $1-B$ & 6 & 51.40 & $4.246060 \mathrm{E}-07$ & 6.18 \\
\hline $01 / 17 / 88$ & $09: 15$ & $1-B$ & 6 & 50.00 & $3.915727 \mathrm{E}-07$ & 8.30 \\
\hline $01 / 17 / 88$ & $12: 11$ & $1-B$ & 6 & 49.60 & $3.921819 \mathrm{E}-07$ & 7.84 \\
\hline $01 / 17 / 88$ & $16: 31$ & $1-B$ & 6 & 49.10 & $3.881412 \mathrm{E}-07$ & 7.77 \\
\hline
\end{tabular}


TABLE B.10 (

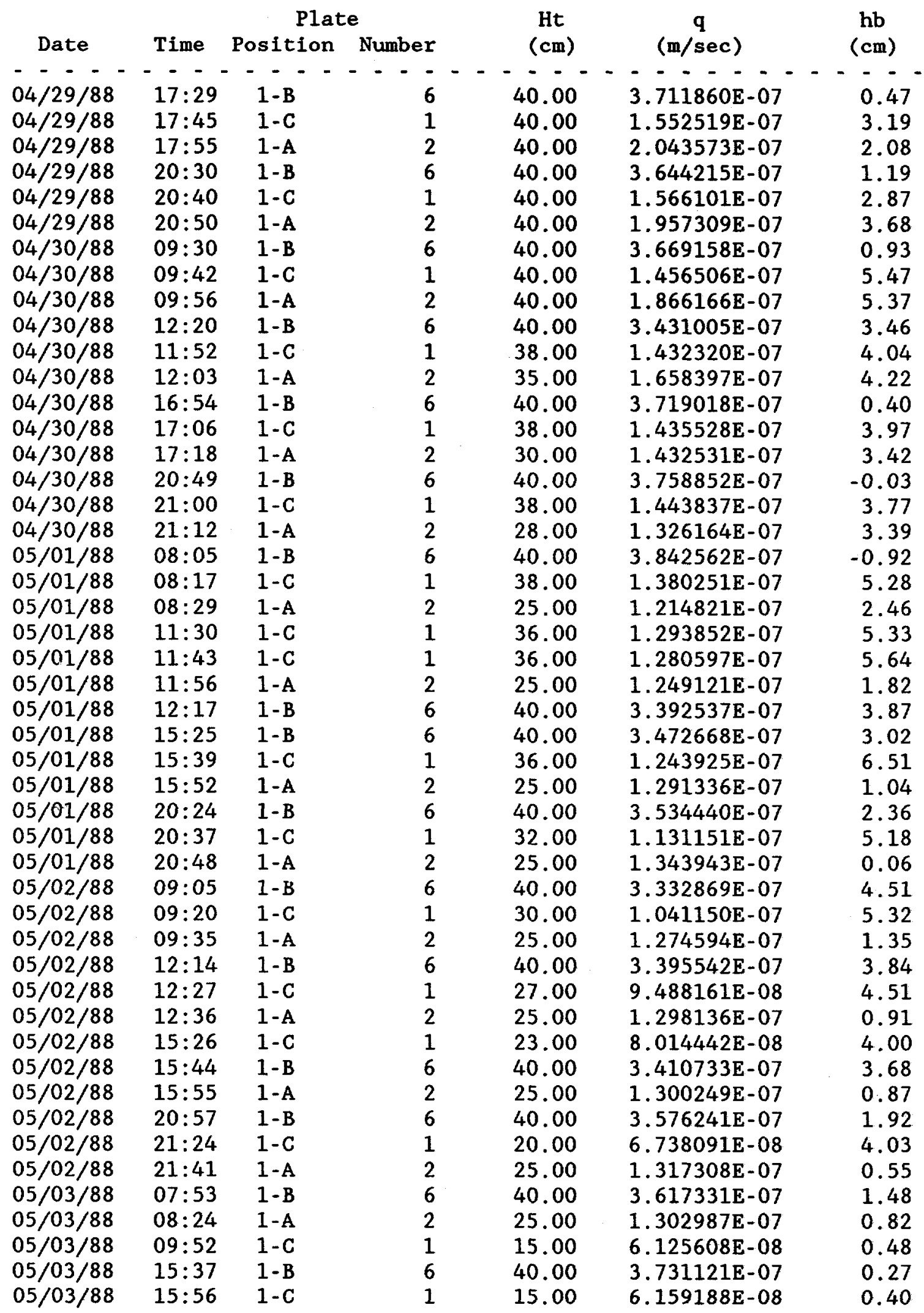


TABLE B.10 (Continued)

\begin{tabular}{|c|c|c|c|c|c|c|}
\hline Date & Time & $\begin{array}{r}\text { Pla } \\
\text { Position }\end{array}$ & Number & $\begin{array}{l}\mathrm{Ht} \\
(\mathrm{cm})\end{array}$ & $\underset{(m / s e c)}{q}$ & $\begin{array}{l}\mathrm{hb} \\
(\mathrm{cm})\end{array}$ \\
\hline 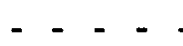 & 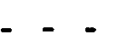 & --- & --- & ---- & $-\ldots-\cdot$ & -- \\
\hline $5 / 03 / 88$ & $15: 54$ & $1-\mathrm{A}$ & 2 & 25.00 & 1. $322111 \mathrm{E}-07$ & 0.46 \\
\hline $05 / 03 / 88$ & $20: 31$ & $1-A$ & 2 & 25.00 & 1. $341561 \mathrm{E}-07$ & 0.10 \\
\hline $05 / 03 / 88$ & $20: 36$ & $1-C$ & 1 & 10.00 & $4.544629 \mathrm{E}-08$ & -0.77 \\
\hline $05 / 03 / 88$ & $20: 47$ & $1-B$ & 6 & 40.00 & $3.505562 \mathrm{E}-07$ & 2.67 \\
\hline $05 / 04 / 88$ & $08: 11$ & $1-\mathrm{B}$ & 6 & 40.00 & $3.710768 \mathrm{E}-07$ & 0.48 \\
\hline $05 / 04 / 88$ & $08: 21$ & $1-C$ & 1 & 10.00 & $4.541229 \mathrm{E}-08$ & -0.77 \\
\hline $05 / 04 / 88$ & $08: 46$ & $1-A$ & 2 & 25.00 & 1. 341561E-07 & 0.10 \\
\hline $05 / 04 / 88$ & $11: 45$ & $1-C$ & 1 & -5.00 & $2.680404 E-08$ & -11.35 \\
\hline $05 / 04 / 88$ & $11: 54$ & $1-\mathrm{A}$ & 2 & 25.00 & $1.366928 \mathrm{E}-07$ & -0.37 \\
\hline $05 / 04 / 88$ & $12: 05$ & 1-B & 6 & 40.00 & $3.804559 \mathrm{E}-07$ & -0.52 \\
\hline $05 / 04 / 88$ & $20: 41$ & $1-C$ & 1 & -5.00 & $2.952989 \mathrm{E}-08$ & -12.00 \\
\hline $05 / 04 / 88$ & $20: 41$ & $1-\mathrm{A}$ & 2 & 25.00 & 1. $375602 E-07$ & -0.53 \\
\hline $05 / 04 / 88$ & $20: 28$ & $1-B$ & 6 & 40.00 & $3.726122 \mathrm{E}-07$ & 0.32 \\
\hline $05 / 05 / 88$ & $08: 34$ & $1-C$ & 1 & -5.00 & $2.639505 \mathrm{E}-08$ & -11.26 \\
\hline $05 / 05 / 88$ & $08: 29$ & $1-\mathrm{A}$ & 2 & 25.00 & $1.325972 \mathrm{E}-07$ & 0.39 \\
\hline $05 / 05 / 88$ & $08: 43$ & $1-B$ & 6 & 40.00 & $3.719538 E-07$ & 0.39 \\
\hline $05 / 05 / 88$ & $12: 56$ & $1-\mathrm{A}$ & 2 & 25.00 & $1.350398 \mathrm{E}-07$ & -0.06 \\
\hline $05 / 05 / 88$ & $12: 20$ & $1-B$ & 6 & 40.00 & $3.625418 \mathrm{E}-07$ & 1.39 \\
\hline $05 / 05 / 88$ & $18: 26$ & $1-C$ & 1 & 0.00 & $5.218347 \mathrm{E}-08$ & -12.37 \\
\hline $05 / 05 / 88$ & $18: 50$ & $1-\mathrm{A}$ & 2 & 25.00 & $1.348533 E-07$ & -0.03 \\
\hline $05 / 05 / 88$ & $19: 02$ & $1-B$ & 6 & 40.00 & $4.469874 E-07$ & -7.60 \\
\hline $05 / 06 / 88$ & $13: 29$ & $1-B$ & 6 & 40.00 & 3.349892E-07 & 4.33 \\
\hline $05 / 06 / 88$ & $13: 57$ & $1-\mathrm{C}$ & 1 & -5.00 & $3.333656 E-08$ & -12.90 \\
\hline $05 / 06 / 88$ & $13: 44$ & $1-\mathrm{A}$ & 2 & 25.00 & $1.296936 \mathrm{E}-07$ & 0.93 \\
\hline $05 / 07 / 88$ & $14: 32$ & $1-B$ & 6 & 40.00 & $3.536107 \mathrm{E}-07$ & 2.34 \\
\hline $05 / 07 / 88$ & $14: 43$ & $1-\mathrm{A}$ & 2 & 25.00 & 1. $270859 \mathrm{E}-07$ & 1.42 \\
\hline $05 / 07 / 88$ & $14: 54$ & $1-C$ & 1 & -5.00 & $2.930464 \mathrm{E}-08$ & -11.95 \\
\hline $05 / 09 / 88$ & $10: 18$ & $1-C$ & 1 & -10.00 & 1. $322313 E-08$ & -13.13 \\
\hline $05 / 09 / 88$ & $10: 26$ & $1-\mathrm{A}$ & 2 & 25.00 & $1.287266 \mathrm{E}-07$ & 1.11 \\
\hline $05 / 09 / 88$ & $10: 13$ & $1-B$ & 6 & 40.00 & $3.618304 \mathrm{E}-07$ & 1.47 \\
\hline $05 / 10 / 88$ & $14: 20$ & $1-C$ & 1 & -10.00 & $1.563402 \mathrm{E}-08$ & -13.71 \\
\hline $05 / 10 / 88$ & $13: 26$ & $1-A$ & 2 & 25.00 & $1.374980 \mathrm{E}-07$ & -0.52 \\
\hline $05 / 10 / 88$ & $13: 14$ & $1-B$ & 6 & 40.00 & $3.293492 \mathrm{E}-07$ & 4.93 \\
\hline $05 / 11 / 88$ & $14: 56$ & $1-C$ & 1 & -10.00 & 1. $316363 E-08$ & -13.12 \\
\hline $05 / 11 / 88$ & $16: 44$ & $1-A$ & 2 & 25.00 & $1.405255 E-07$ & -1.08 \\
\hline $05 / 11 / 88$ & $16: 16$ & $1-B$ & 6 & 40.00 & $3.365574 E-07$ & 4.16 \\
\hline $05 / 12 / 88$ & $15: 00$ & $1-C$ & 1 & -10.00 & $1.416306 \mathrm{E}-08$ & -13.36 \\
\hline $05 / 12 / 88$ & $16: 16$ & $1-A$ & 2 & 25.00 & $1.433177 \mathrm{E}-07$ & -1.60 \\
\hline $05 / 12 / 88$ & $16: 00$ & $1-B$ & 6 & 40.00 & $3.443195 \mathrm{E}-07$ & 3.33 \\
\hline $05 / 13 / 88$ & $15: 32$ & $1-C$ & 1 & -10.00 & 1. $381493 \mathrm{E}-08$ & -13.28 \\
\hline $05 / 13 / 88$ & $17: 01$ & $1-A$ & 2 & 25.00 & $1.405460 \mathrm{E}-07$ & -1.08 \\
\hline $05 / 13 / 88$ & $16: 45$ & $1-B$ & 6 & 40.00 & $3.482922 \mathrm{E}-07$ & 2.91 \\
\hline $05 / 18 / 88$ & $12: 46$ & $1-C$ & 1 & -10.00 & $6.409404 E-09$ & -11.52 \\
\hline $05 / 18 / 88$ & $12: 05$ & $1-A$ & 2 & 25.00 & $1.269851 \mathrm{E}-07$ & 1.43 \\
\hline $05 / 18 / 88$ & $11: 19$ & $1-B$ & 6 & 40.00 & $3.020745 E-07$ & 7.83 \\
\hline $05 / 28 / 88$ & $14: 39$ & $1-B$ & 6 & 40.00 & $2.955604 \mathrm{E}-07$ & 8.53 \\
\hline $05 / 28 / 88$ & $14: 53$ & $1-A$ & 2 & 25.00 & $1.238069 \mathrm{E}-07$ & 2.02 \\
\hline $05 / 30 / 88$ & $08: 57$ & $1-B$ & 6 & 38.00 & $3.126046 \mathrm{E}-07$ & 4.71 \\
\hline
\end{tabular}


TABLE B.10 (

\begin{tabular}{|c|c|c|c|c|c|c|}
\hline & & Pla & & $\mathrm{Ht}$ & $(m / 20-1)$ & hb \\
\hline $\begin{array}{l}\text { Date } \\
---\end{array}$ & $\begin{array}{r}\text { Time } \\
-\quad-\quad-\end{array}$ & Position & Number & (cm) & $\begin{array}{r}(\mathrm{m} / \mathrm{sec}) \\
-\ldots--\end{array}$ & $\begin{array}{r}(\mathrm{cm}) \\
-\quad-\quad-\end{array}$ \\
\hline $05 / 30 / 88$ & $09: 13$ & $1-\mathrm{A}$ & 2 & 25.00 & $1.236449 \mathrm{E}-07$ & 2.05 \\
\hline $05 / 31 / 88$ & $08: 03$ & $1-B$ & 6 & 38.00 & $2.820580 \mathrm{E}-07$ & 7.96 \\
\hline $05 / 31 / 88$ & $08: 17$ & $1-\mathrm{A}$ & 2 & 25.00 & $1.234966 \mathrm{E}-07$ & 2.08 \\
\hline $05 / 31 / 88$ & $08: 21$ & $1-C$ & 1 & -10.00 & $9.016836 \mathrm{E}-09$ & -12.14 \\
\hline $05 / 31 / 88$ & $14: 36$ & 1-B & 5 & 37.50 & $2.271922 \mathrm{E}-07$ & -4.94 \\
\hline $05 / 31 / 88$ & $15: 02$ & $1-\mathrm{C}$ & 4 & -6.00 & $5.304564 \mathrm{E}-08$ & -13.75 \\
\hline $05 / 31 / 88$ & $14: 55$ & $1-A$ & 3 & 25.30 & $6.646474 \mathrm{E}-08$ & -0.49 \\
\hline $06 / 01 / 88$ & $09: 26$ & 1-B & 5 & 37.50 & $2.409473 E-07$ & -7.51 \\
\hline $06 / 01 / 88$ & $09: 44$ & $1-\mathrm{A}$ & 3 & 25.30 & $7.268829 \mathrm{E}-08$ & -2.91 \\
\hline $06 / 02 / 88$ & $08: 10$ & $1-B$ & 5 & 37.50 & $2.378003 E-07$ & -6.92 \\
\hline $06 / 02 / 88$ & $09: 02$ & $1-C$ & 4 & 5.00 & $3.264449 E-08$ & 0.23 \\
\hline $06 / 02 / 88$ & $09: 48$ & $1-\mathrm{A}$ & 3 & 25.30 & $7.423028 E-08$ & -3.51 \\
\hline $06 / 04 / 88$ & $13: 55$ & 1-B & 5 & 37.50 & $2.363852 \mathrm{E}-07$ & -6.65 \\
\hline $06 / 04 / 88$ & $14: 25$ & $1-C$ & 4 & 5.00 & $4.127218 \mathrm{E}-08$ & -1.03 \\
\hline $06 / 04 / 88$ & $14: 56$ & $1-\mathrm{A}$ & 3 & 27.00 & $7.470695 \mathrm{E}-08$ & -1.99 \\
\hline $06 / 05 / 88$ & $15: 01$ & $1-\mathrm{A}$ & 3 & 27.00 & $7.667860 \mathrm{E}-08$ & -2.76 \\
\hline $06 / 06 / 88$ & $15: 54$ & $1-\mathrm{A}$ & 3 & 29.00 & $8.032289 E-08$ & -2.17 \\
\hline $06 / 06 / 88$ & $16: 22$ & $1-\mathrm{C}$ & 4 & 5.00 & $4.018163 E-08$ & -0.87 \\
\hline $06 / 06 / 88$ & $16: 26$ & 1-B & 5 & 37.50 & $2.397386 E-07$ & -7.28 \\
\hline $06 / 07 / 88$ & $08: 09$ & $1-A$ & 3 & 30.00 & $8.421386 \mathrm{E}-08$ & -2.68 \\
\hline 06/07/88 & $08: 19$ & $1-C$ & 4 & 5.00 & $4.317180 \mathrm{E}-08$ & -1.31 \\
\hline $06 / 07 / 88$ & $08: 31$ & $1-B$ & 5 & 37.50 & $2.375153 \mathrm{E}-07$ & -6.86 \\
\hline $06 / 10 / 88$ & $08: 12$ & $1-A$ & 3 & 30.00 & $8.185627 \mathrm{E}-08$ & -1.77 \\
\hline $06 / 10 / 88$ & $08: 53$ & $1-C$ & 4 & 5.00 & $1.098061 \mathrm{E}-07$ & -11.04 \\
\hline $06 / 10 / 88$ & $08: 56$ & $1-B$ & 5 & 37.50 & $2.349621 E-07$ & -6.39 \\
\hline $06 / 10 / 88$ & $19: 47$ & $1-\mathrm{A}$ & 3 & 31.00 & $8.640446 E-08$ & -2.53 \\
\hline $06 / 10 / 88$ & $19: 48$ & $1-C$ & 4 & 6.00 & $1.090441 \mathrm{E}-07$ & -9.93 \\
\hline $06 / 10 / 88$ & $20: 03$ & $1-B$ & 5 & 37.50 & $2.401988 \mathrm{E}-07$ & -7.37 \\
\hline $06 / 12 / 88$ & $11: 59$ & $1-A$ & 3 & 31.00 & $8.088882 E-08$ & -0.39 \\
\hline $06 / 12 / 88$ & $12: 06$ & $1-C$ & 4 & 6.00 & $8.583308 \mathrm{E}-08$ & -6.54 \\
\hline $06 / 12 / 88$ & $12: 18$ & 1-B & 5 & 37.50 & $2.235242 \mathrm{E}-07$ & -4.25 \\
\hline $06 / 13 / 88$ & $09: 34$ & $1-\mathrm{A}$ & 3 & 31.00 & $7.868237 \mathrm{E}-08$ & 0.47 \\
\hline $06 / 13 / 88$ & $09: 46$ & $1-\mathrm{C}$ & 4 & 6.00 & $1.214638 \mathrm{E}-07$ & -11.74 \\
\hline $06 / 13 / 88$ & $09: 56$ & $1-B$ & 5 & 37.50 & $2.181141 E-07$ & -3.24 \\
\hline $06 / 13 / 88$ & $19: 48$ & $1-\mathrm{A}$ & 3 & 30.00 & $8.202287 E-08$ & -1.83 \\
\hline $06 / 13 / 88$ & $20: 07$ & $1-C$ & 4 & 5.00 & $3.359502 E-08$ & 0.09 \\
\hline $06 / 13 / 88$ & $20: 20$ & $1-B$ & 5 & 35.75 & $2.315960 \mathrm{E}-07$ & -7.51 \\
\hline $06 / 14 / 88$ & $08: 00$ & 1-A & 3 & 30.00 & $7.714465 \mathrm{E}-08$ & 0.06 \\
\hline $06 / 14 / 88$ & $08: 20$ & $1-C$ & 4 & 5.00 & $3.717595 E-08$ & -0.43 \\
\hline $06 / 14 / 88$ & $08: 27$ & 1-B & 5 & 35.75 & $2.250555 \mathrm{E}-07$ & -6.29 \\
\hline $06 / 14 / 88$ & $11: 30$ & 1-B & 5 & 35.70 & $2.202417 \mathrm{E}-07$ & -5.44 \\
\hline $06 / 14 / 88$ & $14: 05$ & 1-B & 5 & 35.80 & $2.243962 E-07$ & -6.11 \\
\hline $06 / 15 / 88$ & $08: 44$ & $1-A$ & 3 & 30.00 & $7.904195 E-08$ & -0.67 \\
\hline $06 / 15 / 88$ & $08: 58$ & $1-C$ & 4 & 5.00 & $3.591987 \mathrm{E}-08$ & -0.25 \\
\hline $06 / 15 / 88$ & $09: 05$ & $1-B$ & 5 & 36.20 & $2.280518 \mathrm{E}-07$ & -6.40 \\
\hline $06 / 16 / 88$ & $08: 49$ & 1-A & 3 & 30.00 & $8.210233 E-08$ & -1.86 \\
\hline $06 / 16 / 88$ & $09: 10$ & $1-B$ & 5 & 36.20 & $2.270191 E-07$ & -6.20 \\
\hline $06 / 16 / 88$ & $09: 44$ & 1-B & 5 & 36.20 & $2.592950 \mathrm{E}-07$ & -12.23 \\
\hline
\end{tabular}


TABLE B.10 (Continued)

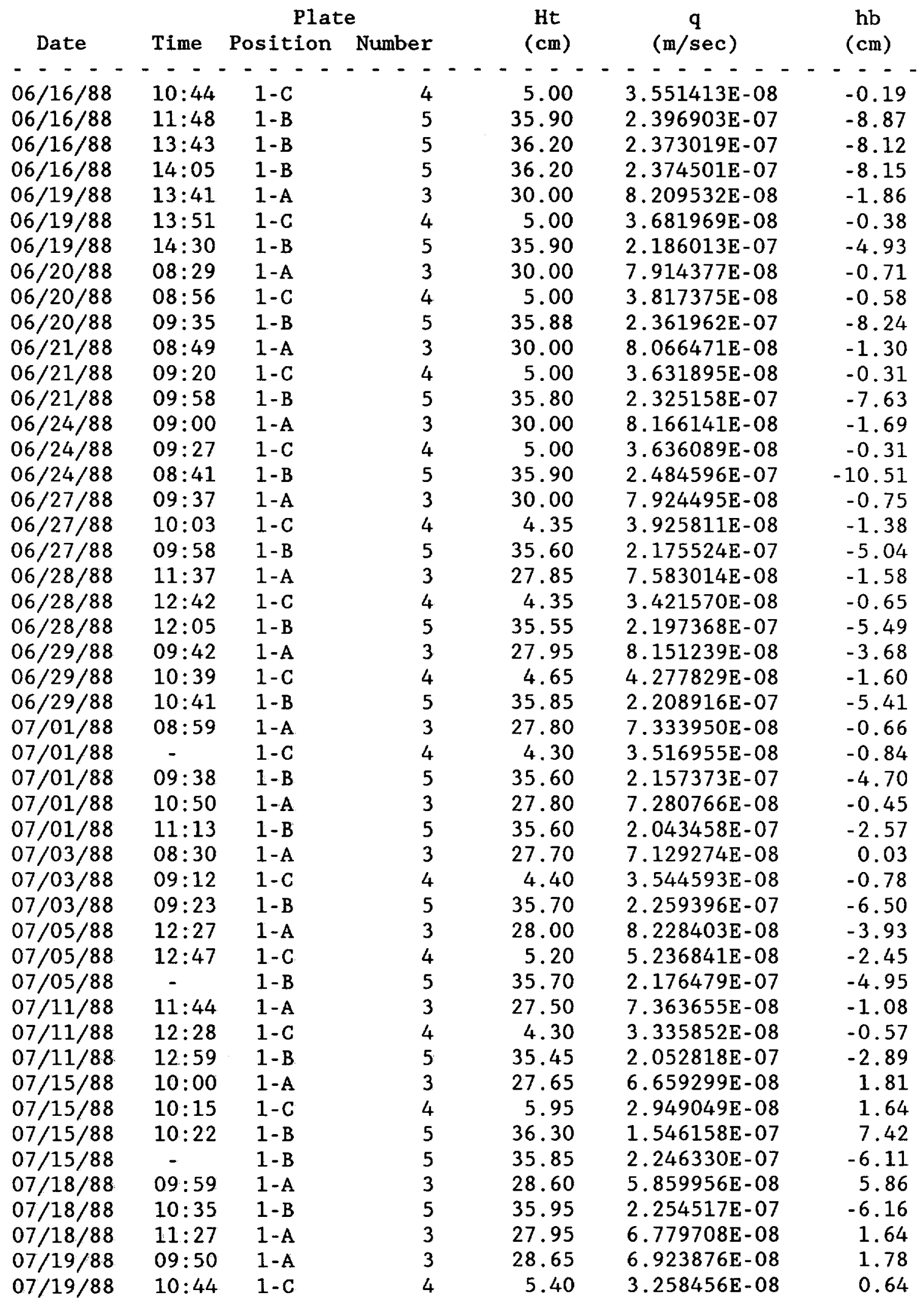


TABLE B.10 (Continued)

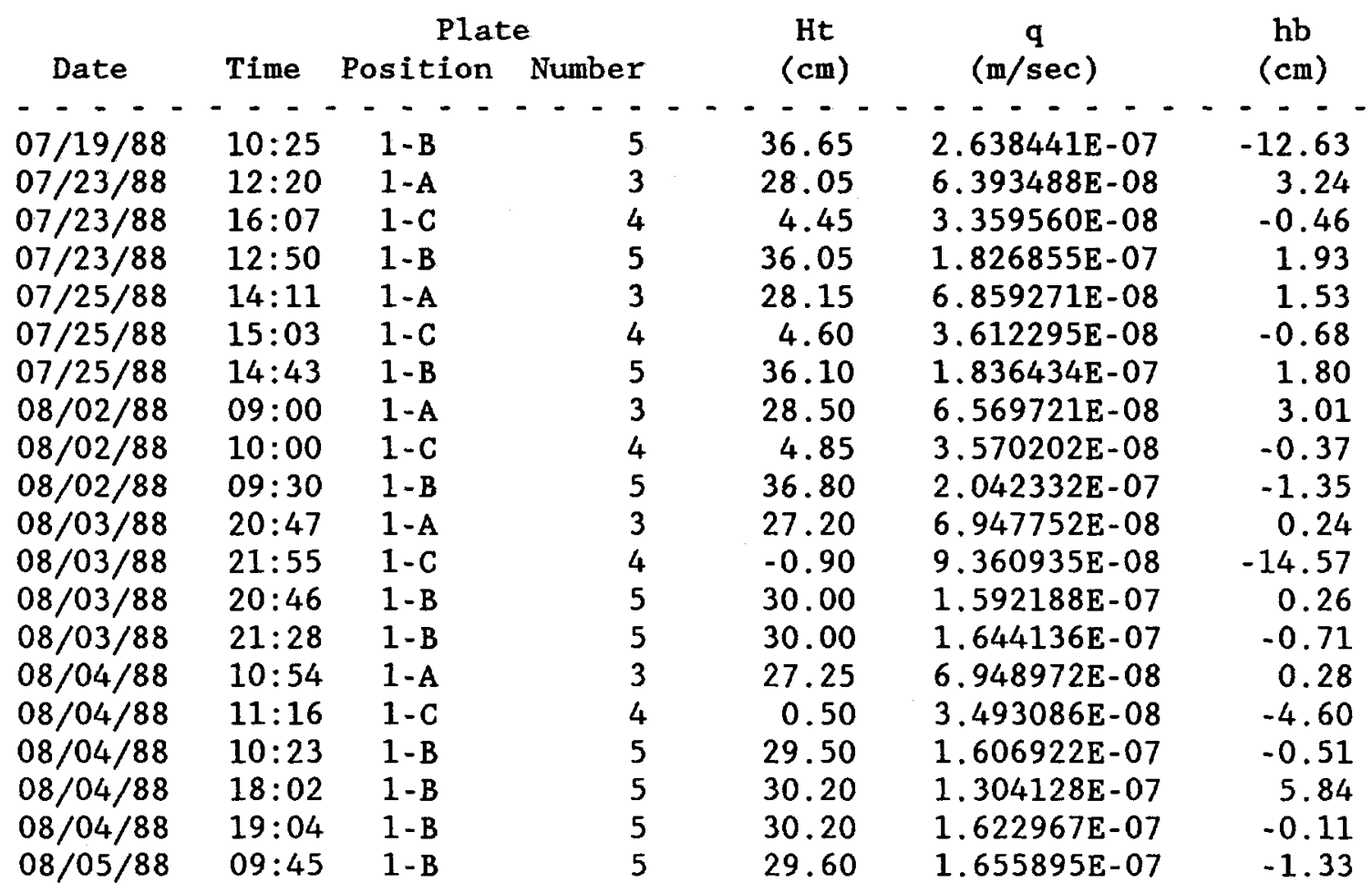


TABLE B.11

TEST BLOCK 1 MOISTURE POTENTIAL DATA TENSIOMETER 1 - JULY 1988

\begin{tabular}{|c|c|c|c|c|c|c|c|}
\hline Date & Time & Port & $\begin{array}{l}\text { Resist. } \\
\text { (kohms) }\end{array}$ & $\begin{array}{l}\text { Air } \\
\text { Temp } \\
\left({ }^{\circ} \mathrm{C}\right)\end{array}$ & $\begin{array}{l}\text { Transd. } \\
\text { Input } \\
\text { (volts) }\end{array}$ & $\begin{array}{c}\text { Transducer } \\
\text { Output } \\
\text { (volts) }\end{array}$ & $\begin{array}{c}\text { Average } \\
\text { Transd. } \\
\text { Output } \\
\text { (volts) }\end{array}$ \\
\hline & -- & & -- & - & -- & $-\ldots$ & $-\quad-\quad-$ \\
\hline $07 / 13 / 88$ & $16: 35$ & 5FUS & - & - & - & $1.360-1.390$ & 1.37500 \\
\hline $07 / 13 / 88$ & $16: 45$ & 5FUC & - & - & $=$ & $1.389-1.392$ & 1.39050 \\
\hline $07 / 13 / 88$ & $16: 49$ & $5 \mathrm{FUC}$ & - & - & - & $1.3957-1.3963$ & 1.39600 \\
\hline $07 / 13 / 88$ & $17: 28$ & 5FLS & - & - & - & - & 1.38550 \\
\hline $07 / 13 / 88$ & $20: 12$ & 5FLC & - & - & - & - & 1.37140 \\
\hline $07 / 13 / 88$ & $20: 20$ & 5FLC & 3.118 & 18.26 & 7.9982 & - & 1.36940 \\
\hline $07 / 13 / 88$ & $20: 49$ & 5FLC & - & - & - & - & 1.36800 \\
\hline $07 / 13 / 88$ & $21: 20$ & 5FLC & 3.068 & 18.63 & 7.9982 & $1.368-1.370$ & 1.36900 \\
\hline $07 / 14 / 88$ & $12: 09$ & $5 F U S$ & 3.128 & 18.19 & 7.9986 & $1.3844-1.3848$ & 1.38450 \\
\hline $07 / 14 / 88$ & $12: 32$ & 5FUC & - & - & - & $1.3832-1.3839$ & 1.38355 \\
\hline $07 / 14 / 88$ & $12: 56$ & 5FLS & - & - & 7.9987 & $1.3741-1.3749$ & 1.37450 \\
\hline $07 / 14 / 88$ & $13: 33$ & 5FLC & 3.078 & 18.55 & 7.9987 & $1.3640-1.3650$ & 1.36450 \\
\hline $07 / 14 / 88$ & $13: 48$ & 5FLC & - & - & - & $1.3627-1.3633$ & 1.36300 \\
\hline $07 / 15 / 88$ & $08: 58$ & 5FLC & 3.220 & 17.52 & 7.9988 & $1.35105-1.35155$ & 1.35130 \\
\hline $07 / 15 / 88$ & $09: 45$ & 5FLS & - & - & - & $1.36510-1.36580$ & 1.36545 \\
\hline $07 / 15 / 88$ & $09: 58$ & 5 FUS & 3.171 & 17.88 & 7.9979 & $1.36950-1.36990$ & 1.36970 \\
\hline $07 / 15 / 88$ & $10: 28$ & $5 \mathrm{FUC}$ & - & - & 7.9977 & $1.36680-1.36720$ & 1.36700 \\
\hline $07 / 15 / 88$ & $10: 50$ & $4 \mathrm{MU}$ & - & - & - & - & 1.38000 \\
\hline $07 / 15 / 88$ & $10: 56$ & 4ML & - & - & - & - & 1.38300 \\
\hline $07 / 15 / 88$ & $11: 07$ & FACE 3 & - & - & - & - & 1.38300 \\
\hline $07 / 15 / 88$ & $11: 15$ & $5 F U C$ & - & - & - & $1.376-1.380$ & 1.37800 \\
\hline $07 / 16 / 88$ & $11: 01$ & 5FUS & 2.860 & 20.16 & 7.9979 & $1.3505-1.3513$ & 1.35800 \\
\hline $07 / 16 / 88$ & $11: 12$ & 5FUC & - & - & - & $1.3405-1.3413$ & 1.34800 \\
\hline $07 / 16 / 88$ & $11: 25$ & 5 FLS & 2.807 & 20.56 & 7.9979 & $1.33500-1.33550$ & 1.33525 \\
\hline $07 / 16 / 88$ & $11: 36$ & 5FLC & - & - & - & $1.31280-1.31320$ & 1.31300 \\
\hline $07 / 24 / 88$ & $20: 59$ & 5FUS & - & - & - & - & 1.30620 \\
\hline $07 / 24 / 88$ & $21: 12$ & 5 FUS & 2.788 & 20.71 & 7.9995 & - & 1.30395 \\
\hline $07 / 24 / 88$ & $21: 44$ & 5FUS & 2.775 & 20.81 & 7.9995 & - & 1.30360 \\
\hline $07 / 25 / 88$ & $08: 37$ & 5 FUS & 3.082 & 18.53 & 7.9996 & - & 1.30400 \\
\hline $07 / 25 / 88$ & $08: 44$ & $5 F U S$ & - & - & - & - & 1.30275 \\
\hline $07 / 25 / 88$ & $09: 01$ & $5 \mathrm{FUS}$ & 3.066 & 18.64 & 7.9996 & - & 1.30200 \\
\hline $07 / 25 / 88$ & $09: 51$ & 5 FUS & - & - & - & - & 1.30100 \\
\hline $07 / 25 / 88$ & $10: 22$ & 5FUS & 2.861 & 20.15 & 7.9996 & - & 1.29830 \\
\hline $07 / 25 / 88$ & $12: 29$ & $5 \mathrm{FUC}$ & 2.810 & 20.54 & 7.9994 & - & 1.27960 \\
\hline $07 / 25 / 88$ & $13: 40$ & $5 \mathrm{FUC}$ & 2.808 & 20.55 & 7.9993 & - & 1.27790 \\
\hline $07 / 25 / 88$ & $14: 32$ & $5 F U C$ & 2.777 & 20.79 & 7.9992 & - & 1.27350 \\
\hline $07 / 25 / 88$ & $15: 07$ & $5 \mathrm{FUC}$ & 2.777 & 20.79 & 7.9993 & - & 1.27210 \\
\hline $07 / 25 / 88$ & $16: 35$ & $5 \mathrm{FUC}$ & 2.740 & 21.08 & 7.9992 & - & 1.26730 \\
\hline $07 / 25 / 88$ & $20: 08$ & 5 FUC & 2.825 & 20.42 & 7.9993 & - & 1.26965 \\
\hline $07 / 25 / 88$ & $20: 18$ & 5FUC & - & - & - & - & 1.27050 \\
\hline $07 / 26 / 88$ & $08: 03$ & 5FUC & 3.068 & 18.63 & 7.9994 & - & 1.27410 \\
\hline $07 / 26 / 88$ & $08: 37$ & 5FLS & 3.029 & 18.91 & 7.9994 & - & 1.27190 \\
\hline $07 / 26 / 88$ & $10: 02$ & 5FLS & - & - & - & - & 1.27340 \\
\hline
\end{tabular}


TABLE B.11 (continued)

\begin{tabular}{|c|c|c|c|c|c|c|c|}
\hline Date & Time & Port & $\begin{array}{l}\text { Resist. } \\
\text { (kohms) }\end{array}$ & $\begin{array}{l}\text { Air } \\
\text { Temp } \\
\left({ }^{\circ} \mathrm{C}\right)\end{array}$ & $\begin{array}{c}\text { Transd. } \\
\text { Input } \\
\text { (volts) }\end{array}$ & $\begin{array}{c}\text { Transducer } \\
\text { Output } \\
\text { (volts) }\end{array}$ & $\begin{array}{c}\text { Average } \\
\text { Transd. } \\
\text { Output } \\
\text { (volts) }\end{array}$ \\
\hline- & $-\quad-$ & -- & --- & -- & & --- & $-\quad--$ \\
\hline $07 / 26 / 88$ & $15: 11$ & 5FLS & 2.833 & 20.36 & 7.9991 & - & 1.26315 \\
\hline $07 / 26 / 88$ & $15: 50$ & 5FLS & - & - & - & - & 1.26120 \\
\hline $07 / 26 / 88$ & $16: 23$ & 5FLS & 2.774 & 20.81 & 7.9991 & - & 1.25970 \\
\hline $07 / 26 / 88$ & $17: 21$ & 5FLS & 2.775 & 20.81 & 7.9991 & - & 1.25780 \\
\hline $07 / 27 / 88$ & $07: 59$ & 5FLS & 3.053 & 18.74 & 7.9993 & - & 1.26460 \\
\hline $07 / 27 / 88$ & $08: 25$ & 5FLS & - & - & - & - & 1.26230 \\
\hline $07 / 27 / 88$ & $09: 45$ & 5FLS & 2.830 & 20.38 & 7.9988 & - & 1.25660 \\
\hline $07 / 27 / 88$ & $10: 40$ & 5FLS & 2.829 & 20.39 & 7.9988 & - & 1.25890 \\
\hline $07 / 27 / 88$ & $10: 58$ & 5FLC & - & - & - & - & 1.24230 \\
\hline $07 / 27 / 88$ & $12: 54$ & 5FLC & 2.810 & 20.54 & 7.9987 & - & 1.24500 \\
\hline $07 / 27 / 88$ & $14: 45$ & 5FLC & 2.782 & 20.75 & 7.9985 & - & 1.2381 \\
\hline $07 / 27 / 88$ & $17: 07$ & 5FLC & 2.759 & 20.93 & 7.9985 & - & 1.2357 \\
\hline $07 / 27 / 88$ & $19: 27$ & $5 F L C$ & 2.818 & 20.47 & 7.9986 & - & 1.2391 \\
\hline $07 / 28 / 88$ & $08: 03$ & 5FLC & 3.038 & 18.85 & 7.9987 & - & 1.2384 \\
\hline $07 / 28 / 88$ & $11: 18$ & 5FLC & - & - & - & - & 1.2405 \\
\hline $07 / 28 / 88$ & $15: 36$ & 5FLC & 2.870 & 20.08 & 7.9990 & - & 1.2311 \\
\hline $07 / 28 / 88$ & $16: 51$ & 5FLC & 2.842 & 20.29 & 7.9990 & - & 1.22860 \\
\hline $07 / 28 / 88$ & $10: 36$ & 5FLC & 2.782 & 20.75 & 7.9989 & - & 1.21560 \\
\hline $07 / 28 / 88$ & $16: 25$ & 5FLC & 2.810 & 20.54 & 7.9988 & - & 1.21100 \\
\hline $07 / 28 / 88$ & $13: 11$ & 5FLC & 3.025 & 18.94 & 7.9989 & - & 1. 2118 \\
\hline $07 / 28 / 88$ & $16: 56$ & 5FLC & 3.033 & 18.88 & 7.9989 & - & 1.2069 \\
\hline $07 / 28 / 88$ & $12: 56$ & 5FLC & 3.060 & 18.69 & 7.9989 & - & 1.1970 \\
\hline $07 / 29 / 88$ & $10: 36$ & $5 F L C$ & 2.782 & 20.75 & 7.9989 & - & 1.21560 \\
\hline $07 / 29 / 88$ & $16: 25$ & 5FLC & 2.810 & 20.54 & 7.9988 & - & 1.21100 \\
\hline $07 / 30 / 88$ & $13: 11$ & 5FLC & 3.025 & 18.94 & 7.9989 & - & 1.21185 \\
\hline $07 / 30 / 88$ & $16: 56$ & 5FLC & 3.033 & 18.88 & 7.9989 & - & 1.2069 \\
\hline $07 / 31 / 88$ & $12: 56$ & 5FLC & 3.060 & 18.69 & 7.9989 & - & 1.1970 \\
\hline $07 / 31 / 88$ & $17: 02$ & 5FLC & 3.070 & 18.61 & 7.9988 & - & 1.1912 \\
\hline $08 / 01 / 88$ & $07: 40$ & $5 F L C$ & 3.040 & 18.83 & 7.9988 & - & 1.1860 \\
\hline $08 / 01 / 88$ & $10: 33$ & 5FLC & 3.036 & 18.86 & 7.9989 & - & 1.1822 \\
\hline $08 / 01 / 88$ & $14: 27$ & 5FLS & 2.865 & 20.12 & 7.9989 & - & 1.2014 \\
\hline $08 / 01 / 88$ & $15: 27$ & 5FLS & 2.880 & 20.01 & 7.9988 & - & 1.2005 \\
\hline $08 / 01 / 88$ & $16: 31$ & 5FLS & 2.860 & 20.16 & 7.9989 & - & 1.1998 \\
\hline $08 / 01 / 88$ & $19: 48$ & 5FLS & 2.851 & 20.22 & 7.9989 & - & 1.1995 \\
\hline $08 / 01 / 88$ & $21: 40$ & 5FUC & 2.781 & 20.76 & 7.9988 & - & 1.1990 \\
\hline $08 / 02 / 88$ & $07: 54$ & 5FUC & 3.052 & 18.74 & 7.9986 & - & 1.2053 \\
\hline $08 / 02 / 88$ & $13: 31$ & 5FUC & 3.029 & 18.91 & 7.9985 & - & 1.2044 \\
\hline $08 / 02 / 88$ & $16: 11$ & 5FUC & 2.888 & 19.95 & 7.9982 & - & 1.1741 \\
\hline $08 / 02 / 88$ & $16: 34$ & $5 F U C$ & - & - & - & - & 1.1721 \\
\hline $08 / 02 / 88$ & $19: 38$ & 5FUC & 2.830 & 20.38 & 7.9993 & - & 1.1966 \\
\hline $08 / 02 / 88$ & $20: 58$ & 5FUC & 3.053 & 18.74 & 7.9992 & - & 1.2003 \\
\hline $08 / 03 / 88$ & $08: 10$ & $5 F U C$ & 3.068 & 18.63 & 7.9994 & - & 1.2056 \\
\hline $08 / 03 / 88$ & $08: 26$ & 5FUS & - & - & - & - & 1.1974 \\
\hline $08 / 03 / 88$ & $09: 55$ & 5FUS & 3.050 & 18.76 & 7.9995 & - & 1.1986 \\
\hline $08 / 03 / 88$ & $11: 00$ & 5 FUS & 3.030 & 18.90 & 7.9994 & - & 1.1925 \\
\hline $08 / 03 / 88$ & $12: 21$ & 5FUS & 2.852 & 20.22 & 7.9994 & - & 1.1904 \\
\hline
\end{tabular}


TABLE B.11 (continued)

\begin{tabular}{|c|c|c|c|c|c|c|c|}
\hline Date & Time & Port & $\begin{array}{l}\text { Resist. } \\
\text { (kohms) }\end{array}$ & $\begin{array}{l}\text { Air } \\
\text { Temp } \\
\left({ }^{\circ} \mathrm{C}\right)\end{array}$ & $\begin{array}{c}\text { Transd. } \\
\text { Input } \\
\text { (volts) }\end{array}$ & $\begin{array}{c}\text { Transducer } \\
\text { Output } \\
\text { (volts) }\end{array}$ & $\begin{array}{c}\text { Average } \\
\text { Transd. } \\
\text { Output } \\
\text { (volts) }\end{array}$ \\
\hline & 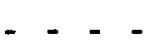 & & --- & -- & --- & $-\quad-$ & $-\ldots$ \\
\hline$/ 03 / 88$ & $15: 10$ & 5FUS & 2.824 & 20.43 & 7.9993 & - & 1.17925 \\
\hline $3 / 03 / 88$ & $17: 41$ & 5FUS & 2.766 & 20.88 & 7.9994 & - & 1.17610 \\
\hline $8 / 03 / 88$ & $19: 49$ & 5FUS & 3.020 & 18.98 & 7.9994 & - & 1.18400 \\
\hline $8 / 03 / 88$ & $21: 55$ & 5FUS & 2.792 & 20.67 & 7. & - & 3060 \\
\hline $8 / 04 / 88$ & $07: 55$ & 5FUS & 2.853 & 20.21 & & - & 3410 \\
\hline $08 / 04 / 88$ & $11: 38$ & 5FUS & 2.841 & 20.30 & & - & 1680 \\
\hline $08 / 04 / 88$ & $15: 20$ & $4 \mathrm{MU}$ & 2.852 & 20.22 & 7. & - & 845 \\
\hline $08 / 04 / 88$ & $17: 30$ & $4 \mathrm{MU}$ & 2.727 & 21.19 & 7. & - & 160 \\
\hline $08 / 04 / 88$ & $21: 51$ & $4 \mathrm{MU}$ & 2.694 & 21.45 & 7.9 & - & 7600 \\
\hline $08 / 04 / 88$ & $22: 57$ & $4 \mathrm{MU}$ & 2.673 & 21.62 & 7.9 & - & 1.17630 \\
\hline $08 / 05 / 88$ & $09: 36$ & 4ML & 2.691 & 21.48 & 7.9 & - & 1.16445 \\
\hline $08 / 05 / 88$ & $11: 05$ & 4ML & 2.674 & 21.62 & 7.9 & - & 1.16400 \\
\hline $08 / 05 / 88$ & $11: 42$ & 4ML & 2.639 & 21.91 & 7.9 & - & 1.16330 \\
\hline $08 / 05 / 88$ & $12: 50$ & 4ML & 2.648 & 21.83 & 7.9991 & - & 1.16370 \\
\hline
\end{tabular}


TABLE B. 12

TEST BLOCK 1 MOISTURE POTENTIAL SUMMARY TENSIOMETER 1 - JULY 1988

\begin{tabular}{|c|c|c|c|c|c|c|c|}
\hline Date & Time & Port & $(\mathrm{cm}$ & $\begin{array}{c}\text { Suction } \\
\text { Applied- } \\
\text { to P1 } \\
\text { water) }\end{array}$ & $\begin{array}{l}\text { Transd. dS } \\
\text { (J.B.Method) }\end{array}$ & $\begin{array}{c}\text { cm of wat } \\
---- \\
\text { Raw } \\
\text { Suction }\end{array}$ & $\begin{array}{l}\text { Cr } \\
\text { Corrected } \\
\quad \text { Suction }\end{array}$ \\
\hline - - & - - & -- & -- & -- & -- & - & $-\cdot-$ \\
\hline $07 / 13 / 88$ & $16: 35$ & 5FUS & & 85.90 & 81.21 & 4.69 & 1.81 \\
\hline $07 / 13 / 88$ & $16: 45$ & $5 F U C$ & & 87.60 & 84.43 & 3.17 & 0.29 \\
\hline $07 / 13 / 88$ & $16: 49$ & $5 F U C$ & & 87.60 & 85.58 & 2.02 & -0.86 \\
\hline $07 / 13 / 88$ & $17: 28$ & 5FLS & & 89.00 & 83.39 & 5.61 & 2.73 \\
\hline $07 / 13 / 88$ & $20: 12$ & 5FLC & & 89.40 & 80.46 & 8.94 & 6.06 \\
\hline $07 / 13 / 88$ & $20: 20$ & 5FLC & & 89.30 & 80.05 & 9.25 & 6.37 \\
\hline $07 / 13 / 88$ & $20: 49$ & $5 F L C$ & & 89.15 & 79.76 & 9.39 & 6.51 \\
\hline $07 / 13 / 88$ & $21: 20$ & 5FLC & & 88.90 & 79.96 & 8.94 & 6.06 \\
\hline $07 / 14 / 88$ & $12: 09$ & 5FUS & & 87.60 & 83.19 & 4.41 & 1.53 \\
\hline $07 / 14 / 88$ & $12: 32$ & $5 F U C$ & & 87.20 & 82.99 & 4.21 & 1.33 \\
\hline $07 / 14 / 88$ & $12: 56$ & 5FLS & & 86.60 & 81.11 & 5.49 & 2.61 \\
\hline $07 / 14 / 88$ & $13: 33$ & 5FLC & & 86.50 & 79.03 & 7.47 & 4.59 \\
\hline $07 / 14 / 88$ & $13: 48$ & 5FLC & & 86.50 & 78.72 & 7.78 & 4.90 \\
\hline $07 / 15 / 88$ & $08: 58$ & 5FLC & & 86.10 & 76.28 & 9.82 & 6.94 \\
\hline $07 / 15 / 88$ & $09: 45$ & 5FLS & & 84.70 & 79.23 & 5.47 & 2.59 \\
\hline $07 / 15 / 88$ & $09: 58$ & 5 FUS & & 84.50 & 80.11 & 4.39 & 1.51 \\
\hline $07 / 15 / 88$ & $10: 28$ & 5 FUC & & 83.80 & 79.55 & 4.25 & 1.37 \\
\hline $07 / 15 / 88$ & $10: 50$ & $4 \mathrm{MU}$ & & 84.70 & 82.25 & 2.45 & -0.43 \\
\hline $07 / 15 / 88$ & $10: 56$ & 4ML & & 84.70 & 82.87 & 1.83 & -1.05 \\
\hline $07 / 15 / 88$ & $11: 07$ & FACE 3 & & 84.50 & 82.87 & 1.63 & -1.25 \\
\hline $07 / 15 / 88$ & $11: 15$ & $5 F U C$ & & 84.30 & 81.83 & 2.47 & -0.41 \\
\hline $07 / 16 / 88$ & $11: 01$ & 5FUS & & 79.40 & 77.68 & 1.72 & -1.16 \\
\hline $07 / 16 / 88$ & $11: 12$ & 5FUC & & 79.10 & 75.60 & 3.50 & 0.62 \\
\hline $07 / 16 / 88$ & $11: 25$ & $5 F L S$ & & 79.10 & 72.95 & 6.15 & 3.27 \\
\hline $07 / 16 / 88$ & $11: 36$ & $5 F L C$ & & 78.90 & 68.32 & 10.58 & 7.70 \\
\hline $07 / 24 / 88$ & $20: 59$ & 5 FUS & & 70.80 & 66.91 & 3.89 & 1.01 \\
\hline $07 / 24 / 88$ & $21: 12$ & 5FUS & & 70.35 & 66.44 & 3.91 & 1.03 \\
\hline $07 / 24 / 88$ & $21: 44$ & 5FUS & & 70.25 & 66.37 & 3.88 & 1.00 \\
\hline $07 / 25 / 88$ & $08: 37$ & $5 \mathrm{FUS}$ & & 70.60 & 66.45 & 4.15 & 1.27 \\
\hline $07 / 25 / 88$ & $08: 44$ & 5FUS & & 70.40 & 66.19 & 4.21 & 1.33 \\
\hline $07 / 25 / 88$ & $09: 01$ & 5 FUS & & 70.10 & 66.04 & 4.06 & 1.18 \\
\hline $07 / 25 / 88$ & $09: 51$ & 5 FUS & & 69.80 & 65.83 & 3.97 & 1.09 \\
\hline $07 / 25 / 88$ & $10: 22$ & 5 FUS & & 69.30 & 65.27 & 4.03 & 1.15 \\
\hline $07 / 25 / 88$ & $12: 29$ & $5 F U C$ & & 67.95 & 61.38 & 6.57 & 3.69 \\
\hline $07 / 25 / 88$ & $13: 40$ & $5 F U C$ & & 67.60 & 61.03 & 6.57 & 3.69 \\
\hline $07 / 25 / 88$ & $14: 32$ & 5FUC & & 66.35 & 60.12 & 6.23 & 3.35 \\
\hline $07 / 25 / 88$ & $15: 07$ & 5 FUC & & 66.15 & 59.82 & 6.33 & 3.45 \\
\hline $07 / 25 / 88$ & $16: 35$ & $5 F U C$ & & 65.20 & 58.83 & 6.37 & 3.49 \\
\hline $07 / 25 / 88$ & $20: 08$ & $5 F U C$ & & 66.20 & 59.31 & 6.89 & 4.01 \\
\hline $07 / 25 / 88$ & $20: 18$ & $5 \mathrm{FUC}$ & & 66.20 & 59.49 & 6.71 & 3.83 \\
\hline $07 / 26 / 88$ & $08: 03$ & 5FUC & & 67.20 & 60.24 & 6.96 & 4.08 \\
\hline $07 / 26 / 88$ & $08: 37$ & 5FLS & & 66.45 & 59.78 & 6.67 & 3.79 \\
\hline $07 / 26 / 88$ & $10: 02$ & 5FLS & & 66.20 & 60.09 & 6.11 & 3.23 \\
\hline
\end{tabular}


TABLE B.12 (continued)

\begin{tabular}{|c|c|c|c|c|c|c|c|}
\hline Date & Time & Port & & $\begin{array}{l}\text { Suction } \\
\text { Applied- } \\
\text { to } \mathrm{Pl} \\
\text { water) }\end{array}$ & $\begin{array}{c}\text { Transd. dS } \\
\text { (J.B.Method) }\end{array}$ & $\begin{array}{c}\mathrm{cm} \text { of wate } \\
---- \\
\text { Raw } \\
\text { Suction }\end{array}$ & $\begin{array}{l}-\bar{r} \\
\text { Corrected } \\
\quad \text { Suction }\end{array}$ \\
\hline & - & 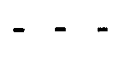 & $\cdot$ & -- & - & -- & $-\cdot-$ \\
\hline $07 / 26 / 88$ & $15: 11$ & 5FLS & & 64.00 & 57.96 & 6.04 & 3.16 \\
\hline $07 / 26 / 88$ & $15: 50$ & 5FLS & & 63.45 & 57.56 & 5.89 & 3.01 \\
\hline $07 / 26 / 88$ & $16: 23$ & 5FLS & & 63.20 & 57.25 & 5.95 & 3.07 \\
\hline $07 / 26 / 88$ & $17: 21$ & $5 F L S$ & & 62.70 & 56.85 & 5.85 & 2.97 \\
\hline $07 / 27 / 88$ & $07: 59$ & 5FLS & & 64.50 & 58.27 & 6.23 & 3.35 \\
\hline $07 / 27 / 88$ & $08: 25$ & $5 F L S$ & & 64.00 & 57.79 & 6.21 & 3.33 \\
\hline $07 / 27 / 88$ & $09: 45$ & $5 F L S$ & & 62.80 & 56.60 & 6.20 & 3.32 \\
\hline $07 / 27 / 88$ & $10: 40$ & $5 F L S$ & & 63.20 & 57.08 & 6.12 & 3.24 \\
\hline $07 / 27 / 88$ & $10: 58$ & 5FLC & & 62.80 & 53.63 & 9.17 & 6.29 \\
\hline $07 / 27 / 88$ & $12: 54$ & $5 F L C$ & & 62.30 & 54.19 & 8.11 & 5.23 \\
\hline $07 / 27 / 88$ & $14: 45$ & $5 \mathrm{FLC}$ & & 61.10 & 52.77 & 8.33 & 5.45 \\
\hline $07 / 27 / 88$ & $17: 07$ & 5FLC & & 60.70 & 52.26 & 8.44 & 5.56 \\
\hline $07 / 27 / 88$ & $19: 27$ & 5FLC & & 61.80 & 52.97 & 8.83 & 5.95 \\
\hline $07 / 28 / 88$ & $08: 03$ & $5 F L C$ & & 62.20 & 52.82 & 9.38 & 6.50 \\
\hline $07 / 28 / 88$ & $11: 18$ & $5 F L C$ & & 62.10 & 53.26 & 8.84 & 5.96 \\
\hline $07 / 28 / 88$ & $15: 36$ & $5 F L C$ & & 59.65 & 51.30 & 8.35 & 5.47 \\
\hline $07 / 28 / 88$ & $16: 51$ & 5FLC & & 59.10 & 50.78 & 8.32 & 5.44 \\
\hline $07 / 28 / 88$ & $10: 36$ & 5FLC & & 57.40 & 48.08 & 9.32 & 6.44 \\
\hline $07 / 28 / 88$ & $16: 25$ & 5FLC & & 56.30 & 47.13 & 9.17 & 6.29 \\
\hline $07 / 28 / 88$ & $13: 11$ & 5FLC & & 57.10 & 47.30 & 9.80 & 6.92 \\
\hline $07 / 28 / 88$ & $16: 56$ & 5FLC & & 56.00 & 46.27 & 9.73 & 6.85 \\
\hline $07 / 28 / 88$ & $12: 56$ & 5FLC & & 54.50 & 44.22 & 10.28 & 7.40 \\
\hline $07 / 29 / 88$ & $10: 36$ & 5FLC & & 57.40 & 48.08 & 9.32 & 6.44 \\
\hline $07 / 29 / 88$ & $16: 25$ & $5 \mathrm{FLC}$ & & 56.30 & 47.13 & 9.17 & 6.29 \\
\hline $07 / 30 / 88$ & $13: 11$ & 5FLC & & 57.10 & 47.30 & 9.80 & 6.92 \\
\hline $07 / 30 / 88$ & $16: 56$ & 5FLC & & 56.00 & 46.27 & 9.73 & 6.85 \\
\hline $07 / 31 / 88$ & $12: 56$ & 5FLC & & 54.50 & 44.22 & 10.28 & 7.40 \\
\hline $07 / 31 / 88$ & $17: 02$ & $5 F L C$ & & 53.40 & 43.02 & 10.38 & 7.50 \\
\hline $08 / 01 / 88$ & $07: 40$ & 5FLC & & 52.90 & 41.93 & 10.97 & 8.09 \\
\hline $08 / 01 / 88$ & $10: 33$ & 5FLC & & 51.80 & 41.14 & 10.66 & 7.78 \\
\hline $08 / 01 / 88$ & $14: 27$ & $5 F L S$ & & 50.80 & 45.13 & 5.67 & 2.79 \\
\hline $08 / 01 / 88$ & $15: 27$ & $5 F L S$ & & 50.60 & 44.94 & 5.66 & 2.78 \\
\hline $08 / 01 / 88$ & $16: 31$ & $5 F L S$ & & 50.40 & 44.80 & 5.60 & 2.72 \\
\hline $08 / 01 / 88$ & $19: 48$ & $5 F L S$ & & 50.40 & 44.74 & 5.66 & 2.78 \\
\hline $08 / 01 / 88$ & $21: 40$ & 5FUC & & 49.80 & 44.63 & 5.17 & 2.29 \\
\hline $08 / 02 / 88$ & $07: 54$ & $5 F U C$ & & 51.20 & 45.94 & 5.26 & 2.38 \\
\hline $08 / 02 / 88$ & $13: 31$ & $5 F U C$ & & 50.10 & 45.75 & 4.35 & 1.47 \\
\hline $08 / 02 / 88$ & $16: 11$ & 5FUC & & 47.70 & 39.47 & 8.23 & 5.35 \\
\hline $08 / 02 / 88$ & $16: 34$ & $5 \mathrm{FUC}$ & & 47.20 & 39.05 & 8.15 & 5.27 \\
\hline $08 / 02 / 88$ & $19: 38$ & $5 \mathrm{FUC}$ & & 49.10 & 44.13 & 4.97 & 2.09 \\
\hline $08 / 02 / 88$ & $20: 58$ & 5 FUC & & 49.30 & 44.90 & 4.40 & 1.52 \\
\hline $08 / 03 / 88$ & $08: 10$ & 5 FUC & & 50.50 & 46.00 & 4.50 & 1.62 \\
\hline $08 / 03 / 88$ & $08: 26$ & 5 FUS & & 49.90 & 44.30 & 5.60 & 2.72 \\
\hline $08 / 03 / 88$ & $09: 55$ & 5 FUS & & 50.10 & 44.55 & 5.55 & 2.67 \\
\hline $08 / 03 / 88$ & $11: 00$ & 5FUS & & 48.90 & 43.28 & 5.62 & 2.74 \\
\hline $08 / 03 / 88$ & $12: 21$ & 5 FUS & & 48.40 & 42.85 & 5.55 & 2.67 \\
\hline
\end{tabular}


TABLE B.12 (continued)

\begin{tabular}{|c|c|c|c|c|c|c|c|}
\hline Date & Time & Port & & $\begin{array}{l}\text { Suction } \\
\text { Applied- } \\
\text { to P1 } \\
\text { water) }\end{array}$ & $\begin{array}{l}\text { Transd. dS } \\
\text { (J.B.Method) }\end{array}$ & $\begin{array}{c}\mathrm{cm} \text { of wat } \\
--- \\
\text { Raw } \\
\text { Suction }\end{array}$ & $\begin{array}{l}\text { er } \\
- \text { Corrected } \\
\quad \text { Suction }\end{array}$ \\
\hline & -- & & -- & - & $-\cdot-$ & $-\cdot-$ & - - \\
\hline $8 / 03 / 88$ & $15: 10$ & 5FUS & & 46.10 & 40.53 & 5.57 & 2.69 \\
\hline $08 / 03 / 88$ & $17: 41$ & 5FUS & & 45.80 & 39.87 & 5.93 & 3.05 \\
\hline $08 / 03 / 88$ & $19: 49$ & 5 FUS & & 47.45 & 41.51 & 5.94 & 3.06 \\
\hline $08 / 03 / 88$ & $21: 55$ & $5 F U S$ & & 46.70 & 40.81 & 5.89 & 3.01 \\
\hline $08 / 04 / 88$ & $07: 55$ & $5 F U S$ & & 47.60 & 41.53 & 6.07 & 3.19 \\
\hline $08 / 04 / 88$ & $11: 38$ & 5FUS & & 45.90 & 40.02 & 5.88 & 3.00 \\
\hline $08 / 04 / 88$ & $15: 20$ & $4 \mathrm{MU}$ & & 46.00 & 42.44 & 3.56 & 0.68 \\
\hline $08 / 04 / 88$ & $17: 30$ & $4 \mathrm{MU}$ & & 43.20 & 41.01 & 2.19 & -0.69 \\
\hline $08 / 04 / 88$ & $21: 51$ & 4MU & & 42.90 & 39.85 & 3.05 & 0.17 \\
\hline $08 / 04 / 88$ & $22: 57$ & 4MU & & 42.70 & 39.91 & 2.79 & -0.09 \\
\hline $08 / 05 / 88$ & $09: 36$ & 4ML & & 41.95 & 37.45 & 4.50 & 1.62 \\
\hline $08 / 05 / 88$ & $11: 05$ & 4ML & & 41.50 & 37.36 & 4.14 & 1.26 \\
\hline $08 / 05 / 88$ & $11: 42$ & 4ML & & 40.90 & 37.21 & 3.69 & 0.81 \\
\hline $08 / 05 / 88$ & $12: 50$ & 4ML & & 40.50 & 37.29 & 3.21 & 0.33 \\
\hline
\end{tabular}


TABLE B.13

\section{MATRIX K AND FRACTURE T - TEST BLOCK 1}

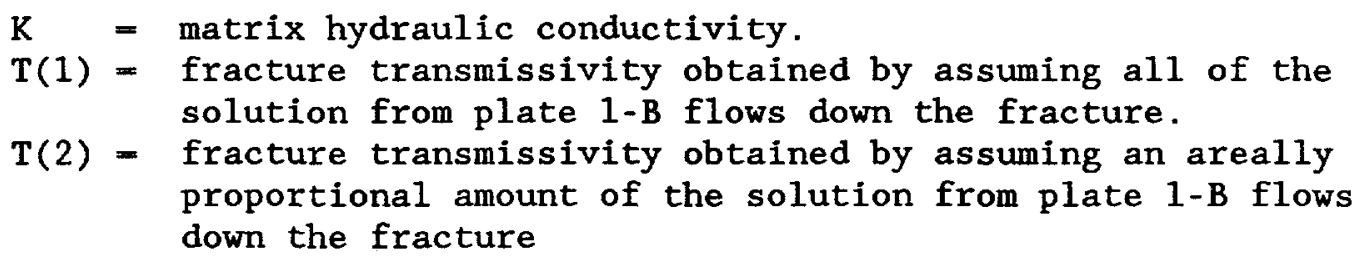

\begin{tabular}{|c|c|c|c|c|c|c|}
\hline & & Pla & & K & $T(1)$ & $\mathrm{T}(2)$ \\
\hline Date & Time & Position & Number & $(\mathrm{m} / \mathrm{sec})$ & $(\mathrm{m} * * 2 / \mathrm{sec})$ & $(\mathrm{m} * * 2 / \mathrm{sec})$ \\
\hline-- & --- & . & $-\quad--$ & $-\overline{-}-\overline{-}$ & ---- & --- \\
\hline $06 / 13 / 88$ & $19: 48$ & $1-\mathrm{A}$ & 3 & $8.52 \mathrm{E}-08$ & - & - \\
\hline $06 / 13 / 88$ & $20: 07$ & $1-\mathrm{C}$ & 4 & $3.35 E-08$ & - & - \\
\hline $06 / 13 / 88$ & $20: 20$ & $1-B$ & 5 & - & $8.25 E-09$ & $6.19 \mathrm{E}-09$ \\
\hline $06 / 14 / 88$ & $08: 00$ & $1-\mathrm{A}$ & 3 & $7.70 \mathrm{E}-08$ & - & - \\
\hline $06 / 14 / 88$ & $08: 20$ & $1-C$ & 4 & $3.75 E-08$ & - & - \\
\hline $06 / 14 / 88$ & $08: 27$ & $1-B$ & 5 & - & $7.79 E-09$ & $5.81 E-09$ \\
\hline $06 / 14 / 88$ & $11: 30$ & $1-B$ & 5 & - & $7.47 \mathrm{E}-09$ & $5.53 \mathrm{E}-09$ \\
\hline $06 / 14 / 88$ & $14: 05$ & 1-B & 5 & - & $7.73 \mathrm{E}-09$ & $5.76 \mathrm{E}-09$ \\
\hline $06 / 15 / 88$ & $08: 44$ & $1-A$ & 3 & $8.01 \mathrm{E}-08$ & - & - \\
\hline $06 / 15 / 88$ & $08: 58$ & $1-\mathrm{C}$ & 4 & $3.61 \mathrm{E}-08$ & - & - \\
\hline $06 / 15 / 88$ & $09: 05$ & 1-B & 5 & - & $7.91 \mathrm{E}-09$ & $5.92 E-09$ \\
\hline $06 / 16 / 88$ & $08: 49$ & $1-\mathrm{A}$ & 3 & $8.53 E-08$ & - & - \\
\hline $06 / 16 / 88$ & $09: 10$ & $1-B$ & 5 & - & $7.84 \mathrm{E}-09$ & $5.81 \mathrm{E}-09$ \\
\hline $06 / 16 / 88$ & $09: 44$ & $1-B$ & 5 & - & $1.04 \mathrm{E}-08$ & $8.05 E-09$ \\
\hline $06 / 16 / 88$ & $10: 44$ & $1-\mathrm{C}$ & 4 & $3.56 \mathrm{E}-08$ & - & - \\
\hline $06 / 16 / 88$ & $11: 48$ & $1-B$ & 5 & - & $8.82 \mathrm{E}-09$ & - \\
\hline $06 / 16 / 88$ & $13: 43$ & $1-B$ & 5 & - & $8.58 \mathrm{E}-09$ & - \\
\hline $06 / 16 / 88$ & $14: 05$ & $1-B$ & 5 & - & $8.59 E-09$ & - \\
\hline $06 / 19 / 88$ & $13: 41$ & $1-A$ & 3 & $8.53 E-08$ & - & - \\
\hline $06 / 19 / 88$ & $13: 51$ & $1-C$ & 4 & $3.71 E-08$ & - & - \\
\hline $06 / 19 / 88$ & $14: 30$ & $1-B$ & 5 & - & $7.33 E-09$ & $5.34 E-09$ \\
\hline $06 / 20 / 88$ & $08: 29$ & $1-A$ & 3 & $8.03 E-08$ & - & - \\
\hline $06 / 20 / 88$ & $08: 56$ & $1-C$ & 4 & $3.86 \mathrm{E}-08$ & - & - \\
\hline $06 / 20 / 88$ & $09: 35$ & $1-B$ & 5 & - & $8.56 E-09$ & $6.43 E-09$ \\
\hline $06 / 21 / 88$ & $08: 49$ & $1-A$ & 3 & $8.29 \mathrm{E}-08$ & - & - \\
\hline $06 / 21 / 88$ & $09: 20$ & $1-C$ & 4 & $3.65 \mathrm{E}-08$ & - & - \\
\hline $06 / 21 / 88$ & $09: 58$ & 1-B & 5 & - & $8.30 \mathrm{E}-09$ & $6.22 E-09$ \\
\hline $06 / 24 / 88$ & $09: 00$ & $1-A$ & 3 & $8.46 \mathrm{E}-08$ & - & - \\
\hline $06 / 24 / 88$ & $09: 27$ & $1-\mathrm{C}$ & 4 & $3.66 \mathrm{E}-08$ & - & - \\
\hline $06 / 24 / 88$ & $08: 41$ & $1-B$ & 5 & - & $9.53 \mathrm{E}-09$ & $7.27 \mathrm{E}-09$ \\
\hline $06 / 27 / 88$ & $09: 37$ & $1-A$ & 3 & $8.05 E-08$ & - & - \\
\hline $06 / 27 / 88$ & $10: 03$ & $1-C$ & 4 & $4.04 \mathrm{E}-08$ & - & - \\
\hline $06 / 27 / 88$ & $09: 58$ & $1-B$ & 5 & - & $7.32 \mathrm{E}-09$ & $5.32 \mathrm{E}-09$ \\
\hline $06 / 28 / 88$ & $11: 37$ & $1-A$ & 3 & $7.83 E-08$ & - & - \\
\hline $06 / 28 / 88$ & $12: 42$ & $1-C$ & 4 & $3.47 E-08$ & - & - \\
\hline $06 / 28 / 88$ & $12: 05$ & $1-B$ & 5 & - & $7.47 E-09$ & $5.60 \mathrm{E}-09$ \\
\hline $06 / 29 / 88$ & $09: 42$ & $1-\mathrm{A}$ & 3 & $8.81 \mathrm{E}-08$ & - & - \\
\hline $06 / 29 / 88$ & $10: 39$ & $1-C$ & 4 & $4.42 E-08$ & - & - \\
\hline $06 / 29 / 88$ & $10: 41$ & $1-B$ & 5 & - & $7.49 \mathrm{E}-09$ & $5.38 \mathrm{E}-09$ \\
\hline
\end{tabular}


TABLE B.13 (continued)

\begin{tabular}{|c|c|c|c|c|c|c|}
\hline Date & Time & $\begin{array}{r}\text { Pla } \\
\text { Position }\end{array}$ & $\begin{array}{l}\text { te } \\
\text { Number }\end{array}$ & $\begin{array}{c}\mathrm{K} \\
(\mathrm{m} / \mathrm{sec})\end{array}$ & $\begin{array}{c}T(1) \\
(m * * 2 / \mathrm{sec})\end{array}$ & $\begin{array}{c}T(2) \\
(\mathrm{m} * * 2 / \mathrm{sec})\end{array}$ \\
\hline- & $--\cdots$ & - & - & -- & $--\cdot-$ & $-\cdot-\cdot$ \\
\hline $07 / 01 / 88$ & $08: 59$ & $1-A$ & 3 & $7.43 \mathrm{E}-08$ & - & - \\
\hline $07 / 01 / 88$ & - & $1-C$ & 4 & $3.58 \mathrm{E}-08$ & - & - \\
\hline $07 / 01 / 88$ & $09: 38$ & 1-B & 5 & - & $7.20 \mathrm{E}-09$ & $5.39 \mathrm{E}-09$ \\
\hline $07 / 01 / 88$ & $10: 50$ & $1-A$ & 3 & $7.35 \mathrm{E}-08$ & - & - \\
\hline $07 / 01 / 88$ & $11: 13$ & 1-B & 5 & - & $6.51 \mathrm{E}-09$ & $4.78 \mathrm{E}-09$ \\
\hline $07 / 03 / 88$ & $08: 30$ & $1-\mathrm{A}$ & 3 & $7.12 \mathrm{E}-08$ & - & - \\
\hline $07 / 03 / 88$ & $09: 12$ & $1-\mathrm{C}$ & 4 & $3.60 \mathrm{E}-08$ & - & - \\
\hline $07 / 03 / 88$ & $09: 23$ & $1-B$ & 5 & - & $7.86 \mathrm{E}-09$ & $6.00 \mathrm{E}-09$ \\
\hline $07 / 05 / 88$ & $12: 27$ & $1-A$ & 3 & $8.94 \mathrm{E}-08$ & - & - \\
\hline $07 / 05 / 88$ & $12: 47$ & $1-C$ & 4 & $5.51 \mathrm{E}-08$ & - & - \\
\hline $07 / 05 / 88$ & - & 1-B & 5 & - & $7.31 \mathrm{E}-09$ & $5.05 \mathrm{E}-09$ \\
\hline $07 / 11 / 88$ & $11: 44$ & $1-\mathrm{A}$ & 3 & $7.53 E-08$ & - & - \\
\hline $07 / 11 / 88$ & $12: 28$ & $1-C$ & 4 & $3.37 E-08$ & - & - \\
\hline $07 / 11 / 88$ & $12: 59$ & $1-B$ & 5 & - & $6.59 \mathrm{E}-09$ & $4.87 E-09$ \\
\hline $07 / 15 / 88$ & $10: 00$ & $1-\mathrm{A}$ & 3 & $6.42 \mathrm{E}-08$ & - & - \\
\hline $07 / 15 / 88$ & $10: 15$ & $1-\mathrm{C}$ & 4 & $2.85 \mathrm{E}-08$ & - & - \\
\hline $07 / 15 / 88$ & $10: 22$ & $1-B$ & 5 & - & $4.06 \mathrm{E}-09$ & $2.80 \mathrm{E}-09$ \\
\hline $07 / 15 / 88$ & - & $1-B$ & 5 & $2.56 \mathrm{E}-07$ & - & - \\
\hline $07 / 18 / 88$ & $09: 59$ & $1-A$ & 3 & $5.24 \mathrm{E}-08$ & - & - \\
\hline $07 / 18 / 88$ & $10: 35$ & $1-B$ & 5 & - & $7.78 \mathrm{E}-09$ & $6.08 \mathrm{E}-09$ \\
\hline $07 / 18 / 88$ & $11: 27$ & $1-A$ & 3 & $6.56 \mathrm{E}-08$ & - & - \\
\hline $07 / 19 / 88$ & $09: 50$ & $1-A$ & 3 & $6.68 \mathrm{E}-08$ & - & - \\
\hline $07 / 19 / 88$ & $10: 44$ & $1-C$ & 4 & $3.22 \mathrm{E}-08$ & - & - \\
\hline $07 / 19 / 88$ & $10: 25$ & $1-B$ & 5 & - & $1.07 \mathrm{E}-08$ & $8.64 E-09$ \\
\hline $07 / 23 / 88$ & $12: 20$ & $1-A$ & 3 & $6.00 \mathrm{E}-08$ & - & - \\
\hline $07 / 23 / 88$ & $16: 07$ & $1-C$ & 4 & $3.39 \mathrm{E}-08$ & - & - \\
\hline $07 / 23 / 88$ & $12: 50$ & $1-B$ & 5 & - & $5.31 \mathrm{E}-09$ & $3.89 \mathrm{E}-09$ \\
\hline $07 / 25 / 88$ & $14: 11$ & $1-\mathrm{A}$ & 3 & $6.65 E-08$ & - & - \\
\hline $07 / 25 / 88$ & $15: 03$ & $1-C$ & 4 & $3.66 \mathrm{E}-08$ & - & - \\
\hline $07 / 25 / 88$ & $14: 43$ & $1-B$ & 5 & - & $5.35 \mathrm{E}-09$ & $3.83 E-09$ \\
\hline $08 / 02 / 88$ & $09: 00$ & $1-A$ & 3 & $6.19 \mathrm{E}-08$ & - & - \\
\hline $08 / 02 / 88$ & $10: 00$ & $1-C$ & 4 & $3.60 E-08$ & - & - \\
\hline $08 / 02 / 88$ & $09: 30$ & $1-B$ & 5 & - & $6.34 \mathrm{E}-09$ & $4.77 \mathrm{E}-09$ \\
\hline $08 / 03 / 88$ & $20: 47$ & $1-A$ & 3 & $6.91 \mathrm{E}-08$ & - & - \\
\hline $08 / 03 / 88$ & $21: 55$ & $1-C$ & 4 & $1.33 \mathrm{E}-07$ & - & - \\
\hline $08 / 03 / 88$ & $20: 46$ & 1 - B & 5 & - & $4.78 \mathrm{E}-09$ & $2.33 E-09$ \\
\hline $08 / 03 / 88$ & $21: 28$ & $1-B$ & 5 & - & $5.04 \mathrm{E}-09$ & $2.54 E-09$ \\
\hline $08 / 04 / 88$ & $10: 54$ & $1-\mathrm{A}$ & 3 & $6.91 \mathrm{E}-08$ & - & - \\
\hline $08 / 04 / 88$ & $11: 16$ & $1-C$ & 4 & $3.85 \mathrm{E}-08$ & - & - \\
\hline $08 / 04 / 88$ & $10: 23$ & $1-B$ & 5 & - & $4.90 E-09$ & $3.31 E-09$ \\
\hline $08 / 04 / 88$ & $18: 02$ & 1-B & 5 & - & $3.52 E-09$ & $2.11 \mathrm{E}-09$ \\
\hline $08 / 04 / 88$ & $19: 04$ & $1-B$ & 5 & - & $4.91 E-09$ & $3.33 \mathrm{E}-09$ \\
\hline $08 / 05 / 88$ & $09: 45$ & $1-B$ & 5 & - & $5.14 \mathrm{E}-09$ & $3.52 E-09$ \\
\hline
\end{tabular}


TABLE B.14

ELECTRONICS DATA SUMMARY - TEST BLOCK 2

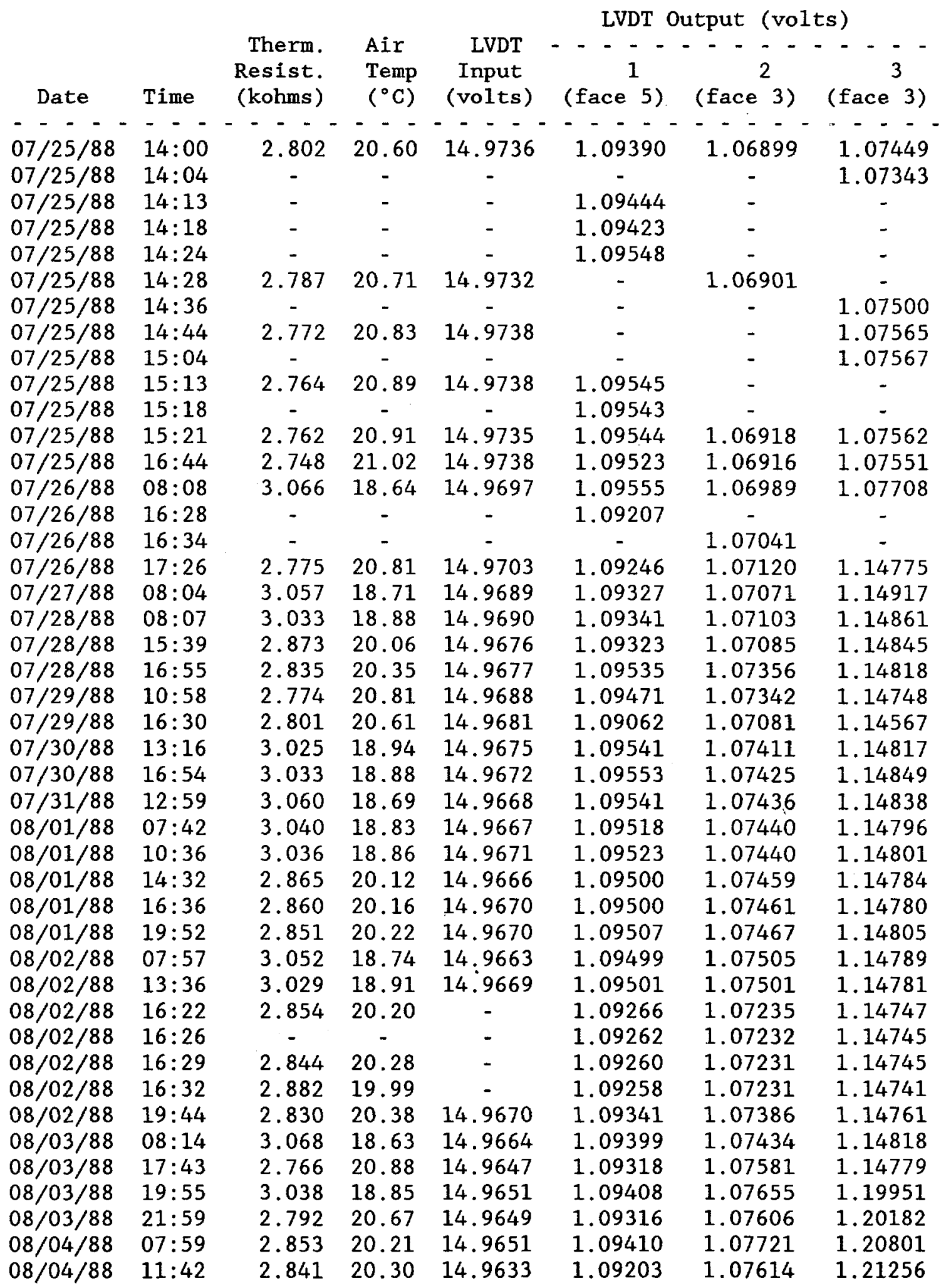


TABLE B.14 (continued)

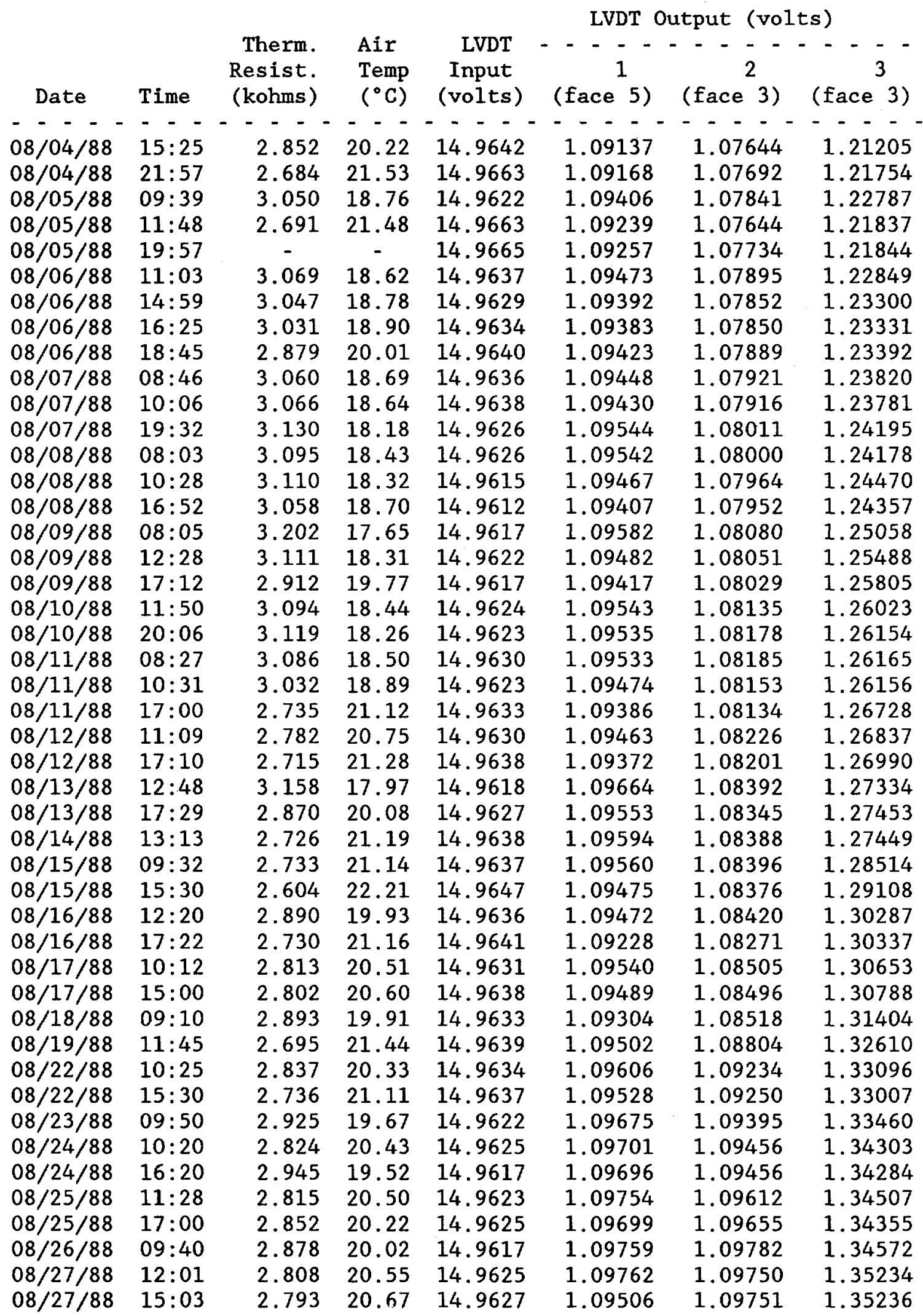


TABLE B.14 (continued)

\begin{tabular}{|c|c|c|c|c|c|c|c|}
\hline & & & & & LVDT & put (vol & 5) \\
\hline Date & Time & $\begin{array}{l}\text { Therm. } \\
\text { Resist. } \\
\text { (kohms) }\end{array}$ & $\begin{array}{l}\text { Air } \\
\text { Temp } \\
\left({ }^{\circ} \mathrm{C}\right)\end{array}$ & $\begin{array}{c}\text { LVDT } \\
\text { Input } \\
\text { (volts) }\end{array}$ & $\begin{array}{c}1 \\
(\text { face } 5)\end{array}$ & $\left(\right.$ face $\left.^{3}\right)$ & $($ face 3$)$ \\
\hline - & -- & $-\quad--$ & - & -- & --- & --- & 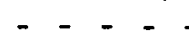 \\
\hline $8 / 88$ & $16: 34$ & 2.850 & 20.23 & 14.9621 & 1.09593 & 1.09943 & 1.35311 \\
\hline $29 / 88$ & $09: 10$ & 3.114 & 18.29 & 14.9611 & 1.09594 & 1.10059 & 1.35319 \\
\hline $8 / 29 / 88$ & $16: 53$ & 2.914 & 19.75 & 14.9625 & 1.09518 & 1.10140 & 1.35840 \\
\hline
\end{tabular}


TABLE B.15

\begin{tabular}{|c|c|c|c|c|c|c|}
\hline Date & Time & $\begin{array}{l}\text { Plate } \\
\text { Position }\end{array}$ & $\begin{array}{r}\text { Plate } \\
\text { Number }\end{array}$ & $\begin{array}{c}\text { Total Head } \\
\text { on Top } \\
\text { of Plate } \\
(-\mathrm{cm})\end{array}$ & $\begin{array}{l}\text { Inflow Rate } \\
\text { (mL/min) }\end{array}$ & $\begin{array}{l}\text { Suction on } \\
\text { Bottom of } \\
\text { Plate (cm) }\end{array}$ \\
\hline & -- & 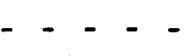 & - & - & ----- & --- \\
\hline $08 / 03 / 88$ & $21: 05$ & $1-C$ & 1 & 4.95 & $3.040993 E-02$ & 11.87 \\
\hline $08 / 03 / 88$ & $21: 48$ & $1-B$ & 6 & 6.20 & $2.401210 \mathrm{E}-02$ & 13.19 \\
\hline $08 / 04 / 88$ & $09: 14$ & $1-\mathrm{C}$ & 1 & 8.50 & $1.905839 \mathrm{E}-02$ & 12.84 \\
\hline $08 / 04 / 88$ & $10: 58$ & $1-B$ & 6 & 6.55 & $1.433051 \mathrm{E}-02$ & 10.72 \\
\hline $08 / 05 / 88$ & $09: 55$ & $1-C$ & 1 & 8.50 & $1.660399 \mathrm{E}-02$ & 12.28 \\
\hline $08 / 05 / 88$ & 12 & 1. & 2 & 12.30 & $1.701230 \mathrm{E}-02$ & 15.33 \\
\hline $08 / 05 / 88$ & 11 & 1 & 6 & 6.50 & 1. $130569 \mathrm{E}-02$ & 9.79 \\
\hline $08 / 06 / 88$ & 10 & 1 & 1 & 16.40 & $1.054737 \mathrm{E}-02$ & 18.80 \\
\hline $08 / 06 / 88$ & 12 & $1-A$ & 2 & 11.90 & $1.490192 \mathrm{E}-02$ & 14.56 \\
\hline $5 / 88$ & 18 & 1 & 6 & 14.00 & $9.459063 E-03$ & 16.75 \\
\hline $08 /$ & 08 & 1 & 1 & 15.00 & $1.109322 \mathrm{E}-02$ & 17.53 \\
\hline $08 / 0$ & $0 \varepsilon$ & 1 & 2 & 12.45 & 1.30080 & 14.77 \\
\hline $08 / 0$ & 08 & 1 & 1 & 13.10 & $1.070801 \mathrm{E}-02$ & 15.54 \\
\hline $08 / 08$ & $0 s$ & $1-\mathrm{A}$ & 2 & 12.40 & $1.211890 \mathrm{E}-02$ & 14.56 \\
\hline $08 / 08 / 88$ & 1] & 1 & 6 & 13.20 & $5.390283 E-03$ & 14.77 \\
\hline $08 / 09 / 88$ & 08 & $1-C$ & 1 & 12.60 & $9.401368 E-03$ & 14.74 \\
\hline $08 / 09 / 88$ & $08: 53$ & A & 2 & 12.30 & $9.705016 \mathrm{E}-03$ & 14.03 \\
\hline $08 / 09 / 88$ & $11: 36$ & 1-B & 6 & 12.82 & $5.182882 \mathrm{E}-03$ & 14.33 \\
\hline $08 / 10 / 88$ & $12: 52$ & $1-C$ & 1 & 10.90 & $9.158348 \mathrm{E}-03$ & 12.9 \\
\hline $08 / 10 / 88$ & $11: 52$ & $1-B$ & 6 & 12.20 & $4.311224 E-03$ & 13.4 \\
\hline $08 / 11 / 88$ & $15: 38$ & $1-C$ & 1 & 15.93 & $1.039400 \mathrm{E}-02$ & 18.30 \\
\hline $08 / 11 / 88$ & $16: 34$ & $1-\mathrm{A}$ & 2 & 15.17 & $9.252756 \mathrm{E}-03$ & 16.82 \\
\hline $08 / 11 / 88$ & $12: 33$ & $1-B$ & 6 & 15.63 & $4.043663 E-03$ & 16.8 \\
\hline $08 / 12 / 88$ & $10: 50$ & $1-\mathrm{C}$ & 1 & 12.32 & $1.016356 \mathrm{E}-02$ & 14.6 \\
\hline $08 / 12 / 88$ & $10: 16$ & $1-\mathrm{A}$ & 2 & 13.50 & $9.604610 \mathrm{E}-03$ & 15.2 \\
\hline $08 / 12 / 88$ & $12: 15$ & 1-B & 6 & 12.96 & $4.017896 \mathrm{E}-03$ & 14.1 \\
\hline $08 / 13 / 88$ & $14: 02$ & $1-\mathrm{C}$ & 1 & 11.74 & $1.124649 \mathrm{E}-02$ & 14.3 \\
\hline $08 / 13 / 88$ & $14: 13$ & $1-A$ & 2 & 13.20 & $9.755184 E-03$ & 14.9 \\
\hline $08 / 13 / 88$ & $15: 34$ & 1-B & 6 & 12.78 & $4.600232 E-03$ & 14.12 \\
\hline $08 / 15 / 88$ & $10: 07$ & $1-\mathrm{C}$ & 1 & 12.62 & $9.885032 E-03$ & 14.87 \\
\hline $08 / 15 / 88$ & $10: 30$ & $1-\mathrm{A}$ & 2 & 14.32 & $8.848310 \mathrm{E}-03$ & 15.90 \\
\hline $08 / 15 / 88$ & $11: 50$ & 1-B & 6 & 13.33 & $4.492286 \mathrm{E}-03$ & 14.6 \\
\hline $08 / 16 / 88$ & $09: 55$ & $1-C$ & 1 & 12.63 & $9.668951 \mathrm{E}-03$ & 14.83 \\
\hline $08 / 16 / 88$ & $09: 33$ & $1-\mathrm{A}$ & 2 & 13.90 & $1.053489 \mathrm{~F}-02$ & 15.7 \\
\hline $08 / 16 / 88$ & $11: 10$ & $1-B$ & 6 & 14.63 & $2.865903 \mathrm{E}-03$ & 15.46 \\
\hline $08 / 17 / 88$ & $10: 05$ & $1-\mathrm{A}$ & 2 & 13.72 & $1.550537 \mathrm{E}-02$ & 16.48 \\
\hline $08 / 17 / 88$ & $11: 01$ & $1-B$ & 6 & 14.54 & $2.510535 \mathrm{E}-03$ & 15.27 \\
\hline $08 / 18 / 88$ & $09: 50$ & $1-C$ & 1 & 13.40 & $1.145015 \mathrm{E}-02$ & 16.01 \\
\hline $08 / 18 / 88$ & $10: 05$ & $1-\mathrm{A}$ & 2 & 13.32 & $1.134170 \mathrm{E}-02$ & 15.34 \\
\hline $08 / 18 / 88$ & $11: 15$ & $1-B$ & 6 & 13.33 & $5.966680 \mathrm{E}-03$ & 15.07 \\
\hline $08 / 19 / 88$ & $10: 25$ & $1-\mathrm{C}$ & 1 & 13.57 & $1.080063 \mathrm{E}-02$ & 16.03 \\
\hline $08 / 19 / 88$ & $10: 30$ & 1-A & 2 & 13.45 & $1.010518 \mathrm{E}-02$ & 15.25 \\
\hline $08 / 19 / 88$ & $11: 40$ & $1-B$ & 6 & 13.98 & $3.475964 E-03$ & 14.99 \\
\hline $08 / 22 / 88$ & $09: 40$ & $1-\mathrm{C}$ & 1 & 12.68 & $1.074487 \mathrm{E}-02$ & 15.13 \\
\hline $08 / 22 / 88$ & $10: 40$ & $1-\mathrm{A}$ & 2 & 13.57 & $8.015565 E-03$ & 15.00 \\
\hline $08 / 22 / 88$ & $11: 15$ & -1 & 6 & 13.72 & $4.443197 \mathrm{E}-03$ & 15.0 \\
\hline
\end{tabular}


TABLE B.15 (continued)

\begin{tabular}{|c|c|c|c|c|c|c|}
\hline Date & Time & $\begin{array}{c}\text { Plate } \\
\text { Position }\end{array}$ & $\begin{array}{r}\text { Plate } \\
\text { Number }\end{array}$ & $\begin{array}{r}\text { Total Hea } \\
\text { on Top } \\
\text { of P1ate } \\
(-\mathrm{cm})\end{array}$ & $\begin{array}{c}\text { Inflow Rate } \\
\text { (mL/min) }\end{array}$ & $\begin{array}{l}\text { Suction on } \\
\text { Bottom of } \\
\text { Plate }(\mathrm{cm})\end{array}$ \\
\hline & & - & - & - & $-\cdots--$ & ---- \\
\hline $08 / 23 / 88$ & $10: 12$ & $1-C$ & 1 & 13.02 & $1.192724 \mathrm{E}-02$ & 15.74 \\
\hline $08 / 23 / 88$ & $10: 42$ & $1-\mathrm{A}$ & 2 & 12.75 & $1.150952 \mathrm{E}-02$ & 14.80 \\
\hline $08 / 23 / 88$ & $11: 55$ & $1-B$ & 6 & 13.43 & $5.603811 E-03$ & 15.06 \\
\hline $08 / 24 / 88$ & $10: 20$ & $1-\mathrm{C}$ & 1 & 13.58 & $1.030874 \mathrm{E}-02$ & 15.93 \\
\hline $08 / 24 / 88$ & $09: 50$ & $1-\mathrm{A}$ & 2 & 12.92 & $1.031500 \mathrm{E}-02$ & 14.76 \\
\hline $08 / 24 / 88$ & $12: 00$ & $1-B$ & 6 & 14.53 & $2.441698 E-03$ & 15.24 \\
\hline $08 / 25 / 88$ & $12: 05$ & $1-\mathrm{C}$ & 1 & 12.50 & $1.681365 \mathrm{E}-02$ & 16.33 \\
\hline $08 / 25 / 88$ & $13: 00$ & $1-A$ & 2 & 13.67 & $7.894549 E-03$ & 15.08 \\
\hline $08 / 25 / 88$ & $12: 10$ & $1-B$ & 6 & 13.84 & $4.552248 E-03$ & 15.16 \\
\hline $08 / 26 / 88$ & $09: 35$ & $1-C$ & 1 & 12.70 & $1.001146 \mathrm{E}-02$ & 14.98 \\
\hline $08 / 26 / 88$ & $10: 07$ & $1-A$ & 2 & 13.00 & $1.881586 \mathrm{E}-02$ & 16.35 \\
\hline $08 / 26 / 88$ & $11: 07$ & $1-B$ & 6 & 12.36 & $5.295211 \mathrm{E}-03$ & 13.90 \\
\hline $08 / 29 / 88$ & $10: 12$ & $1-\mathrm{C}$ & 1 & 12.75 & $2.083406 \mathrm{E}-02$ & 17.49 \\
\hline $08 / 29 / 88$ & $10: 14$ & $1-\mathrm{A}$ & 2 & 12.27 & $1.668344 \mathrm{E}-02$ & 15.24 \\
\hline $08 / 29 / 88$ & $11: 26$ & $1-B$ & 6 & 13.46 & $2.535113 E-03$ & 14.20 \\
\hline
\end{tabular}


TABLE B.16

CONSTANT-HEAD RESERVOIR INFLOW SUMMARY - TEST BLOCK 2

\begin{tabular}{|c|c|c|c|c|c|c|c|}
\hline Date & Time & $\begin{array}{l}\text { Reservoi } \\
\text { Number }\end{array}$ & $\begin{array}{l}r \text { Plate } \\
\text { Number }\end{array}$ & $\begin{array}{c}\text { Total Head } \\
\text { on Top } \\
\text { of Plate } \\
(-\mathrm{cm})\end{array}$ & $\begin{array}{l}\text { Inflow } \\
\text { Volume } \\
\text { (mL) }\end{array}$ & $\begin{array}{l}\text { Flow Rate } \\
(\mathrm{mL} / \mathrm{min})\end{array}$ & $\begin{array}{r}\text { Suction } \\
\text { on Bottom } \\
\text { of Plate } \\
(\mathrm{cm})\end{array}$ \\
\hline 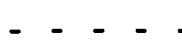 & $-\cdot-$ & --5 & - & - & - - & -- & --- \\
\hline $08 / 05 / 88$ & $09: 50$ & $1-C$ & 1 & 8.00 & 15.00 & $1.412 \mathrm{E}-02$ & 11.22 \\
\hline $08 / 05 / 88$ & $09: 50$ & $1-A$ & 2 & 12.05 & 10.00 & $9.416 \mathrm{E}-03$ & 13.73 \\
\hline $08 / 05 / 88$ & $09: 50$ & $1-B$ & 6 & 5.55 & 10.00 & $1.017 \mathrm{E}-02$ & 8.51 \\
\hline $08 / 06 / 88$ & $11: 07$ & $1-C$ & 1 & 16.20 & 28.00 & $1.846 \mathrm{E}-02$ & 20.40 \\
\hline $08 / 06 / 88$ & $11: 07$ & 1-A & 2 & 11.85 & 28.00 & $1.846 \mathrm{E}-02$ & 15.14 \\
\hline $08 / 06 / 88$ & $11: 07$ & $1-B$ & 6 & 20.50 & 8.00 & $5.274 \mathrm{E}-03$ & 22.03 \\
\hline $08 / 07 / 88$ & $08: 33$ & $1-C$ & 1 & 14.30 & 18.00 & $1.400 \mathrm{E}-02$ & 17.49 \\
\hline $08 / 07 / 88$ & $08: 33$ & $1-\mathrm{A}$ & 2 & 11.90 & 18.00 & $1.400 \mathrm{E}-02$ & 14.39 \\
\hline $08 / 07 / 88$ & $08: 33$ & $1-B$ & 6 & 12.65 & 8.00 & $7.820 \mathrm{E}-03$ & 14.93 \\
\hline $08 / 08 / 88$ & $08: 45$ & $1-C$ & 1 & 12.25 & 18.00 & $1.240 \mathrm{E}-02$ & 15.07 \\
\hline 08/08/88 & $08: 45$ & $1-A$ & 2 & 11.90 & 8.00 & $5.510 \mathrm{E}-03$ & 12.88 \\
\hline $08 / 08 / 88$ & $08: 45$ & $1-B$ & 6 & 12.80 & 8.00 & $5.510 \mathrm{E}-03$ & 14.40 \\
\hline $08 / 09 / 88$ & $12: 24$ & $1-C$ & 1 & 11.90 & 38.00 & $2.274 \mathrm{E}-02$ & 17.08 \\
\hline $08 / 09 / 88$ & $12: 24$ & $1-\mathrm{A}$ & 2 & 11.85 & 38.00 & $2.274 \mathrm{E}-02$ & 15.90 \\
\hline $08 / 09 / 88$ & $12: 24$ & 1-B & 6 & 12.55 & 18.00 & $1.077 \mathrm{E}-02$ & 15.68 \\
\hline $08 / 10 / 88$ & $12: 02$ & $1-\mathrm{C}$ & 1 & 11.30 & 13.00 & $9.168 E-03$ & 13.39 \\
\hline $08 / 10 / 88$ & $12: 02$ & $1-\mathrm{A}$ & 2 & 13.35 & 13.00 & $9.168 \mathrm{E}-03$ & 14.98 \\
\hline $08 / 10 / 88$ & $12: 02$ & 1-B & 6 & 12.40 & 3.00 & $2.116 \mathrm{E}-03$ & 13.02 \\
\hline $08 / 11 / 88$ & $08: 28$ & $1-\mathrm{C}$ & 1 & 14.15 & 18.00 & $1.468 \mathrm{E}-02$ & 17.49 \\
\hline $08 / 11 / 88$ & $08: 28$ & $1-\mathrm{A}$ & 2 & 14.85 & 18.00 & $1.468 \mathrm{E}-02$ & 17.47 \\
\hline $08 / 11 / 88$ & $08: 28$ & $1-B$ & 6 & 14.70 & 3.00 & $2.447 \mathrm{E}-03$ & 15.41 \\
\hline $08 / 12 / 88$ & $11: 00$ & $1-C$ & 1 & 12.90 & 8.00 & $5.025 \mathrm{E}-03$ & 14.04 \\
\hline $08 / 12 / 88$ & $11: 00$ & $1-A$ & 2 & 13.00 & 3.00 & $1.884 \mathrm{E}-03$ & 13.34 \\
\hline $08 / 12 / 88$ & $11: 00$ & $1-B$ & 6 & 13.50 & 8.00 & $5.025 \mathrm{E}-03$ & 14.96 \\
\hline $08 / 13 / 88$ & $12: 45$ & $1-C$ & 1 & 12.00 & 23.00 & $1.489 \mathrm{E}-02$ & 15.39 \\
\hline $08 / 13 / 88$ & $12: 45$ & $1-A$ & 2 & 13.00 & 23.00 & $1.489 \mathrm{E}-02$ & 15.65 \\
\hline $08 / 13 / 88$ & $12: 45$ & $1-B$ & 6 & 13.45 & 0.00 & - & - \\
\hline $08 / 14 / 88$ & $13: 22$ & $1-C$ & 1 & - & 13.00 & $8.802 \mathrm{E}-03$ & - \\
\hline $08 / 14 / 88$ & $13: 22$ & $1-A$ & 2 & - & 13.00 & $8.802 E-03$ & - \\
\hline $08 / 14 / 88$ & $13: 22$ & $1-B$ & 6 & - & 3.00 & $2.031 \mathrm{E}-03$ & - \\
\hline $08 / 15 / 88$ & $09: 55$ & $1-C$ & 1 & 13.13 & 8.00 & $6.488 E-03$ & 14.61 \\
\hline $08 / 15 / 88$ & $09: 55$ & $1-\mathrm{A}$ & 2 & 13.45 & 13.00 & $1.054 \mathrm{E}-02$ & 15.33 \\
\hline $08 / 15 / 88$ & $09: 55$ & 1-B & 6 & 13.75 & 3.00 & $2.433 \mathrm{E}-03$ & 14.46 \\
\hline $08 / 16 / 88$ & $12: 15$ & $1-C$ & 1 & 13.73 & 15.00 & $9.494 \mathrm{E}-03$ & 15.89 \\
\hline $08 / 16 / 88$ & $12: 15$ & $1-A$ & 2 & 14.00 & 28.00 & $1.772 \mathrm{E}-02$ & 17.16 \\
\hline $08 / 16 / 88$ & $12: 15$ & $1-B$ & 6 & 14.20 & 3.00 & $1.899 \mathrm{E}-03$ & 14.75 \\
\hline 08/17/88 & $08: 48$ & $1-C$ & 1 & 12.95 & 16.00 & $1.298 \mathrm{E}-02$ & 15.90 \\
\hline $08 / 17 / 88$ & $08: 48$ & $1-\mathrm{A}$ & 2 & 14.15 & 3.00 & $2.433 \mathrm{E}-03$ & 14.58 \\
\hline 08/17/88 & $08: 48$ & 1-B & 6 & 14.68 & 0.00 & - & - \\
\hline $08 / 18 / 88$ & $09: 00$ & $1-C$ & 1 & 13.75 & 8.00 & $5.510 \mathrm{E}-03$ & 15.00 \\
\hline $08 / 18 / 88$ & $09: 00$ & $1-\mathrm{A}$ & 2 & 13.40 & 8.00 & $5.510 \mathrm{E}-03$ & 14.38 \\
\hline $08 / 18 / 88$ & $09: 00$ & 1-B & 6 & 15.07 & 3.00 & $2.066 \mathrm{E}-03$ & 15.67 \\
\hline 08/19/88 & $11: 45$ & $1-\mathrm{C}$ & 1 & 14.50 & 8.00 & $4.984 \mathrm{E}-03$ & 15.64 \\
\hline $08 / 19 / 88$ & $11: 45$ & $1-A$ & 2 & 13.40 & 23.00 & $1.433 \mathrm{E}-02$ & 15.95 \\
\hline
\end{tabular}


TABLE B.16 (continued)

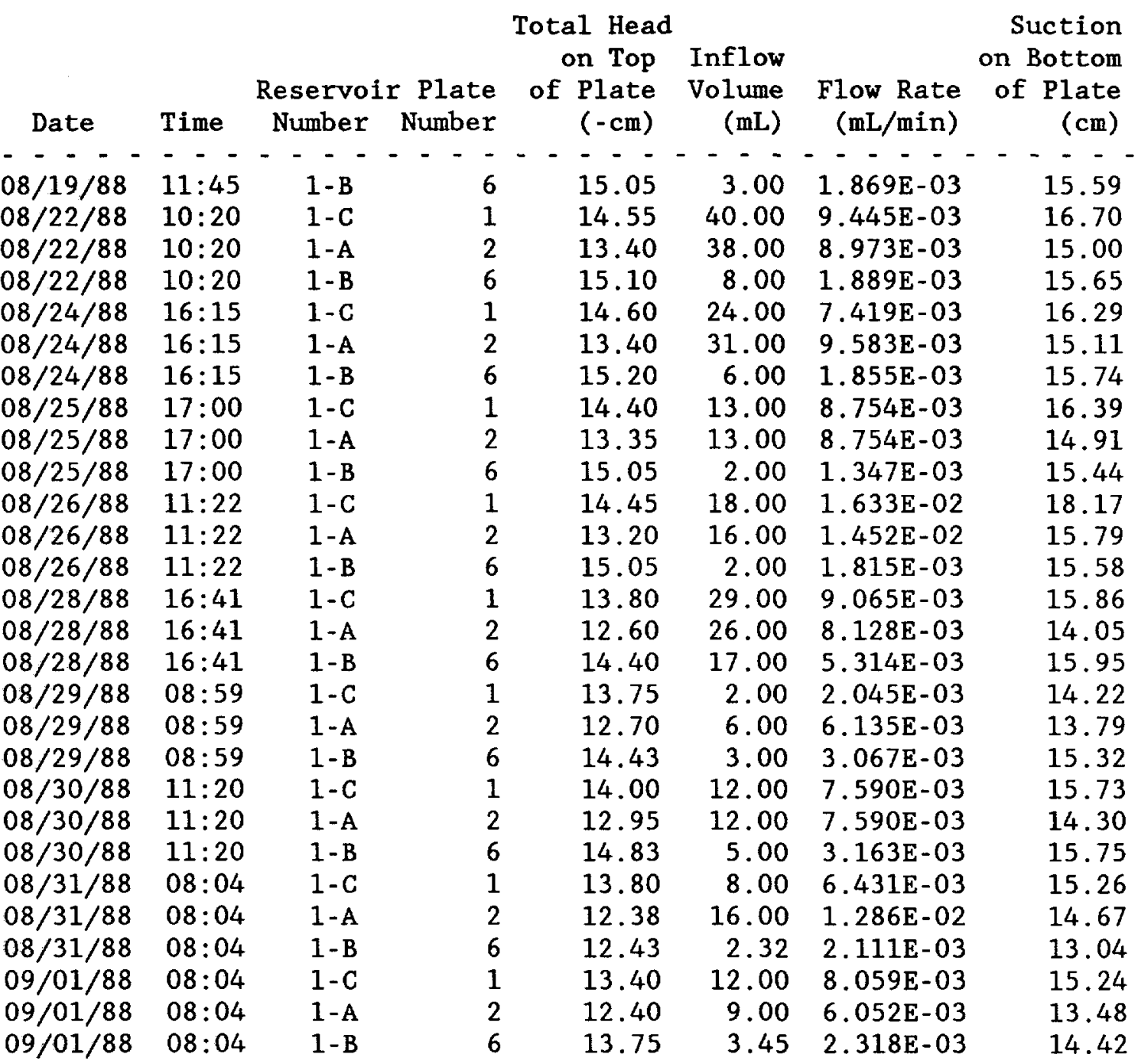


TABLE B.17

WETTING FRONT DATA - TEST BLOCK 2

\section{Depth to Wetting Front at Given Locations \\ (cm from left of face)}

\begin{tabular}{|c|c|c|c|c|c|c|c|}
\hline Date & Time & Face & $2 \mathrm{~cm}$ & $6 \mathrm{~cm}$ & $10 \mathrm{~cm}$ & $14 \mathrm{~cm}$ & $18 \mathrm{~cm}$ \\
\hline$--\cdot-$ & -- & -- & $--\cdot-$ & $--\cdot-$ & $--\cdot-$ & $--\cdot-$ & --- \\
\hline $08 / 03 / 88$ & $22: 37$ & 3 & 0.0 & 0.0 & 2.0 & 1.9 & 1.9 \\
\hline $08 / 03 / 88$ & $22: 42$ & 4 & 0.9 & 2.2 & 0.0 & 0.0 & 0.0 \\
\hline $08 / 03 / 88$ & $22: 41$ & 5 & 1.6 & 0.0 & 1.2 & 0.0 & 0.0 \\
\hline 08/03/88 & $22: 46$ & 6 & 2.3 & 1.6 & 2.2 & 0.0 & 2.5 \\
\hline $08 / 04 / 88$ & $13: 38$ & 3 & 0.0 & 0.0 & 4.1 & 5.8 & 5.3 \\
\hline $08 / 04 / 88$ & $13: 42$ & 4 & 1.6 & 2.7 & 2.0 & 1.3 & 0.0 \\
\hline $08 / 04 / 88$ & $13: 46$ & 5 & 4.3 & 2.2 & 3.6 & 2.5 & 1.7 \\
\hline $08 / 04 / 88$ & $13: 53$ & 6 & 5.2 & 3.9 & 4.2 & 3.4 & 4.6 \\
\hline $08 / 05 / 88$ & $12: 25$ & 3 & 0.7 & 4.0 & 5.8 & 6.9 & 6.6 \\
\hline $08 / 05 / 88$ & $10: 21$ & 4 & 3.3 & 5.8 & 4.0 & 6.1 & 3.0 \\
\hline $08 / 05 / 88$ & $10: 29$ & 5 & 6.4 & 4.1 & 8.4 & 5.8 & 4.6 \\
\hline $08 / 05 / 88$ & $10: 35$ & 6 & 7.0 & 5.6 & 5.6 & 4.8 & 5.8 \\
\hline $08 / 06 / 88$ & $11: 24$ & 3 & 2.0 & 5.2 & 7.3 & 8.3 & 8.6 \\
\hline $08 / 06 / 88$ & $11: 29$ & 4 & 4.5 & 6.8 & 6.6 & 6.5 & 3.5 \\
\hline $08 / 06 / 88$ & $11: 33$ & 5 & 7.9 & 6.4 & 10.3 & 8.4 & 5.7 \\
\hline $08 / 06 / 88$ & $11: 36$ & 6 & 9.2 & 8.5 & 8.6 & 7.7 & 8.6 \\
\hline 08/07/88 & $09: 08$ & 3 & 3.2 & 6.6 & 8.0 & 9.3 & 9.6 \\
\hline $08 / 07 / 88$ & $09: 03$ & 4 & 6.1 & 8.3 & 7.7 & 7.2 & 4.7 \\
\hline 08/07/88 & $09: 00$ & 5 & 9.1 & 8.7 & 10.5 & 9.0 & 7.2 \\
\hline $08 / 07 / 88$ & $08: 57$ & 6 & 12.0 & 11.8 & 10.0 & 9.6 & 8.9 \\
\hline $08 / 08 / 88$ & $09: 24$ & 3 & 6.6 & 8.4 & 10.0 & 10.6 & 10.6 \\
\hline $08 / 08 / 88$ & $09: 20$ & 4 & 6.9 & 8.7 & 8.8 & 10.6 & 6.5 \\
\hline $08 / 08 / 88$ & $09: 18$ & 5 & 10.4 & 9.4 & 11.3 & 10.4 & 8.3 \\
\hline $08 / 08 / 88$ & $09: 07$ & 6 & 10.8 & 10.8 & 10.8 & 10.6 & 10.3 \\
\hline $08 / 10 / 88$ & $14: 05$ & 3 & 8.6 & 9.8 & 11.0 & 12.4 & 13.5 \\
\hline $08 / 10 / 88$ & $14: 00$ & 4 & 10.1 & 10.5 & 10.9 & 9.0 & 8.6 \\
\hline $08 / 10 / 88$ & $13: 50$ & 5 & 11.1 & 11.9 & 11.8 & 11.0 & 10.4 \\
\hline $08 / 10 / 88$ & $13: 58$ & 6 & 12.5 & 12.5 & 12.5 & 12.5 & 12.5 \\
\hline $08 / 11 / 88$ & $16: 44$ & 3 & 9.9 & 10.3 & 12.3 & 13.9 & 15.1 \\
\hline $08 / 11 / 88$ & $16: 48$ & 4 & 11.2 & 11.2 & 11.2 & 11.1 & 9.7 \\
\hline $08 / 11 / 88$ & $16: 52$ & 5 & 13.1 & 13.9 & 13.3 & 14.2 & 11.3 \\
\hline $08 / 11 / 88$ & $16: 54$ & 6 & 17.3 & 16.3 & 14.0 & 13.0 & 12.5 \\
\hline $08 / 14 / 88$ & $13: 34$ & 3 & 11.6 & 12.1 & 13.5 & 15.2 & 17.0 \\
\hline $08 / 14 / 88$ & $13: 32$ & 4 & 11.7 & 11.7 & 11.7 & 11.7 & 11.7 \\
\hline $08 / 14 / 88$ & $13: 31$ & 5 & 15.2 & 14.9 & 14.4 & 13.5 & 12.7 \\
\hline $08 / 14 / 88$ & $13: 29$ & 6 & 19.0 & 19.5 & 16.4 & 15.7 & 15.5 \\
\hline $08 / 16 / 88$ & $13: 11$ & 3 & 14.3 & 14.2 & 14.8 & 15.5 & 18.0 \\
\hline $08 / 16 / 88$ & $13: 07$ & 4 & 14.0 & 14.0 & 14.0 & 14.0 & 14.0 \\
\hline $08 / 16 / 88$ & $13: 09$ & 5 & 16.5 & 15.5 & 14.7 & 14.5 & 13.8 \\
\hline $08 / 16 / 88$ & $13: 05$ & 6 & 19.5 & 19.6 & 17.5 & 16.7 & 16.9 \\
\hline $08 / 19 / 88$ & $15: 30$ & 3 & 16.0 & 15.7 & 16.0 & 17.6 & 19.4 \\
\hline $08 / 19 / 88$ & $15: 30$ & 4 & 16.4 & 16.3 & 15.8 & 15.8 & 16.5 \\
\hline $08 / 19 / 88$ & $15: 30$ & 5 & 18.1 & 17.1 & 16.1 & 15.9 & 15.2 \\
\hline $08 / 19 / 88$ & $15: 30$ & 6 & 21.3 & 21.1 & 20.0 & 19.6 & 19.3 \\
\hline
\end{tabular}


TABLE B.17 (continued)

Depth to Wetting Front at Given Locations (cm from left of face)

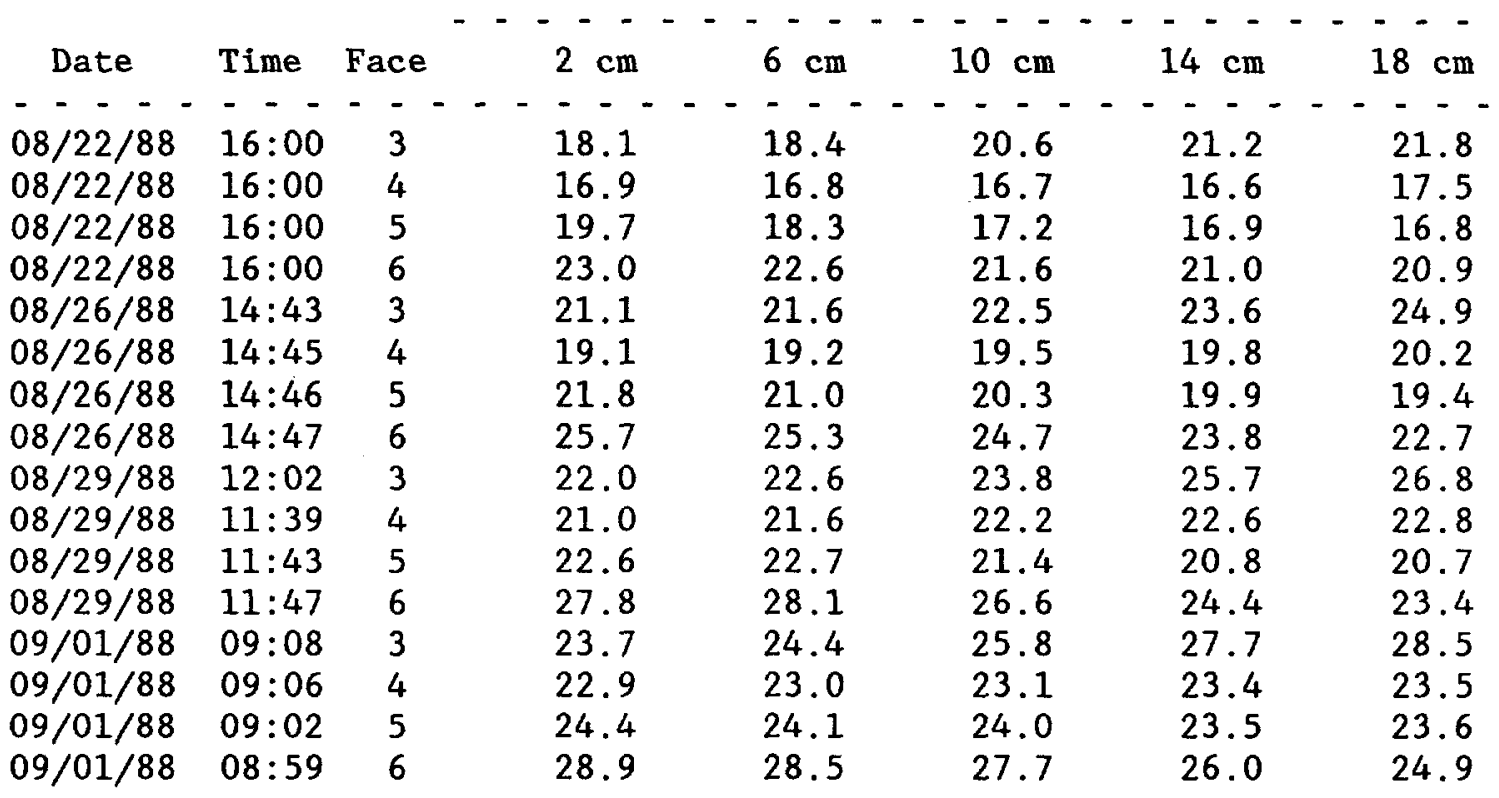

Note: Area of face $1=422.24 \mathrm{sq} . \mathrm{cm}$ 
TABLE B. 18

PHILIP'S EQUATION WETTING FRONT ANALYSIS - TEST BLOCK 2

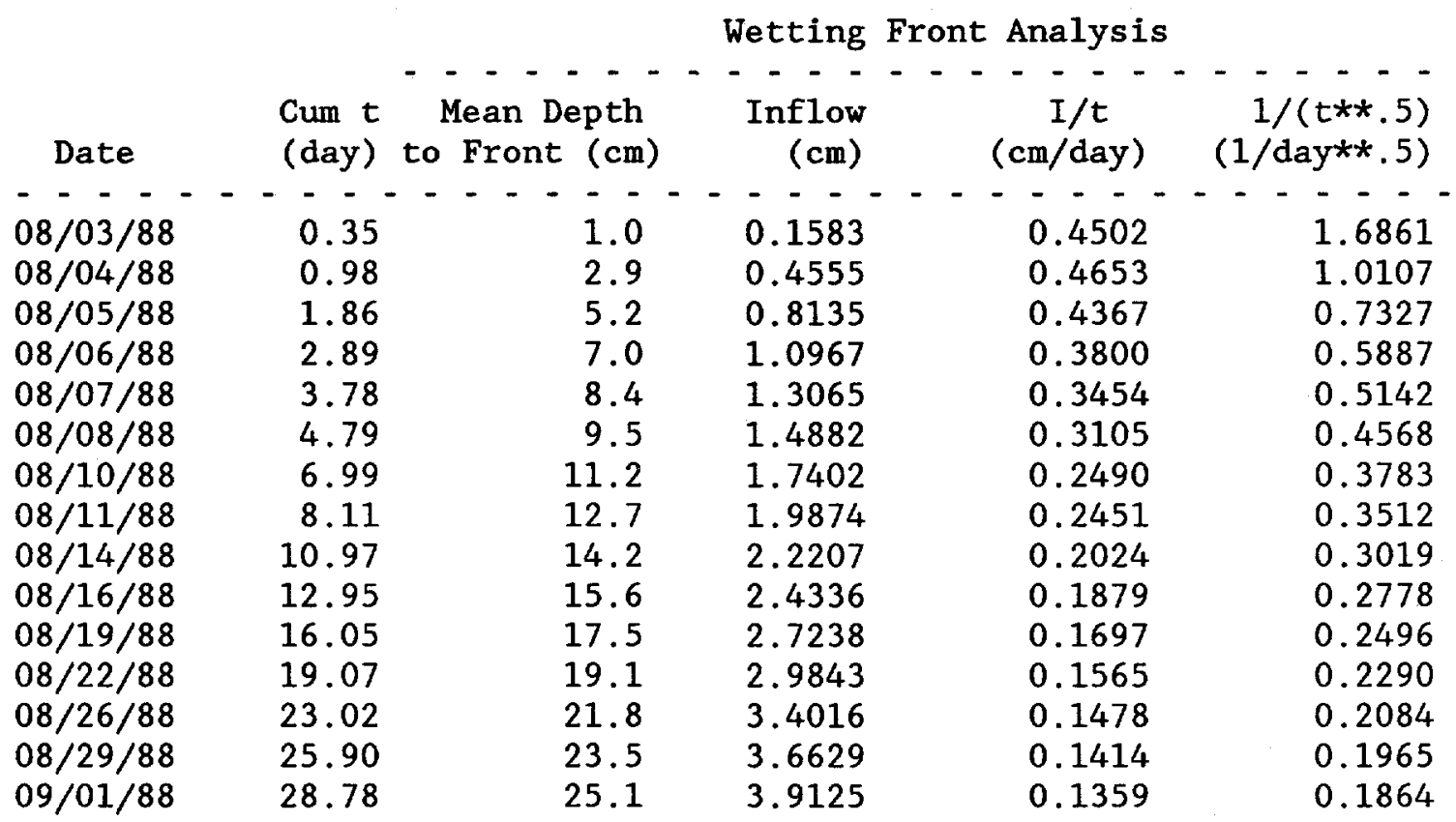


TABLE B.19

PHILIP'S EQUATION INFLOW ANALYSIS - TEST BLOCK 2

Mariotte Bottle Analysis Flow Tube Analysis

\begin{tabular}{|c|c|c|c|c|c|c|c|}
\hline $\begin{array}{l}\text { Cum } t \\
\text { (day) }\end{array}$ & $\begin{array}{r}\text { Inflow } \\
(\mathrm{cm})\end{array}$ & $\begin{array}{l}\text { Front } \\
(\mathrm{cm})\end{array}$ & $\begin{array}{c}I / t \\
(\mathrm{~cm} / \text { day })\end{array}$ & $\begin{array}{c}1 /(t * \star .5) \\
(1 / \text { day**.5) }\end{array}$ & $\begin{array}{l}\text { Inflow } \\
(\mathrm{cm})\end{array}$ & $\begin{array}{c}\mathrm{I} / \mathrm{t} \\
(\mathrm{cm} / \text { day })(\end{array}$ & $\begin{array}{r}1 /(t * * .5) \\
(1 / \text { day**.5) }\end{array}$ \\
\hline 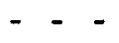 & --- & $--\cdot-$ & --- & $---\cdot-$ & -- & - & 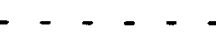 \\
\hline 0.35 & 0.1008 & 0.6 & 0.2865 & 1.6861 & 0.1008 & 0.2865 & 1.6861 \\
\hline 0.98 & 0.2744 & 1.8 & 0.2803 & 1.0107 & 0.2744 & 0.2803 & 1.01 \\
\hline 1.86 & 0.3573 & 2.3 & 0.1918 & 0.7327 & 0.3968 & 0.2130 & 0.732 \\
\hline 2.89 & 0.5088 & 3.3 & 0.1763 & 0.5887 & 0.5103 & 0.1768 & 0 \\
\hline 3.78 & 0.6 & 3.9 & 0.1 & 0.5142 & 0.6 & 0.1614 & 0. \\
\hline 4.79 & 0.6 & 4.4 & 0.1447 & 0.4568 & 0.7156 & 0.1493 & 0.456 \\
\hline 6.99 & 0.9 & 6.3 & 0.1409 & 0.3783 & 0.8974 & 84 & 0. \\
\hline 8.11 & 1.0 & 6.9 & 0.1 & 0.3 & 58 & 16 & 0 . \\
\hline 10.97 & 1.2999 & 8.3 & 0.1185 & 0.7 & 1.2236 & 15 & 0 \\
\hline 12.95 & 1.46 & 9.4 & 0.1131 & 0. & 1.3 & 66 & 0.2 \\
\hline 16.05 & 1.6 & 10.5 & 0.1019 & 0.2 & 1.6572 & 32 & 0.24 \\
\hline 19.07 & 1.8398 & 11.8 & 0.0965 & 0.2290 & 1.8982 & 95 & $0.2+2$ \\
\hline 23.02 & 2.1359 & 13.7 & 0.0928 & 0.2084 & 2.2508 & 0.0978 & $0.2-2$ \\
\hline 25.90 & 2.3324 & 15.0 & 0.0901 & 0.1965 & 2.6001 & 0.1004 & 0.15 \\
\hline 28.78 & 2.5211 & 16.2 & 0.0876 & 0.1864 & & & \\
\hline
\end{tabular}

Legend: $I$ = inflow, measured as height of solution

$t=$ time

Sat'd Front $=$ mean distance to front if all pores are saturated (porosity $=0.156$ ) 
TABLE B. 20

SATURATED HYDRAULIC CONDUCTIVITY DATA

\begin{tabular}{|c|c|c|c|c|c|c|c|}
\hline Date & Time & & Core & $\begin{array}{c}Q \\
(\mathrm{~cm} 3 / \mathrm{min})\end{array}$ & $\begin{array}{c}Q \\
(\mathrm{~m} 3 / \mathrm{sec})\end{array}$ & $\begin{array}{c}\mathrm{L} \\
(\mathrm{m})\end{array}$ & $\stackrel{A}{A}$ \\
\hline & & & & & & . & -- \\
\hline $07 / 06 / 88$ & $01: 12$ & PM & FT-5-A & 0.12897 & $2.150 \mathrm{E}-09$ & 0.0490 & 0.002463 \\
\hline 07/06/88 & $01: 30$ & PM & FT- $5-A$ & 0.12552 & $2.092 \mathrm{E}-09$ & 0.0490 & 0.002463 \\
\hline $07 / 06 / 88$ & $01: 46$ & PM & $F T-5-A$ & 0.12313 & 2.052E-09 & 0.0490 & 0.002463 \\
\hline $07 / 06 / 88$ & $02: 17$ & PM & FT-3-A & 0.09970 & $1.662 \mathrm{E}-09$ & 0.0490 & 0.002463 \\
\hline $07 / 06 / 88$ & $02: 36$ & PM & FT-3-A & 0.09564 & $1.594 \mathrm{E}-09$ & 0.0490 & 0.002463 \\
\hline $07 / 06 / 88$ & $02: 58$ & PM & FT-3-A & 0.09213 & $1.536 \mathrm{E}-09$ & 0.0490 & 0.002463 \\
\hline $07 / 07 / 88$ & $01: 10$ & PM & FT-5-B & 0.06201 & $1.033 \mathrm{E}-09$ & 0.0481 & 0.002463 \\
\hline $07 / 07 / 88$ & $01: 50$ & PM & $F T-5-B$ & 0.06209 & $1.035 E-09$ & 0.0481 & 0.002463 \\
\hline $07 / 07 / 88$ & $02: 20$ & PM & FT-5-B & 0.06088 & $1.015 \mathrm{E}-09$ & 0.0481 & 0.002463 \\
\hline 07/08/88 & $01: 14$ & PM & FT $-3-A$ & 0.10556 & $1.759 \mathrm{E}-09$ & 0.0490 & 02463 \\
\hline 07/08/88 & $01: 38$ & PM & FT-3-A & 0.10538 & $1.756 \mathrm{E}-09$ & 0.0490 & 0.002463 \\
\hline $07 / 08 / 88$ & $02: 03$ & PM & FT-3-A & 0.10511 & $1.752 \mathrm{E}-09$ & 0.0490 & 02463 \\
\hline $07 / 08 / 88$ & $03: 00$ & PM & FT $-5-A$ & 0.12132 & $2.022 E-09$ & 0.0490 & 0.002463 \\
\hline $07 / 08 / 88$ & $03: 19$ & PM & FT $-5-A$ & 0.11879 & 1.980 E -09 & 0.0490 & 0.002463 \\
\hline 07/08/88 & $03: 37$ & PM & FT $-5-A$ & 0.11740 & $1.957 \mathrm{E}-09$ & 0.0490 & 02463 \\
\hline $07 / 11 / 88$ & $12: 21$ & PM & A4A & 0.03059 & $5.099 \mathrm{E}-10$ & 0.0506 & 02539 \\
\hline $07 / 11 / 88$ & $01: 34$ & PM & A4A & 0.02859 & $4.765 E-10$ & 0.0506 & 02539 \\
\hline 07/11/88 & $02: 52$ & PM & A4A & 0.02769 & $4.616 \mathrm{E}-10$ & 0.0506 & 2539 \\
\hline $07 / 12 / 88$ & $01: 05$ & PM & FT $-5-A$ & 0.11634 & $1.939 \mathrm{E}-09$ & 0.0490 & 2463 \\
\hline $07 / 12 / 88$ & $01: 30$ & PM & FT $-5-A$ & 0.11503 & $1.917 \mathrm{E}-09$ & 0.0490 & 2463 \\
\hline $07 / 12 / 88$ & $01: 45$ & PM & FT-5-A & 0.11356 & $1.893 \mathrm{E}-09$ & 0.0490 & 2463 \\
\hline $07 / 12,88$ & $02: 05$ & PM & FT - 5-A & 0.11291 & $1.882 \mathrm{E}-09$ & 0.0490 & 2463 \\
\hline $07 / 12 / 88$ & $02: 20$ & PM & FT $-5-A$ & 0.11184 & $1.864 \mathrm{E}-09$ & 0.0490 & 0.002463 \\
\hline $07 / 12 / 88$ & $02: 50$ & PM & FT $-5-A$ & 0.11085 & $1.847 \mathrm{E}-09$ & 0.0490 & 02463 \\
\hline $07 / 12 / 88$ & $03: 15$ & PM & FT-5-A & 0.11023 & 1.837 E-09 & 0.0490 & 2463 \\
\hline $07 / 12 / 88$ & $03: 30$ & PM & FT $-5-A$ & 0.10993 & $1.832 \mathrm{E}-09$ & 0.0490 & 0.002463 \\
\hline $07 / 13 / 88$ & $12: 02$ & PM & FT-3-A & 0.08982 & $1.497 \mathrm{E}-09$ & 0.0490 & 0.002463 \\
\hline $07 / 13 / 88$ & $12: 59$ & PM & FT-3-A & 0.08615 & $1.436 \mathrm{E}-09$ & 0.0490 & 02463 \\
\hline $07 / 13 / 88$ & $01: 15$ & PM & FT-3-A & 0.08586 & $1.431 \mathrm{E}-09$ & 0.0490 & 0.002463 \\
\hline $07 / 13 / 88$ & $01: 40$ & PM & FT-3-A & 0.08498 & $1.416 \mathrm{E}-09$ & 0.0490 & 0.002463 \\
\hline $07 / 13 / 88$ & $02: 10$ & PM & FT-3-A & 0.08410 & $1.402 \mathrm{E}-09$ & 0.0490 & 0.002463 \\
\hline $07 / 13 / 88$ & $02: 40$ & PM & FT-3-A & 0.08355 & $1.393 \mathrm{E}-09$ & 0.0490 & 0.002463 \\
\hline $07 / 17 / 88$ & $08: 46$ & PM & FT-5-B & 0.04422 & $7.371 \mathrm{E}-10$ & 0.0481 & 0.002463 \\
\hline $07 / 17 / 88$ & $10: 02$ & PM & FT $-5-B$ & 0.03891 & $6.485 E-10$ & 0.0481 & 0.002463 \\
\hline $07 / 17 / 88$ & $02: 40$ & PM & FT - 5-B & 0.03687 & $6.145 E-10$ & 0.0481 & 0.002463 \\
\hline 07/17/88 & $02: 40$ & PM & FT-5-B & 0.03884 & $6.474 E-10$ & 0.0481 & 0.002463 \\
\hline $07 / 17 / 88$ & $02: 40$ & PM & FT-5-B & 0.03843 & $6.406 \mathrm{E}-10$ & 0.0481 & 0.002463 \\
\hline 07/17/88 & $02: 40$ & PM & FT-5-B & 0.03838 & $6.396 \mathrm{E}-10$ & 0.0481 & 0.002463 \\
\hline $07 / 17 / 88$ & $02: 40$ & PM & FT- $5-B$ & 0.03585 & $5.976 \mathrm{E}-10$ & 0.0481 & 0.002463 \\
\hline $07 / 17 / 88$ & $02: 40$ & PM & FT-5-B & 0.03628 & $6.046 E-10$ & 0.0481 & 0.002463 \\
\hline $07 / 23 / 88$ & $03: 11$ & PM & B4A & 0.02267 & $3.778 \mathrm{E}-10$ & 0.0508 & 0.002498 \\
\hline $07 / 24 / 88$ & $06: 50$ & AM & B $4 \mathrm{~A}$ & 0.01705 & $2.842 \mathrm{E}-10$ & 0.0508 & 0.002498 \\
\hline $07 / 24 / 88$ & $12: 22$ & PM & B4A & 0.01622 & $2.704 E-10$ & 0.0508 & 0.002498 \\
\hline $07 / 24 / 88$ & 03:11 & PM & B4A & 0.01514 & $2.524 \mathrm{E}-10$ & 0.0508 & 0.002498 \\
\hline $07 / 24 / 88$ & $08: 53$ & PM & B4A & 0.01511 & $2.519 \mathrm{E}-10$ & 0.0508 & 0.002498 \\
\hline $07 / 25$ & $08: 15$ & AM & B4A & 0.01349 & 0 & 0508 & 8 \\
\hline
\end{tabular}


TABLE B.20 (continued)

\begin{tabular}{|c|c|c|c|c|c|c|c|}
\hline Date & Tim & & Core & $\begin{array}{c}Q \\
(\mathrm{~cm} 3 / \min )\end{array}$ & $\begin{array}{c}Q \\
(\mathrm{~m} 3 / \mathrm{sec})\end{array}$ & $\begin{array}{c}\mathrm{L} \\
(\mathrm{m})\end{array}$ & $\underset{(\mathrm{m} 2)}{\mathrm{A}}$ \\
\hline---- & -- & - & -- & $-\ldots--$ & $-\cdots$ & -- & $--\cdot$ \\
\hline $07 / 25 / 88$ & $09: 45$ & $A M$ & B4A & 0.01344 & $2.241 E-10$ & 0.0508 & 0.002498 \\
\hline $07 / 25 / 88$ & $12: 15$ & PM & B4A & 0.01334 & $2.223 E-10$ & 0.0508 & 0.002498 \\
\hline $07 / 25 / 88$ & $12: 40$ & PM & B4A & 0.01294 & $2.157 E-10$ & 0.0508 & 0.002498 \\
\hline $08 / 24 / 88$ & $11: 05$ & AM & FT - 5-AA & 0.07558 & $1.260 \mathrm{E}-09$ & 0.0543 & 0.002516 \\
\hline $08 / 24 / 88$ & $12: 00$ & PM & FT - 3-AA & 0.13700 & $2.283 E-09$ & 0.0518 & 0.002507 \\
\hline $08 / 24 / 88$ & $12: 32$ & PM & FT - 3-BB & 0.11665 & $1.944 \mathrm{E}-09$ & 0.0516 & 0.002516 \\
\hline $08 / 25 / 88$ & $09: 21$ & AM & A3A & 0.06376 & $1.063 E-09$ & 0.0517 & 0.002588 \\
\hline $08 / 25 / 88$ & $10: 09$ & $\mathrm{AM}$ & B5A-1 & 0.24598 & $4.100 E-09$ & 0.0504 & 0.002498 \\
\hline $08 / 25 / 88$ & $10: 42$ & AM & $\mathrm{B} 6 \mathrm{~A}$ & 0.04630 & $7.716 \mathrm{E}-10$ & 0.0521 & 0.002503 \\
\hline $08 / 25 / 88$ & $11: 24$ & $\mathrm{AM}$ & B5A-2 & 0.20685 & $3.447 \mathrm{E}-09$ & 0.0504 & 0.002498 \\
\hline
\end{tabular}


TABLE B. 21

SATURATED HYDRAULIC CONDUCTIVITY DETERMINATIONS

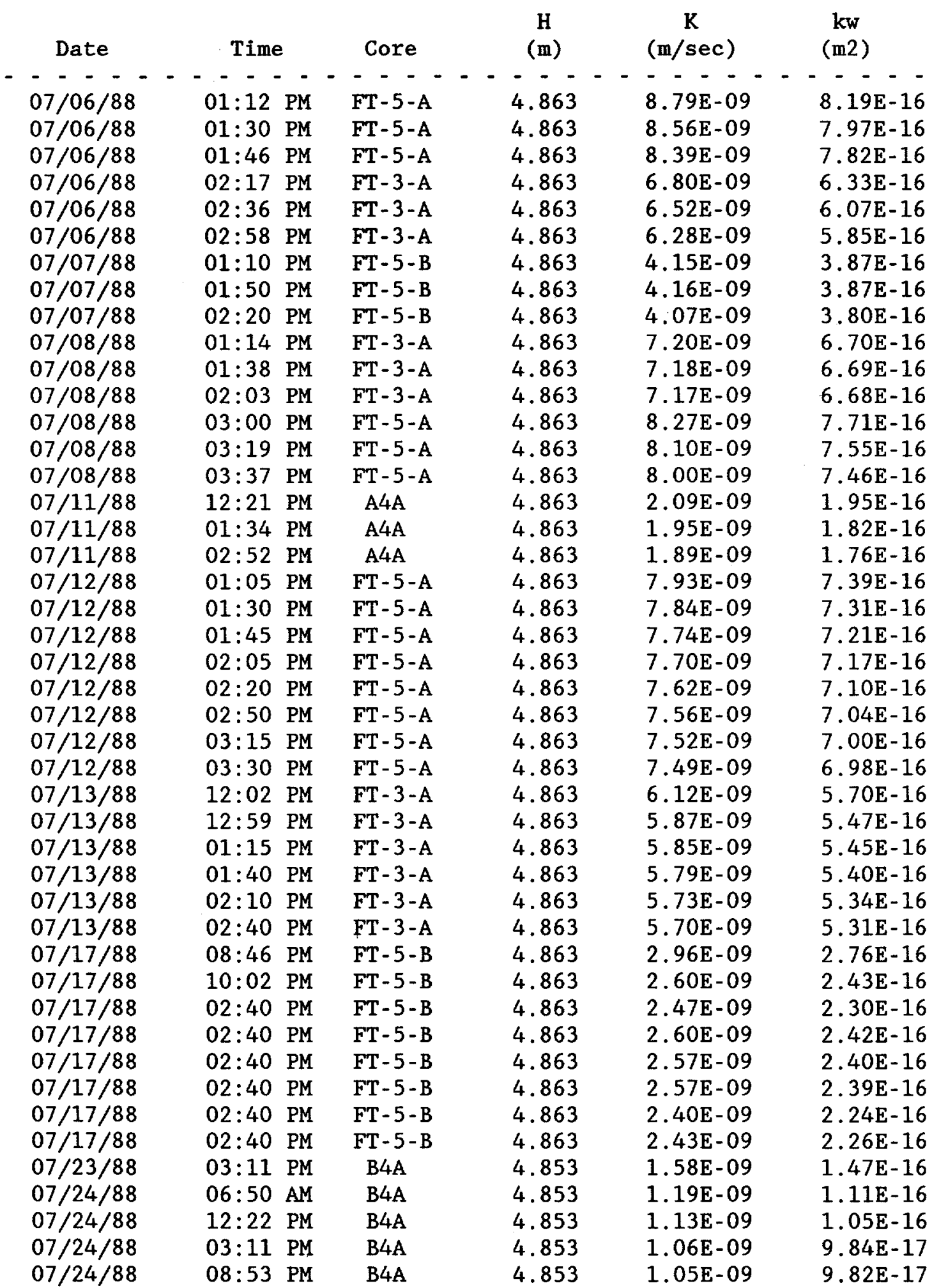


TABLE B.21 (continued)

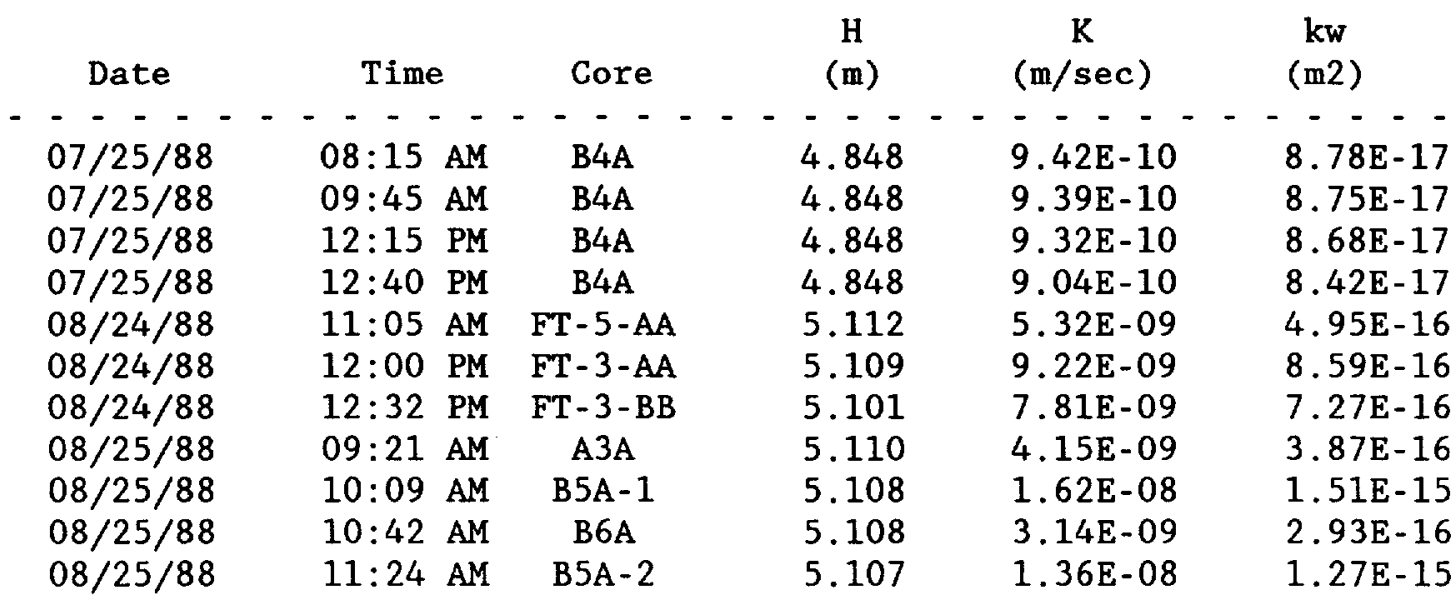

Legend: $\quad H=$ total head on top of core

$\mathrm{K}=$ saturated hydraulic conductivity of core $\mathrm{kw}=$ intrinsic permeability of core 
TABLE B. 22

MOISTURE RELEASE CURVES

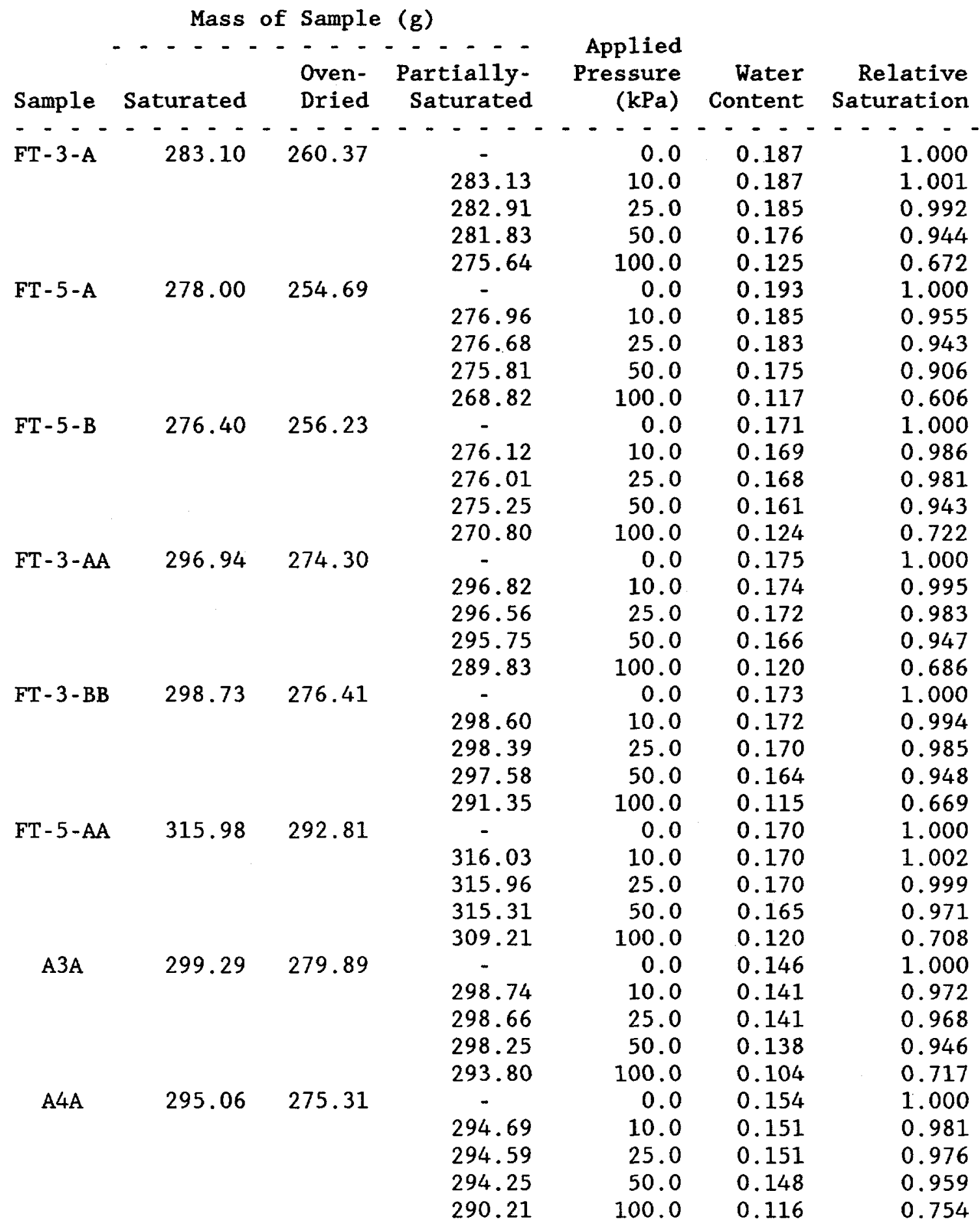


TABLE B.22 (continued)

\begin{tabular}{|c|c|c|c|c|c|c|}
\hline ample & $\begin{array}{l}\text { Mass } \\
---- \\
\text { Saturated }\end{array}$ & $\begin{array}{l}\text { Sample } \\
---- \\
\text { Oven- } \\
\text { Dried }\end{array}$ & $\begin{array}{l}(\mathrm{g}) \\
--- \\
\text { Partially- } \\
\text { Saturated }\end{array}$ & $\begin{array}{r}\text { Applied } \\
\text { Pressure } \\
(\mathrm{kPa})\end{array}$ & $\begin{array}{r}\text { Water } \\
\text { Content }\end{array}$ & $\begin{array}{c}\text { Relative } \\
\text { Saturation }\end{array}$ \\
\hline $\bar{B} 4 \mathrm{~A}$ & $--\overline{-}-\overline{286.92}$ & $-\overline{267.16}$ & $\begin{array}{r}--- \\
- \\
286.44 \\
286.36 \\
286.15 \\
281.68\end{array}$ & $\begin{array}{r}--\overline{0} \\
0.0 \\
10.0 \\
25.0 \\
50.0 \\
100.0\end{array}$ & $\begin{array}{c}--- \\
0.156 \\
0.152 \\
0.152 \\
0.150 \\
0.115\end{array}$ & $\begin{array}{r}- \\
1.000 \\
0.976 \\
0.972 \\
0.961 \\
0.735\end{array}$ \\
\hline B5A & 283.01 & 260.71 & $\begin{array}{c}- \\
283.03 \\
282.88 \\
280.98 \\
274.00\end{array}$ & $\begin{array}{r}0.0 \\
10.0 \\
25.0 \\
50.0 \\
100.0\end{array}$ & $\begin{array}{l}0.178 \\
0.178 \\
0.177 \\
0.162 \\
0.106\end{array}$ & $\begin{array}{l}1.000 \\
1.001 \\
0.994 \\
0.909 \\
0.596\end{array}$ \\
\hline B6A & 300.40 & 281.43 & $\begin{array}{c}- \\
300.25 \\
300.18 \\
299.86 \\
295.79\end{array}$ & $\begin{array}{r}0.0 \\
10.0 \\
25.0 \\
50.0 \\
100.0\end{array}$ & $\begin{array}{l}0.146 \\
0.145 \\
0.144 \\
0.142 \\
0.110\end{array}$ & $\begin{array}{l}1.000 \\
0.992 \\
0.988 \\
0.972 \\
0.757\end{array}$ \\
\hline
\end{tabular}


TABLE B. 23

ROCK CHARACTERISTIC TESTS

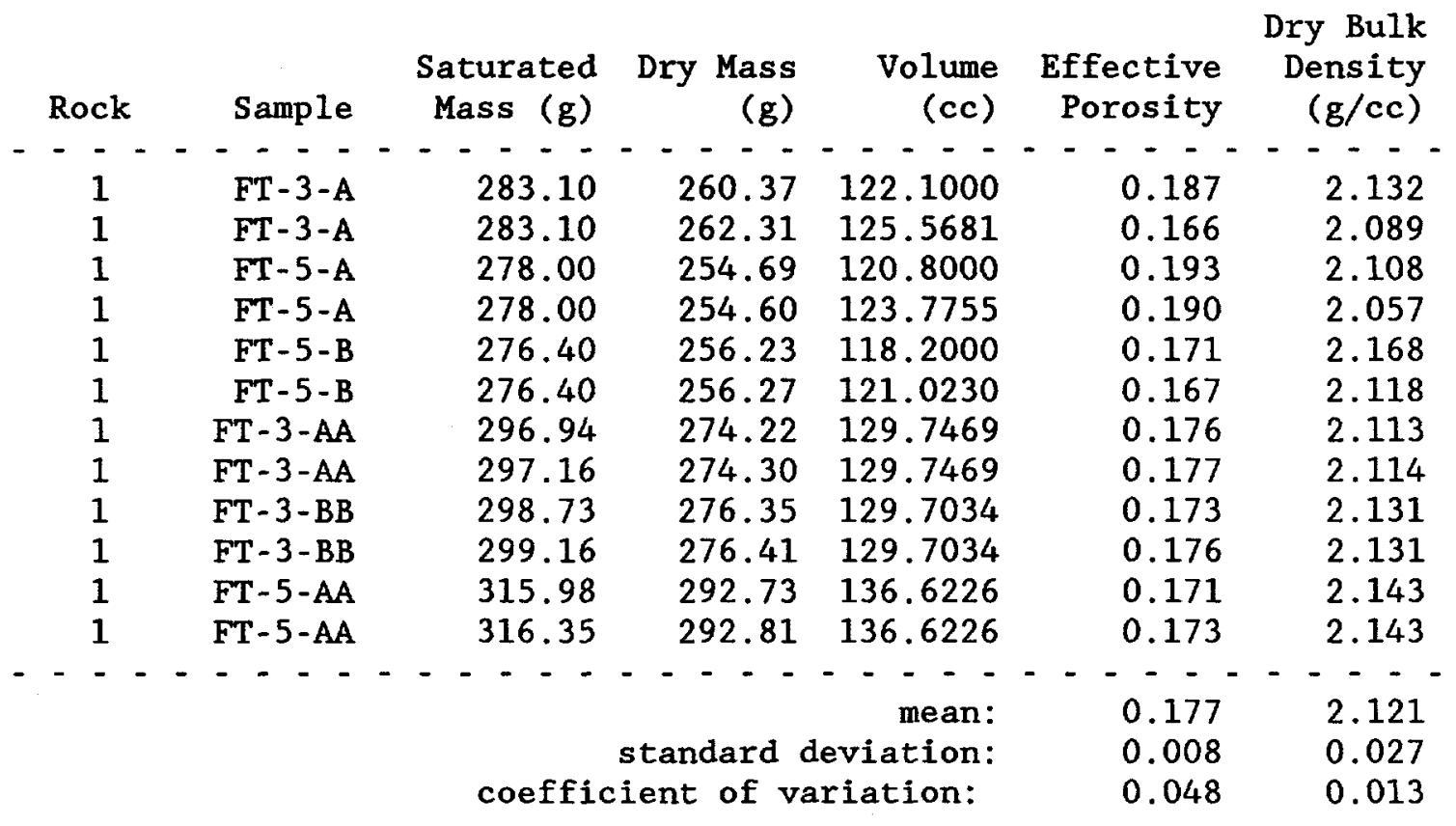

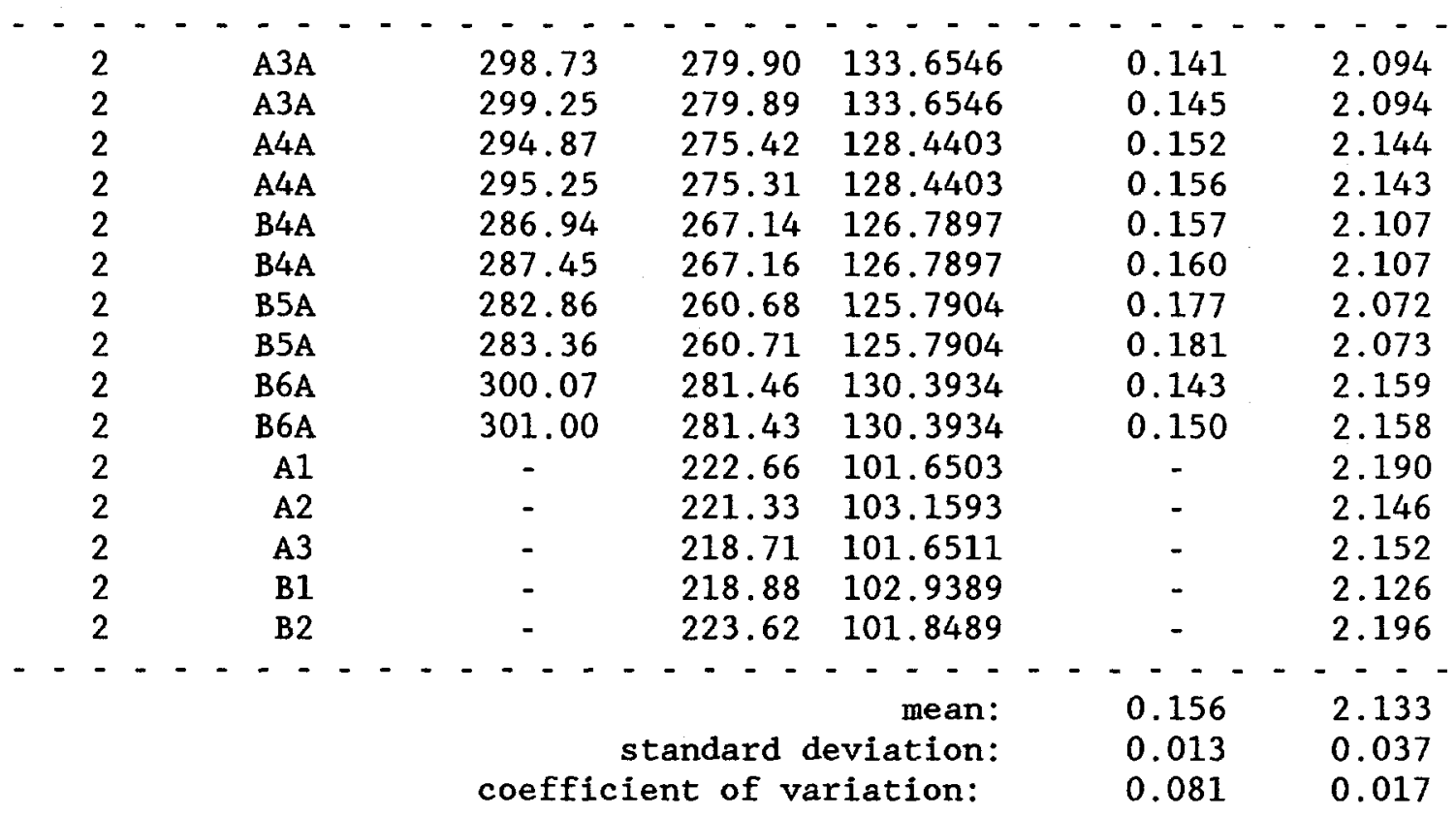


This section presents more detailed mathematical formulations of Philip's (1988) analysis of water penetration into circular-cylindrical cavities. In addition to potential functions, dimensionless flow velocities and stream functions are also discussed. A table of exact and asymptotic maximum dimensionless Kirchhoff potentials ( $v_{\max }$ values) is included.

Philip (1988) defined "water entry" to occur "... [w] hen the Gaussian curvature of air-water interfaces at the mouths of at least some surface pores debouching into the cavity is positive." The suction or moisture potential $\psi$ is then $<0$. The nonlinear equation of steady flow in unsaturated porous medium is:

$$
\nabla(\mathrm{K} \nabla \psi)=\partial \mathrm{K} / \partial \mathrm{z}
$$

where the differentiation is with respect to the physical space coordinates. The quasilinear form of equation C.1 is used instead:

$$
\nabla^{2} \Phi_{\mathrm{p}}=\alpha_{\mathrm{s}} \partial \Phi_{\mathrm{p}} / \partial z
$$

and two special values of $\Phi_{p}$, the Kirchhoff potential, are defined as:

$$
\Phi_{\mathrm{p}, \mathrm{o}}=\int_{-\infty}^{\psi_{\mathrm{o}}} \mathrm{K} \mathrm{d} \psi \quad, \quad \Phi_{\mathrm{p}, \mathrm{m}}=\int_{-\infty}^{0} \mathrm{~K}_{\mathrm{m}} \mathrm{d} \psi
$$

The sorptive number, $\alpha_{s}$, enters the exponential representation of $\mathrm{K}(\psi)$ as:

$$
K(\psi)=K_{0} \exp \left[\alpha_{s}\left(\psi-\psi_{0}\right)\right]
$$$$
0 \geq \psi \geq-\infty
$$

with $\mathrm{K}_{0}$ the conductivity associated with $\psi_{0}$. The seepage velocity, $\mathrm{K}_{0}$, can be any unsaturated conductivity or the saturated conductivity.

Equation C.2 is expressed in dimensionless form as:

$$
\nabla^{2} \mathrm{v}=2 \mathrm{~s} \partial \mathrm{v} / \partial \mathrm{z}
$$

where $\mathrm{v}=\Phi_{\mathrm{p}} / \Phi_{\mathrm{p}, \mathrm{o}}$, with the following two boundary conditions:

$$
\lim _{r \rightarrow \infty} v=1<\Phi_{p, m} / \Phi_{p, o}=v_{m}
$$

corresponding to a $\mathrm{K}_{0}$ far from the cavity; and (2) for no water entry at the cavity surface $A$, the flow velocity normal to $A$ is zero and $\Phi_{p} \leq$ $\Phi_{\mathrm{D}, \mathrm{m}}$. Other dimensionless quantities are defined as:

$$
\mathrm{x} / \mathrm{x}^{*}=\mathrm{y} / \mathrm{y}^{*}=\mathrm{z} / \mathrm{z}^{*}=\mathrm{r} / \mathrm{r}^{*}=1 / 1_{\mathrm{c}} ; \mathrm{s}=0.5 \alpha_{\mathrm{s}} 1_{\mathrm{c}}
$$


For circular cylindrical cavities, the dimensionless radius is $1_{c}$ with physical radius of 1 . Centered at $(x, z)=(0,0)$, in terms of cylindrical polar coordinates $(r, \phi)$ :

$$
\mathrm{x}=\mathrm{r} \sin \phi, z=\mathrm{r} \cos \phi ; \quad-\pi<\phi \leq \pi .
$$

The exact solution to equation C.5 is:

$$
\begin{aligned}
v= & 1+4 \exp [s r \cos \phi] \sum_{j=1}^{\infty} \frac{j I_{1}(s)}{K_{1}(s)} K_{o}(s r)+ \\
& \sum_{n=1}^{\infty}(-1)^{n} K_{n}(s r) \cos (n \phi) \frac{n I_{n}(s)}{K_{n}(s)}+2 \sum_{j=n+1}^{\infty} \frac{j I_{1}(s)}{K_{j}(s)}
\end{aligned}
$$

where $I_{n}$ and $K_{n}$ are the modified Bessel functions of order $n$ of the first and second kinds, respectively. Asymptotic results give:

$$
(1, \pi)=v_{\max }=2 s+2-1 / s+2 / s^{2}-\ldots .
$$

For small $s$ values, $v_{\max }$ is just $1+2 \mathrm{~s}$. The horizontal and vertical flow velocities, $u^{*}$ and $v^{*}$, are normalized with respect to $k_{0}$ :

$$
u=u^{*} / k_{0}=-(1 / 2 s) \partial v / \partial x
$$

and

$$
\mathrm{v}=\mathrm{v}^{*} / \mathrm{K}_{\mathrm{o}}=\mathrm{v}-(1 / 2 \mathrm{~s}) \partial \mathrm{v} / \partial \mathrm{z}
$$

The dimensionless stream functions $\Psi$ are then defined by:

$$
u_{x}=-\partial \Psi / \partial z \quad, \quad u_{z}=\partial \Psi / \partial x
$$

Dimensionless potential and stream functions, and velocity maps can then be plotted for different porous media with different $s$ values. 
Table C.1: Exact and asymptotic values of $v_{\max }$ for seepage about circular-cylindrical cavities (after Philip, 1988).

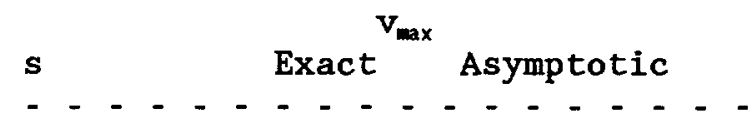

$\begin{array}{lll}0 & 1.0000 & \\ 0.05 & 1.1126 & \\ 0.1 & 1.2361 & \\ 0.15 & 1.3636 & \\ 0.25 & 1.6205 & \\ 0.5 & 2.244 & \\ 0.75 & 2.835 & \\ 1 & 3.403 & 3.000 \\ 1.5 & 4.500 & 4.333 \\ 2 & 5.566 & 5.500 \\ 3 & 7.653 & 7.667 \\ 4 & 9.709 & 9.750 \\ 6 & 13.78 & 13.83 \\ 8 & 17.82 & 17.88 \\ 10 & 21.85 & 21.90 \\ 12 & 25.87 & 25.92 \\ 14 & 29.88 & 29.93 \\ 16 & 33.90 & 33.94\end{array}$


CHLORIDE BREARTHROUGH DATA OF POROUS PLATES

This section presents the chloride breakthrough data of porous plates nos. 1 to 6 . Information on test conditions such as flow rate, head gradient imposed, background and tracer concentrations are included. The effective porosities of the plates are also determined. 
Table D.1: Chloride breakthrough data of porous plate nos. 1 to 6 .

Plate Number: 1

Date: 6-02-88

Flow Rate: $3.03 \times 10^{-9} \mathrm{~m}^{3} / \mathrm{s}$

Head Gradient: $0.50 \mathrm{~m}$

Adjusted Head Gradient: $0.414 \mathrm{~m}$

Test Type: step-up

$$
\begin{gathered}
E_{i}=184 \mathrm{mV} \\
C_{1}=0.00140 \underline{M} \\
E_{0}=127 \mathrm{mV} \\
C_{0}=0.0130 \underline{M}
\end{gathered}
$$

$$
\begin{aligned}
& \text { Elapsed Potential Conc., Conc., } \\
& \text { Time, Difference - min - } \max \\
& t_{\mathrm{E}}(\mathrm{hr}) \quad \mathrm{E}(\mathrm{mV}) \quad(\underline{M}) \quad(\underline{M})
\end{aligned}
$$

$\begin{array}{cccc}- & - & - & - \\ 0.50 & 180-170 & 0.0016 & 0.0024 \\ 1.33 & 180-165 & 0.0016 & 0.0030 \\ 2.00 & 175-160 & 0.0020 & 0.0036 \\ 2.75 & 150-145 & 0.0054 & 0.0066 \\ 3.50 & 150-130 & 0.0054 & 0.0120 \\ 4.25 & 137-130 & 0.0090 & 0.0120 \\ 5.00 & 140-128 & 0.0080 & 0.0125 \\ 6.00 & 135-127 & 0.0096 & 0.0130 \\ 6.92 & 128-127 & 0.0125 & 0.0130\end{array}$

Plate Number: 1

Date: 6-03-88

Flow Rate: $3.15 \times 10^{-9} \mathrm{~m}^{3} / \mathrm{s}$

Head Gradient: $0.50 \mathrm{~m}$

Adjusted Head Gradient: $0.430 \mathrm{~m}$

Test Type: step-down

$$
\begin{gathered}
E_{i}=184 \mathrm{mV} \\
C_{1}=0.00140 \underline{M} \\
E_{o}=127 \mathrm{mV} \\
C_{0}=0.0130 \underline{M}
\end{gathered}
$$

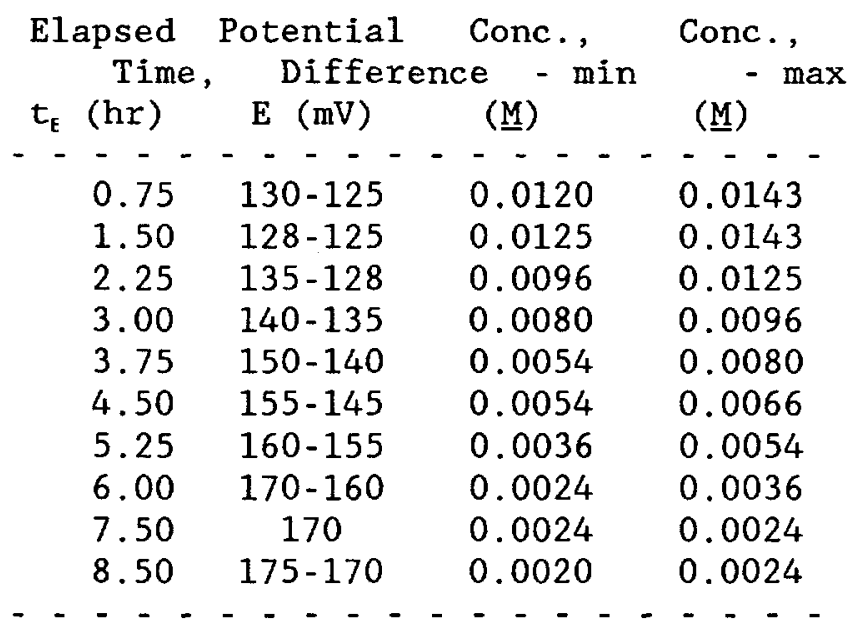


Plate Number: 2

Date: 6-01-88

Flow Rate: $4.08 \times 10^{-9} \mathrm{~m}^{3} / \mathrm{s}$

Head Gradient: $0.50 \mathrm{~m}$

Adjusted Head Gradient: $0.437 \mathrm{~m}$

Test Type: step-up

$$
\begin{aligned}
E_{1} & =185 \mathrm{mV} \\
C_{i_{1}} & =0.00135 \underline{M} \\
E_{0} & =128 \mathrm{mV} \\
C_{0} & =0.0125 \underline{M}
\end{aligned}
$$

\begin{tabular}{|c|c|c|c|}
\hline lapsed & Potential & Conc. & Conc., \\
\hline$t_{E}(h r)$ & $E(m V)$ & (M) & (M) \\
\hline-- & - & $-\overline{0}-$ & $\overline{0}-\overline{-}-\overline{0}$ \\
\hline 1.00 & 175 & 0.0020 & 0.0030 \\
\hline 1.5 & 155 & 0.0044 & 0.0054 \\
\hline 2.00 & & 0.0080 & 0.0080 \\
\hline 2.50 & $13:$ & 0.0096 & 0.0120 \\
\hline 3.00 & 135 & 0.0096 & 0.8 \\
\hline 3.50 & 130 & 0.0120 & 0.0120 \\
\hline
\end{tabular}

Plate Number: 2

Date: 6-01-88

Flow Rate: $4.08 \times 10^{-9} \mathrm{~m}^{3} / \mathrm{s}$

Head Gradient: $0.50 \mathrm{~m}$

\begin{tabular}{|c|c|c|c|}
\hline apsed & Potential & Conc & Conc. \\
\hline Time & Differ & $\mathrm{mi}$ & \\
\hline$t_{E}(h r)$ & $E(m V)$ & ( $\underline{\mathrm{M}})$ & (M) \\
\hline & & -- & \\
\hline 0.25 & 128 & 0.0125 & 0.0125 \\
\hline 0.83 & $132-128$ & 0.0110 & 0.0125 \\
\hline 1.50 & $135-128$ & 0.0096 & 0.0125 \\
\hline 2.25 & $145-135$ & 0.0065 & 0.0096 \\
\hline 3.00 & $145-140$ & 0.0065 & 0.0080 \\
\hline 4.25 & $155-145$ & 0.0044 & 0.0065 \\
\hline 4.75 & $165-160$ & 0.0030 & 0.0036 \\
\hline 5.25 & $170-165$ & 0.0024 & 0.0030 \\
\hline 5.75 & $172-170$ & 0.0023 & 0.0024 \\
\hline 6.25 & $175-170$ & 0.0020 & 0.0024 \\
\hline & $185-180$ & 0.0014 & 0.0016 \\
\hline
\end{tabular}

Adjusted Head Gradient: $0.437 \mathrm{~m}$

Test Type: step-down

$$
\begin{gathered}
\mathrm{E}_{1}=185 \mathrm{mV} \\
\mathrm{C}_{\mathrm{i}_{1}}=0.00135 \underline{\mathrm{M}} \\
\mathrm{E}_{\mathrm{o}}=128 \mathrm{mV} \\
\mathrm{C}_{0}=0.0125 \underline{\mathrm{M}}
\end{gathered}
$$


Plate Number: 3

Date: 5-13-88

Flow Rate: $2.30 \times 10^{-9} \mathrm{~m}^{3} / \mathrm{s}$

Head Gradient: $0.50 \mathrm{~m}$

Adjusted Head Gradient: $0.515 \mathrm{~m}$

$E_{i}=180 \mathrm{mV}$

$\mathrm{C}_{\mathrm{i}}=0.00160 \underline{\mathrm{M}}$

$E_{0}=128 \mathrm{mV}$

$\mathrm{C}_{0}=0.0125 \underline{\mathrm{M}}$

Test Type: step-up

\begin{tabular}{|c|c|c|c|}
\hline Elapsed & Potential & Conc., & Conc., \\
\hline$t_{F}(h r)$ & $E(m V)$ & $(\underline{M})$ & ( $\underline{M})$ \\
\hline$-\quad-$ & $-\quad-$ & - & -- \\
\hline 0.67 & 180 & 0.0016 & 0.0016 \\
\hline 1.42 & 170 & 0.0024 & 0.0024 \\
\hline 2.42 & $160-155$ & 0.0036 & 0.0044 \\
\hline 3.42 & $150-145$ & 0.0054 & 0.0066 \\
\hline 4.42 & 140 & 0.0080 & 0.0080 \\
\hline 5.17 & $137-135$ & 0.0090 & 0.0096 \\
\hline 5.67 & $135-130$ & 0.0096 & 0.0120 \\
\hline 6.17 & $135-130$ & 0.0096 & 0.0120 \\
\hline 6.67 & $130-128$ & 0.0120 & 0.0125 \\
\hline
\end{tabular}

* Shift in calibration curve observed.

Plate Number: 3

Date: 5-13-88

Flow Rate: $2.33 \times 10^{-9} \mathrm{~m}^{3} / \mathrm{s}$

Head Gradient: $0.50 \mathrm{~m}$

Adjusted Head Gradient: $0.515 \mathrm{~m}$

$$
\begin{aligned}
E_{i} & =180 \mathrm{mV} \\
C_{1} & =0.00160 \underline{M} \\
E_{o} & =128 \mathrm{mV} \\
C_{0} & =0.0125 \underline{M}
\end{aligned}
$$

\begin{tabular}{|c|c|c|c|c|}
\hline \multicolumn{2}{|c|}{ Elapsed } & Potential' & Conc., & Conc., \\
\hline & Time & Differ & $-\min$ & $-\max$ \\
\hline$t_{E}$ & (hr) & $\mathrm{E}\langle\mathrm{mV}\rangle$ & $(\underline{M})$ & (M) \\
\hline - - & -- & $-\quad-\quad$ & --- & -- \\
\hline & 0.25 & $135-125$ & 0.0096 & 0.0140 \\
\hline & 0.83 & 135 & 0.0096 & 0.0096 \\
\hline & 1.50 & $140-135$ & 0.0080 & 0.0096 \\
\hline & 2.25 & $145-140$ & 0.0066 & 0.0080 \\
\hline & 3.00 & $150-140$ & 0.0054 & 0.0080 \\
\hline & 4.25 & 150 & 0.0054 & 0.0054 \\
\hline & 4.75 & $155-150$ & 0.0044 & 0.0054 \\
\hline & 5.25 & 155 & 0.0044 & 0.0044 \\
\hline & 5.75 & $160-155$ & 0.0036 & 0.0044 \\
\hline & 6.25 & $165-155$ & 0.0030 & 0.0044 \\
\hline & 6.75 & $165-160$ & 0.0030 & 0.0036 \\
\hline & 7.25 & $168-165$ & 0.0026 & 0.0030 \\
\hline & 7.75 & $175-170$ & 0.0020 & 0.0025 \\
\hline & 8.25 & $175-170$ & 0.0020 & 0.0025 \\
\hline
\end{tabular}

Test Type: step-down

* Shift in calibration curve observed. 
Plate Number: 4

Date: 5-20-88

Flow Rate: $6.00 \times 10^{-9} \mathrm{~m}^{3} / \mathrm{s}$

Head Gradient: $0.50 \mathrm{~m}$

Adjusted Head Gradient: $0.505 \mathrm{~m}$

$$
\begin{aligned}
E_{1} & =185 \mathrm{mV} \\
C_{1_{1}} & =0.00133 \underline{M} \\
E_{0} & =125 \mathrm{mV} \\
C_{0} & =0.0143 \underline{M}
\end{aligned}
$$

\begin{tabular}{|c|c|c|c|}
\hline $\begin{array}{l}\text { Elapsed } \\
\text { Time }\end{array}$ & $\begin{array}{l}\text { Potential } \\
\text { Differe }\end{array}$ & $\begin{array}{l}\text { Conc. , } \\
\text { ce - min }\end{array}$ & $\begin{array}{r}\text { Conc. , } \\
-\max \end{array}$ \\
\hline$t_{E}(h r)$ & $E(m V)$ & $(\underline{M})$ & $(\underline{M})$ \\
\hline$\overline{0.67}$ & $185-175$ & $\overline{0.0013}$ & 0.0020 \\
\hline 1.50 & $155-150$ & 0.0044 & 0.0054 \\
\hline 2.00 & $140-130$ & 0.0080 & 0.0120 \\
\hline 2.50 & 125 & 0.0143 & 0.0143 \\
\hline 3.00 & 125 & 0.0143 & 0.0143 \\
\hline
\end{tabular}

Test Type: step-up

Plate Number: 4

Date: $5-20-88$

Flow Rate: $6.00 \times 10^{-9} \mathrm{~m}^{3} / \mathrm{s}$

\begin{tabular}{|c|c|c|c|}
\hline Elapsed & Potential & Conc. & Conc. \\
\hline Time, & Differe & $\min$ & \\
\hline$t_{E}(h r)$ & $E(m V)$ & (프) & 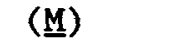 \\
\hline$\overline{0 .} \overline{33}$ & $-\overline{1}^{-}-$ & $\overline{0.0143}$ & $\overline{0.0143}$ \\
\hline 1.00 & $125-120$ & 0.0143 & 0.0170 \\
\hline 1.50 & $140-125$ & 0.0080 & 0.0143 \\
\hline 2.00 & $150-135$ & 0.0054 & 0.0096 \\
\hline 2.50 & $165-155$ & 0.0036 & 0.0044 \\
\hline 3.00 & $170-165$ & 0.0024 & 0.0036 \\
\hline 3.50 & 170 & 0.0024 & 0.0024 \\
\hline 4.00 & $180-175$ & 0.0016 & 0.0020 \\
\hline 4.42 & $175-170$ & 0.0020 & 0.0024 \\
\hline
\end{tabular}

Head Gradient: $0.50 \mathrm{~m}$

$$
\begin{aligned}
E_{1} & =185 \mathrm{mV} \\
C_{1} & =0.00133 \underline{M} \\
E_{0} & =125 \mathrm{mV} \\
C_{0} & =0.0143 \underline{M}
\end{aligned}
$$

Test Type: step-down 
Plate Number: 4

Date: $5-29-88$

Flow Rate: $5.83 \times 10^{-9} \mathrm{~m}^{3} / \mathrm{s}$

Head Gradient: $0.50 \mathrm{~m}$

$E_{1}=183 \mathrm{mV}$

$\mathrm{C}_{1}=0.00145 \mathrm{M}$

Adjusted Head Gradient: $0.491 \mathrm{~m}$

Test Type: step-up

$\mathrm{E}_{\mathrm{o}}=128 \mathrm{mV}$

$\mathrm{C}_{0}=0.0125 \mathrm{M}$

\begin{tabular}{|c|c|c|c|}
\hline $\begin{array}{l}\text { Elapsed } \\
\text { Time }\end{array}$ & $\begin{array}{l}\text { Potential } \\
\text { Differe }\end{array}$ & $\begin{array}{l}\text { Conc. } \\
\text { ce - min }\end{array}$ & $\begin{array}{r}\text { Conc., } \\
-\max \end{array}$ \\
\hline$t_{E}(h r)$ & $E(\mathrm{mV})$ & (M) & (M) \\
\hline$-\overline{-}-\overline{-}$ & $\begin{array}{c}-- \\
185-175\end{array}$ & 0.0013 & $\overline{0.0020}$ \\
\hline 1.00 & $173-165$ & 0.0022 & 0.0036 \\
\hline 1.33 & $165-150$ & 0.0022 & 0.0054 \\
\hline 1.67 & $150-140$ & 0.0054 & 0.0080 \\
\hline 2.00 & $143-138$ & 0.0070 & 0.0085 \\
\hline 2.50 & $135-130$ & 0.0096 & 0.0120 \\
\hline 3.00 & $130-128$ & 0.0120 & 0.0125 \\
\hline 3.50 & 128 & 0.0125 & 0.0125 \\
\hline
\end{tabular}

Plate Number: 4

Date: 5-29-88

Flow Rate: $5.83 \times 10^{-9} \mathrm{~m}^{3} / \mathrm{s}$

Head Gradient: $0.50 \mathrm{~m}$

Adjusted Head Gradient: $0.491 \mathrm{~m}$

Test Type: step-down

$$
\begin{gathered}
E_{1}=183 \mathrm{mV} \\
C_{1}=0.00145 \underline{M} \\
E_{o}=128 \mathrm{mV} \\
C_{0}=0.0125 \underline{M}
\end{gathered}
$$

\begin{tabular}{|c|c|c|c|}
\hline $\begin{array}{c}\text { Elapsed } \\
\text { Time }\end{array}$ & $\begin{array}{l}\text { Potential } \\
\text { Differen }\end{array}$ & $\begin{array}{l}\text { Conc. } \\
\text { ce - min }\end{array}$ & $\begin{array}{r}\text { Conc. } \\
-\max \end{array}$ \\
\hline$t_{E}(h r)$ & $E(\mathrm{mV})$ & (M) & (M) \\
\hline $\begin{array}{l}-\overline{-} \\
0.50\end{array}$ & $128-125$ & $\begin{array}{l}-\overline{-} \\
0.0125\end{array}$ & $\begin{array}{ll}- & - \\
0 & 0143\end{array}$ \\
\hline 1.00 & $128-125$ & 0.0125 & 0.0143 \\
\hline 1.50 & $135-128$ & 0.0096 & 0.0125 \\
\hline 2.00 & $145-140$ & 0.0066 & 0.0080 \\
\hline 2.33 & $157-140$ & 0.0041 & 0.0080 \\
\hline 2.67 & $170-160$ & 0.0024 & 0.0036 \\
\hline 3.17 & $172-160$ & 0.0023 & 0.0036 \\
\hline 3.67 & $182-165$ & 0.0016 & 0.0022 \\
\hline 4.17 & $180-170$ & 0.0016 & 0.0024 \\
\hline 4.67 & $185-176$ & 0.0013 & 0.0020 \\
\hline 5.17 & 180 & 0.0016 & 0.0016 \\
\hline
\end{tabular}


Plate Number: 5

Date: 5-23-88

Flow Rate: $1.83 \times 10^{-9} \mathrm{~m}^{3} / \mathrm{s}$

$E_{\mathrm{i}}=183 \mathrm{mV}$

Head Gradient: $0.50 \mathrm{~m}$

Adjusted Head Gradient: $0.561 \mathrm{~m}$

$C_{1}=0.00145 \underline{M}$

$E_{0}=125 \mathrm{mV}$

$\mathrm{C}_{0}=0.0143 \underline{\mathrm{M}}$

Test Type: step-up

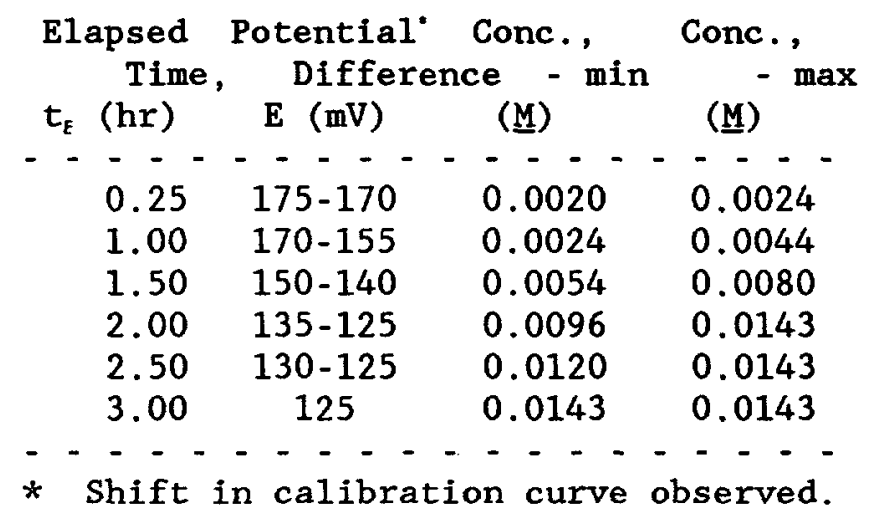

Plate Number: 5

Date: 5-23-88

Flow Rate: $1.83 \times 10^{-9} \mathrm{~m}^{3} / \mathrm{s}$

Head Gradient: $0.50 \mathrm{~m}$

Adjusted Head Gradient: $0.561 \mathrm{~m}$

Test Type: step-down.

$$
\begin{gathered}
E_{1}=183 \mathrm{mV} \\
C_{1}=0.00145 \underline{M} \\
E_{0}=125 \mathrm{mV} \\
C_{0}=0.0143 \underline{M}
\end{gathered}
$$

$$
\begin{aligned}
& \text { Elapsed Potential" Conc., Conc., } \\
& \text { Time, Difference - min - max } \\
& t_{\mathrm{E}}(\mathrm{hr}) \quad \mathrm{E}(\mathrm{mV}) \quad(\underline{M}) \quad(\underline{M}) \\
& \text { - - - - - - - - - . - - - } \\
& \begin{array}{llll}
0.25 & 130-125 & 0.0120 & 0.0143
\end{array} \\
& \begin{array}{llll}
0.75 & 125 & 0.0143 & 0.0143
\end{array} \\
& \begin{array}{llll}
1.25 & 130-125 & 0.0120 & 0.0143
\end{array} \\
& \begin{array}{llll}
2.25 & 150-140 & 0.0054 & 0.0080
\end{array} \\
& \begin{array}{llll}
2.50 & 150-145 & 0.0054 & 0.0065
\end{array} \\
& \begin{array}{llll}
2.75 & 165-150 & 0.0030 & 0.0054
\end{array} \\
& \begin{array}{llll}
3.00 & 160-150 & 0.0036 & 0.0054
\end{array} \\
& \begin{array}{llll}
3.50 & 165-160 & 0.0030 & 0.0036
\end{array} \\
& \begin{array}{llll}
4.00 & 165-160 & 0.0030 & 0.0036
\end{array} \\
& \begin{array}{llll}
4.50 & 170-165 & 0.0024 & 0.0030
\end{array} \\
& \begin{array}{llll}
5.00 & 175-170 & 0.0020 & 0.0024
\end{array}
\end{aligned}
$$




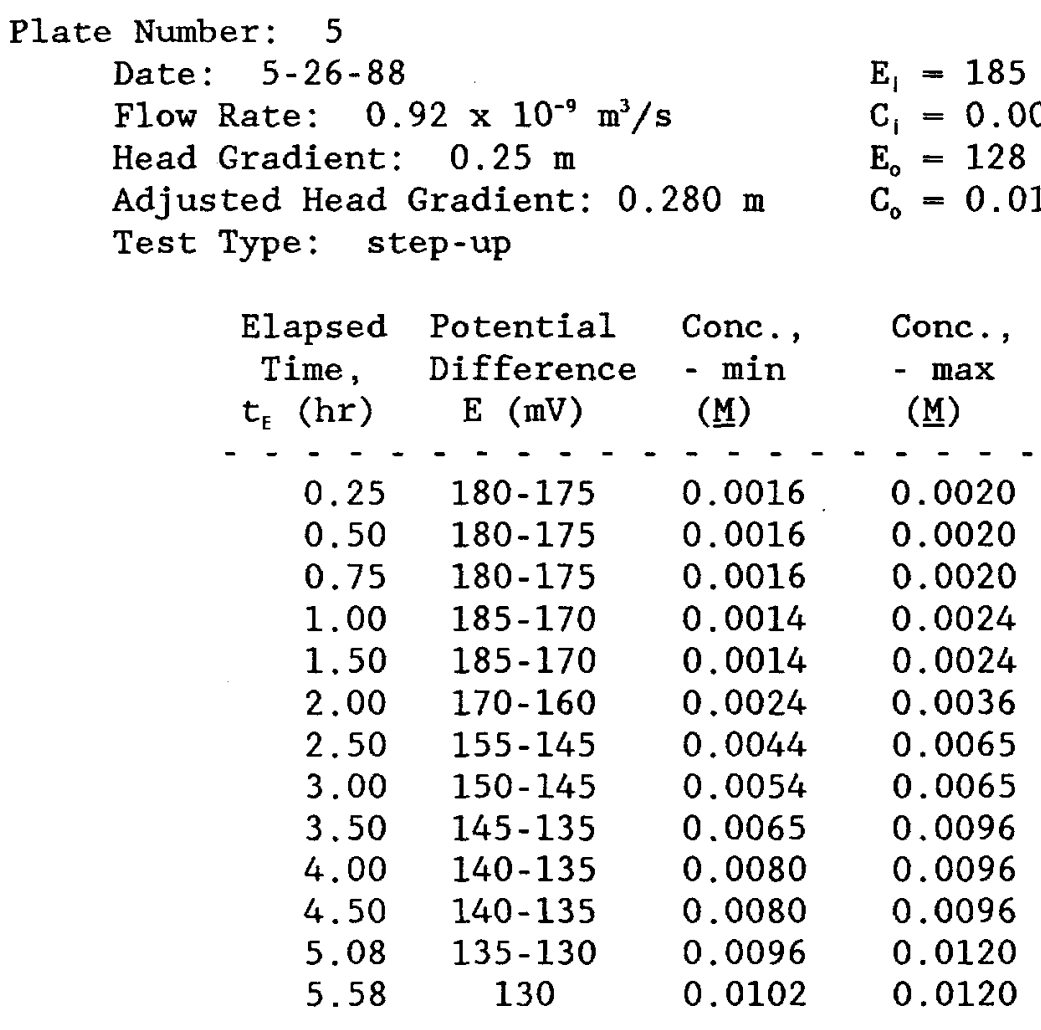


Plate Number: 5

Date: 5-27-88

Flow Rate: $1.00 \times 10^{-9} \mathrm{~m}^{3} / \mathrm{s}$

Head Gradient: $0.25 \mathrm{~m}$

$$
\begin{aligned}
E_{i} & =182 \mathrm{mV} \\
C_{i} & =0.00150 \underline{M} \\
E_{o} & =128 \mathrm{mV} \\
C_{0} & =0.0125 \underline{M}
\end{aligned}
$$

\begin{tabular}{|c|c|c|c|}
\hline lapsed & Potential & Conc. & Conc., \\
\hline Time & Differe & $-\min$ & $-\max$ \\
\hline$t_{E}(h r)$ & $E(m V)$ & (M) & (M) \\
\hline - & $-\quad-$ & - & - - \\
\hline 0.50 & 130 & 0.0120 & 0.0120 \\
\hline 1.00 & $135-130$ & 0.0096 & 0.0120 \\
\hline 1.50 & 130 & 0.0120 & 0.0120 \\
\hline 2.00 & 130 & 0.0120 & 0.0120 \\
\hline 2.50 & $135-130$ & 0.0096 & 0.0120 \\
\hline 3.08 & 135 & 0.0096 & 0.0096 \\
\hline 3.50 & 145 & 0.0065 & 0.0065 \\
\hline 4.00 & 150 & 0.0054 & 0.0054 \\
\hline 4.50 & $155-153$ & 0.0044 & 0.0047 \\
\hline 5.00 & 155 & 0.0044 & 0.0044 \\
\hline 5.50 & 163 & 0.0032 & 0.0032 \\
\hline 6.00 & 165 & 0.0030 & 0.0030 \\
\hline 6.50 & $172-165$ & 0.0023 & 0.0030 \\
\hline 7.25 & $170-165$ & 0.0024 & 0.0030 \\
\hline 8.00 & 172 & 0.0023 & 0.0023 \\
\hline 9.00 & 180 & 0.0016 & 0.0016 \\
\hline
\end{tabular}

Test Type: step-down 
Plate Number: 6

Date: 6-07-88

Flow Rate: $2.25 \times 10^{-9} \mathrm{~m}^{3} / \mathrm{s}$

Head Gradient: $0.50 \mathrm{~m}$

Adjusted Head Gradient: $0.393 \mathrm{~m}$

$$
\begin{gathered}
E_{i}=183 \mathrm{mV} \\
C_{i}=0.00145 \underline{M} \\
E_{o}=128 \mathrm{mV} \\
C_{o}=0.0125 \underline{M}
\end{gathered}
$$

\begin{tabular}{|c|c|c|c|c|}
\hline & apsed & Potential & Conc. & Conc. \\
\hline & Time & Differe & min & $\mathrm{ma}$ \\
\hline$t_{E}$ & $(\mathrm{hr})$ & $\mathrm{E}(\mathrm{mV})$ & $(\underline{M})$ & $(\underline{M})$ \\
\hline & $-\overline{-}-$ & $-\overline{-}-\overline{-}$ & $-\overline{-}-$ & $-\overline{-}-\overline{-}$ \\
\hline & $\begin{array}{l}0.50 \\
1.00\end{array}$ & $\begin{array}{l}180-175 \\
168-160\end{array}$ & $\begin{array}{l}0.0016 \\
0.0027\end{array}$ & $\begin{array}{l}0.0020 \\
0.0036\end{array}$ \\
\hline & 1.50 & $145-135$ & 0.0066 & 0.0096 \\
\hline & 2.00 & $133-130$ & 0.0105 & 0.0120 \\
\hline & 2.50 & 128 & 0.0125 & 0.0125 \\
\hline & 3.00 & 127 & 0.0133 & 0.0133 \\
\hline & & - & & \\
\hline
\end{tabular}

Test Type: step-up

Plate Number: 6

Date: 6-07-88

Flow Rate: $2.42 \times 10^{-9} \mathrm{~m}^{3} / \mathrm{s}$

Head Gradient: $0.50 \mathrm{~m}$

\begin{tabular}{|c|c|c|c|}
\hline Elapsed & Potential & Conc. & Conc. \\
\hline Time, & Differe & $\min$ & - \\
\hline$t_{E}(h r)$ & $\mathrm{E}(\mathrm{mV})$ & $(\underline{M})$ & $(\underline{M})$ \\
\hline-- & & - & - \\
\hline 0.50 & $128-127$ & 0.0125 & 0.0133 \\
\hline 1.00 & $138-130$ & 0.0085 & 0.0120 \\
\hline 1.75 & $155-150$ & 0.0044 & 0.0096 \\
\hline 2.25 & $180-170$ & 0.0016 & 0.0025 \\
\hline 2.75 & $183-180$ & 0.0015 & 0.0016 \\
\hline 3.25 & 180 & 0.0016 & 0.0016 \\
\hline 3.75 & 180 & 0.0016 & 0.0016 \\
\hline
\end{tabular}

$$
\begin{gathered}
E_{i}=186 \mathrm{mV} \\
C_{i}=0.00130 \underline{M} \\
E_{o}=128 \mathrm{mV} \\
C_{o}=0.0125 \underline{M}
\end{gathered}
$$

Adjusted Head Gradient: $0.422 \mathrm{~m}$

Test Type: step-down 
Plate Number: 6

Date: 6-09-88

Flow Rate: $1.00 \times 10^{-9} \mathrm{~m}^{3} / \mathrm{s}$

Head Gradient: $0.25 \mathrm{~m}$

Adjusted Head Gradient: $0.175 \mathrm{~m}$

Test Type: step-up

$$
\begin{aligned}
E_{i} & =185 \mathrm{mV} \\
C_{i} & =0.00135 \underline{M} \\
E_{o} & =127 \mathrm{mV} \\
C_{0} & =0.0133 \underline{M}
\end{aligned}
$$

Elapsed Potential Conc., Conc.,

Time, Difference - min - $\max$

$t_{\mathrm{E}}(\mathrm{hr}) \quad \mathrm{E}(\mathrm{mV}) \quad(\underline{M}) \quad(\underline{M})$

$\begin{array}{cccc}0.50 & 178-175 & 0.0018 & 0.0020 \\ 1.00 & 180-170 & 0.0016 & 0.0025 \\ 1.50 & 170 & 0.0025 & 0.0025 \\ 2.00 & 165-160 & 0.0030 & 0.0036 \\ 2.50 & 145-140 & 0.0066 & 0.0080 \\ 3.00 & 140-135 & 0.0080 & 0.0096 \\ 3.50 & 138-130 & 0.0085 & 0.0120 \\ 4.00 & 130 & 0.0120 & 0.0120 \\ 4.50 & 126 & 0.0135 & 0.0135 \\ 5.00 & 126 & 0.0135 & 0.0135\end{array}$

Plate Number: 6

Date: $6-10-88$

Flow Rate: $1.18 \times 10^{-9} \mathrm{~m}^{3} / \mathrm{s}$

Head Gradient: $0.25 \mathrm{~m}$

\begin{tabular}{|c|c|c|c|}
\hline $\begin{array}{c}\text { Elapsed } \\
\text { Time }\end{array}$ & $\begin{array}{l}\text { Potential } \\
\text { Differe }\end{array}$ & $\begin{array}{l}\text { Conc. } \\
\text { lce - min }\end{array}$ & $\begin{array}{l}\text { Conc. , } \\
-\max \end{array}$ \\
\hline$t_{E}(h r)$ & $\mathrm{E}(\mathrm{mV})$ & $(\underline{M})$ & $(\underline{M})$ \\
\hline--- & $---\overline{-}$ & בחמח & \\
\hline 0.50 & $127-120$ & 0.0097 & 0.0130 \\
\hline 1.00 & $130-120$ & 0.0086 & 0.0130 \\
\hline 1.75 & $125-120$ & 0.0104 & 0.0130 \\
\hline 2.50 & $135-130$ & 0.0070 & 0.0086 \\
\hline 3.25 & $145-140$ & 0.0047 & 0.0016 \\
\hline 4.00 & $160-150$ & 0.0027 & 0.0039 \\
\hline 4.75 & $170-160$ & 0.0018 & 0.0027 \\
\hline 5.50 & $170-165$ & 0.0018 & 0.0022 \\
\hline 6.25 & $170-165$ & 0.0018 & 0.0022 \\
\hline 7.00 & $175-170$ & 0.0014 & 0.0016 \\
\hline 7.75 & 175 & 0.0014 & 0.0014 \\
\hline 8.50 & 175 & 0.0014 & 0.0014 \\
\hline
\end{tabular}

Adjusted Head Gradient: $0.206 \mathrm{~m}$.

$$
\begin{aligned}
E_{i} & =178 \mathrm{mV} \\
C_{1} & =0.00135 \underline{M} \\
E_{o} & =120 \mathrm{mV} \\
C_{o} & =0.0130 \underline{M}
\end{aligned}
$$

Test Type: step-down

* Shift in calibration curve observed. 
Plate Number: 6

Date: 6-09-88

Flow Rate: $2.38 \times 10^{-9} \mathrm{~m}^{3} / \mathrm{s}$

Head Gradient: $0.50 \mathrm{~m}$

$$
\begin{gathered}
\mathrm{E}_{\mathrm{i}}=127 \mathrm{mV} \\
\mathrm{C}_{\mathrm{i}}=0.0130 \mathrm{M} \\
\mathrm{E}_{\mathrm{o}}=80 \mathrm{mV} \\
\mathrm{C}_{\mathrm{o}}=0.086 \mathrm{M}
\end{gathered}
$$

\begin{tabular}{|c|c|c|c|}
\hline Elapsed & Potential & Cone. & Conc., \\
\hline Time, & Differe & $-\min$ & $-\max$ \\
\hline$t_{E}(h r)$ & $\mathrm{E}(\mathrm{mV})$ & (ㅁ) & (M) \\
\hline$-\quad-\quad-$ & -- & 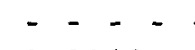 & -2 \\
\hline 0.50 & 127 & 0.0130 & 0.0130 \\
\hline 1.00 & 120 & 0.0175 & 0.0175 \\
\hline 1.50 & $100-90$ & 0.0385 & 0.0570 \\
\hline 2.00 & $85-80$ & 0.0695 & 0.0860 \\
\hline 2.50 & $80-78$ & 0.0860 & 0.0930 \\
\hline 3.00 & $80-78$ & 0.0860 & 0.0930 \\
\hline
\end{tabular}

Test Type: step-up

Plate Number: 6

Date: 6-09-88

Flow Rate: $2.25 \times 10^{-9} \mathrm{~m}^{3} / \mathrm{s}$

Head Gradient: $0.50 \mathrm{~m}$

\begin{tabular}{|c|c|c|c|}
\hline E1apsed & Potential & Conc. & Conc. \\
\hline Time, & Differen & $-\min$ & - ma \\
\hline$t_{E}(h r)$ & $\mathrm{E}(\mathrm{mV})$ & (M) & $(\underline{M})$ \\
\hline & . & --- & - \\
\hline 0.50 & $80-78$ & 0.0860 & 0.0930 \\
\hline 1.00 & 80 & 0.0860 & 0.0860 \\
\hline 1.50 & $95-88$ & 0.0470 & 0.0620 \\
\hline 2.00 & $110-100$ & 0.0260 & 0.0385 \\
\hline 2.50 & $115-110$ & 0.0210 & 0.0260 \\
\hline 3.00 & $120-115$ & 0.0170 & 0.0210 \\
\hline 3.50 & 120 & 0.0170 & 0.0170 \\
\hline 4.00 & $125-120$ & 0.0140 & 0.0170 \\
\hline 4.50 & $128-125$ & 0.0125 & 0.0140 \\
\hline
\end{tabular}

Adjusted Head Gradient: $0.393 \mathrm{~m}$

Test Type: step-down

$$
\begin{aligned}
E_{i} & =130 \mathrm{mV} \\
C_{i} & =0.0120 \underline{M} \\
E_{0} & =80 \mathrm{mV} \\
C_{0} & =0.086 \underline{\mathrm{M}}
\end{aligned}
$$


Plate Number: 6

Date: $6-12-88$

Flow Rate: $2.38 \times 10^{-9} \mathrm{~m}^{3} / \mathrm{s}$

Head Gradient: $0.50 \mathrm{~m}$

Adjusted Head Gradient: $0.415 \mathrm{~m}$

Test Type: step-up

$$
\begin{gathered}
E_{i}=238 \mathrm{mV} \\
C_{i}=0.00016 \mathrm{M} \\
E_{o}=180 \mathrm{mV} \\
C_{o}=0.0016 \mathrm{M}
\end{gathered}
$$

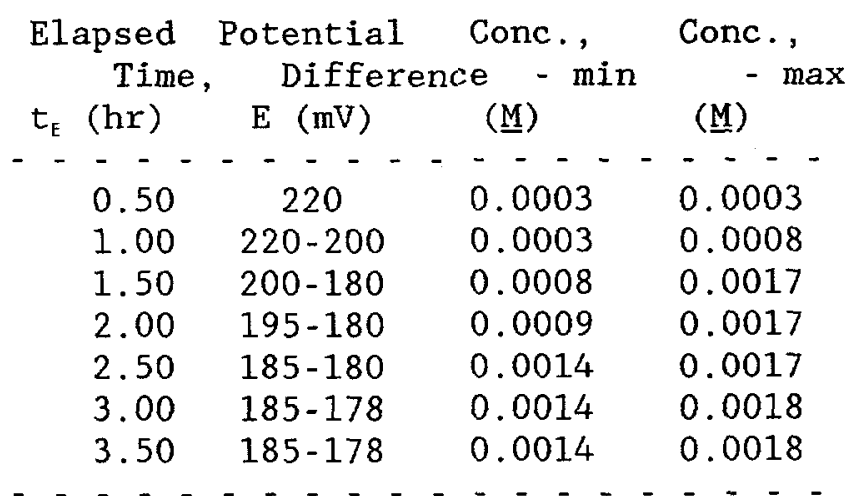

Plate Number: 6

Date: $6-12-88$

Flow Rate: $2.17 \times 10^{-9} \mathrm{~m}^{3} / \mathrm{s}$

Head Gradient: $0.50 \mathrm{~m}$

\begin{tabular}{|c|c|c|c|}
\hline Elapsed & Potential & Cone & Conc. \\
\hline Time & Differe & $\min$ & $-\max$ \\
\hline$t_{E}(h r)$ & $E(m V)$ & (品) & (ㅆ) \\
\hline- & - & --- & -- \\
\hline 0.50 & 178 & 0.0018 & 0.0018 \\
\hline 1.00 & $180-178$ & 0.0016 & 0.0018 \\
\hline 1.50 & $195-180$ & 0.0009 & 0.0016 \\
\hline 2.00 & 200 & 0.0008 & 0.0008 \\
\hline 2.50 & $220-215$ & 0.0003 & 0.0004 \\
\hline 3.00 & $230-215$ & 0.0002 & 0.0004 \\
\hline 3.50 & $225-220$ & 0.0003 & 0.0003 \\
\hline 4.00 & 230 & 0.0002 & 0.0002 \\
\hline 4.50 & $235-230$ & 0.0002 & 0.0002 \\
\hline 5.00 & $235-230$ & 0.0002 & 0.0002 \\
\hline
\end{tabular}

Adjusted Head Gradient: $0.379 \mathrm{~m}$

Test Type: step-down

$$
\begin{aligned}
E_{i} & =238 \mathrm{mV} \\
C_{i} & =0.00016 \mathrm{M} \\
E_{o} & =180 \mathrm{mV} \\
C_{0} & =0.0016 \mathrm{M}
\end{aligned}
$$


P1ate Number: 6

Date: 6-25-88

Flow Rate: $1.67 \times 10^{-9} \mathrm{~m}^{3} / \mathrm{s}$

Head Gradient: $0.36 \mathrm{~m}$

Adjusted Head Gradient: $0.291 \mathrm{~m}$

Test Type: step-up

$$
\begin{aligned}
\mathrm{E}_{\mathrm{i}} & =178 \mathrm{mV} \\
\mathrm{C}_{\mathrm{i}} & =0.0015 \underline{\mathrm{M}} \\
\mathrm{E}_{\mathrm{o}} & =73 \mathrm{mV} \\
\mathrm{C}_{\mathrm{o}} & =0.100 \underline{\mathrm{M}}
\end{aligned}
$$

$$
\begin{aligned}
& \text { Elapsed Potential Conc., Conc., } \\
& \text { Time, Difference - min - } \max \\
& t_{\mathrm{E}}(\mathrm{hr}) \quad \mathrm{E}(\mathrm{mV}) \quad(\underline{\mathrm{M}}) \quad(\underline{M})
\end{aligned}
$$

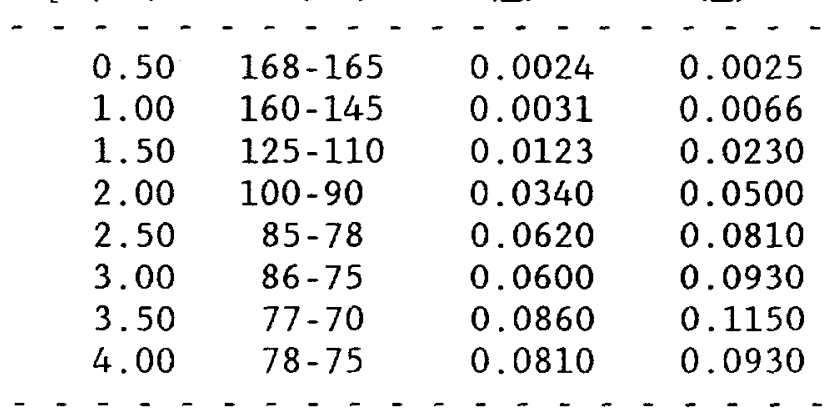

Plate Number: 6

Date: $6-25-88$

Flow Rate: $1.90 \times 10^{-9} \mathrm{~m}^{3} / \mathrm{s}$

Head Gradient: $0.36 \mathrm{~m}$

$$
\begin{aligned}
E_{i} & =178 \mathrm{mV} \\
C_{i} & =0.0015 \underline{M} \\
E_{o} & =73 \mathrm{mV} \\
C_{o} & =0.100 M
\end{aligned}
$$

Adjusted Head Gradient: $0.332 \mathrm{~m}$

Test Type: step-down

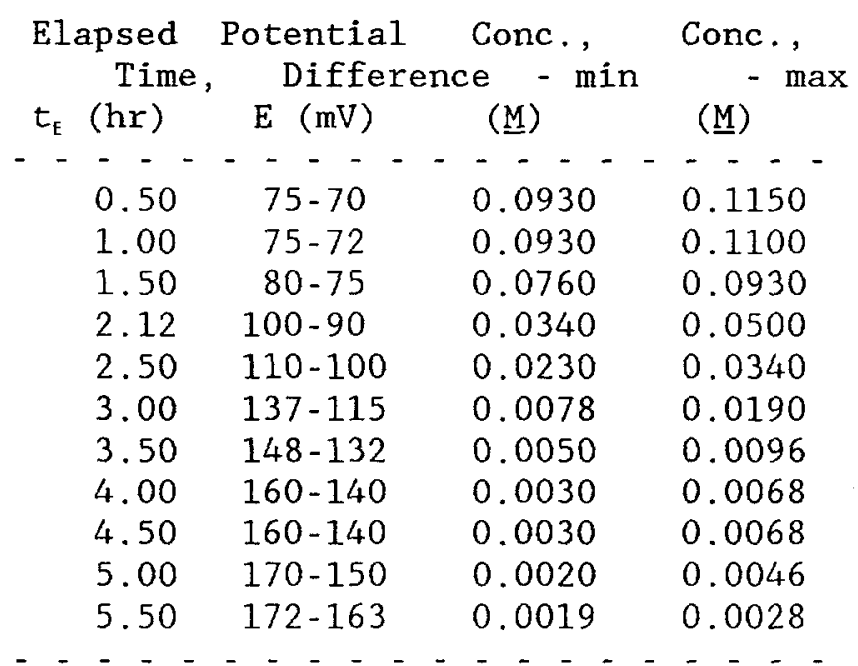


Table D.2: Porous plate effective porosity estimates from chloride breakthrough data.

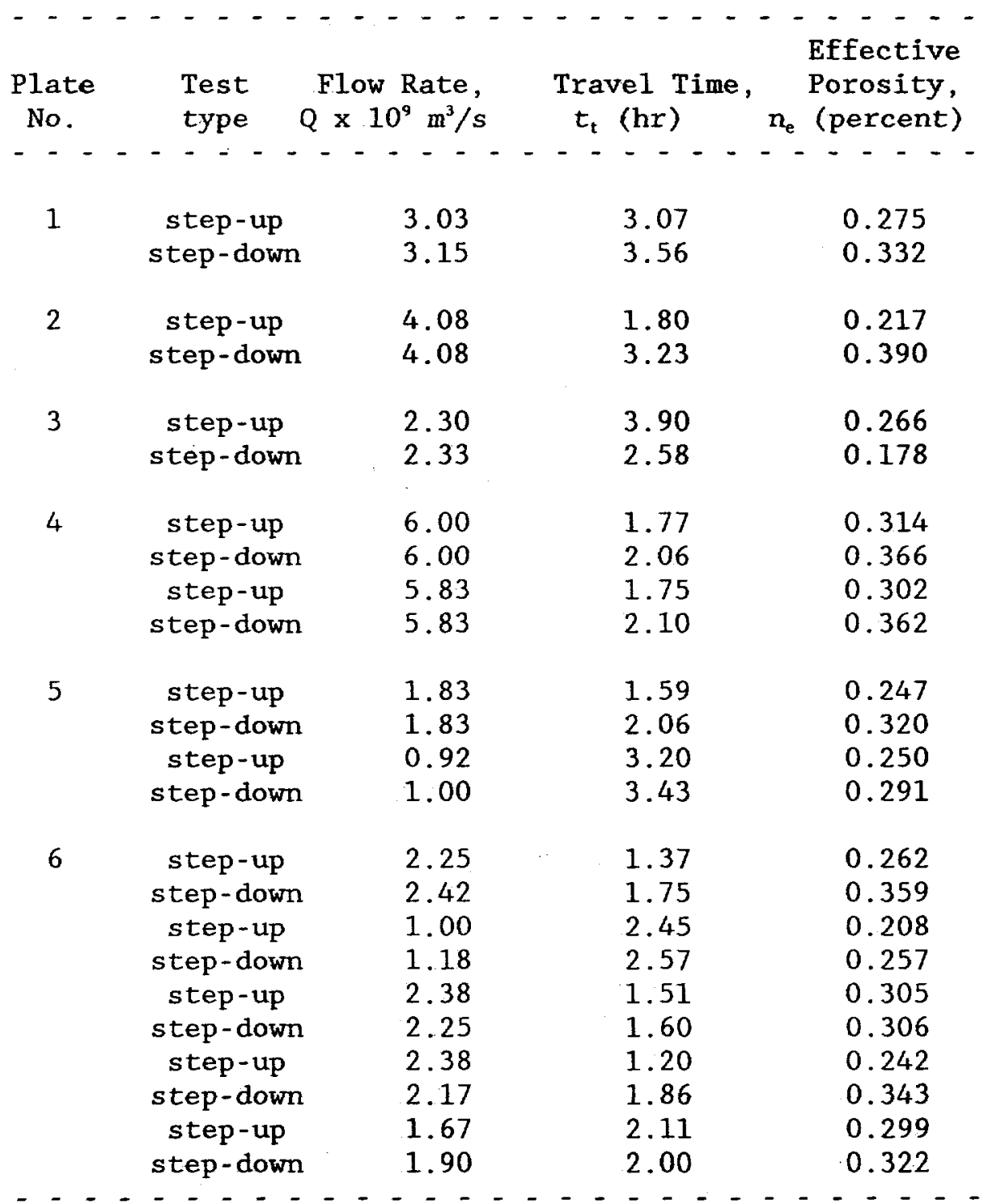


This section includes all the fracture and matrix transport test data. Table E.1 also presents the results of temporal moments analysis for test no. 1 at sampling port 5F-US as an example. The raw data of the spatial distribution of relative concentrations of the fracture surface immediately after fracture transport test no. 3 is presented in Table E.5 and Figure E.1. 
Table E.1: Fracture solute transport test data, and temporal moments analysis data for test no. 1 , sampling port 5F-US.

Sampling Port: 5F-US

Location: $\mathrm{x}=5 \mathrm{~cm} ; \mathrm{z}=10 \mathrm{~cm} \quad$ Face: 5

Date: $6-21-88$ to $6-22-88$

Flow Rate: $1.41 \times 10^{-9} \mathrm{~m}^{3} / \mathrm{s}$

$\mathrm{E}_{\mathrm{i}}=168 \mathrm{mV}$

Head Imposed at Top of Plate: $35.80 \mathrm{~cm}$

$\mathrm{C}_{\mathrm{i}}=0.00150 \underline{\mathrm{M}}$

Test Performed: Slug - $12 \mathrm{hrs}$

Test Duration: 28 hrs

$\mathrm{E}_{\mathrm{o}}=73 \mathrm{mV}$

$\mathrm{C}_{\mathrm{o}}=0.105 \underline{\mathrm{M}}$

Plate no.: 5

\begin{tabular}{|c|c|c|c|c|c|}
\hline $\begin{array}{l}\text { Elapsed } \\
\text { Time, } \\
t_{\mathrm{E}}(\mathrm{hr})\end{array}$ & $\begin{array}{l}\text { Potential } \\
\text { Difference } \\
\text { E (mV) }\end{array}$ & $\begin{array}{l}\text { Conc. } \\
-\min \\
(\underline{M})\end{array}$ & $\begin{array}{l}\text { Conc. , } \\
-\max \\
(\underline{M})\end{array}$ & $\begin{array}{l}\text { Relative } \\
\text { Conc. } \\
\text { - min }\end{array}$ & $\begin{array}{l}\text { Relative } \\
\text { Conc. } \\
\text { - } \max \end{array}$ \\
\hline$\overline{0}-\overline{-}$ & $--_{145}^{-}-$ & $\overline{0}-\overline{-}-\overline{-}$ & $\overline{0}-\overline{0}-\overline{-}$ & 0.034 & $-\overline{0}-\overline{0}-\overline{0}$ \\
\hline 0.75 & 145 & 0.0050 & 0.0050 & 0.034 & 0.034 \\
\hline 1.25 & 145 & 0.0050 & 0.0050 & 0.034 & 0.034 \\
\hline 1.75 & 145 & 0.0050 & 0.0050 & 0.034 & 0.034 \\
\hline 2.25 & $147-145$ & 0.0046 & 0.0050 & 0.030 & 0.034 \\
\hline 2.75 & 145 & 0.0050 & 0.0050 & 0.034 & 0.034 \\
\hline 3.25 & $150-145$ & 0.0040 & 0.0050 & 0.024 & 0.034 \\
\hline 3.75 & $145-140$ & 0.0050 & 0.0063 & 0.034 & 0.046 \\
\hline 4.25 & 145 & 0.0050 & 0.0050 & 0.034 & 0.034 \\
\hline 4.75 & 145 & 0.0050 & 0.0050 & 0.034 & 0.034 \\
\hline 5.25 & 145 & 0.0050 & 0.0050 & 0.034 & 0.034 \\
\hline 5.75 & $148-135$ & 0.0044 & 0.0080 & 0.028 & 0.063 \\
\hline 6.25 & 145 & 0.0050 & 0.0050 & 0.034 & 0.034 \\
\hline 6.75 & 145 & 0.0050 & 0.0050 & 0.034 & 0.034 \\
\hline 7.25 & $138-136$ & 0.0068 & 0.0077 & 0.051 & 0.060 \\
\hline 7.75 & 145 & 0.0050 & 0.0050 & 0.034 & 0.034 \\
\hline 8.25 & 145 & 0.0050 & 0.0050 & 0.034 & 0.034 \\
\hline 8.75 & 128 & 0.0110 & 0.0110 & 0.092 & 0.092 \\
\hline 9.25 & $135-130$ & 0.0080 & 0.0100 & 0.063 & 0.082 \\
\hline 9.75 & $137-133$ & 0.0073 & 0.0088 & 0.056 & 0.071 \\
\hline 10.25 & $135-130$ & 0.0080 & 0.0100 & 0.063 & 0.082 \\
\hline 10.75 & $138-135$ & 0.0068 & 0.0080 & 0.051 & 0.063 \\
\hline 11.25 & $134-132$ & 0.0084 & 0.0092 & 0.067 & 0.074 \\
\hline 11.75 & 128 & 0.0110 & 0.0110 & 0.092 & 0.092 \\
\hline 12.25 & $120-115$ & 0.0152 & 0.0187 & 0.132 & 0.166 \\
\hline 12.75 & $120-117$ & 0.0152 & 0.0175 & 0.132 & 0.155 \\
\hline 13.25 & $123-118$ & 0.0137 & 0.0165 & 0.118 & 0.145 \\
\hline 13.75 & 120 & 0.0152 & 0.0152 & 0.132 & 0.132 \\
\hline 14.25 & $120-115$ & 0.0152 & 0.0187 & 0.132 & 0.166 \\
\hline 14.75 & $115-110$ & 0.0187 & 0.0227 & 0.166 & 0.205 \\
\hline 15.25 & $120-118$ & 0.0152 & 0.0165 & 0.132 & 0.145 \\
\hline 15.75 & 116 & 0.0183 & 0.0183 & 0.162 & 0.162 \\
\hline 16.25 & 120 & 0.0152 & 0.0152 & 0.132 & 0.132 \\
\hline
\end{tabular}


Table E.1: Fracture solute transport test data, and temporal moments analysis data for test no. 1 (continued).

\begin{tabular}{|c|c|c|c|c|c|}
\hline $\begin{array}{l}\text { Elapsed } \\
\text { Time, } \\
\tau_{E}(h r)\end{array}$ & $\begin{array}{l}\text { Potential } \\
\text { Difference } \\
\text { E (mV) }\end{array}$ & $\begin{array}{l}\text { Conc. } \\
-\min \\
(\underline{M})\end{array}$ & $\begin{array}{l}\text { Conc. } \\
-\max \\
(\underline{M})\end{array}$ & $\begin{array}{c}\text { Relative } \\
\text { Conc. } \\
\text { - min }\end{array}$ & $\begin{array}{c}\text { Relative } \\
\text { Conc. } \\
\text { - } \max \end{array}$ \\
\hline$-\quad-$ & - & - & . & $-\quad-$ & $-\quad-\quad-$ \\
\hline 16.75 & $117-115$ & 0.0175 & 0.0187 & 0.155 & 0.166 \\
\hline 17.25 & 107 & 0.0260 & 0.0260 & 0.237 & 0.237 \\
\hline 18.25 & 105 & 0.0280 & 0.0280 & 0.256 & 0.256 \\
\hline 19.25 & $115-110$ & 0.0187 & 0.0227 & 0.166 & 0.205 \\
\hline 20.25 & 110 & 0.0227 & 0.0227 & 0.205 & 0.205 \\
\hline 21.25 & 114 & 0.0193 & 0.0193 & 0.172 & 0.172 \\
\hline 22.25 & $113-107$ & 0.0200 & 0.0260 & 0.179 & 0.237 \\
\hline 23.25 & 113 & 0.0200 & 0.0200 & 0.179 & 0.179 \\
\hline 24.25 & 120 & 0.0152 & 0.0152 & 0.132 & 0.132 \\
\hline 25.25 & 110 & 0.0227 & 0.0227 & 0.205 & 0.205 \\
\hline 26.25 & 113 & 0.0200 & 0.0200 & 0.179 & 0.179 \\
\hline 27.25 & 110 & 0.0227 & 0.0227 & 0.205 & 0.205 \\
\hline 28.25 & 113 & 0.0200 & 0.0200 & 0.179 & 0.179 \\
\hline
\end{tabular}

Results of Temporal Moments Analysis:

\begin{tabular}{|c|c|c|c|c|c|c|}
\hline $\begin{array}{l}\text { Elapsed } \\
\text { Time, }\end{array}$ & $\begin{array}{c}\text { Average } \\
\text { Conc. }\end{array}$ & $\begin{array}{c}t_{E} x \\
\text { Average }\end{array}$ & $\begin{array}{c}\left(t_{E}-t_{m}\right)^{2} x \\
\text { Average }\end{array}$ & $\begin{array}{l}\text { Average } \\
\text { Relative }\end{array}$ & $\begin{array}{l}t_{E} \times \\
\text { Average }\end{array}$ & $\begin{array}{r}\left(t_{E}-t_{m}\right)^{2} x \\
\text { Average }\end{array}$ \\
\hline$t_{E}(h r)$ & (M) & $\mathrm{C}=\mathrm{O}-\mathrm{s}$ & & Conc. & $\operatorname{Re} 1$. & $\operatorname{Re} 1$ \\
\hline$-\overline{0}-$ & $\overline{0}-\overline{-}$ & $\begin{array}{ll}- & - \\
0 & 00\end{array}$ & $-\overline{-}-\overline{1}$ & $-{ }_{0}^{-}-\overline{034}$ & $\begin{array}{ll}- & - \\
0 & 01\end{array}$ & $-{ }_{9}^{-}-{ }^{0}-$ \\
\hline 0.75 & 0.0050 & 0.00 & 1.17 & 0.034 & 0.03 & 8.49 \\
\hline 1.25 & 0.0050 & 0.01 & 1.10 & 0.034 & 0.04 & 7.96 \\
\hline 1.75 & 0.0050 & 0.01 & 1.03 & 0.034 & 0.06 & 7.45 \\
\hline 2.25 & 0.0048 & 0.01 & 0.92 & 0.032 & 0.07 & 6.56 \\
\hline 2.75 & 0.0050 & 0.01 & 0.89 & 0.034 & 0.09 & 6.48 \\
\hline 3.25 & 0.0045 & 0.01 & 0.74 & 0.029 & 0.09 & 5.16 \\
\hline 3.75 & 0.0057 & 0.02 & 0.86 & 0.040 & 0.15 & 6.61 \\
\hline 4.25 & 0.0050 & 0.02 & 0.70 & 0.034 & 0.14 & 5.15 \\
\hline 4.75 & 0.0050 & 0.02 & 0.64 & 0.034 & 0.16 & 4.74 \\
\hline 5.25 & 0.0050 & 0.03 & 0.59 & 0.034 & 0.18 & 4.35 \\
\hline 5.75 & 0.0062 & 0.04 & 0.66 & 0.045 & 0.26 & 5.34 \\
\hline 6.25 & 0.0050 & 0.03 & 0.48 & 0.034 & 0.21 & 3.62 \\
\hline 6.75 & 0.0050 & 0.03 & 0.43 & 0.034 & 0.23 & 3.28 \\
\hline 7.25 & 0.0073 & 0.05 & 0.56 & 0.056 & 0.40 & 4.85 \\
\hline 7.75 & 0.0050 & 0.04 & 0.35 & 0.034 & 0.26 & 2.64 \\
\hline 8.25 & 0.0050 & 0.04 & 0.31 & 0.034 & 0.28 & 2.35 \\
\hline 8.75 & 0.0110 & 0.10 & 0.59 & 0.092 & 0.80 & 5.65 \\
\hline 9.25 . & 0.0090 & 0.08 & 0.42 & 0.072 & 0.67 & 3.91 \\
\hline 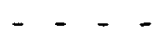 & 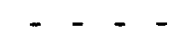 & & 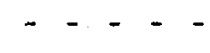 & 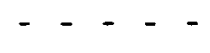 & -- & -5 \\
\hline
\end{tabular}


Table E.1: Fracture solute transport test data, and temporal moments analysis data for test no. 1 (continued).

Results of Temporal Moments Analysis (continued):

\begin{tabular}{|c|c|c|c|c|c|c|}
\hline $\begin{array}{l}\text { Elapsed } \\
\text { Time, }\end{array}$ & $\begin{array}{c}\text { Average } \\
\text { Conc. }\end{array}$ & $\begin{array}{c}t_{E} x \\
\text { Average }\end{array}$ & $\begin{array}{c}\left(t_{\mathrm{E}}-t_{\mathrm{m}}\right)^{2} \mathrm{x} \\
\text { Average }\end{array}$ & $\begin{array}{l}\text { Average } \\
\text { Relative }\end{array}$ & $\begin{array}{l}t_{E} \times \\
\text { Average }\end{array}$ & $\begin{array}{r}\left(t_{E}-t_{m}\right)^{2} x \\
\text { Average }\end{array}$ \\
\hline$t_{E}(h r)$ & (ㅍ) & Conc. & Conc. & Conc. & Rel. Conc. & Re1. Conc. \\
\hline$-\overline{-}-\overline{-}$ & $\overline{0.0081}$ & $\begin{array}{c}-\overline{-}-\overline{-} \\
0.08\end{array}$ & $\begin{array}{c}-\cdot- \\
0.32\end{array}$ & $=-{ }^{-}-\overline{0633}$ & $-\overline{0.62}$ & $-\overline{-}-\overline{-}$ \\
\hline 10.25 & 0.0090 & 0.09 & 0.31 & 0.072 & 0.74 & 2.92 \\
\hline 10.75 & 0.0074 & 0.08 & 0.21 & 0.057 & 0.61 & 1.95 \\
\hline 11.25 & 0.0088 & 0.10 & 0.20 & 0.071 & 0.79 & 2.01 \\
\hline 11.75 & 0.0110 & 0.13 & 0.21 & 0.092 & 1.08 & 2.15 \\
\hline 12.25 & 0.0170 & 0.21 & 0.25 & 0.149 & 1.83 & 2.82 \\
\hline 12.75 & 0.0164 & 0.21 & 0.18 & 0.143 & 1.83 & 2.12 \\
\hline 13.25 & 0.0151 & 0.20 & 0.12 & 0.131 & 1.74 & 1.47 \\
\hline 13.75 & 0.0152 & 0.21 & 0.08 & 0.132 & 1.82 & 1.07 \\
\hline 14.25 & 0.0170 & 0.24 & 0.06 & 0.149 & 2.13 & 0.82 \\
\hline 14.75 & 0.0207 & 0.31 & 0.04 & 0.186 & 2.74 & 0.63 \\
\hline 15.25 & 0.0159 & 0.24 & 0.01 & 0.139 & 2.11 & 0.25 \\
\hline 15.75 & 0.0183 & 0.29 & 0.00 & 0.162 & 2.56 & 0.12 \\
\hline 16.25 & 0.0152 & 0.25 & 0.00 & 0.132 & 2.15 & 0.02 \\
\hline 16.75 & 0.0181 & 0.30 & 0.01 & 0.160 & 2.69 & 0.00 \\
\hline 17.25 & 0.0260 & 0.45 & 0.04 & 0.237 & 4.08 & 0.10 \\
\hline 18.25 & 0.0280 & 0.51 & 0.13 & 0.256 & 4.67 & 0.70 \\
\hline 19.25 & 0.0207 & 0.40 & 0.21 & 0.186 & 3.57 & 1.31 \\
\hline 20.25 & 0.0227 & 0.46 & 0.40 & 0.205 & 4.15 & 2.74 \\
\hline 21.25 & 0.0193 & 0.41 & 0.52 & 0.172 & 3.65 & 3.73 \\
\hline 22.25 & 0.0230 & 0.51 & 0.88 & 0.208 & 4.62 & 6.65 \\
\hline 23.25 & 0.0200 & 0.47 & 1.03 & 0.179 & 4.16 & 7.92 \\
\hline 24.25 & 0.0152 & 0.37 & 1.02 & 0.132 & 3.21 & 7.76 \\
\hline 25.25 & 0.0227 & 0.57 & 1.91 & 0.205 & 5.17 & 15.35 \\
\hline 26.25 & 0.0200 & 0.53 & 2.07 & 0.179 & 4.69 & 16.67 \\
\hline 27.25 & 0.0227 & 0.62 & 2.83 & 0.205 & 5.58 & 23.26 \\
\hline 28.25 & 0.0200 & 0.57 & 2.96 & 0.179 & 5.05 & 24.28 \\
\hline 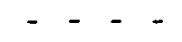 & 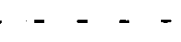 & -- & -- & $-{ }_{-1}$ & -- & $-\cdots$ \\
\hline
\end{tabular}


Table E.1: Fracture solute transport test data, and temporal moments analysis data for test no. 1 (continued).

Summary of Temporal Moments Analysis:

- Average Concentration

Sum Average Conc. =

Sum $\left[t_{\mathrm{E}} \times\right.$ Average Conc. $]=$

Sum $\left[\left(t_{E}-t_{m}\right)^{2} \times\right.$ Average Conc. $]=29.67$

First Moment, $t_{\mathrm{m}}=16.07 \mathrm{hr}$

Second Moment, $t_{\mathrm{var}}=51.02 \mathrm{hr}^{2}$

- Average Relative Concentration

Sum Average Rel. Conc. =

Sum $\left[t_{E} \times\right.$ Average Re1. Conc.] $=$

Sum $\left[\left(t_{E}-t_{m}\right)^{2} \times\right.$ Average Re1. Conc. $]=235.41$

First Moment, $t_{m}=16.59 \mathrm{hr}$

Second Moment, $t_{\mathrm{var}}=47.53 \mathrm{hr}^{2}$ 
Table E.2: Fracture solute transport test data for rest of test no. 1.

Sampling Port: 5F-UC

Location: $\mathrm{x}=10 \mathrm{~cm} ; \mathrm{z}=10 \mathrm{~cm} \quad$ Face: 5

Date: $6-21-88$ to $6-22-88$

Flow Rate: $1.41 \times 10^{-9} \mathrm{~m}^{3} / \mathrm{s}$

$E_{1}=168 \mathrm{mV}$

Head Imposed at Top of Plate:

$\mathrm{C}_{1}=0.00150 \underline{\mathrm{M}}$

Test Performed: Slug - 12 hrs

Test Duration: 28 hrs

$\mathrm{E}_{\mathrm{o}}=73 \mathrm{mV}$

$C_{0}=0.105 \underline{M}$

Plate no.: 5

\begin{tabular}{|c|c|c|c|c|c|}
\hline $\begin{array}{l}\text { Elapsed } \\
\text { Time, } \\
t_{E}(h r)\end{array}$ & $\begin{array}{l}\text { Potential } \\
\text { Difference } \\
\text { E (mV) }\end{array}$ & $\begin{array}{l}\text { Conc. , } \\
- \text { min } \\
(\underline{M})\end{array}$ & $\begin{array}{l}\text { Conc., } \\
-\max \\
(\underline{M})\end{array}$ & $\begin{array}{l}\text { Relative } \\
\text { Conc. } \\
\text { - min }\end{array}$ & $\begin{array}{c}\text { Relative } \\
\text { Conc. } \\
\text { - } \max \end{array}$ \\
\hline$-\overline{-}-$ & $755 \quad 15$ & $-\overline{-}-\overline{-}$ & $-\overline{-}-\overline{-}$ & $--\overline{-}-$ & 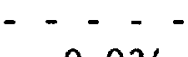 \\
\hline 0.25 & $155-145$ & 0.0031 & 0.0050 & 0.015 & 0.032 \\
\hline 0.75 & 145 & 0.0050 & 0.0050 & 0.034 & 0.034 \\
\hline 1.25 & $150-140$ & 0.0040 & 0.0063 & 0.024 & 0.046 \\
\hline 1.75 & 150 & 0.0040 & 0.0040 & 0.024 & 0.024 \\
\hline 2.25 & $145-140$ & 0.0050 & 0.0063 & 0.034 & 0.046 \\
\hline 2.75 & $135-127$ & 0.0080 & 0.0117 & 0.063 & 0.099 \\
\hline 3.25 & $118-115$ & 0.0165 & 0.0187 & 0.145 & 0.166 \\
\hline 3.75 & $125-118$ & 0.0125 & 0.0165 & 0.106 & 0.145 \\
\hline 4.25 & $130-125$ & 0.0107 & 0.0125 & 0.089 & 0.106 \\
\hline 4.75 & $110-100$ & 0.0227 & 0.0340 & 0.205 & 0.314 \\
\hline 5.25 & 85 & 0.0620 & 0.0620 & 0.585 & 0.585 \\
\hline 5.75 & $93-87$ & 0.0445 & 0.0570 & 0.415 & 0.536 \\
\hline 6.25 & $85-83$ & 0.0620 & 0.0660 & 0.585 & 0.623 \\
\hline 6.75 & $85-83$ & 0.0620 & 0.0660 & 0.585 & 0.623 \\
\hline 7.25 & $85-83$ & 0.0620 & 0.0660 & 0.585 & 0.623 \\
\hline 7.75 & $90-87$ & 0.0505 & 0.0570 & 0.473 & 0.536 \\
\hline 8.25 & $100-95$ & 0.0340 & 0.0415 & 0.314 & 0.386 \\
\hline 8.75 & 85 & 0.0620 & 0.0620 & 0.585 & 0.585 \\
\hline 9.25 & $90-87$ & 0.0505 & 0.0570 & 0.473 & 0.536 \\
\hline 9.75 & $83-80$ & 0.0660 & 0.0755 & 0.623 & 0.715 \\
\hline 10.25 & $85-83$ & 0.0620 & 0.0660 & 0.585 & 0.623 \\
\hline 10.75 & $88-86$ & 0.0550 & 0.0600 & 0.517 & 0.565 \\
\hline 11.25 & $83-78$ & 0.0660 & 0.0810 & 0.623 & 0.768 \\
\hline 11.75 & $82-78$ & 0.0700 & 0.0810 & 0.662 & 0.768 \\
\hline 12.25 & 77 & 0.0840 & 0.0840 & 0.797 & 0.797 \\
\hline 12.75 & 78 & 0.0810 & 0.0810 & 0.768 & 0.768 \\
\hline 13.25 & 83 & 0.0660 & 0.0660 & 0.623 & 0.623 \\
\hline 13.75 & $85-83$ & 0.0620 & 0.0660 & 0.585 & 0.623 \\
\hline 14.25 & 83 & 0.0660 & 0.0660 & 0.623 & 0.623 \\
\hline 14.75 & $87-85$ & 0.0570 & 0.0620 & 0.536 & 0.585 \\
\hline 15.25 & 92 & 0.0470 & 0.0470 & 0.440 & 0.440 \\
\hline 15.75 & 94 & 0.0425 & 0.0425 & 0.396 & 0.396 \\
\hline 16.25 & 95 & 0.0415 & 0.0415 & 0.386 & 0.386 \\
\hline
\end{tabular}


Table E.2: Fracture solute transport test data for rest of test no. 1. Sampling Port: 5F-UC (continued)

\begin{tabular}{|c|c|c|c|c|c|}
\hline $\begin{array}{l}\text { Elapsed } \\
\text { Time, } \\
t_{E}(h r)\end{array}$ & $\begin{array}{l}\text { Potential } \\
\text { Difference } \\
\text { E (mV) }\end{array}$ & $\begin{array}{l}\text { Conc. , } \\
- \text { min } \\
(\underline{M})\end{array}$ & $\begin{array}{l}\text { Conc. , } \\
-\max \\
(\underline{M})\end{array}$ & $\begin{array}{c}\text { Relative } \\
\text { Conc. } \\
\text { - min }\end{array}$ & $\begin{array}{c}\text { Relative } \\
\text { Conc. } \\
\text { - max }\end{array}$ \\
\hline$-\overline{16.75}$ & 96 & $\overline{0}-\overline{0405}$ & 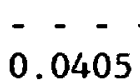 & $-\begin{array}{c}- \\
0.377\end{array}$ & $\begin{array}{c}- \\
0.377\end{array}$ \\
\hline 17.25 & $100-95$ & 0.0340 & 0.0415 & 0.314 & 0.386 \\
\hline 18.25 & $113-110$ & 0.0200 & 0.0227 & 0.179 & 0.205 \\
\hline 19.25 & $110-105$ & 0.0227 & 0.0280 & 0.205 & 0.256 \\
\hline 20.25 & $114-106$ & 0.0193 & 0.0270 & 0.172 & 0.246 \\
\hline 21.25 & 110 & 0.0227 & 0.0227 & 0.205 & 0.205 \\
\hline 22.25 & 117 & 0.0175 & 0.0175 & 0.155 & 0.155 \\
\hline 23.25 & $123-117$ & 0.0137 & 0.0175 & 0.118 & 0.155 \\
\hline 24.25 & $128-125$ & 0.0110 & 0.0125 & 0.092 & 0.106 \\
\hline 25.25 & 115 & 0.0187 & 0.0187 & 0.166 & 0.166 \\
\hline 26.25 & 124 & 0.0127 & 0.0127 & 0.108 & 0.108 \\
\hline 27.25 & 124 & 0.0127 & 0.0127 & 0.108 & 0.108 \\
\hline 28.25 & $135-125$ & 0.0080 & 0.0125 & 0.063 & 0.106 \\
\hline
\end{tabular}


Table E.2: Fracture solute transport test data for rest of test no. 1 .

Sampling Port: 5F-LS

Location: $\mathrm{x}=5 \mathrm{~cm} ; \mathrm{z}=35 \mathrm{~cm} \quad$ Face: 5

Date: 6-21-88 to 6-22-88

Flow Rate: $1.41 \times 10^{-9} \mathrm{~m}^{3} / \mathrm{s}$

$E_{1}=168 \mathrm{mV}$

Head Imposed at Top of Plate: $35.80 \mathrm{~cm} \quad \mathrm{E}_{0}=73 \mathrm{mV}$

Test Performed: Slug - 12 hrs $\quad C_{o}=0.105 \underline{M}$

Test Duration: 28 hrs

Plate no.: 5

\begin{tabular}{|c|c|c|c|c|c|}
\hline $\begin{array}{l}\text { Elapsed } \\
\text { Time, } \\
t_{E}(h r)\end{array}$ & $\begin{array}{l}\text { Potential } \\
\text { Difference } \\
\text { E (mV) }\end{array}$ & $\begin{array}{l}\text { Conc. , } \\
-\min \\
(\underline{M})\end{array}$ & $\begin{array}{l}\text { Conc. , } \\
-\max \\
(\underline{M})\end{array}$ & $\begin{array}{c}\text { Relative } \\
\text { Conc. } \\
\text { - min }\end{array}$ & $\begin{array}{c}\text { Relative } \\
\text { Conc. } \\
\text { - max }\end{array}$ \\
\hline$\overline{0}-\overline{33}$ & ---- & $\overline{0.0063}$ & $\overline{0.0063}$ & $-{ }^{-}-\overline{046}$ & $-\quad-\overline{-}-\overline{0}$ \\
\hline 1.33 & 145 & 0.0050 & 0.0050 & 0.034 & 0.034 \\
\hline 1.83 & 148 & 0.0044 & 0.0044 & 0.028 & 0.028 \\
\hline 2.33 & $152-148$ & 0.0036 & 0.0044 & 0.020 & 0.028 \\
\hline 2.83 & $150-140$ & 0.0040 & 0.0063 & 0.024 & 0.046 \\
\hline 3.33 & $148-145$ & 0.0044 & 0.0050 & 0.028 & 0.034 \\
\hline 3.83 & $135-125$ & 0.0080 & 0.0125 & 0.063 & 0.106 \\
\hline 4.33 & 145 & 0.0050 & 0.0050 & 0.034 & 0.034 \\
\hline 4.83 & 145 & 0.0050 & 0.0050 & 0.034 & 0.034 \\
\hline 5.33 & 145 & 0.0050 & 0.0050 & 0.034 & 0.034 \\
\hline 5.83 & $145-135$ & 0.0050 & 0.0080 & 0.034 & 0.063 \\
\hline 6.33 & 145 & 0.0050 & 0.0050 & 0.034 & 0.034 \\
\hline 6.83 & $155-150$ & 0.0031 & 0.0040 & 0.015 & 0.024 \\
\hline 7.33 & $145-140$ & 0.0050 & 0.0063 & 0.034 & 0.046 \\
\hline 7.83 & $145-140$ & 0.0050 & 0.0063 & 0.034 & 0.046 \\
\hline 8.33 & $145-140$ & 0.0050 & 0.0063 & 0.034 & 0.046 \\
\hline 8.83 & $147-145$ & 0.0046 & 0.0050 & 0.030 & 0.034 \\
\hline 9.33 & 137 & 0.0073 & 0.0073 & 0.056 & 0.056 \\
\hline 9.83 & 135 & 0.0080 & 0.0080 & 0.063 & 0.063 \\
\hline 10.33 & 126 & 0.0120 & 0.0120 & 0.101 & 0.101 \\
\hline 10.83 & 126 & 0.0120 & 0.0120 & 0.101 & 0.101 \\
\hline 11.33 & $133-128$ & 0.0088 & 0.0110 & 0.071 & 0.092 \\
\hline 11.83 & 125 & 0.0125 & 0.0125 & 0.106 & 0.106 \\
\hline 12.33 & 125 & 0.0125 & 0.0125 & 0.106 & 0.106 \\
\hline 12.83 & 123 & 0.0137 & 0.0137 & 0.118 & 0.118 \\
\hline 13.33 & 126 & 0.0120 & 0.0120 & 0.101 & 0.101 \\
\hline 13.83 & 125 & 0.0125 & 0.0125 & 0.106 & 0.106 \\
\hline 14.33 & 116 & 0.0183 & 0.0183 & 0.162 & 0.162 \\
\hline 14.83 & 125 & 0.0125 & 0.0125 & 0.106 & 0.106 \\
\hline 15.33 & $117-115$ & 0.0175 & 0.0187 & 0.155 & 0.166 \\
\hline 15.83 & 120 & 0.0152 & 0.0152 & 0.132 & 0.132 \\
\hline 16.33 & $120-115$ & 0.0152 & 0.0187 & 0.132 & 0.166 \\
\hline 16.83 & $110-107$ & 0.0227 & 0.0260 & 0.205 & 0.237 \\
\hline
\end{tabular}


Table E.2: Fracture solute transport test data for rest of test no. 1. Sampling Port: 5F-LS (continued)

\begin{tabular}{|c|c|c|c|c|c|}
\hline $\begin{array}{l}\text { Elapsed } \\
\text { Time, } \\
t_{\mathrm{E}}(\mathrm{hr})\end{array}$ & $\begin{array}{l}\text { Potential } \\
\text { Difference } \\
\text { E (mV) }\end{array}$ & $\begin{array}{l}\text { Conc. , } \\
- \text { min } \\
(\underline{M})\end{array}$ & $\begin{array}{l}\text { Conc. } \\
-\max \\
(\underline{M})\end{array}$ & $\begin{array}{c}\text { Relative } \\
\text { Conc. } \\
\text { - min }\end{array}$ & $\begin{array}{c}\text { Relative } \\
\text { Conc. } \\
\text { - max }\end{array}$ \\
\hline 17 & 11 & $\overline{0} \overline{0} \overline{0} \overline{07}$ & - - & $--\overline{-}$ & - - - - \\
\hline 18.33 & $120-115$ & 0.0152 & 0.0187 & 0.132 & 0.166 \\
\hline 19.33 & $125-117$ & 0.0125 & 0.0175 & 0.106 & 0.155 \\
\hline 20.33 & 112 & 0.0210 & 0.0210 & 0.188 & 0.188 \\
\hline 21.33 & 106 & 0.0270 & 0.0270 & 0.246 & 0.246 \\
\hline 22.33 & 108 & 0.0245 & 0.0245 & 0.222 & 0.222 \\
\hline 23.33 & $110-105$ & 0.0227 & 0.0280 & 0.205 & 0.256 \\
\hline 24.33 & 115 & 0.0287 & 0.0187 & 0.263 & 0.166 \\
\hline 25.33 & 110 & 0.0227 & 0.0227 & 0.205 & 0.205 \\
\hline 26.33 & $110-107$ & 0.0227 & 0.0260 & 0.205 & 0.237 \\
\hline 27.33 & $115-110$ & 0.0187 & 0.0227 & 0.166 & 0.205 \\
\hline 28.33 & 115 & 0.0187 & 0.0187 & 0.166 & 0.166 \\
\hline & & & & & \\
\hline
\end{tabular}


Table E.2: Fracture solute transport test data for rest of test no. 1 .

Sampling Port: 5F-LC

Location: $x=10 \mathrm{~cm} ; \mathrm{z}=35 \mathrm{~cm} \quad$ Face: 5

Date: $6-21-88$ to $6-22-88$

Flow Rate: $1.41 \times 10^{-9} \mathrm{~m}^{3} / \mathrm{s}$

$E_{\mathrm{i}}=168 \mathrm{mV}$

Head Imposed at Top of Plate:

$\mathrm{C}_{1}=0.00150 \underline{\mathrm{M}}$

Test Performed: slug - 12 hrs

Test Duration: $28 \mathrm{hrs}$

$35.80 \mathrm{~cm}$

$E_{0}=73 \mathrm{mV}$

$\mathrm{C}_{\mathrm{o}}=0.105 \underline{\mathrm{M}}$

Plate no.: 5

\begin{tabular}{|c|c|c|c|c|c|}
\hline $\begin{array}{c}\text { Elapsed } \\
\text { Time, } \\
t_{E}(h r)\end{array}$ & $\begin{array}{l}\text { Potential } \\
\text { Difference } \\
\text { E (mV) }\end{array}$ & $\begin{array}{l}\text { Conc. } \\
- \text { min } \\
(\underline{M})\end{array}$ & $\begin{array}{l}\text { Conc. } \\
-\max \\
(\underline{M})\end{array}$ & $\begin{array}{l}\text { Relative } \\
\text { Conc. } \\
\text { - min }\end{array}$ & $\begin{array}{l}\text { Relative } \\
\text { Conc. } \\
\text { - } \max \end{array}$ \\
\hline $\begin{array}{ll}- & - \\
0 & 33\end{array}$ & $145^{-}$ & $\begin{array}{ll}- & - \\
0 & 0050\end{array}$ & $\overline{0.0050}$ & 0.034 & $-\overline{-}-$ \\
\hline 1.33 & $150-145$ & 0.0040 & 0.0050 & 0.024 & 0.034 \\
\hline 1.83 & $155-150$ & 0.0031 & 0.0040 & 0.015 & 0.024 \\
\hline 2.33 & 145 & 0.0050 & 0.0050 & 0.034 & 0.034 \\
\hline 2.83 & $150-145$ & 0.0040 & 0.0050 & 0.024 & 0.034 \\
\hline 3.33 & $155-150$ & 0.0031 & 0.0040 & 0.015 & 0.024 \\
\hline 3.83 & $155-152$ & 0.0031 & 0.0036 & 0.015 & 0.020 \\
\hline 4.33 & 150 & 0.0040 & 0.0040 & 0.024 & 0.024 \\
\hline 4.83 & $150-145$ & 0.0040 & 0.0050 & 0.024 & 0.034 \\
\hline 5.33 & 145 & 0.0050 & 0.0050 & 0.034 & 0.034 \\
\hline 5.83 & $145-140$ & 0.0050 & 0.0063 & 0.034 & 0.046 \\
\hline 6.33 & 145 & 0.0050 & 0.0050 & 0.034 & 0.034 \\
\hline 6.83 & $150-148$ & 0.0040 & 0.0044 & 0.024 & 0.028 \\
\hline 7.33 & $150-145$ & 0.0040 & 0.0050 & 0.024 & 0.034 \\
\hline 7.83 & $155-150$ & 0.0031 & 0.0040 & 0.015 & 0.024 \\
\hline 8.33 & $150-140$ & 0.0040 & 0.0063 & 0.024 & 0.046 \\
\hline 8.83 & 146 & 0.0049 & 0.0049 & 0.033 & 0.033 \\
\hline 9.33 & $153-148$ & 0.0034 & 0.0044 & 0.018 & 0.028 \\
\hline 9.83 & $150-147$ & 0.0040 & 0.0046 & 0.024 & 0.030 \\
\hline 10.33 & $150-145$ & 0.0040 & 0.0050 & 0.024 & 0.034 \\
\hline 10.83 & 145 & 0.0050 & 0.0050 & 0.034 & 0.034 \\
\hline 11.33 & $145-140$ & 0.0050 & 0.0063 & 0.034 & 0.046 \\
\hline 11.83 & 145 & 0.0050 & 0.0050 & 0.034 & 0.034 \\
\hline 12.33 & $150-145$ & 0.0040 & 0.0050 & 0.024 & 0.034 \\
\hline 12.83 & 145 & 0.0050 & 0.0050 & 0.034 & 0.034 \\
\hline 13.33 & $152-148$ & 0.0036 & 0.0044 & 0.020 & 0.028 \\
\hline 13.83 & $152-148$ & 0.0036 & 0.0044 & 0.020 & 0.028 \\
\hline 14.33 & 145 & 0.0050 & 0.0050 & 0.034 & 0.034 \\
\hline 14.83 & $135-133$ & 0.0080 & 0.0088 & 0.063 & 0.071 \\
\hline 15.33 & $143-135$ & 0.0055 & 0.0080 & 0.039 & 0.063 \\
\hline 15.83 & 145 & 0.0050 & 0.0050 & 0.034 & 0.034 \\
\hline 16.33 & $145-140$ & 0.0050 & 0.0063 & 0.034 & 0.046 \\
\hline 16.83 & $145-140$ & 0.0050 & 0.0063 & 0.034 & 0.046 \\
\hline
\end{tabular}


Table E.2: Fracture solute transport test data for rest of test no. 1. Sampling Port: 5F-LC (continued)

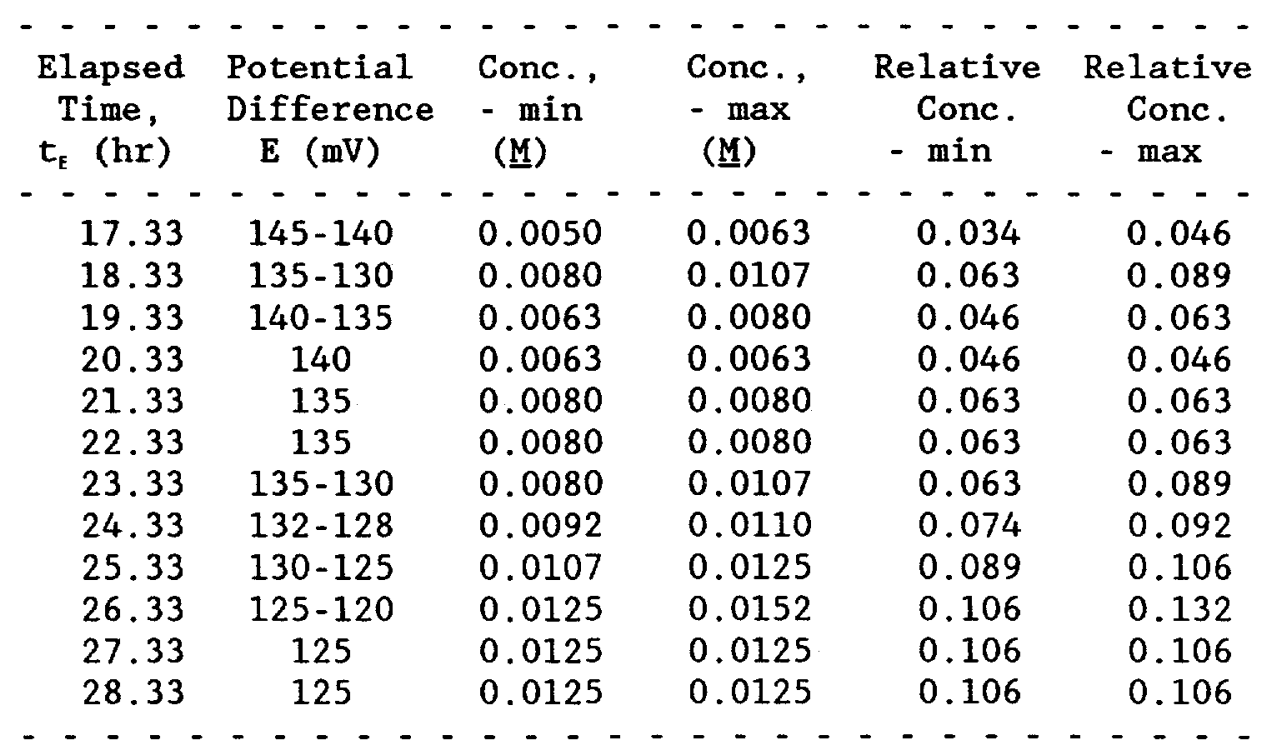


Table E.2: Fracture solute transport test data for rest of test no. 1.

Sampling Location: 6-B

Location: $x=2 \mathrm{~cm} ; \mathrm{z}=50 \mathrm{~cm} \quad$ Face: 2

Date: $6-21-88$ to $6-22-88$

Flow Rate: $1.41 \times 10^{-9} \mathrm{~m}^{3} / \mathrm{s}$

$E_{1}=168 \mathrm{mV}$

Head Imposed at Top of Plate: $35.80 \mathrm{~cm} \quad \mathrm{E}_{0}=73 \mathrm{mV}$

Test Performed: Slug - $12 \mathrm{hrs} \quad \mathrm{C}_{\mathrm{o}}=0.105 \mathrm{M}$

Test Duration: $28 \mathrm{hrs}$

Plate no.: 5

\begin{tabular}{|c|c|c|c|c|c|}
\hline $\begin{array}{l}\text { Elapsed } \\
\text { Time, } \\
t_{E}(h r)\end{array}$ & $\begin{array}{l}\text { Potential } \\
\text { Difference } \\
\text { E (mV) }\end{array}$ & $\begin{array}{l}\text { Conc. , } \\
- \text { min } \\
(\underline{M})\end{array}$ & $\begin{array}{l}\text { Conc. , } \\
-\max \\
(\underline{M})\end{array}$ & $\begin{array}{l}\text { Relative } \\
\text { Conc. } \\
\text { - min }\end{array}$ & $\begin{array}{c}\text { Relative } \\
\text { Conc. } \\
\text { - } \max \end{array}$ \\
\hline$\overline{0}-\overline{42}$ & $-\frac{-}{155}-$ & $\overline{0.0031}$ & $\overline{0.0031}$ & 0.015 & 0.015 \\
\hline 1.42 & $147-145$ & 0.0046 & 0.0050 & 0.030 & 0.034 \\
\hline 2.42 & $150-145$ & 0.0040 & 0.0050 & 0.024 & 0.034 \\
\hline 2.92 & $147-145$ & 0.0046 & 0.0050 & 0.030 & 0.034 \\
\hline 3.42 & $150-145$ & 0.0040 & 0.0050 & 0.024 & 0.034 \\
\hline 3.92 & $147-145$ & 0.0046 & 0.0050 & 0.030 & 0.034 \\
\hline 4.42 & 146 & 0.0049 & 0.0049 & 0.033 & 0.033 \\
\hline 4.92 & $145-140$ & 0.0050 & 0.0063 & 0.034 & 0.046 \\
\hline 5.42 & $145-140$ & 0.0050 & 0.0063 & 0.034 & 0.046 \\
\hline 5.92 & $133-130$ & 0.0088 & 0.0107 & 0.071 & 0.089 \\
\hline 6.42 & $135-130$ & 0.0080 & 0.0107 & 0.063 & 0.089 \\
\hline 6.92 & $133-128$ & 0.0088 & 0.0110 & 0.071 & 0.092 \\
\hline 7.42 & 127 & 0.0117 & 0.0117 & 0.099 & 0.099 \\
\hline 7.92 & 125 & 0.0125 & 0.0125 & 0.106 & 0.106 \\
\hline 8.42 & 125 & 0.0125 & 0.0125 & 0.106 & 0.106 \\
\hline 8.92 & $120-117$ & 0.0152 & 0.0175 & 0.132 & 0.155 \\
\hline 9.42 & 115 & 0.0187 & 0.0187 & 0.166 & 0.166 \\
\hline 9.92 & 115 & 0.0187 & 0.0187 & 0.166 & 0.166 \\
\hline 10.42 & 115 & 0.0187 & 0.0187 & 0.166 & 0.166 \\
\hline 10.92 & 112 & 0.0210 & 0.0210 & 0.188 & 0.188 \\
\hline 11.42 & 110 & 0.0227 & 0.0227 & 0.205 & 0.205 \\
\hline 11.92 & 108 & 0.0245 & 0.0245 & 0.222 & 0.222 \\
\hline 12.42 & 107 & 0.0260 & 0.0260 & 0.237 & 0.237 \\
\hline 12.92 & 107 & 0.0260 & 0.0260 & 0.237 & 0.237 \\
\hline 13.42 & 105 & 0.0280 & 0.0280 & 0.256 & 0.256 \\
\hline 13.92 & 105 & 0.0280 & 0.0280 & 0.256 & 0.256 \\
\hline 14.42 & 106 & 0.0270 & 0.0270 & 0.246 & 0.246 \\
\hline 14.92 & $107-104$ & 0.0260 & 0.0285 & 0.237 & 0.261 \\
\hline 15.42 & 105 & 0.0280 & 0.0280 & 0.256 & 0.256 \\
\hline 15.92 & $112-108$ & 0.0210 & 0.0245 & 0.188 & 0.222 \\
\hline 16.42 & $104-98$ & 0.0285 & 0.0365 & 0.261 & 0.338 \\
\hline 16.92 & 100 & 0.0340 & 0.0340 & 0.314 & 0.314 \\
\hline 17.42 & $108-105$ & 0.0245 & 0.0280 & 0.222 & 0.256 \\
\hline
\end{tabular}


Table E.2: Fracture solute transport test data for rest of test no. 1. Sampling Location: 6-B (continued)

\begin{tabular}{|c|c|c|c|c|c|}
\hline $\begin{array}{l}\text { Elapsed } \\
\text { Time, } \\
t_{\mathrm{f}}(\mathrm{hr})\end{array}$ & $\begin{array}{l}\text { Potential } \\
\text { Difference } \\
\text { E (mV) }\end{array}$ & $\begin{array}{l}\text { Conc. } \\
\text { - min } \\
\text { (M) }\end{array}$ & $\begin{array}{l}\text { Conc. , } \\
-\max \\
(\mathrm{M})\end{array}$ & $\begin{array}{c}\text { Relative } \\
\text { Conc. } \\
\text { - min }\end{array}$ & $\begin{array}{l}\text { Relative } \\
\text { Conc. } \\
\text { - } \max \end{array}$ \\
\hline & & & & 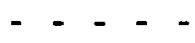 & -- \\
\hline $\begin{array}{l}78.42 \\
19.42\end{array}$ & $\begin{array}{c}110-105 \\
110\end{array}$ & $\begin{array}{l}0.0227 \\
0.0227\end{array}$ & $\begin{array}{l}0.0280 \\
0.0227\end{array}$ & $\begin{array}{l}0.205 \\
0.205\end{array}$ & $\begin{array}{l}0.256 \\
0.205\end{array}$ \\
\hline 20.42 & 107 & 0.0260 & 0.0260 & 0.237 & 0.237 \\
\hline 21.42 & 105 & 0.0280 & 0.0 & 0.256 & 0.256 \\
\hline 22.42 & 115 & 0.0 & 0.0 & 0.166 & 0.166 \\
\hline 23.42 & $114-110$ & 0.0 & 0.0 & 0.172 & 0.205 \\
\hline 24.42 & 115 & 0.0 & 0.0 & 0. & 0.166 \\
\hline 25.42 & $11:$ & 0.0 & 0.0 & 0. & 0.166 \\
\hline 26.42 & 107 & 0.0 & 0.0260 & 0. & 0.237 \\
\hline 27.42 & 110 & 0.0 & 0.0 & & 0.205 \\
\hline 28.42 & $120-115$ & 0.0152 & 0.0187 & 0.132 & 0.166 \\
\hline & & & & & \\
\hline
\end{tabular}


Table E.2: Fracture solute transport test data for rest of test no. 1 .

Sampling Location: 6-M

Location: $x=6 \mathrm{~cm} ; z=50 \mathrm{~cm} \quad$ Face: 2

Date: $6-21-88$ to $6-22-88$

Flow Rate: $1.41 \times 10^{-9} \mathrm{~m}^{3} / \mathrm{s}$

$E_{1}=168 \mathrm{mV}$

Head Imposed at Top of Plate:

Test Performed: Slug - 12 hrs

Test Duration: 28 hrs

$\mathrm{C}_{1}=0.00150 \underline{\mathrm{M}}$

$E_{o}=73 \mathrm{mV}$

$\mathrm{C}_{\mathrm{o}}=0.105 \underline{\mathrm{M}}$

Plate no.: 5

\begin{tabular}{|c|c|c|c|c|c|}
\hline $\begin{array}{l}\text { Elapsed } \\
\text { Time, } \\
t_{E}(h r)\end{array}$ & $\begin{array}{l}\text { Potential } \\
\text { Difference } \\
\text { E (mV) }\end{array}$ & $\begin{array}{l}\text { Conc. } \\
-\min \\
(\underline{M})\end{array}$ & $\begin{array}{l}\text { Conc. , } \\
-\max \\
(\underline{M})\end{array}$ & $\begin{array}{l}\text { Relative } \\
\text { Conc. } \\
\text { - min }\end{array}$ & $\begin{array}{c}\text { Relative } \\
\text { Conc. } \\
\text { - } \max \end{array}$ \\
\hline $\begin{array}{l}- \\
0.42\end{array}$ & $-{ }^{-}--$ & $\overline{0}-\overline{-}-\overline{0}$ & $\begin{array}{l}- \\
0.0034\end{array}$ & $-\overline{0 .}-\overline{0}$ & $-\overline{0}-\overline{0}-\overline{0}$ \\
\hline 1.42 & 152 & 0.0036 & 0.0036 & 0.020 & 0.020 \\
\hline 2.42 & $152-147$ & 0.0036 & 0.0046 & 0.020 & 0.030 \\
\hline 2.92 & 153 & 0.0034 & 0.0034 & 0.018 & 0.018 \\
\hline 3.42 & 150 & 0.0040 & 0.0040 & 0.024 & 0.024 \\
\hline 3.92 & 148 & 0.0044 & 0.0044 & 0.028 & 0.028 \\
\hline 4.42 & 145 & 0.0050 & 0.0050 & 0.034 & 0.034 \\
\hline 4.92 & $140-135$ & 0.0063 & 0.0080 & 0.046 & 0.063 \\
\hline 5.42 & $135-130$ & 0.0080 & 0.0107 & 0.063 & 0.089 \\
\hline 5.92 & $130-128$ & 0.0107 & 0.0110 & 0.089 & 0.092 \\
\hline 6.42 & 125 & 0.0125 & 0.0125 & 0.106 & 0.106 \\
\hline 6.92 & $125-122$ & 0.0125 & 0.0143 & 0.106 & 0.124 \\
\hline 7.42 & 120 & 0.0152 & 0.0152 & 0.132 & 0.132 \\
\hline 7.92 & 117 & 0.0175 & 0.0175 & 0.155 & 0.155 \\
\hline 8.42 & 115 & 0.0187 & 0.0187 & 0.166 & 0.166 \\
\hline 8.92 & 112 & 0.0210 & 0.0210 & 0.188 & 0.188 \\
\hline 9.42 & 111 & 0.0220 & 0.0220 & 0.198 & 0.198 \\
\hline 9.92 & 110 & 0.0227 & 0.0227 & 0.205 & 0.205 \\
\hline 10.42 & 107 & 0.0260 & 0.0260 & 0.237 & 0.237 \\
\hline 10.92 & 107 & 0.0260 & 0.0260 & 0.237 & 0.237 \\
\hline 11.42 & 107 & 0.0260 & 0.0260 & 0.237 & 0.237 \\
\hline 11.92 & 105 & 0.0280 & 0.0280 & 0.256 & 0.256 \\
\hline 12.42 & 105 & 0.0280 & 0.0280 & 0.256 & 0.256 \\
\hline 12.92 & 105 & 0.0280 & 0.0280 & 0.256 & 0.256 \\
\hline 13.42 & 105 & 0.0280 & 0.0280 & 0.256 & 0.256 \\
\hline 13.92 & $105-103$ & 0.0280 & 0.0300 & 0.256 & 0.275 \\
\hline 14.42 & 96 & 0.0405 & 0.0405 & 0.377 & 0.377 \\
\hline 14.92 & $105-103$ & 0.0280 & 0.0300 & 0.256 & 0.275 \\
\hline 15.42 & 105 & 0.0280 & 0.0280 & 0.256 & 0.256 \\
\hline 15.92 & 105 & 0.0280 & 0.0280 & 0.256 & 0.256 \\
\hline 16.42 & $100-97$ & 0.0340 & 0.0385 & 0.314 & 0.357 \\
\hline 16.92 & 105 & 0.0280 & 0.0280 & 0.256 & 0.256 \\
\hline 17.42 & 110 & 0.0227 & 0.0227 & 0.205 & 0.205 \\
\hline
\end{tabular}


Table E.2: Fracture solute transport test data for rest of test no. 1. Sampling Location: 6-M (continued)

\begin{tabular}{|c|c|c|c|c|c|}
\hline $\begin{array}{l}\text { Elapsed } \\
\text { Time, } \\
t_{E}(h r)\end{array}$ & $\begin{array}{l}\text { Potential } \\
\text { Difference } \\
\text { E (mV) }\end{array}$ & $\begin{array}{l}\text { Conc. } \\
- \text { min } \\
(\underline{M})\end{array}$ & $\begin{array}{l}\text { Conc. , } \\
-\max \\
(\underline{M})\end{array}$ & $\begin{array}{c}\text { Relative } \\
\text { Conc. } \\
\text { - min }\end{array}$ & $\begin{array}{l}\text { Relative } \\
\text { Conc. } \\
\text { - max }\end{array}$ \\
\hline 18 20 & - & $-\overline{-}-\overline{7}$ & $-\overline{0}-\overline{1}$ & -- & --- \\
\hline $\begin{array}{l}18.42 \\
19.42\end{array}$ & $\begin{array}{l}106 \\
110\end{array}$ & 0.0227 & 0.0227 & $\begin{array}{l}0.246 \\
0.205\end{array}$ & $\begin{array}{l}0.246 \\
0.205\end{array}$ \\
\hline 20.42 & 120 & 0.0152 & 0.0152 & 0.132 & 0.132 \\
\hline 21.42 & 115 & 0.0187 & 0.0187 & 0.166 & 0.166 \\
\hline 22.42 & 115 & 0.0187 & 0.0187 & 0.166 & 0.166 \\
\hline 23.42 & 116 & 0.0183 & 0.0183 & 0.162 & 0.162 \\
\hline 24.42 & $125-120$ & 0.0125 & 0.0152 & 0.106 & 0.132 \\
\hline 25.42 & 118 & 0.0165 & 0.0165 & 0.145 & 0.145 \\
\hline 26.42 & 120 & 0.0152 & 0.0152 & 0.132 & 0.132 \\
\hline 27.42 & 122 & 0.0143 & 0.0143 & 0.124 & 0.124 \\
\hline 28.42 & 125 & 0.0125 & 0.0125 & 0.106 & 0.106 \\
\hline
\end{tabular}


Table E.2: Fracture solute transport test data for rest of test no. 1 .

Sampling Location: 3-M

Location: $x=12 \mathrm{~cm} ; z=50 \mathrm{~cm} \quad$ Face: 2

Date: $6-21-88$ to $6-22-88$

Flow Rate: $1.41 \times 10^{-9} \mathrm{~m}^{3} / \mathrm{s}$

$E_{1}=168 \mathrm{mV}$
$C_{1}=0.00150 \underline{M}$
$E_{o}=73 \mathrm{mV}$
$C_{o}=0.105 \underline{M}$
Plate no.:

Test Performed: slug - $12 \mathrm{hrs}$

Test Duration: 28 hrs

$E_{1}=168 \mathrm{mV}$
$\quad C_{1}=0.00150 \underline{M}$
$E_{o}=73 \mathrm{mV}$
$C_{o}=0.105 \underline{M}$
Plate no.: 5

\begin{tabular}{|c|c|c|c|c|c|}
\hline $\begin{array}{l}\text { Elapsed } \\
\text { Time, } \\
t_{\mathrm{E}}(\mathrm{h} r)\end{array}$ & $\begin{array}{l}\text { Potential } \\
\text { Difference } \\
\text { E (mV) }\end{array}$ & $\begin{array}{l}\text { Conc., } \\
- \text { min } \\
(\underline{M})\end{array}$ & $\begin{array}{l}\text { Conc. , } \\
-\max \\
(\underline{M})\end{array}$ & $\begin{array}{c}\text { Relative } \\
\text { Conc. } \\
\text { - min }\end{array}$ & $\begin{array}{c}\text { Relative } \\
\text { Conc. } \\
\text { - } \max \end{array}$ \\
\hline$-\overline{0 .}-\overline{-}$ & $--{ }_{150}^{-}-$ & 0.0040 & $\overline{0.0040}$ & 0.024 & 0.024 \\
\hline 1.42 & $153-150$ & 0.0034 & 0.0040 & 0.018 & 0.024 \\
\hline 2.42 & 155 & 0.0031 & 0.0031 & 0.015 & 0.015 \\
\hline 2.92 & $152-150$ & 0.0036 & 0.0040 & 0.020 & 0.024 \\
\hline 3.42 & 155 & 0.0031 & 0.0031 & 0.015 & 0.015 \\
\hline 3.92 & 150 & 0.0040 & 0.0040 & 0.024 & 0.024 \\
\hline 4.42 & 150 & 0.0040 & 0.0040 & 0.024 & 0.024 \\
\hline 4.92 & 145 & 0.0050 & 0.0050 & 0.034 & 0.034 \\
\hline 5.42 & 135 & 0.0080 & 0.0080 & 0.063 & 0.063 \\
\hline 5.92 & 135 & 0.0080 & 0.0080 & 0.063 & 0.063 \\
\hline 6.42 & $130-128$ & 0.0107 & 0.0110 & 0.089 & 0.092 \\
\hline 6.92 & 125 & 0.0125 & 0.0125 & 0.106 & 0.106 \\
\hline 7.42 & 122 & 0.0143 & 0.0143 & 0.124 & 0.124 \\
\hline 7.92 & $125-123$ & 0.0125 & 0.0137 & 0.106 & 0.118 \\
\hline 8.42 & 120 & 0.0152 & 0.0152 & 0.132 & 0.132 \\
\hline 8.92 & 117 & 0.0175 & 0.0175 & 0.155 & 0.155 \\
\hline 9.42 & $116-113$ & 0.0183 & 0.0200 & 0.162 & 0.179 \\
\hline 9.92 & 117 & 0.0175 & 0.0175 & 0.155 & 0.155 \\
\hline 10.42 & 114 & 0.0193 & 0.0193 & 0.172 & 0.172 \\
\hline 10.92 & 108 & 0.0245 & 0.0245 & 0.222 & 0.222 \\
\hline 11.42 & 110 & 0.0227 & 0.0227 & 0.205 & 0.205 \\
\hline 11.92 & 108 & 0.0245 & 0.0245 & 0.222 & 0.222 \\
\hline 12.42 & 107 & 0.0260 & 0.0260 & 0.237 & 0.237 \\
\hline 12.92 & 105 & 0.0280 & 0.0280 & 0.256 & 0.256 \\
\hline 13.42 & 105 & 0.0280 & 0.0280 & 0.256 & 0.256 \\
\hline 13.92 & 105 & 0.0280 & 0.0280 & 0.256 & 0.256 \\
\hline 14.42 & $107-105$ & 0.0260 & 0.0280 & 0.237 & 0.256 \\
\hline 14.92 & 105 & 0.0280 & 0.0280 & 0.256 & 0.256 \\
\hline 15.42 & 105 & 0.0280 & 0.0280 & 0.256 & 0.256 \\
\hline 15.92 & $110-107$ & 0.0227 & 0.0260 & 0.205 & 0.237 \\
\hline 16.42 & 105 & 0.0280 & 0.0280 & 0.256 & 0.256 \\
\hline 16.92 & 104 & 0.0285 & 0.0285 & 0.261 & 0.261 \\
\hline 17.42 & 105 & 0.0280 & 0.0280 & 0.256 & 0.256 \\
\hline
\end{tabular}


Table E.2: Fracture solute transport test data for rest of test no. 1 . Sampling Location: 3-M (continued)

\begin{tabular}{|c|c|c|c|c|c|}
\hline $\begin{array}{l}\text { Elapsed } \\
\text { Time, } \\
t_{\mathrm{E}}(\mathrm{hr})\end{array}$ & $\begin{array}{l}\text { Potential } \\
\text { Difference } \\
\text { E (mV) }\end{array}$ & $\begin{array}{l}\text { Conc., } \\
- \text { min } \\
(\underline{M})\end{array}$ & $\begin{array}{l}\text { Conc., } \\
-\max \\
(\underline{M})\end{array}$ & $\begin{array}{c}\text { Relative } \\
\text { Conc. } \\
\text { - min }\end{array}$ & $\begin{array}{c}\text { Relative } \\
\text { Conc. } \\
-\max \end{array}$ \\
\hline--- & $--\overline{-}$ & $-\overline{-}-$ & -- & $---\overline{-}-$ & $---\overline{-}$ \\
\hline 18.42 & 105 & 0.0280 & 0.0280 & 0.256 & 0.256 \\
\hline 19.42 & $\begin{array}{c}112-108 \\
113\end{array}$ & 0.0 & 5 & & $\begin{array}{l}0.222 \\
0.179\end{array}$ \\
\hline $\begin{array}{l}20.42 \\
21.42\end{array}$ & $\begin{array}{l}113 \\
115\end{array}$ & 0.0 & 0.0 & 0.166 & 0.166 \\
\hline 22.42 & 115 & 0.0187 & 0.0187 & 0.166 & 0.166 \\
\hline 23.42 & 115 & 0.0187 & 0.0187 & 0.166 & 0.166 \\
\hline 24.42 & $117-112$ & 0.0175 & 0.0210 & 0.155 & 0.188 \\
\hline 25.42 & 117 & 0.0175 & 0.0175 & 0.155 & 0.155 \\
\hline 26.42 & 11 & 0.0187 & 0.0187 & 0.166 & 0.166 \\
\hline 27.42 & 115 & 0.0187 & 0.0187 & 0.166 & 0.166 \\
\hline 28.42 & 120 & 0.0152 & 0.0152 & 0.132 & 0.132 \\
\hline
\end{tabular}


Table E.2: Fracture solute transport test data for rest of test no. 1.

Sampling Location: 3-F

Location: $x=18 \mathrm{~cm} ; z=50 \mathrm{~cm} \quad$ Face: 2

Date: $6-21-88$ to $6-22-88$

Flow Rate: $1.41 \times 10^{-9} \mathrm{~m}^{3} / \mathrm{s}$

$E_{1}=168 \mathrm{mV}$

Head Imposed at Top of Plate: $35.80 \mathrm{~cm}$

$\mathrm{C}_{1}=0.00150 \underline{\mathrm{M}}$

Test Performed: Slug - 12 hrs

Test Duration: 28 hrs

$E_{0}=73 \mathrm{mV}$

$\mathrm{C}_{\mathrm{o}}=0.105 \underline{\mathrm{M}}$

Plate no.: 5

\begin{tabular}{|c|c|c|c|c|c|}
\hline $\begin{array}{l}\text { Elapsed } \\
\text { Time, } \\
t_{\mathrm{f}}(\mathrm{hr})\end{array}$ & $\begin{array}{l}\text { Potential } \\
\text { Difference } \\
\text { E (mV) }\end{array}$ & $\begin{array}{l}\text { Conc. , } \\
-\min \\
(\underline{M})\end{array}$ & $\begin{array}{l}\text { Conc. , } \\
-\max \\
(\underline{M})\end{array}$ & $\begin{array}{c}\text { Relative } \\
\text { Conc. } \\
\text { - min }\end{array}$ & $\begin{array}{l}\text { Relative } \\
\text { Conc. } \\
\text { - max }\end{array}$ \\
\hline$-\overline{-}-$ & $-{ }^{-}--$ & $\overline{0.0046}$ & $\overline{0.0046}$ & 0.030 & $0 . \overline{0}$ \\
\hline 1.42 & $150-147$ & 0.0040 & 0.0046 & 0.024 & 0.030 \\
\hline 2.42 & 152 & 0.0036 & 0.0036 & 0.020 & 0.020 \\
\hline 2.92 & $155-152$ & 0.0031 & 0.0036 & 0.015 & 0.020 \\
\hline 3.42 & 153 & 0.0034 & 0.0034 & 0.018 & 0.018 \\
\hline 3.92 & $150-148$ & 0.0040 & 0.0044 & 0.024 & 0.028 \\
\hline 4.42 & 145 & 0.0050 & 0.0050 & 0.034 & 0.034 \\
\hline 4.92 & 140 & 0.0063 & 0.0063 & 0.046 & 0.046 \\
\hline 5.42 & 135 & 0.0080 & 0.0080 & 0.063 & 0.063 \\
\hline 5.92 & 130 & 0.0100 & 0.0100 & 0.082 & 0.082 \\
\hline 6.42 & $130-127$ & 0.0100 & 0.0117 & 0.082 & 0.099 \\
\hline 6.92 & 125 & 0.0125 & 0.0125 & 0.106 & 0.106 \\
\hline 7.42 & 125 & 0.0125 & 0.0125 & 0.106 & 0.106 \\
\hline 7.92 & 125 & 0.0125 & 0.0125 & 0.106 & 0.106 \\
\hline 8.42 & 120 & 0.0152 & 0.0152 & 0.132 & 0.132 \\
\hline 8.92 & 118 & 0.0165 & 0.0165 & 0.145 & 0.145 \\
\hline 9.42 & $125-120$ & 0.0125 & 0.0152 & 0.106 & 0.132 \\
\hline 9.92 & 118 & 0.0165 & 0.0165 & 0.145 & 0.145 \\
\hline 10.42 & $117-115$ & 0.0175 & 0.0187 & 0.155 & 0.166 \\
\hline 10.92 & 110 & 0.0227 & 0.0227 & 0.205 & 0.205 \\
\hline 11.42 & 110 & 0.0227 & 0.0227 & 0.205 & 0.205 \\
\hline 11.92 & 112 & 0.0210 & 0.0210 & 0.188 & 0.188 \\
\hline 12.42 & 110 & 0.0227 & 0.0227 & 0.205 & 0.205 \\
\hline 12.92 & 107 & 0.0260 & 0.0260 & 0.237 & 0.237 \\
\hline 13.42 & $110-105$ & 0.0227 & 0.0280 & 0.205 & 0.256 \\
\hline 13.92 & 105 & 0.0280 & 0.0280 & 0.256 & 0.256 \\
\hline 14.42 & $105-103$ & 0.0280 & 0.0300 & 0.256 & 0.275 \\
\hline 14.92 & 105 & 0.0280 & 0.0280 & 0.256 & 0.256 \\
\hline 15.42 & 103 & 0.0300 & 0.0300 & 0.275 & 0.275 \\
\hline 15.92 & 105 & 0.0280 & 0.0280 & 0.256 & 0.256 \\
\hline 16.42 & $107-105$ & 0.0260 & 0.0280 & 0.237 & 0.256 \\
\hline 16.92 & 103 & 0.0300 & 0.0300 & 0.275 & 0.275 \\
\hline 17.42 & $105-103$ & 0.0280 & 0.0300 & 0.256 & 0.275 \\
\hline
\end{tabular}


Table E.2: Fracture solute transport test data for rest of test no. 1 . Sampling Location: $3-\mathrm{F}$ (continued)

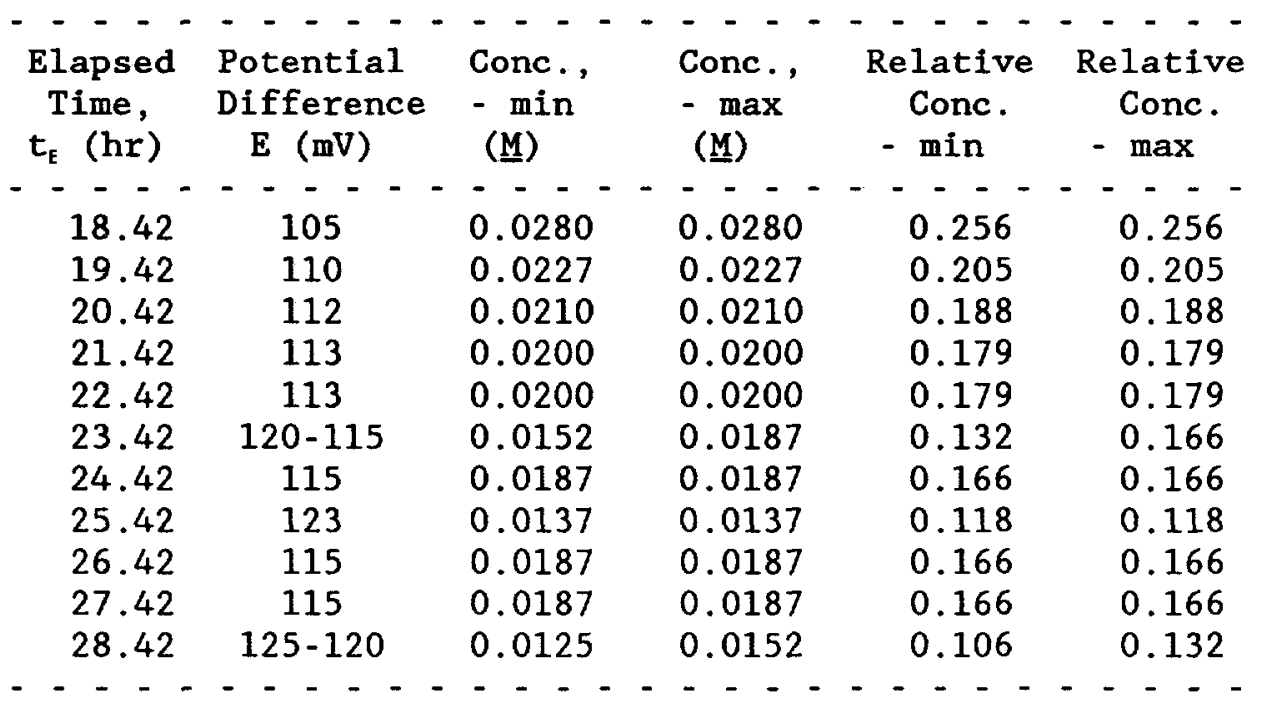


Table E.3: Fracture solute transport test data for test no. 2 .

Sampling Port: 5F-US

Location: $x=5 \mathrm{~cm} ; z=10 \mathrm{~cm} \quad$ Face: 5

Date: $7-12-88$ to $7-18-88$

Flow Rate: $1.36 \times 10^{-9} \mathrm{~m}^{3} / \mathrm{s}$

$E_{\mathbf{l}}=173 \mathrm{mV}$

Head Imposed at Top of Plate: $35.90 \mathrm{~cm} \quad \mathrm{C}_{1}=73 \mathrm{mV}$

Test Performed: Slug - 48 hrs

Test Duration: 150 hrs

$\mathrm{C}_{\mathrm{o}}=0.105 \underline{\mathrm{M}}$

Plate no.: 5

\begin{tabular}{|c|c|c|c|c|c|}
\hline $\begin{array}{l}\text { Elapsed } \\
\text { Time } \\
t_{E}(h r)\end{array}$ & $\begin{array}{l}\text { Potential } \\
\text { Difference } \\
\text { E (mV) }\end{array}$ & $\begin{array}{l}\text { Conc. } \\
- \text { min } \\
(\underline{M})\end{array}$ & $\begin{array}{l}\text { Conc. } \\
-\max \\
(\underline{M})\end{array}$ & $\begin{array}{l}\text { Relative } \\
\text { Conc. } \\
\text { - min }\end{array}$ & $\begin{array}{c}\text { Relative } \\
\text { Conc. } \\
\text { - max }\end{array}$ \\
\hline$-\overline{-}-$ & & - - - - & $-\overline{-}-$ & - - - - - & - - - - \\
\hline $\begin{array}{l}0.25 \\
1.00\end{array}$ & $\begin{array}{c}157-154 \\
160\end{array}$ & $\begin{array}{l}0.0028 \\
0.0025\end{array}$ & $\begin{array}{l}0.0032 \\
0.0025\end{array}$ & $\begin{array}{l}0.015 \\
0.012\end{array}$ & $\begin{array}{l}0.019 \\
0.012\end{array}$ \\
\hline 2.00 & $159-158$ & 0.0026 & 0.0027 & 0.013 & 0.014 \\
\hline 3.00 & $165-158$ & 0.0019 & 0.0027 & 0.006 & 0.014 \\
\hline 4.00 & $156-150$ & 0.0030 & 0.0040 & 0.017 & 0.026 \\
\hline 5.00 & $153-146$ & 0.0034 & 0.0049 & 0.020 & 0.035 \\
\hline 6.00 & $133-130$ & 0.0088 & 0.0107 & 0.072 & 0.090 \\
\hline 7.00 & $140-137$ & 0.0063 & 0.0073 & 0.048 & 0.058 \\
\hline 8.00 & $137-129$ & 0.0073 & 0.0105 & 0.058 & 0.088 \\
\hline 9.00 & $127-125$ & 0.0117 & 0.0125 & 0.100 & 0.107 \\
\hline 10.00 & $126-120$ & 0.0120 & 0.0152 & 0.102 & 0.133 \\
\hline 11.00 & $126-119$ & 0.0120 & 0.0157 & 0.102 & 0.138 \\
\hline 12.00 & $116-112$ & 0.0183 & 0.0210 & 0.162 & 0.188 \\
\hline 13.00 & $118-113$ & 0.0165 & 0.0200 & 0.145 & 0.179 \\
\hline 14.00 & $123-118$ & 0.0137 & 0.0165 & 0.119 & 0.145 \\
\hline 16.00 & $106-105$ & 0.0270 & 0.0280 & 0.245 & 0.255 \\
\hline 18.00 & $114-111$ & 0.0193 & 0.0220 & 0.172 & 0.198 \\
\hline 20.00 & $104-99$ & 0.0285 & 0.0350 & 0.260 & 0.321 \\
\hline 22.00 & $96-93$ & 0.0405 & 0.0445 & 0.374 & 0.412 \\
\hline 24.00 & 95 & 0.0415 & 0.0415 & 0.383 & 0.383 \\
\hline 26.00 & 93 & 0.0445 & 0.0445 & 0.412 & 0.412 \\
\hline 28.00 & 95 & 0.0415 & 0.0415 & 0.383 & 0.383 \\
\hline 30.00 & 101 & 0.0325 & 0.0325 & 0.298 & 0.298 \\
\hline 33.50 & 93-91 & 0.0445 & 0.0495 & 0.412 & 0.460 \\
\hline 43.75 & $89-88$ & 0.0520 & 0.0550 & 0.483 & 0.512 \\
\hline 47.50 & $94-84$ & 0.0425 & 0.0640 & 0.393 & 0,598 \\
\hline 49.00 & $91-89$ & 0.0495 & 0.0520 & 0.460 & 0.483 \\
\hline 50.00 & $89-85$ & 0.0520 & 0.0620 & 0.483 & 0.579 \\
\hline 51.00 & $92-88$ & 0.0470 & 0.0550 & 0.436 & 0.512 \\
\hline 52.00 & $91-87$ & 0.0495 & 0.0570 & 0.460 & 0.531 \\
\hline 53.00 & $89-87$ & 0.0520 & 0.0570 & 0.483 & 0.531 \\
\hline 54.00 & $88-87$ & 0.0550 & 0.0570 & 0.512 & 0.531 \\
\hline 55.00 & $86-85$ & 0.0600 & 0.0620 & 0.560 & 0.579 \\
\hline & & & & & \\
\hline
\end{tabular}


Table E.3: Fracture solute transport test data for test no. 2 .

Sampling Port: 5F-US (continued)

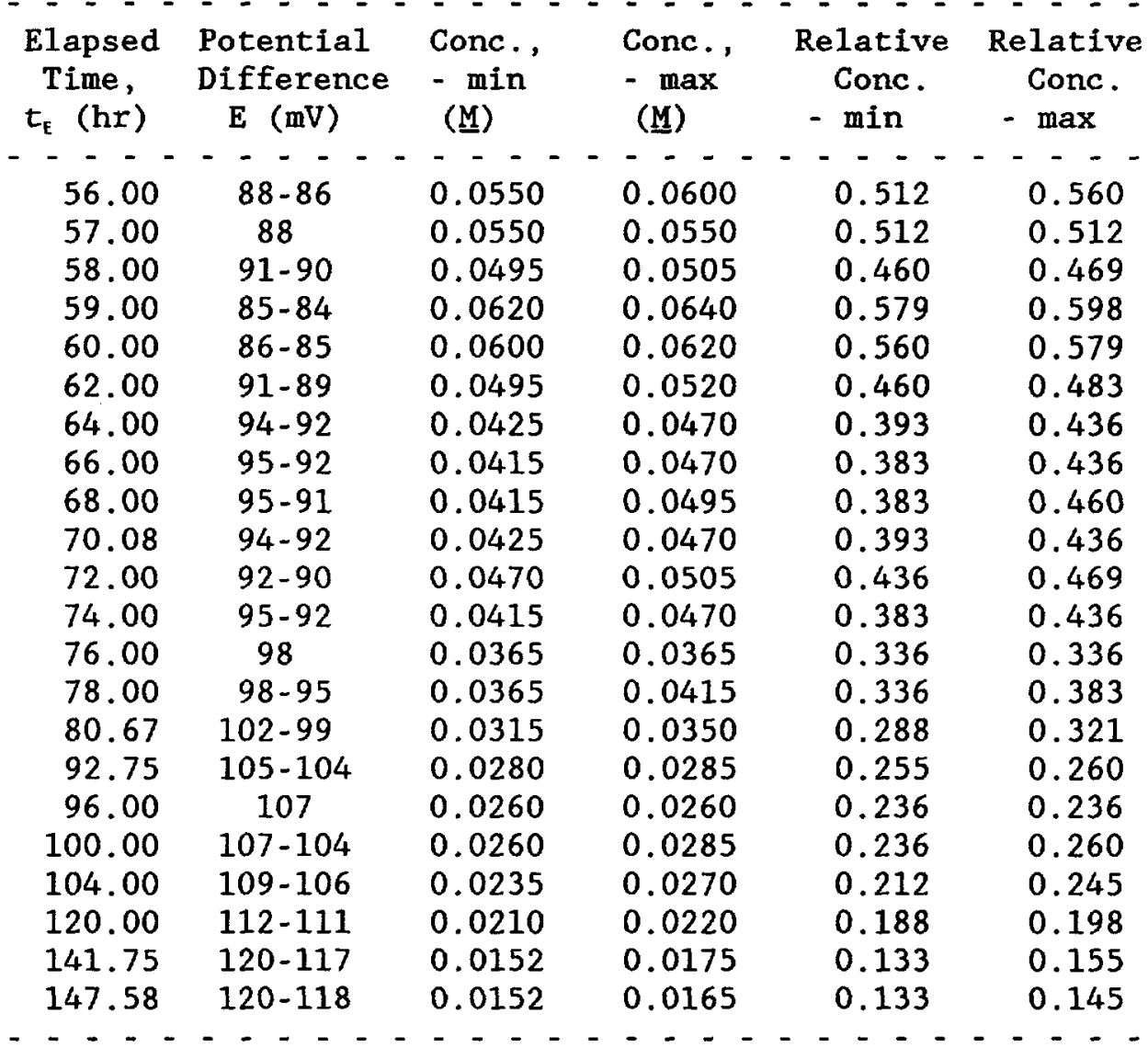


Table E. 3: Fracture solute transport test data for test no. 2 .

Sampling Port: 5F-UC

Location: $x=10 \mathrm{~cm} ; z=10 \mathrm{~cm} \quad$ Face: 5

Date: 7-12-88 to 7-18-88

Flow Rate: $1.36 \times 10^{-9} \mathrm{~m}^{3} / \mathrm{s}$

$E_{1}=173 \mathrm{mV}$

Test Performed: Slug - $48 \mathrm{hrs} \quad \mathrm{C}_{\mathrm{o}}=0.105 \underline{\mathrm{M}}$

Test Duration: 150 hrs

Plate no.: 5

\begin{tabular}{|c|c|c|c|c|c|}
\hline $\begin{array}{l}\text { Elapsed } \\
\text { Time, } \\
t_{E}(h r)\end{array}$ & $\begin{array}{l}\text { Potential } \\
\text { Difference } \\
\text { E (mV) }\end{array}$ & $\begin{array}{l}\text { Conc., } \\
- \text { min } \\
(\underline{M})\end{array}$ & $\begin{array}{l}\text { Conc. } \\
-\max \\
(\underline{M})\end{array}$ & $\begin{array}{c}\text { Relative } \\
\text { Conc. } \\
\text { - min }\end{array}$ & $\begin{array}{l}\text { Relative } \\
\text { Conc. } \\
\text { - max }\end{array}$ \\
\hline$-\quad-\quad-$ & ---- & -- & -- & -- & - \\
\hline $\begin{array}{l}0.25 \\
1.00\end{array}$ & $\begin{array}{l}162-155 \\
167-154\end{array}$ & $\begin{array}{l}0.0023 \\
0.0018\end{array}$ & $\begin{array}{l}0.0031 \\
0.0032\end{array}$ & $\begin{array}{l}0.010 \\
0.005\end{array}$ & $\begin{array}{l}0.018 \\
0.019\end{array}$ \\
\hline 2.00 & $157-151$ & 0.0028 & 0.0038 & 0.015 & 0.024 \\
\hline 3.00 & $153-147$ & 0.0034 & 0.0046 & 0.020 & 0.032 \\
\hline 5.00 & 140 & 0.0063 & 0.0063 & 0.048 & 0.048 \\
\hline 6.00 & $108-107$ & 0.0245 & 0.0260 & 0.221 & 0.236 \\
\hline 7.00 & $110-104$ & 0.0227 & 0.0285 & 0.204 & 0.260 \\
\hline 8.00 & $102-95$ & 0.0315 & 0.0415 & 0.288 & 0.383 \\
\hline 9.00 & $107-99$ & 0.0260 & 0.0355 & 0.236 & 0.326 \\
\hline 10.00 & $92-88$ & 0.0470 & 0.0550 & 0.436 & 0.512 \\
\hline 11.00 & $91-88$ & 0.0495 & 0.0550 & 0.460 & 0.512 \\
\hline 12.00 & $83-82$ & 0.0660 & 0.0700 & 0.617 & 0.655 \\
\hline 13.00 & $86-83$ & 0.0600 & 0.0660 & 0.560 & 0.617 \\
\hline 14.00 & $84-81$ & 0.0640 & 0.0730 & 0.598 & 0.683 \\
\hline 16.00 & 88 & 0.0550 & 0.0550 & 0.512 & 0.512 \\
\hline 18.00 & $97-91$ & 0.0385 & 0.0495 & 0.355 & 0.460 \\
\hline 20.00 & $90-87$ & 0.0505 & 0.0570 & 0.469 & 0.531 \\
\hline 22.00 & $88-83$ & 0.0550 & 0.0660 & 0.512 & 0.617 \\
\hline 24.00 & $88-85$ & 0.0550 & 0.0620 & 0.512 & 0.579 \\
\hline 26.00 & 86 & 0.0600 & 0.0600 & 0.560 & 0.560 \\
\hline 28.00 & $87-85$ & 0.0570 & 0.0620 & 0.531 & 0.579 \\
\hline 30.00 & $85-82$ & 0.0620 & 0.0700 & 0.579 & 0.655 \\
\hline 33.50 & $80-79$ & 0.0755 & 0.0780 & 0.707 & 0.731 \\
\hline 43.75 & $83-82$ & 0.0660 & 0.0700 & 0.617 & 0.655 \\
\hline 47.50 & $81-79$ & 0.0730 & 0.0780 & 0.683 & 0.731 \\
\hline 49.00 & $84-82$ & 0.0640 & 0.0700 & 0.598 & 0.655 \\
\hline 50.00 & 84 & 0.0640 & 0.0640 & 0.598 & 0.598 \\
\hline 51.00 & $84-81$ & 0.0640 & 0.0730 & 0.598 & 0.683 \\
\hline 52.00 & $87-82$ & 0.0570 & 0.0700 & 0.531 & 0.655 \\
\hline 53.00 & $91-89$ & 0.0495 & 0.0520 & 0.460 & 0.483 \\
\hline 54.00 & $89-87$ & 0.0520 & 0.0570 & 0.483 & 0.531 \\
\hline 55.00 & $90-88$ & 0.0505 & 0.0550 & 0.469 & 0.512 \\
\hline 56.00 & $91-89$ & 0.0495 & 0.0520 & 0.460 & 0.483 \\
\hline
\end{tabular}


Table E.3: Fracture solute transport test data for test no. 2 . Sampling Port: 5F-UC (continued)

\begin{tabular}{|c|c|c|c|c|c|}
\hline $\begin{array}{l}\text { Elapsed } \\
\text { Time, } \\
t_{E}(h r)\end{array}$ & $\begin{array}{l}\text { Potential } \\
\text { Difference } \\
\text { E (mV) }\end{array}$ & $\begin{array}{l}\text { Conc. } \\
- \text { min } \\
(\underline{M})\end{array}$ & $\begin{array}{l}\text { Conc. } \\
-\max \\
(\underline{M})\end{array}$ & $\begin{array}{c}\text { Relative } \\
\text { Conc. } \\
\text { - min }\end{array}$ & $\begin{array}{l}\text { Relative } \\
\text { Conc. } \\
\text { - max }\end{array}$ \\
\hline--- & $---\overline{-}$ & $\overline{-}-\overline{1}$ & $-\overline{0}-\overline{0}$ & $-\overline{1}-\overline{203}$ & $-7--\overline{-}$ \\
\hline $\begin{array}{l}57.00 \\
58.00\end{array}$ & $\begin{array}{l}95-91 \\
93-90\end{array}$ & $\begin{array}{l}0.0415 \\
0.0445\end{array}$ & $\begin{array}{l}0.0495 \\
0.0505\end{array}$ & $\begin{array}{l}0.383 \\
0.412\end{array}$ & $\begin{array}{l}0.460 \\
0.469\end{array}$ \\
\hline 59.00 & $95-91$ & 0.0415 & 0.0495 & 0.383 & 0.460 \\
\hline 60.00 & $102-97$ & 0.0315 & 0.0385 & 0.288 & 0.355 \\
\hline 62.00 & $102-98$ & 0.0315 & 0.0365 & 0.288 & 0.336 \\
\hline 64.00 & $97-94$ & 0.0385 & 0.0425 & 0.355 & 0.393 \\
\hline 66.00 & $113-111$ & 0.0200 & 0.0220 & 0.179 & 0.198 \\
\hline 68.00 & $99-96$ & 0.0350 & 0.0405 & 0.321 & 0.374 \\
\hline 70.08 & $101-100$ & 0.0325 & 0.0340 & 0.298 & 0.312 \\
\hline 72.00 & $105-104$ & 0.0280 & 0.0285 & 0.255 & 0.260 \\
\hline 74.00 & $118-113$ & 0.0165 & 0.0200 & 0.145 & 0.179 \\
\hline 76.00 & $105-104$ & 0.0280 & 0.0285 & 0.255 & 0.260 \\
\hline 78.00 & $115-113$ & 0.0187 & 0.0200 & 0.166 & 0.179 \\
\hline 80.67 & $117-115$ & 0.0175 & 0.0187 & 0.155 & 0.166 \\
\hline 92.75 & $118-115$ & 0.0165 & 0.0187 & 0.145 & 0.166 \\
\hline 96.00 & $116-112$ & 0.0183 & 0.0210 & 0.162 & 0.188 \\
\hline 100.00 & $115-113$ & 0.0187 & 0.0200 & 0.166 & 0.179 \\
\hline 104.00 & $110-105$ & 0.0227 & 0.0280 & 0.204 & 0.255 \\
\hline 120.00 & $116-113$ & 0.0183 & 0.0200 & 0.162 & 0.179 \\
\hline 141.75 & $119-118$ & 0.0157 & 0.0165 & 0.138 & 0.145 \\
\hline 147.58 & $117-115$ & 0.0175 & 0.0187 & 0.155 & 0.166 \\
\hline
\end{tabular}


Table E.3: Fracture solute transport test data for test no. 2 .

Sampling Port: 5F-LS

Location: $x=5 \mathrm{~cm} ; \mathrm{z}=35 \mathrm{~cm} \quad$ Face: 5

Date: $7-12-88$ to $7-18-88$

Flow Rate: $1.36 \times 10^{-9} \mathrm{~m}^{3} / \mathrm{s}$

Head Imposed at Top of Plate: $35.90 \mathrm{~cm}$

$$
\begin{aligned}
& E_{1}=173 \mathrm{mV} \\
& C_{1}=0.00125 \underline{M} \\
& E_{0}=73 \mathrm{mV} \\
& C_{0}=0.105 \underline{M} \\
& \text { Plate no.: } 5
\end{aligned}
$$

\begin{tabular}{|c|c|c|c|c|c|}
\hline $\begin{array}{l}\text { Elapsed } \\
\text { Time, } \\
t_{E}(h r)\end{array}$ & $\begin{array}{c}\text { Potential } \\
\text { Difference } \\
\text { E (mV) }\end{array}$ & $\begin{array}{l}\text { Conc. , } \\
- \text { min } \\
(\underline{M})\end{array}$ & $\begin{array}{l}\text { Conc. , } \\
-\max \\
(\underline{M})\end{array}$ & $\begin{array}{c}\text { Relative } \\
\text { Conc. } \\
\text { - min }\end{array}$ & $\begin{array}{c}\text { Relative } \\
\text { Conc. } \\
\text { - max }\end{array}$ \\
\hline $\begin{array}{c}- \\
0.33\end{array}$ & $-\overline{-}-\overline{-}-$ & $\overline{0.0031}$ & $\overline{0}-\overline{0}-\overline{38}$ & 0.018 & 0.024 \\
\hline 1.08 & $159-155$ & 0.0026 & 0.0031 & 0.013 & 0.018 \\
\hline 2.08 & $154-150$ & 0.0032 & 0.0040 & 0.019 & 0.026 \\
\hline 3.08 & $161-154$ & 0.0024 & 0.0032 & 0.011 & 0.019 \\
\hline 4.08 & $161-154$ & 0.0024 & 0.0032 & 0.011 & 0.019 \\
\hline 5.08 & $151-140$ & 0.0038 & 0.0063 & 0.024 & 0.048 \\
\hline 6.08 & $140-137$ & 0.0063 & 0.0073 & 0.048 & 0.058 \\
\hline 7.08 & $140-134$ & 0.0063 & 0.0084 & 0.048 & 0.068 \\
\hline 8.08 & $126-125$ & 0.0120 & 0.0125 & 0.102 & 0.107 \\
\hline 9.08 & $120-117$ & $0: 0152$ & 0.0175 & 0.133 & 0.155 \\
\hline 10.08 & $136-134$ & 0.0077 & 0.0084 & 0.061 & 0.068 \\
\hline 11.08 & $123-118$ & 0.0137 & 0.0165 & 0.119 & 0.145 \\
\hline 12.08 & $128-118$ & 0.0110 & 0.0165 & 0.093 & 0.145 \\
\hline 13.08 & $121-118$ & 0.0147 & 0.0165 & 0.128 & 0.145 \\
\hline 14.08 & $121-111$ & 0.0147 & 0.0220 & 0.128 & 0.198 \\
\hline 16.08 & $119-112$ & 0.0157 & 0.0210 & 0.138 & 0.188 \\
\hline 18.08 & $116-109$ & 0.0183 & 0.0235 & 0.162 & 0.212 \\
\hline 20.08 & $104-101$ & 0.0285 & 0.0325 & 0.260 & 0.298 \\
\hline 22.08 & $122-117$ & 0.0143 & 0.0175 & 0.124 & 0.155 \\
\hline 24.08 & $119-109$ & 0.0157 & 0.0235 & 0.138 & 0.212 \\
\hline 26.08 & 91 & 0.0495 & 0.0495 & 0.460 & 0.460 \\
\hline 28.08 & 97 & 0.0385 & 0.0385 & 0.355 & 0.355 \\
\hline 30.08 & $101-99$ & 0.0325 & 0.0350 & 0.298 & 0.321 \\
\hline 33.58 & $106-98$ & 0.0270 & 0.0365 & 0.245 & 0.336 \\
\hline 43.83 & $98-93$ & 0.0365 & 0.0445 & 0.336 & 0.412 \\
\hline 47.58 & $93-92$ & 0.0445 & 0.0470 & 0.412 & 0.436 \\
\hline 49.08 & $100-99$ & 0.0340 & 0.0355 & 0.312 & 0.326 \\
\hline 50.08 & $96-94$ & 0.0405 & 0.0465 & 0.374 & 0.431 \\
\hline 51.08 & $91-88$ & 0.0495 & 0.0550 & 0.460 & 0.512 \\
\hline 52.08 & $92-88$ & 0.0470 & 0.0550 & 0.436 & 0.512 \\
\hline 53.08 & $96-93$ & 0.0405 & 0.0445 & 0.374 & 0.412 \\
\hline 54.08 & $97-91$ & 0.0385 & 0.0495 & 0.355 & 0.460 \\
\hline 55.08 & $98-97$ & 0.0365 & 0.0385 & 0.336 & 0.355 \\
\hline
\end{tabular}

Test Duration: 150 hrs 
Table E.3: Fracture solute transport test data for test no. 2 . Sampling Port: 5F-LS (continued)

\begin{tabular}{|c|c|c|c|c|c|}
\hline $\begin{array}{l}\text { Elapsed } \\
\text { Time, } \\
t_{\mathrm{F}}(\mathrm{hr})\end{array}$ & $\begin{array}{l}\text { Potential } \\
\text { Difference } \\
\text { E (mV) }\end{array}$ & $\begin{array}{l}\text { Conc. , } \\
- \text { min } \\
(M)\end{array}$ & $\begin{array}{l}\text { Conc. , } \\
-\max \\
(\underline{M})\end{array}$ & $\begin{array}{c}\text { Relative } \\
\text { Conc. } \\
\text { - min }\end{array}$ & $\begin{array}{c}\text { Relative } \\
\text { Conc. } \\
\text { - } \max \end{array}$ \\
\hline$-\overline{5}-\overline{-}$ & $-\cdot--$ & $\overline{0}-\overline{0} \cdot \overline{0}$ & $\overline{0} \overline{0} \overline{15}$ & $-\overline{336}$ & $-\overline{0}-\overline{383}$ \\
\hline $\begin{array}{l}56.08 \\
57.08\end{array}$ & $\begin{array}{l}98-95 \\
93-92\end{array}$ & $\begin{array}{l}0.0365 \\
0.0445\end{array}$ & $\begin{array}{l}0.0415 \\
0.0470\end{array}$ & $\begin{array}{l}0.336 \\
0.412\end{array}$ & $\begin{array}{l}0.383 \\
0.436\end{array}$ \\
\hline 58.08 & $99-92$ & 0.0350 & 0.0470 & 0.321 & 0.436 \\
\hline 59.08 & $96-91$ & 0.0405 & 0.0495 & 0.374 & 0.460 \\
\hline 60.08 & $101-97$ & 0.0325 & 0.0385 & 0.298 & 0.355 \\
\hline 62.08 & $106-99$ & 0.0270 & 0.0 & 0.245 & 0.321 \\
\hline 64.08 & $107-102$ & 0.0260 & 0.0 & 0.236 & 0.288 \\
\hline 66.08 & $105-102$ & 0.0280 & 0.03 & 0.255 & 0.288 \\
\hline 68.08 & $108-103$ & 0.0245 & 0.03 & 0.221 & 0.274 \\
\hline 70.17 & $104-101$ & 0.0285 & 0.0325 & 0.260 & 0.298 \\
\hline 72.08 & $104-99$ & 0.0285 & 0.0 & 0.260 & 0.321 \\
\hline 74.08 & $102-100$ & 0.0315 & 0.0340 & 0.288 & 0.312 \\
\hline 76.08 & $99-96$ & 0.0350 & 0.0405 & 0.321 & 0.374 \\
\hline 78.08 & 101 & 0.0325 & 0.0325 & 0.298 & 0.298 \\
\hline 80.75 & $110-106$ & 0.0227 & 0.0270 & 0.204 & 0.245 \\
\hline 92.83 & $109-106$ & 0.0235 & 0.0270 & 0.212 & 0.245 \\
\hline 96.08 & $111-109$ & 0.0220 & 0.0235 & 0.198 & 0.212 \\
\hline 100.08 & 111 & 0.0220 & 0.0220 & 0.198 & 0.198 \\
\hline 104.08 & $114-113$ & 0.0193 & 0.0200 & 0.172 & 0.179 \\
\hline 120.08 & $116-110$ & 0.0183 & 0.0227 & 0.162 & 0.204 \\
\hline 141.83 & $121-118$ & 0.0147 & 0.0165 & 0.128 & 0.145 \\
\hline 147.67 & $122-120$ & 0.0143 & 0.0152 & 0.124 & 0.133 \\
\hline & & & & & \\
\hline
\end{tabular}


Table E.3: Fracture solute transport test data for test no. 2 .

Sampling Port: 5F-LC

Location: $\mathrm{x}=10 \mathrm{~cm} ; \mathrm{z}=35 \mathrm{~cm} \quad$ Face: 5

Date: $7-12-88$ to $7-18-88$

$E_{i}=173 \mathrm{mV}$

Flow Rate: $1.36 \times 10^{-9} \mathrm{~m}^{3} / \mathrm{s}$

$\mathrm{C}_{\mathrm{i}}=0.00125 \mathrm{M}$

Head Imposed at Top of Plate: $35.90 \mathrm{~cm} \quad \mathrm{E}_{\mathrm{o}}=73 \mathrm{mV}$

Test Performed: Slug - 48 hrs

Test Duration: 150 hrs $\mathrm{C}_{\mathrm{o}}=0.105 \underline{\mathrm{M}}$ Plate no.: 5

\begin{tabular}{|c|c|c|c|c|c|}
\hline $\begin{array}{l}\text { Elapsed } \\
\text { Time } \\
t_{E}(h r)\end{array}$ & $\begin{array}{l}\text { Potential } \\
\text { Difference } \\
\text { E (mV) }\end{array}$ & $\begin{array}{l}\text { Conc. } \\
-\min \\
(\underline{M})\end{array}$ & $\begin{array}{l}\text { Conc. , } \\
-\max \\
(\underline{M})\end{array}$ & $\begin{array}{c}\text { Relative } \\
\text { Conc. } \\
\text { - min }\end{array}$ & $\begin{array}{c}\text { Relative } \\
\text { Conc. } \\
\text { - } \max \end{array}$ \\
\hline & & & - & 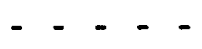 & -- \\
\hline 0.33 & $155-151$ & 0.0031 & 0.0038 & 0.018 & 0.024 \\
\hline 1.08 & $159-155$ & 0.0026 & 0.0031 & 0.013 & 0.018 \\
\hline 2.08 & $154-150$ & 0.0032 & 0.0040 & 0.019 & 0.026 \\
\hline 3.08 & $161-154$ & 0.0024 & 0.0032 & 0.011 & 0.019 \\
\hline 4.08 & $161-154$ & 0.0024 & 0.0032 & 0.011 & 0.019 \\
\hline 5.08 & $151-140$ & 0.0038 & 0.0063 & 0.024 & 0.048 \\
\hline 6.08 & $140-137$ & 0.0063 & 0.0073 & 0.048 & 0.058 \\
\hline 7.08 & $140-134$ & 0.0063 & 0.0084 & 0.048 & 0.068 \\
\hline 8.08 & $126-125$ & 0.0120 & 0.0125 & 0.102 & 0.107 \\
\hline 9.08 & $120-117$ & 0.0152 & 0.0175 & 0.133 & 0.155 \\
\hline 10.08 & $136-134$ & 0.0077 & 0.0084 & 0.061 & 0.068 \\
\hline 11.08 & $123-118$ & 0.0137 & 0.0165 & 0.119 & 0.145 \\
\hline 12.08 & $128-118$ & 0.0110 & 0.0165 & 0.093 & 0.145 \\
\hline 13.08 & $121-118$ & 0.0147 & 0.0165 & 0.128 & 0.145 \\
\hline 14.08 & $121-111$ & 0.0147 & 0.0220 & 0.128 & 0.198 \\
\hline 16.08 & $119-112$ & 0.0157 & 0.0210 & 0.138 & 0.188 \\
\hline 18.08 & $116-109$ & 0.0183 & 0.0235 & 0.162 & 0.212 \\
\hline 20.08 & $104-101$ & 0.0285 & 0.0325 & 0.260 & 0.298 \\
\hline 22.08 & $122-117$ & 0.0143 & 0.0175 & 0.124 & 0.155 \\
\hline 24.08 & $119-109$ & 0.0157 & 0.0235 & 0.138 & 0.212 \\
\hline 26.08 & 91 & 0.0495 & 0.0495 & 0.460 & 0.460 \\
\hline 28.08 & 97 & 0.0385 & 0.0385 & 0.355 & 0.355 \\
\hline 30.08 & $101-99$ & 0.0325 & 0.0350 & 0.298 & 0.321 \\
\hline 33.58 & $106-98$ & 0.0270 & 0.0365 & 0.245 & 0.336 \\
\hline 43.83 & $98-93$ & 0.0365 & 0.0445 & 0.336 & 0.412 \\
\hline 47.58 & $93-92$ & 0.0445 & 0.0470 & 0.412 & 0.436 \\
\hline 49.08 & $100-99$ & 0.0340 & 0.0355 & 0.312 & 0.326 \\
\hline 50.08 & $96-94$ & 0.0405 & 0.0465 & 0.374 & 0.431 \\
\hline 51.08 & $91-88$ & 0.0495 & 0.0550 & 0.460 & 0.512 \\
\hline 52.08 & $92-88$ & 0.0470 & 0.0550 & 0.436 & 0.512 \\
\hline 53.08 & $96-93$ & 0.0405 & 0.0445 & 0.374 & 0.412 \\
\hline 54.08 & $97-91$ & 0.0385 & 0.0495 & 0.355 & 0.460 \\
\hline 55.08 & $98-97$ & 0.0365 & 0.0385 & 0.336 & 0.355 \\
\hline
\end{tabular}


Table E.3: Fracture solute transport test data for test no. 2 . Sampling Port: 5F-LC (continued)

\begin{tabular}{|c|c|c|c|c|c|}
\hline $\begin{array}{l}\text { Elapsed } \\
\text { Time, } \\
t_{E}(h r)\end{array}$ & $\begin{array}{c}\text { Potential } \\
\text { Difference } \\
\text { E (mV) }\end{array}$ & $\begin{array}{l}\text { Conc., } \\
- \text { min } \\
(\underline{M})\end{array}$ & $\begin{array}{l}\text { Conc., } \\
-\max \\
(\underline{M})\end{array}$ & $\begin{array}{l}\text { Relative } \\
\text { Conc. } \\
\text { - min }\end{array}$ & $\begin{array}{c}\text { Relative } \\
\text { Conc. } \\
\text { - max }\end{array}$ \\
\hline $5 \overline{0}-\overline{-}$ & $-\overline{-}-$ & $\begin{array}{ll}- & - \\
0 & 0365\end{array}$ & $\begin{array}{l}-\overline{0} \\
0\end{array}$ & $-\overline{0}-\overline{336}$ & 0.383 \\
\hline 57.08 & $93-92$ & 0.0445 & 0.0470 & 0.412 & 0.436 \\
\hline 58.08 & $99-92$ & 0.0350 & 0.0470 & 0.321 & 0.436 \\
\hline 59.08 & $96-91$ & 0.0405 & 0.0495 & 0.374 & 0.460 \\
\hline 60.08 & $101-97$ & 0.0325 & 0.0385 & 0.298 & 0.355 \\
\hline 62.08 & $106-99$ & 0.0270 & 0.0350 & 0.245 & 0.321 \\
\hline 64.08 & $107-102$ & 0.0260 & 0.0315 & 0.236 & 0.288 \\
\hline 66.08 & $105-102$ & 0.0280 & 0.0315 & 0.255 & 0.288 \\
\hline 68.08 & $108-103$ & 0.0245 & 0.0300 & 0.221 & 0.274 \\
\hline 70.17 & $104-101$ & 0.0285 & 0.0325 & 0.260 & 0.298 \\
\hline 72.08 & $104-99$ & 0.0285 & 0.0350 & 0.260 & 0.321 \\
\hline 74.08 & $102-100$ & 0.0315 & 0.0340 & 0.288 & 0.312 \\
\hline 76.08 & $99-96$ & 0.0350 & 0.0405 & 0.321 & 0.374 \\
\hline 78.08 & 101 & 0.0325 & 0.0325 & 0.298 & 0.298 \\
\hline 80.75 & $110-106$ & 0.0227 & 0.0270 & 0.204 & 0.245 \\
\hline 92.83 & $109-106$ & 0.0235 & 0.0270 & 0.212 & 0.245 \\
\hline 96.08 & $111-109$ & 0.0220 & 0.0235 & 0.198 & 0.212 \\
\hline 100.08 & 111 & 0.0220 & 0.0220 & 0.198 & 0.198 \\
\hline 104.08 & $114-113$ & 0.0193 & 0.0200 & 0.172 & 0.179 \\
\hline 120.08 & $116-110$ & 0.0183 & 0.0227 & 0.162 & 0.204 \\
\hline 141.83 & $121-118$ & 0.0147 & 0.0165 & 0.128 & 0.145 \\
\hline 147.67 & $122-120$ & 0.0143 & 0.0152 & 0.124 & 0.133 \\
\hline
\end{tabular}


Table E.3: Fracture solute transport test data for test no. 2 .

Sampling Location: 6-B

Location: $x=2 \mathrm{~cm} ; z=50 \mathrm{~cm} \quad$ Face: 2

Date: 7-12-88 to 7-18-88

Flow Rate: $1.36 \times 10^{-9} \mathrm{~m}^{3} / \mathrm{s}$

$E_{1}=173 \mathrm{mV}$

Test Performed: Slug $-48 \mathrm{hrs} \quad \mathrm{C}_{0}=0.105 \underline{\mathrm{M}}$

Test Duration: 150 hrs

Plate no.: 5

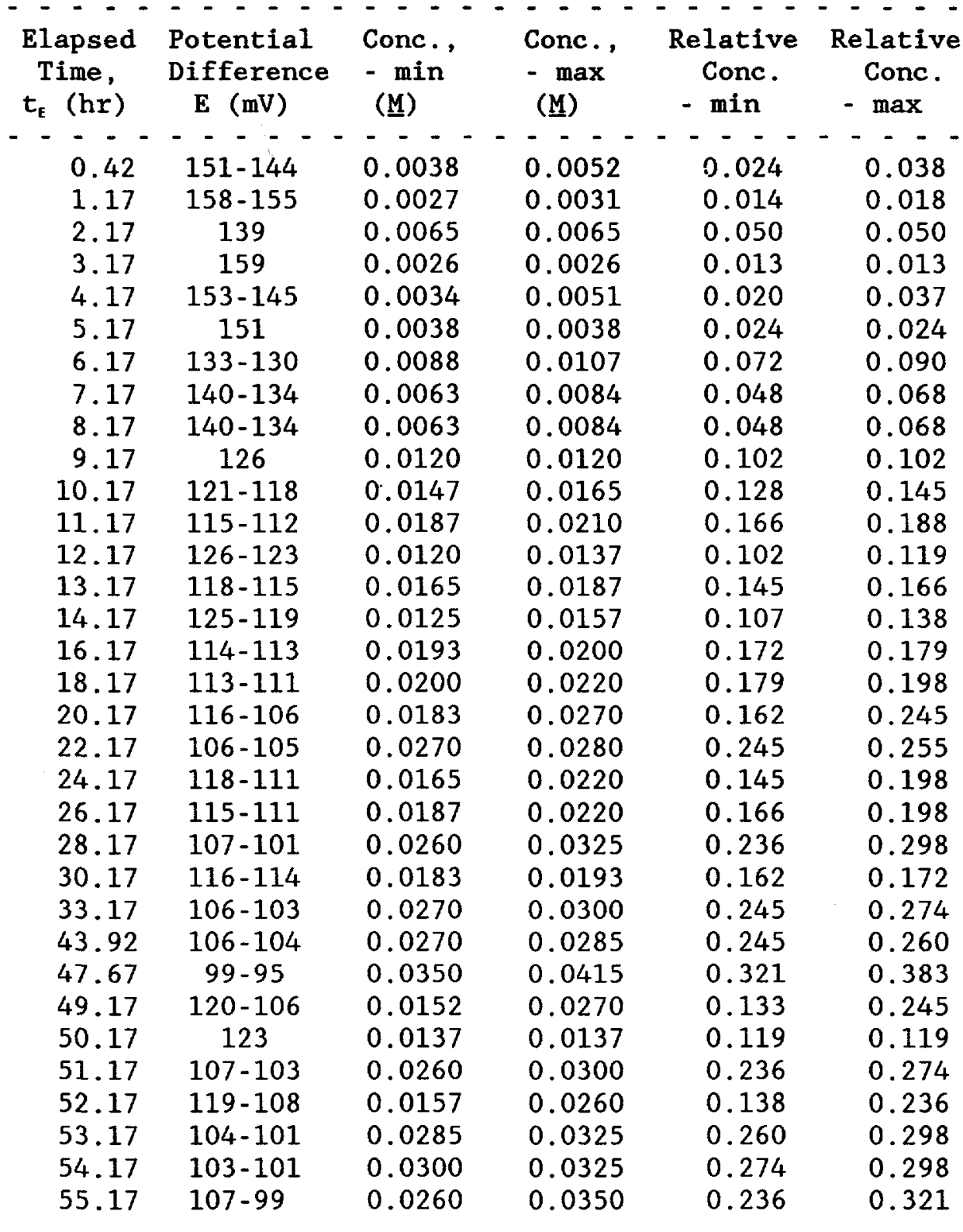


Table E.3: Fracture solute transport test data for test no. 2 . Sampling Location: 6-B (continued)

\begin{tabular}{|c|c|c|c|c|c|}
\hline $\begin{array}{l}\text { Elapsed } \\
\text { Time, } \\
t_{E}(h r)\end{array}$ & $\begin{array}{l}\text { Potential } \\
\text { Difference } \\
\text { E (mV) }\end{array}$ & $\begin{array}{l}\text { Conc. , } \\
- \text { min } \\
\text { (M) }\end{array}$ & $\begin{array}{l}\text { Conc. } \\
-\max \\
(\underline{M})\end{array}$ & $\begin{array}{c}\text { Relative } \\
\text { Conc. } \\
\text { - min }\end{array}$ & $\begin{array}{c}\text { Relative } \\
\text { Conc. } \\
\text { - max }\end{array}$ \\
\hline & ---- & $-\ldots$ & - & $-\ldots$ & - \\
\hline 56.17 & $99-98$ & 0.0350 & 0.0365 & 0.321 & 0.336 \\
\hline 57.17 & $102-101$ & 0.0315 & 0.0325 & 0.288 & 0.298 \\
\hline 58.17 & $120-119$ & 0.0152 & 0.0157 & 0.133 & 0.138 \\
\hline 59.17 & $112-108$ & 0.0210 & 0.0260 & 0.188 & 0.236 \\
\hline 60.17 & $105-102$ & 0.0280 & 0.0315 & 0.255 & 0.288 \\
\hline 62.17 & $112-105$ & 0.0210 & 0.0280 & 0.188 & 0.255 \\
\hline 64.17 & $108-107$ & 0.0260 & 0.0260 & 0.236 & 0.236 \\
\hline 66.17 & $108-106$ & 0.0260 & 0.0270 & 0.236 & 0.245 \\
\hline 68.17 & $109-107$ & 0.0235 & 0.0260 & 0.212 & 0.236 \\
\hline 70.25 & $102-101$ & 0.0315 & 0.0325 & 0.288 & 0.298 \\
\hline 72.17 & $110-106$ & 0.0227 & 0.0270 & 0.204 & 0.245 \\
\hline 74.17 & $112-110$ & 0.0210 & 0.0227 & 0.188 & 0.204 \\
\hline 76.17 & $118-114$ & 0.0165 & 0.0193 & 0.145 & 0.172 \\
\hline 78.17 & $117-113$ & 0.0175 & 0.0200 & 0.155 & 0.179 \\
\hline 80.83 & $122-119$ & 0.0143 & 0.0157 & 0.124 & 0.138 \\
\hline 92.92 & $125-121$ & 0.0125 & 0.0147 & 0.107 & 0.128 \\
\hline 96.1 & $122-120$ & 0.0143 & 0.0152 & 0.124 & 0.133 \\
\hline 100.17 & $125-122$ & 0.0125 & 0.0143 & 0.107 & 0.124 \\
\hline 104.17 & $125-123$ & 0.0125 & 0.0137 & 0.107 & 0.119 \\
\hline 120.17 & $130-129$ & 0.0107 & 0.0105 & 0.090 & 0.088 \\
\hline 141.92 & $134-131$ & 0.0084 & 0.0097 & 0.068 & 0.080 \\
\hline 147.75 & $132-126$ & 0.0092 & 0.0120 & 0.076 & 0.102 \\
\hline- & - & 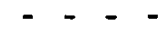 & - & & -- \\
\hline
\end{tabular}


Table E.3: Fracture solute transport test data for test no. 2 .

Sampling Location: 6-M

Location: $x=8 \mathrm{~cm} ; z=50 \mathrm{~cm} \quad$ Face: 2

Date: $7-12-88$ to $7-18-88$

Flow Rate: $1.36 \times 10^{-9} \mathrm{~m}^{3} / \mathrm{s}$

$\mathrm{E}_{1}=173 \mathrm{mV}$

Head Imposed at Top of Plate: $35.90 \mathrm{~cm}$

Test Performed: Slug - 48 hrs

Test Duration: 150 hrs

$C_{\mathrm{i}}=0.00125 \underline{\mathrm{M}}$

$\mathrm{E}_{\mathrm{o}}=73 \mathrm{mV}$

$\mathrm{C}_{0}=0.105 \underline{\mathrm{M}}$

Plate no.: 5

\begin{tabular}{|c|c|c|c|c|c|}
\hline $\begin{array}{l}\text { Elapsed } \\
\text { Time, } \\
t_{E}(h r)\end{array}$ & $\begin{array}{l}\text { Potential } \\
\text { Difference } \\
\text { E (mV) }\end{array}$ & $\begin{array}{l}\text { Conc. , } \\
-\min \\
(\underline{M})\end{array}$ & $\begin{array}{l}\text { Conc. } \\
-\max \\
(\underline{M})\end{array}$ & $\begin{array}{l}\text { Relative } \\
\text { Cone. } \\
\text { - min }\end{array}$ & $\begin{array}{c}\text { Relative } \\
\text { Conc. } \\
\text { - } \max \end{array}$ \\
\hline - & . & - & $-\quad-$ & - & -- \\
\hline 0.42 & $158-155$ & 0.0027 & 0.0031 & 0.014 & 0.018 \\
\hline 1.17 & $156-155$ & 0.0030 & 0.0031 & 0.017 & 0.018 \\
\hline 2.17 & $156-150$ & 0.0030 & 0.0040 & 0.017 & 0.026 \\
\hline 3.17 & $155-151$ & 0.0031 & 0.0038 & 0.018 & 0.024 \\
\hline 4.17 & $158-155$ & 0.0027 & 0.0031 & 0.014 & 0.018 \\
\hline 5.17 & $148-146$ & 0.0044 & 0.0049 & 0.030 & 0.035 \\
\hline 6.17 & 134 & 0.0084 & 0.0084 & 0.068 & 0.068 \\
\hline 7.17 & $130-120$ & 0.0100 & 0.0152 & 0.083 & 0.133 \\
\hline 8.17 & $126-123$ & 0.0120 & 0.0137 & 0.102 & 0.119 \\
\hline 9.17 & $123-122$ & 0.0137 & 0.0143 & 0.119 & 0.124 \\
\hline 10.17 & $120-117$ & 0.0152 & 0.0175 & 0.133 & 0.155 \\
\hline 11.17 & $117-115$ & 0.0175 & 0.0187 & 0.155 & 0.166 \\
\hline 12.17 & $114-111$ & 0.0193 & 0.0220 & 0.172 & 0.198 \\
\hline 13.17 & $114-111$ & 0.0193 & 0.0220 & 0.172 & 0.198 \\
\hline 14.17 & 111 & 0.0220 & 0.0220 & 0.198 & 0.198 \\
\hline 16.17 & 110 & 0.0227 & 0.0227 & 0.204 & 0.204 \\
\hline 18.17 & $111-109$ & 0.0220 & 0.0235 & 0.198 & 0.212 \\
\hline 20.17 & $105-103$ & 0.0280 & 0.0300 & 0.255 & 0.274 \\
\hline 22.17 & 102 & 0.0315 & 0.0315 & 0.288 & 0.288 \\
\hline 24.17 & $100-97$ & 0.0340 & 0.0385 & 0.312 & 0.355 \\
\hline 26.17 & 111 & 0.0220 & 0.0220 & 0.198 & 0.198 \\
\hline 28.17 & $107-103$ & 0.0260 & 0.0300 & 0.236 & 0.274 \\
\hline 30.17 & $104-102$ & 0.0285 & 0.0315 & 0.260 & 0.288 \\
\hline 33.17 & $101-99$ & 0.0325 & 0.0350 & 0.298 & 0.321 \\
\hline 43.92 & $99-97$ & 0.0350 & 0.0385 & 0.321 & 0.355 \\
\hline 47.67 & $95-93$ & 0.0415 & 0.0445 & 0.383 & 0.412 \\
\hline 49.17 & $98-95$ & 0.0365 & 0.0415 & 0.336 & 0.383 \\
\hline 50.17 & $100-97$ & 0.0340 & 0.0385 & 0.312 & 0.355 \\
\hline 51.17 & $102-98$ & 0.0315 & 0.0365 & 0.288 & 0.336 \\
\hline 52.17 & 99 & 0.0350 & 0.0350 & 0.321 & 0.321 \\
\hline 53.17 & $99-98$ & 0.0350 & 0.0365 & 0.321 & 0.336 \\
\hline 54.17 & $99-98$ & 0.0350 & 0.0365 & 0.321 & 0.336 \\
\hline 55.17 & $101-99$ & 0.0325 & 0.0350 & 0.298 & 0.321 \\
\hline
\end{tabular}


Table E.3: Fracture solute transport test data for test no. 2.

Sampling Location: 6-M (continued)

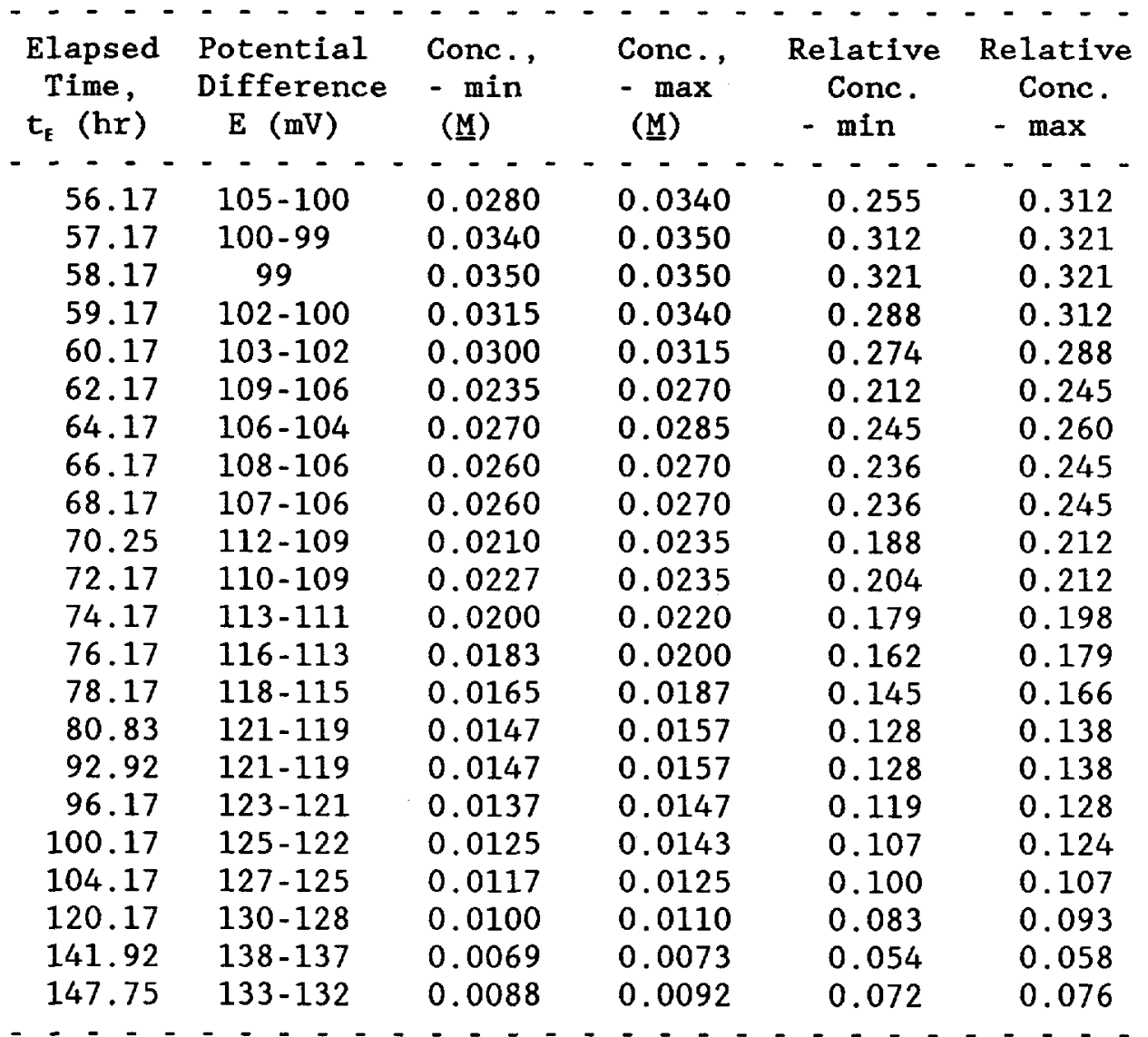


Table E.3: Fracture solute transport test data for test no. 2 .

Sampling Location: 3-M

Location: $\mathrm{x}=12 \mathrm{~cm} ; \mathrm{z}=50 \mathrm{~cm} \quad$ Face: 2

Date: $7-12-88$ to $7-18-88$

Flow Rate: $1.36 \times 10^{-9} \mathrm{~m}^{3} / \mathrm{s}$

$E_{\mathrm{i}}=173 \mathrm{mV}$

Head Imposed at Top of Plate: $35.90 \mathrm{~cm}$ $C_{1}=0.00125 \underline{M}$

Test Performed: Slug - 48 hrs

$E_{0}=73 \mathrm{mV}$

Test Duration: 150 hrs

$\mathrm{C}_{0}=0.105 \underline{\mathrm{M}}$

Plate no.: 5

\begin{tabular}{|c|c|c|c|c|c|}
\hline $\begin{array}{l}\text { Elapsed } \\
\text { Time, } \\
t_{E}(h r)\end{array}$ & $\begin{array}{l}\text { Potential } \\
\text { Difference } \\
\text { E (mV) }\end{array}$ & $\begin{array}{l}\text { Cone. , } \\
- \text { min } \\
(\underline{M})\end{array}$ & $\begin{array}{l}\text { Conc., } \\
-\max \\
(\underline{M})\end{array}$ & $\begin{array}{l}\text { Relative } \\
\text { Conc. } \\
\text { - min }\end{array}$ & $\begin{array}{l}\text { Relative } \\
\text { Conc. } \\
\text { - max }\end{array}$ \\
\hline--- & 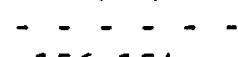 & $--\cdot-$ & $-\overline{-}-$ & - - - - - & $-\cdot--$ \\
\hline 0.42 & $156-154$ & 0.0030 & 0.0032 & 0.017 & 0.019 \\
\hline 1.17 & 160 & 0.0025 & 0.0025 & 0.012 & 0.012 \\
\hline 2.17 & 156 & 0.0030 & 0.0030 & 0.017 & 0.017 \\
\hline 3.17 & $154-151$ & 0.0032 & 0.0038 & 0.019 & 0.024 \\
\hline 4.17 & $157-155$ & 0.0028 & 0.0031 & 0.015 & 0.018 \\
\hline 5.17 & $147-146$ & 0.0046 & 0.0049 & 0.032 & 0.035 \\
\hline 6.17 & $140-137$ & 0.0063 & 0.0073 & 0.048 & 0.058 \\
\hline 7.17 & $134-131$ & 0.0084 & 0.0097 & 0.068 & 0.080 \\
\hline 8.17 & $127-123$ & 0.0117 & 0.0137 & 0.100 & 0.119 \\
\hline 9.17 & $121-120$ & 0.0147 & 0.0152 & 0.128 & 0.133 \\
\hline 10.17 & $119-118$ & 0.0157 & 0.0165 & 0.138 & 0.145 \\
\hline 11.17 & $116-114$ & 0.0183 & 0.0193 & 0.162 & 0.172 \\
\hline 12.17 & 116 & 0.0183 & 0.0183 & 0.162 & 0.162 \\
\hline 13.17 & 111 & 0.0220 & 0.0220 & 0.198 & 0.198 \\
\hline 14.17 & $113-112$ & 0.0200 & 0.0210 & 0.179 & 0.188 \\
\hline 16.17 & 109 & 0.0235 & 0.0235 & 0.212 & 0.212 \\
\hline 18.17 & $110-107$ & 0.0227 & 0.0260 & 0.204 & 0.236 \\
\hline 20.17 & $105-103$ & 0.0280 & 0.0300 & 0.255 & 0.274 \\
\hline 22.17 & 102 & 0.0315 & 0.0315 & 0.288 & 0.288 \\
\hline 24.17 & $102-101$ & 0.0315 & 0.0325 & 0.288 & 0.298 \\
\hline 26.17 & 105 & 0.0280 & 0.0280 & 0.255 & 0.255 \\
\hline 28.17 & $106-104$ & 0.0270 & 0.0285 & 0.245 & 0.260 \\
\hline 30.17 & $105-102$ & 0.0280 & 0.0315 & 0.255 & 0.288 \\
\hline 33.17 & 101 & 0.0325 & 0.0325 & 0.298 & 0.298 \\
\hline 43.92 & $99-97$ & 0.0350 & 0.0385 & 0.321 & 0.355 \\
\hline 47.67 & $98-95$ & 0.0365 & 0.0415 & 0.336 & 0.383 \\
\hline 49.17 & $100-98$ & 0.0340 & 0.0365 & 0.312 & 0.336 \\
\hline 50.17 & $102-98$ & 0.0315 & 0.0365 & 0.288 & 0.336 \\
\hline 51.17 & $103-98$ & 0.0300 & 0.0365 & 0.274 & 0.336 \\
\hline 52.17 & $99-97$ & 0.0350 & 0.0385 & 0.321 & 0.355 \\
\hline 53.17 & $101-97$ & 0.0325 & 0.0385 & 0.298 & 0.355 \\
\hline 54.17 & $98-95$ & 0.0365 & 0.0415 & 0.336 & 0.383 \\
\hline 55.17 & $102-98$ & 0.0315 & 0.0365 & 0.288 & 0.336 \\
\hline
\end{tabular}


Table E.3: Fracture solute transport test data for test No. 2 . Sampling Location 3-M (continued)

\begin{tabular}{|c|c|c|c|c|c|}
\hline $\begin{array}{l}\text { Elapsed } \\
\text { Time, } \\
t_{E}(h r)\end{array}$ & $\begin{array}{l}\text { Potential } \\
\text { Difference } \\
\text { E (mV) }\end{array}$ & $\begin{array}{l}\text { Conc. } \\
- \text { min } \\
(\underline{M})\end{array}$ & $\begin{array}{l}\text { Conc. , } \\
-\max \\
(\underline{\mathrm{M}})\end{array}$ & $\begin{array}{l}\text { Relative } \\
\text { Conc. } \\
\text { - min }\end{array}$ & $\begin{array}{l}\text { Relative } \\
\text { Conc. } \\
\text { - } \max \end{array}$ \\
\hline & & - & - & 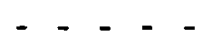 & . \\
\hline 56.17 & $97-95$ & 0.0385 & 0.0415 & 0.355 & 0.383 \\
\hline 57.17 & $98-96$ & 0.0365 & 0.0405 & 0.336 & 0.374 \\
\hline 58.17 & $100-99$ & 0.0340 & 0.0350 & 0.312 & 0.321 \\
\hline 59.17 & $102-99$ & 0.0315 & 0.0350 & 0.288 & 0.321 \\
\hline 60.17 & $102-99$ & 0.0315 & 0.0350 & 0.288 & 0.321 \\
\hline 62.17 & $106-104$ & 0.0270 & 0.0285 & 0.245 & 0.260 \\
\hline 64.17 & 105 & 0.0280 & 0.0280 & 0.255 & 0.255 \\
\hline 66.17 & $107-106$ & 0.0260 & 0.0270 & 0.236 & 0.245 \\
\hline 68.17 & $107-106$ & 0.0260 & 0.0270 & 0.236 & 0.245 \\
\hline 70.25 & $109-106$ & 0.0235 & 0.0270 & 0.212 & 0.245 \\
\hline 72.17 & $111-109$ & 0.0220 & 0.0235 & 0.198 & 0.212 \\
\hline 74.17 & $112-110$ & 0.0210 & 0.0227 & 0.188 & 0.204 \\
\hline 76.17 & $112-111$ & 0.0210 & 0.0220 & 0.188 & 0.198 \\
\hline 78.17 & $112-111$ & 0.0210 & 0.0220 & 0.188 & 0.198 \\
\hline 80.83 & $116-115$ & 0.0183 & 0.0187 & 0.162 & 0.166 \\
\hline 92.92 & $118-116$ & 0.0165 & 0.0183 & 0.145 & 0.162 \\
\hline 96.17 & $109-108$ & 0.0235 & 0.0260 & 0.212 & 0.236 \\
\hline 100.17 & 118 & 0.0165 & 0.0165 & 0.145 & 0.145 \\
\hline 104.17 & 121 & 0.0147 & 0.0147 & 0.128 & 0.128 \\
\hline 120.17 & $125-123$ & 0.0125 & 0.0137 & 0.107 & 0.119 \\
\hline 141.92 & 131 & 0.0097 & 0.0097 & 0.080 & 0.080 \\
\hline 147.75 & $135-134$ & 0.0080 & 0.0084 & 0.064 & 0.068 \\
\hline & & & & & \\
\hline
\end{tabular}


Table E.3: Fracture solute transport test data for test no. 2 .

Sampling Location: $3-\mathrm{F}$

Location: $x=18 \mathrm{~cm} ; \mathrm{z}=50 \mathrm{~cm} \quad$ Face: 2

Date: $7-12-88$ to $7-18-88$

Flow Rate: $1.36 \times 10^{-9} \mathrm{~m}^{3} / \mathrm{s}$

$\mathrm{E}_{\mathrm{i}}=173 \mathrm{mV}$

Head Imposed at Top of Plate: $35.90 \mathrm{~cm} \quad E_{0}=73 \mathrm{mV}$

Test Performed: Slug - 48 hrs

Test Duration: 150 hrs $\mathrm{C}_{0}=0.105 \underline{\mathrm{M}}$ Plate no.: 5

\begin{tabular}{|c|c|c|c|c|c|}
\hline $\begin{array}{l}\text { Elapsed } \\
\text { Time, } \\
t_{E} \text { (hr) }\end{array}$ & $\begin{array}{c}\text { Potential } \\
\text { Difference } \\
\text { E (mV) }\end{array}$ & $\begin{array}{l}\text { Conc., } \\
-\min \\
(\underline{M})\end{array}$ & $\begin{array}{l}\text { Conc. , } \\
-\max \\
(\underline{M})\end{array}$ & $\begin{array}{l}\text { Relative } \\
\text { Conc. } \\
\text { - min }\end{array}$ & $\begin{array}{c}\text { Relative } \\
\text { Conc. } \\
\text { - } \max \end{array}$ \\
\hline$=-\overline{0}$ & $-\overline{154-151}$ & $\overline{0.0032}$ & $\overline{0.0038}$ & $-\overline{0.019}$ & $-\overline{0.024}$ \\
\hline 1.17 & 154 & 0.0032 & 0.0032 & 0.019 & 0.019 \\
\hline 2.17 & $151-147$ & 0.0038 & 0.0046 & 0.024 & 0.032 \\
\hline 3.17 & $154-147$ & 0.0032 & 0.0046 & 0.019 & 0.032 \\
\hline 4.17 & $156-150$ & 0.0030 & 0.0040 & 0.017 & 0.026 \\
\hline 5.17 & 144 & 0.0052 & 0.0052 & 0.038 & 0.038 \\
\hline 6.17 & $144-138$ & 0.0052 & 0.0069 & 0.038 & 0.054 \\
\hline 7.17 & $131-128$ & 0.0097 & 0.0110 & 0.080 & 0.093 \\
\hline 8.17 & 125 & 0.0125 & 0.0125 & 0.107 & 0.107 \\
\hline 9.17 & $122-119$ & 0.0143 & 0.0157 & 0.124 & 0.138 \\
\hline 10.17 & $120-118$ & 0.0152 & 0.0165 & 0.133 & 0.145 \\
\hline 11.17 & $117-114$ & 0.0175 & 0.0193 & 0.155 & 0.172 \\
\hline 12.17 & $114-111$ & 0.0193 & 0.0220 & 0.172 & 0.198 \\
\hline 13.17 & $113-112$ & 0.0200 & 0.0210 & 0.179 & 0.188 \\
\hline 14.17 & $111-109$ & 0.0220 & 0.0235 & 0.198 & 0.212 \\
\hline 16.17 & $112-110$ & 0.0210 & 0.0227 & 0.188 & 0.204 \\
\hline 18.17 & $107-106$ & 0.0260 & 0.0270 & 0.236 & 0.245 \\
\hline 20.17 & $105-104$ & 0.0280 & 0.0285 & 0.255 & 0.260 \\
\hline 22.17 & $105-103$ & 0.0280 & 0.0300 & 0.255 & 0.274 \\
\hline 24.17 & 102 & 0.0315 & 0.0315 & 0.288 & 0.288 \\
\hline 26.17 & $107-104$ & 0.0260 & 0.0285 & 0.236 & 0.260 \\
\hline 28.17 & $106-102$ & 0.0270 & 0.0315 & 0.245 & 0.288 \\
\hline 30.17 & 102 & 0.0315 & 0.0315 & 0.288 & 0.288 \\
\hline 33.17 & $98-97$ & 0.0365 & 0.0385 & 0.336 & 0.355 \\
\hline 43.92 & $99-93$ & 0.0350 & 0.0445 & 0.321 & 0.412 \\
\hline 47.67 & $98-95$ & 0.0365 & 0.0415 & 0.336 & 0.383 \\
\hline 49.17 & $99-97$ & 0.0365 & 0.0385 & 0.336 & 0.355 \\
\hline 50.17 & 97-95 & 0.0385 & 0.0415 & 0.355 & 0.383 \\
\hline 51.17 & $98-95$ & 0.0365 & 0.0415 & 0.336 & 0.383 \\
\hline 52.17 & $99-97$ & 0.0365 & 0.0385 & 0.336 & 0.355 \\
\hline 53.17 & $99-97$ & 0.0365 & 0.0385 & 0.336 & 0.355 \\
\hline 54.17 & $95-94$ & 0.0415 & 0.0425 & 0.383 & 0.393 \\
\hline 55.17 & $101-97$ & 0.0325 & 0.0385 & 0.298 & 0.355 \\
\hline & & & & & \\
\hline
\end{tabular}


Table E.3: Fracture solute transport test data for test no. 2 .

Sampling Location: 3-F (continued)

\begin{tabular}{|c|c|c|c|c|c|}
\hline $\begin{array}{l}\text { Elapsed } \\
\text { Time, } \\
t_{\mathrm{E}}(\mathrm{hr})\end{array}$ & $\begin{array}{l}\text { Potential } \\
\text { Difference } \\
\text { E (mV) }\end{array}$ & $\begin{array}{l}\text { Conc. } \\
- \text { min } \\
(\underline{M})\end{array}$ & $\begin{array}{l}\text { Conc., } \\
-\max \\
(\underline{M})\end{array}$ & $\begin{array}{l}\text { Relative } \\
\text { Conc. } \\
\text { - min }\end{array}$ & $\begin{array}{l}\text { Relative } \\
\text { Conc. } \\
\text { - max }\end{array}$ \\
\hline$-\overline{-}-\overline{-}$ & $---\overline{-}-$ & $-\overline{-}-\overline{-}$ & & $--\overline{0}$ & $--\overline{-}-\overline{-}$ \\
\hline $\begin{array}{l}56.17 \\
57.17\end{array}$ & $\begin{array}{l}98-95 \\
97-96\end{array}$ & $\begin{array}{l}0.0365 \\
0.0385\end{array}$ & $\begin{array}{l}0.0415 \\
0.0405\end{array}$ & $\begin{array}{l}0.336 \\
0.355\end{array}$ & $\begin{array}{l}0.383 \\
0.374\end{array}$ \\
\hline 58.17 & $101-99$ & 0.0325 & 0.0365 & 0.298 & 0.336 \\
\hline 59.17 & $100-97$ & 0.0340 & 0.0385 & 0.312 & 0.355 \\
\hline 60.17 & $101-99$ & 0.0325 & 0.0365 & 0.298 & 0.336 \\
\hline 62.17 & $103-102$ & 0.0300 & 0.0315 & 0.274 & 0.288 \\
\hline 64.17 & $106-104$ & 0.0270 & 0.0285 & 0.245 & 0.260 \\
\hline 66.17 & $107-106$ & 0.0260 & 0.0270 & 0.236 & 0.245 \\
\hline 68.17 & $109-102$ & 0.0235 & 0.0315 & 0.212 & 0.288 \\
\hline 70.25 & $106-105$ & 0.0270 & 0.0280 & 0.245 & 0.255 \\
\hline 72.17 & $108-106$ & 0.0260 & 0.0270 & 0.236 & 0.245 \\
\hline 74.17 & $109-106$ & 0.0235 & 0.0270 & 0.212 & 0.245 \\
\hline 76.17 & $111-104$ & 0.0220 & 0.0285 & 0.198 & 0.260 \\
\hline 78.17 & $110-109$ & 0.0227 & 0.0235 & 0.204 & 0.212 \\
\hline 80.83 & $109-105$ & 0.0235 & 0.0280 & 0.212 & 0.255 \\
\hline 92.92 & $117-115$ & 0.0175 & 0.0187 & 0.155 & 0.166 \\
\hline 96.17 & $118-116$ & 0.0165 & 0.0183 & 0.145 & 0.162 \\
\hline 100.17 & $120-118$ & 0.0152 & 0.0165 & 0.133 & 0.145 \\
\hline 104.17 & $119-115$ & 0.0157 & 0.0187 & 0.138 & 0.166 \\
\hline 120.17 & $117-115$ & 0.0175 & 0.0187 & 0.155 & 0.166 \\
\hline 141.92 & $124-123$ & 0.0130 & 0.0137 & 0.112 & 0.119 \\
\hline 147.75 & 125 & 0.0125 & 0.0125 & 0.107 & 0.107 \\
\hline
\end{tabular}


Table E.4: Fracture solute transport test data for test no. 3 .

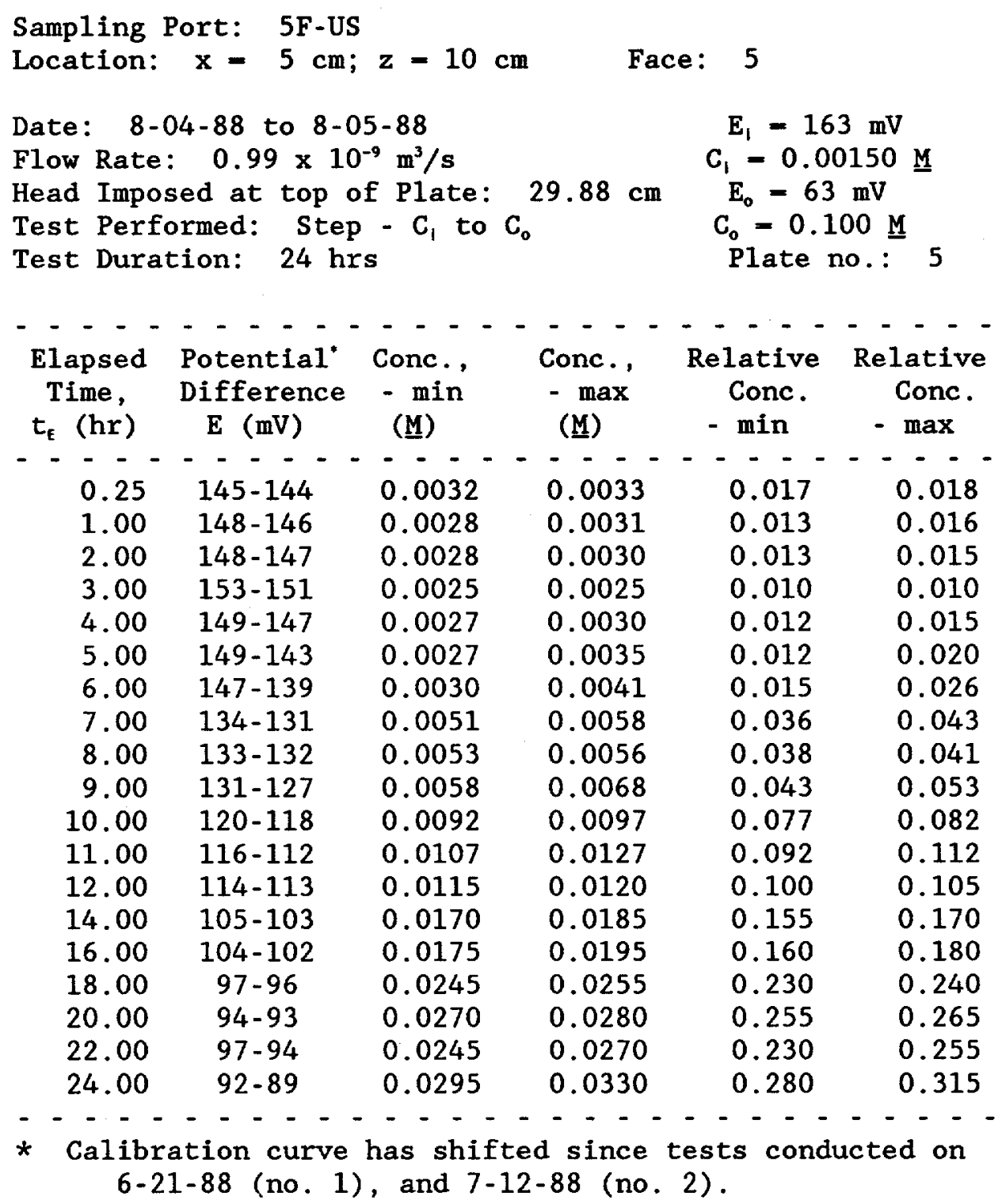


Table E.4: Fracture solute transport test data for test no. 3 .

Sampling Port: 5F-UC

Location: $\mathrm{x}=10 \mathrm{~cm} ; \mathrm{z}=10 \mathrm{~cm} \quad$ Face: 5

Date: $8-04-88$ to $8-05-88$

$E_{1}=163 \mathrm{mV}$

Flow Rate: $0.99 \times 10^{-9} \mathrm{~m}^{3} / \mathrm{s}$

$C_{1}=0.00150 \underline{M}$

Head Imposed at Top of Plate: $29.88 \mathrm{~cm} \quad E_{0}=63 \mathrm{mV}$

Test Performed: Step - $C_{1}$ to $C_{0} \quad C_{0}=0.100 \underline{M}$

Test Duration: 24 hrs

Plate no.: 5

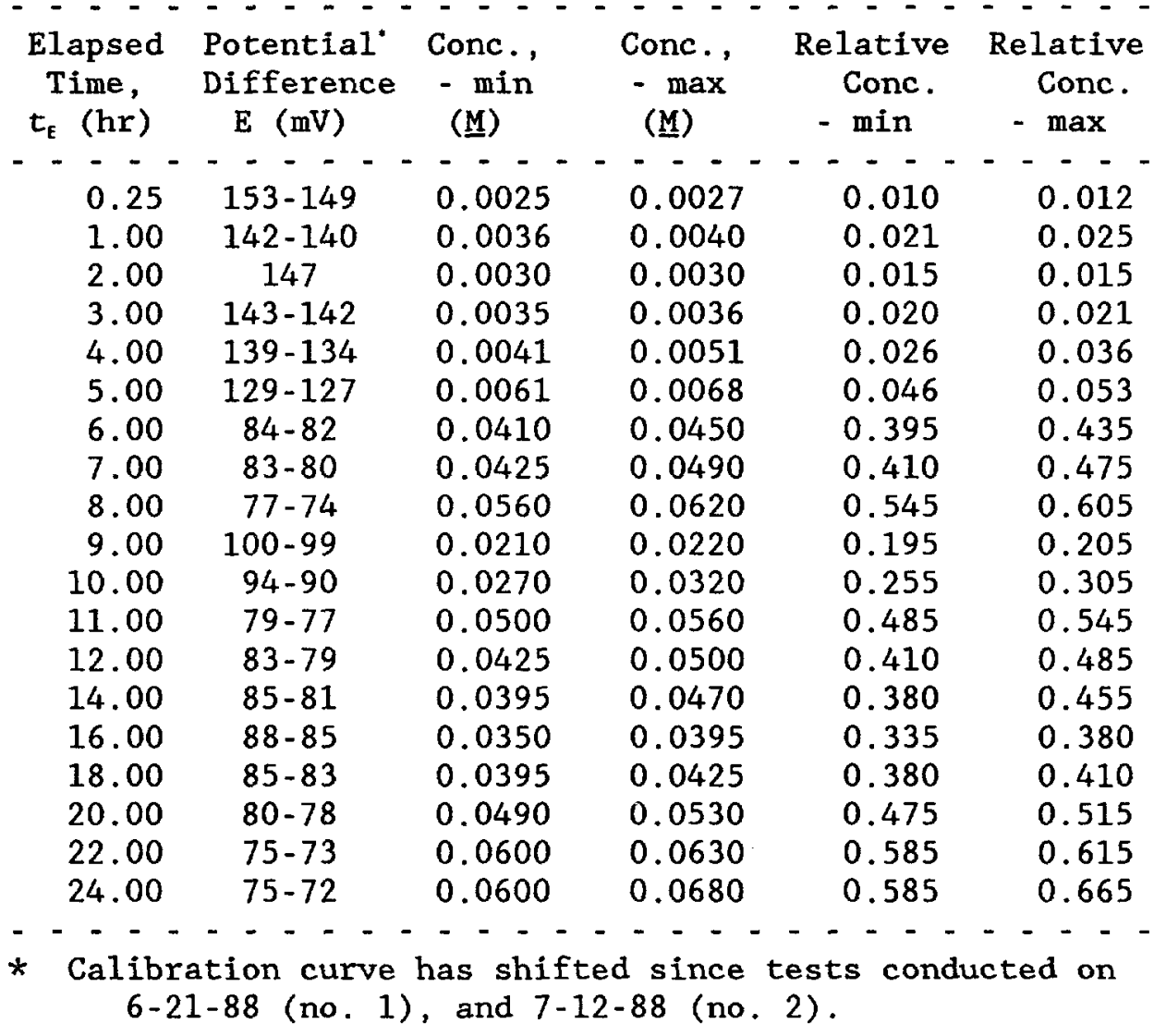


Table E.4: Fracture solute transport test data for test no. 3 .

Sampling Port: 5F-LS

Location: $x=5 \mathrm{~cm} ; z=35 \mathrm{~cm} \quad$ Face: 5

Date: $8-04-88$ to $8-05-88$

Flow Rate: $0.99 \times 10^{-9} \mathrm{~m}^{3} / \mathrm{s}$

$\mathrm{E}_{1}=163 \mathrm{mV}$

Head Imposed at Top of Plate: $29.88 \mathrm{~cm}$

Test Performed: Step $-C_{1}$ to $C_{0}$

Test Duration: 24 hrs $\mathrm{C}_{1}=0.00150 \underline{\mathrm{M}}$

$E_{o}=63 \mathrm{mV}$ $\mathrm{C}_{\mathrm{o}}=0.100 \underline{\mathrm{M}}$

Plate no.: 5

\begin{tabular}{|c|c|c|c|c|c|}
\hline $\begin{array}{l}\text { Elapsed } \\
\text { Time, } \\
t_{\mathrm{F}}(\mathrm{hr})\end{array}$ & $\begin{array}{l}\text { Potential } \\
\text { Difference } \\
\text { E (mV) }\end{array}$ & $\begin{array}{l}\text { Conc. } \\
-\min \\
(\mathrm{M})\end{array}$ & $\begin{array}{l}\text { Conc. , } \\
-\max \\
(\mathrm{M})\end{array}$ & $\begin{array}{c}\text { Relative } \\
\text { Conc. } \\
\text { - min }\end{array}$ & $\begin{array}{c}\text { Relative } \\
\text { Conc. } \\
\text { - } \max \end{array}$ \\
\hline 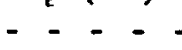 & $--\cdot$ & & & -- & $-\quad-\cdot$ \\
\hline 0.33 & $146-145$ & 0.0031 & 0.0032 & 0.016 & 0.017 \\
\hline 1.08 & $138-134$ & 0.0043 & 0.0051 & 0.028 & 0.036 \\
\hline 2.08 & $140-139$ & 0.0040 & 0.0041 & 0.025 & 0.026 \\
\hline 3.08 & $137-134$ & 0.0045 & 0.0051 & 0.030 & 0.036 \\
\hline 4.08 & $136-134$ & 0.0047 & 0.0051 & 0.032 & 0.036 \\
\hline 5.08 & $143-142$ & 0.0035 & 0.0036 & 0.020 & 0.021 \\
\hline 6.0 & $146-143$ & 0.0031 & 0.0035 & 0.016 & 0.020 \\
\hline 7.08 & $135-134$ & 0.0049 & 0.0051 & 0.034 & 0.036 \\
\hline 8.08 & $136-131$ & 0.0047 & 0.0058 & 0.032 & 0.043 \\
\hline 9.08 & $135-129$ & 0.0049 & 0.0061 & 0.034 & 0.046 \\
\hline 10.08 & $136-134$ & 0.0047 & 0.0051 & 0.032 & 0.036 \\
\hline 11.08 & $130-128$ & 0.0060 & 0.0065 & 0.045 & 0.050 \\
\hline 12.08 & $127-125$ & 0.0068 & 0.0074 & 0.053 & 0.059 \\
\hline 14.08 & $133-120$ & 0.0053 & 0.0092 & 0.038 & 0.077 \\
\hline 16.08 & $121-118$ & 0.0088 & 0.0097 & 0.073 & 0.082 \\
\hline 18.08 & $118-116$ & 0.0097 & 0.0107 & 0.082 & 0.092 \\
\hline 20.08 & $116-114$ & 0.0107 & 0.0115 & 0.092 & 0.100 \\
\hline 22.08 & $123-121$ & 0.0079 & 0.0088 & 0.064 & 0.073 \\
\hline 24.08 & $110-109$ & 0.0140 & 0.0145 & 0.125 & 0.130 \\
\hline & & 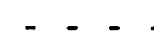 & & 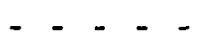 & - \\
\hline
\end{tabular}


Table E.4: Fracture solute transport test data for test no. 3 .

Sampling Port: 5F-LC

Location: $\mathrm{x}=10 \mathrm{~cm} ; \mathrm{z}=35 \mathrm{~cm} \quad$ Face: 5

Date: $8-04-88$ to 8-05-88

Flow Rate: $0.99 \times 10^{-9} \mathrm{~m}^{3} / \mathrm{s}$

Head Imposed at Top of Plate: $29.88 \mathrm{~cm}$

Test Performed: Step $-\mathrm{C}_{1}$ to $\mathrm{C}_{0}$

Test Duration: 24 hrs

$$
\begin{gathered}
E_{1}=163 \mathrm{mV} \\
C_{1}=0.00150 \mathrm{M} \\
E_{0}=63 \mathrm{mV} \\
\mathrm{C}_{0}=0.100 \mathrm{M} \\
\text { Plate no.: } 5
\end{gathered}
$$

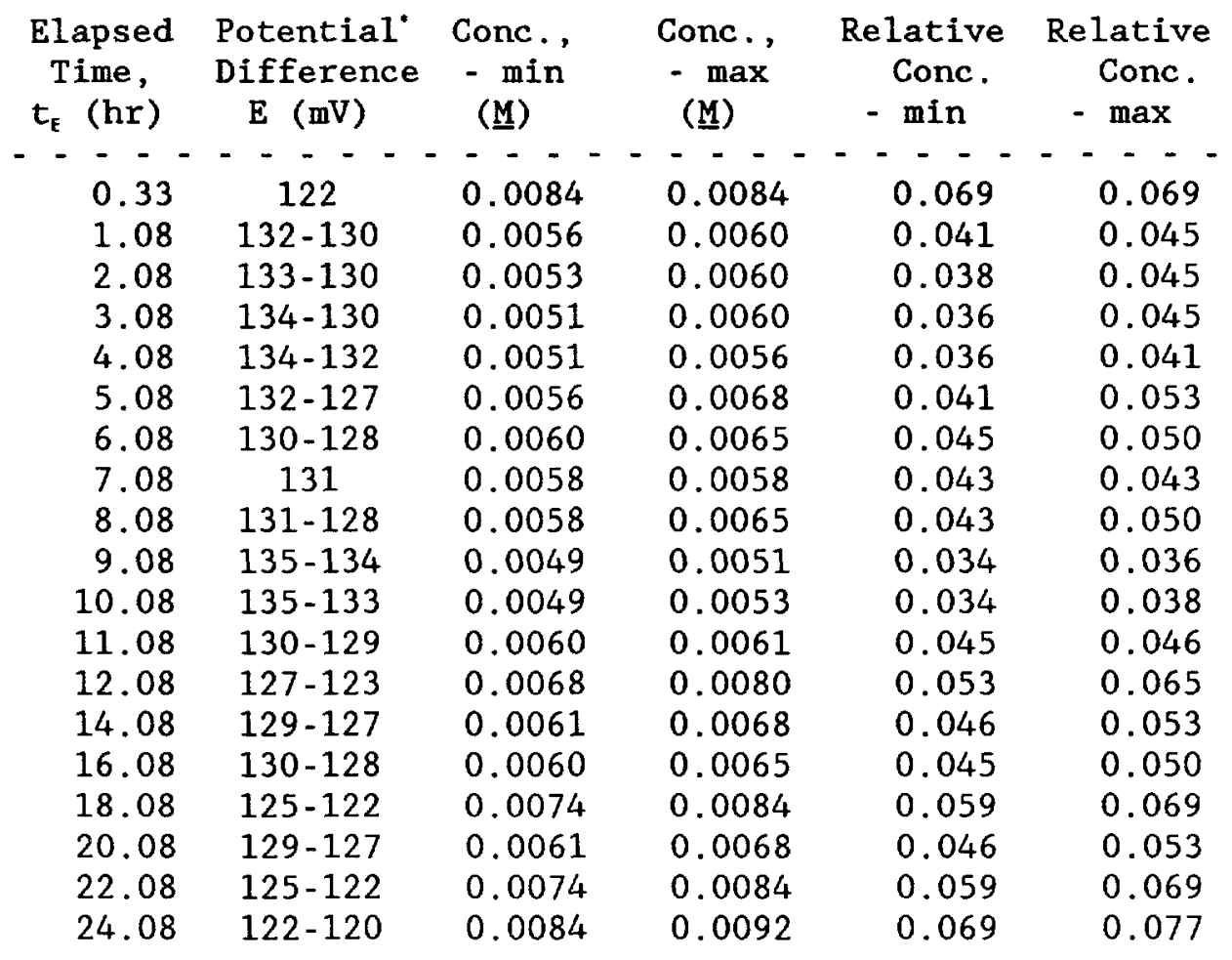

* Calibration curve has shifted since tests conducted on 6-21-88 (no. 1), and 7-12-88 (no. 2). 
Table E.4: Fracture solute transport test data for test no. 3 .

Sampling Location: 6-B

Location: $x=2 \mathrm{~cm} ; \mathrm{z}=50 \mathrm{~cm} \quad$ Face: 2

Date: $8-04-88$ to 8-05-88

Flow Rate: $0.99 \times 10^{-9} \mathrm{~m}^{3} / \mathrm{s}$

$\mathrm{E}_{1}=163 \mathrm{mV}$

Head Imposed at Top of Plate:

$C_{1}=0.00150 \underline{M}$

$E_{0}=63 \mathrm{mV}$

Test Performed: Step $-C_{1}$ to $C_{0}$

Test Duration: 24 hrs

$\mathrm{C}_{\mathrm{o}}=0.100 \underline{\mathrm{M}}$

Plate no.: 5

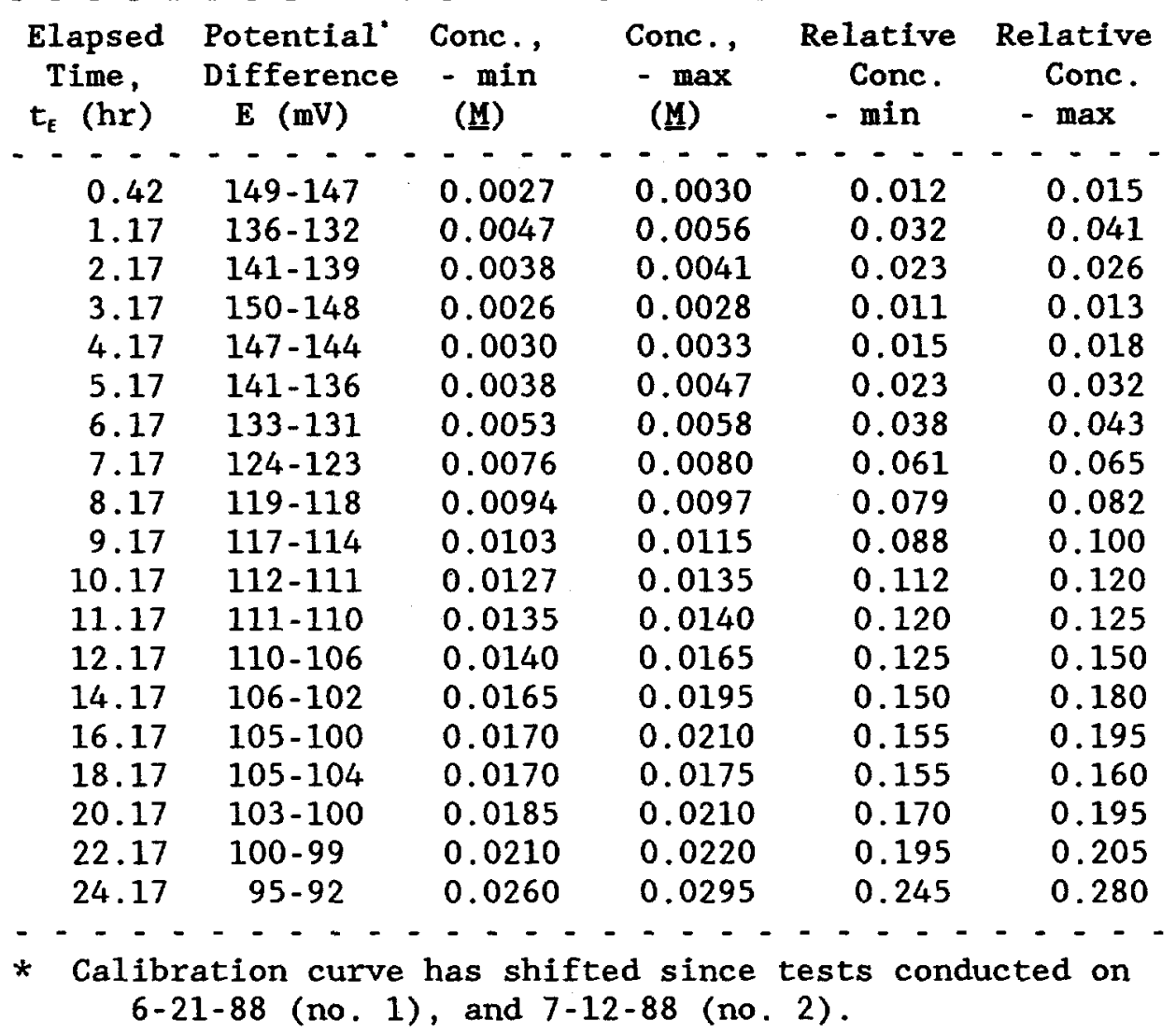


Table E.4: Fracture solute transport test data for test no. 3 .

Sampling Location: 6-M

Location: $x=8 \mathrm{~cm} ; \mathrm{z}=50 \mathrm{~cm} \quad$ Face: 2

Date: $8-04-88$ to $8-05-88$

Flow Rate: $0.99 \times 10^{-9} \mathrm{~m}^{3} / \mathrm{s}$

$E_{1}=163 \mathrm{mV}$

Head Imposed at Top of Plate:

$C_{1}=0.00150 \underline{M}$

$E_{0}=63 \mathrm{mV}$

Test Performed: Step - $C_{1}$ to $C_{0}$

Test Duration: 24 hrs

$\mathrm{C}_{\mathrm{o}}=0.100 \underline{\mathrm{M}}$

Plate no.: 5

\begin{tabular}{|c|c|c|c|c|c|}
\hline $\begin{array}{l}\text { Elapsed } \\
\text { Time, } \\
t_{\mathrm{F}}(\mathrm{hr})\end{array}$ & $\begin{array}{l}\text { Potential* } \\
\text { Difference } \\
\text { E (mV) }\end{array}$ & $\begin{array}{l}\text { Conc. , } \\
-\min \\
\text { (M) }\end{array}$ & $\begin{array}{l}\text { Conc. , } \\
-\max \\
(\mathrm{M})\end{array}$ & $\begin{array}{c}\text { Relative } \\
\text { Conc. } \\
\text { - min }\end{array}$ & $\begin{array}{c}\text { Relative } \\
\text { Conc. } \\
\text { - } \max \end{array}$ \\
\hline & & & & - & - \\
\hline 0.42 & $142-140$ & 0.0036 & 0.0040 & 0.021 & 0.025 \\
\hline 1.17 & $143-139$ & 0.0035 & 0.0041 & 0.020 & 0.026 \\
\hline 2.17 & 143 & 0.0035 & 0.0035 & 0.020 & 0.020 \\
\hline 3.17 & 144 & 0.0033 & 0.0033 & 0.018 & 0.018 \\
\hline 4.17 & $147-139$ & 0.0030 & 0.0041 & 0.015 & 0.026 \\
\hline 5.17 & $140-137$ & 0.0040 & 0.0045 & 0.025 & 0.030 \\
\hline 6.17 & $129-126$ & 0.0061 & 0.0071 & 0.046 & 0.056 \\
\hline 7.17 & $121-119$ & 0.0088 & 0.0094 & 0.073 & 0.079 \\
\hline 8.17 & $119-117$ & 0.0094 & 0.0103 & 0.079 & 0.088 \\
\hline 9.17 & $119-115$ & 0.0094 & 0.0110 & 0.079 & 0.095 \\
\hline 10.17 & $115-113$ & 0.0110 & 0.0120 & 0.095 & 0.105 \\
\hline 11.17 & $114-113$ & 0.0115 & 0.0120 & 0.100 & 0.105 \\
\hline 12.17 & $112-109$ & 0.0127 & 0.0145 & 0.112 & 0.130 \\
\hline 14.17 & $113-111$ & 0.0120 & 0.0135 & 0.105 & 0.120 \\
\hline 16.17 & $104-102$ & 0.0175 & 0.0195 & 0.160 & 0.180 \\
\hline 18.17 & $110-108$ & 0.0140 & 0.0150 & 0.125 & 0.135 \\
\hline 20.17 & $104-103$ & 0.0175 & 0.0185 & 0.160 & 0.170 \\
\hline 22.17 & $109-107$ & 0.0145 & 0.0155 & 0.130 & 0.140 \\
\hline 24.17 & $106-104$ & 0.0165 & 0.0175 & 0.150 & 0.160 \\
\hline & & & & -- & $\begin{array}{l}- \\
\text { ed on }\end{array}$ \\
\hline
\end{tabular}


Table E.4: Fracture solute transport test data for test no. 3 .

Sampling Location: $3-\mathrm{M}$

Location: $x=12 \mathrm{~cm} ; z=50 \mathrm{~cm} \quad$ Face: 2

Date: $8-04-88$ to $8-05-88$

Flow Rate: $0.99 \times 10^{-9} \mathrm{~m}^{3} / \mathrm{s}$

$E_{1}=163 \mathrm{mV}$

Head Imposed at Top of Plate: $29.88 \mathrm{~cm}$

Test Performed: Step $-\mathrm{C}_{1}$ to $\mathrm{C}_{0}$

Test Duration: 24 hrs

$\mathrm{C}_{1}=0.00150 \underline{\mathrm{M}}$

$\mathrm{E}_{\mathrm{o}}=63 \mathrm{mV}$

$\mathrm{C}_{\mathrm{o}}=0.100 \mathrm{M}$

Plate no.: 5

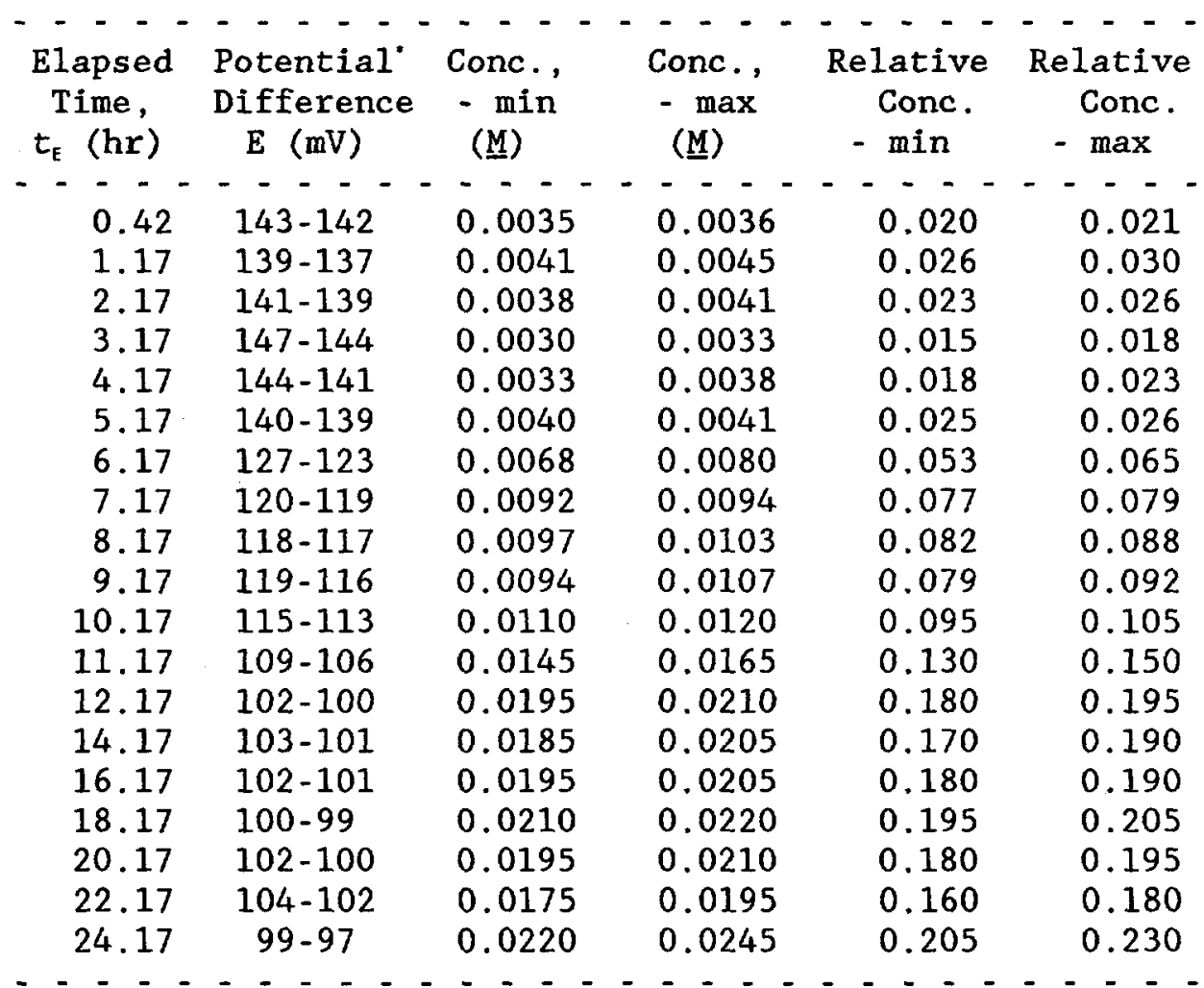

* Calibration curve has shifted since tests conducted on 6-21-88 (no. 1), and 7-12-88 (no. 2). 
Table E.4: Fracture solute transport test data for test no. 3 .

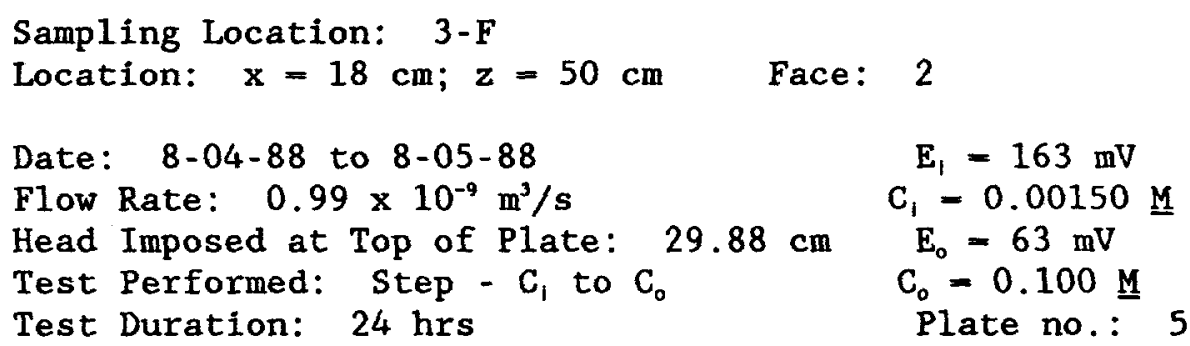

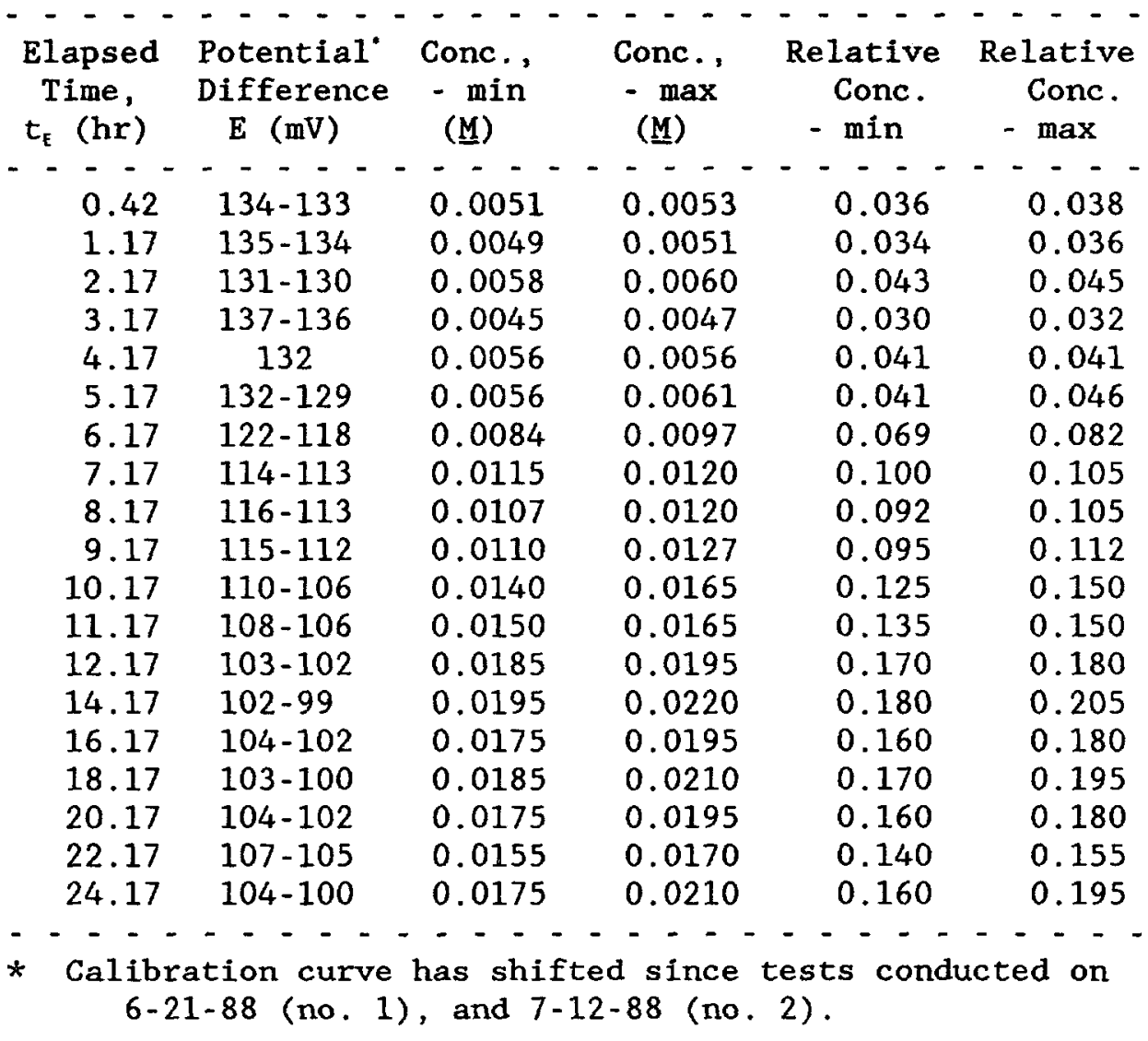


Table E.5: Spatial distribution of relative concentrations of fracture surface immediately after 24-hour step input fracture transport test (no. 3).

\begin{tabular}{|c|c|c|c|c|c|}
\hline Lateral & Longitudinal & Potential & Chloride & Relative $\mathrm{Co}$ & ncentration \\
\hline $\begin{array}{l}\text { Distance, } \\
\times(\mathrm{cm})\end{array}$ & $\begin{array}{l}\text { Distance, } \\
\mathrm{z}(\mathrm{cm})\end{array}$ & $\begin{array}{l}\text { Difference } \\
(\mathrm{mV})\end{array}$ & $\begin{array}{c}\text { Concentration } \\
(\underline{M})\end{array}$ & $\begin{array}{c}\text { At } \\
\text { Location }\end{array}$ & $\begin{array}{l}\text { Laterally } \\
\text {-Averaged }\end{array}$ \\
\hline$-\frac{-}{13}-$ & $-\cdots \frac{-}{4}-\cdots$ & $---\cdot-$ & 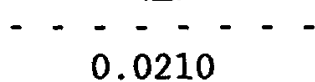 & $-\cdot-\cdot-$ & $---\cdot-$ \\
\hline 17 & 4 & 70 & 0.0730 & 0.726 & 0.462 \\
\hline 4 & 9 & 90 & 0.0320 & 0.310 & \\
\hline 7 & 9 & 70 & 0.0730 & 0.726 & \\
\hline 10 & 9 & 95 & 0.0260 & 0.249 & \\
\hline 13 & 9 & 85 & 0.0400 & 0.390 & \\
\hline 15 & 8 & 85 & 0.0400 & 0.390 & 0.413 \\
\hline 3 & 16 & 83 & 0.0420 & 0.411 & \\
\hline 7 & 16 & 90 & 0.0320 & 0.310 & \\
\hline 11 & 16 & 90 & 0.0320 & 0.310 & \\
\hline 14 & 16 & 87 & 0.0370 & 0.360 & \\
\hline 17 & 16 & 87 & 0.0370 & 0.360 & 0.350 \\
\hline 3 & 24 & 125 & 0.0074 & 0.060 & \\
\hline 6 & 24 & 80 & 0.0490 & 0.482 & \\
\hline 10 & 24 & 87 & 0.0370 & 0.360 & \\
\hline 12 & 24 & 110 & 0.0140 & 0.127 & \\
\hline 15 & 24 & 105 & 0.0170 & 0.157 & 0.237 \\
\hline 5 & 35 & 100 & 0.0210 & 0.198 & \\
\hline 8 & 35 & 120 & 0.0092 & 0.078 & \\
\hline 10 & 35 & 125 & 0.0074 & 0.060 & \\
\hline 14 & 35 & 123 & 0.0080 & 0.066 & \\
\hline 18 & 35 & 115 & 0.0113 & 0.099 & 0.100 \\
\hline 2 & $42^{(1)}$ & 95 & 0.0260 & 0.249 & \\
\hline 5 & 42 & 95 & 0.0260 & 0.249 & \\
\hline 8 & 42 & 90 & 0.0320 & 0.310 & \\
\hline 10 & 42 & 90 & 0.0320 & 0.310 & \\
\hline 13 & 42 & 100 & 0.0210 & 0.19 .8 & \\
\hline 15 & 42 & 100 & 0.0210 & 0.198 & 0.252 \\
\hline 3 & 48 & 95 & 0.0260 & 0.249 & \\
\hline 6 & 48 & 115 & 0.0113 & 0.099 & \\
\hline 10 & 48 & 97 & 0.0240 & 0.228 & \\
\hline 13 & 48 & 87 & 0.0370 & 0.360 & \\
\hline 18 & 48 & 113 & 0.0120 & 0.107 & 0.209 \\
\hline
\end{tabular}

(1) Longitudinal distance of $z=42 \mathrm{~cm}$ actually ranged between $\mathrm{z}=39$ $\mathrm{cm}$ at $\mathrm{x}=2 \mathrm{~cm}$ to $z=44 \mathrm{~cm}$ at $\mathrm{x}=15 \mathrm{~cm}$. 


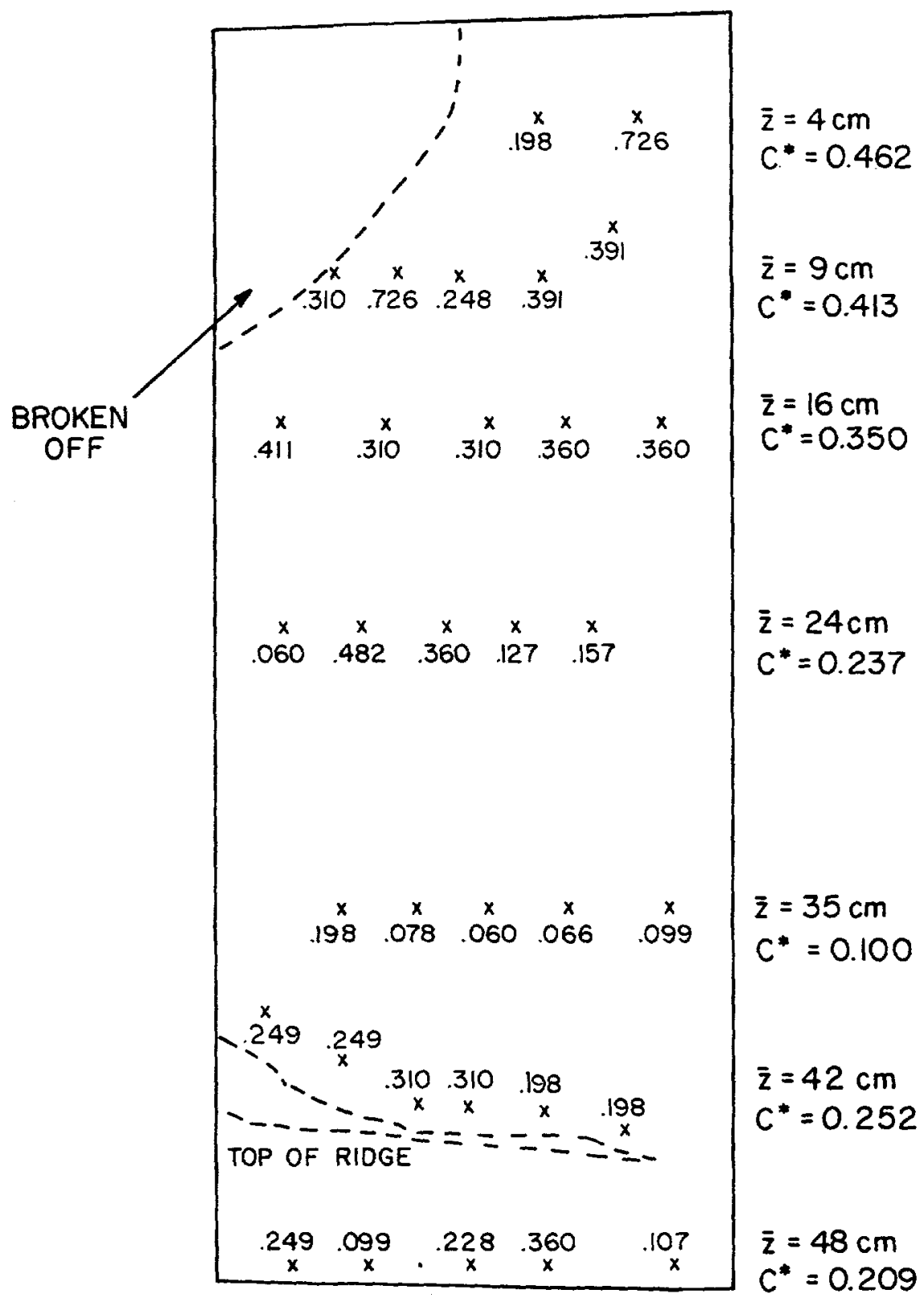

Figure E.1: Location and concentration of samples taken of solution remaining on fracture surface immediately after fracture test 3 . 
Table E.6: Matrix solute transport test data.

Sampling port: $4 \mathrm{M}-\mathrm{U}$

Location: $x=5 \mathrm{~cm} ; z=5 \mathrm{~cm} \quad$ Face: 4

Date: 6-14-88 to 8-05-88

Flow Rate: $0.60 \times 10^{-9} \mathrm{~m}^{3} / \mathrm{s}$

Head Imposed at Top of Plate: $5.0 \mathrm{~m}$

$E_{1}=172 \mathrm{mV}$
$C_{1}=0.00130 \underline{M}$
$E_{o}=125 \mathrm{mV}$
$C_{o}=0.0125 \underline{M}$
Plate no.: 4

Test Duration: $1222.25 \mathrm{hrs} \quad$ Plate no.: 4

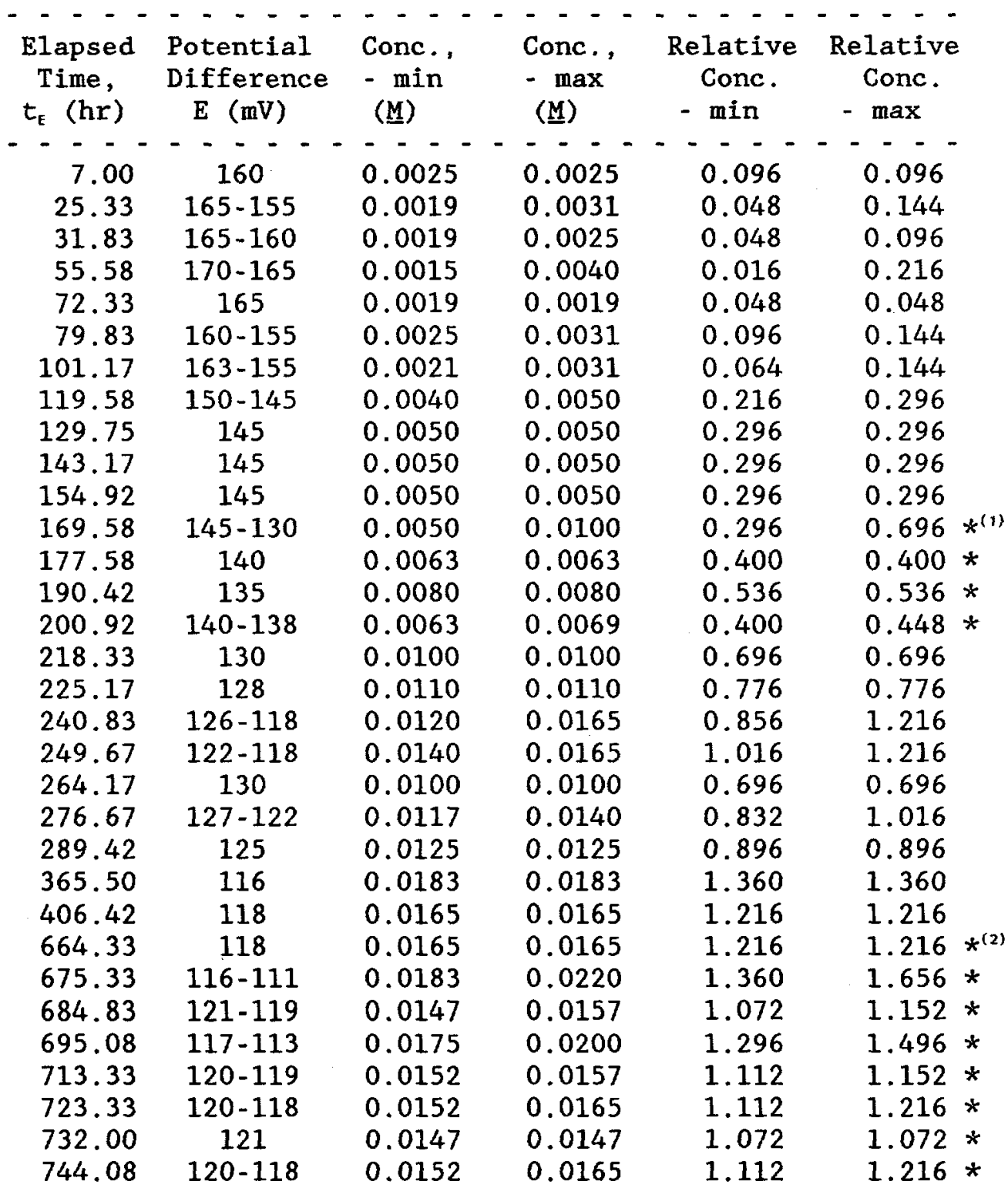

(1) Period corresponding to fracture transport test no. 1 .

(2) Period corresponding to fracture transport test no. 2. 
Table E.6: Matrix solute transport test data.

Sampling Port: 4M-U (continued)

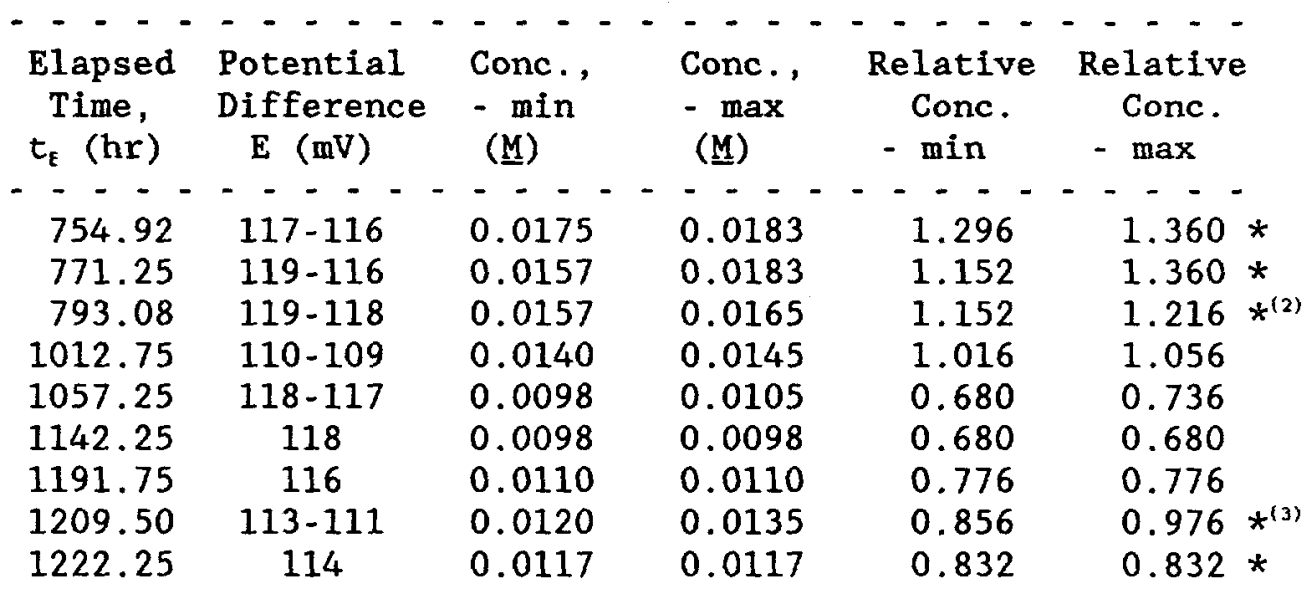

(2) Period corresponding to fracture transport test no. 2 .

(3) Period corresponding to fracture transport test no. 3. 
Table E.6: Matrix solute transport test data.

Sampling Port: 4M-L

Location: $\mathrm{x}=5 \mathrm{~cm} ; \mathrm{z}=30 \mathrm{~cm} \quad$ Face: 4

Date: $6-14-88$ to $8-05-88$

Flow Rate: $0.60 \times 10^{-9} \mathrm{~m}^{3} / \mathrm{s}$

Head Imposed at Top of Plate: $5.0 \mathrm{~cm}$

$E_{1}=172 \mathrm{mV}$
$C_{1}=0.00130 \mathrm{M}$
$E_{0}=125 \mathrm{mV}$
$C_{0}=0.0125 \mathrm{M}$
Plate no.: 4

Test Performed: Step $\mathrm{C}_{1}$ to $\mathrm{C}_{0}$

Test Duration: 1222.25 hrs

\begin{tabular}{|c|c|c|c|c|c|c|}
\hline $\begin{array}{l}\text { Elapsed } \\
\text { Time, } \\
t_{E}(h r)\end{array}$ & $\begin{array}{c}\text { Potential } \\
\text { Difference } \\
E(\mathrm{mV})\end{array}$ & $\begin{array}{l}\text { Conc., } \\
- \text { min } \\
(\underline{M})\end{array}$ & $\begin{array}{l}\text { Conc. } \\
-\max \\
(\underline{M})\end{array}$ & $\begin{array}{c}\text { Relative } \\
\text { Conc. } \\
\text { - min }\end{array}$ & $\begin{array}{c}\text { Relative } \\
\text { Conc. } \\
\text { - } \max \end{array}$ & \\
\hline$\overline{406.50}$ & 140 & $\overline{0.0063}$ & $\overline{0.0063}$ & 0.400 & 0.400 & \\
\hline 664.33 & $129-122$ & 0.0105 & 0.0143 & 0.736 & 1.040 & $*^{(2)}$ \\
\hline 675.33 & $132-120$ & 0.0092 & 0.0152 & 0.632 & 1.112 & * \\
\hline 684.83 & $133-127$ & 0.0088 & 0.0117 & 0.600 & 0.832 & * \\
\hline 695.08 & $135-126$ & 0.0080 & 0.0120 & 0.536 & 0.856 & * \\
\hline 713.33 & $139-134$ & 0.0065 & 0.0084 & 0.416 & 0.568 & * \\
\hline 723.33 & $137-129$ & 0.0073 & 0.0105 & 0.480 & 0.736 & * \\
\hline 732.00 & $135-130$ & 0.0080 & 0.0107 & 0.536 & 0.752 & * \\
\hline 744.08 & $130-127$ & 0.0107 & 0.0117 & 0.752 & 0.832 & * \\
\hline 754.92 & $130-129$ & 0.0107 & 0.0105 & 0.752 & 0.736 & * \\
\hline 771.25 & $133-129$ & 0.0088 & 0.0105 & 0.600 & 0.736 & * \\
\hline 793.08 & $136-134$ & 0.0077 & 0.0084 & 0.512 & 0.568 & * \\
\hline 1012.75 & $110-109$ & 0.0140 & 0.0145 & 1.016 & 1.056 & \\
\hline 1057.25 & $118-116$ & 0.0098 & 0.0110 & 0.680 & 0.776 & \\
\hline 1142.25 & $118-116$ & 0.0098 & 0.0110 & 0.680 & 0.776 & \\
\hline 1191.75 & $118-117$ & 0.0098 & 0.0103 & 0.680 & 0.720 & \\
\hline 1209.50 & $114-113$ & 0.0117 & 0.0120 & 0.832 & 0.856 & $*^{(3)}$ \\
\hline
\end{tabular}

(2) Period corresponding to fracture transport test no. 2.

(3) Period corresponding to fracture transport test no. 3. 
Table E.7: Assumed input function for porous plate no. 4 - temporal moments analysis.

\begin{tabular}{|c|c|c|c|}
\hline Time, & Relative & $t_{E} \times$ & $\left(t_{E}-t m\right)^{2}$ \\
\hline$-2-$ & $-\ldots$ & _. - . & - - - - \\
\hline 7.00 & 0.068 & 0.48 & 14927.9 \\
\hline 25.33 & 0.827 & 20.95 & 167622.1 \\
\hline 31.83 & 1.000 & 31.83 & 196876.5 \\
\hline 55.58 & 1.000 & 55.58 & 176364.4 \\
\hline 72.33 & 1.000 & 72.33 & 162576.4 \\
\hline 79.83 & 1.000 & 79.83 & 156584.5 \\
\hline 101.17 & 1.000 & 101.17 & 140151.1 \\
\hline 119.58 & 1.000 & 119.58 & 126705.8 \\
\hline 129.75 & 1.000 & 129.75 & 119569.1 \\
\hline 143.17 & 1.000 & 143.17 & 110468.2 \\
\hline 154.92 & 1.000 & 154.92 & 102795.7 \\
\hline 169.58 & 1.000 & 169.58 & 93610.1 \\
\hline 177.58 & 1.000 & 177.58 & 88778.8 \\
\hline 190.42 & 1.000 & 190.42 & 81292.1 \\
\hline 200.92 & 1.000 & 200.92 & 75414.8 \\
\hline 218.33 & 1.000 & 218.33 & 66155.8 \\
\hline 225.17 & 1.000 & 225.17 & 62684.0 \\
\hline 240.83 & 1.000 & 240.83 & 55087.7 \\
\hline 249.67 & 1.000 & 249.67 & 51016.2 \\
\hline 264.17 & 1.000 & 264.17 & 44676.3 \\
\hline 276.67 & 1.000 & 276.67 & 39548.3 \\
\hline 289.42 & 1.000 & 289.42 & 34639.8 \\
\hline 365.50 & 1.000 & 365.50 & 12108.3 \\
\hline 406.42 & 1.000 & 406.42 & 4777.2 \\
\hline 664.33 & 1.000 & 664.33 & 35642.6 \\
\hline 675.33 & 1.000 & 675.33 & 39917.0 \\
\hline 684.83 & 1.000 & 684.83 & 43803.3 \\
\hline 695.08 & 1.000 & 695.08 & 48198.9 \\
\hline 713.33 & 1.000 & 713.33 & 56545.2 \\
\hline 723.33 & 1.000 & 723.33 & 61401.1 \\
\hline 732.00 & 1.000 & 732.00 & 65772.9 \\
\hline 744.08 & 1.000 & 744.08 & 72115.0 \\
\hline 754.92 & 1.000 & 754.92 & 78054.5 \\
\hline 771.25 & 1.000 & 771.25 & 87445.8 \\
\hline 793.08 & 1.000 & 793.08 & 100833.2 \\
\hline 1012.75 & 1.000 & 1012.75 & 288597.1 \\
\hline 1057.25 & 1.000 & 1057.25 & 338389.3 \\
\hline 1142.25 & 1.000 & 1142.25 & 444505.4 \\
\hline 1191.75 & 1.000 & 1191.75 & 512960.2 \\
\hline 1209.50 & 1.000 & 1209.50 & 538700.8 \\
\hline 1222.25 & 1.000 & 1222.25 & 557579.4 \\
\hline & --- & --- & --- \\
\hline Sum $=$ & 39.895 & 18971.57 & 5554892.4 \\
\hline
\end{tabular}




\section{SELECTED REFERENCES}

Ammann, D., 1986, Ion-Selective Electrodes, Principles, Design and Application, Springer-Verlag Berlin Heidelberg, 346 pp.

Bear, J., 1979, Hydraulics of Groundwater, McGraw-Hi11, 569 pp.

Biggar, J.W. and D.R. Nielsen, 1962, "Miscible Displacement in Soils: II. Behavior of Tracers," Soil Sci. Soc. Amer. Proc., 26:125-128.

Cattrall, R.W. and H. Freiser, 1971, "Coated Wire Ion Selective Electrodes," Analytical Chemistry, 43(13):1905-1906.

Cattra11, R.W. and I.C. Hamilton, 1984, "Coated-Wire Ion-Selective Electrodes," Ion-Selective Electrode Rev., 6:125-172.

Chuang, Y., 1988, Solute Transport Measurement by Ion-Selective Electrodes in Fracture Tuff, M.S. Thesis, University of Arizona, Tucson, Arizona.

Covington, A.K. (ed.), 1979, Ion-Selective Electrode Methodology, Vo1. I, CRC Press, Inc., 257 pp.

Dagan, G., 1984, "Solute Transport in Heterogeneous Porous Formations," J. Fluid Mech., 145:151-177.

Davis, S.N., and R.J.M. De Wiest, 1966, Hydrogeology, John Wiley and Sons, $463 \mathrm{pp}$.

de Marsily, G., 1986, Quantitative Hydrogeology, Groundwater Hydrology for Engineers, Academic Press, Inc., $440 \mathrm{pp}$.

Engelder, T., and C.H. Scholz, 1981, Fluid Flow Along Very Smooth Joints at Effective Pressure up to 200 Megapascals, in Mechanical Behavior of Crustal Rocks, Geophysical Monograph Series, AGU, $24: 147-152$.

Evans, D.D., 1988, Unsaturated Flow and Transport Through Fractured Rock Related to High-level Waste Repositories, NRC 04-86-114 Progress Report.

Fischer, H.B., E.J. List, R.C.Y. Koh, J. Imberger and N.H. Brooks, 1979, Mixing in Inland and Coastal Waters, Academic Press, $483 \mathrm{pp}$.

Freeze, R.A. and J.A. Cherry, 1979, Groundwater, Prentice-Hall, Inc., $604 \mathrm{pp}$.

Freiser, H. (ed.), 1978, Ion-Selective Electrodes in Analytical Chemistry, Vols. I and II, Plenum Press, 439 pp. and 291 pp.

Freyberg, D.L., 1986, "A Natural Gradient Experiment of Solute Transport in a Sand Aquifer 2. Spatial Moments and the Advection and Dispersion of Nonreactive Tracers," Water Resour. Res., $22(13): 2031-2046$.

Gale, J.E., 1982, The Effects of Fracture Type (Induced Versus Natural) on the Stress-Fracture Closure-Fracture Permeability Relationships, in Proceedings at 23rd Symposium on Rock Mechanics, Berkeley, CA, 290-298.

Gale, J.E., A. Rouleau, and L.C. Atkinson, 1985, Hydraulic Properties of Fractures, in International Association of Hydrogeologists Memoires of Congress on Hydrology of Rocks of Low Permeability, Tucson, $\mathrm{AZ}, 17(1): 1-16$.

Gelhar, L.W. and C.L. Axness, 1983, "Three-Dimensional Stochastic Analysis of Macrodispersion in Aquifers," Water Resour. Res., $19(1): 161-180$.

Gelhar, L.W., A.L. Gutjahr and R.L. Naff, 1979, "Stochastic Analysis of Macrodispersion in a Stratified Aquifer," Water Resour. Res., 15(6): 1387-1397. 
Glover, R.E., 1953, Flow from a Test-hole Located Above Groundwater Level, in Theory and Problems of Water Percolation, U.S. Bureau of Reclamation Engineering Monograph No. 8, 66-71.

Grisak, G.E. and J.F. Pickens, 1980, "Solute Transport Through Fractured Media 1. The Effect of Matrix Diffusion," Water Resour. Res., $16(4): 719-730$.

Grisak, G.E., J.F. Pickens and J.A. Cherry, 1980, "Solute Transport Through Fractured Media 2. Column Study of Fractured Till, "Water Resour. Res., 16(4):731-739.

Haldeman, W.R., 1988, "Water Flow Through Variably Saturated Fractured Tuff: A Laboratory Study," M.S. Thesis, University of Arizona, 219 pp.

Hillel, D., 1971, Soil and Water, Physical Principles and Processes, Academic Press, Inc., 288 pp.

Hillel, D., 1980, Fundamentals of Soil Physics, Academic Press, Inc., $413 \mathrm{pp}$.

Iwai, K., 1976, Fundamental Studies of Fluid Flow Through a Single Fracture, Ph.D. Dissertation, University of California, Berkeley, California.

James, H., G. Carmack and H. Freiser, 1972, "Coated Wire Ion Selective Electrodes," Analytical Chemistry, 44(4):586-587.

James, R.V. and J. Rubin, 1972, "Accounting for Apparatus-Induced Dispersion in Analysis of Miscible Displacement Experiments," Water Resour. Res., 8(3):717-721.

Kemper, W.D., 1960, "Water and Ion Movement in Thin Films as Influenced by the Electrostatic Charge and Diffuse Layer of Cations Associated with Clay Mineral Surfaces," Soil Sci. Soc. Amer.

Kilbury, R.K., 1984, Water Intake at the Atmosphere-Earth Interface in a Fractured Rock System Near Patagonia, Arizona, M.S. Thesis, University of Arizona, Tucson, Arizona.

Kilbury, R.K., T.C. Rasmussen, D.D. Evans, and A.W. Warrick, 1986, Water and Air Intake of Surface-Exposed Rock Fractures in Situ, Water Resources Research, 22(10):1431-1443.

Klute, A., and C. Kirksen, 1986, Hydraulic Conductivity and Diffusivity: Laboratory Methods, in Methods of Soil Analysis, Part 1, Physical and Mineralogical Methods, American Society of Agronomy, Soil Science Society of America, Number 9, pp. 687-734.

Lomize, G.M., 1951, Flow in Fracture Rocks (in Russian), Gosenergoizdat, Moscow, U.S.S.R., $127 \mathrm{pp}$.

Louis, C., 1969, A Study of Groundwater Flow in Jointed Rock and Its Influence on the Stability of Rock Masses, Rock Mechanics Research Report 10, Imperial College, London, England, $90 \mathrm{pp}$.

Mercado, A., 1967, "The Spreading Pattern of Injected Water in a Permeability-Stratified Aquifer," Proc. Int'1 Assoc. Sci. Hydrol. Symp., Haifa Pub. 72 , p. 23.

Minnich, M.M. and M.B. McBride, 1987, "Copper Activity in Soil Solution: I. Measurement by Ion-selective Electrode and Donnan Dialysis," Soil Sci. Soc. Am. J., 51:568-572.

Moreno, L., I. Neretnieks and T. Eriksen, 1985, "Analysis of Some Laboratory Tracer Runs in Natural Fissures," Water Resour. Res., 21(7):951-958.

Neretnieks, I., T. Eriksen and P. Tahtinen, 1982, "Tracer Movement in a Single Fissure in Granitic Rock: Some Experimental Results and Their Interpretation," Water Resour. Res., 18(4):849-858. 
Neuman, S.P., 1987, "Stochastic Continuum Representation of Fractured Rock Permeability as an Alternative to the REV and Fracture Network Concepts," 28th U.S. Symposium on Rock Mechanics, Tucson, Arizona, 29 June $-1 \mathrm{July}$, pp. 553-561.

Nielsen, D.R. and J.W. Biggar, 1961, "Miscible Displacement in Soils: I. Experimental Information," Soil Sci. Sco. Amer. Proc., 25:1-5.

Nielsen, D.R. and J.W. Biggar, 1962, "Miscible Displacement in Soils: III. Theoretical Considerations," Soil Sci. Sco. Amer. Proc., $26: 216-221$.

Peterson, D.W., 1961, Dacitic Ash-Flow Sheet Near Superior and Globe, Arizona, Ph.D. Dissertation, Stanford University, Stanford, California.

Peterson, D.W., 1968, Zoned Ash-Flow Sheet in the Region Around Superior, Arizona, in Southern Arizona Guidebook III, Geological Society of America, pp. 215-222.

Philip, J.R., 1969, Theory of Infiltration, Advances in Hydroscience, $5: 216-296$.

Philip, J.R., 1985, Approximate Analysis of the Borehole Permeameter in Unsaturated Soil, Water Resources Research, 21(7):1025-1033.

Philip, J.R., 1988, "Water Penetration From Downward Seepage into Macropores, Cavities, and Tunnels, "International Conference and Workshop on the Validation of Flow and Transport Models for the Unsaturated Zone, Ruidoso, New Mexico, May 22-25, 1988, pp. $306-$ 319 .

Rahi, K.A., 1986, Hydraulic Conductivity Assessment for a VariablySaturated Rock Matrix, M.S. Thesis, University of Arizona, Tucson, Arizona.

Rasmussen, T.C., and D.D. Evans, 1987, Unsaturated Flow and Transport Through Fractured Rock Related to High-Level Waste Repositories, Nuclear Regulatory Commission, Washington, D.C., NUREG/CR-4655.

Rasmussen, T.C., 1988, "Fluid Flow and Solute Transport Through ThreeDimensional Networks of Variably Saturated Discrete Fractures," Ph.D. Dissertation, University of Arizona, 327 pp.

Rasmussen, T.C., and D.D. Evans, 1989, Fluid Flow and Solute Transport Modeling Through Three-Dimensional Networks of Variably Saturated Discrete Fractures, Nuclear Regulatory Commission, Washington, D.C., NUREG/CR-5239.

Reda, D.C., and G.R. Hadley, 1986, Saturated Permeability Measurements On Pumice and Welded-Tuffaceous Materials, Environ. Geol. Water Sci., 8(3):137-145.

Reginato, R.J., and C.H.M. Van Bave1, 1964, Soil Water Measurement with Gamma Attenuation, Soil Sci. Soc. Proc., 28:721-724.

Schrauf, T.W., 1984, Relationship Between the Gas Conductivity and Geometry of a Natural Fracture, M.S. Thesis, University of Arizona, Tucson, Arizona.

Schrauf, T.W., and D.D. Evans, 1986, Laboratory Studies of Gas Flow Through a Single Natural Fracture, Water Resources Research, $22(7): 1038-1050$.

Sharp, J.C., 1970, Fluid Flow Through Fissure Media, Ph.D. Dissertation, University of London, Imperial College of Science and Technology, London, England.

Smith, L., C.W. Mase, and R.W. Schwartz, 1987, Estimation of Fracture Aperture Using Hydraulic and Tracer Tests, in 28th U.S. Symposium on Rock Mechanics, Tucson, AZ, 453-463. 
Smith, S., 1987, Geologic Isolation of High-Level Radioactive Waste:

Putting It Away Forever, Water Well Journal, 41(4):31-39.

Stumm, W. and J.J. Morgan, 1981, Aquatic Chemistry, 2nd Ed., John Wiley and Sons, $780 \mathrm{pp}$.

Trautz, R.C., 1984, Rock Fracture Aperture and Gas Conductivity Measurements In Situ, M.S. Thesis, University of Arizona, Tucson, Arizona.

Tsang, Y.W., 1984, The Effect of Tortuosity on Fluid Flow Through a Single Fracture, Water Resources Research, 20(9):1209-1215.

Tsang, Y.W. and C.F. Tsang, 1987, "Channel Model of Flow Through Fractured Media," Water Resour. Res., 23(3):467-479.

Tsang, Y.W. and P.A. Witherspoon, 1985, "Effects of Fracture Roughness on Fluid Flow Through a Single Deformable Fracture," Hydrogeology of Rocks of Low Permeability, Vo1. 2: Proceedings of 17 th International Congress of International Association of Hydrogeologists, Tucson, Arizona, January 7 - 12, pp. 683-694.

Walter, G.R., 1985, "The Effects of Molecular Diffusion on Groundwater Solute Transport Through Fractured Tuff," Ph.D. Dissertation, University of Arizona, $187 \mathrm{pp}$.

Wang. J.S.Y. and T.N. Narasimhan, 1985, "Hydrologic Mechanisms Governing Fluid Flow in a Partially Saturated, Fractured, Porous Medium," Water Resour. Res., 21(12):1861-1874.

Weber, D.S., 1987, Stable Isotopes of Authigenic Minerals in VariablySaturated Fractured Tuff, M.S. Thesis, University of Arizona, Tucson, Arizona.

Wilson, M.L. and A.L. Dudley, 1987, "Radionuclide Transport in an Unsaturated Fractured Medium," Flow and Transport through Unsaturated Fractured Rock, Geophysical Monograph 42:23-30. 
Laboratory Analysis of Fluid Flow and Solute Transport Through a Variably Saturated Fracture Emibedded in Porous Tuff

5. AUTHOR(S)

Y. Chuang, W. R. Haldeman, T. C. Rasmussen, D. D. Evans

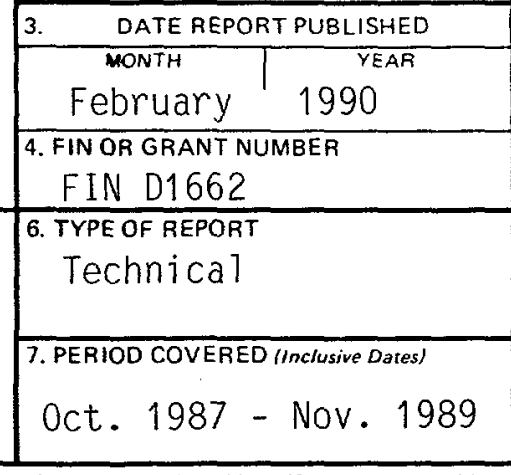

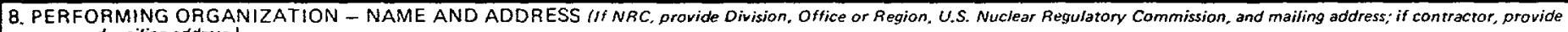
name and mailing address.)

Department of Hydrology and Water Resources

University of Arizona

Tucson, Arizona 85721

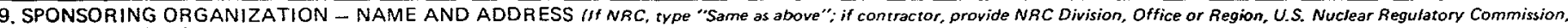
and mailing address.'

Division of Engineering

Office of Nuclear Regulatory Research

U.S. Nuclear Regulatory Commission

Washington, DC 20555

10. SUPPLEMENTARY NOTES

11. ABSTRACT 200 words or less)

Laboratory techniques are developed that allow concurrent measurement of unsaturated matrix hydraulic conductivity and fracture transmissivity of fractured rock blocks. Two Apache Leap tuff blocks with natural fractures were removed from near Superior, Arizona, shaped into rectangular prisms, and instrumented in the laboratory. Porous ceramic plates provided solution to block tops at regulated pressures. Infiltration tests were performed on both test blocks. Steady flow testing of the saturated first block provided estimates of matrix hydraulic conductivity and fracture transmissivity. Fifteen centimeters of suction applied to the second block top showed that fracture flow was minimal and matrix hydraulic conductivity was an order of magnitude less than the first block saturated matrix conductivity. Coated-wire ion-selective electrodes monitored aqueous chlorided breakthrough concentrations. Minute samples of tracer solution were collected with filter paper. The techniques worked well for studying transport behavior at nearsaturated flow conditions and also apper to be promising for unsaturated conditions. Breakthrough curves in the fracture and matrix, and a concentration map of chloride. concentrations within the fracture, suggest preferential flows paths in the fracture and substantial diffusion into the matrix. Average travel velocity, disperion coefficient and longitudinal dispersivity in the fracture are obtained.

12. KEY WORDS/DESCR!PTORS (List words or phrases that will assist researchers in locating the report.)

average travel velocity boundary integral method breakthrough curves dispersion coefficient fracture transmissivity infiltration tests ion-selective electrodes hydraulic conductivity matrix hydraulic conductivity preferential flow paths steady-state flow tests temporal moments analysis unsaturated fracture flow solute transport

\begin{tabular}{|l|} 
13. AVAILABILITY STATEMENT \\
Un 1 imited \\
\hline 14. SFrIHAITY CLASSIFICATION \\
ithis Page' \\
Unclassified \\
\hline ithis Report \\
Unclassified \\
\hline 15. NUMBER OF PAGES \\
\hline 16. PRICE
\end{tabular}

